Ground-Water Levels

in the United States

$1956-60$

Southwestern States

Prepared under the direction of O. M. HACKETT, Chief, Ground Water Branch

Geological Survey Water-Supply Paper 1770

Prepared in cooperation with the States of Arizona, California, Hawaii, Nevada, and New Mexico, and with other agencies

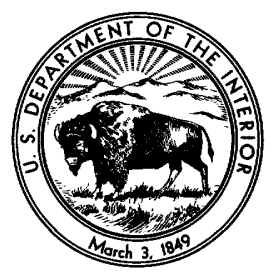

United States Government Printing Office, Washington: 1963 


\section{UNITED STATES DEPARTMENT OF THE INTERIOR}

Stewart L. Udall, Secretary

GEOLOGICAL SURVEY

Thomas B. Nolan, Director

For sale by the Superintendent of Documents, U.S. Government Printing Office Washington, D.C., 20402 - Price 60 cents 


\section{PREFACE}

This report was prepared by the Geological Survey in cooperation with the States of Arizona, California, Hawaii, Nevada, and New Mexico, and with other agencies, by personnel of the Water Resources Division under the direction of:

Luna B. Leopold . . . . . . . . . . . . . . . . Chief Hydraulic Engineer

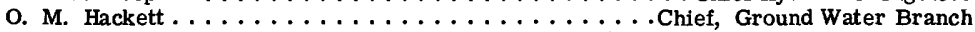
P. E. Dennis. . . . . . . . . . District Geologist (Ground Water), Tucson, Arizona Fred Kunkel. . . . . . . . District Geologist (Ground Water), Sacramento, California

D. A. Davis. . . . . . . . . . District Geologist (Ground Water), Honolulu, Hawaii

o. J. Loeltz . . . . . . . . District Engineer (Ground Water), Carson City, Nevada

s. W. West . . . . . . . District Geologist (Ground Water), Albuquerque, New Mexico 



\section{CONTENTS}

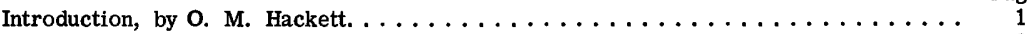

Arizona, by M. B. Booher and E. K. Morse. . . . . . . . . . . . .

California, by E. J. McClelland, W. R. Moyle, Jr., and R. E. Evenson . . . . . 26

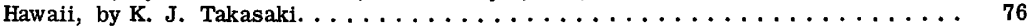

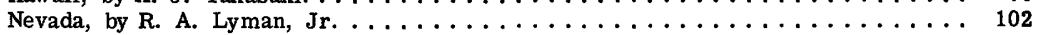

New Mexico, by H. O. Reeder and W. C. Ballance . . . . . . . . . . . 124

\section{ILLUSTRATIONS}

Figure 1. Outline map of the United States, showing States included in this volume. . 2

2. Location of observation wells in Arizona, $1956-60 . \ldots \ldots \ldots \ldots \ldots \ldots$

3. Location of observation wells in California, $1956-60 \ldots \ldots \ldots \ldots \ldots \ldots \ldots$

4. Location of observation wells in Hawaii, $1956-60 \ldots \ldots \ldots \ldots \ldots \ldots$

5. Location of observation wells in Nevada, $1956-60 \ldots \ldots \ldots \ldots \ldots \ldots \ldots \ldots$

6. Location of observation wells in New Mexico, 1956-60. . . . . . . . . 126 


\section{GROUND-WATER LEVELS IN \\ THE UNITED STATES}

1956-60

\section{SOUTHWESTERN STATES}

\section{INTRODUCTION}

By O. M. Hackett

Publication of records of water levels in the United States in the annual series of watersupply papers was begun by the Geological Survey in 1935 . From 1935 through 1939 , 5 volumes were issued as Water-Supply Papers 777, 817, 840, 845, and 886. From 1940 through 1955, 6 volumes, each for a separate section of the United States as shown in figure 1, were published annually. The following table gives the numbers of water-supply papers by year and section.

\begin{tabular}{|c|c|c|c|c|c|c|}
\hline Year & $\begin{array}{l}\text { North- } \\
\text { eastern } \\
\text { (1) }\end{array}$ & $\begin{array}{l}\text { South- } \\
\text { eastern } \\
\text { (2) }\end{array}$ & $\begin{array}{l}\text { North- } \\
\text { central } \\
\text { (3) }\end{array}$ & $\begin{array}{l}\text { South- } \\
\text { central } \\
\text { (4) }\end{array}$ & $\begin{array}{l}\text { North- } \\
\text { western } \\
\text { (5) }\end{array}$ & $\begin{array}{l}\text { South- } \\
\text { western } \\
\text { (6) }\end{array}$ \\
\hline 1940 & 906 & 907 & 908 & 909 & 910 & 911 \\
\hline 1941 & 936 & 937 & 938 & 939 & 940 & 941 \\
\hline 1942 & 944 & 945 & 946 & 947 & 948 & 949 \\
\hline 1943 & 986 & 987 & 988 & 989 & 990 & 991 \\
\hline 1944 & 1016 & 1017 & 1018 & 1019 & 1020 & 1021 \\
\hline 1945 & 1023 & 1024 & 1025 & 1026 & 1027 & 1028 \\
\hline 1946 & 1071 & 1072 & 1073 & 1074 & 1075 & 1076 \\
\hline 1947 & 1096 & 1097 & 1098 & 1099 & 1100 & 1101 \\
\hline 1948 & 1126 & 1127 & 1128 & 1129 & 1130 & 1131 \\
\hline 1949 & 1156 & 1157 & 1158 & 1159 & 1160 & 1161 \\
\hline 1950 & 1165 & 1166 & 1167 & 1168 & 1169 & 1170 \\
\hline 1951 & 1191 & 1192 & 1193 & 1194 & 1195 & 1196 \\
\hline 1952 & 1221 & 1222 & 1223 & 1224 & 1225 & 1226 \\
\hline 1953 & 1265 & 1266 & 1267 & 1268 & 1269 & 1270 \\
\hline 1954 & 1321 & 1322 & 1323 & 1324 & 1325 & 1326 \\
\hline 1955 & 1404 & 1405 & 1406 & 1407 & 1408 & 1409 \\
\hline 1956 & $\ldots$ & ... & 1456 & $\ldots$ & $\ldots$ & $\cdots$ \\
\hline $1956-57$ & 1537 & $\ldots$ & $\ldots$ & $\ldots$ & $\ldots$ & $\cdots$ \\
\hline $1956-58$ & $\ldots$ & 1538 & $\ldots$ & $\ldots$ & $\ldots$ & $\ldots$ \\
\hline $1956-59$ & $\ldots$ & $\cdots$ & $\ldots$ & 1549 & $\ldots$ & $\ldots$ \\
\hline $1956-60$ & $\ldots$ & $\ldots$ & $\ldots$ & $\ldots$ & 1760 & $\ldots$ \\
\hline $1956-60$ & $\ldots$ & $\ldots$ & $\ldots$ & $\ldots$ & . & 1770 \\
\hline
\end{tabular}

The program begun in 1956 provides for the publication of 5 years of record in 1 volume for each geographic section. During a 4-year transitional period, however, 4 of the volumes will not contain 5 years of record, as shown in the following schedule:

$\begin{array}{ll}\text { North-central: } & 1956,1957-61,1962-66, \text { etc. } \\ \text { Northeastern: } & 1956-57,1958-62,1963-67, \text { etc. } \\ \text { Southeastern: } & 1956-58,1959-63,1964-68, \text { etc. } \\ \text { South-central: } & 1956-59,1960-64,1965-69, \text { etc. } \\ \text { Northwestern: } & 1956-60,1961-65,1966-70, \text { etc. } \\ \text { Southwestern: } & 1956-60,1961-65,1966-70, \text { etc. }\end{array}$


Moreover, beginning in 1956, only water-level data from a basic network of observation wells has been published. The minimum basic network provides observation wells so located that the most significant data are obtained from the fewest wells in the most important aquifers. In some States the basic network forms the skeletal index of a more comprehensive observationwell program. Publications that contain substantial additional water-level data are listed at the end of each State report in this volume.

Water-level measurements in this report are given in feet with reference to either mean sea level (msl) or land-surface datum (lsd). Mean sea level is the datum plane on which the national network of precise levels is based; land-surface datum is a precise datum plane that is approximately at land surface at each well. If known, the altitude of the land-surface datum above mean sea level is given in the well description. The height of the measuring point (MP) above or below land-surface datum is given in each well description. A plus sign $(+)$ is placed before a measurement if the level is above the plane of reference. Measurements of water levels in wells equipped with recording gages are given for every fifth day and the end of each month (eom).

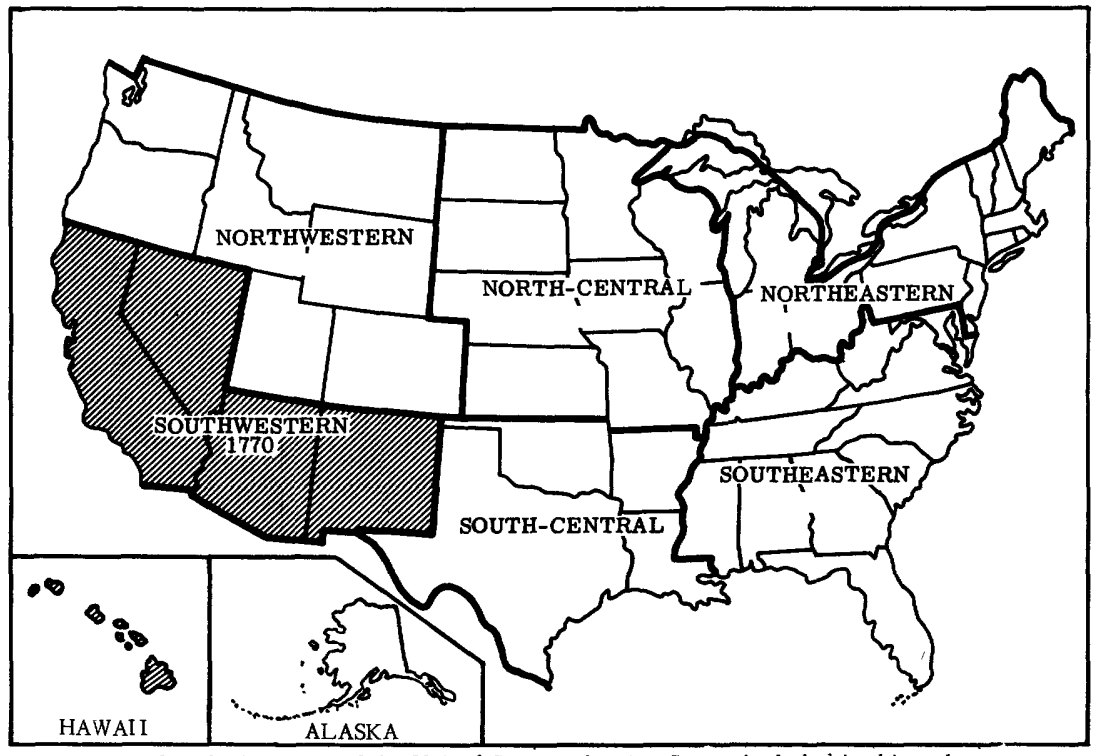

Figure 1.--Outline map of the United States, showing States included in this volume.

In the collection of water-level data to serve the objectives of the observation-well program, the problem generally is to measure changes in water level accurately, rather than to determine the absolute position of the water surface. Because the measurements are made in many types of wells, under varying conditions of access and at different temperatures, neither the method of measuring nor the equipment can be standardized. At each observation well, however, the best possible combination of measuring technique and equipment is selected, and so long as it is not changed, the measurements at each well will be consistent.

Water levels are reported to as many significant figures as can be justified by the local conditions and the end use of the information. For example, in a measurement of a depth to water of several hundred feet, the er ror in determining the absolute value of the total depth to water may be a few tenths of a foot, whereas the error in determining the net change of water level between successive measurements may be only a hundredth or a few hundredths of a foot. For lesser depths to water the accuracy is greater. Accordingly, in view of the principal use to which the data are put, most measurements are reported to a hundredth of a foot, but some are given only to a tenth of a foot or a larger unit.

Joy L. Charbonneau was responsible for the compilation of this report; James $O$. Runyan was responsible for the illustrations. 


\section{ARIZONA}

By M. B. Booher and E. K. Morse

The observation-well program in Arizona, continued during 1956 through 1960, is an integral part of the Statewide ground-water survey conducted by the U.S. Geological Survey, Ground Water Branch, in cooperation with the Arizona State Land Department.

Irrigation districts, cities, well drillers, water and power companies, government agencies, and individuals have been helpful in providing data. Figure 2 shows the location of observation wells included in this report.

\section{Well-Numbering System}

The well numbers used by the U.S. Geological Survey in Arizona are in accordance with the Bureau of Land Management's system of land subdivision. The land survey in Arizona is based on the Gila and Salt River meridian and base line, which divide the State into four quadrants. These quadrants are designated counterclockwise by the capital letters $A, B, C$, and $D$. All land north and east of the point of origin is in A quadrant, that north and west in B quadrant, that south and west in $C$ quadrant, and that south and east in D quadrant. The first digit of a well number indicates the township, the second the range, and the third the section in which the well is situated. The lowercase letters $a, b$, $c$, and $d$ after the section number indicate the well location within the section. The first letter denotes a particular 160-acre tract, the second the 40 -acre tract, and the third the 10-acre tract. These letters also are assigned in a counterclockwise direction, beginning in the northeast quarter. If the location is known within a 10-acre tract, three lowercase letters are shown in the well number. For example, well number (D-4-5) 19 caa designates the well in $\mathrm{NE}_{\frac{1}{4}} \mathrm{NE}_{4}^{\frac{1}{4} \mathrm{SW}} \frac{1}{4}$ sec. 19, T. $4 \mathrm{~S}$., R. $5 \mathrm{E}$. Where there is more than one well within a 10-acre tract, consecutive numbers beginning with 1 are added as suffixes,

\section{Well Descriptions and Water-Level Measurements}

Water levels are reported in feet below land-surface datum, unless otherwise indicated.

\section{Apache County}

(A-13-28)29ca. E. L. Johns. Drilled domestic water-table well in gravel, diam 12 in, depth $50 \mathrm{ft}$, cased to 50 . Lsd 5, $775 \mathrm{ft}$ above msl. MP top of casing, $1.60 \mathrm{ft}$ above lsd. Highest water level 8.4 below Isd, Aug. 7, 1950; lowest 30.9 below lsd, May 3, 1956. Records available: 1944-58, 1960. No measurement made in 1959. May 3, 1956, 30. 9; Oct. 23, 24. 2; May 15, 1957, 28. 2; Oct. 21, 22.8; Apr. 21, 1958, 23.3; Oct. 17, 1958, 23. 0; Feb. 12, 1960, 22. 9 .

\section{Cochise County}

(D-12-27)32aab. Creighton. Drilled stock water-table well in alluvial fill, diam 6 in, reported depth $250 \mathrm{ft}$. Lsd about $3,980 \mathrm{ft}$ above msl. MP top of casing, 2.00 ft above lsd. Highest water level 132.5 below 1sd, Dec. 20, 1956; lowest 137.2 below 1sd, Dec. 12, 1954. Records available: 1952-54, 1956-57, 1959-60. No measurement made in 1955 or 1958.

\begin{tabular}{|c|c|c|c|c|c|c|c|}
\hline Date & $\begin{array}{c}\text { Water } \\
\text { level }\end{array}$ & Date & $\begin{array}{r}\text { Water } \\
\text { level }\end{array}$ & Dat & $\begin{array}{c}\text { Water } \\
\text { level }\end{array}$ & Date & $\begin{array}{c}\text { Water } \\
\text { level }\end{array}$ \\
\hline $\begin{array}{lrl}\text { Jan. } & 21, & 1952 \\
\text { Jan. } & 12, & 1953 \\
\text { Mar. } & 6 \\
\text { Jan. } & 27, & 1954 \\
\text { Nov. } & 8 \\
\end{array}$ & \begin{tabular}{|r}
133.4 \\
133.1 \\
133.1 \\
133.0 \\
$a 132.8$ \\
\end{tabular} & $\begin{array}{lr}\text { Dec. } & 12,1954 \\
\text { May } & 22,1956 \\
\text { July } & 12 \\
\text { Aug. } & 7 \\
\text { Sept. } & 12 \\
\end{array}$ & $\begin{array}{r}137.2 \\
133.2 \\
\mathrm{a} 133.8 \\
133.1 \\
133.0 \\
\end{array}$ & $\begin{array}{lrl}\text { Oct. } & 9, & 1956 \\
\text { Nov. } & 15 & \\
\text { Dec. } & 20 & \\
\text { Jan. } & 21, & 1957 \\
\text { Mar. } & 8 & \\
\end{array}$ & $\begin{array}{r}134.9 \\
133.1 \\
132.5 \\
\mathrm{a} 134.6 \\
133.8 \\
\end{array}$ & \begin{tabular}{ll|} 
July 17,1957 \\
Sept. & $\mathbf{1 1}$ \\
Dec. & 9 \\
Jan. & $\mathbf{2 1}, 1959$ \\
Jan. & $\mathbf{2 0}, 1960$ \\
\end{tabular} & $\begin{array}{l}135.6 \\
135.4 \\
134.9 \\
132.9 \\
133.8 \\
\end{array}$ \\
\hline
\end{tabular}

a Well being pumped. 


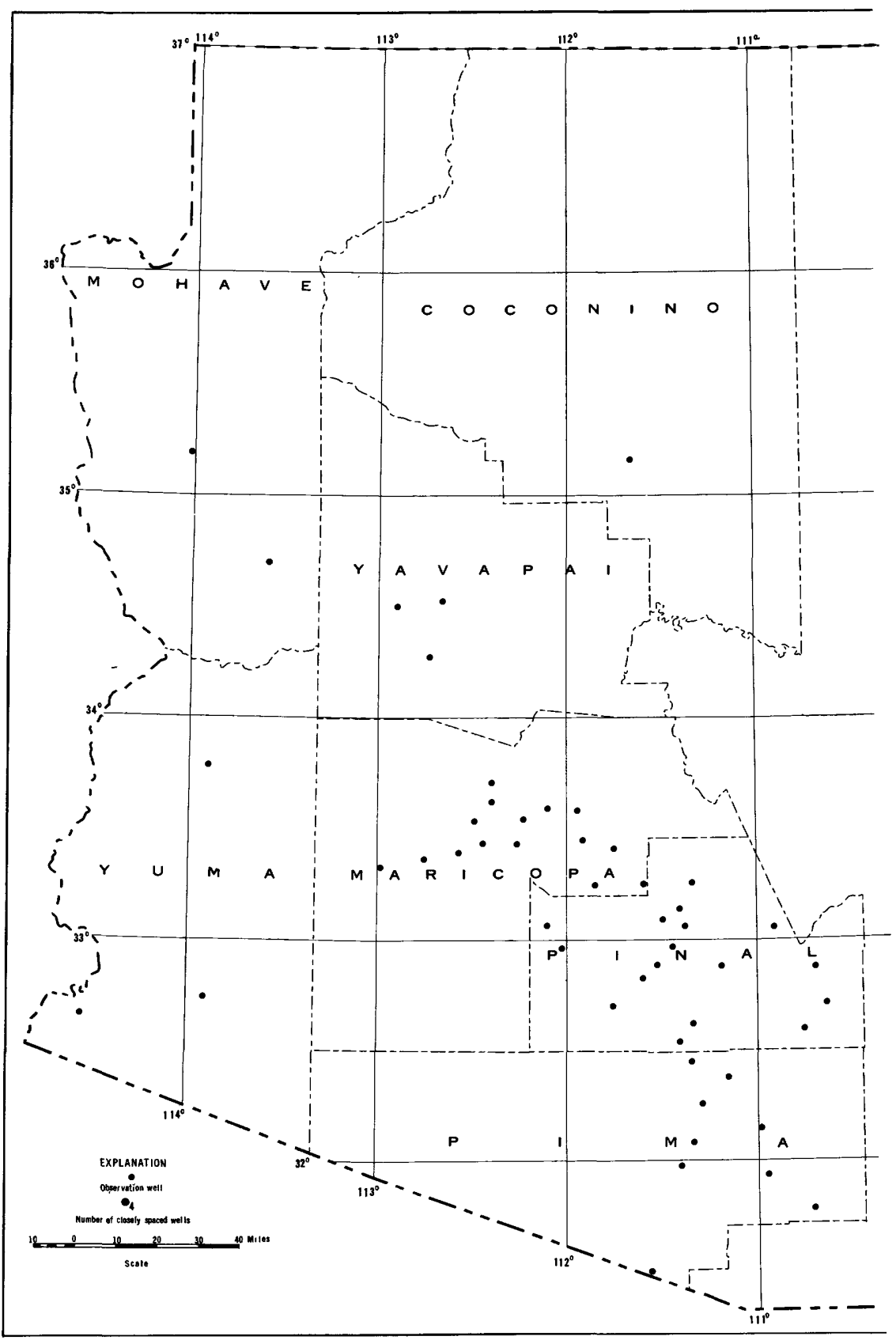

Figure 2. --Location of observation 


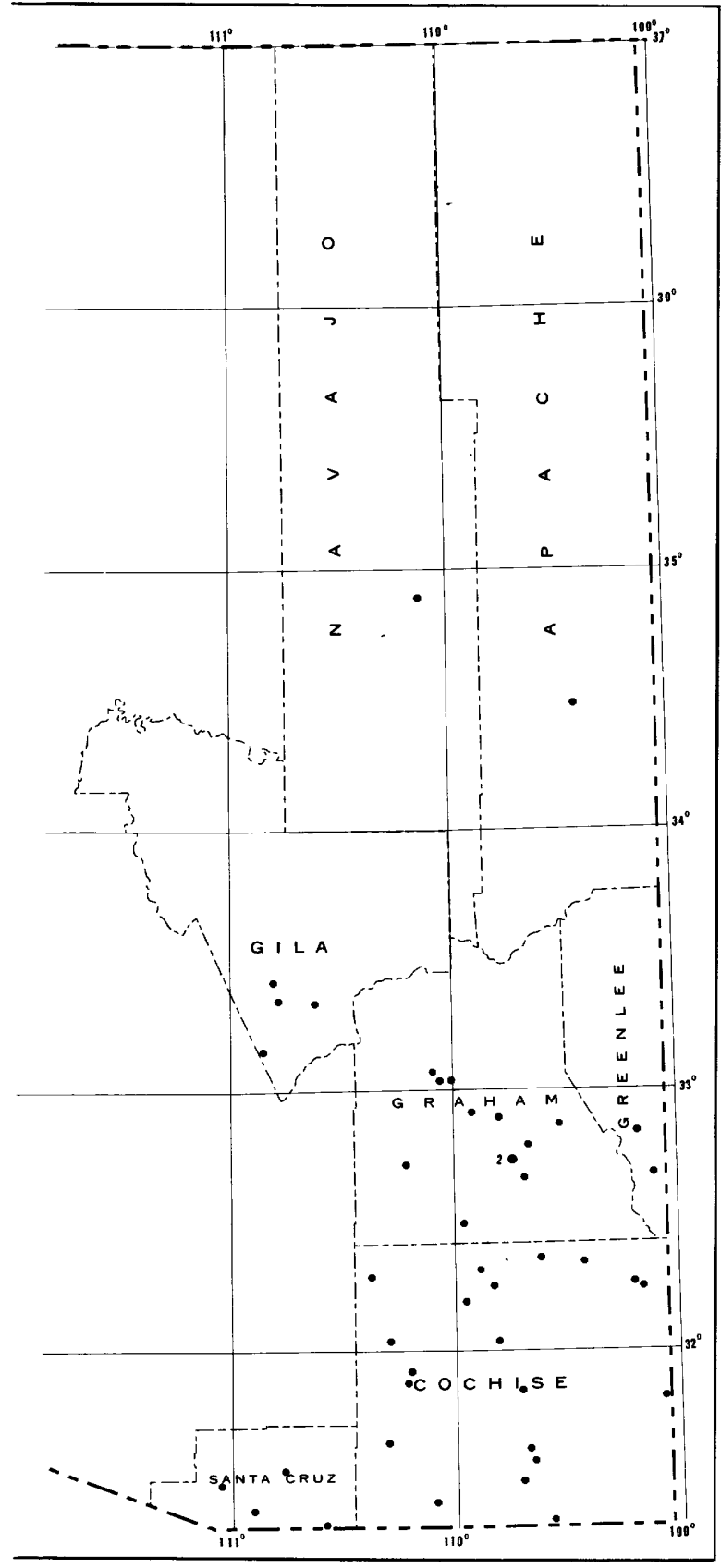

wells in Arizona, 1956-60. 
(D-13-19)23dad. Reed. Drilled stock water-table well in alluvial fill, diam 6 in, reported depth $85 \mathrm{ft}$. Lsd about $3,150 \mathrm{ft}$ above $\mathrm{msl}$. MP top of casing, $3.50 \mathrm{ft}$ above $1 \mathrm{sd}$. Highest water level 37.1 below 1sd, Feb. 24, 1960; lowest 49.8 below 1sd, May 28, 1954 . Records available: $1949-60$.

\begin{tabular}{|c|c|c|c|c|c|c|c|}
\hline Date & $\begin{array}{l}\text { Water } \\
\text { level }\end{array}$ & Date & $\begin{array}{l}\text { Water } \\
\text { level }\end{array}$ & Date & $\begin{array}{l}\text { Water } \\
\text { level }\end{array}$ & Date & $\begin{array}{r}\text { Water } \\
\text { level }\end{array}$ \\
\hline Dec. $\quad 7,1949$ & 40.2 & Jan. 19,1953 & b37.4 & 7,1955 & $\mathrm{a} 49.1$ & Nov. 19,1956 & 42.8 \\
\hline Apr. $\quad 4,1950$ & 41.2 & Apr. 16 & a39. 3 & Jan. 27,1956 & b42. 7 & Dec. 17 & 43.7 \\
\hline Sept. 20 & 39.1 & July 23 & 39.4 & May 26 & b48. 8 & Mar. $\quad 5,1957$ & 45.2 \\
\hline Dec. 18 & 41.9 & Nov. & 46.1 & June 18 & 41.7 & July & a51. 2 \\
\hline Apr. 11,1951 & 41.0 & Feb. 23,1954 & 42.4 & July 16 & b46. 2 & Sept. & a47. 7 \\
\hline Dec. 19 & 46.1 & May 28 & 49.8 & Aug. 13 & 43.0 & Apr. 9,1958 & 45.2 \\
\hline Apr. 16,1952 & 39.3 & Aug. 25 & 45.9 & Sept. 10 & 44.0 & Feb. 11,1959 & 38.1 \\
\hline $\begin{array}{l}\text { Sept. } 9 \\
\text { Nov. } 18\end{array}$ & $\begin{array}{l}\text { b36. } 8 \\
\text { b37. } 5\end{array}$ & Nov. 19 & $a 49.5$ & Oct. & 43.6 & Feb. 24,1960 & 37.1 \\
\hline
\end{tabular}

a Well being pumped.

b Well pumped recently.

(D-13-24)16bbb. State of Arizona. Drilled observation water-table well in alluvial fill, diam $16 \mathrm{in}$, depth $1,356 \mathrm{ft}$. Lsd about $4,210 \mathrm{ft}$ above msl. MP top of casing, $1.70 \mathrm{ft}$ above lsd (since Feb. 25, 1952). Highest water level 29.7 below lsd, May 13, 1942; lowest 122.5 below lsd, Sept. 20, 1960. Records available: 1942, 1944-60. Recorder installed Feb. 22, 1952.

\begin{tabular}{|c|c|c|c|c|c|c|c|c|c|}
\hline $\begin{array}{ll}\text { May } 1 \\
\end{array}$ & 13,1942 & 29.7 & Dec. 11,1946 & 32.6 & Feb. 28, 1949 & 39.5 & Feb. & 19,1951 & 44.7 \\
\hline June & 8,1944 & 31.0 & Feb. 25,1947 & 33.6 & July 7 & 41.1 & Apr. & 26 & 45.1 \\
\hline Oct. & 16,1945 & 31.9 & June 11 & 34.1 & Oct. 14 & 42.4 & May & 28 & 45.6 \\
\hline Tan. 2 & 24,1946 & 32.1 & Aug. 27 & 35.0 & Dec. 15 & 42.1 & July & 17 & 46.8 \\
\hline Mar. 2 & 26 & 32.1 & Sept. 24 & 35.2 & 8,1950 & 41.9 & Oct. & 2 & 8.1 \\
\hline May & 3 & 32.2 & June 16,1948 & 37.1 & June 26 & 43.5 & & 10 & 3 \\
\hline ane 1 & 10 & 32.4 & Aug. 12 & 37.9 & Sept. & 44.3 & Jan. & 3,1952 & 48.1 \\
\hline aly & 26 & 32.8 & Oct. 13 & 38.9 & Dec. 14 & 45.0 & & 29 & 48.0 \\
\hline Oct. 1 & 15 & 32.2 & & & & & & & \\
\hline
\end{tabular}

Highest water level for the day, from recorder graph, 1952

\begin{tabular}{|c|c|c|c|c|c|c|c|c|c|c|c|c|}
\hline Day & Jan. & Feb. & Mar. & Apr. & May & June & July & Aug. & Sept. & Oct. & Nov. & Dec. \\
\hline 5 & $\ldots$ & & 48.2 & 48.2 & 48.7 & 49.4 & 50.2 & 50.9 & 51.7 & 52.4 & 52.8 & 52.6 \\
\hline 10 & $\ldots$ & $\ldots$ & 48.2 & 48. 3 & 48.8 & 49.4 & 50.3 & 51.1 & 51.9 & 52.5 & 52.8 & 52.6 \\
\hline 15 & $\ldots$ & $\ldots$ & 48.1 & 48.4 & 48.9 & 49.6 & 50.5 & 51.2 & 52.1 & 52.6 & 52.7 & 52.5 \\
\hline 20 & $\ldots$ & & 48.2 & 48. 4 & 49.0 & 49.8 & 50.6 & 51.3 & 52.2 & 52.7 & 52.7 & 52.4 \\
\hline 25 & $\ldots$ & 48.3 & 48.1 & 48.5 & 49.1 & 49.9 & 50.7 & 51.4 & 52.3 & 52.7 & 52.7 & 52.4 \\
\hline Eom & $\ldots$ & … & 48. 2 & 48. 6 & 49.3 & 50.0 & 50.8 & 51.6 & 52. 3 & 52.8 & 52.6 & 52.4 \\
\hline \multicolumn{13}{|c|}{1953} \\
\hline 5 & 52.4 & $\cdots$ & 52.0 & 52.3 & $5 \overline{3} .5$ & 54.8 & 56.0 & 57.0 & 58.2 & $\ldots$ & 58.8 & \\
\hline 10 & 52.4 & & 52.0 & 52.6 & 53.6 & 55.0 & 55.8 & 57.0 & 58.5 & & 58.8 & 58.3 \\
\hline 15 & 52.3 & 52.1 & 52.0 & 52.8 & 53.7 & 54.9 & 55.9 & 57.3 & 58.9 & 59.0 & $\cdots$ & 58.3 \\
\hline 20 & & 52.1 & 52.0 & 53.0 & 53.8 & 55 & 56.1 & 57.6 & & 59.0 & & 58.2 \\
\hline 25 & $\ldots$ & 52.1 & 52.0 & 53.2 & 54.1 & 55.4 & 56.6 & 57.9 & ... & 58.9 & ... & 58.2 \\
\hline Eom & $\ldots$ & $\ldots$ & 52. 1 & 53.2 & 54.5 & 55.7 & 56.6 & 58.1 & $\ldots$ & 58.9 & $\ldots$ & 58.1 \\
\hline \multicolumn{13}{|c|}{1954} \\
\hline 5 & 58.0 & 57.6 & 57.4 & 57.9 & $59 . \overline{3}$ & 60.6 & 61.9 & 62.1 & 63.6 & 64.2 & 64.2 & 63.6 \\
\hline 10 & 58.0 & 57.6 & 57.3 & 58.4 & 59.5 & 60.8 & 62.3 & 62.1 & 63.8 & 64.3 & 64.1 & 63.4 \\
\hline 15 & 57.8 & 57.5 & 57.3 & 58.5 & 59.6 & 61.2 & 62.5 & 62.3 & 64.2 & 4.3 & 63.9 & 63.4 \\
\hline 20 & 57.8 & 57.5 & 57.5 & 59.0 & 59.6 & 61.5 & 62.4 & 62.4 & 64.1 & 64.3 & 63.9 & 63.2 \\
\hline 25 & 57.7 & 57.4 & 57.4 & 59.0 & 59.9 & 61.6 & 61.8 & 63.0 & 64.2 & 64.3 & 63.8 & 63.1 \\
\hline Eom & 57.7 & $\cdots$ & 57.5 & 59 & 60.2 & 61.8 & 61.9 & 63.3 & 64.1 & 64.2 & 63.7 & 63.0 \\
\hline \multicolumn{13}{|c|}{1955} \\
\hline 5 & 62.8 & 62.3 & 62.1 & 63.2 & 66.4 & 73.2 & 76.1 & 78.6 & 80.8 & 79.7 & 76.4 & 74.4 \\
\hline 10 & 62.8 & 62.2 & 62.2 & 64.0 & 66.5 & 73.9 & 77.3 & 79.4 & 79.6 & 79.0 & 76.0 & 74.1 \\
\hline 15 & 62.7 & 62.1 & 62.3 & 65.0 & 68.3 & 73.0 & 78.4 & 79.3 & 81.3 & 78.4 & 75.7 & 73.8 \\
\hline 20 & 62.6 & 62.0 & 62.3 & 66.1 & 70.2 & 72.4 & 78.7 & 80.1 & 81.8 & 77.7 & 75.4 & 73.6 \\
\hline 25 & 62.5 & 62.0 & 62.4 & 66.6 & 71.9 & 72.2 & 78.8 & 79.1 & 81.0 & 77.3 & 75.1 & 73.4 \\
\hline Eom & 62.4 & $\ldots$ & 62.6 & 66.5 & 71.9 & 74.5 & 77.7 & 79.5 & 80.3 & 76.9 & 74.7 & 73.3 \\
\hline \multicolumn{13}{|c|}{1956} \\
\hline 5 & 73.0 & 71.7 & 70.7 & 76.7 & 80.9 & 80.7 & 86.9 & 88.1 & 90.1 & 88.1 & 83.7 & 81.0 \\
\hline 10 & 72.7 & 71.5 & 70.6 & 78.0 & 81.3 & 80.9 & 87.3 & 88.0 & 91.2 & 87.2 & 83.1 & 80.7 \\
\hline 15 & 72.5 & 71.3 & 70.6 & 78.9 & 82.5 & 83.1 & 87.8 & 89.1 & 91.7 & 86.5 & 82.6 & 80.4 \\
\hline 20 & 72.3 & 71.2 & 70.6 & 79.6 & 82. 2 & 84.7 & 87.9 & 89.2 & 91.1 & 85.8 & 82.2 & 80.1 \\
\hline 25 & 72.2 & 71.0 & 73.1 & 80.3 & 81.1 & 85.9 & 87.9 & 89.6 & 91.4 & 85.0 & 81.8 & 79.8 \\
\hline Eom & 72.0 & $\because$ & 75.5 & 80.4 & 80.8 & 86.2 & 88.4 & 89.9 & 89.9 & 84.4 & 81.4 & 79.5 \\
\hline
\end{tabular}


(D-13-24)16bbb--Continued.

\begin{tabular}{|c|c|c|c|c|c|c|c|c|c|c|c|c|}
\hline \multicolumn{13}{|c|}{1957} \\
\hline$\overline{\text { Day }}$ & Jan. & Feb. & Mar. & Apr. & May & June & July & Aug. & Sept. & Oct. & Nov. & Dec. \\
\hline 5 & 79.1 & 77.6 & 77.3 & 81.4 & 86.0 & 91.0 & 88.4 & 86.7 & 88.9 & 91.0 & 86.0 & 83.5 \\
\hline 10 & 78.8 & 77.4 & 77.4 & 81.8 & 86.9 & e90. 6 & 87.9 & 86.9 & 90.7 & 89.5 & 85.5 & 83.3 \\
\hline 15 & 78.5 & 77.2 & 78.2 & 81.8 & 88.0 & e90.1 & 88.0 & 88.7 & 92.8 & 88.8 & 85.0 & 83.0 \\
\hline 20 & 78.3 & 77.2 & 78.8 & 82.2 & 88.9 & e89.7 & 88.7 & 89.0 & 94.0 & 88.0 & 84.7 & 82.9 \\
\hline 25 & 78.0 & 77.0 & 78.8 & 84.2 & 89.6 & e89. 3 & 89.5 & 88.6 & 94.1 & 87.3 & 84.2 & 82.8 \\
\hline Eom & 77.8 & a. & 79.5 & 85.6 & 89.8 & e88. 8 & 87.6 & 88.9 & 92.6 & 86.6 & 83.9 & 82.6 \\
\hline \multicolumn{13}{|c|}{1958} \\
\hline 5 & 82.0 & 81.0 & 79.6 & 81.1 & 86.3 & 92.9 & 99.9 & 105.2 & 105.0 & 99.8 & 91.6 & 88.1 \\
\hline 10 & 82.0 & 81.0 & 79.3 & 82.0 & 87.2 & 95.0 & 99.6 & 105.2 & 105.3 & 98.3 & 91.0 & 87.5 \\
\hline 15 & 81.6 & 80.8 & 79.1 & 83.6 & 88.1 & 97.0 & 101.9 & 106.3 & 104.8 & 96.8 & 90.2 & 87.8 \\
\hline 20 & 81.3 & 80.5 & 79.0 & 83.7 & 89.0 & 96.6 & 103.2 & 107.0 & 104.2 & 95.3 & 89.7 & 88.1 \\
\hline 25 & 81.0 & 80.0 & 78.9 & 84.5 & 90.3 & 97.2 & 105.2 & 105.8 & 102.8 & 94.1 & 88.9 & 87.6 \\
\hline Eom & 80.8 & & 80.0 & 85.4 & 91.1 & 99.2 & 105.8 & 105.8 & 101.3 & 93.0 & 88.5 & 87.6 \\
\hline \multicolumn{13}{|c|}{1959} \\
\hline 5 & 86.9 & 87.3 & 91.5 & 102.3 & 107.2 & 112.2 & 105.8 & 111.7 & 108.4 & 108.3 & 99.2 & 95.4 \\
\hline 10 & 86.5 & 88.3 & 92.5 & 104.9 & 105.2 & 112.2 & 105.1 & 111.3 & 109.2 & 107.1 & 98.3 & 94.9 \\
\hline 15 & 86.2 & 88.4 & 93.2 & 104.4 & 106.5 & 111.2 & 107.6 & 110.8 & 110.2 & 105.7 & 97.5 & 94.3 \\
\hline 20 & 85.8 & 89.4 & 93.2 & 105.2 & 108.6 & 109.7 & 109.7 & 110.2 & 111.0 & 103.7 & 96.7 & 93.8 \\
\hline 25 & 86.0 & 90.4 & 93.6 & 107.0 & 110.1 & 109.3 & 110.8 & 107.4 & 110.8 & 101.8 & 96.3 & 93.3 \\
\hline Eom & 87.1 & & 97.1 & 108.9 & 112.1 & 109.2 & 112.0 & 107.4 & 109.4 & 100.6 & 95.9 & 92.9 \\
\hline \multicolumn{13}{|c|}{1960} \\
\hline 5 & 92.5 & 92.2 & 96.8 & 105.0 & 109.8 & 115.4 & 114.8 & 117.6 & 120.3 & 121.6 & 110.9 & 103.7 \\
\hline 10 & 92.1 & 93.2 & 98.0 & 105.9 & 110.4 & 114.7 & 115.1 & 117.6 & 121.1 & 119.5 & 109.0 & 103.1 \\
\hline 15 & 91.7 & 93.7 & 99.2 & 107.8 & 112.5 & 114.0 & 116.4 & 117.4 & 121.8 & 117.4 & 107.5 & 102.4 \\
\hline 20 & 91.6 & 93.8 & 100,6 & 107.2 & 112.6 & 113.8 & 117.8 & 118.5 & 122.5 & 115.2 & 106.3 & 101.9 \\
\hline 25 & 91.2 & 94.7 & 102.0 & 108.5 & 113.5 & 113.9 & 118.8 & 119.4 & 121.9 & 112.9 & 105.3 & 101.4 \\
\hline Eom & 90.9 & $\ldots$ & 103.8 & 109.5 & 115.2 & 114.2 & 117.1 & 120.0 & 122.4 & 112.1 & 104.5 & 100.9 \\
\hline
\end{tabular}

(D-13-29)6ccc. A. R. Spikes. Drilled stock and irrigation artesian well in sand and gravel, diam 6 in, reported depth $835 \mathrm{ft}$. Lsd about $3,675 \mathrm{ft}$ above msl. MP top east side of casing, $0.20 \mathrm{ft}$ above lsd. Highest water level 9.5 below lsd, May 2, 1941; lowest 116. 1 below lsd, Jan. 22, 1960, Records available: 1941-42, 1944, 1946-47, 1949-52, 1954-57, 1959-60. No measurement made in 1958. Jan. 12, 1956, 87.9; Apr. 12, 107. 2; Jan. 24, 1957, 110.1; Dec. 11, 109. 0; Jan. 22, 1959, 111.5; Jan. 22, 1960, 116.1.

(D-13-31)30cdc. Elmer Franklin. Drilled domestic water-table well in sand and gravel, diam $4 \mathrm{in}$, depth $72 \mathrm{ft}$. Lsd about $3,610 \mathrm{ft}$ above $\mathrm{msl}$. MP top of $4-\mathrm{x}$ 4-in pipe clamp, $1.70 \mathrm{ft}$ above 1sd. Highest water level 58.7 below lsd, Nov. 2, 1949; lowest 70.1 below lsd, July 1 , 1955. Records available: $1940-42,1944,1946-57,1959-60$. No measurement made in 1958 Jan. 12, 1956, 64. 6; Apr. 12, 65.4; Jan. 23, 1957, 67.7; Dec. 17, 64. 8; Jan. 23, 1959, 64.7; Jan. 22, 1960, 66.1.

(D-14-23)36baa. Fay Proctor. Drilled domestic and stock water-table well in sand and gravel, diam $6 \mathrm{in}$, depth $50 \mathrm{ft}$. Lsd about 4, $210 \mathrm{ft}$ above msl. MP top of 4- $\mathrm{x}$ 4-in clamp, $1.20 \mathrm{ft}$ above lsd. Highest water level 36.0 below lsd, May 13, 1942; lowest 43.5 below lsd, July 15, 1957. Records available: 1942, 1944-60.

\begin{tabular}{|c|c|c|c|c|c|c|c|}
\hline Date & $\begin{array}{l}\text { Water } \\
\text { level }\end{array}$ & Date & $\begin{array}{c}\text { Water } \\
\text { level }\end{array}$ & Date & $\begin{array}{r}\text { Water } \\
\text { level }\end{array}$ & e & \\
\hline $\begin{array}{lr}\text { Apr. } & 9,1956 \\
\text { May } & \mathbf{2 1} \\
\text { July } & 9\end{array}$ & $\begin{array}{l}42.3 \\
41.4 \\
42.1\end{array}$ & $\begin{array}{l}\text { Aug. } 6,1956 \\
\text { Nov. } 13 \\
\text { Dec. } 21\end{array}$ & $\begin{array}{r}42.1 \\
a 42.9 \\
42.6\end{array}$ & $\begin{array}{lll}\text { Jan. } & 22, & 1957 \\
\text { July } & 15 \\
\text { Sept. } & 10\end{array}$ & $\begin{array}{l}42.4 \\
43.5 \\
42.9\end{array}$ & $\begin{array}{lr}\text { Mar. } & 24,1958 \\
\text { Jan. } & 7,1959 \\
\text { Jan. } & 18,1960\end{array}$ & $\begin{array}{l}42.2 \\
42.6\end{array}$ \\
\hline
\end{tabular}

a Well being pumped.

(D-14-25)6cac. E. T. Dunlap. Drilled domestic water-table well in sand and gravel, diam $6 \mathrm{in}$, depth $34 \mathrm{ft}$. Lsd about $4,166 \mathrm{ft}$ above msl. MP top of concrete curb, $0.20 \mathrm{ft}$ above 1 sd. Highest water level 9.8 below lsd, Oct. 21, 1955; lowest 21.2 below 1sd, Jan. 7, 1959. Records available: 1942, 1944-57, 1959-60. No measurement made in 1958.

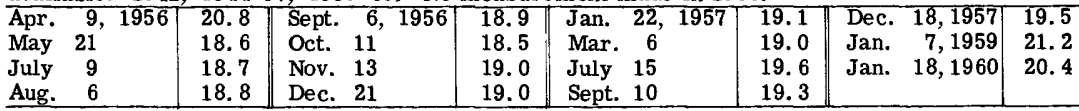

(D-14-31)3ddd. A. G. Pierce. Drilled unused artesian well in sand, diam 8 in, reported depth $400 \mathrm{ft}$. Lsd about $3,690 \mathrm{ft}$ above msl. MP top of casing, $1.60 \mathrm{ft}$ above $1 \mathrm{sd}$. Highest water level 17.2 below lsd, Apr. 24, 1942; lowest 72.9 below 1sd, July 20, 1959. Records available: 1941-42, 1946-60. Recorder removed July 20, 1960. 
(D-14-31)3ddd-- Continued.

Highest water level for the day, from recorder graph, 1956

\begin{tabular}{|c|c|c|c|c|c|c|c|c|c|c|c|c|}
\hline Day & Jan. & Feb. & Mar. & Apr. & May & June & July & Aug. & Sept. & Oct. & Nov. & Dec. \\
\hline 5 & 49.7 & 48.7 & 48.3 & 53.2 & 55.2 & 57.4 & 59.8 & 61.4 & 62.9 & 61.3 & 58.4 & 55.9 \\
\hline 10 & 49.5 & 48.5 & 49.0 & 53.7 & 55.9 & 57.8 & 60.2 & 61.7 & 63.0 & 60.5 & 58.0 & 55.4 \\
\hline 15 & 49.2 & 48.3 & 49.8 & 54.0 & 55.6 & 57.6 & 60.6 & 62.2 & 63.2 & 60.0 & 57.6 & 54.8 \\
\hline 20 & 49.0 & 48.2 & 50.8 & 54.1 & 55.4 & 58.2 & 60.6 & 62.1 & 63.4 & 60.0 & 57.2 & 54.4 \\
\hline 25 & 49.0 & 48.2 & 51.7 & 54.6 & 56.3 & 58.8 & 60.9 & 62.3 & 62.9 & 59.3 & 56.7 & 54.0 \\
\hline Eom & 48.9 & $\cdots$ & 52.4 & 54.9 & 56.7 & 59.5 & 61.1 & 62.5 & 62.1 & 58.9 & 56.3 & 53.5 \\
\hline \multicolumn{13}{|c|}{1957} \\
\hline 5 & 53.0 & 50.9 & 50.4 & 50.9 & 54.5 & 55.8 & 59.9 & 60.3 & 59.9 & 60.1 & $5 \overline{6} .7$ & 55.7 \\
\hline 10 & 52.6 & 50.6 & 50.7 & 51.2 & 54.8 & 56.9 & 60.5 & 60.6 & 59.5 & 59.5 & 56.3 & 55.2 \\
\hline 15 & 52.2 & 50.3 & 50.9 & 52.2 & 55.0 & 57.7 & 60.7 & 60.6 & 59.8 & 58.9 & 55.9 & 54.7 \\
\hline 20 & 51.8 & 50.1 & 50.8 & 53.2 & 54.6 & 58.4 & 60.9 & 60.6 & 60.5 & 58.3 & 56.2 & 54.2 \\
\hline 25 & 51.5 & 50.2 & 50.7 & 53.9 & 54.5 & 59.2 & 61.3 & 60.4 & 61.0 & 57.8 & 56.2 & 53.9 \\
\hline Eom & 51.2 & & 50.9 & 54.5 & 55.3 & 59.6 & 60.7 & 60.3 & 60.6 & 57.3 & 56.1 & 53.6 \\
\hline \multicolumn{13}{|c|}{1958} \\
\hline 5 & $5 \overline{3.2}$ & 51.7 & 50.8 & 52.2 & 56.8 & 59.6 & 62.6 & 64.1 & 65.4 & 62.2 & $5 \overline{8.8}$ & 56.6 \\
\hline 10 & 53.0 & 51.5 & 50.6 & 53.8 & 57.2 & 60.1 & 63.0 & 64.4 & 65.6 & 61.6 & 58.5 & 56.3 \\
\hline 15 & 52.7 & 51.4 & 50.5 & 54.9 & 57.6 & 60.7 & 63.2 & 64.8 & 65.1 & 60.9 & 58.0 & 56.0 \\
\hline 20 & 52. 4 & 51.2 & 50.4 & 55.6 & 58.0 & 61.1 & 63.2 & 65.0 & 64.3 & 60.3 & 57.7 & 55.7 \\
\hline 25 & 52.1 & 51.0 & 50.4 & 56.0 & 58.5 & 61.7 & 63.6 & 65.0 & 63.5 & 59.7 & 57.3 & 55.4 \\
\hline Eom & 51.9 & $\ldots$ & 50.9 & 56.4 & 59.0 & 62.2 & 63.9 & 65.1 & 62.9 & 59.3 & 57.0 & 55.2 \\
\hline \multicolumn{13}{|c|}{1959} \\
\hline 5 & 54.8 & 54.1 & 53.0 & 57.3 & 59.9 & 68.6 & 68.4 & 70.4 & 71.0 & 66.1 & 62.6 & 60.4 \\
\hline 10 & 54.6 & 54.2 & 53.0 & 58.1 & 61.1 & 65.5 & 66. 8 & 68.0 & 70.6 & 65.2 & 62.1 & 60.1 \\
\hline 15 & 54.4 & 54.1 & 53.3 & 58.8 & 64. 6 & 65.0 & 66. 2 & 68.6 & 70.0 & 64.7 & 61.7 & 59.8 \\
\hline 20 & 54.1 & 53.6 & 54.0 & 59.6 & 63. 3 & 64.8 & 72.9 & 67.6 & 70.2 & 64.0 & 61.4 & 59.5 \\
\hline 25 & 53.9 & 53.2 & 55. 4 & 60.6 & 62. 6 & 66.0 & 68.5 & 68.6 & 69.4 & 63.5 & 61.0 & 59.2 \\
\hline Eom & 55.8 & & 56.3 & 59.7 & 62.7 & 66.9 & 68.9 & 67.4 & 67.2 & 63.1 & 60.6 & 58.9 \\
\hline \multicolumn{13}{|c|}{1960} \\
\hline 5 & 58.7 & 57.1 & 56.4 & 63.5 & 64.9 & 66.2 & & $\ldots$ & & & & \\
\hline 10 & 58.3 & 56.9 & 56.3 & 62.4 & 64.9 & 65.7 & $\because$ & $\cdots$ & & & $\cdots$ & $\ldots$ \\
\hline 15 & 58.0 & 56.8 & 57. 6 & 62.0 & 63.9 & 67.6 & . & $\cdots$ & $\cdots$ & $\cdots$ & $\cdots$ & $\ldots$ \\
\hline 20 & 57.8 & 56.6 & 57.2 & 62.0 & 65.5 & 67.3 & $\cdots$ & $\cdots$ & $\cdots$ & $\cdots$ & $\cdots$ & $\ldots$ \\
\hline 25 & 57. & 56.6 & 71.6 & 61.6 & 68.4 & $\cdots$ & $\cdots$ & & & $\cdots$ & $\cdots$ & $\ldots$ \\
\hline Eon & 57.3 & $\ldots$ & 64.4 & 61.3 & 66.0 & $\ldots$ & $\cdots$ & $\ldots$ & $\ldots$ & $\ldots$ & $\cdots$ & $\ldots$ \\
\hline
\end{tabular}

(D-16-20)34acd. L. A. Scott. Drilled domestic and stock water-table well in sand and gravel, diam 6 in, depth $98 \mathrm{ft}$. MP top of casing, $1.50 \mathrm{ft}$ above lsd. Highest water level 70.4 below 1sd, June 12, 1941; lowest 85. 6 below 1sd, May 28, 1954. Records available: 1941-42, 1944-60. Jan. 23, 1956, 84.1; Sept. 28, 82. 7; Sept. 6, 1957, 80.4; Apr. 9, 1958, 83. 8; Feb. 11, 1959, 81. 7; Feb. 24, 1960, 78.6.

(D-16-25)16add. W. D. Wear. Drilled stock water-table well in sand and gravel, diam $6 \mathrm{in}$, depth $65 \mathrm{ft}$. Lsd about $4,190 \mathrm{ft}$ above msl. MP top of casing, $1.20 \mathrm{ft}$ above lsd. Highest water level 34.0 below 1sd, June 7, 1944; lowest 43.6 below lsd, Dec. 19, 1957. Records available: 1942, 1944-57, 1959-60. No measurement made in 1958. Jan. 10, 1956, 38. 3; Apr. 10, 40. 9; Jan. 23, 1957, 40. 8; Dec. 19, 43. 6; Jan. 6, 1959, 41. 2; Jan. 21, 1960, 39. 5.

(D-17-21)32bad. Boquillas Cattle Co. Drilled domestic and stock artesian well in sand and gravel, diam 6 in, reported depth $520 \mathrm{ft}$, cased to 500 . Lsd about 3, $075 \mathrm{ft}$ above msl. MP top of casing, $1.30 \mathrm{ft}$ above 1sd. Highest water level 16.9 below lsd, Dec. 9, 1946; lowest 25. 2 below Isd, Nov. 19, 1956 . Records available: 1944-60.

\begin{tabular}{|c|c|c|c|c|c|c|c|}
\hline Date & $\begin{array}{l}\text { Water } \\
\text { level }\end{array}$ & Date & $\begin{array}{l}\text { Water } \\
\text { level }\end{array}$ & Date & $\begin{array}{l}\text { Water } \\
\text { level }\end{array}$ & Date & $\begin{array}{l}\text { Water } \\
\text { level }\end{array}$ \\
\hline $\begin{array}{ll}\text { Jan. } & 26,1956 \\
\text { May } & 25 \\
\text { June } & 18 \\
\text { July } & 16\end{array}$ & $\begin{array}{r}21.4 \\
\text { a40.8 } \\
\text { a26. } \\
\text { a35.5 }\end{array}$ & $\begin{array}{l}\text { Aug. } 13,1956 \\
\text { Sept. } 10 \\
\text { Oct. } 8 \\
\text { Nov. } 19\end{array}$ & $\begin{array}{r}22.6 \\
233.6 \\
22.1 \\
25.2\end{array}$ & $\begin{array}{lrl}\text { Dec. } & 17, & 1956 \\
\text { Mar. } & 5,1957 \\
\text { July } & 10 \\
\text { Sept. } & 9\end{array}$ & $\begin{array}{r}21.6 \\
b 30.2 \\
23.7 \\
22.2\end{array}$ & $\begin{array}{ll}\text { Apr. } & 10,1958 \\
\text { Feb. } & 13,1959 \\
\text { Jan. } & 20,1960\end{array}$ & $\begin{array}{r}22.8 \\
b 24.2 \\
21.6\end{array}$ \\
\hline
\end{tabular}

a Well being pumped.

b Well pumped recently.

(D-18-21)6aab. Walter Haymore. Drilled domestic water-table well in sand and gravel, diam 4 in, depth $60 \mathrm{ft}$, cased to 60 . Lsd about $3,620 \mathrm{ft}$ above msl. MP top of casing, $1.20 \mathrm{ft}$ above Isd. Highest water level 25.6 below 1sd, Mar. 30, 1946; lowest 39.7 below 1sd, May 28, 1954. Records available: 1944-58, 1960. No measurement made in 1959. Jan. 28, 1956, 33.9 (well being pumped); Sept. 27, 37.9; Sept. 5, 1957, 35. 0; Apr. 10, 1958, 31.4; Feb. 24, $1960,28.8$. 
(D-18-26)28aaa. Frank Geers. Drilled stock water-table well in sand and gravel, diam $6 \mathrm{in}$, depth $140 \mathrm{ft}$. Lsd 4, 267. $8 \mathrm{ft}$ above msl. MP top of casing, $1.70 \mathrm{ft}$ above lsd. Highest water level 70. 6 below lsd, Dec. 21, 1949; lowest 83.1 below 1sd, Feb. 17, 1960. Records available: $1946-60$.

\begin{tabular}{|c|c|c|c|c|c|c|c|}
\hline Date & $\begin{array}{c}\text { Water } \\
\text { level }\end{array}$ & Date & $\begin{array}{c}\text { Water } \\
\text { level }\end{array}$ & Date & $\begin{array}{c}\text { Water } \\
\text { level }\end{array}$ & $\mathbf{D a}$ & $\begin{array}{c}\text { Water } \\
\text { level }\end{array}$ \\
\hline $\begin{array}{lll}\text { Jan. } & 11,1956 \\
\text { Apr. } & 25 \\
\text { May } & 24 \\
\text { June } & 19 \\
\text { July } & 17\end{array}$ & $\begin{array}{r}78.1 \\
78.5 \\
\text { b78. } 6 \\
79.4 \\
79.0\end{array}$ & $\begin{array}{l}\text { Aug. } 15,1956 \\
\text { Sept. } 12 \\
\text { Oct. } 17 \\
\text { Nov. } \mathbf{2 1} \\
\text { Dec. } 18\end{array}$ & $\begin{array}{r}79.2 \\
79.6 \\
79.6 \\
280.2 \\
280.0\end{array}$ & 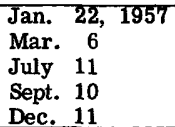 & $\begin{array}{l}79.2 \\
79.1 \\
80.4 \\
80.7 \\
80.8\end{array}$ & $\begin{array}{ll}\text { Apr. } & 22,1958 \\
\text { Jan. } & 20,1959 \\
\text { Dec. } & 15 \\
\text { Feb. } & 17,1960\end{array}$ & $\begin{array}{l}81.0 \\
81.7 \\
83.0 \\
83.1\end{array}$ \\
\hline
\end{tabular}

a Well being pumped.

b Well pumped recently.

(D-18-32)33bca. Darnell. Drilled unused water-table well in alluvial fill, diam 14 in, reported depth $200 \mathrm{ft}$. Lsd about 4,250 ft above msl. MP top of casing, at lsd. Highest water level 107.1 below 1sd, Mar. 20, 1951; lowest 132.0 below 1sd, Jan. 21, 1960. Records available: $1950-51,1953-57,1959-60$. No measurement made in 1952 or 1958.

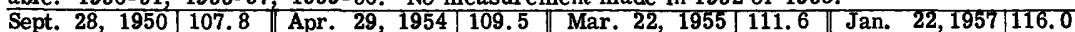
\begin{tabular}{l|l|l|l||lc||l|l|l|l|} 
Mar. 20, 1951 & 107.1 & Aug. 19 & 110.1 & Oct. 4 & 112.9 & Dec. 10 & 118.3 \\
Dec. 15, 1953 & 108.5 & Nov. 25 & 110.0 & Dec. 14 & 113.4 & Jan. 22, 1959 & 126.3
\end{tabular}

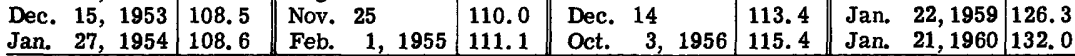

(D-20-20)32cdb. Lon Hunt. Drilled unused water-table well in sand and gravel, diam $6 \mathrm{in,}$ depth $125 \mathrm{ft}$. Lsd about $4,280 \mathrm{ft}$ above msl. MP top of casing, $2.10 \mathrm{ft}$ above lsd. Highest water level 86. 2 below lsd, Apr. 2, 1941; lowest 96.6 below 1sd, May 24, 1954. Records available: 1941-43, 1945-60. Jan. 27, 1956, 90. 0; Sept. 27, 90. 8; Sept. 5, 1957, 92. 3; Apr. 10, 1958, 93.0; Feb. 13, 1959, 94. 3; Jan. 18, 1960, 95. 7.

(D-21-26)2baa. Randel and Hunt. Drilled unused water-table well in sand and gravel, diam $20 \mathrm{in}$, depth $110 \mathrm{ft}$, cased $0-100$. Lsd about 4,130 ft above msl. MP top of casing, 3. $00 \mathrm{ft}$ above 1sd. Highest water level 36.6 below lsd, Aug. 26, 1947; lowest 61.7 below 1sd, Oct. 15Nov. 30, 1960. Records available: 1947-60.

Highest water level for the day, from recorder graph, 1956

\begin{tabular}{|c|c|c|c|c|c|c|c|c|c|c|c|c|}
\hline$\overline{\text { Day }}$ & Jan. & Feb. & Mar. & Apr. & May & June & July & Aug. & Sept. & Oct. & Nov. & Dec. \\
\hline 5 & 5.0 & 54.7 & 54.4 & 54.4 & 55.4 & 55.9 & 56.4 & 57.2 & 57.9 & 58.2 & 58.4 & 58.2 \\
\hline 10 & 54.9 & 54.6 & 54.3 & 54.5 & 55.6 & 56.0 & 56.5 & 57.4 & 58.0 & 57.9 & 58.4 & 58.2 \\
\hline 15 & 54.9 & 54.6 & 54.3 & 54.7 & 55.7 & 56.2 & 56.6 & 57.6 & 58.0 & 58.2 & 58.4 & 58.1 \\
\hline 20 & 54.8 & 54.5 & 54.3 & 54.9 & 55.7 & 56.3 & 56.8 & 57.5 & 58.2 & 58.4 & 58.4 & 58.0 \\
\hline 25 & 54.8 & 54.5 & 54.3 & 55,0 & 55.8 & 56.4 & 56.9 & 57.6 & 58.3 & 58.4 & 58,3 & 0 \\
\hline Eom & 7 & $\ldots$ & 5 & 55.2 & 55.9 & 56.3 & 57.1 & 57.8 & 58.4 & 58.5 & 58.3 & 57.9 \\
\hline \multicolumn{13}{|c|}{1957} \\
\hline 5 & 57.8 & 57.4 & 7.0 & 56.7 & 57.5 & 58.2 & 58.6 & 59.0 & 58.6 & 59.2 & 59.0 & 58.6 \\
\hline 10 & 57.7 & 57.3 & 56.9 & 56.7 & 57.7 & 58.3 & 58.4 & 59.1 & 58.4 & 59.2 & 8.9 & 3.5 \\
\hline 15 & 57.7 & 57.2 & 56.9 & 56.8 & 57.9 & 58 & 58.7 & 59.2 & 58.3 & 59.2 & 8.8 & 8. 4 \\
\hline 20 & 57.6 & 57.2 & 56.8 & 57.0 & 58.0 & 58.6 & 58.8 & 59.0 & 58.7 & 59.2 & 58.8 & 58.4 \\
\hline 25 & 57.5 & 57.1 & 56.8 & 57.1 & 58.0 & 58.7 & 58.9 & 58.7 & 59.0 & 59.1 & 58.7 & 58.3 \\
\hline Eom & 57.4 & $\ldots$ & 56.7 & 57.3 & 58.0 & 58.8 & 59.0 & 58.7 & 59.1 & 59.0 & 58.6 & 58.3 \\
\hline \multicolumn{13}{|c|}{1958} \\
\hline 5 & 58.2 & 57.8 & 7.4 & 57.2 & 57.4 & 57.2 & 56.5 & 57.2 & 57.2 & 56.7 & 57.1 & 57.2 \\
\hline 10 & 58.1 & 57.8 & 57.4 & 57.2 & 57.5 & 57.0 & 56.8 & 57.3 & 57.1 & 56.8 & 57.2 & 57.2 \\
\hline 15 & 58.1 & 57.7 & 57.3 & 57.2 & 57.4 & 56.7 & 57.1 & 57.3 & 56.6 & 56.9 & 57.2 & 57.2 \\
\hline 20 & 58.0 & 57.6 & 57.3 & 57.2 & 57.3 & 56.4 & 57.1 & 57.2 & 56.5 & 57.0 & 57.2 & 57.2 \\
\hline 25 & 58.0 & 57.5 & 57.2 & 57.2 & 57.3 & 56.0 & 57.1 & 57.2 & 5 & 57.1 & 57.2 & 57.1 \\
\hline Eom & 57.9 & 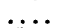 & 57.2 & 57.3 & 57.3 & 56.1 & 57.2 & 57.2 & 56.5 & 57.1 & 57.2 & 57.1 \\
\hline \multicolumn{13}{|c|}{1959} \\
\hline 5 & 57.1 & 56.9 & 56.8 & 56.7 & 57.2 & 57.6 & 58.0 & 58.5 & 59.0 & $5 \overline{8.8}$ & 59.1 & 59.0 \\
\hline 10 & 57.1 & 56.9 & 56.8 & 56.8 & 57.2 & 57.7 & 57.9 & 58.6 & 9.1 & 58.9 & 99.0 & 59.1 \\
\hline 15 & 57.0 & 56.9 & 56.7 & 56.8 & 57.3 & 57.8 & 58.0 & 58.7 & 58.9 & 58.9 & 9.1 & 59.1 \\
\hline 20 & 57.0 & 56.8 & 56.7 & 56.9 & 57.3 & 57.9 & 58.1 & 58.8 & 58.7 & 59.0 & 59.1 & 59.0 \\
\hline 25 & 57.0 & 56.8 & 56.7 & 57.0 & 57.4 & 58.1 & 58.3 & 58.9 & 58.6 & 59.0 & 59.1 & 59.0 \\
\hline Eom & 57.0 & & 56.7 & 57.1 & 57.5 & 58.2 & 58.4 & 59.0 & 58.7 & 59.1 & 59.1 & 59.0 \\
\hline \multicolumn{13}{|c|}{1960} \\
\hline 5 & 59.0 & 58.7 & 58.4 & 58.5 & 59.2 & 59 & 60.5 & 60.8 & 61.3 & 61.6 & 61.7 & 61.6 \\
\hline 10 & 58.9 & 58.6 & 58. 4 & 58.6 & 59.4 & 59.9 & 60.6 & 60.9 & 61.4 & 61.6 & 61.7 & 61.6 \\
\hline 15 & 58.9 & 58.6 & 58.4 & 58.7 & 59.5 & 60.0 & 60.6 & 60.9 & 61.4 & 61.7 & 61.7 & 61.5 \\
\hline 20 & 58.8 & 58.6 & 58.3 & 58.8 & 59.5 & 60.1 & 60.4 & 61.0 & 61.5 & 61.7 & 61.7 & 61.5 \\
\hline 25 & 58.8 & 58. & 58.3 & 59.0 & 59.6 & 60.3 & 60.4 & 61.1 & 61.5 & 61.7 & 61.7 & 61.4 \\
\hline Eom & 58.7 & & 58.4 & 59.1 & 59.7 & 60.4 & 60.7 & 61.2 & 61.6 & 61.7 & 61.7 & 61.4 \\
\hline
\end{tabular}


(D-21-26)24baa. McNeal Cemetery. Drilled domestic water-table well in sand and gravel, diam $8 \mathrm{in}$, depth $196 \mathrm{ft}$. Lsd 4, 195.8 ft above msl. MP top of casing, 2. $00 \mathrm{ft}$ above lsd. Highest water level 112.0 below lsd, Jan. 31, 1946; lowest 137.9 below lsd, Jan. 19 , 1960. Records available: 1946-58, 1960. No measurement made in 1959.

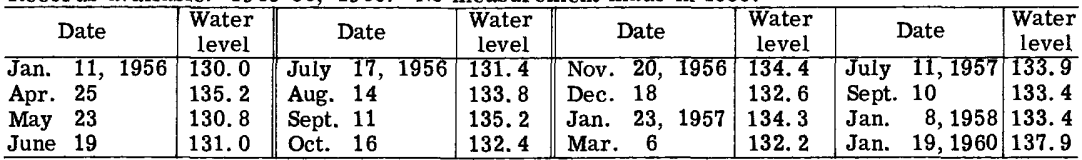

(D-22-26)28bab2. J. E. Brophy. Drilled irrigation water-table well in sand and gravel, diam $8 \mathrm{in}$, depth $90 \mathrm{ft}$. Lsd 4,026.6 ft above msl. MP top of concrete foundation under pump, $1.20 \mathrm{ft}$ above lsd. Highest water level 26. 4 below lsd, July 25, 1946; lowest 44.4 below lsd, Jan. 20, 1959. Records available: 1946-47, 1949-51, 1953-60.

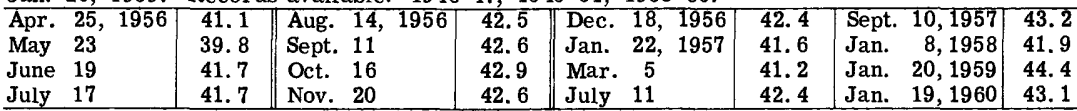

(D-23-22)33ded. U. S. Government. Drilled observation water-table well in alluvial fill, diam 12 to $8 \mathrm{in}$, depth $92 \mathrm{ft}$, cased 0-92. Lsd about 4, $200 \mathrm{ft}$ above msl. MP top of casing, $3.40 \mathrm{ft}$ above lsd. Highest water level 11.5 below lsd, Aug. 25, 1955; lowest 17.9 below lsd, June 20, 1959. Records available: 1954-60. Recorder installed July 1954. July 1, $1954,15.0$.

\begin{tabular}{|c|c|c|c|c|c|c|c|c|c|c|c|c|}
\hline$\overline{\text { Day }}$ & Ta & Foh & & & & & & & & & & \\
\hline Day & Ja & Feb. & Ind & Ap. & May & June & $\mathrm{Ju}$ & Aug. & Sept. & Oct. & Nov. & Dec. \\
\hline 5 & $\ldots$ & $\cdots$ & $\ldots$ & $\cdots$ & $\cdots$ & $\ldots$ & $\ldots$ & $\ldots$ & 13.0 & 13.5 & 13.6 & 13.5 \\
\hline 10 & $\ldots$ & $\ldots$ & $\ldots$ & $\ldots$ & $\ldots$ & $\ldots$ & $\ldots$ & $\ldots$ & $\ldots$ & 13.6 & 13.6 & 13.5 \\
\hline 15 & $\ldots$ & $\ldots$ & $\ldots$ & $\ldots$ & $\ldots$ & $\ldots$ & 14. 7 & $\ldots$ & 13. 3 & 13.6 & 13. 6 & 13.5 \\
\hline 20 & $\ldots$ & $\ldots$ & $\ldots$ & $\ldots$ & $\ldots$ & $\ldots$ & 14. 3 & 12.7 & 13. 4 & 13.6 & 13.5 & 13.5 \\
\hline 25 & $\ldots$ & $\ldots$ & $\ldots$ & $\ldots$ & $\ldots$ & $\ldots$ & 13. 9 & 12.7 & 13.4 & 13.6 & 13.5 & 13.5 \\
\hline Eom & $\ldots$ & $\ldots$ & $\ldots$ & $\ldots$ & $\ldots$ & $\ldots$ & 13. 8 & 13.0 & 13.5 & 13.6 & 13.5 & 13. 5 \\
\hline \multicolumn{13}{|c|}{1955} \\
\hline 5 & 13.5 & 13.5 & 13.6 & 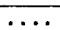 & $\cdots$ & $\ldots$ & & & 12.7 & 13.2 & $1 \overline{3.3}$ & $13 . \overline{3}$ \\
\hline 10 & 13.5 & 13.5 & 13. 5 & $\ldots$ & . & $\ldots$ & & & 12.9 & 13.3 & 13. 3 & 13. 3 \\
\hline 15 & 13.5 & 13.6 & $\ldots$ & $\ldots$ & $\ldots$ & $\ldots$ & & $\ldots$ & 13.0 & 13.3 & 13.3 & 13. 3 \\
\hline 20 & 13.5 & 13. 6 & $\ldots$ & $\ldots$ & . & $\ldots$ & $\ldots$ & $\ldots$ & 13. 2 & 13.2 & 13. 3 & 13. 3 \\
\hline 25 & 13. 5 & 13.5 & & $\ldots$ & 16.6 & 17. 6 & 13.9 & 11.5 & 13.3 & 13. 2 & 13. 3 & 13. 3 \\
\hline Eom & 13.5 & $\ldots$ & 15.5 & 16.4 & . & $\ldots$ & $\ldots$ & 12.4 & 13. 3 & 13.3 & 13. 3 & 13. 3 \\
\hline \multicolumn{13}{|c|}{1956} \\
\hline 5 & 13.3 & 13.3 & 13.4 & 13.4 & 13.7 & 13.9 & 13.9 & 13.9 & 13.8 & 14.2 & 14.0 & 14.0 \\
\hline 10 & 13. 3 & 13. 4 & 13. 4 & 13.4 & 13.7 & 13.9 & 13.9 & 14. 2 & 13.9 & 14. 6 & 14. 3 & 14.0 \\
\hline 15 & 13.3 & 13.3 & 13. 4 & 13.5 & 13.7 & 14.0 & 13.7 & 14.0 & 13. 8 & 14.9 & 14.5 & 14.0 \\
\hline 20 & 13. 3 & 13. 4 & 13.5 & 13.5 & 13.8 & 14.1 & 13. 6 & 13.8 & 13. 8 & 14. 6 & 14. 2 & 13.9 \\
\hline 25 & 13. 3 & 13. 4 & 13.5 & 13.6 & 13.8 & 14.1 & 13.5 & 14.1 & 14. 1 & 14.4 & 14.0 & 13.9 \\
\hline Eom & 13.3 & $\cdots$ & 13.5 & 13.6 & 13.8 & 14.0 & 13.5 & 13.7 & 14. 2 & 14. 2 & 14.0 & 13.9 \\
\hline \multicolumn{13}{|c|}{1957} \\
\hline 5 & 13.9 & 14.1 & 14.2 & 14.1 & 14.6 & 14.2 & 14.3 & 13.6 & 13.4 & 13.8 & 13.8 & 13.8 \\
\hline 10 & 13.9 & 14.2 & 14.1 & 14.0 & 14.4 & 14.5 & 14.0 & 13.6 & 13.5 & 13.8 & 13.8 & 13.8 \\
\hline 15 & 13.8 & 14.5 & 14.3 & 14. 2 & 14. 7 & 14.7 & 14.1 & 13.5 & 13. 6 & 13.8 & 13.8 & 13. 8 \\
\hline 20 & 13.9 & 14.1 & 14.2 & 14. 3 & 14.5 & 14.7 & 14.0 & 13.0 & 13.7 & 13. 8 & 13. 8 & 13.7 \\
\hline 25 & 13.8 & 14.3 & 14.0 & 14.4 & 14. 3 & 14.7 & 13. 8 & 13.2 & 13. 8 & 13.8 & 13.8 & 13.7 \\
\hline Eom & 13.9 & $\ldots$ & 14.0 & 14.3 & 14. 2 & 14.4 & 13. 9 & 13.3 & 13.8 & 13.7 & 13.8 & 13.8 \\
\hline \multicolumn{13}{|c|}{1958} \\
\hline 5 & 13.8 & $1 \overline{3} . \overline{8}$ & $1 \overline{3} . \overline{7}$ & 14.0 & 14.3 & 14.8 & 14.0 & 12.6 & 12.8 & 12.8 & 13.3 & 13.4 \\
\hline 10 & 13. 8 & 13.8 & 13.7 & 14. 1 & 14.0 & 14.8 & 15. 6 & 12.9 & 12. 8 & 12.9 & 13.4 & 13.4 \\
\hline 15 & 13.7 & 13. 8 & 13.7 & 14.1 & 15. 2 & 14. 6 & 14. 6 & 12.7 & 12. 7 & 13.0 & 13.5 & 13.4 \\
\hline 20 & 13.7 & 13.7 & 13.8 & 14.2 & 15.4 & 14.4 & 14. 1 & 12.5 & 13. 0 & 13. 2 & 13. 3 & 13. 4 \\
\hline 25 & 13. 7 & 13.7 & 13.9 & 14.2 & 14.5 & 15.2 & 14.0 & 12. 4 & 12.8 & 13. 2 & 13.3 & 13.3 \\
\hline Eom & 13. 8 & $\ldots$ & 14.2 & 14.3 & 14. 7 & 14.5 & 13.8 & 12.7 & 12. 8 & 13.3 & 13. 3 & 13.2 \\
\hline \multicolumn{13}{|c|}{1959} \\
\hline 5 & $1 \overline{3} . \overline{2}$ & 13.4 & 13.8 & 13.8 & 14.1 & 14.5 & 14.2 & 13.1 & 13.2 & 13.6 & 13.5 & 13.4 \\
\hline 10 & 13. 3 & 13.3 & 13. 6 & 14.0 & 14.0 & 14.9 & 14. 2 & 13.2 & 13. 6 & 13.9 & 13. 4 & 13. 4 \\
\hline 15 & 13. 3 & 13.3 & 13.7 & 13.6 & 14. 4 & 15.3 & 14. 6 & 12.8 & 13. 8 & 13.9 & 13. 4 & 13.4 \\
\hline 20 & 13. 3 & 13.3 & 14.0 & 13. 6 & 14. 2 & 17.9 & 13.9 & 12.9 & 13.7 & 14.0 & 13.4 & 13.4 \\
\hline 25 & 13. 3 & 13.3 & 14.0 & 14.0 & 14.1 & 17.0 & 14. 2 & 13. 2 & 13. 6 & 13. 8 & 13.4 & 13.4 \\
\hline Eom & 13.4 & $\ldots$ & 13. 8 & 14. 2 & 14.4 & 14.2 & 13.0 & 13.0 & 13.5 & 13.6 & 13.4 & 13.4 \\
\hline \multicolumn{13}{|c|}{1960} \\
\hline 5 & 13.3 & 13.2 & 13.4 & 13.3 & $13 . \overline{3}$ & 14. 2 & 14.1 & 13.9 & 14.5 & 14.0 & 14.0 & 14.0 \\
\hline 10 & 13. 3 & 13.2 & 13.7 & 13. 3 & 13.4 & 14. 2 & 14. 2 & 13.9 & 13. 8 & 14.1 & 14.2 & 13.9 \\
\hline 15 & 13.1 & 13. 2 & 13. 7 & 13. 3 & 13. 6 & 14.2 & 14. 4 & 13.5 & 13. 8 & 14.0 & 14.3 & 14.0 \\
\hline 20 & 13.2 & 13.2 & 13.5 & 13. 3 & 14.0 & 14.2 & 14.5 & 13.9 & 14.0 & 13.9 & 14.1 & 14.0 \\
\hline 25 & 13. 2 & 13.2 & 13. 3 & 13.2 & 14. 1 & 14.0 & 14.0 & 14.2 & 14. 1 & 13.9 & 14.0 & 13.9 \\
\hline Eom & 13.2 & $\ldots$ & 13. 4 & 13. 2 & 14. 2 & 13.9 & 13.9 & 14.0 & 14.1 & 14.0 & 14.0 & 13.9 \\
\hline
\end{tabular}


(D-24-27)5bdb. Fred Price. Dug stock water-table well in sand and gravel, diam $8 \mathrm{ft}$, depth $82 \mathrm{ft}$, lined with concrete. Lsd about 3,996 ft above msl. MP top of 2- x 12-in board, $1.00 \mathrm{ft}$ above 1sd. Highest water level 54.3 below 1sd, May 26, 1942; lowest 65.2 below lsd, Jan. 19, 1960. Records available: 1942, 1944-57, 1959-60. No measurement made in 1958. Jan. 9, 1956, 63. 0; Sept. 4, 1957, 64. 8; Dec. 10, 64. 5; Jan. 19, 1959, 64.6; Jan. 19, 1960, 65.2 .

\section{Coconino County}

(A-22-6)26aaa. City of Flagstaff. Drilled unused water-table well in Coconino Sandstone of Permian age, diam $16 \mathrm{in}$, reported depth 1,021 ft, cased 0-1,021. Lsd about 7, $335 \mathrm{ft}$ above msl. MP top of casing, at lsd. Highest water level 129.7 below lsd, Sept. 28, 1945; lowest 132. 1 below isd, Dec. 19, 1957. Records available: 1944-57, 1960. No measurement made in 1958 or 1959 . June 19, 1956, 131.6; Oct. 25, 131. 8; May 30, 1957, 132. 0; Dec. 19, 132.1; Apr. 13, 1960, 131.9.

\section{Gila County}

(A-1-15)9aad. Kenneth Hoopes. Drilled industrial water-table well in sand and gravel, diam 12 in, depth $160 \mathrm{ft}$. Lsd 3,237.5 ft above msl. MP hole in casing, $2.50 \mathrm{ft}$ above lsd. Highest water level 39.2 below Isd, Apr. 9, 1952; lowest 95.9 below 1sd, Feb. 10, 1959. Records available: 1945-60. Sept. 18, 1956, 93.4; Sept. 11, 1957, 80.9; Mar. 25, 1958, 88.5; Feb. 10, 1959, 95. 9; Feb. 9, 1960, 63.0.

(D-1-15)13bad. Schniffen. Drilled unused water-table well in sand and gravel, diam 6 in, depth $105 \mathrm{ft}$. Lsd about 3,800 ft above msl. MP top of casing, $0.20 \mathrm{ft}$ above $1 \mathrm{sd}$. Highest water level 3.5 below Isd, May 5, 1949; lowest 38.9 below 1sd, Apr. 11, 1951. Records available: $1946-55,1957,1959-60$. No measurement made in 1956 or 1958 . Sept. 11, 1957, 25. 2; Feb. 10, 1959, 33.5; Feb. 9, 1960, 7.1.

(D-1-17)10bd. U. S. Indian Service. Drilled unused water-table well in alluvial fill, diam $8 \mathrm{in}$, reported depth $125 \mathrm{ft}$. Lsd about $2,995 \mathrm{ft}$ above msl. MP top of casing, $0.50 \mathrm{ft}$ above lsd. Highest water level 18.6 below lsd, Nov. 7, 1951; lowest 19.4 below 1sd, Mar. 25, 1958, Feb. 10, 1959, Feb. 9, 1960. Records available: 1951-60.

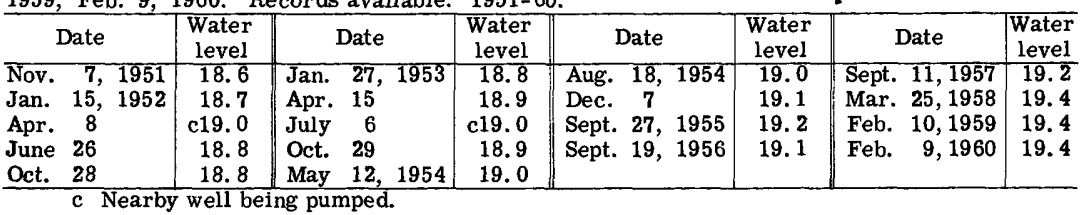

(D-3-15)20ddb. Marvin Dodge. Drilled irrigation water-table well in Gila Conglomerate of Pliocene and Pleistocene age, diam $20 \mathrm{in}$, reported depth $300 \mathrm{ft}$, cased $0-193$. Lsd about 2,730 ft above msl. MP slot in casing, at lsd (since Sept. 21, 1956). Highest water level 81.6 below 1sd, Feb. 25, 1960; lowest 102. 2 below 1sd, Sept. 11, 1957. Records available: 1952-60. \begin{tabular}{l|c||c|c||c|c||c|c|}
\hline May 22, 1952 & 91.9 & Mar. 1, 1954 & 98.5 & Sept. 28, 1955 & 97.1 & Mar. 26, 1958 & 95.7 \\
\hline
\end{tabular} \begin{tabular}{l|l|ll|l|l||l|l|l|l|} 
Apr. 3, 1953 & 94.0 & May 11 & 97.6 & Sept. 21, 1956 & 101.0 & Feb. 6, 1959 & 99.8
\end{tabular} \begin{tabular}{l|r|rr|r|r|r|r|r|r|} 
July & 2 & 97.7 & Dec. 8 & 97.5 & Sept. 11, 1957 & 102.2 & Feb. 25, 1960 & 81.6 \\
\hline
\end{tabular}

$\frac{\text { Dec. } 8}{\text { a Well being pumped. }}$

\section{Graham County}

(D-4-22)13dba. Aubrey Rabb. Drilled irrigation water-table well in sand and gravel, diam $16 \mathrm{in,} \mathrm{reported} \mathrm{depth} 100 \mathrm{ft}$. Lsd about 2, $640 \mathrm{ft}$ above msl. MP hole in east side of pumpbase, $1.80 \mathrm{ft}$ above Isd. Highest water level 13.2 below 1sd, Jan. 26, 1960; lowest 29.0 below 1sd, Aug. 31, 1953. Records available: 1953-60.

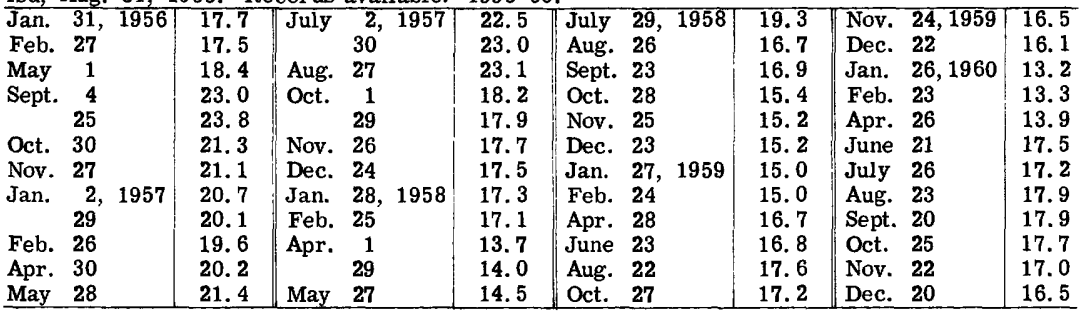


(D-4-22)35ddd. Pat Hinton. Drilled stock water-table well in sand and gravel, diam 6 in, depth $75 \mathrm{ft}$, cased $0-75$. Lsd 2,859.5 ft above msl. MP top of casing, $2.00 \mathrm{ft}$ above lsd. Highest water level 17.1 below 1sd, Feb. 11, 1943; lowest 39.4 below 1sd, Mar. 29, 1940. Records available: $1939-44,1946-60$.

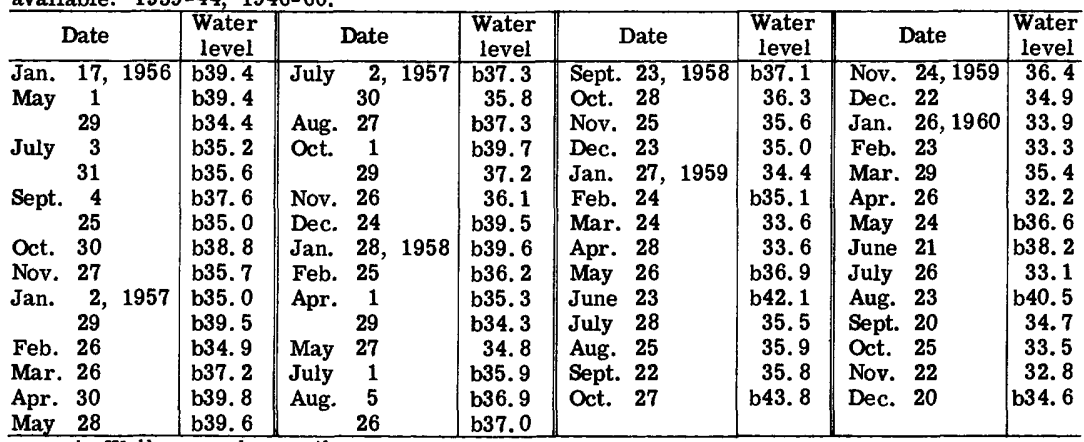

b Well pumped recently.

(D-4-23)29adc. T. D. Motes. Formerly Silas Jarvis. Drilled irrigation water-table well in sand and gravel, diam $16 \mathrm{in}$, depth $83 \mathrm{ft}$, cased to 83, perforated 53-73. Lsd 2,705. $7 \mathrm{ft}$ above msl. MP hole in pumpbase, $0.90 \mathrm{ft}$ above 1sd. Highest water level 46.1 below lsd, Mar. 18, 1941; lowest 63.2 below 1sd, Feb. 15, 1948. Records available: 1940-60.

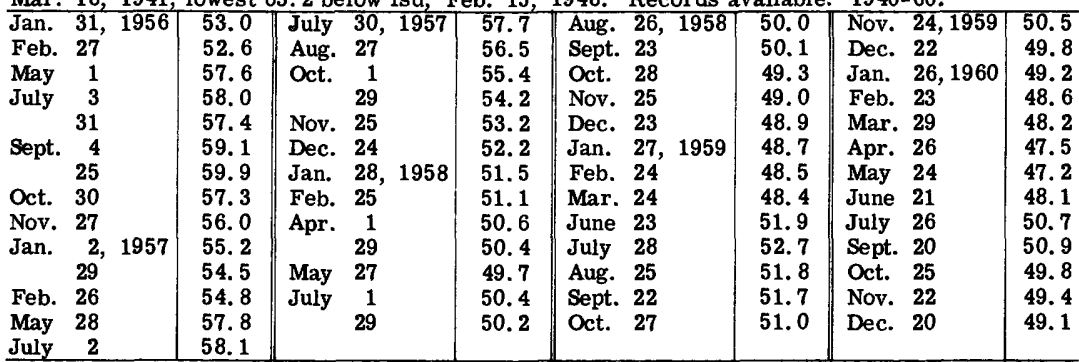

(D-6-24)5acc. M. J. Ferguson. Formerly Eldon Palmer. Drilled irrigation water-table well in sand and gravel, diam $16 \mathrm{in}$, depth $64 \mathrm{ft}$, cased 0-64. Lsd 2,779.6 ft above msl. MP hole in casing, $1.50 \mathrm{ft}$ above lsd. Highest water level 38.9 below lsd, May 29, 1941; lowest 57.7 below 1sd, June 28, 1955 . Records available: 1940-60.

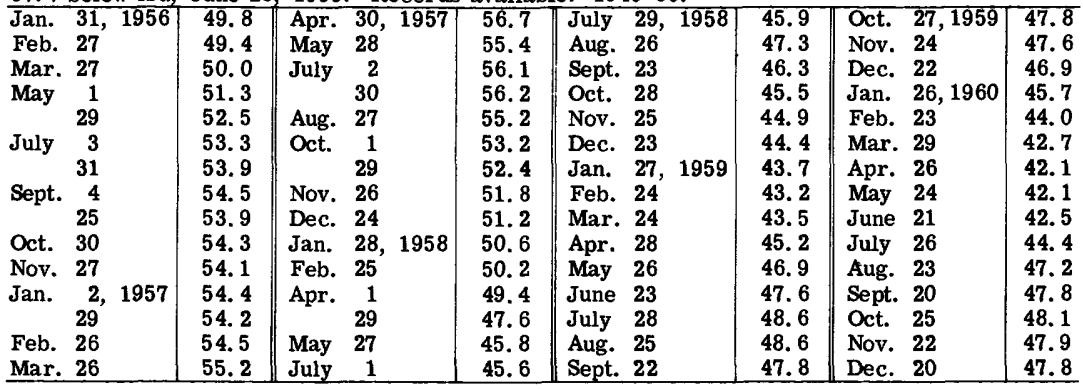

(D-6-25)20aaa. Vance Marshall. Drilled irrigation water-table well in sand and gravel, diam 16 in, depth $46 \mathrm{ft}$, cased $0-46$. Lsd 2,821. $6 \mathrm{ft}$ above msl. MP top of casing, $0.30 \mathrm{ft}$ above Isd (since Apr. 26, 1955). Highest water level 10. 8 below 1sd, May 26, 1941; lowest 29.6 below 1sd, Oct. 30, 1956. Records available: 1939-46, 1948-50, 1952-60.

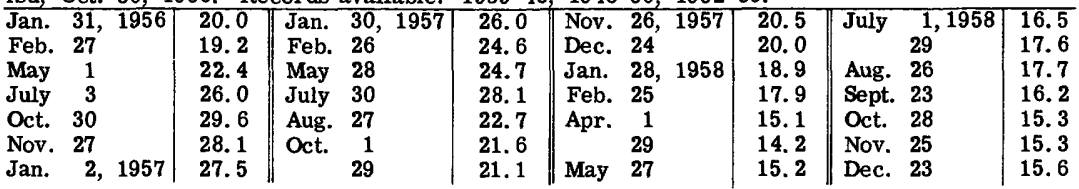


(D-6-25)20aaa--Continued.

\begin{tabular}{l|r||l|c||l|l||c|c}
\hline \multicolumn{1}{c|}{ Date } & $\begin{array}{c}\text { Water } \\
\text { level }\end{array}$ & Date & $\begin{array}{r}\text { Water } \\
\text { level }\end{array}$ & Date & $\begin{array}{c}\text { Water } \\
\text { level }\end{array}$ & Date & $\begin{array}{c}\text { Water } \\
\text { level }\end{array}$ \\
\hline Jan. 27, 1959 & 15.1 & July 28, 1959 & c18.4 & Jan. 26, 1960 & 13.9 & July 26, 1960 & 16.5 \\
Feb. 24 & 15.0 & Aug. 25 & 16.7 & Feb. 23 & 13.6 & Aug. 23 & 18.2 \\
Mar. 24 & c18.2 & Sept. 22 & 16.6 & Mar. 29 & 12.5 & Sept. 20 & 18.1 \\
Apr. 28 & c18.1 & Oct. 27 & 17.2 & Apr. 26 & 13.5 & Oct. 25 & 16.4 \\
May 26 & c18.7 & Nov. 24 & 15.6 & May 24 & 14.6 & Nov. 22 & 16.0 \\
June 23 & 17.5 & Dec. 22 & 15.3 & June 21 & 16.6 & Dec. 20. & 15.9 \\
\hline
\end{tabular}

(D-6-28)31aab. J. W. Irvin. Drilled unused water-table well in Gila Conglomerate, diam $16 \mathrm{in}$, depth $540 \mathrm{ft}$. Lsd about $3,150 \mathrm{ft}$ above msl. MP top of casing, $0.80 \mathrm{ft}$ above 1 sd. Highest water level 23. 6 below lsd, June 2, 1952; lowest 43.1 below lsd, Jan. 7, 1957. Records available: 1951-60.

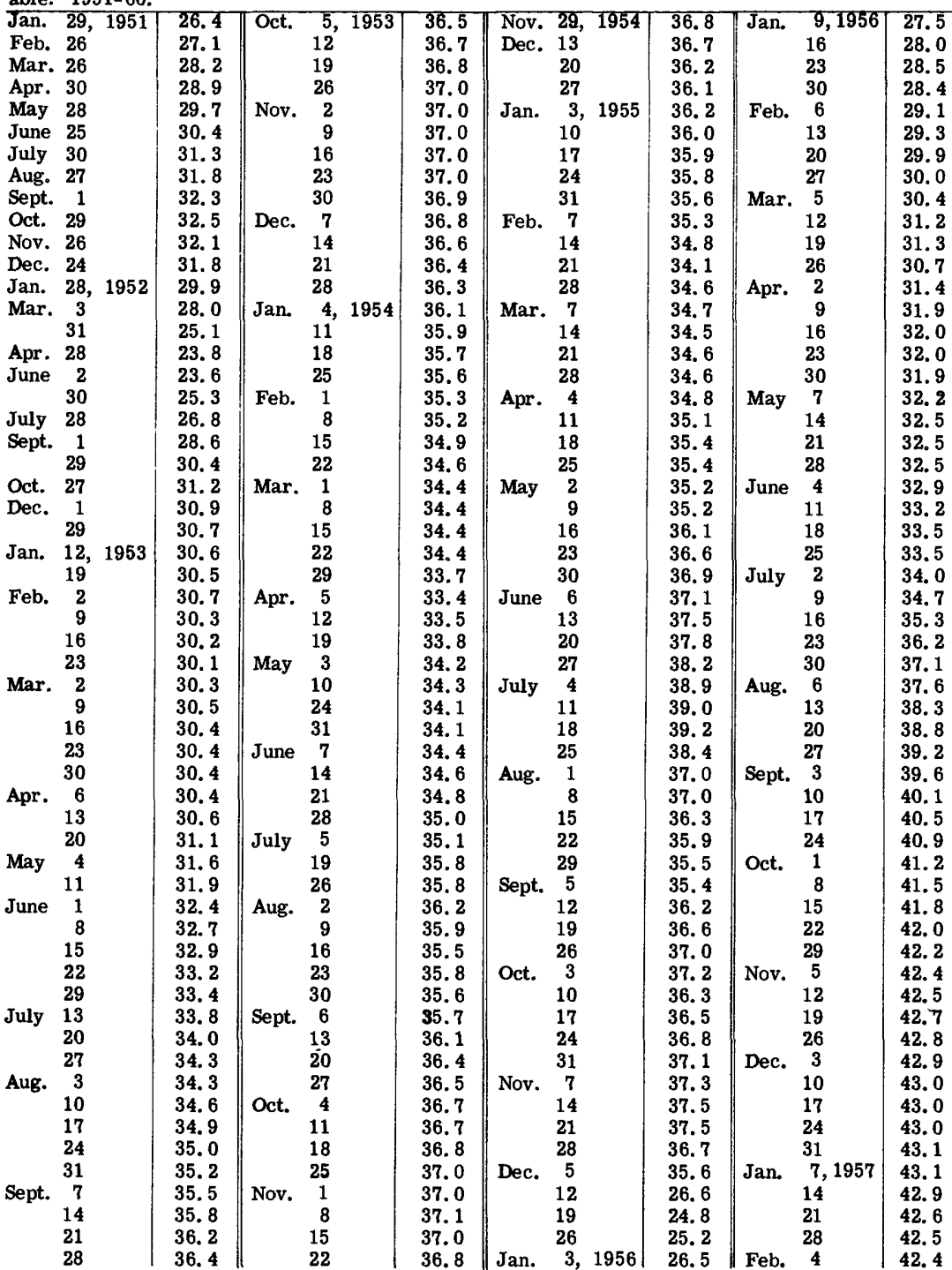


(D-6-28)31aab--Continued.

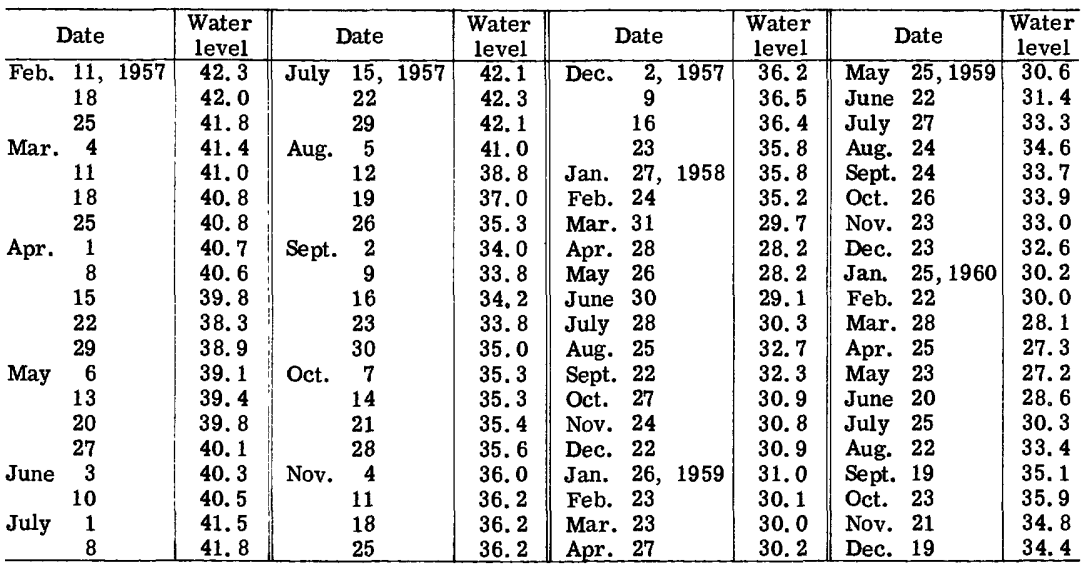

(D-7-26)22bac. Lee Johns. Drilled irrigation water-table well in sand and gravel, diam $16 \mathrm{in}$, depth $90 \mathrm{ft}$, cased to 90 . Lsd 2, 950.3 ft above msl. MP top of casing, $0.50 \mathrm{ft}$ above lsd (since Apr. 30, 1956). Highest water level 20.3 below 1sd, May 25, 1942; lowest 68.2 below 1sd, July 1, 1957. Records available: 1940-60.

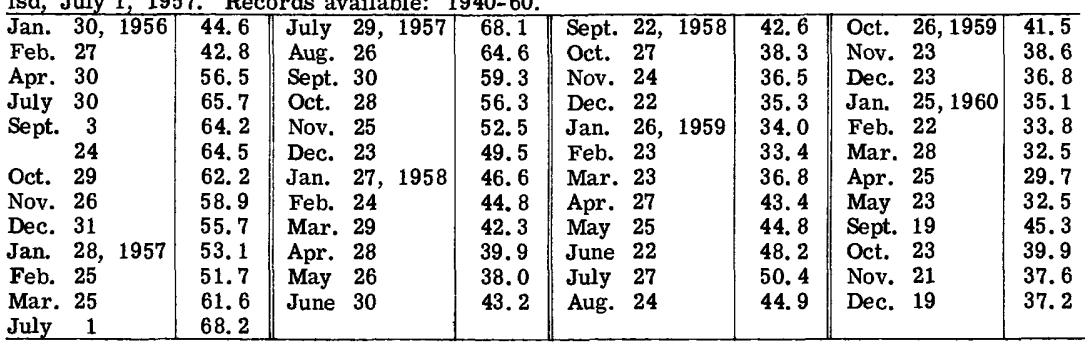

(D-8-21)18dad. J. D. Williams. Drilled irrigation water-table well in alluvial fill, diam $12 \mathrm{in}$, reported depth $140 \mathrm{ft}$. Lsd about $3,880 \mathrm{ft}$ above msl. MP hole in north side of pumpbase, $1.50 \mathrm{ft}$ above lsd. Highest water level 32.3 below lsd, Feb. 3, 1960; lowest 43.1 below lsd, Dec. 18, 1957. Records available: 1955-57, 1959-60. No measurement made in 1958 .

\begin{tabular}{|c|c|c|c|c|c|c|c|}
\hline $\begin{array}{l}\text { Feb. 1, 1955 } \\
\text { Mar. } 23 \\
\text { Dec. } 18\end{array}$ & $\begin{array}{l}36.2 \\
36.6 \\
38.3\end{array}$ & $\begin{array}{lll}\text { May } & 23, & 1956 \\
\text { July } & 11 \\
\text { Sept. } & 10\end{array}$ & $\begin{array}{l}39.4 \\
40.0 \\
40.0\end{array}$ & $\begin{array}{l}\text { Nov. } 14,1956 \\
\text { Dec. } 19 \\
\text { Jan. } 25,1957\end{array}$ & $\begin{array}{l}40.4 \\
40.2 \\
38.1\end{array}$ & \begin{tabular}{lr|} 
Dec. & 18,1957 \\
Feb. & 12,1959 \\
Feb. & 3,1960
\end{tabular} & $\begin{array}{l}43.1 \\
38.5 \\
32.3\end{array}$ \\
\hline
\end{tabular}

(D-8-25)1 2acc. Andrew Angle. Drilled unused artesian well in alluvial fill, diam 4 in. Lsd about $3,215 \mathrm{ft}$ above msl. MP top of casing, $4.00 \mathrm{ft}$ above lsd. Highest water level $3.8 \mathrm{be}-$ low lsd, Dec. 28, 1946; lowest 26.2 below lsd, June 30, 1956, Sept. 19, 1960 . Records available: $1946-60$.

\begin{tabular}{|c|c|c|c|c|c|c|c|c|c|c|c|}
\hline Nov. & $\begin{array}{l}18,1946 \\
28\end{array}$ & $\begin{array}{l}3 . \overline{9} \\
3.8\end{array}$ & Aug. & $\begin{array}{l}3,1953 \\
2\end{array}$ & $\begin{array}{l}22.7 \\
20.0\end{array}$ & Jan. & 1,1957 & 18.3 & June & 30,1958 & 18.7 \\
\hline $\begin{array}{l}\text { Dec. } \\
\text { Mar. }\end{array}$ & 10,1947 & $\begin{array}{l}\text {.. } 0 \\
4.5\end{array}$ & $\begin{array}{l}\text { Nov. } \\
\text { Feb. }\end{array}$ & $\begin{array}{ll}2 & \\
9 & 1954\end{array}$ & $\begin{array}{l}20.0 \\
20.1\end{array}$ & Feb. & $\begin{array}{l}29 \\
28\end{array}$ & $\begin{array}{l}17.4 \\
17.2\end{array}$ & Aug. & $\begin{array}{c}4 \\
26\end{array}$ & $\begin{array}{l}10.5 \\
18.5\end{array}$ \\
\hline uly & 29 & 10.2 & Apr. 1 & 19 & 18.3 & Mar. & 25 & 17.1 & Sept. & 23 & 19.2 \\
\hline eb. & 17,1948 & 10.0 & July 1 & 12 & 22.1 & Apr. & 29 & 17.9 & Oct. & 27 & 18.5 \\
\hline Apr. & 30 & 9.7 & Oct. & 18 & 21.3 & May & 21 & 17. & Nov. & 24 & 18.6 \\
\hline Feb. & 1,1949 & 14.4 & Jan. 2 & 34,1955 & 18.3 & July & 2 & 18.2 & Dec. & 22 & 18.4 \\
\hline uly & 15 & 15.1 & Apr. 1 & 19 & 20.5 & & 30 & 18.4 & Jan. & 26,1959 & 18.7 \\
\hline Det. & 28 & 15.6 & July 1 & 12 & 26.0 & Aug. & 27 & 18.1 & Feb. & 23 & 18.4 \\
\hline ar. & 1950 & 15.6 & Oct. 17 & 17 & 22.0 & Sept. & 30 & 17.8 & Mar. & 23 & 19.4 \\
\hline lly & 11 & 16.3 & Jan. & 23,1956 & 19.5 & Oct. & 28 & 17.5 & Apr. & 27 & 19.3 \\
\hline lar. & 5,1951 & 16.5 & May 2 & 28 & 24.2 & Nov. & 26 & 17.3 & May & 25 & 19.8 \\
\hline Iay & 21 & 19.0 & June 31 & 30 & 26.2 & Dec. & 23 & 17.6 & June & 22 & 20.6 \\
\hline an. & 28,1952 & 20.3 & July & 30 & 22.4 & Jan. & 27,1958 & 17.6 & July & 27 & 21.0 \\
\hline lay & 8 & 17.1 & Sept. & 1 & 23.0 & Feb. & 24 & 17.5 & Aug. & 24 & 21.3 \\
\hline ec. & 4 & 18.6 & & 22 & 24.1 & Mar. & 31 & 17.5 & Sept. & 24 & 21.5 \\
\hline n. & 53 & 17.9 & Oct. & 30 & 23.1 & Apr. & 28 & 18.2 & Oct. & 26 & 22.1 \\
\hline & 7 & 20.5 & Nov. & 26 & 21.7 & May & 26 & 18.6 & Nov. & 23 & 1.6 \\
\hline
\end{tabular}


(D-8-25)12acc--Continued.

\begin{tabular}{c|c||c|c||c|c||c|c}
\hline \multicolumn{1}{c|}{ Date } & $\begin{array}{c}\text { Water } \\
\text { level }\end{array}$ & \multicolumn{1}{|c|}{ Date } & $\begin{array}{c}\text { Water } \\
\text { level }\end{array}$ & \multicolumn{1}{|c|}{ Date } & $\begin{array}{c}\text { Water } \\
\text { level }\end{array}$ & \multicolumn{1}{|c|}{ Date } & $\begin{array}{c}\text { Water } \\
\text { level }\end{array}$ \\
\hline Dec. 22, 1959 & $\mathbf{2 1 . 5}$ & Apr. 25, 1960 & $\mathbf{2 2 . 2}$ & July 25, 1960 & $\mathbf{2 4 . 6}$ & Oct. 23, 1960 & $\mathbf{2 5 . 5}$ \\
Jan. 25, 1960 & $\mathbf{2 1 . 8}$ & May 23 & $\mathbf{2 3 . 0}$ & Aug. 22 & $\mathbf{2 5 . 6}$ & Nov. 21 & $\mathbf{2 5 . 4}$ \\
Feb. 22 & $\mathbf{2 2 . 0}$ & June 20 & $\mathbf{2 3 . 5}$ & Sept. 19 & $\mathbf{2 6 . 2}$ & Dec. 19 & $\mathbf{2 5 . 1}$ \\
Mar. 28 & $\mathbf{2 2 . 3}$ & & & & & & \\
\hline
\end{tabular}

(D-8-25)12dba. Andrew Angle. Drilled irrigation water-table well in alluvial fill, diam $12 \mathrm{in}$, reported depth $400 \mathrm{ft}$, cased to 300 , perforated 50-300. Lsd about 3, $235 \mathrm{ft}$ above msl. MP hole in casing, $0.30 \mathrm{ft}$ above lsd. Highest water level 55.6 below lsd, Mar. 13, 1950; lowest 102. 2 below lsd, June 20, 1960. Records available: 1950-60.

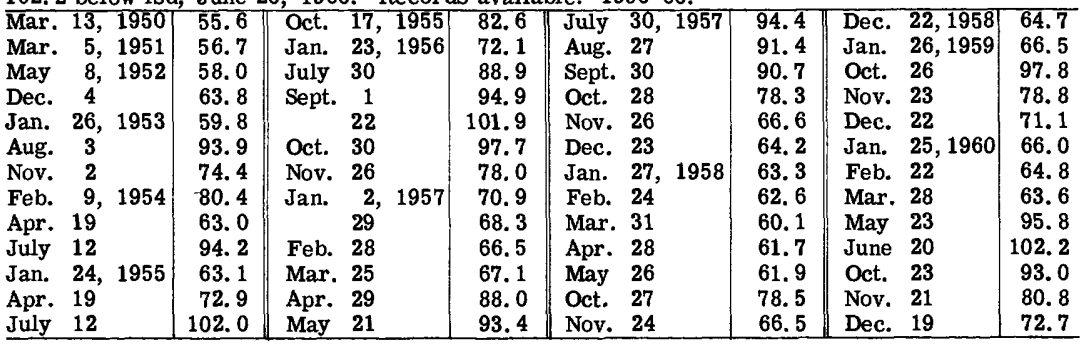

(D-8-26)33ccc. R. G. Layton. Drilled irrigation artesian well in alluvial fill, diam 16 in, reported depth $400 \mathrm{ft}$. Lsd about $3,340 \mathrm{ft}$ above msl. MP hole in pumpbase, $1.70 \mathrm{ft}$ above $1 \mathrm{sd}$. Highest water level 17. 0 below 1sd, Mar. 13, 1950; lowest 50.6 below 1sd, July 25, 1960. Records available: $1950-60$.

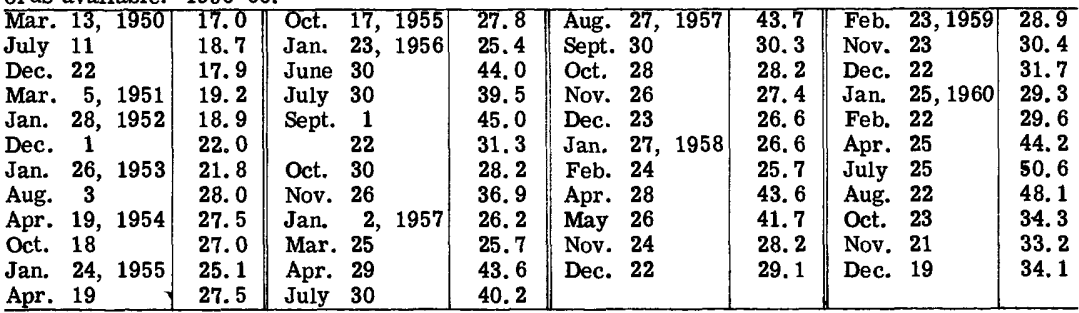

(D-10-23)35acb. 76 Ranch. Drilled stock water-table well in alluvial fill, diam 6 in, reported depth $350 \mathrm{ft}$. Lsd about 4, 465 ft above msl. MP top of casing, $1.80 \mathrm{ft}$ above lsd. Highest water level 233.1 below lsd, July 2, 1946; lowest 250.5 below lsd, Sept. 25, 1957. Records available: $1946,1949-57,1959-60$. No measurement made in 1958.

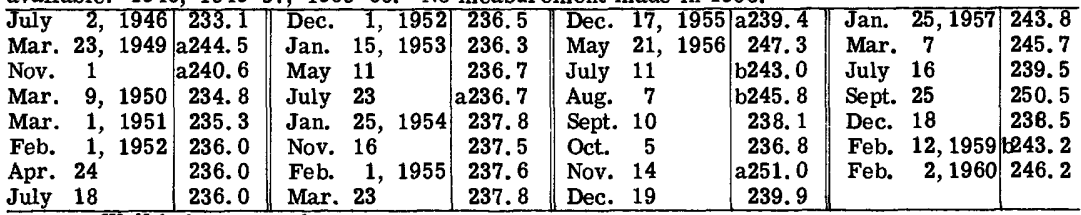

a Well being pumped.

b Well pumped recently.

\section{Greenlee County}

(D-7-31)4bcc. J. C. Merritt. Formerly Barney \& Frazier. Drilled irrigation watertable well in sand and gravel, diam $20 \mathrm{in}$, depth $75 \mathrm{ft}$. Lsd 3,544.4 ft above msl. MP hole in pumpbase, $0.60 \mathrm{ft}$ above lsd. Highest water level 24.2 below 1sd, May 5, 1941; lowest 41.0 below isd, Aug. 8, 1956. Records available: 1939-43, 1945-60.

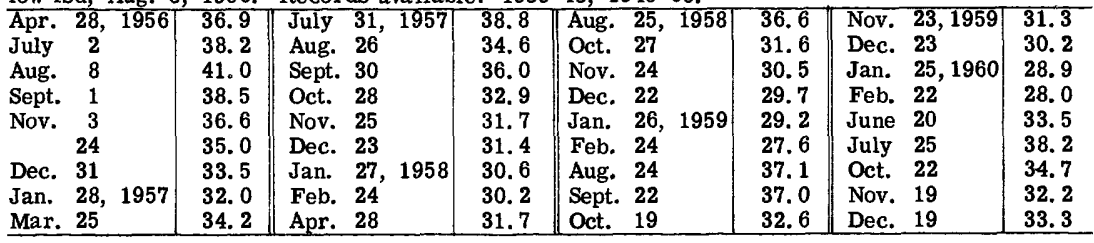


(D-8-32)32cda. Delbert Meyers. Formerly Lavar Merrill. Drilled domestic water-table well in sand and gravel, diam $4 \mathrm{in}$, depth $110 \mathrm{ft}$. Lsd 3, 716.0 ft above msl. MP top of casing, $0.50 \mathrm{ft}$ above lsd. Highest water level 22.7 below lsd, Mar. 15, 1945; lowest 38.6 below lsd, Aug. 27, 1951. Records available: 1939-60.

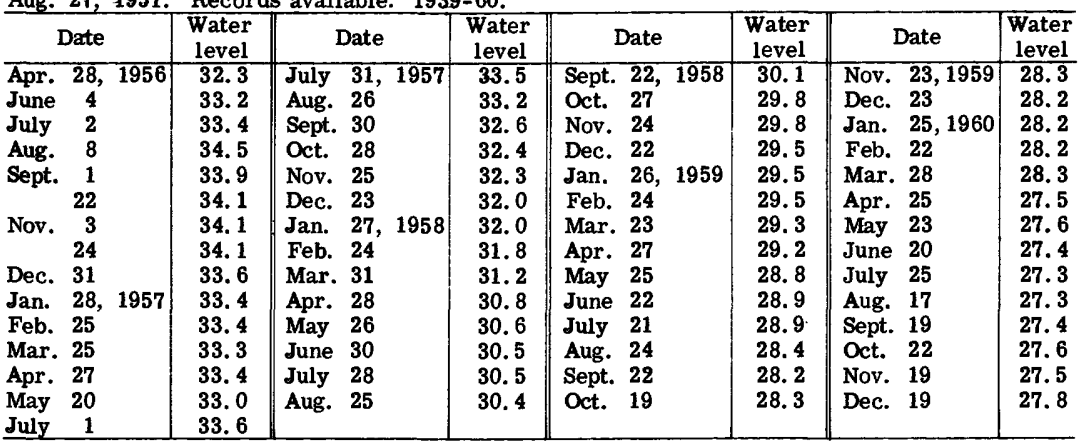

\section{Maricopa County}

(A-1-1)4aaa2. Isabell-Hartner Ranches. Drilled irrigation water-table well in sand and gravel, diam $20 \mathrm{in}$, depth $514 \mathrm{ft}$, cased to 365 , perforated 150-355. Lsd about $1,025 \mathrm{ft}$ above msl. MP below pumpbase, $0.50 \mathrm{ft}$ above lsd. Highest water level 111.1 below lsd, Feb. 2, 1953; lowest 169.4 below 1sd, Feb. 3, 1960. Records available: 1953-60. Jan. 10, 1956, 153.5 (nearby well being pumped); May 7, 153.1 (nearby well being pumped); Aug. 2, 156. 0 (nearby well being pumped); Feb. 8, 1957, 152. 0 (nearby well being pumped); Jan. 13, 1958, 166. 1; Jan. 8, 1959, 152.9; Feb. 3, 1960, 169.4.

(A-1-4)11bcb. J. B. House. Drilled domestic water-table well in sand and gravel, diam $6 \mathrm{in}$, depth $201 \mathrm{ft}$. Lsd about $1,185 \mathrm{ft}$ above msl. MP top of casing, $0.30 \mathrm{ft}$ above lsd. Highest water level 36.8 below 1sd, Feb. 21, 1946; lowest 134. 0 below 1sd, Aug. 2, 1956. Records available: 1946-57. No measurement made in 1958, 1959 or 1960. Jan. 18, 1956, 123. 7; May 4, 129.1; Aug. 2, 134.0; Nov. 2, 129.5; Feb. 13, 1957, 128.8.

(A-1-6)23daa. Logan Stillwell. Drilled domestic water-table well in sand and gravel, diam $10 \mathrm{in}$, depth $408 \mathrm{ft}$, cased to 328 . Lsd $1,375.7 \mathrm{ft}$ above msl. MP top of pumpbase, $0.50 \mathrm{ft}$ above lsd. Highest water level 229.2 below lsd, Mar. 19, 1946; lowest 418.6 below 1sd, Feb. 19, 1960. Records available: 1946, 1948-60. Feb. 28, 1956, 359.0; Aug. 2, 376.8; Nov. 2, 382.5; Feb. 12, 1957, 376.1; Jan. 14, 1958, 391.0; Jan. 6, 1959, 402.3; Feb. 19, 1960, 418.6.

(A-3-1)35baa. Otis Cook. Drilled domestic water-table well in sand and gravel, diam $6 \mathrm{in}$, depth $217 \mathrm{ft}$. Lsd about 1,130 ft above msl. MP top of casing, $0.30 \mathrm{ft}$ above lsd. Highest water level 54.5 below 1sd, Mar. 20, 1946; lowest 166. 6 below lsd, Feb. 2, 1960. Records available: $1946-60$.

\begin{tabular}{l|c|c||l|l|l|l|l|l|l|}
\hline Jan. 10,1956 & 126.9 & Aug. & 1,1956 & 133.9 & Feb. 6,1957 & 134.4 & Jan. & 7, 1959 & 153.1 \\
May 4 & 131.1 & Oct. 31 & 134.4 & Jan. 10,1958 & 145.2 & Feb. 2,1960 & 166.6 \\
\hline
\end{tabular}

(A-3-2)12caa. John M. Jacobs. Drilled unused water-table well in sand and gravel, diam $20 \mathrm{in}$, depth $809 \mathrm{ft}$. Lsd 1,309. $7 \mathrm{ft}$ above msl. MP bottom edge notch even with concrete base, $0.50 \mathrm{ft}$ above lsd. Highest water level 254. 0 below lsd, Feb. 21, 1949; lowest 414.9 below lsd, Jan. 6, 1959. Records available: 1948-59. No measurement made in 1960.

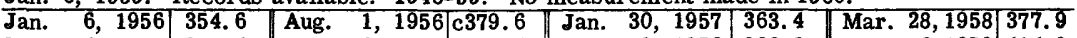
\begin{tabular}{l|l|l|l|ll|l|l|l|} 
May 4 & c375.4 & Nov. 2 & c371.4 & Jan. 9,1958 & 380.2 & Jan. 6,1959 & 414.9 \\
\hline c Nearby well being pumped.
\end{tabular}

(A-3-4)15ddd. David and Leona Gooze. Drilled unused water-table well in sand and gravel, diam 6 in, depth $193 \mathrm{ft}$, uncased. Lsd about 1, 390 ft above msl. MP top of casing, $0.50 \mathrm{ft}$ above lsd. Highest water level 165. 8 below lsd, Mar. 24, 1946; lowest 174. 7 below lsd, Aug. 2, 1956. Records available: 1946-54, 1956-59. No measurement made in 1960 . Jan. 3, 1956, 174. 5; May 4, 169.7; Aug. 2, 174. 7; Nov. 2, 170. 2; Feb. 12, 1957, 170. 3; Jan. 24, 1958, 171.1; Jan. 21, 1959, 172. 0 .

(B-1-2)13acd. Roosevelt Irrigation District. Drilled irrigation water-table well in sand and gravel, diam $20 \mathrm{in}$, depth $155 \mathrm{ft}$, perforated 40-130. Lsd $958.9 \mathrm{ft}$ above msl. MP on pumpbase, at lsd. Highest water level 39.0 below lsd, Apr. 30, 1928; lowest 81.6 below lsd, Jan. 21, 1959. Records available: 1928-31, 1934-41, 1944-45, 1947-60.

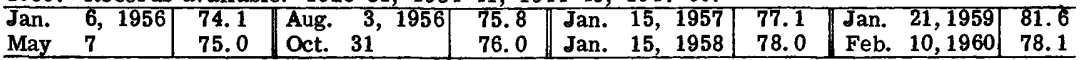


(B-1-3)34bbb. Roosevelt Irrigation District. Drilled irrigation water-table well in sand and gravel, diam $20 \mathrm{in}$, depth $200 \mathrm{ft}$, perforated 74-176. Lsd 916.7 ft above msl. MP top of casing, at lsd. Highest water level 54.2 below 1sd, June 1, 1944; lowest 77.1 below lsd, Feb. 10, 1960. Records available: $1928-58,1960$. No measurement made in 1959. Jan. 10, 1956, 68. 8; May 7, 71.5; Aug. 3, 76.1; Oct. 31, 70. 3; Jan. 16, 1957, 70.7; Feb. 11, 1958, 72. 3; Feb. 10, $1960,77.1$.

(B-2-2)4dcb. Maricopa County Municipal Water Conservation District No. 1. Drilled unused water-table well in sand and gravel, diam $20 \mathrm{in}$, depth $500 \mathrm{ft}$, perforated 204-484. Lsd about 1, $205 \mathrm{ft}$ above msl. MP top of casing, $1.00 \mathrm{ft}$ above $1 \mathrm{sd}$. Highest water level $183.7 \mathrm{below}$ lsd, May 17, 1940; lowest 291. 4 below lsd, Feb. 9, 1960. Records available: 1940-42, 19461960.

\begin{tabular}{c|c||c|c||c|c||c|c}
\hline Date & $\begin{array}{c}\text { Water } \\
\text { level }\end{array}$ & Date & $\begin{array}{c}\text { Water } \\
\text { level }\end{array}$ & Date & $\begin{array}{c}\text { Water } \\
\text { level }\end{array}$ & Date & $\begin{array}{c}\text { Water } \\
\text { level }\end{array}$ \\
\hline Mar. 6, 1956 & $\mathbf{2 6 4 . 1}$ & Aug. 2, 1956 & $\mathbf{2 6 9 . 0}$ & Jan. 25, 1957 & $\mathbf{2 6 6 . 2}$ & Jan. 20, 1959 & $\mathbf{2 8 4 . 7}$ \\
May 4 & $\mathbf{2 6 6 . 1}$ & Oct. 31 & $\mathbf{2 7 1 . 2}$ & Jan. 21, 1958 & $\mathbf{2 7 6 . 8}$ & Feb. 9, 1960 291.4 \\
\hline
\end{tabular}

(B-3-1)8abb. R. I. C. Manning. Drilled irrigation water-table well in sand and gravel, diam $20 \mathrm{in}$, reported depth $800 \mathrm{ft}$, perforated 200-800. Lsd 1, 217.4 ft above msl. MP on pumpbase, at lsd. Highest water level 246.1 below lsd, Jan. 29, 1952; lowest 310.4 below lsd, Jan. 23, 1958. Records available: 1951-58. No measurement made in 1959 or 1960 . Feb. 14, 1956, 267.8; Jan. 23, 1957, 303.5; Jan. 23, 1958, 310. 4.

(B-4-1)8daa. Maricopa County Municipal Water Conservation District No. 1. Drilled unused water-table well in sand and gravel, diam 20 in, depth $500 \mathrm{ft}$, perforated 182-484. Lsd about 1, $335 \mathrm{ft}$ above msl. MP top of cover, $4.00 \mathrm{ft}$ above $1 \mathrm{sd}$. Highest water level 180.0 below lsd, Nov. 28, 1938; lowest 287. 2 below lsd, Feb. 5, 1960. Records available: 1938, 1940-42, 1944, 1946-60. Feb. 21, 1956, 255. 5; May 4, 250. 2; Oct. 31, 253. 3; Jan. 23, 1957, 262. 4; Jan. 23, 1958, 266. 2; Jan. 16, 1959, 276.5; Feb. 5, 1960, 287.2.

(C-1-5)1aab. Charles Yokum. Drilled stock water-table well in sand and gravel, diam $6 \mathrm{in}$, depth $185 \mathrm{ft}$. Lsd $918.8 \mathrm{ft}$ above msl. MP top of casing, at lsd. Highest water level 62.8 below lsd, Oct. 25, 1946; lowest 99.2 below lsd, Aug. 29, 1960. Records available: 1946-60.

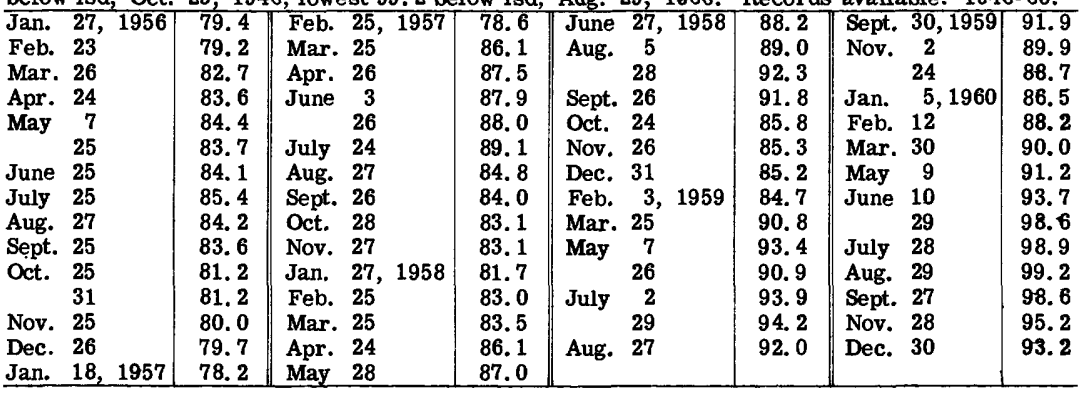

(C-1-7)15bbb. Lee C. Underdown. Drilled unused water-table well in sand and gravel, diam $20 \mathrm{in}$, depth $650 \mathrm{ft}$, perforated 164-254. Lsd $948.9 \mathrm{ft}$ above msl. MP top of casing, $0.30 \mathrm{ft}$ above lsd. Highest water level 178. 2 below lsd, Mar. 4, 1949; lowest 193.8 below lsd, Apr. 21, 1960. Records available: $1949-60$.

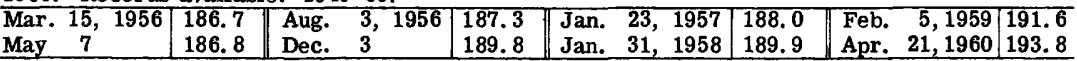

(D-2-5)15bbb. Jack Cornett. Formerly L. S. Breckler. Drilled domestic water-table well in sand and gravel, diam 6 in, depth $400 \mathrm{ft}$ (deepened from $200 \mathrm{ft}$ in 1956). Lsd 1, 214.0 ft above msl. MP top of casing, $0.80 \mathrm{ft}$ above lsd. Highest water level 40.2 below lsd, Mar. 23, 1945; lowest 144. 4 below lsd, Jan. 15, 1959. Records avallable: 1945-60. Jan. 17, 1956, 107.1; May 3, 110.5; Nov. 2, 111.3; Feb. 14, 1957, 143.3 (nearby well pumped recently); Jan. 16, 1958, 134. 3; Jan. 15, 1959, 144.4; Jan. 28, 1960, 131. 2.

(D-2-7)12ddd. L. M. Mecham. Drilled irrigation water-table well in sand and gravel, diam 20 in, depth $600 \mathrm{ft}$, perforated 250-585. Lsd 1,456.6 ft above msl. MP top of concrete base, $0.30 \mathrm{ft}$ above lsd. Highest water level 177.0 below lsd, Feb. 28, 1948; lowest 296.2 below lsd, Feb. 18, 1960. Records avallable: 1948-60. Mar. 7, 1956, 285. 8; Feb. 6, 1957, 275.9; Jan. 10, 1958, 284. 8; Jan. 9, 1959, 283.5; Feb. 18, 1960, 296. 2. 


\section{Mohave County}

(B-15-13)2ab. Formerly (B-16-13)34dd. Dr. A. E. Carter. Dug stock and domestic water-table well in sand and gravel, diam $4 \mathrm{ft}$, depth $20 \mathrm{ft}$, cased to 20 . Lsd about $1,855 \mathrm{ft}$ above msl. MP top of manhole cover, $1.00 \mathrm{ft}$ above lsd. Highest water level 11.4 below lsd, Nov. 8, 1955; lowest 18.5 below lsd, Aug. 3, 1951. Records available: 1945-60. June 18, 1956, 14. 4; Oct. 23, 16. 2; May 31, 1957, 15. 2; Feb. 17, 1958, 16.3; Jan. 28, 1959, 16.5; Mar. 8, 1960, 15.9.

(B-21-17)24cd. E. A. Kier. Drilled domestic water-table well in sand and gravel, diam $6 \mathrm{in}$, depth $120 \mathrm{ft}$. Lsd about $5,500 \mathrm{ft}$ above msl. MP top of $6-\mathrm{x} 6$-in timber, $1.00 \mathrm{ft}$ above lsd. Highest water level 101. 5 below lsd, Aug. 14, 1944; lowest 114.7 below lsd, Mar. 9, 1960. Records available: 1944-60. June 18, 1956, 110. 4; Oct. 24, 111. 3; May 31, 1957, 111.3; Feb. 18, 1958, 111.7; Jan. 29, 1959, 112.6; Mar. 9, 1960, 114.7.

\section{Navajo County}

(A-17-21)7bb. Arizona State Highway Department. Drilled unused water-table well in Coconino Sandstone, diam $10 \mathrm{in}$, depth $110 \mathrm{ft}$. Lsd 5,110.5 ft above msl. MP top west side of casing, $0.30 \mathrm{ft}$ above 1sd. Highest water level 39.5 below 1sd, June 3, 1948; lowest 44.1 below lsd, July 2, 1952. Records available: 1944-58. Measurement discontinued. May 3, 1956, 40. 41; Oct. 22, 40.41; May 17, 1957, 40.6; Oct. 18, 40.6; Apr. 17, 1958, 40. 3.

\section{Pima County}

(D-11-10)22add. Tom Greenfield. Drilled domestic and irrigation water-table well in sand and gravel, diam $20 \mathrm{in}$, reported depth $600 \mathrm{ft}$, cased to 600 , perforated 145-582. Lsd 1,914. $6 \mathrm{ft}$ above msl. MP north side of pumpbase, $0.90 \mathrm{ft}$ below lsd. Highest water level 140.7 below lsd, Feb. 28, 1940; lowest 214.9 below lsd, Dec. 30, 1957. Records available: 1940, 1942, 1945-48, 1950-57. No measurement made in 1958, 1959 or 1960. Jan. 5, 1956, 198. 5; Jan. 29, 1957, 204. 2; Dec. 30, 214. 9.

(D-12-12)16bad. Cortaro Water Users Association. Drilled unused water-table well in sand and gravel, diam 24 to $18 \mathrm{in}$, reported depth $300 \mathrm{ft}$, cased to 292, perforated 75-285. Lsd about 2, $085 \mathrm{ft}$ above msl. MP top of casing, at lsd. Highest water level 74.7 below lsd, Feb. 20, 1940; lowest 123.3 below lsd, Aug. 25, 1948. Records available: 1939-57, 1959-60. No measurement made in 1958.

\begin{tabular}{|c|c|c|c|c|c|c|c|}
\hline Date & $\begin{array}{c}\text { Water } \\
\text { level }\end{array}$ & Date & $\begin{array}{l}\text { Water } \\
\text { level }\end{array}$ & Date & $\begin{array}{l}\text { Water } \\
\text { level }\end{array}$ & Date & $\begin{array}{l}\text { Water } \\
\text { level }\end{array}$ \\
\hline $\begin{array}{lc}\text { Jan. } & 4,1956 \\
\text { Apr. } & 10\end{array}$ & $\begin{array}{l}106.8 \\
103.9\end{array}$ & $\begin{array}{l}\text { Aug. } 7,1956 \\
\text { Jan. } 29,1957\end{array}$ & $\begin{array}{l}114.5 \\
105.0\end{array}$ & $\begin{array}{l}\text { July } 26,1957 \\
\text { Dec. } 27\end{array}$ & $\begin{array}{l}113.0 \\
105.7\end{array}$ & $\begin{array}{lr}\text { Jan. } & 30,1959 \\
\text { Jan. } & 6,1960\end{array}$ & $\begin{array}{r}99.8 \\
102.4\end{array}$ \\
\hline
\end{tabular}

(D-13-11)31ccc. Jones-Avra Farms. Drilled irrigation water-table well in alluvial fill, diam $20 \mathrm{in}$, reported depth $900 \mathrm{ft}$, cased to 736. Lsd 2, $210 \mathrm{ft}$ above msl. MP hole in pumpbase, $2.50 \mathrm{ft}$ above lsd (since Aug. 6, 1953). Highest water level 276.2 below lsd, Mar. 6, 1953; lowest 327. 6 below lsd, Jan. 7, 1960. Records available: 1953-57, 1959-60. No measurement made in 1958.

\begin{tabular}{l|l||l|l|l||lr||r|r|r|r}
\hline Mar. 6, 1953 & 276.2 & Feb. 11, 1954 & 296.0 & Nov. 11, 1955 & 321.1 & Dec. 30, 1957 & 321.2 \\
Aug. 6 & 293.6 & May & 6 & 305.4 & Jan. & 6,1956 & 304.7 & Jan. 29, 1959 & 322.7 \\
Nov. 3 & 292.2 & Nov. 22 & & 307.0 & Jan. 31, 1957 & 309.6 & Jan. 7, 1960 & 327.6 \\
\hline
\end{tabular}

(D-15-10)35aaa. State of Arizona. Drilled unused water-table well in sand and gravel, diam $6 \mathrm{in}$, depth $295 \mathrm{ft}$, cased to 295 . Lsd about 2, $530 \mathrm{ft}$ above msl. MP top of casing, at lsd. Highest water level 212. 2 below lsd, Oct. 7, 1948; lowest 223. 4 below lsd, Nov. 14, 1955. Records available: 1940-42, 1944, 1946-57, 1959-60. No measurement made in 1958. Jan. 13, 1956, 219. 2; Aug. 13, 218. 2; Feb. 1, 1957, 218. 8; July 22, 219.0; Dec. 27, 219. 7; Jan. 29, 1959, 220. 3; Jan. 7, 1960, 221. 6.

(D-15-13)2cca. City of Tucson. Dug and drilled unused water-table well in sand and gra vel, diam $12 \mathrm{in}$, depth $104 \mathrm{ft}$. Lsd about $2,430 \mathrm{ft}$ above msl. MP top of casing, $3.90 \mathrm{ft}$ above 1sd. Highest water level 31.7 below lsd, July 29, 1942; lowest 71.0 below lsd, July 25-31, 1960. Records available: $1942-60$.

Highest water level for the day, from recorder graph, 1956

\begin{tabular}{l|l|l|l|l|l|l|l|l|l|l|l|l}
\hline Day & Jan. & Feb. & Mar. & Apr. & May & June & July & Aug. & Sept. & Oct. & Nov. & Dec. \\
\hline 5 & 50.4 & 48.8 & $\mathbf{4 8 . 3}$ & 48.7 & 48.8 & 52.1 & 54.2 & 52.1 & 57.6 & 60.2 & 60.3 & 59.9 \\
10 & 50.3 & 48.8 & 48.5 & 48.8 & 49.3 & 52.7 & 54.8 & 54.0 & 58.1 & 60.5 & 60.3 & 59.6 \\
15 & 49.9 & 48.5 & 48.7 & 49.0 & 49.9 & 53.4 & 51.6 & 54.4 & 58.6 & 60.4 & 60.3 & 59.6 \\
20 & 49.7 & 48.3 & 48.5 & 48.9 & 50.3 & 54.0 & 52.6 & 55.0 & 59.0 & 60.7 & 60.3 & 59.2 \\
25 & 49.5 & 48.3 & 48.3 & 49.1 & 51.0 & 54.8 & 53.6 & 56.1 & 59.5 & 60.6 & 60.0 & 59.0 \\
Eom & 49.4 & $\ldots .$. & 48.6 & 48.9 & 51.4 & $\mathbf{5 5 . 2}$ & 50.6 & 56.7 & 59.9 & 60.3 & 60.1 & 58.8 \\
\hline
\end{tabular}


(D-15-13)2cca--Continued.

1957

\begin{tabular}{|c|c|c|c|c|c|c|c|c|c|c|c|c|}
\hline Day & Jan. & Feb. & Mar. & Apr. & May & June & July & Aug. & Sept. & Oct. & Nov. & Dec. \\
\hline 5 & 58.6 & 57.8 & 57.2 & 58.1 & 60.8 & 62.4 & 64.5 & 64.0 & 60.5 & 64.9 & 60.4 & 60.9 \\
\hline 10 & 58.4 & 57.7 & 57.4 & 58.6 & 61.1 & 62.7 & 64.4 & e63. 6 & 62.2 & 64.5 & 61.0 & 60.9 \\
\hline 15 & 58.3 & 57.7 & 57.5 & 59.2 & 60.9 & 63.0 & 64.9 & 63.2 & 62.3 & 63.8 & 60.9 & 60.9 \\
\hline 20 & 58.2 & 57.5 & 57.6 & 59.7 & 61.2 & 63.5 & 64.2 & 61.1 & 64.1 & 63.2 & 60.9 & 60.8 \\
\hline 25 & 58.1 & 57.3 & 57.6 & 59.6 & 61.5 & 63.7 & 64.5 & 61.3 & 64.3 & 62.7 & 60.9 & 60.8 \\
\hline Eom & 57.9 & $\ldots$ & 57.6 & 60.0 & 61.9 & 64.2 & 64.2 & 62.4 & 64.9 & 62.0 & 61.0 & 60.8 \\
\hline \multicolumn{13}{|c|}{1958} \\
\hline 5 & 60.8 & 60.1 & 59.9 & 59.3 & 60.7 & 64.1 & 64.8 & 61.8 & 58.1 & 62.0 & 62.8 & 62.2 \\
\hline 10 & 60.6 & 59.8 & 59.6 & 59.2 & 61.1 & 64.5 & 63.6 & 57.6 & 58.8 & 61.8 & 62.8 & 62.1 \\
\hline 15 & 60.7 & 59.8 & 59.6 & 59.2 & 61.6 & 64.7 & 64.7 & 55.6 & 59.6 & 62.2 & 62.5 & 62.0 \\
\hline 20 & 60.8 & 59.8 & 59.4 & 59.5 & 62.4 & 65.2 & 63.9 & 57.3 & 61.0 & 62.6 & 62.2 & 62.0 \\
\hline 25 & 60.5 & 59.8 & 59.4 & 60.1 & 63.0 & 65.4 & 64.6 & 57.6 & 61.6 & 63.2 & 62.3 & 61.9 \\
\hline Eom & 60.2 & $\cdots$ & 59.2 & 60.4 & 63.6 & 65.6 & 60.7 & 57.9 & 61.2 & 63.2 & 62.2 & 61.9 \\
\hline \multicolumn{13}{|c|}{1959} \\
\hline 5 & 61.8 & 61.2 & 62.2 & 63.7 & 65.4 & 66.9 & 68.7 & $65 . \overline{6}$ & 63.6 & 68.1 & 67.6 & 66.4 \\
\hline 10 & 61.7 & 61.2 & 62.6 & 64.0 & 65.6 & 67.2 & 69.0 & 64.9 & 65.4 & 68.4 & 67.3 & 66.1 \\
\hline 15 & 61.6 & 61.4 & 62.8 & 64.4 & 65.9 & 67.6 & 69.2 & 63.7 & 66.2 & 68.4 & 67.0 & 66.0 \\
\hline 20 & 61.5 & 61.6 & 63.0 & 64.5 & 66.0 & 67.9 & 67.9 & 62.5 & 67.0 & 68.5 & 66.6 & 65.8 \\
\hline 25 & 61.4 & 61.8 & 63.3 & 64.9 & 66. 3 & 68.3 & 66.0 & 61.0 & 67.5 & 68.4 & 66.4 & 65.6 \\
\hline Eom & 61.2 & 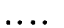 & 63.5 & 65.1 & 66.6 & 68.6 & 66.1 & 61.8 & 67.8 & 68.1 & 66.3 & 65.3 \\
\hline \multicolumn{13}{|c|}{1960} \\
\hline$\overline{5}$ & 65.2 & 61.3 & 62.0 & 64.0 & 66.0 & 68.1 & 69.8 & 70.2 & 65.4 & 68.1 & 67.9 & 68.5 \\
\hline 10 & 64.3 & 61.5 & 62.5 & 64.6 & 66. 3 & 68.3 & 70.3 & 67.8 & 66.0 & 66.8 & 68.2 & 68.4 \\
\hline 15 & 60.3 & 61.9 & 63.2 & 65.1 & 66.8 & 68.8 & 70.6 & 64.7 & 65.0 & 64.9 & 68.8 & 68.4 \\
\hline 20 & 58.1 & 61.6 & 63.3 & 65.3 & 67.1 & 69.2 & 70.9 & 66.0 & 66.4 & 65.7 & 69.0 & 68.6 \\
\hline 25 & 59.4 & 61.6 & 63.6 & 65.6 & 67.3 & 69.6 & 71.0 & 65.3 & 67.0 & 66.2 & 68.9 & 68.5 \\
\hline Eom & 60.4 & $\cdots$ & 63.6 & 65.8 & 67.5 & 69.8 & 71.0 & 65.4 & 67.8 & 66.6 & 68.7 & 68.1 \\
\hline
\end{tabular}

(D-17-9)1bcc. King Investment Co. Drilled domestic water-table well in alluvial fill, diam 6 in, reported depth $217 \mathrm{ft}$. Lsd about $2,760 \mathrm{ft}$ above msl. MP top of casing, $1.00 \mathrm{ft}$ above 1sd. Highest water level 182.9 below Isd, May 14, 1957; lowest 186.7 below Isd, Dec. 13, 1951. Records available: 1946, 1949-58. Measurement discontinued.

\begin{tabular}{|c|c|c|c|c|c|c|c|}
\hline Date & $\begin{array}{l}\text { Water } \\
\text { level }\end{array}$ & Date & $\begin{array}{l}\text { Water } \\
\text { level }\end{array}$ & Date & $\begin{array}{c}\text { Water } \\
\text { level }\end{array}$ & Date & $\begin{array}{c}\text { Water } \\
\text { level }\end{array}$ \\
\hline Aug. 22,1946 & 185.6 & Aug. 21,1952 & 183.9 & 3,1953 & 183.5 & Sept. 20,1955 & 184.3 \\
\hline Nov. 15,1949 & 183.9 & Nov. 26 & 183.4 & 9,1954 & a186. 9 & Aug. 13,1956 & 184.0 \\
\hline Oct. 18,1950 & 183. 8 & Jan. 22, 1953 & 183.4 & May & 183.8 & May 14,1957 & 182.9 \\
\hline Dec. 13,1951 & 186.7 & Apr. $\mathbf{2 3}$ & 183.5 & Aug. & 183.9 & Sept. $\mathbf{5}$ & 183.0 \\
\hline May 12,1952 & 183. 7 & July 14 & 183.7 & Nov. 23 & 185.0 & Mar. 21, 1958 & 183.1 \\
\hline
\end{tabular}

a Well being pumped.

(D-17-14)18cab. Arizona State Highway Dept. Dug observation water-table well in sand and gravel, diam 36 to $12 \mathrm{in}$, depth $124 \mathrm{ft}$, cased to 124 . Lsd about 2, $710 \mathrm{ft}$ above msl. MP top of concrete curb, $1.00 \mathrm{ft}$ above lsd. Highest water level 52.2 below lsd, Jan. 2, 1940; lowest 83. 1 below isd, June 10-25, 1960. Records available: 1939-60.

Highest water level for the day, from recorder graph, 1956

\begin{tabular}{|c|c|c|c|c|c|c|c|c|c|c|c|c|}
\hline \\
\hline & Jan. & Feb. & Mar. & Apr. & May & June & & & & Oct. & Nov. & Dec. \\
\hline 5 & 71.0 & 70.8 & 73.2 & 76.7 & 77.3 & $77 . \overline{9}$ & 78.3 & 77.6 & 75.6 & 75.6 & 75.0 & 75.4 \\
\hline 10 & 71.0 & 70.9 & 73.7 & 77.1 & 77.0 & 78.0 & 78.3 & 77.3 & 75.4 & 75.7 & 75.2 & 75.5 \\
\hline 15 & 71.2 & 71.1 & 74.4 & 77.5 & 76.9 & 78.1 & 78.3 & 76.9 & 75.3 & 75.4 & 75.3 & 75.6 \\
\hline 20 & 71.1 & 71.8 & 75.0 & 77.7 & 77.3 & 78.2 & 78.2 & 76.5 & 75.2 & 2 & 75.4 & 75.6 \\
\hline 25 & 71.0 & 72.3 & 75.5 & 77.8 & 77.6 & 78.3 & 78.0 & 76.2 & 75,3 & 75.1 & 75.4 & 75.7 \\
\hline Eom & 70.8 & .. & 76.1 & 77.7 & 77.8 & 78.4 & 77.9 & 75.9 & 75.4 & 75.0 & 75.4 & 75.7 \\
\hline \multicolumn{13}{|c|}{1957} \\
\hline 5 & 75.8 & 75.8 & 77.8 & 79.6 & 78.1 & 77.9 & 79.4 & 78.1 & 78.9 & 79.1 & 78.5 & 78.3 \\
\hline 10 & 75.8 & 75.9 & 78.3 & 79.3 & 78.0 & 78.3 & 79.6 & 79.0 & 79.1 & 78.9 & 78.4 & 78.4 \\
\hline 15 & 75.8 & 76.1 & 78.8 & 79.1 & 77.8 & 78.6 & 79.7 & 79.0 & 79.3 & 78.8 & 78.4 & 79.0 \\
\hline 20 & 75.7 & 76.4 & 79.2 & 78.9 & 77.6 & 78.9 & 79.4 & 78.7 & 79.6 & 78.8 & 78.3 & 79.1 \\
\hline 25 & 75.6 & 77.0 & 79.6 & 78.7 & 77.4 & 79.1 & 79.1 & 78.5 & 79.6 & 78.7 & 78.3 & 79.2 \\
\hline Eom & 75.7 & & 79.8 & 78.4 & 77.5 & 79.2 & 78.8 & 78.4 & 79.2 & 78.6 & 78.3 & 79.3 \\
\hline \multicolumn{13}{|c|}{1958} \\
\hline 5 & 79.6 & 80.5 & 81.0 & 81.7 & 81.1 & 79.1 & 78.3 & 76.9 & 76.6 & 75.7 & 74.8 & 75.2 \\
\hline 10 & 80.0 & 80.5 & 81.1 & 81.8 & 80.7 & 79.0 & 78.0 & 76.8 & 76.6 & 75.4 & 74.8 & 75.3 \\
\hline 15 & 80.1 & 80.6 & 81.2 & 81.7 & 80.3 & 78.9 & 77.7 & 76.6 & 76.4 & 75.3 & 74.9 & 75.4 \\
\hline 20 & 80.2 & 80.8 & 81.4 & 81.7 & 79.9 & 78.8 & 77.5 & 76.6 & 76.2 & 75.2 & 75.0 & 75.4 \\
\hline 25 & 80.4 & 80.8 & 81.5 & 81.6 & 79.6 & 78.6 & 77.4 & 76.6 & 76.1 & 75.1 & 75.1 & 75.6 \\
\hline Eom & 80.4 & . & 81.6 & 81.5 & 79.3 & 78.5 & 77.2 & 76.6 & 75.9 & 75.0 & 75.2 & 75.8 \\
\hline
\end{tabular}


(D-17-14)18cab--Continued.

\begin{tabular}{|c|c|c|c|c|c|c|c|c|c|c|c|c|}
\hline \multicolumn{13}{|c|}{1959} \\
\hline Day & Jan. & Feb. & Mar. & Apr. & May & June & July & Aug. & \begin{tabular}{|l} 
Sept. \\
.
\end{tabular} & Oct. & Nov. & Dec. \\
\hline 5 & 76.1 & 77.4 & 78.9 & 80.2 & 81.0 & 79.4 & 78.7 & 78.4 & 77.9 & 77.3 & 76.3 & 76.3 \\
\hline 10 & 76.3 & 77.7 & 79.2 & 80.4 & 80.8 & 79.2 & 78.6 & 78.4 & 77.9 & 77.2 & 76.1 & 76.4 \\
\hline 15 & 76.4 & 78.0 & 79.5 & 80.6 & 80.5 & 79.0 & 78.5 & 78.2 & 77.8 & 77.0 & 76.1 & 76.5 \\
\hline 20 & 76.6 & 78.2 & 79.5 & 80.8 & 80.2 & 78.8 & 78.4 & 78.1 & 77.8 & 76.7 & 76.1 & 76.6 \\
\hline 25 & 76.8 & 78.4 & 79.7 & 81.0 & 79.9 & 78.8 & 78.4 & 78.0 & 77.7 & 76.5 & 76.1 & 76.7 \\
\hline Eom & 77.0 & & 79.9 & 81 & 79.7 & 78.7 & 7 & 78.0 & 77.5 & 76.4 & 76.2 & 76.8 \\
\hline \multicolumn{13}{|c|}{1960} \\
\hline 5 & 77.1 & 77.9 & 79.3 & 81.4 & 82.7 & 83.0 & 82.9 & 82.6 & 81.8 & 81.8 & 81.5 & 81.7 \\
\hline 10 & 77.2 & 78.1 & 79.6 & 81.6 & 82.7 & 83.1 & 82.8 & 82.6 & 81.7 & 82.0 & 81.5 & 81.7 \\
\hline 15 & 77.3 & 78.4 & 80.0 & 81.9 & 82. 8 & 83.1 & 82.8 & 82.5 & 81. 6 & 81.9 & 81.5 & 81.8 \\
\hline 20 & 77.5 & 78.6 & 80.4 & 82.1 & 82.9 & 83.1 & 82.8 & 82.4 & 81.5 & 81.8 & 81.5 & 81.9 \\
\hline 25 & 77.6 & 78.8 & 80.7 & 82.3 & 83.0 & 83.1 & 82.7 & 82.3 & 81.6 & 81.7 & 81.6 & 82.1 \\
\hline Eom & 77.8 & $\ldots$ & 81. 0 & 82.5 & 83.0 & 82.9 & 82.6 & 82.1 & 81.7 & 81.6 & 81.6 & 82.4 \\
\hline
\end{tabular}

(D-19-16)10ddd. W. W. Foskett. Drilled stock water-table well in alluvial fill, diam 6 in, reported depth $300 \mathrm{ft}$. Lsd about $4,980 \mathrm{ft}$ above msl. MP top of casing, $1.00 \mathrm{ft}$ above lsd. Highest water level 205.1 below lsd, Sept. 21, 1955; lowest 220.3 below 1sd, Sept. 26, 1956. Records available: $1952-60$.

\begin{tabular}{|c|c|c|c|c|c|c|c|}
\hline Date & $\begin{array}{c}\text { Water } \\
\text { level }\end{array}$ & Date & $\begin{array}{l}\text { Water } \\
\text { level }\end{array}$ & Date & $\begin{array}{l}\text { Water } \\
\text { level }\end{array}$ & Date & $\begin{array}{c}\text { Water } \\
\text { level }\end{array}$ \\
\hline $\begin{array}{lll}\text { Jan. } & 30, & 1952 \\
\text { May } & 20 \\
\text { Sept. } & 17 \\
\text { Dec. } & 11 \\
\text { Jan. } & 13, & 1953\end{array}$ & $\begin{array}{l}206.6 \\
209.0 \\
207.5 \\
207.6 \\
211.5\end{array}$ & $\begin{array}{ll}\text { Apr. } & \mathbf{8}, 1953 \\
\text { Aug. } & 3 \\
\text { Feb. } & 2,1954 \\
\text { Aug. } & 24\end{array}$ & $\begin{array}{r}213.3 \\
2214.2 \\
2219.0 \\
219.8\end{array}$ & $\begin{array}{lll}\text { Nov. } & 29,1954 \\
\text { Sept. } 21,1955 \\
\text { Jan. 25, } 1956 \\
\text { Sept. } 26\end{array}$ & $\begin{array}{r}2216.2 \\
205.1 \\
213.9 \\
220.3\end{array}$ & $\begin{array}{lr}\text { Sept. } & 4,1957 \\
\text { Mar. } & 22,1958 \\
\text { Feb. } & 5,1959 \\
\text { Feb. } & 4,1960\end{array}$ & $\begin{array}{l}219.2 \\
214.6 \\
216.9 \\
218.6\end{array}$ \\
\hline
\end{tabular}

(D-22-8)19dbc. Rancho de la Osa. Drilled domestic and stock water-table well in alluvial fill, diam 8 in, reported depth $160 \mathrm{ft}$. Lsd about $3,610 \mathrm{ft}$ above msl. MP top of casing, $1.00 \mathrm{ft}$ above Isd (since July 14, 1953). Highest water level 91.2 below lsd, Oct. 18, 1950; lowest 96.2 below lsd, Jan. 5, 1960. Records available: 1950-60.

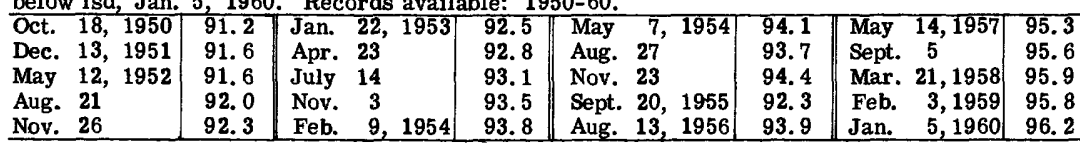

\section{Pinal County}

(D-2-10)8ccc. E. M. Little. Drilled unused water-table well in sand and gravel, diam $8 \mathrm{in}$, depth $437 \mathrm{ft}$. Lsd $1,902.5 \mathrm{ft}$ above msl. MP top of casing, $0.20 \mathrm{ft}$ above 1 sd. Highest water level 396. 8 below 1sd, Jan. 22, 1946; lowest 413.6 below lsd, Feb. 19, 1960. Records available: $1939-60$.

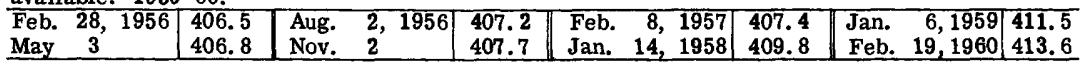

(D-4-2)13ccc. Berry. Drilled unused water-table well in alluvial fill, diam 20 in, reported depth $408 \mathrm{ft}$, cased to 408 . Lsd $1,175 \mathrm{ft}$ above msl. MP top of casing, at lsd. Highest water level 67.7 below lsd, Feb. 17, 1953; lowest 81.4 below lsd, Sept. 17, 1957. Records available: 1953-60.

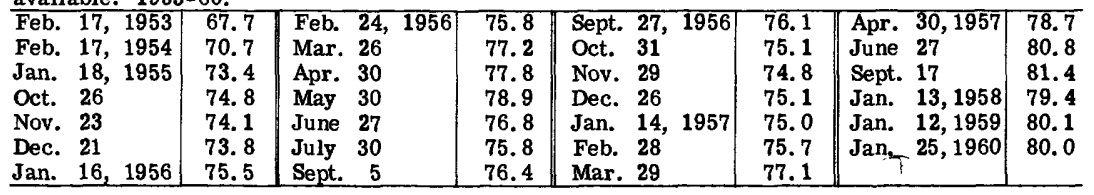

(D-4-8)2ccc. Arizona Ranches, Inc. Drilled unused water-table well in sand and gravel, diam $20 \mathrm{in}$, depth $237 \mathrm{ft}$. Lsd 1,530.5 ft above msl. MP top of casing, $0.50 \mathrm{ft}$ above $1 \mathrm{sd}$. Highest water level 158.0 below lsd, June 12, 1941; lowest 237. 6 below lsd, Jan. 14, 1958. Records available: 1941-60.

\begin{tabular}{|l|l|l|l|l|l||l|l||}
\hline Mar. 6, 1956 & 231.7 & Aug. 2, 1956 & 233.3 & Feb. 8, 1957 & 234.7 & Jan. 13, 1959 236.0
\end{tabular} \begin{tabular}{l|l|lll|l|l||l|l|l|l|} 
May 3 & 232.3 & Nov. 2 & 234.2 & Jan. 14, 1958 & 237.6 & Feb. 18, 1960 & 237.0 \\
\hline
\end{tabular} 
(D-4-9)25bcc. State of Arizona. Drilled observation water-table well in alluvial fill, diam $8 \mathrm{in}$, depth $250 \mathrm{ft}$. Lsd about $1,480 \mathrm{ft}$ above $\mathrm{msl}$. MP top of casing, $3.40 \mathrm{ft}$ above Isd. Highest water level 77. 6 below Isd, Feb. 20, 1950; lowest 123. 6 below Isd, Jan. 26, 1960 . Records available: $1950-60$.

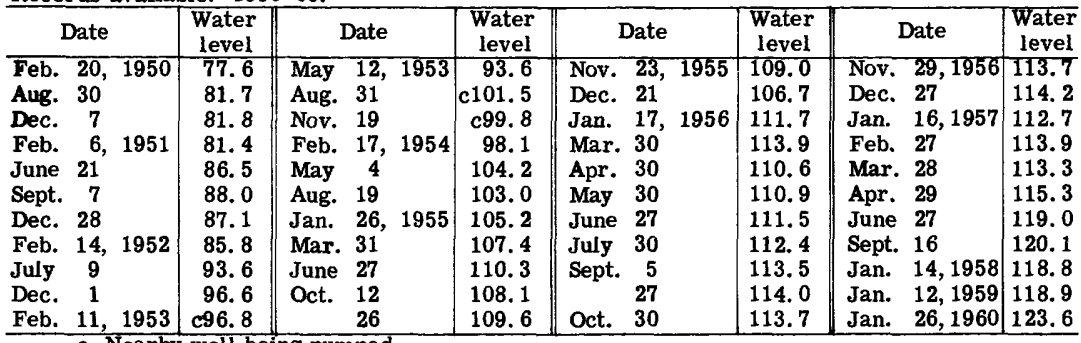

c Nearby well being pumped.

(D-4-14)21bdd. Wilkens. Drilled unused water-table well in alluvial fill, diam 20 in, reported depth $80 \mathrm{ft}$, cased to 76 . Lsd about $1,790 \mathrm{ft}$ above msl. MP top of casing, $1.00 \mathrm{ft}$ above Isd. Highest water level 5.7 below isd, Feb. 6, 1959; lowest 8.3 below lsd, Dec. 8, 1953.

Records available: $1952-55,1957-60$. No measurement made in 1956.

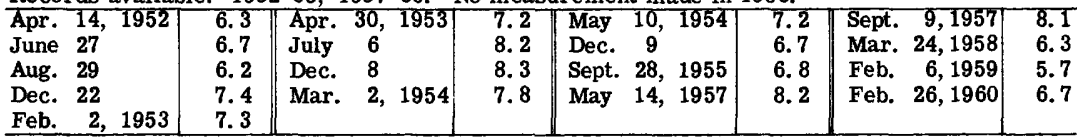

(D-5-3)25add. Decker and Miller. Drilled irrigation water-table well in sand and gravel, diam $20 \mathrm{in}$, depth $550 \mathrm{ft}$, cased to 550 . Lsd about 1, $240 \mathrm{ft}$ above $\mathrm{msl}$. MP hole in pumpbase, $0.60 \mathrm{ft}$ above lsd. Highest water level 119. 4 below Isd, Feb. 2, 1951; lowest 211.3 below isd, Jan. 17, 1957. Records available: 1951-52, 1954-58, 1960. No measurement made in 1959.

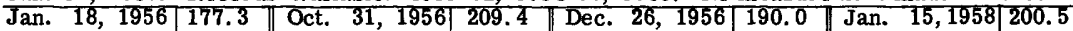
\begin{tabular}{l|l|l|l|l|l|l|l|l|l|l} 
Apr. 10 & b206.2 & Nov. 29 & 193.1 & Jan. 17, 1957 & 211.3 & Jan. 27, 1960 201.0 \\
\hline
\end{tabular} b Well pumped recently.

(D-5-9)29ada. Bureau of Indian Affairs well 76. Drilled unused water-table well in sand and gravel, diam $16 \mathrm{in}$, reported depth $616 \mathrm{ft}$, perforated 134-440. Lsd 1,520.0 ft above mśl. MP top of casing, $1.50 \mathrm{ft}$ above 1sd. Highest water level 114. 2 below Isd, Feb. 16, 1944; lowest 205.9 below 1sd, Oct. 30, 1956. Records available: 1942-57, 1959-60. No measurement made in 1958. Jan. 16, 1956, 175. 4; Apr. 18, 197.0; Oct. 30, 205.9; Jan. 16, 1957, 177. 2; Jan. 12, 1959, 197. 0; Jan. 25, 1960, 198.8.

(D-6-8)21 dcc. W. H. Simmons. Drilled irrigation water-table well in alluvial fill, diam $20 \mathrm{in}$, reported depth $400 \mathrm{ft}$. Lsd about $1,470 \mathrm{ft}$ above msl. MP hole in pumpbase, $1.50 \mathrm{ft}$ above Isd. Highest water level 117. 3 below Isd, Feb. 5, 1952; lowest 171. 6 below isd, Oct. 12, 1955. Records available: 1952-56, 1960. No measurement made in 1957 . 1958 or 1959.

\begin{tabular}{lr|r||l|l|l|l|l|l|l|l|}
\hline Feb. & 5,1952 & 117.3 & Jan. 25, 1955 & 139.6 & Oct. 12, 1955 & 171.6 & Apr. 18, 1956 166.5 \\
Feb. 18, 1953 & 130.4 & Mar. 31 & 166.0 & Jan. 19,1956 & 152.8 & Jan. 26, 1960 & 156.2 \\
Feb. 17, 1954 & 148.7 & & & & & & & & \\
\hline
\end{tabular}

Feb. 17, 1954 148.7

b Well pumped recently.

(D-6-11)27bbd. Grathan and Carney. Drilled stock water-table well in alluvial fill, diam $4 \mathrm{in}$, reported depth $300 \mathrm{ft}$. Lsd about 2, $300 \mathrm{ft}$ above msl. MP top of casing, $1.00 \mathrm{ft}$ above $1 \mathrm{sd}$. Highest water level 28.5 below Isd, Feb. 10, 1960; lowest 33. 1 below 1sd, July 7, 1953, Feb. 25, 1954. Records available: $1953-54,1956,1960$. No measurement made in 1955, 1957, 1958 or 1959.

\begin{tabular}{|c|c|c|c|c|c|c|c|}
\hline $\begin{array}{lll}\text { Jan. } & 8, & 1953 \\
\text { July } & 7 \\
\text { Dec. } & 9\end{array}$ & $\begin{array}{l}32.1 \\
33.1 \\
32.5\end{array}$ & $\begin{array}{l}\text { Feb. } 25,1954 \\
\text { May } 13\end{array}$ & $\begin{array}{r}33.1 \\
234.6\end{array}$ & $\begin{array}{l}\text { Aug. } 19,1954 \\
\text { Dec. } 6\end{array}$ & $\begin{array}{l}32.3 \\
29.6\end{array}$ & $\mid \begin{array}{ll}\text { Sept. } & 25,1956 \\
\text { Feb. } & 10,1960\end{array}$ & $\begin{array}{l}30.4 \\
28.5\end{array}$ \\
\hline
\end{tabular}

$$
\text { a Well being pumped. }
$$

(D-6-16)33bad. Fred Ash. Drilled unused water-table well in alluvial fill, diam 12 in, reported depth $60 \mathrm{ft}$. Lsd about $2,100 \mathrm{ft}$ above msl. MP top of casing, $1.00 \mathrm{ft}$ above $1 \mathrm{sd}$. Highest water level 8.2 below Isd, Jan. 27, 1956; lowest 18.5 below lsd, Dec. 7, 1953. Records available: $1949-60$.

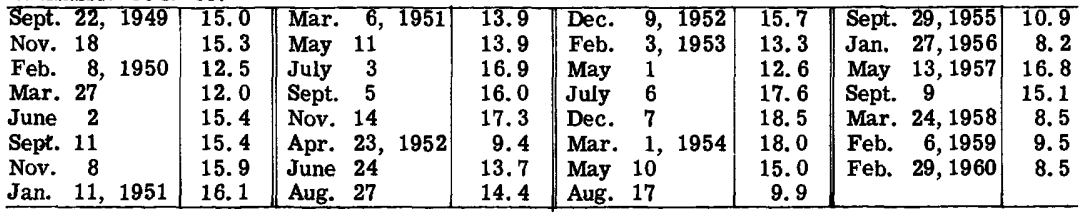


(D-7-7)11cdd. E. C. Grasty. Drilled irrigation water-table well in sand and gravel, diam $20 \mathrm{in}$, depth $460 \mathrm{ft}$, perforated 100-430. Lsd 1,498.2 ft above msl. MP at pumpbase and concrete curb, 2. $00 \mathrm{ft}$ above lsd. Highest water level 85.9 below lsd, Mar. 11, 1942; lowest 222.6 below lsd, Jan. 27, 1960. Records available: 1942-57, 1959-60. No measurement made in 1958. Jan. 18, 1956, 204. 8; Jan. 17, 1957, 202. 2; Jan. 13, 1959, 217. 5; Jan. 27, 1960, 222.6.

(D-8-6)30dad. Chiu Chuischu Ranch. Drilled irrigation water-table well in sand and gravel, diam 20 in, depth $296 \mathrm{ft}$, perforated 69-200. Lsd about $1,510 \mathrm{ft}$ above msl. MP top of casing, at 1sd. Highest water level 108.6 below 1sd, July 28, 1948; lowest 187. 3 below 1sd, Jan. 28, 1960. Records available: 1948-52, 1954-60. Jan. 18, 1956, 150.9; Apr. 10, 179. 1; July 12, 171. 2; Jan. 17, 1957, 167. 4; Jan. 17, 1958, 174.4; Jan. 16, 1959, 178. 8; Jan. 28, 1960, 187.3.

(D-8-17)19dad. Carmen and Edward Mungaray. Drilled domestic water-table well in alluvial fill, diam 6 in, reported depth $46 \mathrm{ft}$. Lsd about $2,400 \mathrm{ft}$ above $\mathrm{msl}$. MP top of casing, $1.00 \mathrm{ft}$ above 1sd. Highest water level 5.7 below lsd, Jan. 26, 1956; lowest 18. 2 below lsd, Dec. 7, 1953. Records available: 1949-58. No measurement made in 1959 or 1960.

\begin{tabular}{|c|c|c|c|c|c|c|c|}
\hline Date & $\begin{array}{l}\text { Water } \\
\text { level }\end{array}$ & Date & $\begin{array}{l}\text { Water } \\
\text { level }\end{array}$ & Date & $\begin{array}{l}\text { Water } \\
\text { level }\end{array}$ & Date & $\begin{array}{l}\text { Water } \\
\text { level }\end{array}$ \\
\hline Sept. 21, 1949 & 11.6 & Jan. 11,1951 & 14.8 & June 24,1952 & 15.2 & May 10,1954 & 17.3 \\
\hline Nov. 18 & 13.6 & Mar. 6 & 14.9 & Aug. 26 & 13.7 & Dec. 9 & 7 \\
\hline Feb. $\quad 8,1950$ & 12.1 & May 11 & 15.2 & Dec. & 16.5 & Sept. 29,1955 & 8 \\
\hline Mar. 27 & 12.3 & July & & 3, 1953 & 16 & Jan. 26 & \\
\hline June & 14.3 & Sept. & 13.7 & May & 16.6 & May 13,1957 & 13 \\
\hline Sept. 11 & 12.3 & Nov. 14 & 12.2 & July & b19. 1 & Sept. 9 & 13.1 \\
\hline Nov. & 14.6 & Apr. 24,1952 & 13.4 & Dec. & 18.2 & Mar. 24, 1958 & 9.4 \\
\hline
\end{tabular}

(D-9-10)19aaa. Clemens Cattle Co. Drilled unused water-table well in alluvial fill, diam $20 \mathrm{in}$, depth $600 \mathrm{ft}$, cased to 486, perforated $240-486$. Lsd about $1,830 \mathrm{ft}$ above msl. MP top of casing, $1.50 \mathrm{ft}$ above lsd. Highest water level 181.1 below 1sd, Mar. 28, 1955; lowest 208.7 below lsd, July 5, 1956. Records available: 1953-60.

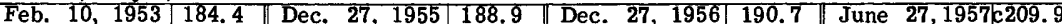

\begin{tabular}{l|l|l|l|l|l|l|l|l|l|} 
Feb. 11, 1954 & 187.6 & Apr. 17, 1956 & 200.6 & Jan. 18, 1957 & 191.0 & Sept. 16 & c210.5
\end{tabular}

\begin{tabular}{l|l|l|l|l|l|l|l|l|l|} 
Jan. 12, 1955 & 188.0 & July & 5 & 208.7 & Feb. 27 & c204.1 & Jan. 3, 1958191.5
\end{tabular}

\begin{tabular}{l|l|l|l|l|l|l|l|l|} 
Mar. 28 & 181.1 & Oct. 30 & 191.9 & Mar. 28 & c202.7 & Jan. 16, 1959 193.0
\end{tabular}

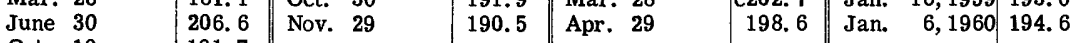

\begin{tabular}{l|l|} 
Oct. 10 & 191.7 \\
\hline c Nearby well being pumped.
\end{tabular}

(D-9-13)7ccc. Foreman. Drilled stock water-table well in alluvial fill, diam 6 in, reported depth $275 \mathrm{ft}$. Lsd about $3,115 \mathrm{ft}$ above msl. MP top of casing, $1.00 \mathrm{ft}$ above lsd. Highest water level 43.0 below lsd, Sept. 23, 1955; lowest 111.5 below lsd, Jan. 6, 1953. Records available: $1952-60$.

\begin{tabular}{|c|c|c|c|c|c|c|c|c|}
\hline $\begin{array}{l}\text { Oct. } \\
\text { Jan. } \\
\text { July } \\
\text { Dec. }\end{array}$ & $\begin{array}{ll}2, & 1952 \\
6, & 1953 \\
7 & \\
9 & \\
\end{array}$ & $\begin{array}{r}59.6 \\
111.5 \\
87.7 \\
82.0 \\
\end{array}$ & \begin{tabular}{lc|} 
Feb. & 25,1954 \\
May & 13 \\
Aug. & 20 \\
Dec. & 6
\end{tabular} & $\begin{array}{r}996.8 \\
88.0 \\
77.8 \\
101.5 \\
\end{array}$ & $\begin{array}{l}\text { Sept. 23, } 1955 \\
\text { Sept. 25, } 1956 \\
\text { Sept. 13, } 1957\end{array}$ & $\begin{array}{l}43.0 \\
96.0 \\
74.7\end{array}$ & $\begin{array}{lr}\text { Mar. } & 26,1958 \\
\text { Feb. } & 4,1959 \\
\text { Feb. } & 10,1960\end{array}$ & $\begin{array}{l}49.5 \\
65.2 \\
50.8\end{array}$ \\
\hline
\end{tabular}

(D-9-15)36bbc. A. I. Kendall. Dug domestic water-table well in granitic rocks, diam $48 \mathrm{in}$, reported depth $92 \mathrm{ft}$. Lsd about $4,500 \mathrm{ft}$ above $\mathrm{msl}$. MP top of floor board, $0.40 \mathrm{ft}$ above 1sd. Highest water level 61. 2 below lsd, Feb. 26, 1960; lowest 82.1 below Isd, Aug. 16, 1954. Records available: $1949-60$.

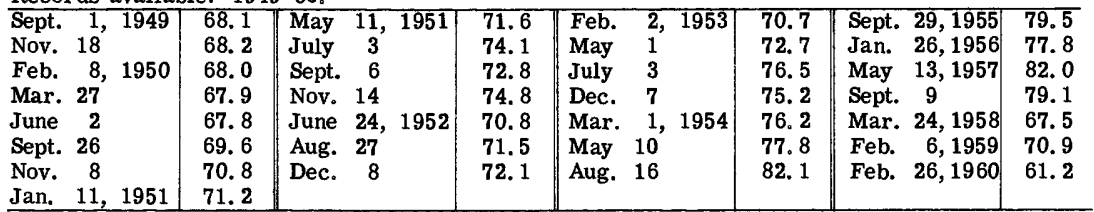

(D-10-9)16ccc. L. Kinney. Drilled unused water-table well in alluvial fill, diam 20 in, depth $400 \mathrm{ft}$, cased to 392 , perforated $170-378$. Lsd about $1,780 \mathrm{ft}$ above msl. MP top of casing, $2.00 \mathrm{ft}$ above 1sd. Highest water level 209.8 below 1sd, Feb. 10, 1954; lowest 260.0 below 1sd, Jan. 7, 1960. Records available: 1954-60.

\begin{tabular}{l|l|l|l||ll||l|l|l|}
\hline Feb. 10, 1954 & 209.8 & Apr. 30, 1956 & 227.2 & Oct. 30, 1956 & 231.5 & Apr. 29, 1957 236.7
\end{tabular}

Jan. 12, 1955 217.9 May 30

Oct. $24 \quad 234.0$ June 27

\begin{tabular}{l|l|ll} 
Nov. 22 & 224.6 & July & 30
\end{tabular}

Dec. 21

Feb. 24, 1956

224. 4 Sept. 5

Mar. 26

231.7

June 27 238. 5

229.2

230.8

Nov. 29

232. 0

Jan. 18, 1957

231.2

Sept. 16

244.4

232. 7

Feb. 27

8 Jan. 3, 1958232.0

231.4 Jan. 16, 1959 253. 2

\begin{tabular}{|l|l|l|l|l|l|l|l|}
226.6 & & & 236.1 & Jan. 7, 1960 260.0
\end{tabular}




\section{Santa Cruz County}

(D-22-13)35dcd. T. T. Pendleton. Drilled irrigation water-table well in sand and gravel, diam $20 \mathrm{in}$, depth $88 \mathrm{ft}$, cased. Lsd about 3, $430 \mathrm{ft}$ above msl. MP south side of $1 \frac{1}{2}-$ in pipe, $0.50 \mathrm{ft}$ above $1 \mathrm{sd}$. Highest water level 9.9 below lsd, Feb. 8, 1960; lowest 53.7 below lsd, Feb. 9, 1954. Records available: 1939-57, 1959-60. No measurement made in 1958.

\begin{tabular}{|c|c|c|c|c|c|c|c|}
\hline Date & $\begin{array}{c}\text { Water } \\
\text { level }\end{array}$ & Date & $\begin{array}{c}\text { Water } \\
\text { level }\end{array}$ & Date & $\begin{array}{c}\text { Water } \\
\text { level }\end{array}$ & Date & $\begin{array}{c}\text { Water } \\
\text { level }\end{array}$ \\
\hline $\begin{array}{l}\text { an. } 6,1956 \\
\text { eb. } 22 \\
\text { ar. } 23\end{array}$ & $\begin{array}{l}10.2 \\
10.6 \\
11.0\end{array}$ & $\begin{array}{l}\text { Apr. } 23,1956 \\
\text { May } 23 \\
\text { Aug. } 10\end{array}$ & $\begin{array}{l}12.1 \\
13.0 \\
15.7\end{array}$ & $\begin{array}{l}\text { Nov. } 7,1956 \\
\text { Dec. } 6 \\
\text { Jan. } 31,1957\end{array}$ & $\begin{array}{l}20.9 \\
22.2 \\
25.4\end{array}$ & $\begin{array}{l}\text { Dec. } 2 \\
\text { Jan. } 2 \\
\text { Feb. }\end{array}$ & $\begin{array}{r}40.2 \\
23.5 \\
9.9\end{array}$ \\
\hline
\end{tabular}

(D-22-15)12aad. V. Farley. Drilled domestic water-table well in alluvial fill, diam 8 in, reported depth $60 \mathrm{ft}$, cased to 60 . Lsd about 4,035 ft above msl. MP top of concrete base, $1.00 \mathrm{ft}$ above lsd (since Jan, 13, 1953). Highest water level 10.1 below lsd, Jan. 25, 1956; lowest 26.0 below lsd, June 8, 1954. Records available: 1949, 1952-60.

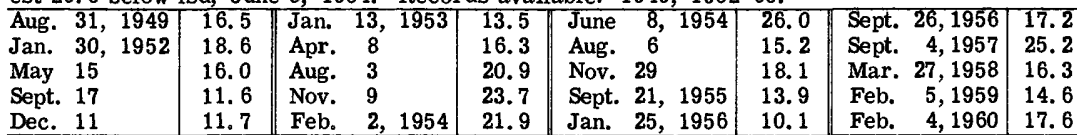

(D-23-14)27baa. Ramon Michelena. Dug unused water-table well in sand and gravel, diam $5 \mathrm{ft}$, depth $36 \mathrm{ft}$, concrete casing to 9, open-hole 9-36. Lsd about 3,550 ft above msl. MP top of concrete casing, $0.30 \mathrm{ft}$ above lsd. Highest water level 12.6 below lsd, Jan. 6, 1960; lowest 21.8 below lsd, July 28, 1948. Records available: 1939-57, 1959-60. No measurement made in 1958 .

\begin{tabular}{|c|c|c|c|c|c|c|c|c|}
\hline $\begin{array}{l}\text { Jan. } \quad 6,1956 \\
\text { Feb. } 22 \\
\text { Mar. } 23 \\
\text { Apr. } 23\end{array}$ & $\begin{array}{l}16.7 \\
16.6 \\
16.5 \\
16.5\end{array}$ & $\begin{array}{lr}\text { May } & 23,1956 \\
\text { June } & 21 \\
\text { Aug. } & 10 \\
\text { Oct. } & 9\end{array}$ & $\begin{array}{l}16.9 \\
17.8 \\
16.1 \\
18.1\end{array}$ & $\begin{array}{lcc}\text { Nov. } & 7,1956 \\
\text { Dec. } & 6 & \\
\text { Jan. } & 31,1957 \\
\text { July } & 17 & \\
\end{array}$ & $\begin{array}{l}18.0 \\
17.5 \\
16.9 \\
19.1\end{array}$ & $\begin{array}{l}\text { Dec } \\
\text { Jan. } \\
\text { Jan. }\end{array}$ & $\begin{array}{r}23,1957 \\
28,1959 \\
6,1960\end{array}$ & $\begin{array}{l}19.1 \\
17.5 \\
12.6\end{array}$ \\
\hline
\end{tabular}

(D-24-17)11 cab. U.S. Geol. Survey. Driven observation water-table well in alluvial fill, diam 1 in, depth $23 \mathrm{ft}$, cased to 23 . Lsd about 4,650 ft above msl. MP top of casing, $1.40 \mathrm{ft}$ above lsd. Highest water level 1.8 below lsd, Dec. 30, 1955; lowest 7.8 below lsd, July 1 , 1960. Records available: $1949-60$.

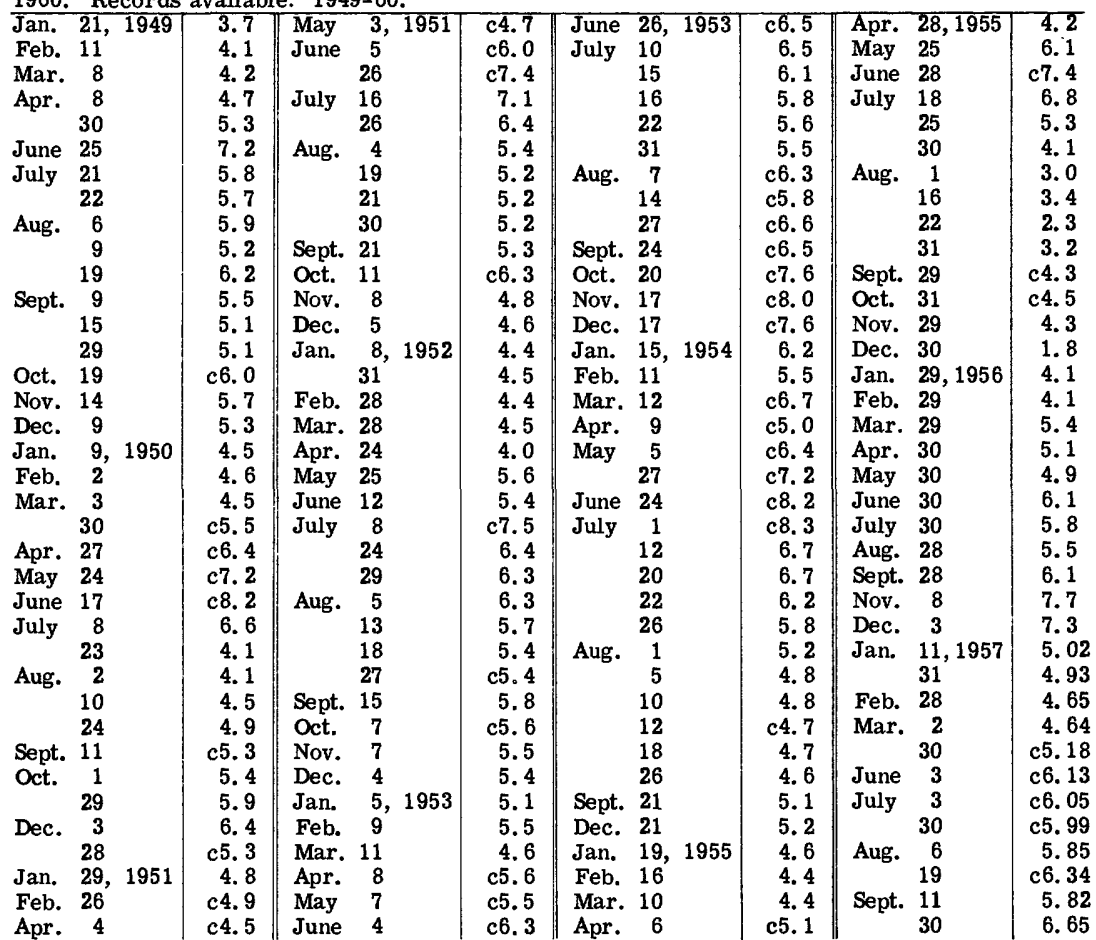


(D-24-17)11 cab--Continued.

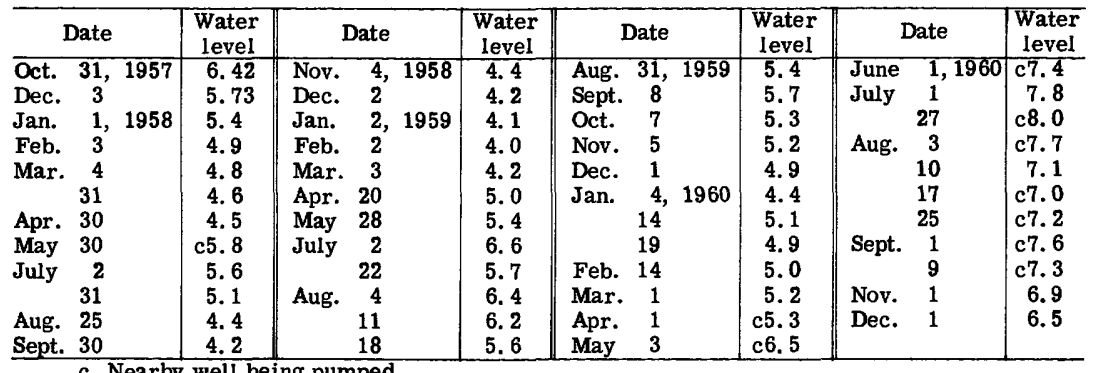

c Nearby well being pumped.

\section{Yavapai County}

(B-11-5)25dab. Mr. Towne. Drilled unused water-table well in sand and gravel, diam $8 \mathrm{in}$, depth $212 \mathrm{ft}$, cased to 55 . Lsd about $4,485 \mathrm{ft}$ above msl. MP top of casing, $1.40 \mathrm{ft}$ above Isd. Highest water level 23.6 below Isd, July 16, 1952; lowest 40.8 below Isd, Aug. 4, 1951. Records available: 1946, 1948-49, 1951-57, 1959-60. No measurement made in 1958. June 21, 1956, 34.6; Oct. 22, 36.6; May 28, 1957, 36.5; Dec. 18, 37. 2; Mar. 3, 1959, 34. 3; Mar. 15, $1960,32.2$.

(B-13-6)9dd. J. W. Ropeter. Dug irrigation water-table well in sand and gravel, diam $6 \mathrm{ft}$, depth $22 \mathrm{ft}$ (well has been deepened), wood-lined. Lsd about 2, $500 \mathrm{ft}$ above msl. MP through bolt hole of pumpbase, at 1sd. Highest water level 14.0 below lsd, Jan. 17, 1945; lowest 18. 1 below led, June 1, 1955. Records available: 1945-49, 1951-57, 1959-60. No measurement made in 1958. June 21, 1956, 29. 7 (well being pumped); Oct. 23, 32.8 (well being pumped); May 28, 1957, 17. 2; Dec. 18, 17. 2; Mar. 3, 1959, 16.8; Mar. 15, 1960, 15. 9.

(B-14-4)33ab. C. C. McLain. Drilled unused water-table well in sand and gravel, diam $16 \mathrm{in}$, depth $85 \mathrm{ft}$ (well has been deepened). Lsd about 4,340 ft above msl. MP hole in casing, $0.50 \mathrm{ft}$ above Isd. Highest water level 11.0 below Isd, Apr. 14, 1945; lowest 19.1 below Isd, Oct. 23, 1956. Records available: 1944-49, 1951-57, 1959-60. No measurement made in 1958. June 21, 1956, 18. 2; Oct. 23, 19.1; May 28, 1957, 17.8; Dec. 18, 19.0; Mar. 3, 1959, 18.0; Mar. 15, 1960, 15.2 .

\section{Yuma County}

(B-5-16)10ddd. Crowder Cattle Co. Drilled unused water-table well in sand and gravel, diam 16 in, depth $164 \mathrm{ft}$. Lsd about $730 \mathrm{ft}$ above msl. MP top of caeing, $0.50 \mathrm{ft}$ above Isd. Highest water level 112. 6 below Isd, Feb. 21, 1946; lowest 114. 4 below Isd, Jan. 22, 1957, Jan. 30, 1958. Records available: 1946, 1948-60. Mar. 16, 1956, 114. 0; Jan. 22, 1957, 114. 4; Jan. 30, 1958, 114. 4; Jan. 29, 1959, 114.3; Apr. 19, 1960, 114. 3.

(C-8-16)28bda. Bob Anderson. Drilled domestic water-table well in sand and gravel, diam 16 in, depth $419 \mathrm{ft}$, cased to 416 . Lsd about $330 \mathrm{ft}$ above msl. MP top of casing, at isd. Highest water level 63.0 below Isd, Mar. 3, 1960; lowest 87.0 below Isd, Mar. 3, 1954. Records available: 1945-47, 1949-60. Feb. 2, 1956, 84. 2; Jan. 31, 1957, 80. 2; Jan. 21, 1958, 75. 6; Feb. 10, 1959, 73.1; Mar. 3, 1960, 63.0.

(C-9-22)17ddd. Archie J. Griffin. Drilled unused water-table well in sand and gravel, diam $16 \mathrm{in}$, depth $195 \mathrm{ft}$. Lsd $210.5 \mathrm{ft}$ above msl. MP top of casing, $1.50 \mathrm{ft}$ above Isd. Highest water level 52.5 below Isd, Nov. 15, 1960; lowest 97.6 below Isd, Sept. 5, 1946. Records available: $1945-58,1960$. No measurement made in 1959 . All 1960 measurements made by U. S. Bureau of Reclamation. Feb. 1, 1956, 68.1; Jan. 30, 1957, 66.0; Jan. 20, 1958, 64.6; Jan. 15, 1960, 56. 8; July 13, 55. 8; Nov. 15, 52.5.

\section{References}

Armstrong, C. A., and Yost, C. B., Jr., 1958, Geology and ground-water resources of the Palomas Plain-Dendora Valley area, Maricopa and Yuma Counties, Arizona: Arizona State Land Dept., Water Resources Rept. 4, 49 p.

Brown, R. H. , Harshbarger, J. W., and Thomas, H. E., 1956, Analysis of basic data concerning ground water in the Yuma area, Arizona: U.S. Geol. Survey open-file rept., 117 p. 
Coates, D. R., 1955, Memorandum on the geology and ground-water resources of Dripping Springs basin, Gila and Pinal Counties, Arizona: U. S. Geol. Survey open-file report, $11 \mathrm{p}$.

Coates, D. R., and Cushman, R. L., 1955, Geology and ground-water resources of the Douglas basin, Arizona: U. S. Geol. Survey Water-Supply Paper 1354, 56 p.

Cosner, O. J., 1962, Ground water in the Wupatki and Sunset Crater National Monuments, Coconino County, Arizona: U. S. Geol. Survey Water-Supply Paper 1475-J, p. 357-374.

Hardt, W. F., Cahill, J. M. , and Booher, M. B., 1958, Annual report on ground water in Arizona--spring 1957 to spring 1958: Arizona State Land Dept., Water Resources Rept. 5, $60 \mathrm{p}$.

Hardt, W. F., Stulik, R. S., and Booher, M. B., 1959, Annual report on ground water in Arizona--spring 1958 to spring 1959: Arizona State Land Dept., Water Resources Rept. 6, $61 \mathrm{p}$. 1960, Annual report on ground water in Arizona--spring 1959 to spring 1960: Arizona State Land Dept., Water Resources Rept. 7, 73 p.

Harshbarger, J. W., and others, 1957, Annual report on ground water in Arizona--spring 1956 to spring 1957: Arizona State Land Dept., Water Resources Rept. 2, 42 p.

Heindl, L. A., 1955, Memorandum on geology and ground-water resources in the vicinity of Oracle, Pinal County, Arizona: U. S. Geol. Survey open-file report, 11 p. 1955, Ground-water conditions between Oracle and Oracle Junction, Pinal County, Arizona: U.S. Geol. Survey open-file report, $5 \mathrm{p}$.

Heindl, L. A., and McCullough, R. A., 1960, Geology and the availability of water in the lower Bonita Creek area, Graham County, Arizona: U. S. Geol. Survey open-file report, 81 p.

Johnson, P. W. , and Cahill, J. M., 1955, Ground-water resources and geology of the Gila Bend and Dendora areas, Maricopa County, Arizona: U.S. Geol. Survey open-file report, 43 p.

Kam, William, 1957, Interim report on the ground-water resources of the McMullen valley area, Maricopa, Yavapai, and Yuma Counties, Arizona: U. S. Geol. Survey open-file report, $27 \mathrm{p}$.

Metzger, D. G. , 1957, Geology and ground-water resources of the Harquahala Plains area, Maricopa and Yuma Counties, Arizona: Arizona State Land Dept., Water Resources Rept. 3, $40 \mathrm{p}$.

Wolcott, H. N., Skibitzke, H. E., and Halpenny, L. C. , 1956, Water resources of the Bill Williams River valley pear Alamo, Arizona: U.S. Geol. Survey Water-Supply Paper 1360-D, p. 291-319. 


\section{CALIFORNIA}

By E. J. McClelland, W. R. Moyle, Jr., and R. E. Evenson

The observation-well program in California was continued in the period 1956 through 1960 in cooperation with the California Department of Water Resources and Santa Barbara County. Figure 3 shows the location of observation wells included in this report.

\section{We11-Numbering System}

The well-numbering system shows the location of wells according to the rectangular system of public-land surveys. Water-Supply Paper 991 contains a cross-reference table of previous numbers and location symbols. For well 5/10-6N1, in Antelope Valley in Los Angeles County, the segment of the number before the hyphen indicates the township and range (T. 5 N., R. $10 \mathrm{~W}$.). Letters indicating cardinal directions appear in this part of the symbol if a basin or area spans two or more quadrants of a particular base and meridian. The digits between the hyphen and the letter indicate the section (sec. 6), and the letter indicates the 40-acre tract within the section as shown by the following diagram. Within the 40 -acre tract, the wells are numbered serially as indicated by the final digit of the symbol. Thus, well 5/10-6N1 was the first well listed by the U.S. Geological Survey in the $S W \frac{1}{4} S W \frac{1}{4} \sec .6, T$. 5 N., R. 10 W. For a well located only approximately, the symbol is shortened to the designation of township, range and section only. For areas which have never been subdivided by public-land surveys, the rectangular system has been projected, commonly after private surveys or after projections made by local officials for purposes of land assessment. The description and records are given by counties in alphabetical sequence and for each county by valleys or ground-water basins. Thus, each group of data pertains to a distinct ground-water area as indicated by subheadings in the report. Under each subhead the records are presented in numerical order of the location symbols.

\begin{tabular}{|c|c|c|c|}
\hline D & C & B & A \\
\hline E & F & G & H \\
\hline M & L & K & J \\
\hline N & P & Q & R \\
\hline
\end{tabular}

Well Descriptions, and Water-Level Measurements

Water levels are reported in feet below land-surface datum, unless otherwise indicated.

\section{Del Norte County}

\section{Smith River Basin}

16/1W-17K1. Dan Berquist. $1.5 \mathrm{mi}$ north of Crescent City. Drilled domestic water-table well in Battery Formation of Pleistocene age, diam 6 in, depth $40 \mathrm{ft}$, perforated 34-39. Lsd $49 \mathrm{ft}$ above msl. MP top of casing, $1.00 \mathrm{ft}$ above lsd. Highest water level 11.4 below lsd, Feb. 18, 1959; lowest 24.0 below Isd, Jan. 27, 1960. Records available: 1953-54, 1958-60. No measurement made 1955-57.

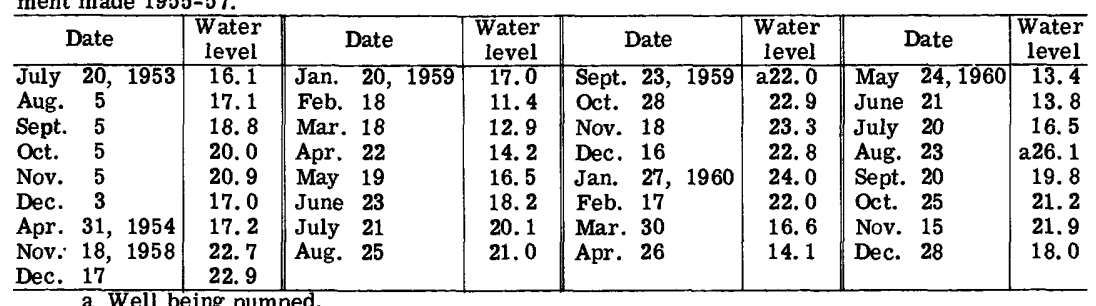


17/1W-2P1. Homer Martin. 1.6 mi north of Ft. Dick. Drilled domestic water-table well in undifferentiated flood-plain deposits, diam $8 \mathrm{in}$, depth $26 \mathrm{ft}$. Lsd $31 \mathrm{ft}$ above msl. MP top of casing, $0.20 \mathrm{ft}$ above 1sd. Highest water level 10.9 below 1sd, Mar. 30, 1960; lowest 23.1 below lsd, Oct. 15, 1958. Records available: 1952, 1958-60. No measurement made 1953-57.

\begin{tabular}{|c|c|c|c|c|c|c|c|}
\hline Date & $\begin{array}{l}\text { Water } \\
\text { level }\end{array}$ & Date & $\begin{array}{l}\text { Water } \\
\text { level }\end{array}$ & Date & $\begin{array}{l}\text { Water } \\
\text { level }\end{array}$ & Date & Water \\
\hline 28,1952 & 19.2 & Apr. 22, 1959 & 18.6 & Nov. 18,1959 & 22.1 & June 21,1960 & a19.2 \\
\hline 15, & 23.1 & May 19 & & Dec. 16 & & July 20 & 1 \\
\hline 18 & 1 & June 23 & 3 & Jan. 27,1960 & & Aug. 2 & 8 \\
\hline 18 & 19.4 & July 21 & 22.0 & Feb. 17 & 1 & Sept. 20 & 22.3 \\
\hline 20,1959 & 16.5 & Aug. 25 & 21.8 & Mar. 30 & 9 & Oct. 25 & 22.8 \\
\hline Feb. 18 & 11.7 & Sept. 23 & 22.1 & Apr. 26 & 17.2 & Nov. 15 & 21.2 \\
\hline Mar. 18 & 17.7 & Oct. 28 & 21.9 & May 24 & 15.5 & Dec. 28 & 17.5 \\
\hline
\end{tabular}

a Well being pumped.

18/1W-26P1. Merlin Goodlin. 0.75 mi south of Smith River. Drilled domestic, stock, and irrigation water-table well in Pleistocene terrace deposits of Quaternary age, diam 12 in, depth $28 \mathrm{ft}$. Lsd $38 \mathrm{ft}$ above msl. MP top of casing, at lsd. Highest water level 8.5 below lsd, May 28, 1952; lowest 26.9 below 1sd, Oct. 15, 1958. Records available: 1952-54, 1958-60. No measurement made 1955-57.

\begin{tabular}{ll||l|c||l|l||l|l|}
\hline May 28, 1952 & 8.5 & Apr. 22, 1959 & 17.2 & Nov. 18, 1959 & 19.8 & June 21, 1960 & 18.0 \\
July 23, 1953 & 10.5 & May 19 & 18.3 & Dec. 16 & 19.1 & July 20 & 19.0 \\
Aug. 20 & 12.8 & June 23 & 19.0 & Jan. 27, 1960 & 16.1 & Aug. 23 & 22.0 \\
Aug. 20, 1954 & 13.4 & July 21 & (a) & Feb. 17 & 14.6 & Sept. 20 & 24.5 \\
Oct. 15, 1958 & 26.9 & Aug. 25 & (a) & Mar. 30 & 12.4 & Oct. 25 & 22.7 \\
Dec. 18 & 16.9 & Sept. 23 & 21.2 & Apr. 26 & 16.5 & Nov. 15 & 19.4 \\
Jan. 20, 1959 & 13.8 & Oct. 28 & 19.1 & May 24 & 15.0 & Dec. 28 & 16.6 \\
Mar. 18 & 16.0 & & & & & & \\
\hline
\end{tabular}

a Well being pumped.

\section{Humboldt County \\ Eureka- Fortuna Area}

3/1W-34J1. City of Fortuna. Drilled unused artesian well in Carlotta Formation of Ogle (1953) of Pliocene to Pleistocene age, diam 12 in, depth $496 \mathrm{ft}$, perforated 182-226, 285-365. Lsd $60 \mathrm{ft}$ above msl. MP $\frac{1}{2}$-in hole in plate welded to casing, $1.00 \mathrm{ft}$ above $1 \mathrm{sd}$. Highest water level 23.3 below lsd, Sept. 5, 1952; lowest 36.1 below 1sd, Nov. 17, 1959. Records available: 1951-53, 1958-60. No measurement made 1954-57.

\begin{tabular}{|c|c|c|c|c|c|c|c|c|}
\hline June & 8,1951 & 25.7 & Jan. 20,1959 & 32.5 & Oct. 27,1959 & 35.9 & June 21,1960 & 33.2 \\
\hline May & 11,1952 & 27.3 & Mar. 18 & 31.9 & Nov. 17 & 36.1 & July 19 & 34.1 \\
\hline Aug. & 4 & 24.7 & Apr. 22 & 32.5 & Dec. 15 & 36.0 & Aug. 23 & 35.1 \\
\hline Sept. & $\mathbf{5}$ & 23.3 & May 19 & 33.4 & Jan. 26,1960 & 34.7 & Sept. 20 & 35.5 \\
\hline Apr. 2 & 26,1953 & 27.2 & June 23 & 34.3 & Feb. 16 & 31.4 & Oct. 25 & 5.7 \\
\hline Aug. 2 & 29 & 24.3 & July 21 & 35.0 & Mar. 29 & 31.6 & Nov. 15 & 35.8 \\
\hline Nov. 1 & 18,1958 & 35.5 & Aug. 25 & 35.7 & Apr. 26 & 32.1 & Dec. 28 & 33.0 \\
\hline Dec. 1 & 17 & 35.5 & Sept. 23 & 35.7 & May 24 & 32.7 & & \\
\hline
\end{tabular}

3/2W-26R1. Winfred Goble. About $4.5 \mathrm{mi}$ west of Fortuna. Drilled irrigation water-table well in alluvium of Quaternary age, diam $14 \mathrm{in}$, depth $30 \mathrm{ft}$. Lsd $20 \mathrm{ft}$ above msl. MP top south side of casing, $2.00 \mathrm{ft}$ above isd. Highest water level 0.00 , Feb. 18, 1959; lowest 14.5 below lsd, Nov. 11, 1952. Records available: 1951-54, 1958-60. No measurement made 1955-57.

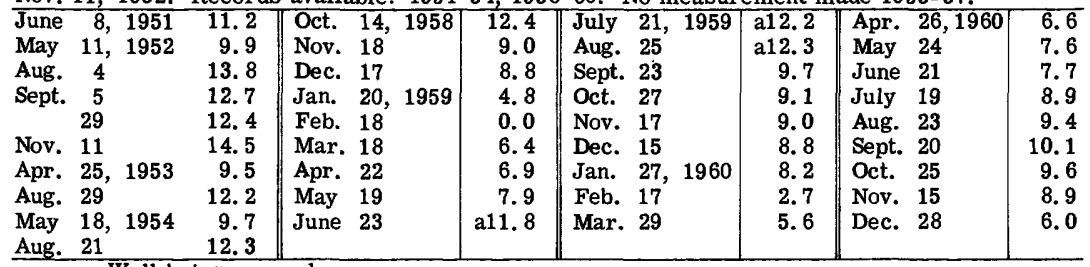

a Well being pumped.

6/1E-29P1. Golden State Co., Ltd. Arcata. Drilled industrial water-table well in alluvium of Quaternary age, diam 24 in, depth $46 \mathrm{ft}$. Lsd $25 \mathrm{ft}$ above msl. MP top of concrete pump foundation, $2.00 \mathrm{ft}$ above lsd. Highest water level 7. 3 below 1sd, Feb. 18, 1959; lowest 43.0 below Isd, June 7, 1951. Records available: 1951-54, 1958-60. No measurement made 1955-57.

\begin{tabular}{lrl||l|l||l|l|l||l|l|}
\hline June & 7,1951 & 43.0 & Apr. 27, 1953 & 29.4 & Dec. 17, 1958 & 9.7 & June 23, 1959 & 10.5 \\
May & 10,1952 & 20.8 & Aug. 28 & 28.0 & Jan. 20, 1959 & 8.2 & July 21 & 12.3 \\
Aug. & 5 & 26.7 & May 18, 1954 & 22.0 & Feb. 18 & 7.3 & Aug. 25 & 13.7 \\
Sept. 5 & 23.6 & Aug. 20 & 23.2 & Mar. 18 & 7.9 & Sept. 23 & 11.4 \\
& 29 & 18.4 & Oct. 15, 1958 & 11.8 & Apr. 22 & 9.7 & Oct. 27 & 12.0 \\
Nov. 11 & 22.7 & Nov. 18 & 9.5 & May 19 & 9.0 & Nov. 17 & 10.8
\end{tabular}




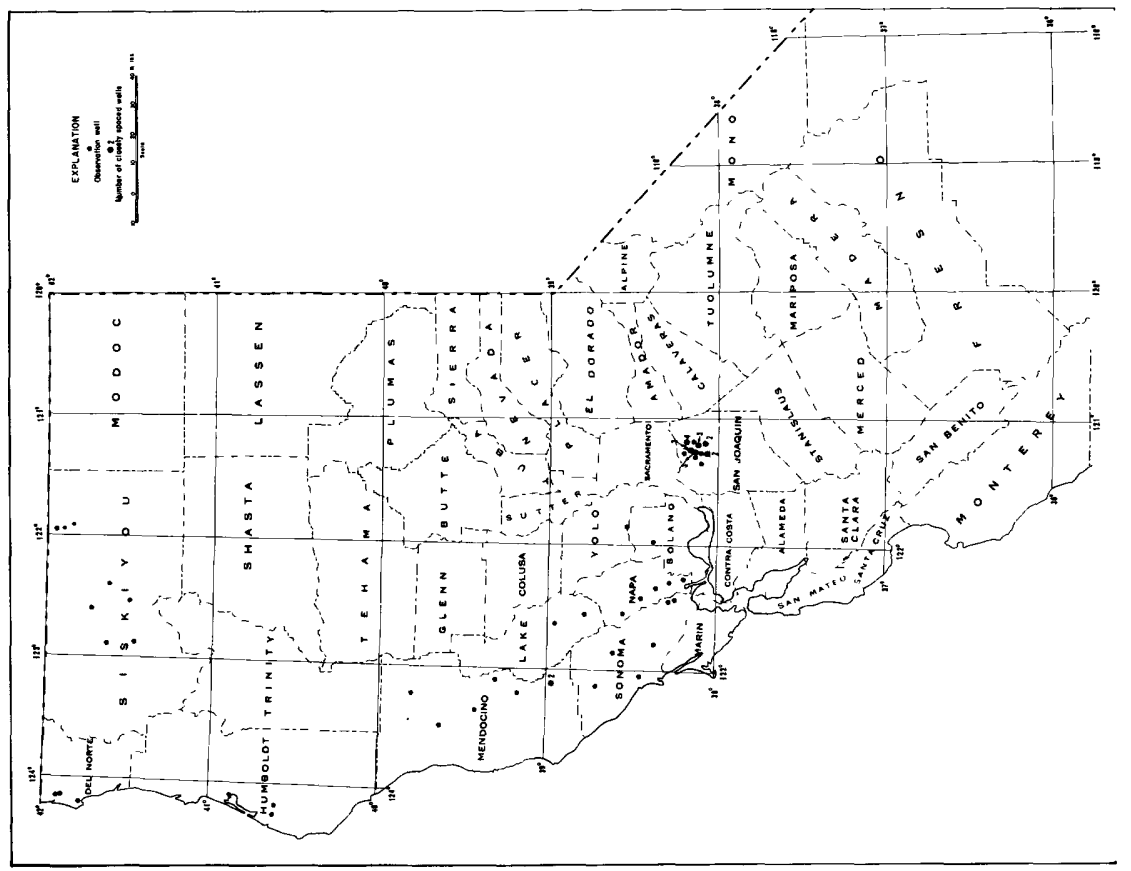




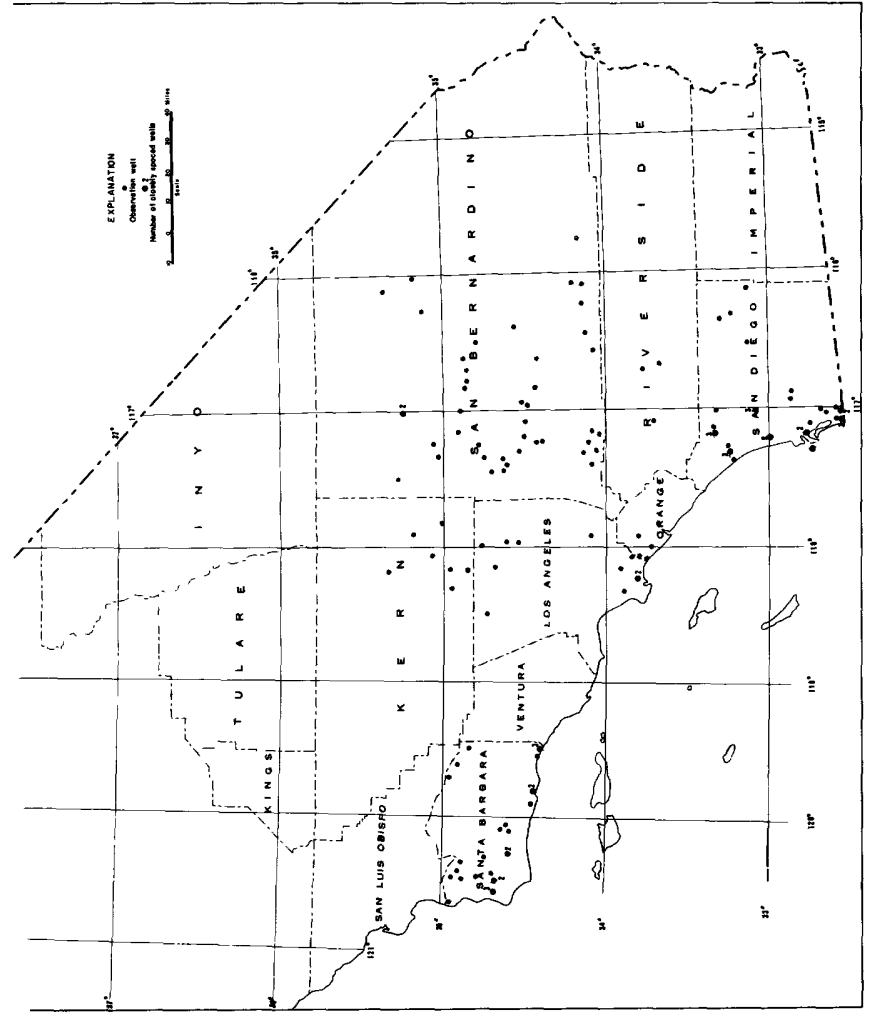

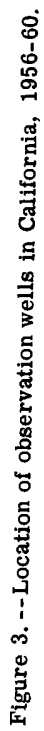


6/1 E-29P1--Continued.

\begin{tabular}{|c|c|c|c|c|c|c|c|}
\hline Date & $\begin{array}{l}\text { Water } \\
\text { level }\end{array}$ & Date & $\begin{array}{l}\text { Water } \\
\text { level }\end{array}$ & Date & $\begin{array}{l}\text { Water } \\
\text { level }\end{array}$ & Date & $\begin{array}{l}\text { Water } \\
\text { level }\end{array}$ \\
\hline $\begin{array}{lll}\text { Dec. } & 16,1959 \\
\text { Jan. } & 27,1960 \\
\text { Feb. } & 17 \\
\text { Mar. } 30 & \end{array}$ & $\begin{array}{r}11.9 \\
9.0 \\
7.9 \\
7.6\end{array}$ & $\begin{array}{lll}\text { Apr. } & 26,1960 \\
\text { May } & 24 \\
\text { June } & 21\end{array}$ & $\begin{array}{l}7.5 \\
7.7 \\
8.1\end{array}$ & $\begin{array}{lll}\text { July } 19,1960 \\
\text { Aug. } & 23 \\
\text { Sept. } & 20\end{array}$ & $\begin{array}{r}9.8 \\
10.5 \\
12.2\end{array}$ & $\begin{array}{ll}\text { Oct. } & 25,1960 \\
\text { Nov. } 15 \\
\text { Dec. } 28\end{array}$ & $\begin{array}{r}10.3 \\
10.1 \\
7.8\end{array}$ \\
\hline
\end{tabular}

\section{$\underline{\text { Kern County }}$}

\section{Antelope Valley--Lancaster Basin}

9/12-21D3. T. R. Loomis. Rosamond. Drilled domestic water-table well in alluvium of Pleistocene age, diam $9 \mathrm{in}$, depth $107 \mathrm{ft}$, cased with steel. Lsd about 2, $350 \mathrm{ft}$ above msl. MP hole in pumpbase, $1.80 \mathrm{ft}$ above 1sd. Highest water level 60.73 below 1sd, Feb. 14, 1952; lowest 80.20 below 1sd, Nov. 10, 1960. Records available: 1951-54, 1956-60. No measurement made in 1955.

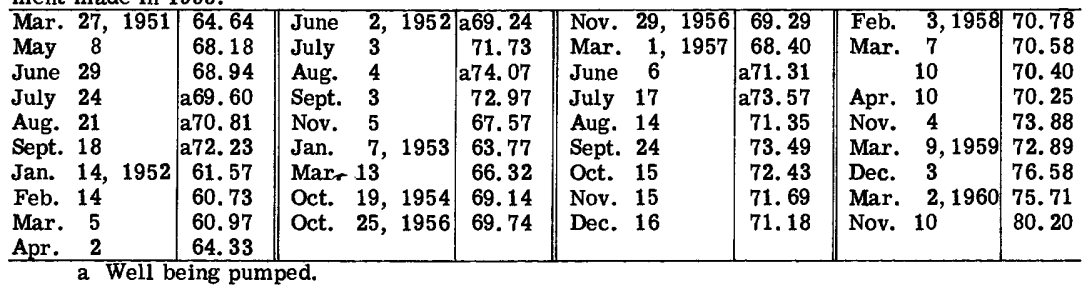

\section{Antelope Valley--Gloster Area}

10/12-20C3. O. J. Backus. South of Mojave. Drilled domestic water-table well in alluvium of Pleistocene age, diam $8 \mathrm{in}$, depth about $128 \mathrm{ft}$, cased with steel. Lsd about $2,645 \mathrm{ft}$ above msl. MP bottom of pumpbase, at isd. Highest water level 93.12 below 1sd, Mar. 4, 1958; lowest 94.60 below lsd, Nov. 10,1960 . Records available: 1952, 1958-60. No measurement made 1953-57. Nov. 13, 1952, 93.32; Mar. 4, 1958, 93.12; Nov. 4, 93.86; Mar. 9, 1959 , 93.50; Dec. 1, 94.23; Mar. 2, 1960, 94. 20; Nov. 10, 94.60.

\section{Antelope Valley--Willow Springs Area}

10/13-19M1. Dewey Butler. North of Willow Springs. Drilled unused water-table well in alluvium of Pleistocene age, diam $16 \mathrm{in}$, depth about $770 \mathrm{ft}$, cased with steel. Lsd about $2,905 \mathrm{ft}$ above msl. MP hole in casing, $1.00 \mathrm{ft}$ above $1 \mathrm{sd}$. Highest water level 291.61 below lsd, Jan. 20, 1953; lowest 309.66 below isd, Nov. 10, 1960. Records available: 1953-60.

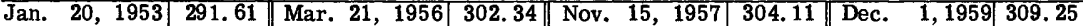

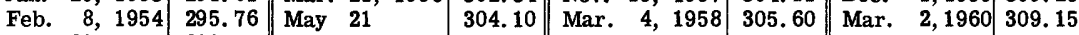
Nov. $29 \quad 299.20$ Sept. 26 \begin{tabular}{l|r|rr||l|l|l|l|} 
Mar. 1, 1955 & 299.12 & Mar. 5, 1957 & 303.97 & Mar. 9, 1959 & 306.61 \\
\hline
\end{tabular}

\section{Antelope Valley-- North Muroc Basin}

11/9-34A1. F. J. Schultz. Thundering Herd Ranch. East of Edwards. Drilled domestic water-table well in alluvium of Pleistocene age, diam $10 \mathrm{in}$, depth $194 \mathrm{ft}$, cased with steel. Lsd about 2, $303 \mathrm{ft}$ above msl. MP top of casing, $0.40 \mathrm{ft}$ above lsd. Highest water level 93.11 below Isd, Jan. 25, 1951; lowest 102.60 below lsd, Nov. 16, 1960. Records available: 195152 , 1955-60. No measurement made 1953-54.

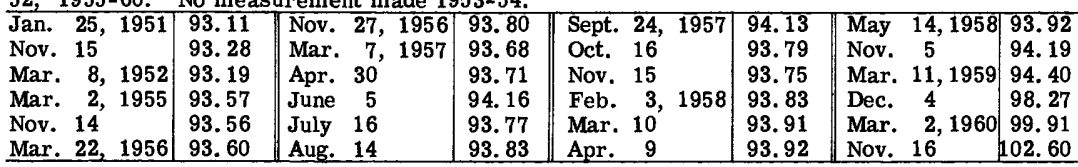

\section{Fremont Valley--Chaffee Area}

11/11-8D1. Zepha Thorning. East side of Mojave. Drilled unused water-table well in alluvium of Pleistocene age, diam $12 \mathrm{in}$, depth $204 \mathrm{ft}$, cased with steel. Lsd 2,624.5 ft above msl. MP bottom hole in casing, $1.00 \mathrm{ft}$ above lsd. Highest water level 198. 00 below lsd, Dec. 1, 1959; lowest 218. 60 below lsd, Sept. 28, 1929. Records available: 1929-30, 1952, 1954-60. 
11/11-8D1-- Continued.

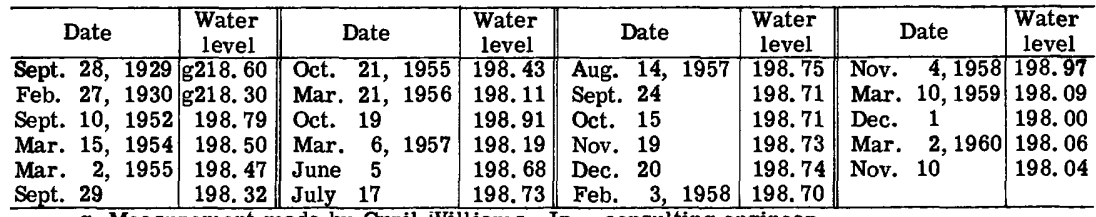

g Measurement made by Cyril Williams, Jr., consulting engineer.

\section{Fremont Valley--Koehn Lake}

30/39-8A1. Owner unknown. East of Koehn Lake. Drilled unused water-table well in alluvium of Pleistocene age, diam $12 \mathrm{in}$, depth $268 \mathrm{ft}$, cased with steel. Lsd about $2,075 \mathrm{ft}$ above msl. MP top of casing, $1.00 \mathrm{ft}$ above lsd. Highest water level 136.55 below lsd, Mar, 2, 1955; lowest 140. 20 below 1sd, Nov. 10, 1960. Records available: 1953-60.

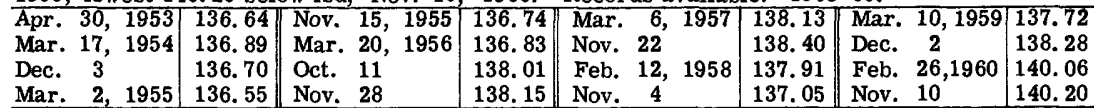

31/37-35N1. M. \& R. Conklin Ranch. North of California City. Drilled unused watertable well in alluvium of Pleistocene age, diam $16 \mathrm{in}$, depth about $439 \mathrm{ft}$, cased with steel. Lsd about $2,320 \mathrm{ft}$ above msl. MP hole in casing cover, $0.10 \mathrm{ft}$ below lsd. Highest water level 230. 89 below lsd, Jan. 22, 1953; lowest 247.92 below 1sd, Dec. 2, 1959. Records available: 1953 , 1958-60. No measurement made 1954-57. \begin{tabular}{|l||l|l|l||l|l|l|l|}
\hline Jan. 22, 1953 & 230.89 & Mar. 4, 1958 & 243.72 & Mar. 10, 1959 246.04 & Mar. 2, 1960 247.38 \\
\hline
\end{tabular} \begin{tabular}{l|l|l|l|l|l|l|l|l|} 
Jan. 30,1958 & 244.08 & Nov. 5 & 245.82 & Dec. 2 & 247.92 & Nov. 10 & 247.60 \\
\hline
\end{tabular}

\section{Lake County}

\section{Lower Lake-Middletown Area}

11/6-19G1. Roy Trimble. La-Z-2 Ranch. Northeast of Middletown in Coyote Valley. Drilled domestic water-table well in alluvium of Quaternary age, diam $8 \mathrm{in}$, depth $50 \mathrm{ft}$. Lsd $960 \mathrm{ft}$ above msl. MP top south side of casing, $0.90 \mathrm{ft}$ above 1sd. Highest water level $7.6 \mathrm{be}$ low lsd, Feb. 15, 1960; lowest 16.6 below lsd, Oct. 11, 1950. Records available: 1950-54, 1956-60. No measurement made in 1955.

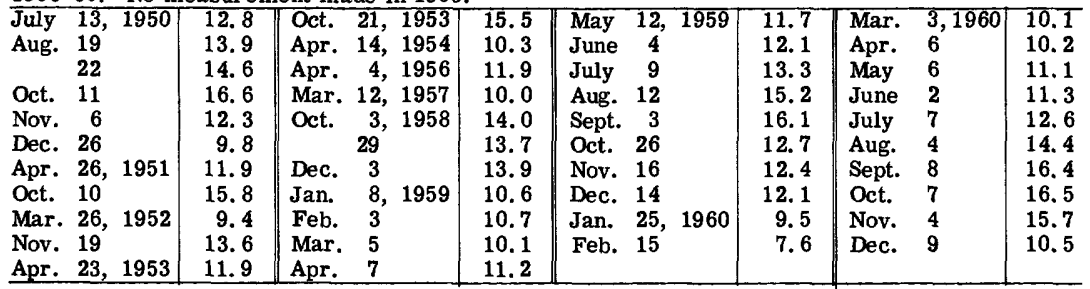

13/7-15Q1. F. Bartrum. Northeast of Clearlake Highlands. Burns Valley. Drilled unused water-table well in Cache Formation of Anderson (1936), diam 12 in, depth $172 \mathrm{ft}$. Lsd $1,385 \mathrm{ft}$ above msl. MP top east side of casing, $1.00 \mathrm{ft}$ above lsd. Highest water level $0.3 \mathrm{be}-$ low lsd, Mar. 26, 1952; lowest 12.4 below 1sd, Oct. 11, 1950. Records available: 1949-54, 1956-60. No measurement made in 1955.

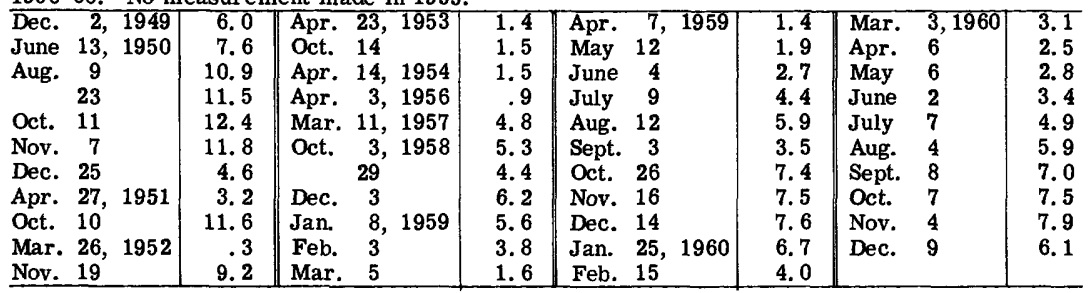




\section{Los Angeles County}

\section{Antelope Valley}

5/10-6N1. Little Rock Irrigation District. Near Little Rock. Drilled unused water-table well in alluvium, diam 14 in, depth $250+\mathrm{ft}$, cased with steel. Lsd 2, 777. $2 \mathrm{ft}$ above msl. MP top of access pipe, $0.20 \mathrm{ft}$ above lsd. Highest water level 85.0 below lsd, Mar. 2, 1945; lowest 147.30 below lsd, July 17, 1956. Records available: 1938, 1940-60. Records furnished by Los Angeles County Flood Control District.

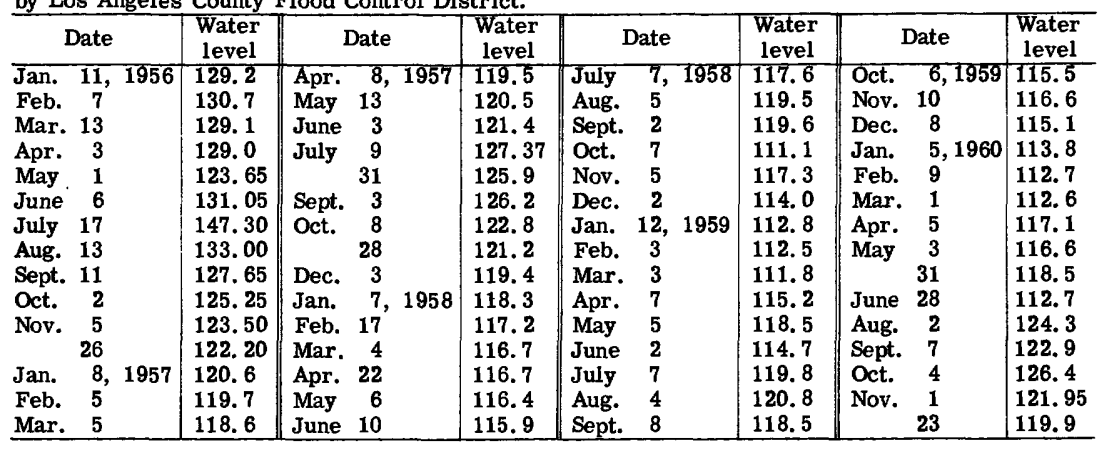

6/10-9Q1. Rhodes-Cogburn. Formerly Henry Winters. Near Alpine Butte. Drilled irrigation and domestic water-table well in alluvium, diam $16 \mathrm{in}$, reported depth $275 \mathrm{ft}$, cased with steel. Lsd about 2,596 ft above msl. MP hole in casing under pumpbase at top concrete block, $2.50 \mathrm{ft}$ above lsd. Highest water level 142.1 below lsd, Dec. 2, 1941; lowest 164.5 below lsd, Nov. 22, 1960. Records available: 1940-48, 1950-60. Records furnished by Los Angeles County Flood Control District. Nov. 27, 1956, 159.9; Oct. 30, 1957, 160.9; Nov. 14, 1958, 162.0; Oct. 23, 1959, 161. 2; Nov. 22, 1960, 164. 5.

7/12-15F2. Los Angeles County Water District, well 4. Tenth and Date Sts., Lancaster. Drilled public-supply water-table well in alluvium, diam 16 to $12 \mathrm{in}$, reported depth $600 \mathrm{ft}$, cased, 16-in to 244, 12-in to 372 . Lisd 2, 354 ft above msl. MP top of casing, $0.50 \mathrm{ft}$ above lsd. Highest water level 42.9 below lsd, Feb. 28, 1945; lowest 124.4 below 1sd, Oct. 26, 1959. Records available: $1943-45,1947-55,1957-60$. No measurement made in 1956. Records furnished by Los Angeles County Flood Control District. Mar. 8, 1957, 102. 03; Nov. 12, 108.99; Mar. 10, 1958, 105.46; Nov. 4, 118.58; Oct. 26, 1959, 124.4; Nov. 28, 1960, 121.7.

8/10-19Q1. Union Trust \& Savings Bank. East of Redman School. Drilled unused watertable well in alluvium, diam $12 \mathrm{in}$, reported depth $750 \mathrm{ft}$, cased with steel. Lsd about $2,342 \mathrm{ft}$ above msl. MP top of casing, $0.36 \mathrm{ft}$ above 1sd. Highest water level 29.7 below 1sd, Apr. 9, 1941; lowest 147.56 below lsd, July 16, 1957. Records available: 1939-42, 1944-48, $1950-60$. \begin{tabular}{ll|l|l|l|l||l|l|l|}
\hline Mar. 7, 1956 & 104.4 & June 6, 1957 & 138.80 & Mar. 11, 1958 & 91.88 & Mar. 12, 1959 93.56
\end{tabular}

\begin{tabular}{l|l|l|l||l|l||l|l|} 
Oct. 23 & 105.7 & July 16 & 147.56 & Apr. 10 & 90.92 & Dec. 8 & 102.49 \\
Feb. 28, 1957 & 103.38 & Sept. 25 & 141.53 & May 15 & 93.58 & Mar. 4, 1960 & 101.77 \\
Apr. 30 & 138.22 & Oct. 23 & 121.47 & Nov. 6 & 104.51 & Nov. 15 & 106.43 \\
\hline
\end{tabular}

8/15-33G1. J. Alesso. Near Fairmont. Drilled domestic water-table well in alluvium, diam $12 \mathrm{in}$, depth $282 \mathrm{ft}$, cased with steel. Lsd about $2,930 \mathrm{ft}$ above msl. MP top of casing, $1.00 \mathrm{ft}$ above lsd. Highest water level 194. 0 below lsd, Jan. 23, 1946; lowest 269.2 below lsd, Oct. 28, 1960. Records available: 1946-60. Records furnished by California State Division of Water Resources.

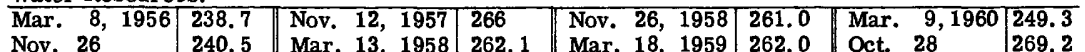

\section{San Gabriel River Basin}

1S/10-18. Baldwin Park. Drilled observation water-table well tapping fine sand to coarse gravel in alluvial deposits, diam 16 in, depth $200 \mathrm{ft}$, perforated 74-174. Lsd 387 ft above msl. Highest water level 56. 0 below lsd, May 19, 1916; lowest 150. 82 below lsd, Oct. 31, 1960. Records available: $1903-60$.

Mean water level for the day, from recorder graph, 1956

\begin{tabular}{|c|c|c|c|c|c|c|c|c|c|c|c|c|}
\hline Day & Jan. & Feb. & Mar. & Apr. & May & June & July & Aug. & Sept. & Oct. & Nov. & Dec. \\
\hline 5 & 137.14 & 136.57 & 135.51 & 135.76 & 135.28 & 135.85 & 137.87 & 140.12 & 141.6 & 142.81 & 142.23 & 142.30 \\
\hline 10 & 137.05 & 136.37 & 135.53 & 135 & 135.35 & 13 & 138.25 & 140.41 & 141 & 142. 5 & 142.42 & 142.15 \\
\hline 15 & 136.99 & 136.08 & 135.59 & 135.63 & 135.32 & 136.44 & 138.65 & 140.70 & 142.21 & 42.42 & 42.4 & 142.10 \\
\hline & 137.04 & 135.89 & 135.67 & 135.45 & 135.61 & 136.70 & 139.05 & 140.9 & 142.48 & 142. 31 & 142. 38 & 142. 09 \\
\hline & 137 & 135. 71 & 135.77 & 135 & 13 & 13 & 13 & 14 & 142.69 & 42. & 142.33 & 142.06 \\
\hline & & & & & & & & & & & & 42.03 \\
\hline
\end{tabular}


1s/10-18--Continued.

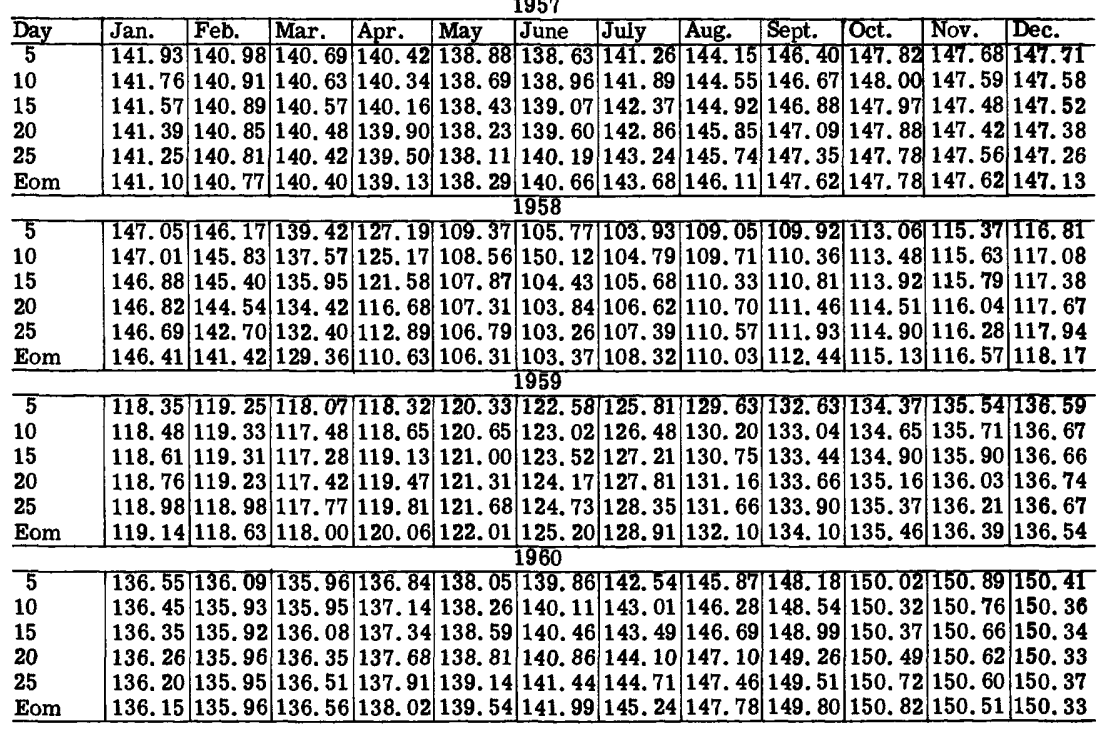

Coastal Plain

3S/12-8L3. Los Angeles County Farm. Near Downey. Drilled unused artesian well in Gaspur water-bearing zone of Recent age and underlying deposits of Pleistocene age, diam 8 in, depth $248 \mathrm{ft}$, cased with steel. Lsd about $92 \mathrm{ft}$ above msl. MP hole in recorder floor, top of platform over well, $5.00 \mathrm{ft}$ below lsd. Highest water level 14. 45 below lsd, Mar. 20, 1930; lowest 80.50 below lsd, July 25, 1960. Records available: 1930-60. Records furnished by San Gabriel Valley Protective Association.

\begin{tabular}{|c|c|c|c|c|c|c|c|}
\hline Date & $\begin{array}{l}\text { Water } \\
\text { level }\end{array}$ & Date & $\begin{array}{l}\text { Water } \\
\text { level }\end{array}$ & Date & $\begin{array}{l}\text { Water } \\
\text { level }\end{array}$ & Date & $\begin{array}{c}\text { Water } \\
\text { level }\end{array}$ \\
\hline $\begin{array}{lll}\text { Jan. } & 30,1956 \\
\text { Feb. } & 27 \\
\text { Mar. } 26 \\
\text { Apr. } 30 \\
\text { May } 28 \\
\text { June } 25 \\
\text { July } 30 \\
\text { Aug. } 27 \\
\text { Sept. } 24 \\
\text { Oct. } 29 \\
\text { Nov. } 26 \\
\text { Dec. } 31 \\
\text { Jan. } 7, \quad 1957 \\
& 28 \\
\text { Feb. } & 25 \\
\text { Mar. } 25 & \end{array}$ & $\begin{array}{l}62.69 \\
62.62 \\
65.80 \\
64.11 \\
66.20 \\
67.47 \\
69.74 \\
69.75 \\
69.75 \\
68.19 \\
68.84 \\
68.38 \\
67.83 \\
67.10 \\
67.59 \\
68.60 \\
\end{array}$ & $\begin{array}{lll}\text { Apr. } & 29, & 1957 \\
\text { May } & 27 \\
\text { June } & 3 \\
\text { July } & 1 \\
\text { Aug. } & 5 \\
\text { Sept. } & 2 \\
\text { Oct. } & 7 \\
\text { Nov. } & 4 \\
\text { Dec. } & 2 \\
\text { Jan. } & 6,1958 \\
\text { Feb. } & 3 \\
\text { Mar. } & 3 \\
\text { Apr. } & 7 \\
\text { May } & 5 \\
\text { June } & 2\end{array}$ & $\begin{array}{l}69.63 \\
69.72 \\
71.35 \\
73.35 \\
74.44 \\
73.74 \\
73.52 \\
71.84 \\
71.84 \\
71.39 \\
70.95 \\
71.59 \\
70.37 \\
72.89 \\
74.70\end{array}$ & $\begin{array}{lll}\text { July } & 7, & 1958 \\
\text { Aug. } & 4 & \\
\text { Sept. } & 1 & \\
\text { Oct. } & 6 & \\
\text { Nov. } & 3 & \\
\text { Dec. } & 1 & \\
\text { Jan. } & 26, & 1959 \\
\text { Feb. } & 23 & \\
\text { Mar. } & 23 \\
\text { Apr. } & 27 \\
\text { May } & 25 \\
\text { June } & 29 \\
\text { July } & 27 \\
\text { Aug. } & 31 \\
\text { Sept. } & 28\end{array}$ & $\begin{array}{l}78.58 \\
76.01 \\
75.39 \\
77.35 \\
75.79 \\
75.07 \\
74.20 \\
73.58 \\
74.79 \\
75.33 \\
76.64 \\
77.02 \\
78.14 \\
78.18 \\
77.58\end{array}$ & $\begin{array}{ll}\text { Oct. } 26,1959 \\
\text { Nov. } 30 \\
\text { Dec. } 21 \\
\text { Jan. } 25,1960 \\
\text { Feb. } 29 \\
\text { Mar. } 28 \\
\text { Apr. } 25 \\
\text { May } & 30 \\
\text { June } & 27 \\
\text { July } & 25 \\
\text { Aug. } 29 \\
\text { Sept. } 26 \\
\text { Oct. } 31 \\
\text { Nov. } 28 \\
\text { Dec. } 26\end{array}$ & $\begin{array}{l}77.41 \\
77.35 \\
76.53 \\
75.48 \\
75.17 \\
76.40 \\
77.68 \\
78.13 \\
79.06 \\
80.50 \\
80.30 \\
80.47 \\
80.10 \\
78.83 \\
78.57\end{array}$ \\
\hline
\end{tabular}

3S/14-21B1. Southern California Water Co. Rosecrans plant well 2 (previously reported well 1). Near Hawthorne. Drilled public-supply artesian well in sand and gravel deposits of Pleistocene age, diam $16 \mathrm{in,}$ depth $500 \mathrm{ft}$, cased with steel. Lsd about $63 \mathrm{ft}$ above msl. Fighest water level 66 below 1sd, May 1, 1931; lowest 134 below 1sd, Oct. 7, Nov. 7, Dec. 7, Dec. 28, 1958, Jan. 7, 1959. Records available: 1931-37, 1939-60. Records furnished by Southern California Water Co.

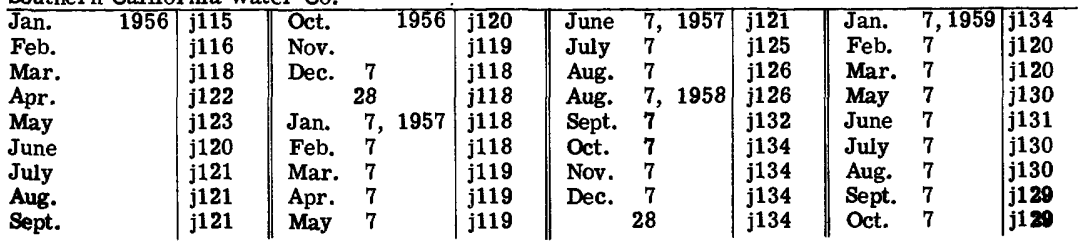


3S/14-21B1-- Continued.

\begin{tabular}{|c|c|c|c|c|c|c|c|}
\hline Date & $\begin{array}{l}\text { Water } \\
\text { level }\end{array}$ & Date & $\begin{array}{c}\text { Water } \\
\text { level }\end{array}$ & Date & $\begin{array}{c}\text { Water } \\
\text { level }\end{array}$ & Date & $\begin{array}{l}\text { Water } \\
\text { lepel }\end{array}$ \\
\hline $\begin{array}{lll}\text { Nov. } & 7, & 1959 \\
\text { Dec. } & 7 & \\
\text { Jan. } & 7, & 1960 \\
\text { Feb. } & 7 & \\
\end{array}$ & $\begin{array}{l}\mathrm{j} 124 \\
\mathrm{j} 122 \\
\mathrm{j} 118 \\
\mathrm{j} 118\end{array}$ & $\begin{array}{lll}\text { Mar. } & 7,1960 \\
\text { Apr. } & 7 & \\
\text { May } & 7 \\
\text { June } & 7\end{array}$ & $\begin{array}{l}\mathbf{j 1 1 5} \\
\mathbf{j 1 2 2} \\
\mathbf{j 1 2 3} \\
\mathrm{j} 124\end{array}$ & $\begin{array}{lll}\text { July } & 7,1960 \\
\text { Aug. } & 7 \\
\text { Sept. } & 7\end{array}$ & $\begin{array}{l}\mathrm{j} 127 \\
\mathrm{j} 129 \\
\mathrm{j} 127\end{array}$ & $\begin{array}{ll}\text { Oct. } & 7,1960 \\
\text { Nov. } & 7 \\
\text { Dec. } & 7\end{array}$ & $\begin{array}{l}j 126 \\
j 123 \\
j 120\end{array}$ \\
\hline
\end{tabular}

4S/11-5D1. V. Capovilla. Near Norwalk. Drilled domestic artesian well in deposits of Pleistocene age, diam $10 \mathrm{in}$, depth $270 \mathrm{ft}$, cased with steel. Lisd $44.70 \mathrm{ft}$ above msl. MP hole in pumpbase, $0.54 \mathrm{ft}$ above lsd. Highest water level 3.41 below lsd, Mar. 17, 1933; lowest 86. 37 below lsd, Aug. 25, 1959. Records available: 1930-60. Records furnished by Orange County Flood Control District.

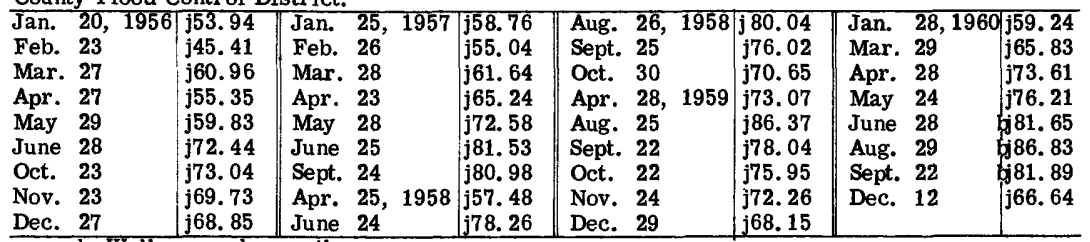

b Well pumped recently.

j Water level below sea level.

4S/13-14L1. Southern California Edison Co., Ltd. Long Beach. Drilled unused artesian well in Gaspur water-bearing zone of Recent age, diam $10 \mathrm{in}$, depth $114 \mathrm{ft}$, perforated 90-114. Lsd 28. $55 \mathrm{ft}$ above msl. MP top of platform, 2.21 ft above lsd. Highest water level 20.62 below lsd, Apr. 5, 1941; lowest 54. 46 below lsd, Dec. 26, 1960. Records available: 1930-60. Records furnished by city of Long Beach.

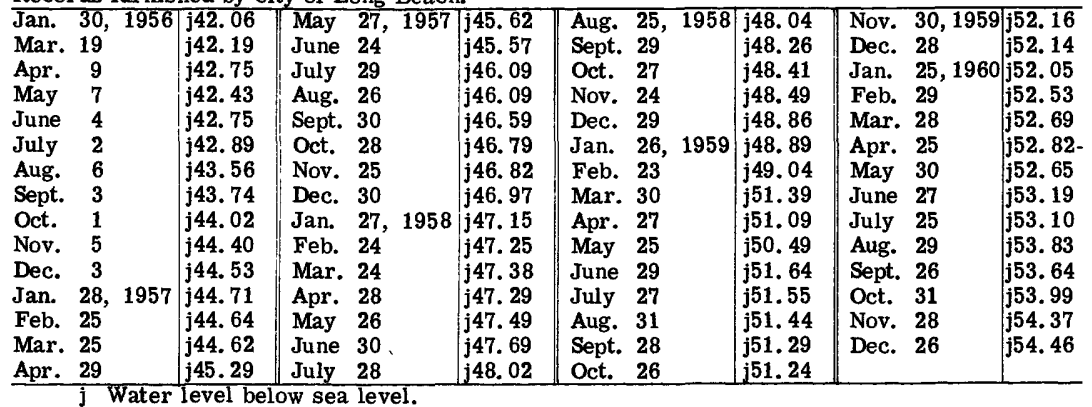

4S/13-23G2. City of Long Beach. Drilled unused artesian well in Silverado Formation of Pleistocene age, diam 26 to $16 \mathrm{in}$, depth $1,074 \mathrm{ft}$, perforated 650-900. Lsd $23.23 \mathrm{ft}$ above msl (previously reported $24.50 \mathrm{ft}$ above $\mathrm{msl}$ ). MP hole in pumpbase, $1.30 \mathrm{ft}$ above $1 \mathrm{sd}$. Highest water level 52.93 below 1sd, Feb. 6, 1939; lowest 131.75 below 1sd, Jan. 20, 1953. Records available: 1932-60. Records furnished by city of Long Beach.

\begin{tabular}{|c|c|c|c|c|c|c|c|c|c|c|c|}
\hline Jan. & 30,1956 & $\mathrm{j104.80}$ & Apr. & 29,1957 & j102.50 & July & 28,1958 & j116.25 & Oct. & 26,1959 & $\mathrm{j112.40}$ \\
\hline Feb. & 27 & j102. 85 & May & 27 & j109.10 & Aug. & 25 & & Nov. & 30 & \\
\hline Mar. & 26 & 7 & une & 24 & 12 & Sept. & 29 & & Dec. & 28 & \\
\hline & 30 & & uly & 29 & & Oct. & 17 & & Jan. & 25,1960 & \\
\hline & 28 & & Aug. & 26 & & Nov. & & & Feb. & 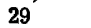 & \\
\hline & 26 & & iept. & 30 & & Dec. & 29 & & Mar. & & \\
\hline & 30 & & Oct. & 28 & & Jan. & 26,1959 & & Apr. & & \\
\hline . & 27 & & Nov. & 2 & & Feb. & 23 & & May & & \\
\hline Sept. & 24 & & Dec. & 30 & & Mar. & 30 & & June & & \\
\hline Oct. & 29 & & Jan. & 27, & & Apr. & 7 & & July & & \\
\hline Nov. & 26 & & Feb. & 24 & & May & 28 & & Aug. & & \\
\hline Dec. & & & Mar. & 31 & & June & O & & Sept. & & \\
\hline & 28 , & & Apr. & 28 & & & n & & Oct. & & \\
\hline & 25 & & $\mathrm{Ma}$ & & & & & & Nov. & & \\
\hline ar. & 25 & j100.85 & June & 30 & & Sept. & 28 & .00 & Dec. & 26 & 9.88 \\
\hline
\end{tabular}




\section{Mendocino County}

\section{Sanel Valley}

13/11-19P1. Charles Ashurst. About $0.4 \mathrm{mi}$ south of Hopland. Dug and drilled irrigation water-table well in alluvium of Recent age, size $4 \times 5 \mathrm{ft}$, depth $44 \mathrm{ft}, 12$-in casing to 44, perforated 24-44. Lsd $488 \mathrm{ft}$ above msl. MP top of plank cover at intake, $0.50 \mathrm{ft}$ above 1sd. Highest water level 1.3 below 1sd, Feb. 9, 1960; lowest 21.0 below 1sd, Oct. 2, 1958. Records available: 1953-55, 1958-60. No measurement made 1956-57.

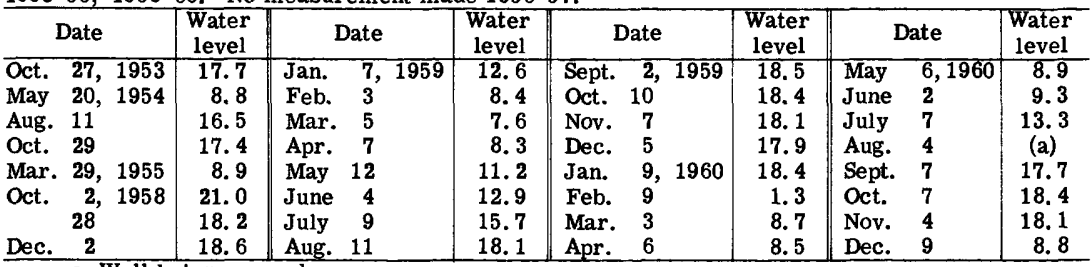

a Well being pumped.

13/11-20G1. I. Bliss. $0.5 \mathrm{mi}$ east of East Hopland. Drilled domestic well in alluvium of Recent age, diam $6 \mathrm{in}$, depth $135 \mathrm{ft}$. Lsd $515 \mathrm{ft}$ above msl. MP top north side of casing, 2. $00 \mathrm{ft}$ above 1sd. Highest water level 2.3 below lsd, Feb. 9, 1960; lowest 21. 2 below 1sd, Oct. 2, 1958. Records available: $1953-55,1958-60$. No measurement made 1956-57.

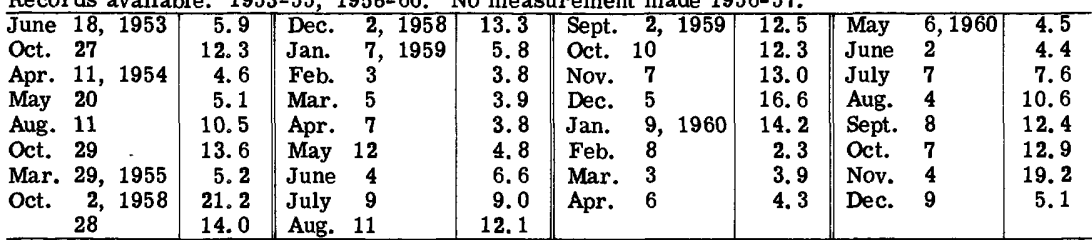

Ukiah Area

15/12-8L1. Arnold Bogner. About $1 \mathrm{mi}$ north of Ukiah. Drilled domestic water-table well in terrace deposits of Recent age, diam $12 \mathrm{in}$, depth $62 \mathrm{ft}$. Lsd $665 \mathrm{ft}$ above msl. MP top east side of casing, $1.00 \mathrm{ft}$ above 1sd. Highest water level 14.6 below 1sd, Mar. 5, 1959, Mar. 3, 1960; lowest 30.6 below 1sd, Dec. 5, 1959. Records available: 1951-55, 1958-60. No measurement made 1956-57.

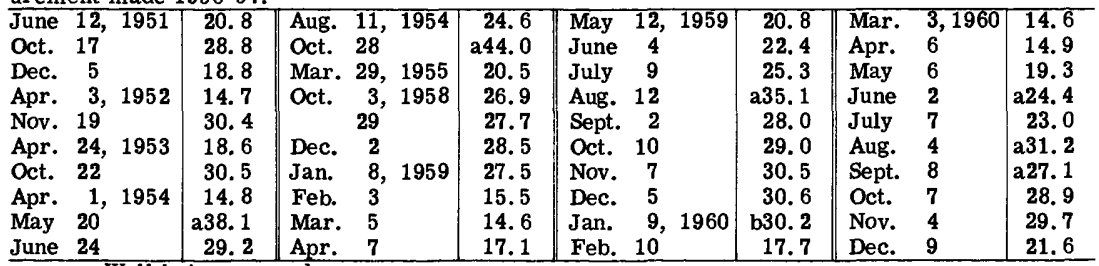

a Well being pumped.

b Well pumped recently.

\section{Potter Valley}

17/11-29P1. Harrison. About $2 \mathrm{mi}$ south of Potter Valley. Drilled stock water-table well in Pliocene gravels, diam $8 \mathrm{in}$, depth $104 \mathrm{ft}$, perforated 21-24, 41-43, 89-100. Lsd $940 \mathrm{ft}$ above msl. MP top west side of casing, $1.00 \mathrm{ft}$ above 1sd. Highest water level 18. 2 below lsd, Feb. 19, 1959; lowest 27.5 below 1sd, Aug. 24, 1959. Records available: 1951-55, 1958-60. No measurement made 1956-57. Measurement discontinued.

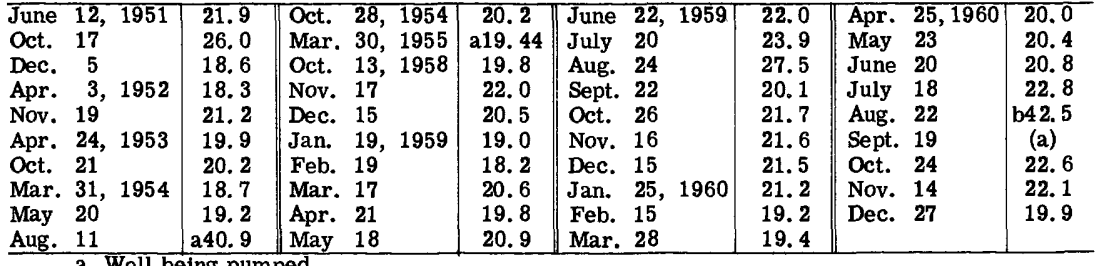

b Well pumped recently. 


\section{Little Lake Valley}

18/13-18E1. Northwestern Pacific RR. Willits. Drilled unused artesian well in alluvium of Recent age and continental deposits of Pliocene and Pleistocene age, diam $12 \mathrm{in}$, depth $493 \mathrm{ft}$. Lsd 1, $350 \mathrm{ft}$ above msl. MP center of well cap, $1.60 \mathrm{ft}$ above lsd. Highest water level 25.7 below 1sd, Mar. 17, Apr. 21, 1959; lowest 37.6 below 1sd, Oct. 24, 1960. Records available: 1958-60.

\begin{tabular}{|c|c|c|c|c|c|c|c|}
\hline Date & $\begin{array}{c}\text { Water } \\
\text { level }\end{array}$ & Date & $\begin{array}{c}\text { Water } \\
\text { level }\end{array}$ & Date & $\begin{array}{c}\text { Water } \\
\text { level }\end{array}$ & Date & $\begin{array}{c}\text { Water } \\
\text { level }\end{array}$ \\
\hline $\begin{array}{lll}\text { Dec. } & 15, & 1958 \\
\text { Jan. } & 19, & 1959 \\
\text { Feb. } & 19 & \\
\text { Mar. } & 17 \\
\text { Apr. } & \mathbf{2 1} \\
\text { May } & 18 & \\
\text { June } & \mathbf{2 2} & \end{array}$ & $\begin{array}{l}28.9 \\
26.3 \\
26.2 \\
25.7 \\
25.7 \\
26.2 \\
26.9\end{array}$ & $\begin{array}{lll}\text { July } & 20,1959 \\
\text { Aug. } & 24 \\
\text { Sept. } & 22 \\
\text { Oct. } & 27 \\
\text { Nov. } & 17 \\
\text { Dec. } & 15\end{array}$ & $\begin{array}{l}29.6 \\
32.7 \\
32.1 \\
31.2 \\
30.9 \\
31.4\end{array}$ & $\begin{array}{ll}\text { Jan. } & 26,1960 \\
\text { Feb. } & 16 \\
\text { Mar. } 28 \\
\text { Apr. } 25 \\
\text { May } 23 \\
\text { June } 20\end{array}$ & $\begin{array}{l}28.2 \\
27.2 \\
26.2 \\
26.0 \\
26.5 \\
26.7\end{array}$ & $\begin{array}{ll}\text { July } & 18,1960 \\
\text { Aug. } & 22 \\
\text { Sept. } & 19 \\
\text { Oct. } & 24 \\
\text { Nov. } & 14 \\
\text { Dec. } & 27\end{array}$ & $\begin{array}{l}27.4 \\
31.2 \\
33.4 \\
37.6 \\
30.6 \\
27.9\end{array}$ \\
\hline
\end{tabular}

\section{Laytonville Area}

21/14-30M1. Mary E. Tracy. About $2 \mathrm{mi}$ south of Laytonville. Dug domestic and irrigation water-table well in alluvium of Recent age, size $5 \times 5 \mathrm{ft}$ to $19-\mathrm{ft}$ depth, $2 \mathrm{ft}$ to 29 -ft depth. Lsd 1, $688 \mathrm{ft}$ above msl. MP top of pumphouse floor, $1.50 \mathrm{ft}$ above isd. Highest water level 3.4 below Isd, Mar. 31, 1954; lowest 20.0 below Isd, Aug. 25, 1959. Records available: 1952-55, 1958-60. No measurement made 1956-57.

\begin{tabular}{l|r|ll|r||l|r||l|r|}
\hline Nov, 19, 1952 & 16.2 & Oct. 13, 1958 & 15.9 & July 20, 1959 & 14.6 & Apr. 26, 1960 & 6.5 \\
June 3, 1953 & 4.7 & Nov. 18 & 16.1 & Aug. 25 & 20.0 & May 24 & 7.3 \\
Oct. 23 & 15.3 & Dec. 17 & 15.5 & Sept. 22 & 16.7 & June 21 & 9.7 \\
Mar. 31, 1954 & 3.4 & Jan. 19, 1959 & 6.6 & Oct. 27 & 17.1 & July 19 & 13.0 \\
May 19 & 7.0 & Feb. 19 & 3.5 & Nov. 17 & 18.3 & Aug. 22 & 15.2 \\
July 16 & b17.4 & Mar. 17 & 5.4 & Dec. 15 & 17.4 & Sept. 19 & 220.4 \\
Sept. 24 & 16.8 & Apr. 22 & 5.5 & Jan. 26, 1960 & 12.7 & Oct. 24 & 17.2 \\
Mar. 29, 1955 & 5.0 & May 18 & 8.5 & Feb. 16 & 7.1 & Nov. 14 & 17.3 \\
May 14, 1958 & 8.6 & June 23 & 12.0 & Mar. 29 & 6.0 & Dec. 27 & $\mathbf{7 . 8}$ \\
\hline
\end{tabular}

May 14,1958 8.6 June 23

a Well being pumped.

b Well pumped recently.

\section{Round Valley}

22/12-4B1. C. Rohn. 2. $5 \mathrm{mi}$ east of Covelo. Drilled irrigation artesian well in alluvium of Recent age, diam 12 in, depth $200 \mathrm{ft}$. Lsd 1, $351 \mathrm{ft}$ above msl. MP top north side of casing, $1.00 \mathrm{ft}$ above isd. Highest water level 3.7 below 1sd, Feb. 19, 1959; lowest 17. 7 below lsd, Dec. 15,1959 . Records available: 1951-60.

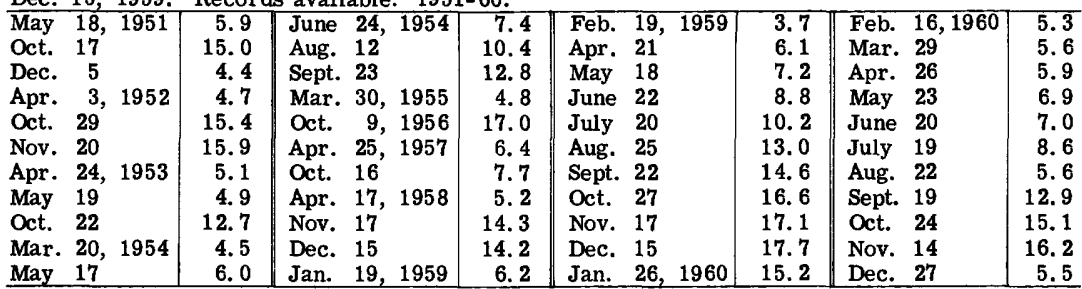

\section{Napa County}

\section{Napa Valley}

4/4-13E1. G. Lawrence. About $6.5 \mathrm{mi}$ south of Napa. Drilled stock water-table well in younger and older alluvium, diam $8 \mathrm{in}$, depth $98 \mathrm{ft}$. Lsd $41.57 \mathrm{ft}$ above msl. MP top south side of casing, at isd. Highest water level 10.1 below 1sd, Mar. 28, 1952; lowest 17.0 below lsd, Oct. 9 , 1950. Records available: 1930-32, 1949-60. No measurement made 1933-48.

\begin{tabular}{|c|c|c|c|c|c|c|c|}
\hline Mar. 25, 1930 & 13.6 & Nov. 22,1931 & 14.7 & June 6,1950 & 13.8 & Mar. 28,1952 & 10.1 \\
\hline Oct. 28 & 14.1 & Dec. 19 & 14.6 & July & 14.3 & Nov. 18 & 13.0 \\
\hline lar. 20, 1931 & 14.6 & Jan. 22, 1932 & 13.8 & Aug. 11 & 15.2 & Apr. 13,1953 & 11.3 \\
\hline Apr. 21 & 14.7 & Feb. 26 & 13.9 & Sept. & 15.8 & Oct. 27 & 12.5 \\
\hline May 21 & 16.1 & Mar. 27 & 13.4 & Oct. & 17.0 & Apr. 13,1954 & 11.6 \\
\hline June 22 & 15.0 & Dec. 21,1949 & 15.5 & Nov. & 15.4 & Mar. 28,1955 & 11.4 \\
\hline July & 15.3 & Jan. 25,1950 & 15.2 & Feb. 28, 1951 & 13.9 & 3,1956 & 12.2 \\
\hline Aug. 21 & 15.7 & Mar. 1 & 13.8 & May 26 & 12.4 & Mar. 13,1957 & 13.6 \\
\hline Sept. 28 & 14.6 & Apr. & 13.3 & Oct. & 11.6 & Sept. 30,1958 & 14.7 \\
\hline ct. 20 & 13.8 & May & 13.2 & Dec. & 12.8 & Oct. & 13.6 \\
\hline
\end{tabular}


4/4-13E1--Continued.

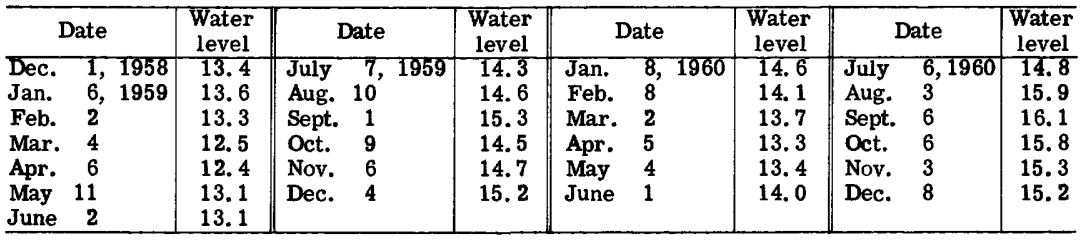

5/4-11M1. DeWitt Machine Shop. Napa. Drilled industrial water-table well in younger and older alluvium, diam $8 \mathrm{in}$, depth $77 \mathrm{ft}$. Lsd $13 \mathrm{ft}$ above msl. MP top north side of casing, $0.30 \mathrm{ft}$ above lsd. Highest water level 5.2 below 1sd, Mar. 27, 1952; lowest 10.5 below lsd, Sept. 8, 1950. Records available: 1950-60.

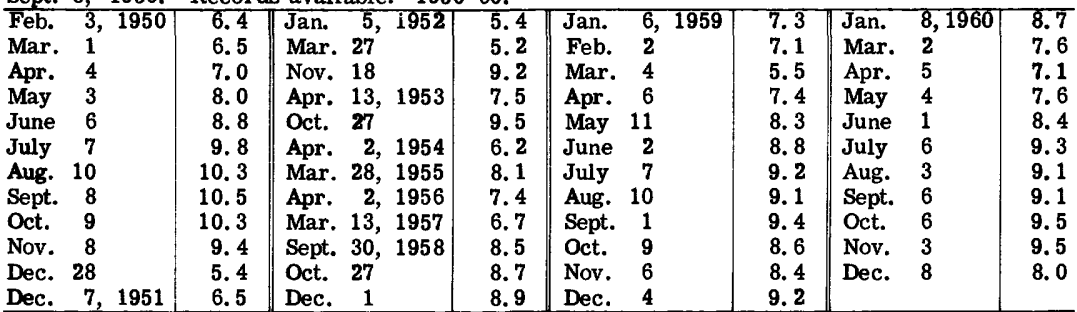

6/4-17A1. R. Ohlandt. North of Napa. Drilled irrigation water-table well in younger and older alluvium, diam $12 \mathrm{in}$, depth $250 \mathrm{ft}$, cased to 250 . Lsd $67 \mathrm{ft}$ above msl. MP top south side of casing, $0.50 \mathrm{ft}$ above lsd. Highest water level 0.7 below lsd, Mar. 27, 1952; lowest 23.7 below lsd, Oct. 6, 1960. Records available: 1949-60.

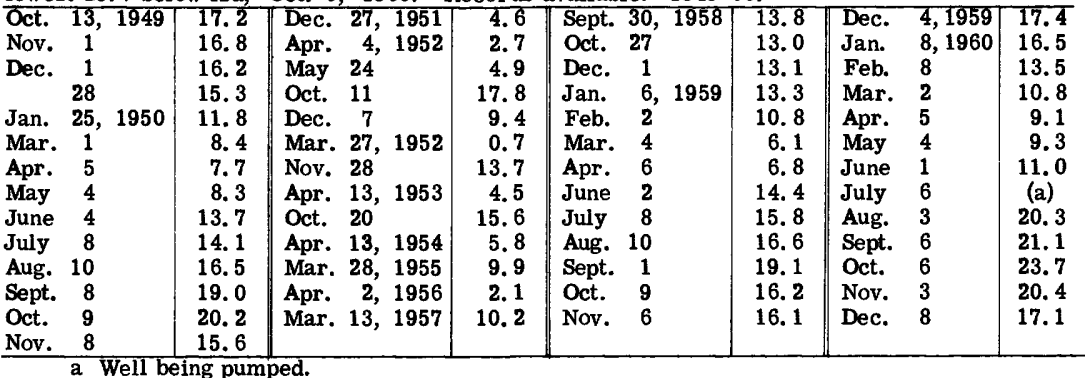

7/5-16B2. W. E. Lawson. Northeast of Rutherford. Drilled unused water-table well in younger and older alluvium, diam $10 \mathrm{in}$, depth $232 \mathrm{ft}$. Lsd $155 \mathrm{ft}$ above msl. MP top south side of casing, $0.50 \mathrm{ft}$ above lsd. Highest water level 4.0 below lsd, Mar. 27, 1952; lowest 20.9 below lsd, July 8, 1959. Records available: 1949-5s, 1955-60. No measurement made in 1954.

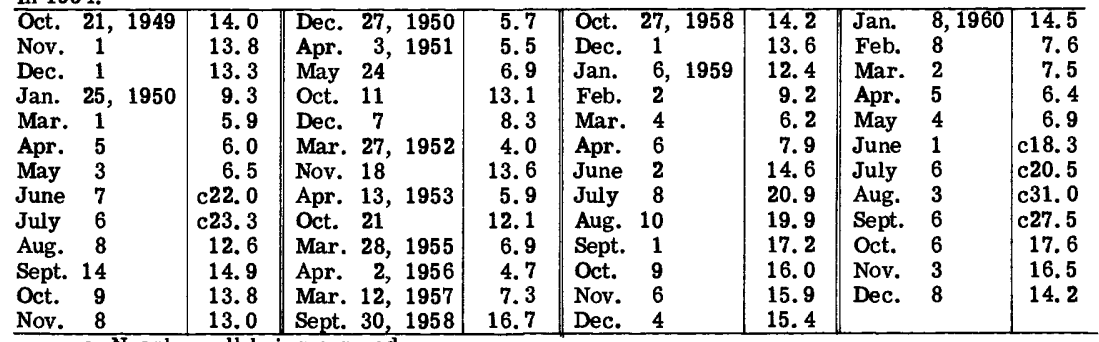

8/6-10Q1. Marolf Bros. Southeast of Callistoga. Drilled stock and irrigation watertable well in younger and older alluvium, diam $10 \mathrm{in}$, depth $184 \mathrm{ft}$. Lsd $290 \mathrm{ft}$ above msl. MP $\frac{1}{2}$-in hole in east side of pumpbase, $0.60 \mathrm{ft}$ above lsd. Highest water level 0.8 below lsd, Mar. 26, 1952, Apr. 14, 1954; lowest 40.8 below lsd, Sept. 14, 1950 . Records available: 1949-60. 
8/6-10Q1--Continued.

\begin{tabular}{|c|c|c|c|c|c|c|c|c|}
\hline Date & $\begin{array}{l}\text { Water } \\
\text { level }\end{array}$ & Date & $\begin{array}{l}\text { Water } \\
\text { level }\end{array}$ & Date & $\begin{array}{l}\text { Water } \\
\text { level }\end{array}$ & \multicolumn{2}{|c|}{ Date } & $\begin{array}{l}\text { Water } \\
\text { level }\end{array}$ \\
\hline$\overline{\text { Sept. } 30,1949}$ & 10.4 & Dec. 27,1950 & 1.4 & Sept. 30,1958 & 6.5 & Nov. & 6,1959 & 10.4 \\
\hline Nov. 2 & 17.0 & Apr. 3, 1951 & 1.3 & Oct. 27 & 6.8 & Jan. & 8,1960 & 8.3 \\
\hline Dec. & 11.5 & May 24 & 2.6 & Dec. & 6.1 & Feb. & 8 & 1.2 \\
\hline Jan. 25, 1950 & 4.4 & Oct. 11 & a63.7 & 6,1959 & 5.0 & Mar. & 2 & 1.8 \\
\hline Mar. 1 & 1.3 & Dec. 7 & 4.7 & Feb. & 2.6 & Apr. & 5 & 1.5 \\
\hline Apr. & 1.4 & Mar. 26, 1952 & 0.8 & Mar. & 1.8 & May & 4 & 2.3 \\
\hline May & 2.0 & Nov. 18 & 24.3 & Apr. & 2.0 & June & 1 & 3.7 \\
\hline June 21 & 6.8 & Apr. 3,1953 & 1.4 & May 11 & 3.5 & July & 6 & 4.9 \\
\hline July & 5.7 & Oct. 21 & 18.5 & June & 4.4 & Aug. & 3 & 6.8 \\
\hline Aug. & a65. 1 & Apr. 14, 1954 & 0.8 & July & 6.9 & Sept. & 6 & 7.9 \\
\hline Sept. 14 & 40.8 & Mar. 28, 1955 & 1.8 & Aug. 10 & 9.1 & Oct. & 6 & 9.8 \\
\hline Oct. & a74. 2 & Apr. $\quad 2,1956$ & 2.5 & Sept. 1 & 9.9 & Nov. & 4 & 10.1 \\
\hline Nov. 7 & 18.2 & Mar. 12,1957 & 1.0 & Oct. & 9.2 & Dec. & 8 & 9.0 \\
\hline
\end{tabular}

\section{Orange County}

\section{Coastal Plain}

4S/10-22L2. Halderman \& Callens. Near Anaheim. Drilled irrigation artesian and water-table well in sand and gravel of Pleistocene age, diam $16 \mathrm{in}$, depth $472 \mathrm{ft}$ (previously reported $475 \mathrm{ft}$ ), perforated 140-158, 370-401, 410-457. Lsd $135.99 \mathrm{ft}$ above $\mathrm{msl}$. MP hole in pumpbase, $0.10 \mathrm{ft}$ above Isd. Highest water level 97.16 below lsd, May 3, 1945; lowest 156.41 below lsd, Sept. 13, 1957. Records available: 1928-60. Measurements made by Orange County Flood Control District.

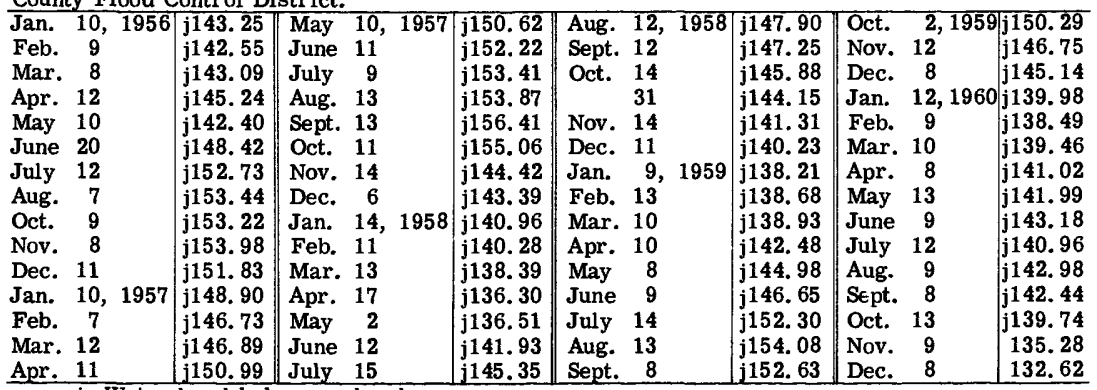

Apr. $\mathrm{j}$ Water level below sea level.

4S/11-19K1. Los Alamitos Sugar Co. Near Los Alamitos. Drilled unused artesian well in deposits of Pleistocene age, diam 12 in, depth $448 \mathrm{ft}$, perforated 440-460. Lsd 28.50 ft above msl. MP hole in recorder-shelter platform, $0.22 \mathrm{ft}$ above 1sd. Highest water level flowing, 1901; lowest 73.53 below Isd, July 22, 1957. Records available: 1901, 1903, 1929-60.

Measurements made by city of Long Beach.

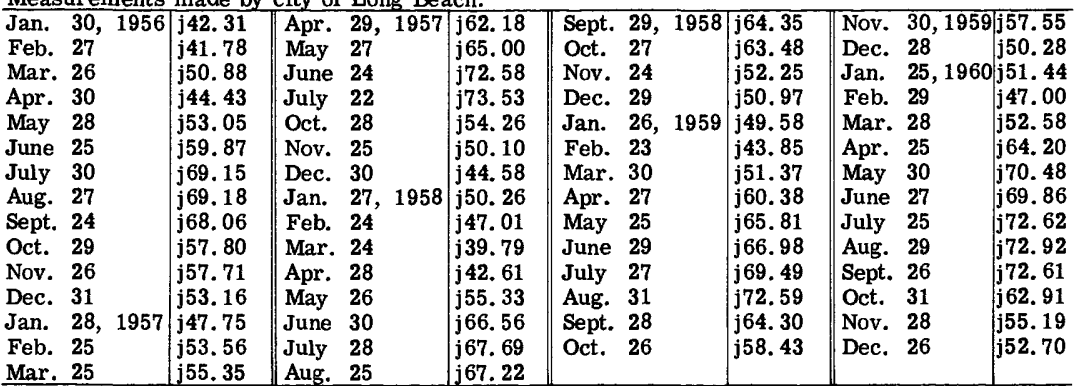

j Water level below sea level.

5S/12-12P1. U. S. Naval Ammunition and Net Depot. Near Seal Beach. Drilled unused artesian well in deposits of Pleistocene age, diam $12 \mathrm{in}$, depth $185 \mathrm{ft}$, cased with steel. Lsd $15.97 \mathrm{ft}$ above msl. MP top of cover plate over casing, $0.82 \mathrm{ft}$ above lsd. Highest water level 6. 26 below Isd, Mar. 13, 1933; lowest 42. 25 below 1sd, Aug. 9, 1957. Records available: 1930-60. Records furnished by city of Long Beach. 
5S/12-12P1--Continued.

\begin{tabular}{|c|c|c|c|c|c|c|c|}
\hline Date & $\begin{array}{l}\text { Water } \\
\text { level }\end{array}$ & Date & $\begin{array}{l}\text { Water } \\
\text { level }\end{array}$ & Date & $\begin{array}{l}\text { Water } \\
\text { level }\end{array}$ & Date & $\begin{array}{l}\text { Water } \\
\text { level }\end{array}$ \\
\hline Jan. 20,1956 & $j 34.18$ & Jan. 11,1957 & $j 36.40$ & May 23,1958 & j31.53 & Sept. 18,1958 & j40.13 \\
\hline Feb. 9 & j32. 68 & Feb. 21 & j38. 13 & June 13 & j33. 88 & Oct. 30 & $\mathrm{j} 37.95$ \\
\hline Mar. & j33. 16 & Mar. 15 & j38. 40 & July 25 & j36.90 & Nov. 20 & $\mathrm{j} 38.38$ \\
\hline 23 & $\mathrm{j} 35.50$ & Apr. 26 & j38. 48 & Aug. 15 & j37. 18 & Dec. 31 & $\mathrm{j} 36.25$ \\
\hline Apr. 13 & $\mathrm{j} 37.08$ & May 17 & j37. 28 & Sept. 26 & j36. 36 & Jan. 27, 1960 & $j 35.02$ \\
\hline May & $\mathrm{j} 32.91$ & June 28 & $\mathrm{j} 39.61$ & Oct. 17 & j35. 76 & Feb. 11 & j34. 85 \\
\hline 25 & j32. 26 & July 19 & $\mathrm{j} 40.83$ & Nov. 28 & j30.86 & Mar. 25 & $\mathrm{j} 35.44$ \\
\hline June 15 & j33. 86 & Aug. & j42. 25 & Dec. 19 & j33. 60 & Apr. 15 & $\mathrm{j} 36.36$ \\
\hline July & $\mathrm{j} 34.89$ & Sept. 13 & $\mathrm{j} 40.74$ & Jan. 30,1959 & j34. 60 & May 27 & j37. 26 \\
\hline 27 & $\mathrm{j} 38.03$ & Oct. 25 & j37. 40 & Feb. 20 & j32. 97 & June 17 & 08 \\
\hline Sept. 7 & j38. 74 & Nov. 15 & j36. 42 & Mar. 13 & j34. 01 & July 29 & $\mathrm{j} 39.08$ \\
\hline 28 & j38.65 & Dec. 27 & j34. 31 & Apr. 24 & j35. 99 & Aug. 19 & $\mathrm{j} 38.68$ \\
\hline Oct. & j39. 16 & Jan. 17,1958 & $\mathrm{j} 34.32$ & May 15 & j36. 57 & Sept. 30 & $\mathrm{j} 39.68$ \\
\hline Nov. & j36. 48 & Feb. 28 & j32. 96 & June 26 & j38. 76 & Oct. 21 & $\mathrm{j} 39.87$ \\
\hline 30 & $\mathrm{j} 37.06$ & Mar. 21 & $\mathrm{j} 30.78$ & July 17 & $j 40.00$ & Dec. 2 & $j 34.88$ \\
\hline Dec. 21 & $\mathrm{j} 35$ & Apr. 11 & j27.89 & Aug. 28 & $\mathrm{j} 40.78$ & 23 & $\mathrm{j} 33.99$ \\
\hline
\end{tabular}

6S/11-13G2. Surf Land \& Water Co. East of Huntington Beach. Drilled unused artesian well in Talbert water-bearing zone, diam $12 \mathrm{in,} \mathrm{depth} 154 \mathrm{ft}$, cased with steel, Lsd $3.30 \mathrm{ft}$ (previously reported $2.85 \mathrm{ft}$ ) above msl. MP top of recorder floor, $3.50 \mathrm{ft}$ above $1 \mathrm{sd}$. Highest water level 1.65 above 1sd, Apr. 21, 1941; lowest 17.09 below 1sd, Feb. 21, 1951. Records available: 1930-60. Records furnished by Orange County Flood Control District.

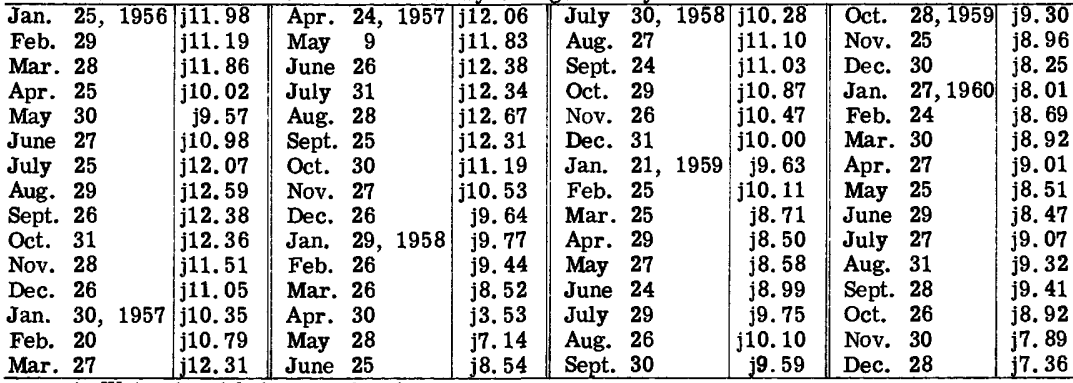

j Water level below sea level.

\section{Riverside County}

\section{San Jacinto Valley}

5/2W-27E2. Fred Harvey. Winchester. Drilled domestic water-table well in alluvium, diam 9 in, depth unknown, cased with steel. Lsd about $1,477 \mathrm{ft}$ above msl. MP top of $2-\mathrm{x}$ 8-in plank, $0.80 \mathrm{ft}$ above 1sd. Highest water level 25. 20 below 1sd, Mar. 4, 1931; lowest 60.31 below lsd, Dec. 5, 1960. Records available: 1930-60. Records furnished by Riverside County Flood Control and Conservation District.

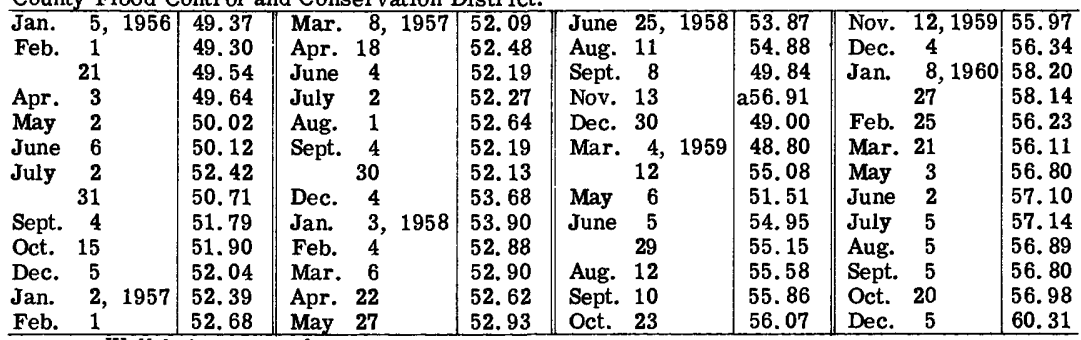

a Well being pumped.

6/3-3H1. James Miner. Menifee Valley. Drilled irrigation water-table well in alluvium, diam and depth unknown, cased with steel. Lsd about $1,428 \mathrm{ft}$ above msl. MP top of $1 \frac{\mathrm{k}}{2}$-in pipe, flush with slab south of pumpbase, at 1sd. Highest water level 126. 60 below Isd, Apr. 17, 1958; lowest 157.59 below Isd, Dec. 5, 1960. Records available: 1958-60. Records furnished by Riverside County Flood Control and Conservation District. 
6/3-3H1--Continued.

\begin{tabular}{|c|c|c|c|c|c|c|c|}
\hline Date & $\begin{array}{c}\text { Water } \\
\text { level }\end{array}$ & Date & $\begin{array}{l}\text { Water } \\
\text { level }\end{array}$ & Date & $\begin{array}{c}\text { Water } \\
\text { level }\end{array}$ & Date & $\begin{array}{l}\text { Water } \\
\text { level }\end{array}$ \\
\hline $\begin{array}{lr}\text { Jan. } & 3,1958 \\
\text { Feb. } & 14 \\
\text { Mar. } & 8 \\
\text { Apr. } 17 & \end{array}$ & $\begin{array}{l}135.18 \\
133.72 \\
129.42 \\
126.60\end{array}$ & $\begin{array}{lrr}\text { Aug. } & 12, & 195 \overline{8} \\
\text { Dec. } & 13 & \\
\text { Mar. } & 4, & 1959 \\
& 12 & \end{array}$ & $\begin{array}{l}143.98 \\
154.40 \\
144.22 \\
150.37\end{array}$ & $\begin{array}{lll}\text { June } & \text { 4, } & 1959 \\
\text { Dec. } & \mathbf{2} & \\
\text { Jan. } & \mathbf{8 ,} & 1960\end{array}$ & $\begin{array}{l}150.12 \\
155.83 \\
153.97\end{array}$ & $\begin{array}{lr}\text { Jan. } & 28,1960 \\
\text { Feb. } & 25 \\
\text { Dec. } & 5\end{array}$ & $\begin{array}{l}153.00 \\
153.97 \\
157.59\end{array}$ \\
\hline
\end{tabular}

4/3W-32E1. James Malcolm. Fourth and F Sts., Perris. Drilled water-table well in alluvium, diam $16 \mathrm{in}$, depth unknown. Lsd about $1,433 \mathrm{ft}$ above $\mathrm{msl}$. MP top of casing,

$1.70 \mathrm{ft}$ above lsd. Highest water level 62.45 below lsd, Apr. 26, 1948; lowest 89.90 below lsd, Mar. 12, 1959. Records available: 1929-43, 1945-60. Records furnished by Riverside County Flood Control and Water Conservation District.

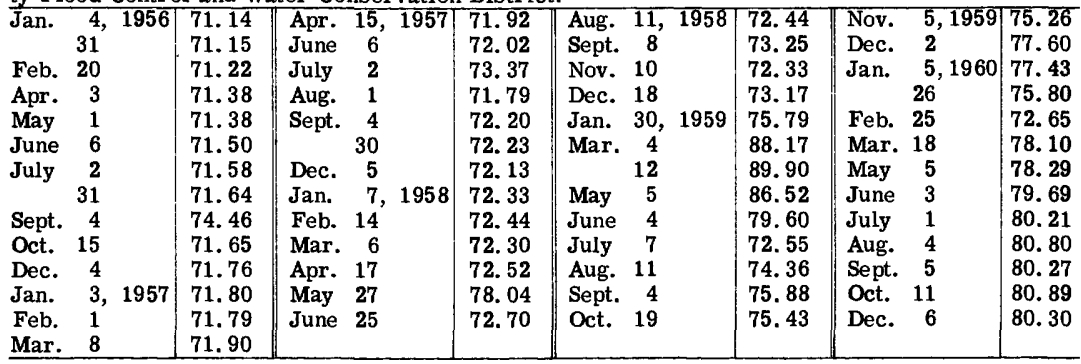

\section{San Bernardino County}

Chino Basin

1S/5-22E1. Citizens' Land \& Water Co. Bloomington. Drilled unused water-table well in alluvium of Pleistocene age, diam $14 \mathrm{in}$, depth $620 \mathrm{ft}$, cased with steel. Lsd 1,107.0 ft above msl. MP top of 2 -in access pipe, $1.00 \mathrm{ft}$ below lsd. Highest water level 236.8 below lsd, Oct. 7, 1927; lowest 275. 00 below 1sd, Dec. 9, 1959. Records available: 1927-60. Records furnished by San Bernardino County Flood Control District.

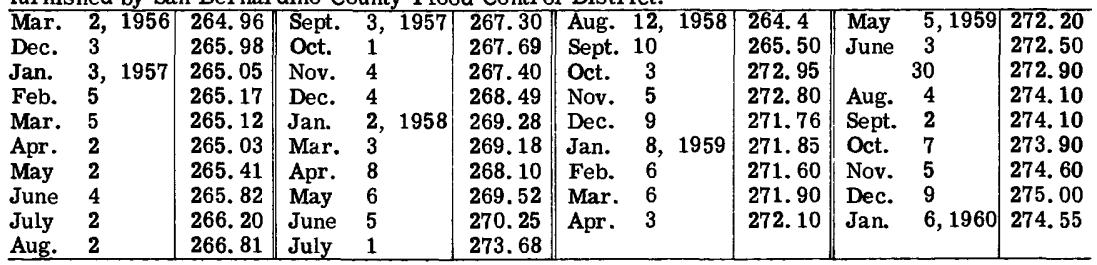

\section{Mojave River Basin}

Upper Basin

3/3W-6E2. Mike Spranger. Near Mojave River southeast of Hesperia. Drilled domestic water-table well in alluvium, diam 12 in, reported depth $61 \mathrm{ft}$, cased with steel. Lsd about $2,950 \mathrm{ft}$ above msl. MP top of access pipe, $0.96 \mathrm{ft}$ above lsd. Highest water level 3.95 below lsd, Mar. 24, 1958; lowest 57.27 below lsd, Nov. 26, 1951. Records available: 1948-60. Records furnished by San Bernardino County Flood Control District.

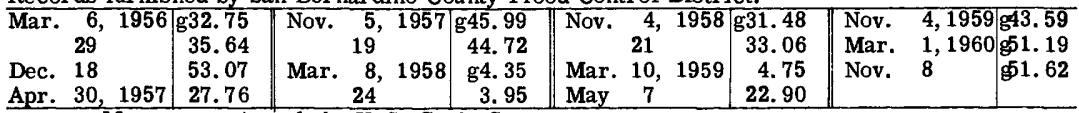

$\mathrm{g}$ Measurement made by U.S. Geol. Survey.

4/3W-18E1. Owner unknown. Near Mojave River east of Hesperia. Drilled irrigation water-table well in alluvium, diam $12 \mathrm{in}$, reported depth $100 \mathrm{ft}$, cased with steel. Lsd about 2, $867 \mathrm{ft}$ above msl. MP hole in pumpbase, $1.00 \mathrm{ft}$ above lsd. Highest water level 14. 36 below lsd, May 2, 1945; lowest 50.15 below lsd, Dec. 1, 1960. Records available: 1930-32, 1935, 1938-60. Records furnished by San Bernardino County Flood Control District. 
4/3W-18E1--Continued.

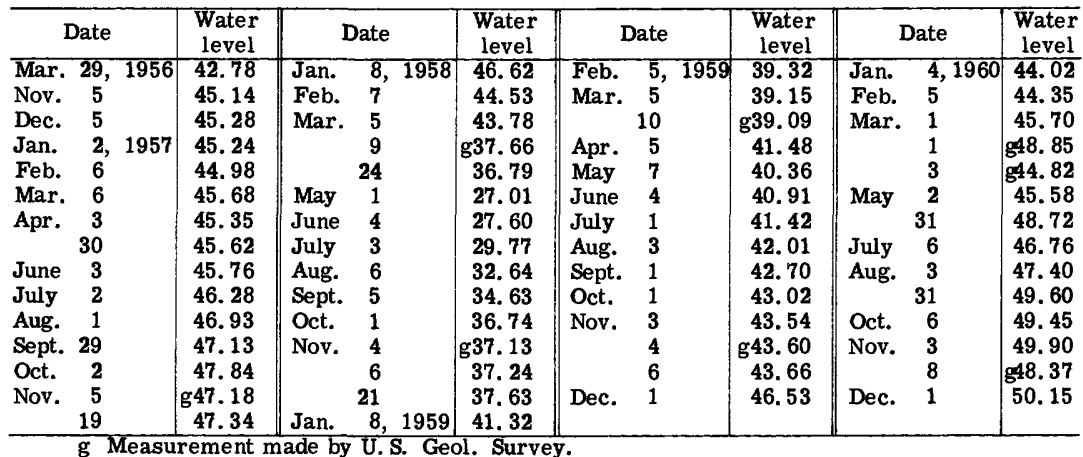

5/3W-22A1. Curtis Marshall. Apple Valley southeast of Victorville. Drilled domestic and ir rigation water-table well in alluvium, diam $12 \mathrm{in}$, depth $267 \mathrm{ft}$, cased with steel. Lsd about 2,924 ft above msl. MP top of $1 \frac{1}{4}$-in pipe, $1.20 \mathrm{ft}$ above 1sd. Highest water level 88. 87 below lsd, May 7, 1948; lowest 102.40 below lsd, Nov. 16, 1954. Records available: 1948-60. Records furnished by San Bernardino County Flood Control District.

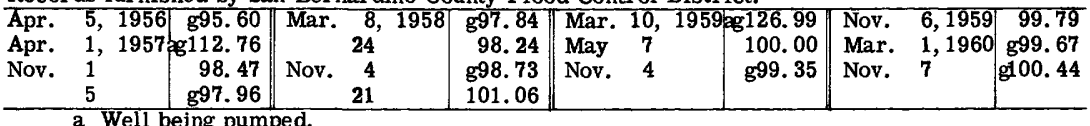

\section{a Well being pumped.}

g Measurement made by U.S. Geol. Survey.

5/4W-11P1. Mr. Pratt. Near Victorville. Drilled domestic water-table well in alluvium, diam $8 \mathrm{in}$, reported depth $65 \mathrm{ft}$, cased with steel. Lsd about 2, $786 \mathrm{ft}$ above msl. MP top of casing, $1.60 \mathrm{ft}$ above lsd. Highest water level 50.20 below lsd, Dec. 8, 1955 (previously reported 53.77 below lsd, Dec. 13, 1944); lowest 62.40 below lsd, May 11, 1954. Records available: 1931-32, 1935, 1937-60. Records furnished by San Bernardino County Flood Control District.

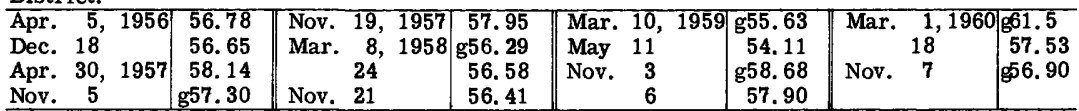

\begin{tabular}{l|l|l|l|} 
Nov. 5 & g57.30 & Nov. 21 & 56.41 \\
\hline g Measurement made by U. S. Geol. Survey
\end{tabular}

6/6-32K1. F. Reinke. El Mirage Valley. Drilled stock water-table well in deposits of Pleistocene age, diam 12 in, depth $226 \mathrm{ft}$, cased with steel. Lsd 3,047. $8 \mathrm{ft}$ above msl. MP hole in casing, $0.88 \mathrm{ft}$ above lsd. Highest water level 131.0 below lsd, Feb. 20, 1918; lowest 167. 10 below 1sd, Dec. 5, 1955. Records available: 1918, 1947, 1949-60.

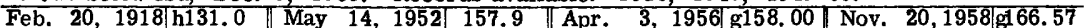

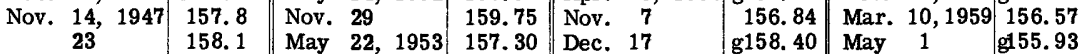

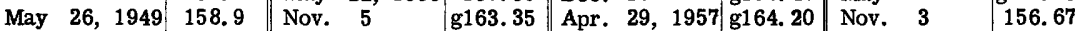

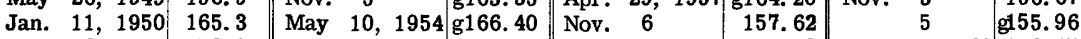

\begin{tabular}{l|l|l|l|l|l|l|l|l|l|} 
May 8 & 158.0 & Nov. 15 & g161.35 & Mar. 8, 1958 & 156.68 & Feb. 29, 1960 156.47
\end{tabular}

\begin{tabular}{l|l|l|l|l|l|l|l|l|l|l|} 
Nov. 16 & 156.9 & Apr. 11, 1955 & g161.25 & Apr. & $\mathbf{7}$ & g157.30 & Mar. 18 & g156.55
\end{tabular}

\begin{tabular}{|l|l|l|l|ll|l|l|l|l|l|l|l|l|} 
May 15,1951 & 156.4 & Dec. 5 & g167.10 & Nov. 4 & 156.57 & Nov. 7 & 156.52 \\
\hline
\end{tabular}

Nov. 27 $156.7 \mid$\begin{tabular}{l|l|l|l|}
150 \\
\hline
\end{tabular}

h Measurement made by California Department of Engineering.

\section{Middle Basin}

6N/4W-6E6. R. D. Workman. South of LaDelta. Drilled unused water-table well in alluvium, diam $6 \mathrm{in}$, depth $104 \mathrm{ft}$, cased with steel. Lsd about 2,580 ft above msl. MP top of casing, $1.00 \mathrm{ft}$ above lsd. Highest water level 42.88 below lsd, Mar. 3, 1960; lowest 44.54 below lsd, Nov. 17, 1960. Records available: 1958-60. Nov. 7, 1958, 44.53; Mar. 12, 1959 , 42.90; Nov. 30, 44. 20; Mar. 3, 1960, 42. 88; Nov. 17, 44. 54.

6N/5W-8F1. Lingren. North of Adelanto. Drilled domestic water-table well in alluvium, diam 8 in, depth about $90 \mathrm{ft}$, cased with steel. Lsd about $2,780 \mathrm{ft}$ above msl. MP hole in west side of casing, $0.65 \mathrm{ft}$ above lsd. Highest water level 77.13 below lsd, Dec. 17, 1956; lowest 82. 76 below Isd, Nov. 29, 1952. Records available: 1947-60. 
$6 \mathrm{~N} / 5 \mathrm{~W}-8 \mathrm{~F} 1--$ Continued.

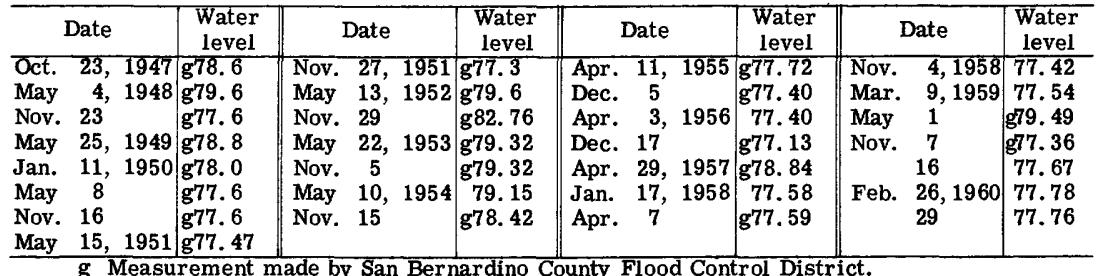

6/5-14M1. Owner unknown. North of George Air Force Base. Dug unused water-table well in alluvium of Pleistocene age, diam $45 \mathrm{in}$, depth $164 \mathrm{ft}$, cribbed with wood. Lsd about $2,825 \mathrm{ft}$ above msl. MP top of wood curbing, $0.80 \mathrm{ft}$ above lsd. Highest water level 116. 41 below 1sd, Feb. 29, 1960; lowest 120.7 below 1sd, May 8, 1950. Records available: 1947-60.

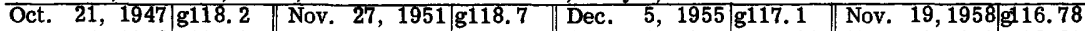

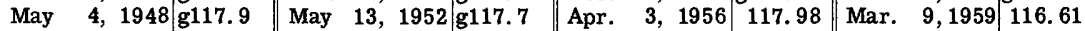
\begin{tabular}{l|l|l|l|l|l|l|l|l|l|l|l|l|l|} 
Nov. 23 & g118.2 & Nov. 29 & g117.95 & Dec. 17 & g117.20 & May 1 & g1 16.61
\end{tabular} \begin{tabular}{l|l|l|l|l|l|l|l|l|} 
May 25, 1949 & g117.7 & May 22, 1953 g117.37 & Apr. 29, 1957 & g118.46 & Nov. 16 & 116.50
\end{tabular} \begin{tabular}{l|l|l|l|ll||l|l|l|} 
Jan. 11, 1950 & g118. & Nov. 5 & g117. 70 & Oct. 11 & 116.91 & Feb. 29, 1960 116.41
\end{tabular}

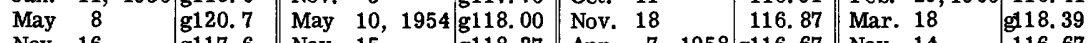
\begin{tabular}{l|l|l|l|l|l|l|l|l|l|} 
Nov. 16 & g117. & Nov. 15 & g118.27 & Apr. 7,1958 & g116.67 & Nov. 14 & 116.67 \\
May 15, 1951 & g117.65 & Apr. 11, 1955 & g118.30 & Nov. 4 & 116.60 & & \\
\hline
\end{tabular}

$7 \mathrm{~N} / 5 \mathrm{~W}-7 \mathrm{N1}$. Bob's Smoke Shop. North of Adelanto. Drilled domestic water-table well in alluvium of Pleistocene age, diam 6 in, depth about $178 \mathrm{ft}$, cased with steel. Lsd about $2,780 \mathrm{ft}$ above msl. MP top of casing, $0.80 \mathrm{ft}$ above $1 \mathrm{sd}$. Highest water level 145. $80 \mathrm{below}$ Isd, Feb. 29, 1960; lowest 157.2 below 1sd, Oct. 26, 1950. Records available: 1950, 195860. Oct. 26, 1950, 157.2 (measurement made by U. S. Bureau of Reclamation); Feb. 27, $1958,150.00$; Nov. 4, 148.18; Mar. 9, 1959, 147.63; Nov. 17, 146. 62; Feb. 29, 1960, 145. 80; Nov. 14, 150.13.

8/4W-12Q1. Holcomb Bros. Near Wild Crossing. Drilled irrigation water-table well in alluvium, diam $48 \mathrm{in}$, depth unknown, cased. Lsd about 2, $329 \mathrm{ft}$ above msl. MP top of casing under discharge, 2.50 ft above 1sd. Highest water level 7.16 below lsd, May 13, 1954 (pre-viously reported 8.16 below lsd); lowest 28.88 below lsd, June 3,1957 . Records available: 1931-32, 1935-37, 1939-41, 1943-60. Records furnished by San Bernardino County Flood Control District.

\begin{tabular}{|c|c|c|c|c|c|c|c|c|c|c|}
\hline Mar & $50-1056$ & 1777 & 2.1057 & 30 & & 17058 & 57 & & 1960 & 51566 \\
\hline Nov. & 5 & $\begin{array}{l}11.77 \\
13.12\end{array}$ & $\begin{array}{lll}\text { Dec. } & 2,1957 \\
\text { Jan. } & 8,1958\end{array}$ & $\begin{array}{l}28.10 \\
13.64\end{array}$ & Dec. & $\begin{array}{l}1,1958 \\
7,1959\end{array}$ & $\begin{array}{l}11.57 \\
11.99\end{array}$ & $\begin{array}{l}\text { Feb. } \\
\text { Mar. }\end{array}$ & 2,1960 & $\begin{array}{l}15.56 \\
15.99\end{array}$ \\
\hline Dec. & 5 & 12.80 & Feb. & 12.68 & Feb. & 5 & 12.28 & & 2 & g. 5.89 \\
\hline Jan. & 2, 1957 & 12.97 & Mar. & 21.49 & Mar. & 5 & 11.53 & May & 2 & 17.22 \\
\hline Feb. & 6 & 12.30 & 24 & 12.10 & May & 4 & 14.07 & & 31 & 32.14 \\
\hline Mar. & 6 & 12.54 & May & 19.07 & Nov. & 3 & 16.46 & July & 6 & 232.00 \\
\hline Apr. & 3 & 28.39 & June & 17.47 & & 11 & 15.15 & Oct. & 6 & 21.20 \\
\hline May & 1 & 15.71 & 10 & g16.96 & Dec. & 1 & g15. 45 & Nov. & 3 & 21.15 \\
\hline June & 3 & 28.88 & July & 17.93 & & 1 & 16.46 & & 17 & 920.85 \\
\hline Aug. & 29 & 17.65 & Sept. & 20.52 & Jan. & 4, 1960 & 15.85 & Dec. & 1 & 20.60 \\
\hline Oct. & 1 & 17.67 & Nov. & 11. 36 & & & & & & \\
\hline
\end{tabular}

8/4W-31R1. Fred Orebaugh. Near Helendale. Dug and drilled unused water-table well, diam $14 \mathrm{in}$. Lsd about 2, $449 \mathrm{ft}$ above msl. MP top of casing, $12.72 \mathrm{ft}$ below $1 \mathrm{sd}$. Highest water level 14.10 below lsd, Apr. 25, 1946 (previously reported 5.14 below Isd, Jan. 16, 1953); lowest 27.41 below Isd, Nov. 11, 1959. Records available: 1930-32, 1934-48, 195060. Records furnished by San Bernardino County Flood Control District. Corrected measurements shown for 1953-55.

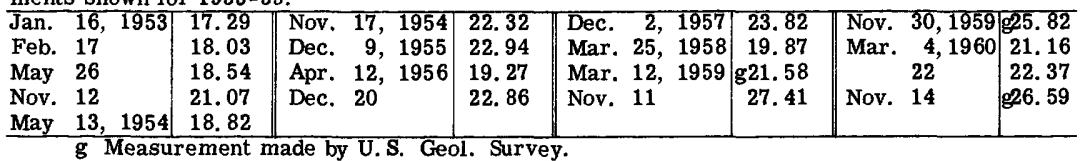

9N/1W-5D1. Tex Bernett. Barstow. Drilled industrial water-table well in alluvium, diam $10 \mathrm{in}$, depth $60 \mathrm{ft}$, cased with steel. Lsd about 2,115 ft above msl. MP top of casing, $2.00 \mathrm{ft}$ above $1 \mathrm{sd}$. Highest water level 24.01 below 1sd, June 19, 1958; lowest 27. 91 below lsd, Nov. 16, 1960. Records available: 1958-60. June 19, 1958, 24.01; Dec. 1, 1959, 27.90; May 2, 1960, 27.18; Nov. 16, 27.91. 
10N/3W-35N1. Edward Horton. Near Hinkley. Drilled domestic water-table well in alluvium, diam 8 in, depth $144 \mathrm{ft}$, cased. Lsd about 2, $220 \mathrm{ft}$ above $\mathrm{msl}$. MP top of casing, $0.95 \mathrm{ft}$ above lsd. Highest water level 51.1 below lsd, Dec, 19, 1930; lowest 105. 41 below lsd, Mar. 2, 1960. Records avallable: 1930-32, 1958-60.

\begin{tabular}{|c|c|c|c|c|c|c|c|}
\hline Date & $\begin{array}{c}\text { Water } \\
\text { level }\end{array}$ & Date & $\begin{array}{l}\text { Water } \\
\text { level }\end{array}$ & Date & $\begin{array}{l}\text { Water } \\
\text { level }\end{array}$ & Date & $\begin{array}{c}\text { Water } \\
\text { level }\end{array}$ \\
\hline $\begin{array}{l}\text { Sept. } 19,1930 \\
\text { Dec. } 19 \\
\text { Aug. } \quad 6, \quad 1931 \\
\end{array}$ & \begin{tabular}{|l|}
52.0 \\
51.1 \\
54.0 \\
\end{tabular} & $\begin{array}{l}\text { Mar. } 4,1932 \\
29 \\
\text { Apr. } 21\end{array}$ & $\begin{array}{l}52.8 \\
53.4 \\
54.1 \\
\end{array}$ & $\begin{array}{lll}\text { July } & 26, & 1932 \\
\text { Dec. } & 22 \\
\text { Nov. } & 20, & 1958\end{array}$ & $\begin{array}{l}55.4 \\
54.4 \\
94.08 \\
\end{array}$ & $\begin{array}{|lr|}\text { Dec. } & 1,1959 \\
\text { Mar. } & 2,1960 \\
\text { Nov. } & 16\end{array}$ & \begin{tabular}{|r|}
96.81 \\
105.41 \\
93.34 \\
\end{tabular} \\
\hline
\end{tabular}

Lower Basin

8/4E-12L1. Mojave Camp Service Station. East of Troy Dry Lake. Drilled public-supply water-table well in alluvium, diam $10 \mathrm{in}$, reported depth $205 \mathrm{ft}$, cased with steel. Lsd about $1,810 \mathrm{ft}$ above $\mathrm{msl}$. MP top of casing, $1.00 \mathrm{ft}$ above lsd. Highest water level 30.39 below lsd, Mar. 27, 1958; lowest 33. 58 below lsd, Nov. 24, 1942. Records available: 1930, 1932, 1935-45, 1947-60. Records furnished by San Bernardino County Flood Control District.

\begin{tabular}{|c|c|c|c|c|c|c|}
\hline $\begin{array}{l}\text { Apr. } 17,1956 \\
\text { Dec. } 26 \\
\text { May } \quad 3,1957\end{array}$ & $\begin{array}{l}31.20 \\
31.40 \\
30.70\end{array}$ & $\begin{array}{l}\text { Dec. } 4,1957 \\
\text { Mar. 27, } 1958 \\
\text { Dec. } 3\end{array}$ & $\begin{array}{l}31.26 \\
30.39 \\
31.17\end{array}$ & $\begin{array}{l}\text { May } 5,1959 \\
\text { June } 17 \\
\text { Nov. } 12\end{array}$ & $\begin{array}{r}31.63 \\
\mathrm{~g} 31.21 \\
31.20\end{array}$ & \begin{tabular}{l|l|l|} 
Mar. 24,1960 & 31.56 \\
Nov. 15 & $\$ 31.86$
\end{tabular} \\
\hline
\end{tabular}
\begin{tabular}{l|l|l|l|l|l|} 
May 3,1957 & 30.70 & Dec. 3 & 31.17 \\
\hline
\end{tabular}

9/1E-13E2. California Electric Power Co. Formerly Getz. Drilled unused water-table well in alluvium, diam $12 \mathrm{in}$, depth $170 \mathrm{ft}$, cased with steel, Lsd about 1,950 ft above msl. MP top of casing, $0.70 \mathrm{ft}$ above isd. Highest water level 54.46 below isd, Apr. 26, 1944; lowest 86. 20 below lsd, Aug. 31, 1960. Records available: 1925-27, 1930-33, 1935-60. Records furnished by San Bernardino County Flood Control District.

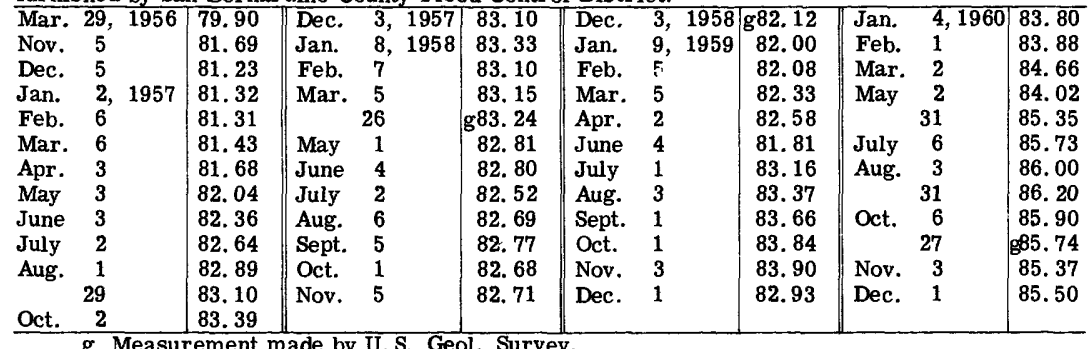

9/2E-20Q1. Daggett Airport. Minneola Crossing. Drilled domestic water-table well in alluvium, diam 12 in, reported depth $142 \mathrm{ft}$, cased with steel. Lsd about $1,922 \mathrm{ft}$ above $\mathrm{msl}$. MP hole drilled at southwest side of pumpbase, plugged with redwood, $0,40 \mathrm{ft}$ above isd. Highest water level 41,81 below lsd, Nov, 15, 1945; lowest 66, 60 below 1sd, Mar. 24, 1960. Records available: 1932, 1941-48, 1952-60. Records furnished by San Bernardino County Flood Control District.

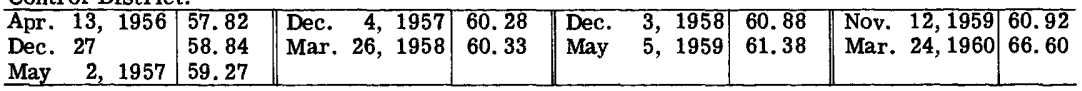

9/3E-12E1. Tankersley. Northeast of Troy Dry Lake. Drilled domestic and irrigation water-table well in alluvium, diam $10 \mathrm{in}$, depth unknown, cased with steel. Lsd about $1,801 \mathrm{ft}$ above msl. MP top of casing, 2.60 ft below lsd. Highest water level 23. 84 below lsd, Mar. 23, 1922; lowest 35.60 below lsd, May 19, 1954. Records available: 1922, 1930-33, 1935, 193744, 1946-60. Records furnished by San Bernardino County Flood Control District.

\begin{tabular}{|c|c|c|c|c|c|c|c|c|c|c|c|}
\hline & & & & & & & & & & & \\
\hline Mar. & 29,1956 & 34.32 & Oct. & 2,1957 & 33.72 & Jan. & 8,1959 & 31.82 & Dec. & 1,1959 & 32.81 \\
\hline Nov. & 5 & 32.49 & Dec. & & 31. 63 & Feb. & 5 & 30.68 & Jan. & 4,1960 & 32.45 \\
\hline Dec. & & 27.20 & Jan. & 3,1958 & 32.00 & Mar & 5 & 31.02 & Feb. & 1 & 33.27 \\
\hline Jan. & 2,1957 & 32.25 & & 8 & 33.59 & Apr. & 2 & 31.27 & Mar. & 2 & 32.18 \\
\hline Feb. & 6 & 31.58 & Feb. & 7 & 32.35 & May & 5 & 32.51 & & 24 & 31.80 \\
\hline Mar. & 6 & 31.58 & Mar. & 5 & 32.34 & June & 4 & 32.56 & May & 2 & 32.23 \\
\hline Apr. & 3 & 31.90 & May & 1 & 31.96 & July & 1 & 32.50 & & 31 & 33.65 \\
\hline May & 2 & 32.30 & June & 4 & 32.36 & Aug. & 3 & 32.53 & July & 6 & 34.12 \\
\hline & 3 & 32.40 & July & 3 & 34.07 & Sept & 1 & 33.38 & Aug. & 31 & 34.50 \\
\hline June & 3 & 32.08 & Sept. & 5 & 32.93 & Oct. & 1 & 33.35 & Oct. & 6 & 35.00 \\
\hline July & 2 & 32.59 & Oct. & 1 & 32.95 & Nov. & 3 & 33.19 & Nov. & 3 & 34.17 \\
\hline Aug. & 1 & 34.02 & Nov. & 6 & 34.38 & & 12 & 32.83 & Dec. & 1 & 33.70 \\
\hline & 29 & 33.15 & Dec. & 3 & 32.17 & & 18 & $\mathrm{~g} 32.92$ & & & \\
\hline
\end{tabular}

$\mathrm{g}$ Measurement made by U. S. Geol. Survey. 
9/3E-19P1. J. G. Frey. Near Newberry. Drilled unused artesian well in alluvium, diam $12 \mathrm{in}$, reported depth $151 \mathrm{ft}$, cased with steel. Lsd about $1,859 \mathrm{ft}$ above msl. MP top of casing, 2. $00 \mathrm{ft}$ above lsd. Highest water level flowing, Jan. 3, May 10, Nov. 16, 1945, May 2, 1946, May 23, 1947; lowest 9.88 below Isd, May 5, 1959. Records available: 1919, 1922, 1930-35, 1938-47, 1951-60. Records furnished by San Bernardino County Flood Control District.

\begin{tabular}{|c|c|c|c|c|c|c|c|}
\hline Date & $\begin{array}{l}\text { Water } \\
\text { level }\end{array}$ & Date & $\begin{array}{c}\text { Water } \\
\text { level }\end{array}$ & Date & $\begin{array}{c}\text { Water } \\
\text { level }\end{array}$ & Date & $\begin{array}{l}\text { Water } \\
\text { level }\end{array}$ \\
\hline $\begin{array}{lrr}\text { Apr. } & 4, & 1956 \\
\text { Dec. } & 26 & \\
\text { May } & 3, & 1957\end{array}$ & $\begin{array}{l}6.70 \\
6.25 \\
7.60\end{array}$ & $\begin{array}{lrl}\text { Dec. } & 4,1957 \\
\text { Mar. } & 26,1958 \\
\text { Dec. } & 3\end{array}$ & $\begin{array}{r}7.79 \\
7.30 \\
\text { g8. } 96\end{array}$ & $\begin{array}{lrl}\text { May } & 5,1959 \\
\text { Nov. } 12 & \end{array}$ & $\begin{array}{l}9.88 \\
8.39\end{array}$ & $\begin{array}{lr}\text { Dec. } & 4,1959 \\
\text { Mar. } & 24,1960\end{array}$ & $\begin{array}{l}9.19 \\
8.40\end{array}$ \\
\hline
\end{tabular}

\section{Santa Ana River Basin}

1N/4-32N1. City of San Bernardino. Baseline well. Northwest of San Bernardino. Drilled observation water-table well in alluvium of Pleistocene age, diam $20 \mathrm{in}$, depth $581 \mathrm{ft}$, cased with steel. Lsd about $1,184 \mathrm{ft}$ above msl. MP top of casing, $1.80 \mathrm{ft}$ above lsd. Highest water level 49.4 below lsd, Mar. 6, 1947; lowest 174. 2 below lsd, Sept. 27, 1960. Records available: 1946-60. Records furnished by owner.

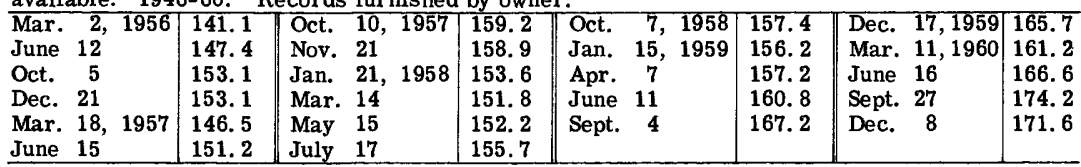

1S/3-17C3. Harold Daniels. Northwest of Redlands. Drilled observation water-table well in alluvium of Pleistocene age, diam $8 \mathrm{in}$, depth $296 \mathrm{ft}$, cased with steel. Lsd 1, 175. $83 \mathrm{ft}$ above msl. MP top of $1 \frac{1}{4}$-in pipe, $0.30 \mathrm{ft}$ above lsd. Highest water level 101.95 below lsd, July 1, 1958; lowest 152.65 below 1sd, Oct. 29, 1960. Records available: 1957-60. Records furnished by Gage Canal Co.

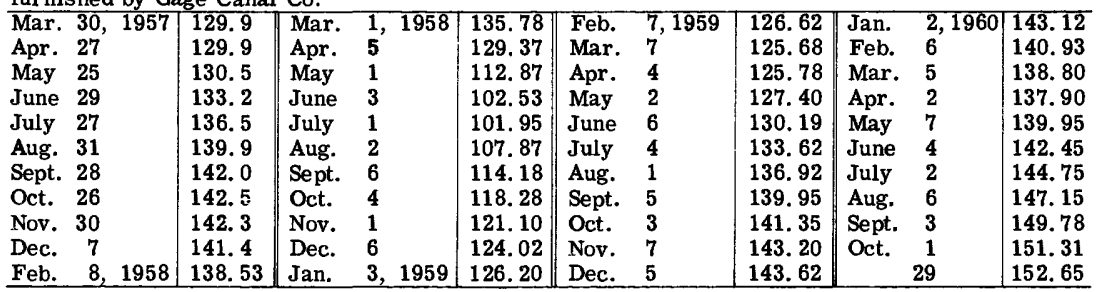

1S/3-32D1. Hinckley. Windmill well. Near San Timoteo Canyon west of Redlands. Drilled unused well in alluvium of Pliocene (?) and Pleistocene age, diam $10 \mathrm{in}$, depth $720 \mathrm{ft}$, cased with steel. Lsd 1, 206.9 ft above msl. MP top of casing, $0.60 \mathrm{ft}$ above isd. Highest water level 35.1 below 1sd, Mar. 2, 1932 (previously reported 1923); lowest 197. 5 below lsd, Aug. 3, 1960. Records available: $1900,1904-07,1910-60$. Records furnished by San Bernardino Valley Water Conservation District.

\begin{tabular}{|c|c|c|c|c|c|c|c|c|c|c|c|}
\hline Jan. & 17,1956 & 137.3 & Aug. & 20,1957 & 170.3 & Aug. & 8,1958 & 163.7 & \multicolumn{3}{|c|}{\begin{tabular}{l|l|}
5,1959 & 176.6
\end{tabular}} \\
\hline Mar & 26 & 134.8 & Oct. & 2 & 172.4 & Sept. & 9 & 166.0 & Sept. & 17 & 179.0 \\
\hline July & 19 & 156.2 & Nov. & 6 & 162.9 & Oct. & 6 & 171.7 & Nov. & 5 & 177.9 \\
\hline Oct. & 1 & 164.3 & Dec. & 3 & 161.6 & Nov. & 13 & 168.0 & Dec. & 2 & 180.7 \\
\hline Dec. & 28 & 162.5 & Jan. 1 & 10,1958 & 150.3 & Dec. & 20 & 166.3 & Jan. & 12,1960 & 173.7 \\
\hline Jan. & 1957 & 151.2 & Feb. & 11 & 147.0 & Jan. & 30,1959 & 163.0 & Feb. & 23 & 171.2 \\
\hline Feb. & 18 & 142.4 & Mar. & 10 & 143.0 & Mar. & 16 & 148.1 & Mar. & 24 & 6.5 \\
\hline Mar & 21 & 137.2 & Apr. & 10 & 138.7 & Apr. & 14 & 158.0 & Apr. & 25 & 166.1 \\
\hline Apr. & 3 & 137.1 & May & 1 & 140.4 & May & 14 & 166.3 & May & 24 & 178.1 \\
\hline May & 23 & 146.1 & June 1 & 10 & 147.4 & June & 11 & 168.2 & June & 30 & 195.1 \\
\hline July & 27 & 164.9 & July 1 & 19 & 160.0 & July & 15 & 174.2 & Aug. & 3 & 197.5 \\
\hline
\end{tabular}

1S/4-1A6. Riverside Water Co. McCrary tract well 4. East of San Bernardino. Drilled public-supply water-table well in alluvium of Pleistocene age, diam $10 \mathrm{in}$, reported depth $648 \mathrm{ft}$, cased with steel. Lsd 1, 096. $0 \mathrm{ft}$ above msl. MP $1.56 \mathrm{ft}$ above lsd. Highest water level 15.94 (previously reported 51.94) above lsd, Mar. 25, 1917; lowest 84.45 below lsd, Sept. 9, 1960. Records available: 1915-60. Records furnished by owner.

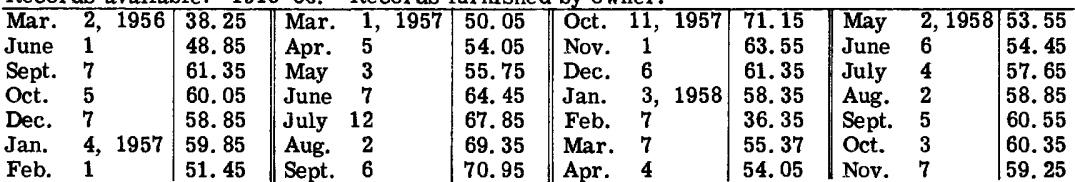


1S/4-1A6--Continued.

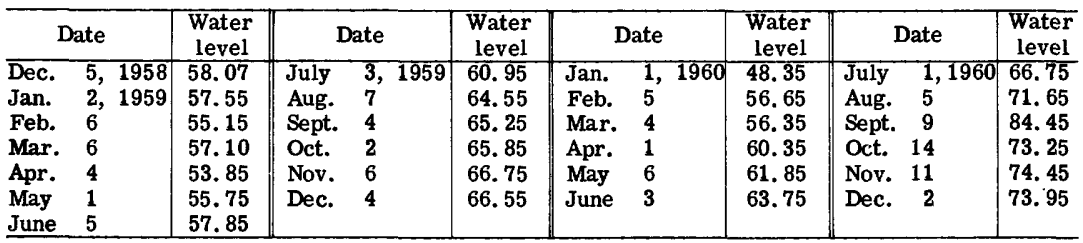

1S/4-29H2. Riverside Water Co., flume 1. South of Colton. Drilled irrigation watertable well in alluvium of Pleistocene and Recent age, diam 20 in, reported depth $189 \mathrm{ft}$, cased with steel. Lsd about $934 \mathrm{ft}$ above msl. MP top of casing, $4.00 \mathrm{ft}$ above lsd. Highest water level 3.5 below lsd, June 3, 1938; lowest 98.80 below lsd, Oct. 7, 1960. Records available: 1928-60. Records furnished by owner.

\begin{tabular}{|c|c|c|c|c|c|c|c|c|c|c|c|}
\hline Jan. & 6,1956 & 66.00 & Feb. & $7, \mathbf{1 9 5 8}$ & 65.20 & Feb. & 6,1959 & 70.50 & Feb. & \multicolumn{2}{|c|}{\begin{tabular}{l|l|}
5,1960 & 84.60
\end{tabular}} \\
\hline Apr. 1 & 14 & 62.80 & Mar. & 7 & 62.50 & Mar. & 6 & 65.25 & Mar. & 4 & 80.80 \\
\hline July 1 & 13 & 71.00 & Apr. & 4 & 49.00 & Apr. & 10 & 72.90 & Apr. & 8 & al12. 00 \\
\hline Dec. & 7 & 66.80 & May & 2 & 41.40 & May & 1 & a104. 75 & May & 6 & 81.80 \\
\hline Jan. & 4,1957 & 74.4 & June & 4 & 49.40 & June & 5 & al11. 70 & June & 3 & al17.90 \\
\hline Feb. & 1 & 60.9 & July & 12 & 57.20 & July & 3 & 85.50 & July & 1 & al 18.90 \\
\hline Mar. & 1 & 50.1 & Aug. & 1 & 59.00 & Aug. & 7 & 2120.90 & Aug. & 5 & ald 21.50 \\
\hline Apr. 1 & 2 & 58.70 & Sept. & 26 & 65.57 & Sept. & 4 & 93.40 & Sept. & 1 & 95.65 \\
\hline Oct. 1 & 8 & 79.60 & Oct. & 3 & 67.80 & Oct. & 2 & 92.40 & Oct. & 7 & 98.80 \\
\hline Nov. & 8 & 77.00 & Nov. & 7 & 70.42 & Nov. & 6 & 97.10 & Nov. & 4 & 98.30 \\
\hline Dec. & 6 & 75.40 & Dec. & & 70.45 & Dec. & 4 & 97.00 & Dec. & 2 & 94.70 \\
\hline In. & 58. & 70.10 & Jan. & 2,1959 & 69.60 & Jan. & 360 & 90.60 & & & \\
\hline
\end{tabular}

\section{Yucca Valley-Twentynine Palms Area}

1N/7E-26N1. Clyde Rollard. Near south end of Copper Mountain. Drilled domestic watertable well in alluvium of Pleistocene age, diam 8 in, depth $208 \mathrm{ft}$, cased with steel. Lsd about 2, $475 \mathrm{ft}$ above msl. MP top of casing, $1.50 \mathrm{ft}$ above $1 \mathrm{sd}$. Highest water level 159.0 below lsd, Apr. 21, 1954; lowest 175.12 below lsd, Nov. 13, 1951. Records available: 1946-60.

Nov. 15, 1946 Apr. 16, 1947 g169.00 Nov. 13 g175.12 Apr. 25, 1956 g168.10 Mar. 11, 1959 168.90

\begin{tabular}{l|l|l|l|l|l|l|l|l|l|l|l|l|l|l|} 
Nov. 17 & g169. 00 & Apr. 14, 1952 & g165.48 & Jan. 17, 1957 & g168.12 & May 13 & 168.08
\end{tabular}

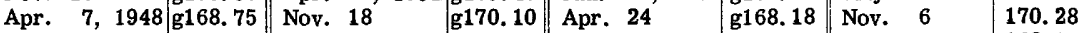

Nov. 16 g168.40 May 25, 1953 g168.50 Apr. 8, 1958 172.91 Dec. 9 168.05

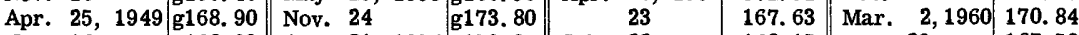

\begin{tabular}{l|l|l|l|l|l|l|l|} 
Nov. 16 & g168. 93 & Apr. 21, 1954 & g159. 0 & July 22 & 168.15 & 29 & g67.50
\end{tabular}

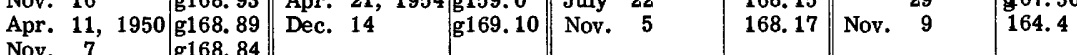

Nov, 7 Measurement made by San Bernardino County Flood Control District.

1N/9E-5G1. U. S. Dept. of the Navy. South edge of Mesquite Dry Lake. Drilled publicsupply water-table well in alluvium of Pleistocene age, diam $14 \mathrm{in}$, depth $500 \mathrm{ft}$, perforated 266-284, 324-330, 358-365, 390-396, 415-428. Lsd 1,779. $2 \mathrm{ft}$ above msl. MP hole in pumpbase, $0.80 \mathrm{ft}$ above $1 \mathrm{sd}$. Highest water level 4.98 below lsd, Apr. 28, 1952; lowest 6. 52 below lsd, Sept. 1, 1960. Records available: 1952-60.

\begin{tabular}{|c|c|c|c|c|c|c|c|c|c|c|c|}
\hline Feb. & 13,1952 & 5.48 & May & 17,1955 & 5.07 & Anr & 3.1957 & 5.16 & Mar & 111959 & 534 \\
\hline Apr. & 28 & 4.98 & June & 17 & b5. 35 & May & 7 & 5.12 & Apr. & 7 & 5.20 \\
\hline Dec. & 5 & a20.31 & July & 21 & 5.23 & June & 6 & 5.26 & May & 11 & 5.27 \\
\hline Jan. & 23,1953 & a12.82 & Aug. & 24 & 5.22 & July & 5 & 5.36 & June & 10 & 5.58 \\
\hline eb. & 15 & 5.18 & Sept. & 23 & a39.75 & Aug. & 6 & 5.32 & July & 7 & 5.32 \\
\hline & 16 & a12.55 & Oct. & 18 & 5.25 & Sept. & 9 & 5.71 & Oct. & 6 & 5.40 \\
\hline Apr. & 30 & a8. 49 & Nov. & 23 & 5.29 & Oct. & 7 & 5.27 & Nov & 7 & 5.30 \\
\hline May & 29 & a21. 95 & Dec. & 21 & 5. 29 & Nov. & 7 & 5.24 & Dec. & 9 & 6.00 \\
\hline Aug. & 8 & 5.00 & Jan. & 23,1956 & 5. 26 & Dec. & 9 & 5.21 & Jan. & 4,1960 & 6.21 \\
\hline Aug. & 24, & 250 & Mar. & 1 & 5.30 & Jan. & 10,1958 & 5.19 & Feb. & 1 & b7. 12 \\
\hline Sept. & 24 & 5.16 & May & 1 & 5.29 & Feb. & 10 & 5.54 & Mar & 2 & 6.24 \\
\hline Oct. & 21 & 5.16 & July & 2 & 5.35 & Mar. & 11 & 5.16 & Apr & 3 & 6.14 \\
\hline Nov. & 20 & 5.18 & Sept. & 5 & 6.13 & Apr. & 16 & 5.17 & May & 3 & 52.9 \\
\hline Dec. & 21 & 5.15 & Oct. & 4 & 5. 35 & May & 14 & 5.19 & Sept & 1 & 6.52 \\
\hline an. & 20 , & 5.15 & Dec. & 3 & 5.25 & June & 14 & 5.69 & Oct. & 3 & 6.41 \\
\hline Feb. & 20 & 5.13 & Jan. & 9,1957 & b5. 85 & Aug. & 12 & 5.32 & Nov & 9 & 6.42 \\
\hline Mar. & 16 & 5.09 & Feb. & 7 & 5.30 & Jan. & 6,1959 & 5.21 & Dec. & 5 & 6.33 \\
\hline pr. & 15 & 5.09 & Mar. & 5 & .32 & Feb. & 6 & 5.38 & & & \\
\hline
\end{tabular}

\section{a Well being pumped.}

b Well pumped recently. 
1N/9E-30Q1. Mathias Laufer (Mission Inn). West of Twentynine Palms. Drilled unused water-table well in alluvium of Pleistocene age, diam $8 \mathrm{in}$, depth $144 \mathrm{ft}$, cased with steel. Lsd 2, 091. $6 \mathrm{ft}$ above msl. MP top of casing, $3.40 \mathrm{ft}$ above $1 \mathrm{sd}$. Highest water level 101.80 below lsd, May 24, 1953; lowest 105.24 below 1sd, Nov.9, 1960. Records available: 1946-60.

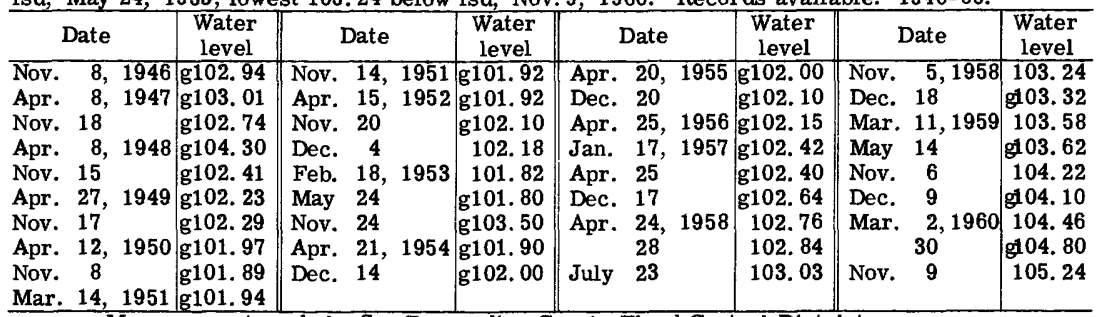

g Measurement made by San Bernardino County Flood Control District.

1S/5E-2B1. Owner unknown. Yucca Valley. Drilled unused water-table well in alluvium of Pleistocene age, diam $8 \mathrm{in}$, depth $240 \mathrm{ft}$, cased with steel. Lsd about $3,240 \mathrm{ft}$ above msl. MP top of casing, at 1sd. Highest water level 161.18 below lsd, Nov. 13, 1947; lowest 179.48 below lsd, Nov. 8, 1960. Records available: 1947-60.

Apr. 15, 1947 g161.90 $\mid$ Mar. 12, 1951 g165.38|| Apr. 19, 1955 g170.20|| Mar. 11, 1959 176.13

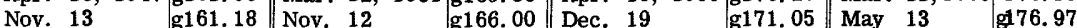

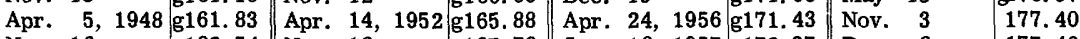

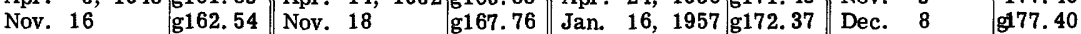

Apr. 27, 1949 g163.12 May 22, 1953 g167.90 Apr. 23

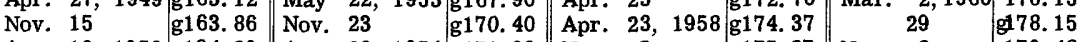

\begin{tabular}{l|r||l|l|l|l|l|l|l|} 
Apr. 10, 1950 & g164. 29 & Apr. 20, 1954 & g171.00 & Nov. 5 & 175.27 & Nov. 8 & 179.48 \\
Nov. 6 & g164.96 & Dec. 13 & g172.70 & Dec. 17 & g175.25 & & & \\
\hline
\end{tabular}

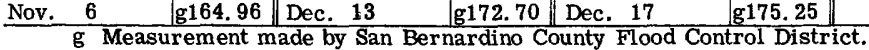

\section{Morongo Valley}

1S/4E-22J1. Clara V. Livingstone. Morongo Valley. Drilled domestic water-table well in alluvium of Pleistocene age, diam $8 \mathrm{in}$, depth $235 \mathrm{ft}$, cased with steel. Lsd about 2, $750 \mathrm{ft}$ above msl. MP top of casing, $0.85 \mathrm{ft}$ above lsd. Highest water level 154.10 below lsd, Apr. 20, 1954; lowest 172.15 below 1sd, Mar. 29, 1960. Records available: 1947-60.

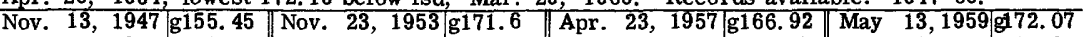

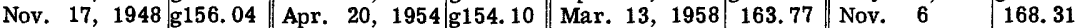
Nov. 15, 1949 g157. 33 Dec. 13 g161.70 Apr. 22 g169.45

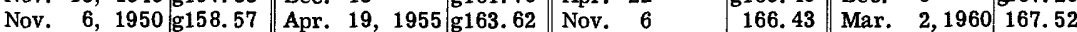
Nov. 12, 1951 g160.47 Dec. 19 19 g164.70

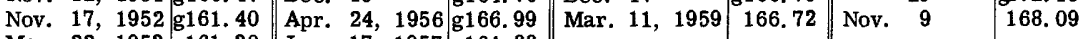
May 22, 1953 g161.30 Jan. 17, 1957 g164.66

g Measurement made by San Bernardino County Flood Control District.

\section{Dale Valley}

1N/12E-20D1. Dale Chemical Co. Northwest of Dale Lake on Amboy Rd. Drilled unused water-table well in alluvium of Pleistocene age, diam 12 in, depth unknown, cased with steel. Lsd about 1, $220 \mathrm{ft}$ above msl. MP bottom of I-beam, $0.50 \mathrm{ft}$ above lsd. Highest water level 27. 88 below 1sd, Mar. 30, 1960; lowest 30.78 below lsd, Dec. 15, 1954 . Records available: 1953-60.

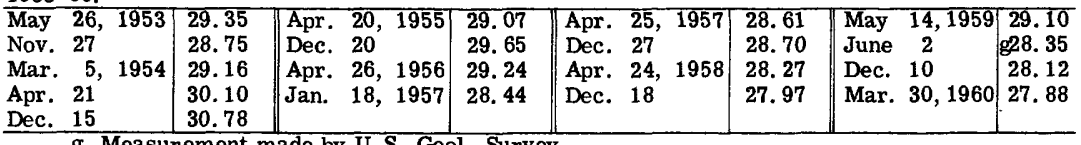

g Measurement made by U.S. Geol. Survey.

\section{Johnson Valley}

4N/3E-24Q1. Owner unknown. Northeast of Old Woman Springs. Drilled unused watertable well in alluvium of Pleistocene age, diam $12 \mathrm{in,} \mathrm{depth} 242 \mathrm{ft}$, cased with steel. Lsd about 2, $835 \mathrm{ft}$ above msl. MP top of casing, $1.00 \mathrm{ft}$ above lsd. Highest water level 54.60 below lsd, May 6, 1954; lowest 55. 28 below 1sd, Nov. 4, 1959. Records available: 1954-60.

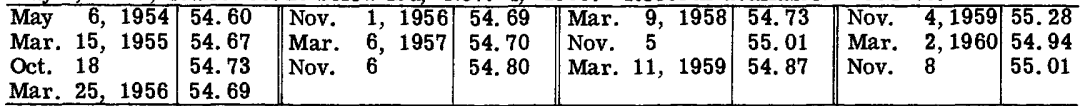




\section{Lucerne Valley Area}

5N/1W-1R3. Hards. North of Lucerne Lake. Drilled unused water-table well in alluvium of Pleistocene age, diam $12 \mathrm{in}$, depth about $165 \mathrm{ft}$, cased with steel. Lsd about 2, $890 \mathrm{ft}$ above msl. MP top of casing, $0.60 \mathrm{ft}$ above 1 sd. Highest water level 75.9 below $1 \mathrm{sd}$, Nov. 8, 1954; lowest 92.11 below lsd, Nov. 8, 1960. Records available: 1954-60.

\begin{tabular}{|c|c|c|c|c|c|c|c|}
\hline Date & $\begin{array}{c}\text { Water } \\
\text { level }\end{array}$ & Date & $\begin{array}{c}\text { Water } \\
\text { level }\end{array}$ & Date & $\begin{array}{c}\text { Water } \\
\text { level }\end{array}$ & Date & $\begin{array}{l}\text { Water } \\
\text { level }\end{array}$ \\
\hline $\begin{array}{lrl}\text { Nov. } & 8,1954 \\
\text { Mar. } 14, & 1955 \\
\text { Nov. } 9 & \\
\text { Mar. } 24, & 1956\end{array}$ & $\begin{array}{l}75.9 \\
76.95 \\
79.24 \\
78.78\end{array}$ & $\begin{array}{l}\text { Nov. } 1,1956 \\
\text { Mar. } 7,1957 \\
\text { Nov. } 14\end{array}$ & $\begin{array}{l}83.03 \\
81.63 \\
85.17\end{array}$ & $\begin{array}{l}\text { Mar. } 9,1958 \\
\text { Nov. } 5 \\
\text { Mar. } 11,1959\end{array}$ & $\begin{array}{l}83.99 \\
88.26 \\
86.31\end{array}$ & $\begin{array}{ll}\text { Nov. } & 5,1959 \\
\text { Mar. } & 1,1960 \\
\text { Nov. } & 8\end{array}$ & $\begin{array}{l}89.85 \\
89.02 \\
92.11\end{array}$ \\
\hline
\end{tabular}

5/1W-35Q1. Owner unknown. South of Lucerne Lake. Drilled irrigation water-table well in alluvium of Pleistocene age, diam $12 \mathrm{in,} \mathrm{depth} \mathrm{about} 300 \mathrm{ft}$, cased with steel. Lsd about 2, $855 \mathrm{ft}$ above $\mathrm{msl}$. MP hole in pumpbase, $1.00 \mathrm{ft}$ above lsd. Highest water level 34.99 below 1sd, Mar. 14, 1955; lowest 48.29 below isd, Sept. 23, 1954. Records available: 1954-60.

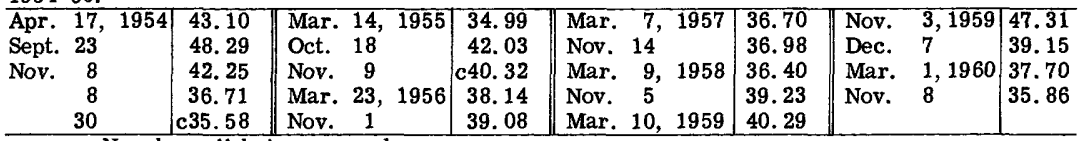

c Nearby well being pumped.

4N/1E-13M1. Wilson. Eastern part of Lucerne Valley. Drilled domestic water-table well in alluvium of Pleistocene age, diam $12 \mathrm{in}$, depth about $270 \mathrm{ft}$, cased with steel to $8 \pm$. Lsd about $3,020 \mathrm{ft}$ above $\mathrm{msl}$. MP hole in pumpbase, $0.40 \mathrm{ft}$ above 1 sd. Highest water level 170.9 below lsd, Mar. 3, 1960; lowest 172.34 below 1sd, Mar. 6, 1957. Records available: 1954-60.

\begin{tabular}{l|l||lrr|r||l|l|l||ll|l}
\hline Nov. 19, 1954 & 171.00 & Nov. 1, 1956 & 171.35 & Mar. 9, 1958 & 171.56 & Nov. & 5, 1959 & 171.9 \\
Mar. 14, 1955 & 171.05 & Mar. 6, 1957 & 172.34 & Nov. 5 & 171.69 & Mar. 3,1960 & 170.9 \\
Nov. 9 & 171.10 & Nov. 14 & 171.50 & Mar. 11, 1959 & 171.90 & Nov. & 8 & 172.19 \\
Mar. 25, 1956 & 171.24 & & & & & & & & \\
\hline
\end{tabular}

\section{Harper Valley Area}

11/3-7D1. Owner unknown. Northeast of Harper Lake. Drilled unused water-table well in alluvium of Pleistocene age, diam $12 \mathrm{in}$, depth $123 \mathrm{ft}$, cased with steel. Lsd about 2,082 ft above msl. MP top of casing, $2.00 \mathrm{ft}$ above $1 \mathrm{sd}$. Highest water level 55.55 below 1sd, June 16, 1954; lowest 62. 42 below 1sd, Apr. 26, 1953. Records available: 1953-60.

\begin{tabular}{lr|r||l|l||l|l||l|l|}
\hline Apr. 26, 1953 & 62.42 & Mar. 23, 1956 & 56.89 & Mar. 11, 1958 & 58.45 & Nov. 18, 1959 & 59.69 \\
June 16, 1954 & 55.55 & Nov. 2 & 57.28 & Nov. 6 & 58.91 & Mar. 2, 1960 & 59.90 \\
Mar. 4, 1955 & 56.09 & Mar. 6, 1957 & 60.30 & Mar. 12, 1959 & 59.21 & Nov. 16 & 60.33 \\
Nov. 17 & 56.62 & Nov. 8 & 58.15 & & & & & \\
\hline
\end{tabular}

11/4-29R1. Lockhart Ranch. Drilled unused water-table well in alluvium of Pleistocene age, diam unknown, depth about $500 \mathrm{ft}$, cased with steel. Lsd about 2, $085 \mathrm{ft}$ above msl. MP top of casing, $1.00 \mathrm{ft}$ above 1sd. Highest water level 83.46 below 1sd, Nov. 17, 1960; lowest 98. 85 below 1sd, Nov. 1, 1956. Records available: 1953-60.

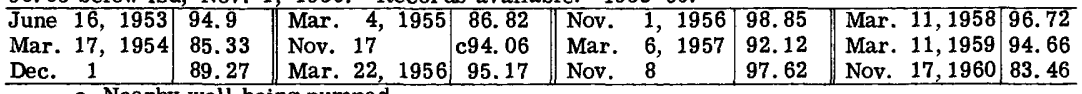

\section{c Nearby well being pumped.
Superior Valley Area}

31/46-12P1. Owner unknown. East edge of Superior Lake. Drilled unused water-table well in alluvium of Pleistocene age, diam $10 \mathrm{in}$, depth $154 \mathrm{ft}$, cased with steel. Lsd about 3, $004 \mathrm{ft}$ above msl. MP top of casing, $3.00 \mathrm{ft}$ above 1sd. Highest water level 84.0 below 1sd, Feb. 10, 1954; lowest 84.78 below lsd, Oct. 30, 1956, Nov. 7, 1957. Records available: 1954-59. Measurement discontinued; replaced by well 31/46-2M1.

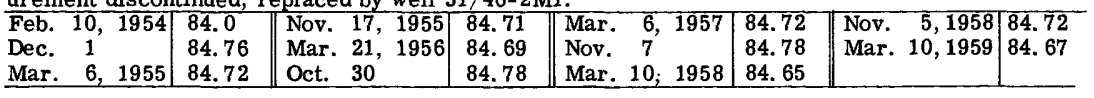

31/46-2M1. U. S. Government. Northeast edge of Superior Dry Lake. Drilled unused water-table well in alluvium of Pleistocene (?) age, diam $8 \mathrm{in}$, depth $117 \mathrm{ft}$, cased with steel. Lsd about 3,020 ft above ms1. MP top of casing, $1.00 \mathrm{ft}$ above 1sd. Highest water level 99.01 below 1sd, Mar. 1, 1960; lowest 105 below 1sd, 1920. Records available: 1920, 1954-60. 
31/46-2M1--Continued.

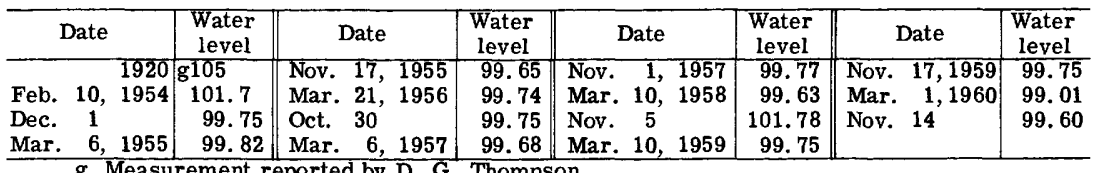

g Measurement reported by D. G. Thompson.

\section{Cuddeback Valley Area}

30/42-20D1. Owner unknown. West edge of Cuddeback Lake. Drilled unused water-table well in alluvium of Pleistocene age, diam 6 in, depth unknown, cased with steel. Lsd about $2,630 \mathrm{ft}$ above msl. MP top of metal cover, at lsd. Highest water level 91.22 below lsd, Nov. 14, 1960; lowest 93.20 below lsd, Oct. 30, 1956. Records available: 1953, 1955-60. \begin{tabular}{l|l||l|l||l||l|l|}
\hline Apr. 29, 1953 & 91.25 & Mar. 20, 1956 & 92.58 & Mar. 10, 1958 92.00 & Nov. 17, 1959 91.47
\end{tabular} \begin{tabular}{l||l|l|l|l||l|l|l|} 
Mar. 5, 1955 c99.74 & Oct. 30 & 93.20 & Nov. 4 & 91.78 & Mar. 1, 1960 91.23
\end{tabular} \begin{tabular}{l|l|l|l|l|l|l|l|l|l|} 
May 8 & c98. 89 & Mar. 5, 1957 & 93.10 & Mar. 10, 1959 & 91.58 & Nov. 14 & 91.22 \\
\hline
\end{tabular} \begin{tabular}{l|l|l|} 
Nov. 16 & 92.39 & Nov. 6 \\
\hline c Nearby well being pumped.
\end{tabular}

\section{Coyote Valley Area}

12/2-31A1. U. S. Army. Camp Irwin. West of Coyote Lake. Drilled unused watertable well in alluvium of Pleistocene age, diam $14 \mathrm{in}$, depth $584 \mathrm{ft}$, perforated 150-552. Lsd $1,789.5 \mathrm{ft}$ above $\mathrm{msl}$. MP hole in casing cover, $1.88 \mathrm{ft}$ above $1 \mathrm{sd}$. Highest water level 55.82 below lsd, Mar. 6, 1957; lowest 56.57 below lsd, Jan. 7, 1955. Records available: 1955-60. \begin{tabular}{ll||l|l|l||l||l|l|}
\hline Jan. 1, 1955 & 56.24 & Mar. 1, 1955 & 55.88 & Mar. 6, 1957 & 55.82 & Nov. 19, 1959 55.93
\end{tabular}

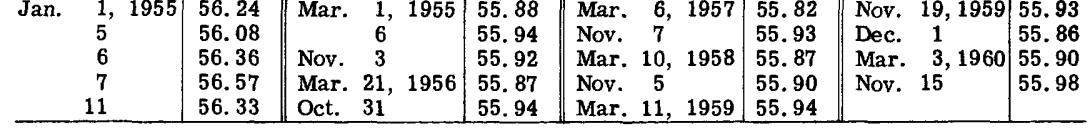

Soda Lake Valley Area

13N/9E-20J1. Fred Twisselman. East edge of Soda Lake. Drilled unused water-table well in alluvium of Pleistocene age, diam $16 \mathrm{in}$, depth about $400 \mathrm{ft}$, cased with steel. Lsd about $980 \mathrm{ft}$ above $\mathrm{msl}$. MP hole in pumpbase, $1.10 \mathrm{ft}$ above 1 sd. Highest water level 65.35 below lsd, Dec. 2, 1959; lowest 65.89 below lsd, Oct. 27, 1954. Records available: 1954-56, 1958-60. No measurement made in 1957.

\begin{tabular}{lr|r||l|l||l|l||l|l|}
\hline Feb. 18, 1954 & 65.82 & Nov. 3, 1955 & 65.83 & Mar. 11, 1959 & 65.55 & Mar. 3, 1960 & 65.56 \\
Oct. 27 & & 65.89 & Mar. 22, 1956 & 65.70 & Dec. 2 & 65.35 & Nov. 16 & 65.65 \\
Mar. 2, 1955 & 65.71 & Nov. 6, 1958 & 65.74 & & & & & \\
\hline
\end{tabular}

\section{Silver Lake Valley Area}

15N/8E-22R1. Death Valley Panamint Mining Co. Northeast edge of Silver Lake. Drilled domestic and industrial water-table well in alluvium of Pleistocene age, diam 12 in, depth about $120 \mathrm{ft}$, cased with steel. Lsd about $930 \mathrm{ft}$ above msl. MP hole in pumpbase, $0.81 \mathrm{ft}$ above lsd. Highest water level 55.84 below lsd, Mar. 12, 1958; lowest 56.12 below lsd, Oct. 31, 1956. Records available: $1954-60$.

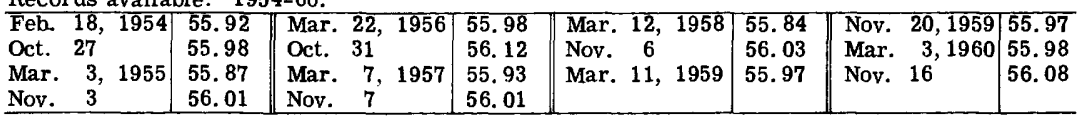

Cronise Lake Area

12N/7E-18R1. Owner unknown. South edge of East Cronise Lake. Drilled unused watertable well in alluvium of Pleistocene age, diam $12 \mathrm{in,} \mathrm{depth} 138 \mathrm{ft}$, cased with steel. Lsd about $1,075 \mathrm{ft}$ above msl. MP top of casing, $3.60 \mathrm{ft}$ above isd. Highest water level 15.36 below lsd, Feb. 19, 1954; lowest 18.53 below lsd, Nov. 16, 1960. Records available: 1954-60.

\begin{tabular}{l|l|l||l|l|l|l|l||l|l|l|}
\hline Feb. 19, 1954 & 15.36 & Mar. 3, 1956 & 16.09 & Mar. 11, 1958 & 16.98 & Dec. 2, 1959 & 18.11 \\
Oct. 26 & 15.51 & Oct. 31 & 16.22 & Nov. 5 & 17.24 & Mar. 3, 1960 & 18.26 \\
Mar. 2, 1955 & 15.73 & Mar. 7, 1957 & 16.48 & Mar. 11, 1959 & 17.55 & Nov. 16 & 18.53 \\
Nov. 3 & 15.87 & Nov. 7 & 16.79 & & & & & & \\
\hline
\end{tabular}




\section{San Diego County}

\section{San Diego River Basin}

15/1E-17H6. La Mesa, Lemon Grove, and Spring Valley Irrigation District. Near Lakeside. Drilled unused water-table well in alluvium of Recent age, diam $14 \mathrm{in}$, depth $61 \mathrm{ft}$, perforated 39-61. Lsd 434.4 ft above msl. MP hole in casing cover, $1.00 \mathrm{ft}$ above 1sd. Highest water level 0.2 below lsd, May 18, 1930; lowest dry, Nov. 19, 1959. Records available: 192932, 1934-59. Well dry in 1960.

\begin{tabular}{c|c||c|c||c|c||c|c}
\hline \multicolumn{1}{c|}{ Date } & $\begin{array}{c}\text { Water } \\
\text { level }\end{array}$ & Date & $\begin{array}{c}\text { Water } \\
\text { level }\end{array}$ & Date & $\begin{array}{c}\text { Water } \\
\text { level }\end{array}$ & Date & $\begin{array}{c}\text { Water } \\
\text { level }\end{array}$ \\
\hline Feb. 21, 1956 & $\mathbf{5 1 . 7 0}$ & Feb. 26, 1957 & $\mathbf{5 1 . 1 7}$ & Feb. 25, 1958 & $\mathbf{5 0 . 3 2}$ & Feb. 26, 1959 48.10 \\
May 17 & $\mathbf{5 1 . 1 8}$ & May 28 & $\mathbf{4 9 . 8 8}$ & May 28 & $\mathbf{4 9 . 4 7}$ & May 27 & $\mathbf{5 5 . 3 0}$ \\
Aug. 29 & $\mathbf{5 2 . 8 0}$ & Aug. 29 & $\mathbf{5 1 . 9 0}$ & Aug. 28 & $\mathbf{4 9 . 7 0}$ & Nov. 19 & (f) \\
Nov. 20 & $\mathbf{5 2 . 7 7}$ & Nov. 26 & $\mathbf{5 1 . 4 8}$ & Nov. 25 & $\mathbf{4 8 . 6 0}$ & & \\
\hline
\end{tabular}

15/1W-13R4. Owner unknown. Lakeside. Drilled unused water-table well in alluvium of Recent age, diam $12 \mathrm{in}$, depth $107 \mathrm{ft}$, cased with steel. Lsd $386.5 \mathrm{ft}$ above msl. MP top of board cover, at lsd. Highest water level 34.75 below lsd, Feb. 24, 1954; lowest 45. 33 below lsd, Aug. 29, 1956. Records available: 1953-56, 1958-60. No measurement made in 1957.

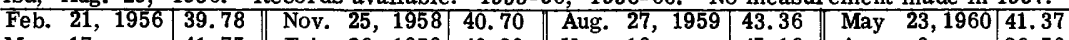
\begin{tabular}{ll|l|l|l|l|l|l|l|l|l|} 
May 17 & 41.75 & Feb. 26, 1959 & 40.30 & Nov. 19 & 45.16 & Aug. 9 & 36.50
\end{tabular}

\begin{tabular}{|l|l||l|l|l||l|l|l|l|l|l|} 
Aug. 29 & 45.33 & May 27 & 43.55 & Feb. 18, 1960 & 41.36 & Nov. 16 & 39.86 \\
\hline
\end{tabular}

Nov, 20

16/2W-19D1. Woodward Sand Co. Mission Valley. Drilled unused water-table well in alluvium of Recent age, diam 6 in, depth $57 \mathrm{ft}$, cased with steel. Lsd $47.2 \mathrm{ft}$ above msl. MP top of flange, $5.00 \mathrm{ft}$ above isd (since Feb. 26, 1957). Highest water level 14.80 below 1sd, May 28, 1958; lowest 24.50 below Isd, Aug. 29, 1956. Records available: 1953-60.

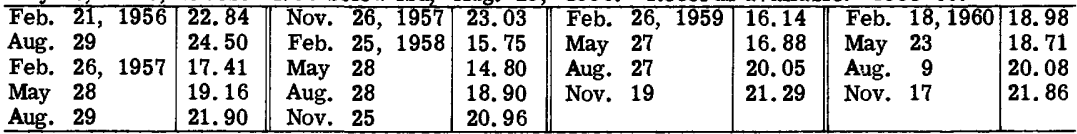

16/3W-21R1. Beratino. Near San Diego. Drilled domestic water-table well in alluvium of Recent age, diam 8 in, depth $27 \mathrm{ft}$. Lsd about $15 \mathrm{ft}$ above msl. MP top north side of casing, at lsd. Highest water level 8.20 below lsd, Aug. 24, 1954; lowest 13.92 below lsd, Nov. 20, 1956. Records available: 1953-58. Measurement discontinued.

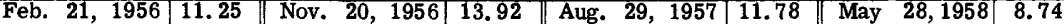

\begin{tabular}{l|l|l|l|l|l|l|l|l|l|l|l|l|l|} 
May 17 & 11.35 & Feb. 26, 1957 & 10.60 & Nov. 26 & 11.95 & Aug. 28 & 9.95 \\
\hline
\end{tabular}

Aug. 29

10.83 Feb. 25, 1958 12.5

16/3W-23H1. W. W. Miller. Mission Valley. Drilled irrigation water-table well in alluvium of Recent age, diam $16 \mathrm{in}$, depth $54 \mathrm{ft}$. Lsd about $30 \mathrm{ft}$ above msl. MP bottom of hole in casing, $0.50 \mathrm{ft}$ above lsd. Highest water level 10.94 below lsd, Feb. 23, 1954; lowest 17.12 below 1sd, Nov. 26, 1957. Records available: 1953-59. Measurement discontinued.

\begin{tabular}{l|c||l|l||ll|l|l||l|l|l|}
\hline Feb. 21, 1956 & (a) & Feb. 26, 1957 & (a) & Feb. 25, 1958 & 16. 20 & Feb. 26, 1959 & 12.00 \\
May 17 & 16.70 & May 28 & (a) & May 28 & (a) & May 27 & (a) \\
Aug. 29 & (a) & Aug. 29 & (a) & Aug. 28 & 14.20 & Aug. 27 & 12.60 \\
Nov. 20 & (a) & Nov. 26 & 17.12 & Nov. 25 & 15.25 & & & \\
\hline
\end{tabular}

Nov. 20

a Well being pumped.

\section{San Dieguito River Basin}

12/1W-31 H2. City of San Diego. San Pasqual Valley. Drilled domestic water-table well in alluvial deposits, diam $12 \mathrm{in}$, depth $46 \mathrm{ft}$, cased with steel. Lsd $357.4 \mathrm{ft}$ above msl. MP top of casing, $0.15 \mathrm{ft}$ below $1 \mathrm{sd}$. Highest water level 3.59 below lsd, Feb. 22, 1933; lowest 16. 69 below Isd, Nov. 16, 1960 . Records available: 1929-60.

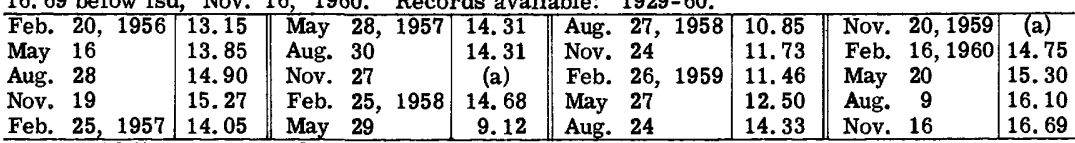

$\frac{\text { Feb. 25, } 1957}{\text { a Well being pumped. }}$

12/1W-33J1. F. B. Gierman. San Pasqual Valley. Dug unused water-table well in alluvium of Recent age, diam $10 \mathrm{in}$, depth $17 \mathrm{ft}$, lined with concrete. Lsd $391.8 \mathrm{ft}$ above msl. MP top of casing, at lsd. Highest water level 0.00, Mar. 30, 1944; lowest 13.05 below lsd, Feb. 25, 1958. Records available: 1943-58. Measurement discontinued. 
12/1W-33J1--Continued.

\begin{tabular}{c|c||c|c||l|c||c|c}
\hline \multicolumn{1}{|c|}{ Date } & $\begin{array}{c}\text { Water } \\
\text { level }\end{array}$ & \multicolumn{1}{|c|}{ Date } & $\begin{array}{c}\text { Water } \\
\text { level }\end{array}$ & \multicolumn{1}{|c|}{ Date } & $\begin{array}{c}\text { Water } \\
\text { level }\end{array}$ & \multicolumn{1}{|c|}{ Date } & $\begin{array}{c}\text { Water } \\
\text { level }\end{array}$ \\
\hline Feb. 20, 1956 & $\mathbf{7 . 1 2}$ & Nov. 19, 1956 & 10.76 & Aug. 30, 1957 & 12.47 & May 29, 1958 & 5.59 \\
May 16 & 7.10 & Feb. 25, 1957 & 10.60 & Nov. 27 & 12.76 & Aug. 27 & 7.98 \\
Aug. 28 & 9.65 & May 28 & 11.23 & Feb. 25, 1958 & 13.05 & Nov. 24 & $\mathbf{8 . 5 2}$ \\
\hline
\end{tabular}

13/2W-1J1. Owner unknown. Lake Hodges, near gaging station. Drilled unused watertable well in alluvium of Recent age, diam $14 \mathrm{in}$, depth $86 \mathrm{ft}$, cased with steel. Lsd about $335 \mathrm{ft}$ above msl. MP hole in south side of casing, $2.00 \mathrm{ft}$ above lsd. Highest water level 2. 27 below 1sd, May 14, 1954; lowest 38. 60 below lsd, Nov. 16, 1960. Records available: 19531960.

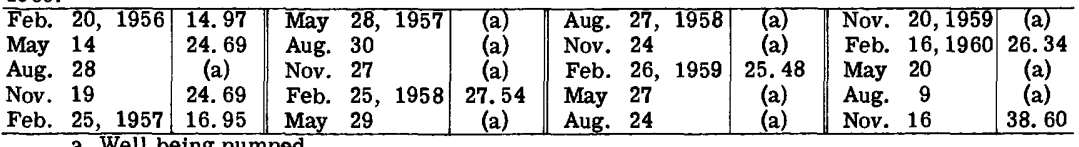
a Well being pumped.

13/3W-33C1. Owner unknown. Near Rancho Santa Fe. Drilled unused water-table well in alluvium of Recent age, diam $12 \mathrm{in}$, depth $66 \mathrm{ft}$, cased with steel. Lsd about $40 \mathrm{ft}$ above msl. MP top south side of casing, $1.00 \mathrm{ft}$ above lsd. Highest water level 30.88 below lsd, Dec. 7, 1953; lowest 46. 75 below 1sd, Feb. 25, 1958. Records available: 1953-59. Measurement discontinued.

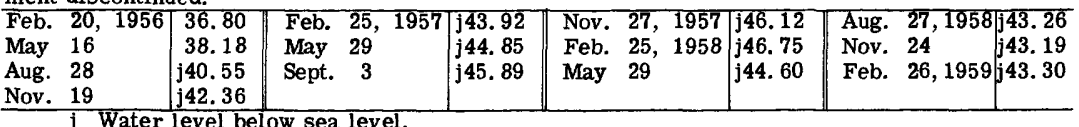

13/3W-33M1. Griset Bros. Near Rancho Santa Fe. Drilled unused water-table well in alluvium of Recent age, diam $12 \mathrm{in}$, depth $90 \mathrm{ft}$, cased with steel. Lsd about $35 \mathrm{ft}$ above msl. MP top north side of casing, at Isd. Highest water level 28. 22 below Isd, Jan. 8, 1954; lowest 54.87 below lsd, Nov. 16,1960 . Records available: 1954-60.

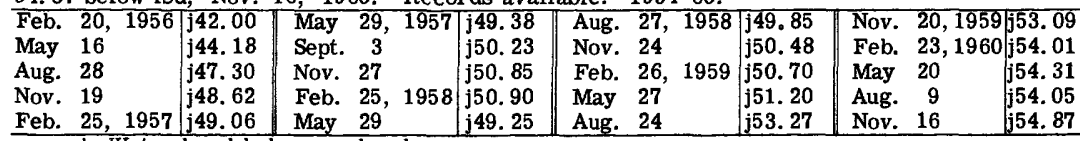

$\frac{\text { Feb. } 25,1957 \text { j49.06 May } 29}{\mathrm{j} \text { Water level below sea level. }}$

\section{San Luis Rey River Basin}

10/1W-8P1. H. N. Berger. Pauma Valley. Drilled unused water-table well in alluvium of Recent age, diam $14 \mathrm{in}$, depth $198 \mathrm{ft}$, cased with steel. Lsd about $725 \mathrm{ft}$ above msl. MP top of casing, 1.50 ft above lsd (since Feb. 19, 1960). Highest water level 10. 28 below lsd, May 14, 1954; lowest 36.50 below Isd, Nov. 16, 1960. Records available: 1954-58, 1960. No measurement made in 1959.

\begin{tabular}{l|c|c|c||l|l||ll|}
\hline Feb. 15, 1956 & 13.25 & Feb. 25, 1957 & 17.77 & Feb. 26, 1958 & 21.30 & Feb. 19, 1960 & 22.48 \\
May 16 & (a) & May 27 & 20.32 & May 29 & (a) & May 24 & (a) \\
Aug. 28 & (a) & Aug. 27 & (a) & Aug. 27 & (a) & Aug. 10 & (a) \\
Nov. 19 & (a) & Nov. 27 & 24.80 & Nov. 28 & (a) & Nov. 16 & 36.50 \\
\hline
\end{tabular}

a Well being pumped.

10/2W-6F2. San Luis Rey Ranch. Monserate Narrows, near gaging station. Drilled unused water-table well in alluvium of Recent age, diam 12 in, depth $109 \mathrm{ft}$, cased with steel. Lsd 282. $36 \mathrm{ft}$ above msl. MP top of casing, 2.40 ft above lsd (since Nov. 19, 1960). Highest water level 5.18 below 1sd, Mar. 15, 1943; lowest 32.67 below 1sd, Nov. 16, 1960 . Records available: $1937-60$.

\begin{tabular}{l|r|ll||l||l|l||l|l|}
\hline Feb. 15, 1956 & 9.27 & May 27, 1957 & (a) & Aug. 27, 1958 & 10.60 & Nov. 20, 1959 21.73 \\
May 16 & 9.08 & Aug. 27 & (a) & Nov. 28 & 12.86 & Feb. 19, 1960 & 20.97 \\
Aug. 28 & 15.00 & Nov. 27 & 22.54 & Feb. 25, 1959 & 10.20 & May 24 & (a) \\
Nov. 19 & 18.83 & Feb. 26, 1958 & 17.67 & May 27 & 12.64 & Aug. 10 & (a) \\
Feb. 26, 1957 & $\mathbf{1 7 . 7 8}$ & May 29 & 9.72 & Aug. 26 & 18.10 & Nov. 16 & 32.67 \\
\hline
\end{tabular}

Feb. 26, $1957 \mid 17.78$ May

10/3W-11G1. Fallbronk Public Utility District. Near Bonsall. Drilled unused watertable well in alluvium of Recent age, diam 8 in, depth $66 \mathrm{ft}$, cased with steel. Lsd 236.91 ft above msl. MP top of 2-in coupling, $3.20 \mathrm{ft}$ above lsd. Highest water level 2.33 below lsd, Mar. 15, 1943; lowest 35.73 below lsd, Nov. 27, 1957. Records available: 1939-59. Measurement discontinued. 
10/3W-11G1--Continued.

\begin{tabular}{|c|c|c|c|c|c|c|c|}
\hline Date & $\begin{array}{l}\text { Water } \\
\text { level }\end{array}$ & Date & $\begin{array}{l}\text { Water } \\
\text { level }\end{array}$ & Date & $\begin{array}{c}\text { Water } \\
\text { level }\end{array}$ & Date & \\
\hline $\begin{array}{|lll|}\text { Feb. } & 15, & 1956 \\
\text { May } & 16 \\
\text { Aug. } & 28\end{array}$ & $\begin{array}{l}21.50 \\
24.04 \\
28.65\end{array}$ & $\begin{array}{lll}\text { Nov. } & 19,1956 \\
\text { Feb. } & 25,1957 \\
\text { May } 27 & \end{array}$ & $\begin{array}{l}30.84 \\
30.02 \\
31.15\end{array}$ & $\begin{array}{lll}\text { Aug. } & 27, & 1957 \\
\text { Nov. } 27 & \\
\text { Feb. } 26, & 1958\end{array}$ & $\begin{array}{l}35.70 \\
35.73 \\
34.26\end{array}$ & $\begin{array}{l}\text { Aug. } 27,1958 \\
\text { Nov. } 28 \\
\text { Feb. } 25,1959\end{array}$ & $\begin{array}{l}15.60 \\
21.82 \\
20.04\end{array}$ \\
\hline
\end{tabular}

10/3W-16F1. San Luis Rey Mutual Heights Water Co. Bonsall and Monserate Rds. Drilled unused water-table well in alluvium of Recent age, diam $12 \mathrm{in}$, depth $59 \mathrm{ft}$, cased with steel. Lsd about $190 \mathrm{ft}$ above msl. MP top of casing, $5.00 \mathrm{ft}$ above $1 \mathrm{sd}$. Highest water level 28.10 below 1sd, Feb. 25, 1959; lowest 48.17 below 1sd, Aug. 27, 1957. Records available: 1953-59. Measurement discontinued.

\begin{tabular}{l|l||l|l|l|l|l|l|l}
\hline Feb. 15, 1956 & 38.05 & Feb. 25, 1957 & 34.90 & Nov. 27, 1957 & 47.59 & Aug. 27, 1958 & 38.00 \\
May 16 & 41.60 & May 27 & 39.83 & Feb. 26, 1958 & 39.33 & Nov. 28 & 37.34 \\
Aug. 28 & 45.48 & Aug. 27 & 48.17 & May 29 & 30.85 & Feb. 25, 1959 & 28.10 \\
Nov. 19 & $\mathbf{4 5 . 9 5}$ & & & & & & & \\
\hline
\end{tabular}

10/3W-30K1. Fallbrook Public Utility District. Near Bonsall. Drilled unused watertable well in alluvium of Recent age, diam $8 \mathrm{in}$, reported depth $80 \mathrm{ft}$, cased with steel. Lsd $149.76 \mathrm{ft}$ above msl. MP top of 2-in coupling, $3.00 \mathrm{ft}$ above $1 \mathrm{sd}$. Highest water level 6.09 below lsd, Mar. 17, 1941; lowest 22. 10 below lsd, Nov. 19, 1956. Records available: 1939-60. \begin{tabular}{l|l|l|l|l|l|l|l|l|l|l|}
\hline Feb 15,1956 & 21.70 & May 27, 1957 & 21.61 & Aug. 27, 1958 & 11.65 & Nov, 20, 1959 13.46
\end{tabular}

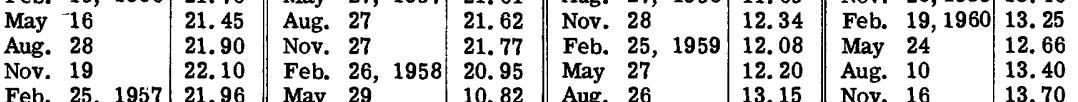

11/4W-2D1. Winston Properties. Near San Luis Rey. Drilled irrigation water-table well in alluvium of Recent age, diam $12 \mathrm{in}$, reported depth $110 \mathrm{ft}$, cased with steel. Lsd about $95 \mathrm{ft}$ above msl. MP bottom northeast side of pumpbase, at lsd. Highest water level 36.69 below 1sd, Feb. 23, 1954; lowest 72.57 below 1sd, Nov. 27, 1957. Records available: 1953-59. Measurement discontinued.

\begin{tabular}{l|c||c||l|l|l||l|l|l}
\hline Feb. 15, 1956 & 57.62 & Nov. 19, 1956 & 72.30 & Aug. 27, 1957 & 72.22 & Aug. 27, 1958 & (a) \\
May 16 & (a) & Feb. 25, 1957 & 67.88 & Nov. 27 & 72.57 & Nov. 28 & 54.96 \\
Aug. 28 & (a) & May 27 & 72.22 & Feb. 26, 1958 & 69.75 & Feb. 25, 1959 & 54.14 \\
\hline
\end{tabular}

a Well being pumped.

11/4W-9F1. City of Oceanside. Near San Luis Rey. Drilled observation well in alluvium of Recent age, diam 4 in, reported depth $199 \mathrm{ft}$. Lsd about $63 \mathrm{ft}$ above msl. MP top of casing, 4. $40 \mathrm{ft}$ above $1 \mathrm{sd}$. Highest water level 3.47 below $1 \mathrm{sd}$, Mar. 14, 1943; lowest 73.35 below lsd, Jan. 6, 1958. Records available: 1940-60. Records furnished by city of Oceanside.

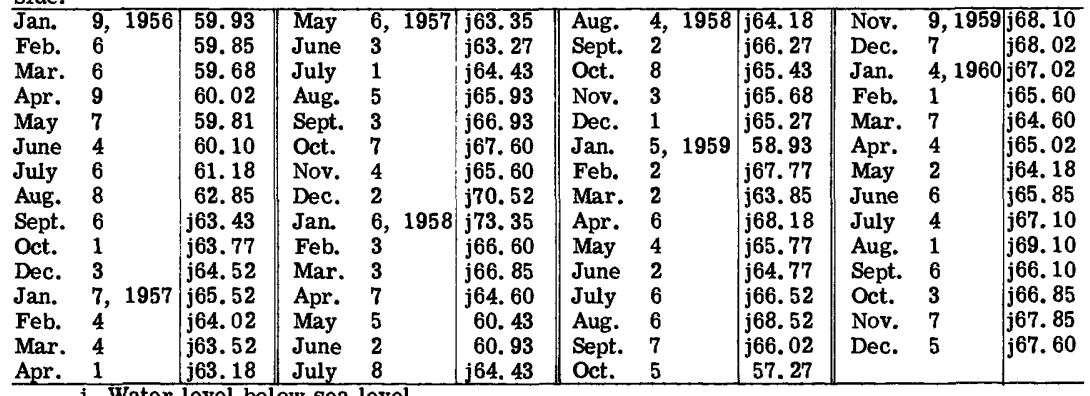

11/4W-18C2. Robert Slaught. Near San Luis Rey. Drilled unused artesian well in alluvium of Recent age, diam $12 \mathrm{in}$, reported depth $134 \mathrm{ft}$, cased with steel. Lsd about $37 \mathrm{ft}$ above msl. MP hole in cover, $0.50 \mathrm{ft}$ above lsd. Highest water level 34.72 below 1sd, Mar. 4, 1952; lowest 71.18 below 1sd, Aug. 27, 1957. Records available: 1952-59. Measurement discontinued.

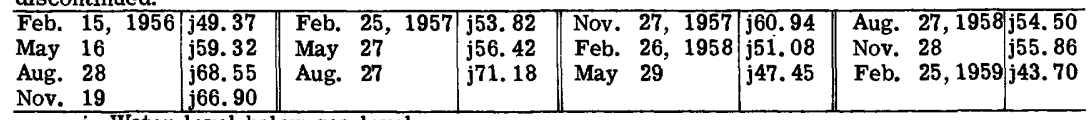

j Water level below sea level. 
11/4W-18L3. Carlsbad Mutual Water Co. Near Oceanside. Drilled observation artesian well in alluvium of Recent age, diam 6 in, reported depth $115 \mathrm{ft}$, cased with steel. Lsd $35.34 \mathrm{ft}$ above msl. MP top of casing, $1.10 \mathrm{ft}$ above Isd. Highest water level 5.97 below Isd, Apr. 14, 1941; lowest 67.63 below Isd, Nov. 19, 1956. Records available: 1939-59. Measurement discontinued. Records furnished by owner.

\begin{tabular}{|c|c|c|c|c|c|c|c|}
\hline Date & $\begin{array}{l}\text { Water } \\
\text { level }\end{array}$ & Date & $\begin{array}{c}\text { Water } \\
\text { level }\end{array}$ & Date & $\begin{array}{c}\text { Water } \\
\text { level }\end{array}$ & Date & $\begin{array}{c}\text { Water } \\
\text { level }\end{array}$ \\
\hline $\begin{array}{lll}\text { Feb. } & 15,1956 \\
\text { Nov. } & 19 \\
\text { Feb. } & 25,1957 \\
\end{array}$ & $\begin{array}{l}j 52.80 \\
j 67.63 \\
j 63.85 \\
\end{array}$ & $\begin{array}{ll}\text { May } 27,1957 \\
\text { Aug. } 27 \\
\text { Nov. } 27 \\
\end{array}$ & $\begin{array}{l}j 60.92 \\
j 66.79 \\
j 58.71\end{array}$ & $\begin{array}{lll}\text { Feb. } & 26,1958 \\
\text { May } & 29 & \\
\text { Aug. } & 27 & \\
\end{array}$ & $\begin{array}{l}j 50.90 \\
j 51.24 \\
j 56.03\end{array}$ & $\begin{array}{ll}\text { Nov. } & 2 \\
\text { Feb. } & 2\end{array}$ & \\
\hline
\end{tabular}

Feb. 25, 1957 j63.85

j Water level below sea level.

11/5W-13N1. City of Oceanside. Near airport. Drilled unused artesian well in alluvium of Recent age, diam $14 \mathrm{in}$, depth $160 \mathrm{ft}$. Lsd $16.26 \mathrm{ft}$ above msl. MP top of casing, $4.50 \mathrm{ft}$ above Isd. Highest water level 1.61 below Isd, Mar. 15, 1943; lowest 30.50 below Isd, Jan. 6, 1958. Records available: 1939-60. Records furnished by city of Oceanside.

\begin{tabular}{|c|c|c|c|c|c|c|c|c|c|c|c|}
\hline Jan. & 9,1956 & $\lcm{j 24.67}$ & May & 6,1957 & j25.17 & Aug. & 6,1958 & $j 18.00$ & Nov. & \multicolumn{2}{|c|}{$9,1959 \mid \jmath 22.17$} \\
\hline Feb. & 6 & j23.67 & June & 3 & j25.67 & Sept. & 2 & $j 19.08$ & Dec. & & j23.58 \\
\hline Mar. & 6 & j24.50 & July & 1 & j26.58 & Oct. & 8 & j19.75 & Jan. & & $0 . j 21.50$ \\
\hline Apr. & 9 & j25.58 & Aug. & 5 & j27. 42 & Nov. & 3 & j19.50 & Feb. & 1 & j20.67 \\
\hline May & 7 & j26.00 & Sept. & 3 & j28. 25 & Dec. & 1 & j18.33 & Mar & 7 & jig. 50 \\
\hline June & 4 & j26. 50 & Oct. & 7 & j27. 83 & Jan. & 5,1959 & j19.08 & Apr. & 4 & j20.25 \\
\hline uly & 6 & j28. 17 & Nov. & 4 & j26.92 & Feb. & 2 & j19. 33 & May & 2 & $\mathrm{j} 30.42$ \\
\hline Aug. & 8 & j28. 15 & Dec. & 2 & j27. 67 & Mar. & 2 & j20.58 & June & 6 & j20. 33 \\
\hline Sept. & 6 & j29.00 & Jan. & 6,1958 & j30.50 & Apr. & 6 & j19. 25 & July & 4 & j22.08 \\
\hline Oct. & 1 & $\mathrm{j} 29.42$ & Feb. & 3 & $\mathrm{j} 25.42$ & May & 4 & $\mathrm{j} 20.13$ & Aug. & 1 & $\mathrm{j} 21.42$ \\
\hline Dec. & & j28. 67 & Mar. & 3 & j24.08 & June & 2 & j18. 67 & Sept & 6 & $\mathrm{j} 21.17$ \\
\hline Jan. & 7 , & j27. 67 & Apr. & 7 & 12.42 & July & 6 & $\mathrm{j} 21.08$ & Oct. & 3 & j22.00 \\
\hline Feb. & 4 & $\mathrm{j} 26.42$ & May & 5 & 16.21 & Aug. & 6 & j22.00 & Nov. & 7 & j21.08 \\
\hline Mar. & 4 & j25.58 & June & 2 & 16.21 & Sept. & 7 & j22. 42 & Dec. & 5 & $j 20.67$ \\
\hline Apr. & 1 & j25. 17 & July & 8 & j16. 33 & Oct. & 5 & j22.00 & & & \\
\hline
\end{tabular}

j Water level below sea level.

\section{Otay River Basin}

18/1W-19D1. W. Ellerson. Near Otay. Drilled domestic water-table well in marine sand and gravel of Pliocene age, diam $10 \mathrm{in}$, reported depth $182 \mathrm{ft}$, cased with steel. Lsd about $130 \mathrm{ft}$ above $\mathrm{msl}$. MP hole in west side of plate, $0.50 \mathrm{ft}$ above Isd. Highest water level 55.40 below lsd, May 18, 1954; lowest 71.50 below Isd, Nov. 25, 1958. Records available: 1953-59. Measurement discontinued.

\begin{tabular}{l|l|l|l|l|ll|l|l|l}
\hline Feb. 21, 1956 & 61.50 & Feb. 26, 1957 & 62.65 & Nov. 26, 1957 & 70.85 & Aug. 28, 1958 & 68.68 \\
May 17 & 65.40 & May 28 & 62.39 & Feb. 24, 1958 & 64.39 & Nov. 25 & 71.50 \\
Aug. 31 & 66.45 & Aug. 29 & & 65.33 & May 28 & 66.15 & Feb. 25, 1959 & 71.48 \\
Nov. 20 & 65.87 & & & & & & & & \\
\hline
\end{tabular}

18/2W-22F1. Western Aire Motel. Near Otay. Drilled domestic water-table well in alluvium of Recent age, diam $10 \mathrm{in}$, reported depth $150 \mathrm{ft}$, cased with steel. Lsd about $40 \mathrm{ft}$ above msl. MP top of 2-in access pipe, $2.40 \mathrm{ft}$ above Isd. Highest water level 18.40 below Isd, Apr. 10, 1922; lowest 48. 30 below Isd, Dec. 6, 1955. Records available: 1916-60.

\begin{tabular}{|l||l|l|l||l||l|l|}
\hline Feb. 21, 1956 & j43. 48 & May 28, 1957 & 36.07 & Aug. 28, 1958 & 39.00 & Nov. 19, 1959 j41.30
\end{tabular} \begin{tabular}{l|l|l|l|l|l|l|l|} 
May 17 & j41.59 & Aug. 29 & j41.39 & Nov. 25 & 39.95 & Feb. 17, 1960 39.13
\end{tabular} \begin{tabular}{l|l|l|l|l|l|l|l|l|l|l|l|l|l|} 
Aug. 31 & j44.77 & Nov. 26 & j41.11 & Feb. 25, 1959 & 39.10 & May 23 & 39.75
\end{tabular} \begin{tabular}{l|l|l|l|l|l|l|l|l|l|l|} 
Nov. 20 & j47.17 & Feb. 24, 1958 & 35.99 & May 28 & j40.30 & Aug. 9 & 39.15 \\
\hline
\end{tabular} \begin{tabular}{l|l|l|l|l|l|l|l|l|l|} 
Feb. 26, 1957 & 38.95 & May 28 & 37.10 & Aug. 27 & 39.87 & Nov. 17 & j41.00 \\
\hline
\end{tabular} $j$ Water level below sea level.

\section{Sweetwater River Basin}

17/1W-19Q2. California Water \& Telephone Co. Near Sunnyside. Drilled unused watertable well in alluvium of Recent age, diam $12 \mathrm{in}$, depth $40 \mathrm{ft}$, cased with steel. Lsd about $85 \mathrm{ft}$ above msl. MP top of 2-in coupling, $3.70 \mathrm{ft}$ above Isd. Highest water level 1.51 below Isd, Dec. 23, 1943; lowest 38. 29 below Isd, Dec. 28, 1949. Records available: 1943-59. Measurement discontinued.

\begin{tabular}{|c|c|c|c|c|c|c|c|}
\hline $\begin{array}{l}\text { Feb. } 21,1956 \\
\text { May } 17 \\
\text { Aug. } 31 \\
\text { Nov. } 20\end{array}$ & $\begin{array}{l}8.15 \\
8.05 \\
8.64 \\
9.17\end{array}$ & $\begin{array}{lll}\text { Feb. } & 26, & 1957 \\
\text { May } & 28 \\
\text { Aug. } & 29\end{array}$ & $\begin{array}{l}5.22 \\
5.30 \\
7.04\end{array}$ & $\begin{array}{lll}\text { Nov. } & 26,1957 \\
\text { Feb. } 24, & 1958 \\
\text { May } 28 & \end{array}$ & $\begin{array}{l}7.5 \overline{3} \\
4.93 \\
4.85\end{array}$ & $\begin{array}{ll}\text { Aug. } & 28,1958 \\
\text { Nov. } & 25 \\
\text { Feb. } & 25,1959\end{array}$ & $\begin{array}{l}5.97 \\
5.02 \\
4.58\end{array}$ \\
\hline
\end{tabular}

17/2W-35E1. Owner unknown. Near Chula Vista. Drilled domestic water-table well in alluvium of Recent age, diam $12 \mathrm{in}$, depth $58 \mathrm{ft}$, cased with steel. Lsd about $35 \mathrm{ft}$ above msl. MP hole in pumpbase, $2.00 \mathrm{ft}$ above lsd. Highest water level 8.62 below Isd, Nov. 10, 1954; lowest 15. 29 below Isd, Nov. 19, 1959. Records available: 1953-60. 
17/2W-35E1--Continued.

\begin{tabular}{|c|c|c|c|c|c|c|c|}
\hline Date & $\begin{array}{c}\text { Water } \\
\text { level }\end{array}$ & Date & $\begin{array}{c}\text { Water } \\
\text { level }\end{array}$ & Date & $\begin{array}{l}\text { Water } \\
\text { level }\end{array}$ & Date & $\begin{array}{c}\text { Water } \\
\text { level }\end{array}$ \\
\hline Feb. 21, & 13.00 & May 28,1957 & 13.21 & Aug. 28,1958 & 13.50 & Nov. 19,1959 & 15.29 \\
\hline May 17 & 13.23 & Aug. 29 & 14.34 & Nov. 25 & 14.35 & Feb. 17,1960 & 10.15 \\
\hline Aug. 31 & 14.39 & Nov. 26 & 14.38 & Feb. 25,1959 & 10.52 & May 23 & 11.55 \\
\hline Nov. 20 & 14.49 & Feb. 24,1958 & 14.05 & May 28 & 12.14 & Aug. & 11.70 \\
\hline Feb. 26,1957 & 12.14 & May 28 & 10.47 & Aug. 27 & 14.60 & Nov. 17 & 11.50 \\
\hline
\end{tabular}

Tia Juana River Basin

18/2W-33L1. Robert Egger. Near Nestor. Driven observation water-table well in alluvium of Recent age, diam $2 \mathrm{in}$, depth $29 \mathrm{ft}$, cased. Lsd $19.04 \mathrm{ft}$ above msl. MP top of casing, $3.20 \mathrm{ft}$ above 1sd. Highest water level 5.16 below lsd, May 15, 1941; lowest 28.57 below lsd, Dec. 6, 1955. Records available: 1927-59. Measurement discontinued.

\begin{tabular}{l|l||ll|l|l|l||l|l|l|l|}
\hline Feb. 21, 1956 & j27.02 & Feb. 26, 1957 & j27. 24 & Nov. 26, 1957 & j25.89 & Aug. 28, 1958 & j24. 17 \\
May 17 & j26.42 & May 28 & j25. 39 & Feb. 24, 1958 & j24.91 & Nov. 25 & j22.95 \\
Aug. 31 & j28.32 & Aug. 29 & j27.15 & May 28 & & j20.85 & Feb. 25, 1959 j21.70 \\
Nov. 20 & j28.26 & & & & & & & & & \\
\hline
\end{tabular}

\begin{tabular}{l|l|} 
Nov. 20 & j28.26 \\
\hline $\mathrm{j}$ Water level below sea level.
\end{tabular}

18/2W-34J1. C. Iguchi. Near Nestor. Driven observation water-table well in alluvium of Recent age, diam 2 in, depth $28 \mathrm{ft}$, cased. Lsd $30.97 \mathrm{ft}$ above msl. MP top of casing, $3.00 \mathrm{ft}$ above 1sd. Highest water level 3.01 below 1sd, Jan. 26, 1944; lowest 27. 05 below 1sd, Nov. 20, 1956. Records available: 1927-58. Measurement discontinued.

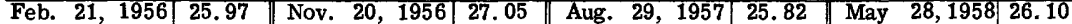

\begin{tabular}{l|l|l|l|l|l|l|l|l|l|} 
May 17 & 17 & 26.22 & Feb. 26, 1957 & 22.66 & Nov. 26 & 26.00 & Aug. 28 & 26.00 \\
\hline
\end{tabular}

\begin{tabular}{l|l|l|l|l|l|l|l|l|l|} 
Aug. 31 & 26.34 & May 28 & 26.30 & Feb. 24, 1958 & 25.65 & Nov. 25 & 25.85 \\
\hline
\end{tabular}

19/2W-1N1. State of California. Near San Ysidro. Drilled observation water-table well in alluvium of Recent age, diam $8 \mathrm{in}$, depth $68 \mathrm{ft}$, cased with steel. Lsd about $50 \mathrm{ft}$ above msl. MP top of casing, 2. $50 \mathrm{ft}$ above 1sd. Highest water level 8.32 below lsd, May 18, 1954; lowest 34. 73 below lsd, Nov. 17, 1960. Records available: 1953-60.

\begin{tabular}{l|l||l|l||l|l||l|l}
\hline Feb. 21, 1956 & 15.98 & May 28, 1957 & 19.11 & Aug. 28, 1958 & 22.60 & Nov. 19, 1959 & 28.67 \\
May 17 & 15.34 & Aug. 29 & 21.90 & Feb. 25, 1959 & 25.35 & Feb. 17, 1960 & 26.58 \\
Aug. 31 & 18.67 & Nov. 26 & 22.71 & May 28 & 24.30 & May 23 & 26.93 \\
Nov. 20 & 21.38 & Feb. 24, 1958 & 23.08 & Aug. 27 & 27.13 & Nov. 17 & 34.73 \\
Feb. 26, 1957 & 19.10 & May 28 & 18.60 & & & & \\
\hline
\end{tabular}

19/2W-4A6. California Water \& Telephone Co. Near Nestor. Drilled unused artesian well in alluvium of Recent age, diam $12 \mathrm{in}$, depth $90 \mathrm{ft}$, cased with steel. Lsd about $25 \mathrm{ft}$ above msl. MP top of plate on casing, $4.00 \mathrm{ft}$ above lsd. Highest water level 19.87 below lsd, May 18, 1954; lowest 38.22 below lsd, Aug. 27, 1959. Records available: 1953-60.

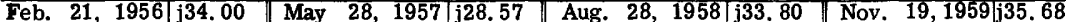
\begin{tabular}{ll|l|l|l|l|l|l|} 
May 17 & j30.76 & Aug. 29 & j32. 80 & Nov. 25 & j32.00 & Feb. 17, 1960 j30.60
\end{tabular}

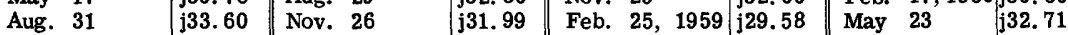

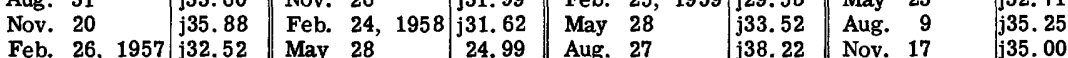

Feb. 26, 1957 j32.52 May 28

j Water level below sea level.

\section{Borrego Valley Area}

10S/6E-21A1. DiGiorgio Fruit Corp. North of Borrego. Drilled unused water-table well in alluvium of Pleistocene age, diam $12 \mathrm{in}$, depth $322 \mathrm{ft}$, cased with steel. Lsd about $664 \mathrm{ft}$ above msl. MP top of casing, $0.50 \mathrm{ft}$ above lsd. Highest water level 130.55 below 1sd, Jan. 3, 1953; lowest 174.2 below isd, June 16, 1960. Records available: 1952-60. All measurements made by Surface Water Branch, U.S. Geol. Survey, from recorder charts, except as indicated.

\begin{tabular}{|c|c|c|c|c|c|c|c|c|c|c|c|c|}
\hline ne & 24 & 1952 & h136.36 & Mar. & 16,1955 & 149.4 & May & 3,1956 & 157.8 & May & 27,1957 & 162.7 \\
\hline & 3 , & 195 & 55 & Apr. & 14 & c156. 2 & June & 6 & 161.1 & June & 26 & 166.3 \\
\hline dy & 3 & & 142.48 & May & 19 & 159.4 & July & 2 & 161.12 & July & 24 & 167.9 \\
\hline & 25 & & 14 & June & 29 & 16 & Aug. & 2 & 162.0 & Aug. & 22 & 167.9 \\
\hline ug. & 3 & & 78 & July & 20 & & Sept. & 4 & 15 & Sept. & 26 & .5 \\
\hline Feb. & 24 & 1954 & h138. 79 & Aug. & 3 & g160. & Oct. & 3 & 153.3 & Nov. & 6 & 5.6 \\
\hline pr. & 15 & & 38 & Sept. & 20 & 15 & Nov. & 1 & 151.2 & & 26 & hil \\
\hline ug. & 27 & & c15 & Oct. & 25 & 15 & & 16 & .99 & Dec. & 11 & .0 \\
\hline ct. & 21 & & 148.7 & Nov. & 28 & h148.12 & Dec. & 3 & 14 & Jan. & 7 & 152.1 \\
\hline ov. & 9 & & h147. 68 & & 29 & 147.0 & Jan. & 3 , & 7 & Feb. & 11 & 3.5 \\
\hline & 19 & & 14 & Jan. & 4,195 & & Feb. & 4 & & Mar. & 15 & .94 \\
\hline ec. & 17 & & 14 & Feb. & 7 & 14 & Mar. & 1 & & Mar. & & 2.1 \\
\hline & 12 , & 55 & & Mar. & 8 & & & 15 & & Apr. & 21 & .0 \\
\hline & 10 & & & & 18 & & & 2 & & May & 5 & .5 \\
\hline ar & 7 & & h1 & Apr & 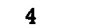 & & Apr. & 25 & 161.5 & June & 23 & 67.3 \\
\hline
\end{tabular}


10S/6E-21A1-- Continued.

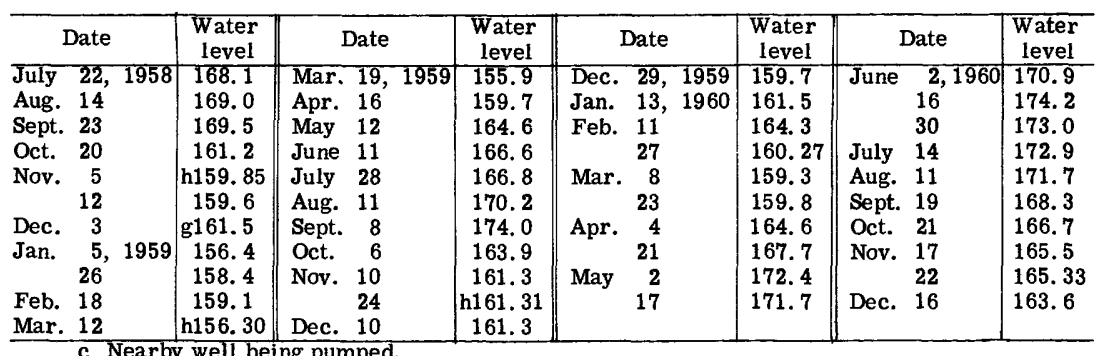

c Nearby well being pumped.

g Measurement made by California Dept. of Water Resources.

h Tape measurement.

11S/6E-11D2. Kosky and Hagenbuck. North of Borrego. Drilled unused water-table well in alluvium of Pleistocene age, diam $14 \mathrm{in}$, depth $218 \mathrm{ft}$, cased with steel. Lsd about $486 \mathrm{ft}$ above msl. MP top of casing cover, $1.05 \mathrm{ft}$ above $1 \mathrm{sd}$. Highest water level 17.53 below lsd, Nov. 16, 1953; lowest 37.91 below lsd, Nov. 5, 1958. Records available: 1953-60.

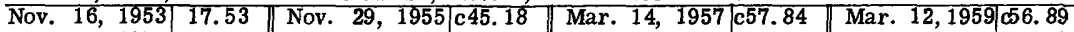

\begin{tabular}{|l|l|l|l||l|l|l|l|} 
Feb. 24, 1954 & 19.49 & Mar. 18, 1956 24.07 & Nov. 27 & c57.48 & Nov. 24 & 31.59
\end{tabular}

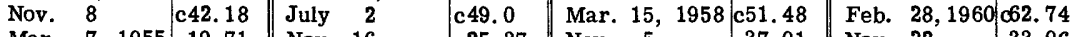

\begin{tabular}{l|l|l|l|l|l|l|l|} 
Mar. 7, 1955 & 19.71 & Nov. 16 & 25.37 & Nov. 5 & 37.91 & Nov. 22 & 33.96 \\
\hline
\end{tabular}

c Nearby well being pumped.

San Felipe Valley Area

12S/4E-24K1. Orville Cummings. San Felipe Ranch. Northeast of Julian. Drilled stock water-table well in alluvium of Pleistocene age, diam $12 \mathrm{in}$, depth $97 \mathrm{ft}$, cased. Lsd about $2,430 \mathrm{ft}$ above msl. MP top of casing, $1.15 \mathrm{ft}$ above $1 \mathrm{sd}$. Highest water level 18.48 below Isd, Nov. 24, 1953; lowest 30.56 below 1sd, Nov. 22, 1960. Records available: 1953, 1956-60.

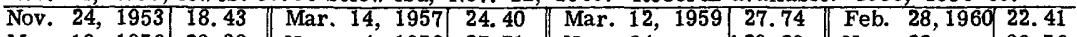
\begin{tabular}{l|l|l|l|l|l|l|l|} 
Mar. 19, 1956 & 23.30 & Nov. 4,1958 & 27.71 & Nov. 24 & b29.29 & Nov. 22 & 30.56 \\
\hline
\end{tabular} b Well pumped recently.

\section{Ocotillo Valley Area}

12S/8E-22E1. A. F. Demes. South of Ocotillo. Drilled domestic and irrigation watertable well in alluvium of Pleistocene age, diam 16 in, depth about $226 \mathrm{ft}$, cased with steel. Lsd about $120 \mathrm{ft}$ above msl. MP bottom edge southeast side of pumpbase, 1. $40 \mathrm{ft}$ above 1 sd. Highest water level 101.83 below 1sd, Nov. 10, 1954; lowest 108.92 bel ow 1sd, Nov. 23, 1960. Records available: $1953-60$.

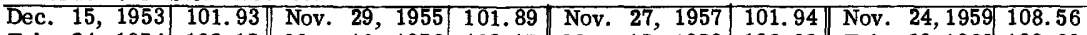

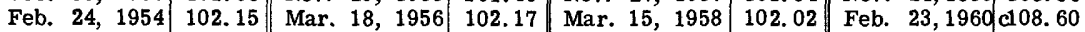
\begin{tabular}{lrl|l|l|l|l|l|l|l|l|l} 
Nov. 10 & 101.83 & Nov. 16 & 101.92 & Nov. 4 & & 105.61 & Nov. 23 & 108.92 \\
Mar. 6, 1955 & 101.89 & Mar. 15, 1957 & 101.87 & Mar. 12, 1959 & 105.86 & & & \\
\hline
\end{tabular} $\frac{\text { Mar. } 6,1955101.89}{\text { c Nearby well being pumped. }}$

\section{San Joaquin County}

Mokelumne River Basin

3N/6-3K11. F. B. Mills Estate. About $1.8 \mathrm{mi}$ west of Lodi. Drilled irrigation watertable well in Victor Formation of Pleistocene age, diam $12 \mathrm{in}$, depth $120 \mathrm{ft}$. Lsd $41.03 \mathrm{ft}$ above msl. MP top southwest side of casing, $0.50 \mathrm{ft}$ above lsd. Highest water level 10.95 below lsd, May 1, 1958; lowest 23. 76 below lsd, Sept. 1, 1960. Records available: 1947-60.

\begin{tabular}{|c|c|c|c|c|c|c|c|c|c|c|c|}
\hline & & & & & & & & & & & \\
\hline Jan. & 3,1956 & 19.96 & Feb & 1,1957 & 19.44 & Jan. & 3,1958 & 20.44 & Dec. & 1,195 & 17.07 \\
\hline Feb. & 1 & 18.22 & Mar & & 19.44 & Feb. & & 20.47 & Jan. & 2,195 & 3 \\
\hline Mar. & & 16.91 & Apr & 1 & 19.98 & Mar & & 20.10 & $\mathrm{Fe}$ & 2 & 6 \\
\hline pr. & 2 & 17.34 & May & 1 & 19. & Apr. & 1 & $\begin{array}{l}18.56 \\
10.95\end{array}$ & Mar. & 2 & $\begin{array}{l}18.00 \\
18.33\end{array}$ \\
\hline Ia & 2 & 16.33 & June & 1 & $\begin{array}{c}18.47 \\
\text { (a) }\end{array}$ & $M$ & $\frac{1}{2}$ & 10.90 & Apr. & 1 & 18,25 \\
\hline Sept. & $\begin{array}{l}1 \\
4\end{array}$ & 1780 & Jury & 1 & 23,10 & July & 1 & 18,60 & June & 1 & (a) \\
\hline ct. & 3 & 17.66 & Sept & 3 & 21. 36 & Aug. & 1 & (a) & July & 1 & (a) \\
\hline Tov. & 1 & 17.71 & $\mathrm{Oc}$ & 1 & 20.46 & Sept & 2 & 16.75 & Aug. & 4 & 22.65 \\
\hline ec. & 3 & 16.38 & Nov & 1 & 19.85 & Oct. & 1 & 16.67 & Sept. & 1 & 21.64 \\
\hline an. & 3 & 19. & Dec & 2 & 20.15 & Nov. & 3 & 17.19 & Oct. & 1 & 20.27 \\
\hline
\end{tabular}


3N/6-3K11- -Continued.

\begin{tabular}{|c|c|c|c|c|c|c|c|}
\hline Date & $\begin{array}{l}\text { Water } \\
\text { level }\end{array}$ & Date & $\begin{array}{l}\text { Water } \\
\text { level }\end{array}$ & Date & $\begin{array}{l}\text { Water } \\
\text { level }\end{array}$ & Date & $\begin{array}{l}\text { Water } \\
\text { level }\end{array}$ \\
\hline $\begin{array}{lll}\text { Nov. } & 3,1959 \\
\text { Dec. } & 1 & \\
\text { Jan. } & 4, & 1960 \\
\text { Feb. } & 1\end{array}$ & $\begin{array}{l}19.74 \\
19.36 \\
19.73 \\
19.27\end{array}$ & $\begin{array}{ll}\text { Mar. } & 1,1960 \\
\text { Apr. } & 1 \\
\text { May } & 2 \\
\text { June } & 1\end{array}$ & $\begin{array}{l}20.37 \\
20.60 \\
20.28 \\
\text { (a) }\end{array}$ & $\begin{array}{lll}\text { July } & 1,1960 \\
\text { Aug. } & 1 \\
\text { Sept. } & 1\end{array}$ & $\begin{array}{c}\text { (a) } \\
23.54 \\
23.76\end{array}$ & $\begin{array}{ll}\text { Oct. } & 3,1960 \\
\text { Nov. } & 1 \\
\text { Dec. } & 1\end{array}$ & $\begin{array}{l}22.50 \\
22.35 \\
21.80\end{array}$ \\
\hline
\end{tabular}

a Well being pumped.

3N/6-17D11. A. Delu. About $4.5 \mathrm{mi}$ southwest of Lodi. Drilled irrigation water-table well in Victor Formation of Pleistocene age, diam $12 \mathrm{in}$, depth $93 \mathrm{ft}$. Lsd $23.80 \mathrm{ft}$ above msl. MP top north side of casing, $10.60 \mathrm{ft}$ below lsd. Highest water level 7.46 below lsd, May 1, 1952; lowest 26.40 below lsd, Aug. 1, Oct. 3, 1960. Records available: 1949-60.

\begin{tabular}{|c|c|c|c|c|c|c|c|c|c|c|c|}
\hline Jan. & 3.1956 & 19.07 & $\mathrm{Apr}$ & 1.1957 & 1711 & Sept. & 2,1958 & 19.13 & Nov. & 3,1959 & 21.19 \\
\hline Feb. & 1 & 15.58 & May & 1 & 17.66 & $\begin{array}{l}\text { sept. } \\
\text { Oct. }\end{array}$ & 1 & $\begin{array}{l}18.48 \\
18.40\end{array}$ & Dec. & & 21.05 \\
\hline Mar. & 1 & 12.89 & June & 1 & 17.32 & Nov. & 3 & (a) & Jan. & 4,1960 & 20.23 \\
\hline Apr. & 2 & 13.00 & July & 1 & 21. 87 & Dec. & 1 & 16.49 & Feb. & 1 & 19.60 \\
\hline May & 2 & 13.16 & Aug. & 1 & j24. 90 & Jan. & 2, 1959 & 15.86 & Mar. & 1 & 18.45 \\
\hline June & 1 & 15.69 & Sept. & 3 & j25. 52 & Feb. & 2 & 15.35 & Apr. & 1 & 18.05 \\
\hline July & 2 & 19.39 & Oct. & 1 & j24.00 & Mar. & 2 & 14.84 & May & 2 & (a) \\
\hline Aug. & 1 & 22.73 & Nov. & 1 & 21.88 & Apr. & 1 & 14.37 & June & 1 & (a) \\
\hline Sept. & 4 & 23.16 & Dec. & 2 & 20.67 & May & 1 & 16.25 & July & 1 & (a) \\
\hline Oct. & 3 & 21.13 & Jan. & 3,1958 & 19.83 & June & 1 & (a) & Aug. & 1 & j26.40 \\
\hline Nov. & 1 & 19.88 & Feb. & 3 & 18.94 & July & 1 & 21.88 & Sept. & 1 & (a) \\
\hline Dec. & & 18.83 & Mar. & 3 & 16.74 & Aug. & 4 & j25. 46 & Oct. & 3 & j26. 40 \\
\hline Jan. & 4,1957 & 18.13 & Apr. & 1 & 13.69 & Sept. & 1 & j25. 00 & Nov. & 1 & $\mathrm{j} 24.54$ \\
\hline Feb. & 1 & 17.68 & July & 1 & 14.37 & Oct. & 1 & 22.63 & Dec. & 1 & 23.13 \\
\hline Mar. & 1 & 17.32 & Aug. & 1 & 16.97 & & & & & & \\
\hline
\end{tabular}

3N/6-25R11. E. E. Morse Estate. About $4.4 \mathrm{mi}$ south of Lodi. Drilled domestic watertable well in Victor Formation of Pleistocene age, diam $10 \mathrm{in}$, depth $93 \mathrm{ft}$. Lsd $40.55 \mathrm{ft}$ above msl. MP lower edge of pumpbase, at slot, $0.50 \mathrm{ft}$ above lsd. Highest water level 26.15 below lsd, Jan. 5, 1953; lowest 44.86 below Isd, Oct. 5, 1960. Records available: 1948-60.

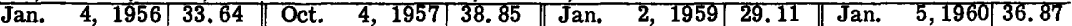
\begin{tabular}{lll|ll|ll||ll||l|l|l|l|l|} 
Oct. & 5 & & 33.27 & Jan. & 3, & 1958 & 35.37 & Oct. & 5 & 39.81 & Oct. & 5 & j44. 86 \\
\hline
\end{tabular} $\frac{\text { Jan. } 4,1957 \mid 30.93}{j \text { Water level below sea level. }}$

3N/6-36R2. Leland W. Bunch. About $5.5 \mathrm{mi}$ south of Lodi. Drilled domestic watertable well in Victor Formation of Pleistocene age, diam $8 \mathrm{in}$, depth $85 \mathrm{ft}$. Lsd $37.97 \mathrm{ft}$ above msl. MP arrow on floor of concrete pit, $7.60 \mathrm{ft}$ below lsd. Highest water level 11.72 below lsd, Apr. 8, 1938; lowest 44.20 below lsd, Oct. 5, 1960. Records available: 1926-29, 1935-60.

\begin{tabular}{|c|c|c|c|c|c|c|c|c|c|c|c|}
\hline Jan. & 4,1956 & $\overline{31.17}$ & Apr. & 1,1957 & 29.03 & July & 1,1958 & 24.80 & Oct. & \multicolumn{2}{|c|}{\begin{tabular}{l|l|}
5,1959 & 35.79
\end{tabular}} \\
\hline Feb. & 1 & 28.27 & May & 1 & 30.08 & Aug. & $\overrightarrow{1}$ & (a) & Nov. & 3 & 36.41 \\
\hline Mar. & $\mathbf{1}$ & 27.04 & June & 1 & 31.72 & Sept. & 2 & 28.75 & Dec. & 1 & 36.51 \\
\hline Apr. & 2 & 27.43 & July & 1 & (a) & Oct. & 3 & 29.32 & Jan. & 5,1960 & 36.05 \\
\hline May & 2 & 27.77 & Aug. & 1 & 36.91 & Nov. & 3 & 28.74 & Feb. & 1 & 35.54 \\
\hline June & 1 & 28.50 & Sept. & 3 & 37.63 & Dec. & 1 & 27.69 & Mar. & 1 & 35.12 \\
\hline July & 2 & a30.12 & Oct. & 4 & 37.84 & Jan. & 2, 1959 & 27.19 & Apr. & 1 & 35.45 \\
\hline Aug. & 1 & 30.87 & Nov. & 1 & 36.19 & Feb. & 2 & 27.00 & May & 2 & 37.74 \\
\hline Sept. & 4 & 30.00 & Dec. & 2 & 34.78 & Mar. & 2 & 26.58 & June & 1 & j39. 58 \\
\hline Oct. & 5 & 30.18 & Jan. & 6,1958 & 34.17 & Apr. & 1 & 27.14 & July & 1 & j41. 75 \\
\hline Nov. & 1 & 30.04 & Feb. & 3 & 33.45 & May & 1 & 28.18 & Aug. & 1 & (a) \\
\hline Dec. & & 29.20 & Mar & 3 & 31.59 & June & 1 & (a) & Sept. & 1 & j43. 58 \\
\hline Jan. & 1957 & 29.05 & Apr. & 1 & 28.47 & July & 1 & 34.40 & Oct. & 5 & j44. 20 \\
\hline Feb. & 1 & 29.09 & May & 1 & 21. 46 & Aug. & 4 & 37.83 & Nov. & 1 & j43. 76 \\
\hline Mar. & 1 & 29.14 & June & 2 & 23.34 & Sept. & 1 & j39. 80 & Dec. & 1 & $j 41.09$ \\
\hline
\end{tabular}

a Well being pumped.

j Water level below sea level.

3N/7-3C1. Jacob Knoll. About 4. 0 mi east of Lodi. Drilled observation water-table well in Victor Formation of Pleistocene age, diam 8 in, depth $48 \mathrm{ft}$, cased to 48 , perforated 38-48. Lsd 80. $45 \mathrm{ft}$ above msl. MP top of casing, $1.40 \mathrm{ft}$ above lsd. Highest water level 25.31 below lsd, June 2, 1943; lowest 44.94 below 1sd, Dec. 1, 1960 . Records available: $1935-60$

\begin{tabular}{ll|l||lll|l||lll|l||ll|l}
\hline Jan. & 5,1956 & 41.94 & May & 2,1956 & 38.38 & Sept. & 4,1956 & 37.56 & Jan. & 5,1957 & 39.54 \\
Feb. & 1 & 39.56 & June & 1 & & 36.61 & Oct. & 8 & & 38.46 & Feb. & 1 & 39.90 \\
Mar. & 1 & 38.49 & July & 2 & & 35.10 & Nov. & 1 & & 38.89 & Mar. & 1 & 40.32 \\
Apr. & 2 & 38.33 & Aug. & 1 & & 36.20 & Dec. & 3 & & 39.22 & Apr. & 1 & 40.37
\end{tabular}


3N/7-3C1--Continued.

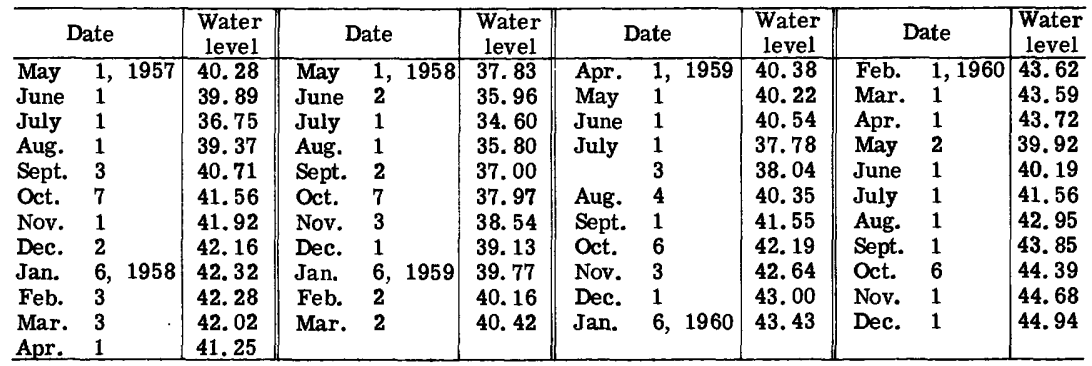

3N/7-6M8. R. E. and Ruth F. Coker. About $215 \mathrm{ft}$ east of Cherokee Lane and $2 \mathrm{ft}$ north of Southern Pacific RR. right-of-way. Lodi. Drilled observation water-table well in Victor Formation of Pleistocene age, diam 4 in, depth $40 \mathrm{ft}$, cased to 40, perforated 30-40. Lsd $53.35 \mathrm{ft}$ above msl. MP top of casing, $0.40 \mathrm{ft}$ above lsd. Highest water level 17.82 below 1sd, Apr. 30, 1943; lowest dry, May 1, 1959 through 1960. Records available: 1935-60.

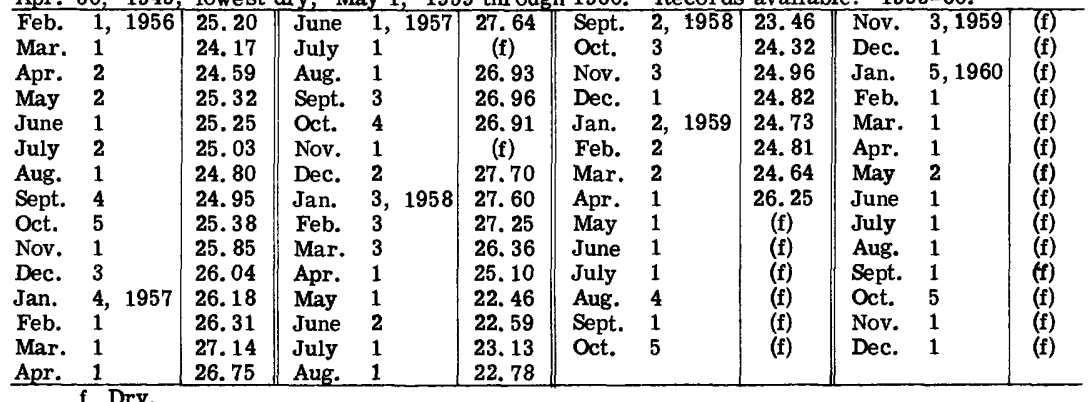

3N/7-7M1. J. and Rachel K. Goetken. East of Cherokee Lane. Lodi. Drilled irrigation water-table well in Victor Formation of Pleistocene age, diam $10 \mathrm{in}$, depth $49 \mathrm{ft}$. Lsd 52. $63 \mathrm{ft}$ above msl. MP top of 2-in floor flange, $0.10 \mathrm{ft}$ above $1 \mathrm{sd}$. Highest water level 24.51 below Isd, Apr. 6, 1938; lowest 42.52 below 1sd, May 1, 1950. Records available: 1935-60.

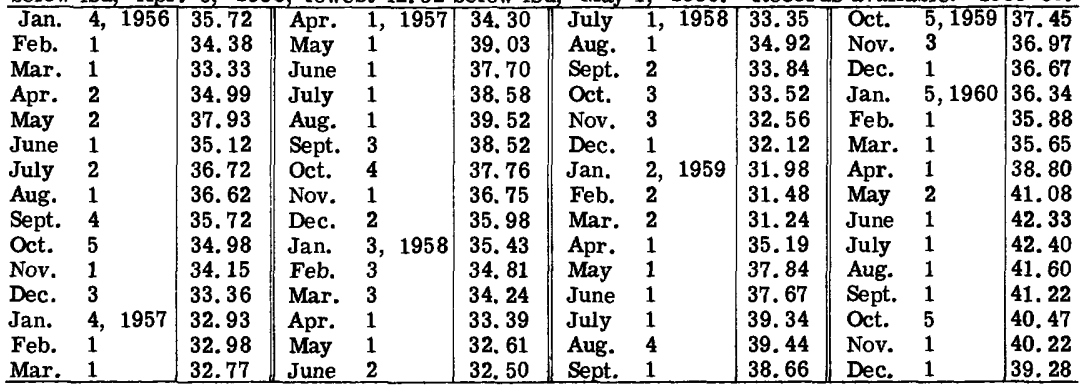

3N/7-10L3. Edward Preszler. About 4. 0 mi east of Lodi. Drilled observation watertable well in Victor Formation of Pleistocene age, diam $10 \mathrm{in}$, depth $57 \mathrm{ft}$, cased to 57 , perforated 47-57. Lsd 72.59 ft above msl. MP top of casing, $1.20 \mathrm{ft}$ above lsd. Highest water level 35.33 below Isd, Jan. 12, 1939; lowest 68.44 below lsd, Aug. 1, 1960. Records available: $1935-60$.

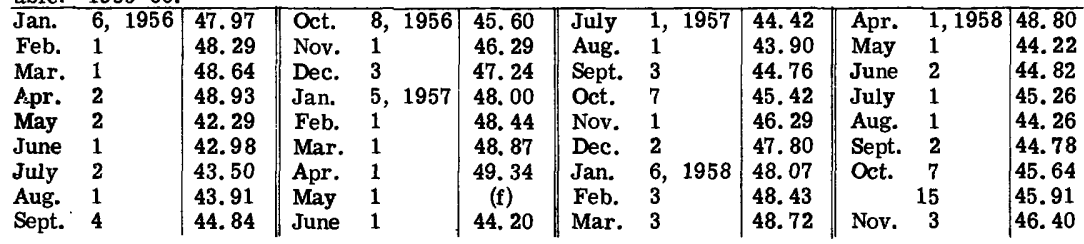


3N/7-10L3--Continued.

\begin{tabular}{|c|c|c|c|c|c|c|c|}
\hline Date & $\begin{array}{l}\text { Water } \\
\text { level }\end{array}$ & Date & $\begin{array}{l}\text { Water } \\
\text { level }\end{array}$ & Date & $\begin{array}{l}\text { Water } \\
\text { level }\end{array}$ & Date & $\begin{array}{l}\text { Water } \\
\text { level }\end{array}$ \\
\hline $\begin{array}{ll}\text { Dec. } & 1,1958 \\
\text { Jan. } & 6,1959 \\
\text { Feb. } & 2 \\
\text { Mar. } & 2 \\
\text { Apr. } & 1 \\
\text { May } & 1 \\
\text { June } & 1 \\
\end{array}$ & $\begin{array}{r}47.06 \\
47.63 \\
47.98 \\
48.15 \\
c 48.67 \\
43.15 \\
43.39\end{array}$ & $\begin{array}{ll}\text { July } & 1,1959 \\
\text { Aug. } & 4 \\
\text { Sept. } & 1 \\
\text { Oct. } & 6 \\
\text { Nov. } & 3 \\
\text { Dec. } & 1\end{array}$ & \begin{tabular}{|r|}
$c 43.85$ \\
43.05 \\
43.89 \\
45.06 \\
45.95 \\
47.07
\end{tabular} & $\begin{array}{lll}\text { Jan. } & 6, & 1960 \\
\text { Feb. } & 1 & \\
\text { Mar. } & 1 \\
\text { Apr. } & 1 \\
\text { May } & 2 \\
\text { June } & 1 & \end{array}$ & $\begin{array}{c}47.95 \\
48.54 \\
49.05 \\
(\mathrm{f}) \\
45.54 \\
45.20\end{array}$ & $\begin{array}{ll}\text { July } & 1,1960 \\
\text { Aug. } & 1 \\
\text { Sept. } & 1 \\
\text { Oct. } & 6 \\
\text { Nov. } & 1 \\
\text { Dec. } & 1\end{array}$ & $\begin{array}{l}66.70 \\
68.44 \\
68.40 \\
66.89 \\
65.18 \\
63.16\end{array}$ \\
\hline
\end{tabular}

3N/7-10L4. Edward Preszler. About 4. 0 mi east of Lodi. Drilled observation watertable well in Victor Formation of Pleistocene age, Arroyo Seco Gravel of Pleistocene age, and Laguna Formation of Pliocene (?) age, diam 12 to $10 \mathrm{in}$, depth $190 \mathrm{ft}$, cased to 190 . Lsd $72.37 \mathrm{ft}$ above msl. MP top of 2-in plank support, $0.87 \mathrm{ft}$ above lsd. Highest water level 35.13 below lsd, Jan. 12, 1939; lowest 70.87 below 1sd, July 1, 1960. Records available: 1935-60.

\begin{tabular}{|c|c|c|c|c|c|c|c|c|c|c|c|}
\hline & & & & & & & & & & & \\
\hline Jan. & 6,1956 & 54.03 & Apr & 1,1957 & 52.90 & July & 1,1958 & 59.87 & Oct. & 6,1959 & 61.35 \\
\hline Feb. & 1 & 52.91 & May & 1 & 59.93 & Aug. & 1 & 62.61 & Nov. & & 59.84 \\
\hline Iar. & 1 & 51.87 & Jun & 1 & 58.69 & Sept. & 2 & 59 & Dec. & & 3 \\
\hline Apr. & 2 & 5 & July & 1 & 6 & $\mathrm{O}$ & $\begin{array}{l}7 \\
3\end{array}$ & & Ja & 0,1960 & $\begin{array}{l}57.42 \\
56.43\end{array}$ \\
\hline Iuye & $\begin{array}{l}2 \\
1\end{array}$ & 5 & Aug & 3 & 6421 & Nov. & i & & Mar & 1 & \\
\hline uly & 2 & 60.86 & Oct. & 7 & 60,06 & Jan. & 6. 1959 & & Apr. & 1 & 63 \\
\hline tug. & 1 & 62.69 & Nov & 1 & & Feb. & 2 & & $\mathrm{M}$ & 2 & \\
\hline ept. & 4 & 59 & Dec & & 66 & Mar. & 2 & 51.53 & June & 1 & \\
\hline ct. & 8 & 56.92 & Jan. & 6,1958 & 55.43 & Apr. & 1 & & July & 1 & 70.87 \\
\hline Tov. & 1 & 55.09 & Feb & 3 & 54.48 & May & 1 & 64.32 & Aug. & 1 & (a) \\
\hline lec. & & 53.48 & Mar & 3 & 53.57 & June & 1 & 61.63 & Sept. & 1 & 7 \\
\hline an. & 5,1 & 52 & Apr & 1 & & July & 1 & & Oct. & 6 & \\
\hline eb. & 1 & 52. & May & 1 & 01 & Aug. & 4 & 69.30 & Nov. & 1 & 64 \\
\hline Mar. & 1 & 52.46 & June & 2 & 54.70 & Sept. & 1 & 65.21 & Dec. & 1 & \\
\hline
\end{tabular}

a Well being pumped.

3N/7-18N12. Joe Garnero. About $2.5 \mathrm{mi}$ south of Lodi. Drilled domestic water-table well in Victor Formation of Pleistocene age, diam 6 in, depth $78 \mathrm{ft}$. Lsd $47.44 \mathrm{ft}$ above msl. MP top east side of casing, $0.30 \mathrm{ft}$ above $1 \mathrm{sd}$. Highest water level 29.86 below 1sd, Feb. 2, 1953; lowest 47.67 below 1sd, July 1, 1960. Records available: 1946-60.

\begin{tabular}{|c|c|c|c|c|c|c|c|c|c|c|c|}
\hline & & & & & & & & & & & \\
\hline Jan. & 4,1956 & 36.42 & Apr. & 1,1957 & 33.72 & July & 1,1958 & 38.56 & Oct. & 5,1959 & 38.73 \\
\hline Feb. & 1 & 35.61 & May & 1 & 36.57 & Aug. & 1 & 38.61 & Nov. & & 37.98 \\
\hline Mar. & 1 & 34.46 & June & 1 & 38.33 & Sept. & 2 & 35.23 & Dec. & 1 & 37.52 \\
\hline Apr. & 2 & 34.19 & July & 1 & 42. 33 & Oct. & 3 & 33.30 & Jan. & 5,1960 & 37.54 \\
\hline May & 2 & 36.65 & Aug. & 1 & 43.30 & Nov. & 3 & 32.54 & Feb. & 1 & 36.93 \\
\hline June & 1 & 37.31 & Sept. & 4 & 41.01 & Dec. & 1 & 32.67 & Mar. & 1 & 36.55 \\
\hline July & 2 & 39.50 & Oct. & 5 & 39.25 & Jan. & 2,1959 & 33.18 & Apr. & 1 & 37 \\
\hline Aug. & 1 & 40.33 & Nov. & 1 & 38.25 & Feb. & 2 & 32.73 & May & 2 & 41.73 \\
\hline Sept. & 4 & 38.49 & Dec. & 2 & 37.58 & Mar. & 2 & 33.26 & June & 1 & 44.17 \\
\hline Oct. & 5 & 36.33 & Jan. & 3,1958 & 36.68 & Apr. & 1 & 34.15 & July & 1 & j47. 67 \\
\hline Nov. & 1 & 35.13 & Feb. & 3 & 35.98 & May & 1 & 37.12 & Aug. & 1 & 46.86 \\
\hline Dec. & 3 & 34.13 & Mar. & 3 & 35.50 & June & 1 & 39.51 & Sept. & 1 & 44.62 \\
\hline Jan. & 4,1957 & 33.53 & Apr. & 1 & 34.72 & July & 1 & 42.22 & Oct. & 5 & 42.70 \\
\hline Feb. & 1 & 33.96 & May & 1 & 32.59 & Aug. & 4 & 42.11 & Nov. & 1 & 41.96 \\
\hline Mar. & 1 & 34.18 & June & 2 & 32.85 & Sept. & 1 & 40.20 & Dec. & 1 & 41.14 \\
\hline
\end{tabular}

$\mathrm{j}$ Water level below sea level.

3N/7-22C11. John Nietschke. About $4.6 \mathrm{mi}$ southeast of Lodi. Drilled domestic watertable well, largely in Victor Formation of Pleistocene age, but probably penetrating into underlying Arroyo Seco Gravel of Pleistocene age, and Laguna Formation of Pliocene (?) age, diam $8 \mathrm{in}$, depth $137 \mathrm{ft}$. Lsd $66.43 \mathrm{ft}$ above msl. MP top southeast side of casing, $0.80 \mathrm{ft}$ above $1 \mathrm{sd}$. Highest water level 50.21 below lsd, Mar. 2, 1953; lowest 73.99 below lsd, Sept. 1, 1960. Records available: 1952-60.

\begin{tabular}{|c|c|c|c|c|c|c|c|c|c|c|c|}
\hline Jan. & 6,1956 & 60.08 & Nov & 1,1956 & 60.94 & Sept. & 3,1957 & j68.56 & July & 1,1958 & 61.46 \\
\hline Feb & 1 & 58.12 & Dec & 3 & 59.26 & Oct. & 7 & 65.73 & Aug. & & 64.08 \\
\hline Mar & 1 & 57.48 & Jan. & 5, 1957 & 58.55 & Nov. & 1 & 63.87 & Sept. & 2 & 63.23 \\
\hline Apr & 2 & 57.70 & Feb & 1 & 56.86 & Dec. & 2 & 62.13 & Oct. & 6 & 60.97 \\
\hline May & 2 & 59.85 & Mar & 1 & 55.93 & Jan. & 6,1958 & 60.64 & Nov. & 3 & .43 \\
\hline une & 1 & c64. 83 & Apr & 1 & 55.64 & Feb. & 3 & 59.39 & Dec. & 1 & 58.33 \\
\hline July & 2 & 62.99 & May & 1 & 58.68 & Mar. & 3 & 58.38 & Jan. & 6,1959 & 56.73 \\
\hline Aug & 1 & 65.17 & June & 1 & 60.57 & Apr. & 1 & 57.28 & Feb. & 2 & 55.73 \\
\hline Sept & 4 & 65.44 & July & 1 & 64. 86 & May & 1 & 56.74 & Mar. & 2 & .66 \\
\hline Oct. & 8 & 62.63 & Aug & 1 & j68. 41 & June & 2 & a) & Apr. & 1 & .73 \\
\hline
\end{tabular}


3N/7-22C11--Continued.

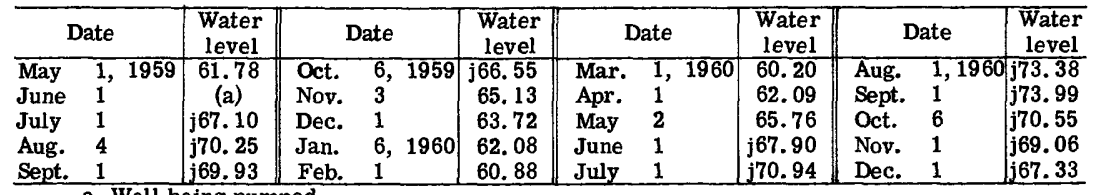

a Well being pumped.

c Nearby well being pumped.

j Water level below sea level.

3N/7-27F3. John F. Heitzmann. About $5.5 \mathrm{mi}$ southeast of Lodi. Drilled observation water-table well in Victor Formation of Pleistocene age, diam 8 in, depth $46 \mathrm{ft}$, deepened in May 1949 to $91 \mathrm{ft}$, cased to 63 . Lsd $59.42 \mathrm{ft}$ above msl. MP top of casing, $1.70 \mathrm{ft}$ above $1 \mathrm{sd}$. Highest water level 26.12 below lsd, Mar. 31, 1943; lowest 67. 25 below lsd, Sept. 1, 1960. Records available: 1935-60.

\begin{tabular}{|c|c|c|c|c|c|c|c|c|c|c|c|}
\hline Jan. & 5,1956 & 58.04 & Apr. & 1,1957 & 52.39 & July & 1,1958 & 55.26 & Oct. & \multicolumn{2}{|c|}{61959176295} \\
\hline Feb. & 1 & 56.82 & May & 1 & 52.85 & Aug. & 1 & 57.50 & Noy. & 3 & j62. 33 \\
\hline Mar. & 1 & 55.16 & June & 1 & 55. 35 & Sept. & 2 & j60.28 & Dec. & 1 & j61. 63 \\
\hline Apr. & 2 & 54.31 & July & 1 & 58.35 & Oct. & 6 & j59. 75 & Jan. & 6,1960 & $j 59.49$ \\
\hline May & 2 & 54.50 & Aug. & 1 & j63. 47 & Nov. & 3 & 56.63 & Feb. & 1 & 59.30 \\
\hline June & 1 & 55.56 & Sept. & 3 & j63. 07 & Dec. & 1 & 56 & Mar. & 1 & 58.40 \\
\hline July & 2 & 58.72 & Oct. & 7 & j62. 15 & Jan. & 6,1959 & 53.40 & Apr. & 1 & 57.45 \\
\hline Aug. & 1 & j61.05 & Nov. & 1 & j60.70 & Feb. & 2 & 52.17 & May & 2 & 58.98 \\
\hline Sept. & 4 & j62. 79 & Dec. & 2 & 59.36 & Mar. & 2 & 51.00 & June & 1 & j61. 35 \\
\hline Oct. & 8 & $\mathrm{j} 60.83$ & Jan. & 6,1958 & 57.95 & Apr. & 1 & 50.39 & July & 1 & 10 \\
\hline Nov. & 1 & j59. 45 & Feb. & 3 & 56.76 & May & 1 & 52.77 & Aug. & 1 & 70 \\
\hline Dec. & & 57.67 & Mar. & 3 & 55.67 & June & 1 & 55.08 & Sept. & 1 & $\mathrm{j} 67.25$ \\
\hline Jan. & 4,1957 & 56.06 & Apr. & 1 & 54.14 & July & 1 & j59. 68 & Oct. & 6 & $\mathrm{j} 66.03$ \\
\hline Feb. & 1 & 54.77 & May & 1 & 53.85 & Aug. & 4 & j62. 84 & Nov. & 1 & 19 \\
\hline & 1 & 53.64 & June & 2 & 54.65 & Sept. & 1 & 54.48 & Dec. & 1 & 18 \\
\hline
\end{tabular}

4N/6-12R11. A. T. Carlson. About $4.5 \mathrm{mi}$ north of Lodi. Drilled domestic and irrigation water-table well in Victor, Arroyo Seco, and Laguna Formations, diam 8 in, reported depth $150 \mathrm{ft}$. Lsd $57.95 \mathrm{ft}$ above msl. MP lower edge of $1 \frac{1}{2}$-in pipe in concrete pump foundation, $0.60 \mathrm{ft}$ above 1sd. Highest water level 38.84 below 1sd, May 1, 1952; lowest 69. 25 below lsd, Sept. 1, 1960. Records available: 1948-60.

\begin{tabular}{|c|c|c|c|c|c|c|c|c|c|c|c|}
\hline Jan. & 4,1956 & 50.28 & Apr. & 1,1957 & 47.10 & July & 1,1958 & 55.65 & Oct. & \multicolumn{2}{|c|}{$5,1959 \mid j 61.37$} \\
\hline Feb. & 1 & 48.73 & May & 1 & 50.72 & Aug. & 1 & j58.03 & Nov. & 3 & $j 60.26$ \\
\hline Mar. & 1 & 46.96 & June & 1 & 52. 69 & Sept. & 2 & j59.07 & Dec. & 1 & 56.34 \\
\hline Apr. & 2 & 47.10 & July & 1 & 57.38 & Oct. & 3 & 57.37 & Jan. & 5,1960 & 54.15 \\
\hline Iay & 2 & 48.23 & Aug. & 1 & j61. 27 & Nov. & 3 & 55.47 & Feb. & 1 & 52.47 \\
\hline June & 1 & 52.64 & Sept. & 3 & $\mathrm{j} 62.58$ & Dec. & 1 & 53.92 & Mar. & 1 & 51.48 \\
\hline July & 2 & j58.54 & Oct. & 4 & j59. 59 & Jan. & 2, 1959 & 48.67 & Apr. & 1 & 53.52 \\
\hline Aug. & 1 & $j 61.20$ & Nov. & 1 & 56.72 & Feb. & 2 & 47.45 & May & 2 & 55.19 \\
\hline Sept. & 4 & $j 60.14$ & Dec. & 2 & 52.81 & Mar. & 2 & 46.28 & June & 1 & $\mathrm{j} 60.29$ \\
\hline Oct. & 5 & $\mathrm{j} 58.55$ & Jan. & 3,1958 & 50.63 & Apr. & 1 & 49.87 & July & 1 & 00 \\
\hline Nov. & 1 & 53.39 & Feb. & 3 & 49.08 & May & 1 & 53.00 & Aug. & 1 & $\mathrm{j} 67.60$ \\
\hline Dec. & 3 & 52.79 & Mar. & 3 & 48.01 & June & 1 & 55.60 & Sept. & 1 & $j 69.25$ \\
\hline Jan. & 4, 1957 & 49.21 & Apr. & 1 & 46.68 & July & 1 & j59. 31 & Oct. & 5 & j67.97 \\
\hline Feb. & 1 & 48.09 & May & 1 & 49.65 & Aug. & 4 & j64.09 & Nov. & 1 & j66.33 \\
\hline Mar. & 1 & 46.54 & June & 2 & 50.82 & Sept. & 1 & $\mathrm{j} 64.30$ & Dec. & 1 & $\mathrm{j} 60.22$ \\
\hline
\end{tabular}

4N/6-36D1. D. D. Smith and S. H. and I. Zimmerman. About 1.6 mi north of Lodi. Drilled unused water-table well in Victor Formation of Pleistocene age, diam 6 in, depth $35 \mathrm{ft}$. Lsd $49.90 \mathrm{ft}$ above msl. MP top of casing, $0.16 \mathrm{ft}$ below $1 \mathrm{sd}$. Highest water level 15.02 below lsd, Mar. 31, 1943; lowest dry at 35.00 below lsd, May 1, 1946. Records available: 1926-29, 1935-60.

\begin{tabular}{|c|c|c|c|c|c|c|c|c|c|c|c|}
\hline Jan. & 4,1956 & 24.03 & Dec. & 3,1956 & 23.45 & Nov & 1,1957 & 24.59 & Oct. & 2,1958 & 21.59 \\
\hline Feb. & 1 & 22.12 & Jan. & 4, 1957 & 24.19 & Dec & & 24.92 & Nov. & 3 & 21.62 \\
\hline Mar. & 1 & 21.80 & Feb. & & 24.25 & Jan. & 3, 1958 & 25.39 & Dec. & 1 & 21.70 \\
\hline Apr. & 2 & 21.21 & Mar & 1 & 24.55 & Feb & 3 & 25.30 & Jan. & 2,1959 & 22.00 \\
\hline May & 2 & 21.67 & Apr. & 1 & 23.52 & Mar & 3 & 24.62 & Feb. & 2 & 22.78 \\
\hline June & 1 & c26.23 & May & 1 & 23.96 & Apr & 1 & 23.28 & Mar. & 2 & 22.57 \\
\hline July & 2 & 24.95 & June & 1 & c29. 61 & May & 1 & 17.93 & Apr. & 1 & 26.64 \\
\hline Aug. & 1 & 24.42 & July & 1 & 27.51 & June & 2 & 23.72 & May & 1 & 28.83 \\
\hline Sept. & 4 & 22.85 & Aug. & 1 & 27.99 & July & 1 & 23.56 & June & 1 & 28.46 \\
\hline Oct. & 4 & 22.45 & Sept. & 3 & 25.28 & Aug & 1 & 22.03 & July & 1 & 28.03 \\
\hline Nov. & 1 & 22.60 & Oct. & 2 & 24.74 & Sept & 2 & 21.51 & Aug. & 4 & 28.30 \\
\hline
\end{tabular}


4N/6-36D1-- Continued.

\begin{tabular}{|c|c|c|c|c|c|c|c|}
\hline Date & $\begin{array}{l}\text { Water } \\
\text { level }\end{array}$ & Date & $\begin{array}{c}\text { Water } \\
\text { level }\end{array}$ & Date & $\begin{array}{c}\text { Water } \\
\text { level }\end{array}$ & Date & $\begin{array}{l}\text { Water } \\
\text { level }\end{array}$ \\
\hline $\begin{array}{lll}\text { Sept. } & 1,1959 \\
\text { Oct. } & 2 & \\
\text { Nov. } & 3 & \\
\text { Dec. } & 1 & \end{array}$ & $\begin{array}{l}26.16 \\
25.81 \\
25.70 \\
25.40\end{array}$ & $\begin{array}{lll}\text { Jan. } & 5,1960 \\
\text { Feb. } & 1 & \\
\text { Mar. } & 1 & \\
\text { Apr. } & 1 & \end{array}$ & $\begin{array}{l}25.18 \\
25.81 \\
26.09 \\
25.52\end{array}$ & $\begin{array}{lll}\text { May } & 2,1960 \\
\text { June } & 1 & \\
\text { July } & 1 & \\
\text { Aug. } & 1 & \end{array}$ & $\begin{array}{l}29.40 \\
30.76 \\
30.98 \\
30.46\end{array}$ & $\begin{array}{ll}\text { Sept. } & 1,1960 \\
\text { Oct. } & 4 \\
\text { Nov. } & 1 \\
\text { Dec. } & 1\end{array}$ & $\begin{array}{l}28.42 \\
27.50 \\
27.63 \\
28.16\end{array}$ \\
\hline
\end{tabular}

4N/7-15B3. Robert L. Carter. About 6.0 mi northeast of Lodi. Drilled observation water-table well in Victor Formation of Pleistocene age, diam $8 \mathrm{in}$, depth $85 \mathrm{ft}$, cased to 69. Lsd $92.05 \mathrm{ft}$ above msl. MP top of casing, $1.00 \mathrm{ft}$ above 1sd. Highest water level 32.11 below lsd, Sept. 1, 1939; lowest dry several times, 1957-59. Records available: 1935-59. Well abandoned; measurement discontinued.

\begin{tabular}{|c|c|c|c|c|c|c|c|c|c|c|c|}
\hline Jan. & 6,1956 & $j 57.44$ & Dec. & 3,1956 & 71.58 & Dec. & 2,1957 & 73.09 & Dec. & 1,1 & 73.49 \\
\hline & 27 & $\mathrm{j} 57.76$ & Jan. & 8,1957 & 70.42 & Jan. & 7,1958 & 71.96 & Jan. & 8,1959 & 72.09 \\
\hline Feb. & 1 & $\mathrm{j} 58.16$ & Feb. & 1 & 69.73 & Feb. & 3 & 70.88 & Feb. & 2 & 71.21 \\
\hline Mar. & 1 & 67.29 & Mar. & 1 & 68.98 & Mar. & 3 & j56. 70 & Mar. & 2 & $\mathrm{j} 59.35$ \\
\hline Apr. & 2 & j58.10 & Apr. & 1 & 68.17 & Apr. & 1 & j55.26 & Apr. & 1 & 70.21 \\
\hline May & 2 & 68.01 & May & 1 & 68.99 & May & 1 & 69.23 & May & 1 & 69.15 \\
\hline June & 1 & 69.27 & June & 1 & 69.57 & June & 2 & 70.55 & June & 1 & (f) \\
\hline July & 2 & 73.14 & July & 1 & 73.73 & July & 1 & 73.45 & July & 1 & (f) \\
\hline Aug. & 1 & 75.04 & Aug. & 1 & (f) & Aug. & 1 & (f) & Aug. & 4 & $\mathrm{j} 65.54$ \\
\hline Sept. & 4 & 75.82 & Sept. & 3 & (f) & Sept. & 2 & (f) & Sept. & 1 & $\mathrm{j} 59.57$ \\
\hline Oct. & 9 & 74.75 & Oct. & 8 & 75.48 & Oct. & 7 & (f) & Oct. & 7 & (f) \\
\hline Nov. & 1 & 73.23 & Nov. & 1 & 74.18 & Nov. & 3 & 74.62 & Nov. & 3 & (f) \\
\hline
\end{tabular}

j Water leaking into well.

4N/7-22Q4. Adolphus Eddlemon. About $4.9 \mathrm{mi}$ northeast of Lodi. Drilled observation water-table well in Victor Formation of Pleistocene age, and underlying unclassified sand and gravel, diam $10 \mathrm{in}$, depth $51 \mathrm{ft}$, cased to 51, perforated 39-49. Lsd $83.61 \mathrm{ft}$ above msl. MP top of casing, $0.80 \mathrm{ft}$ above lsd. Highest water level 35.95 below Isd, Apr. 30, 1943; lowest 67. 43 below lsd, Sept. 1, 1960. Records available: 1935-60.

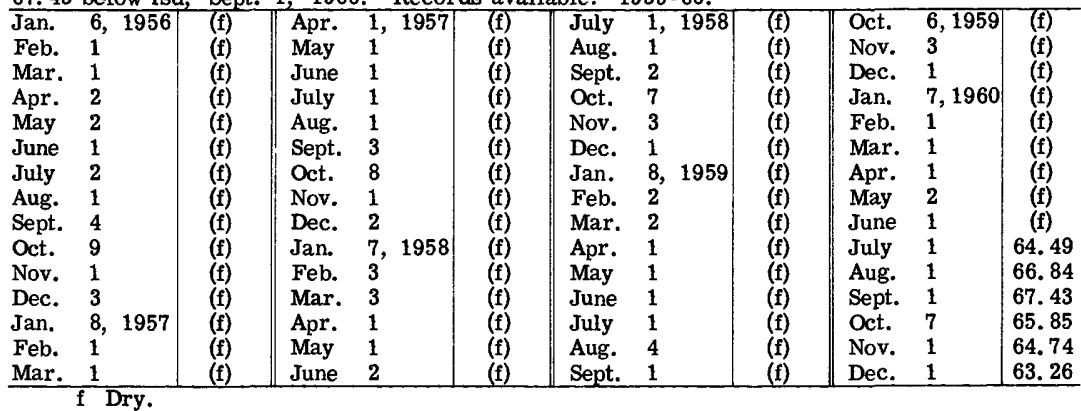

4N/7-22Q5. Adolphus Eddlemon. About 4.9 mi northeast of Lodi. Drilled observation artesian well in Victor, Arroyo Seco, and Laguna Formations, diam $10 \mathrm{in,} \mathrm{depth} 266 \mathrm{ft}$, cased 0-79, 129-149. Lsd 83.83 ft above msl. MP top of casing, $0.20 \mathrm{ft}$ above lsd. Highest water level 36. 34 below lsd, Mar. 31, 1943; lowest 71.90 below lsd, July 1, 1960 . Records available: 1935-60.

\begin{tabular}{|c|c|c|c|c|c|c|c|c|c|c|c|}
\hline Jan. & 6,1956 & 54.90 & Apr. & 1,1957 & 52.92 & July & 1,1958 & 63.01 & Oct. & 6,1959 & 62.60 \\
\hline Feb. & 1 & 53.72 & May & 2 & c59.57 & Aug. & 1 & 61.68 & Nov. & 3 & 61.55 \\
\hline Mar. & 1 & 52.60 & June & 1 & 59.53 & Sept. & 2 & 60.23 & Dec. & 1 & 60.38 \\
\hline Apr. & 2 & 54.56 & July & 1 & 65.32 & Oct. & 7 & 57.91 & Jan. & 7,1960 & 59.22 \\
\hline May & 2 & 58.19 & Aug. & 1 & c67.64 & Nov. & 3 & 56.40 & Feb. & 1 & 58.40 \\
\hline June & 1 & 57.34 & Sept. & 3 & 63. 35 & Dec. & 1 & 55.55 & Mar. & 1 & 58.05 \\
\hline July & 2 & 62.40 & Oct. & 8 & 60.17 & Jan. & 8,1959 & 54.50 & Apr. & 1 & 58.48 \\
\hline Aug. & 1 & 63.36 & Nov. & 1 & 58.67 & Feb. & 2 & 54.00 & May & 2 & 66.89 \\
\hline Sept. & 4 & 59.74 & Dec. & 2 & 57.47 & Mar. & 2 & 53.40 & June & 1 & 68.60 \\
\hline Oct. & 9 & 57.44 & Jan. & 7,1958 & 56.33 & Apr. & 1 & 55.16 & July & 1 & 71.90 \\
\hline Nov. & 1 & 56.12 & Feb. & 3 & 55.48 & May & 1 & c64.55 & Aug. & 1 & 71.76 \\
\hline Dec. & 3 & 54.66 & Mar. & 3 & 54. 69 & June & 1 & c63. 83 & Sept. & 1 & 70.70 \\
\hline Jan. & 8,1957 & 53.78 & Apr. & 1 & 53.66 & July & 1 & 67.82 & Oct. & 7 & 67.70 \\
\hline Feb. & 1 & 53.37 & May & 1 & 53. 12 & Aug. & 4 & c68. 43 & Nov. & 1 & 66.30 \\
\hline Mar. & 1 & 52.93 & June & 2 & c57.26 & Sept. & 1 & c67. 18 & Dec. & 1 & 64.16 \\
\hline
\end{tabular}

c Nearby well being pumped. 
4N/7-27P1. Frank H. and Leonard W. Buck. About 4.4 mi northeast of Lodi. Drilled observation water-table well in Victor Formation of Pleistocene age, diam $10 \mathrm{in,} \mathrm{depth} 49 \mathrm{ft}$, cased to 49, perforated 39-49. Lsd $81.20 \mathrm{ft}$ above msl. MP top of casing, $0.90 \mathrm{ft}$ above $1 \mathrm{sd}$. Highest water level 24.60 below lsd, June 3, 1938; lowest 40.79 below lsd, Dec. 1, 1960. Records available: 1935-60.

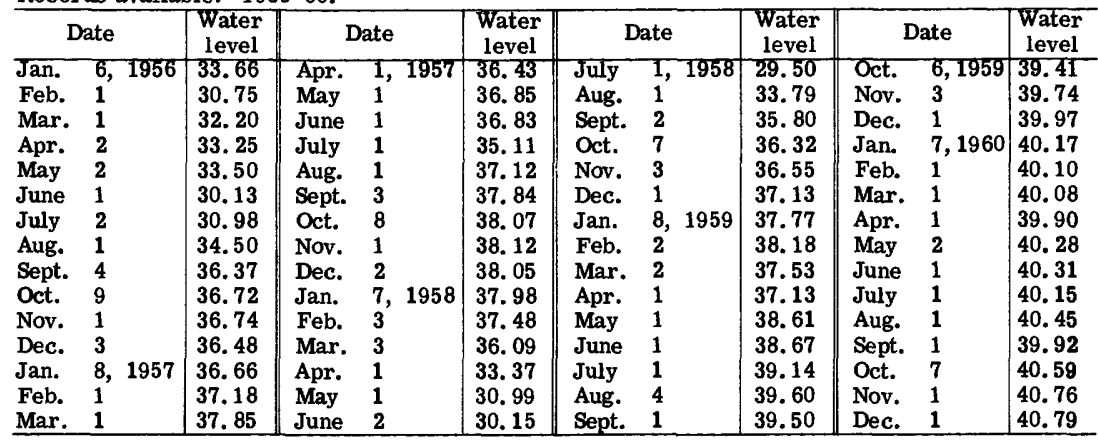

4N/7-30E4. Charles Weber. About $2.5 \mathrm{mi}$ north of Lodi. Drilled unused water-table well in Victor Formation of Pleistocene age, diam $6 \mathrm{in}$, depth $76 \mathrm{ft}$. Lsd $57.18 \mathrm{ft}$ above msl. MP top of casing, $0.50 \mathrm{ft}$ above lsd. Highest water level 26. 35 below lsd, Jan. 4, 1944; lowest 51. 96 below lsd, Aug. 1, 1960. Records available: 1941-60.

\begin{tabular}{|c|c|c|c|c|c|c|c|c|c|c|c|}
\hline $\begin{array}{l}\text { Jan. } \\
\text { Feb. } \\
\text { Mar. } \\
\text { Apr. } \\
\text { May } \\
\text { June } \\
\text { July } \\
\text { Aug. } \\
\text { Sept. } \\
\text { Oct. } \\
\text { Nov. } \\
\text { Dec. } \\
\text { Jan. } \\
\text { Feb. } \\
\text { Mar. } \\
\text { Apr. }\end{array}$ & $\begin{array}{ll}4, & 1956 \\
1 & \\
1 & \\
2 & \\
2 & \\
1 & \\
2 & \\
1 & \\
4 & \\
5 & \\
1 & \\
3 & \\
4, & 1957 \\
1 & \\
1 & \\
1 & \\
\end{array}$ & $\begin{array}{l}38.76 \\
37.47 \\
36.02 \\
35.06 \\
37.78 \\
37.35 \\
41.37 \\
41.44 \\
41.62 \\
37.27 \\
36.42 \\
35.70 \\
35.63 \\
35.18 \\
34.80 \\
34.88\end{array}$ & $\begin{array}{l}\text { May } \\
\text { June } \\
\text { July } \\
\text { Aug. } \\
\text { Sept. } \\
\text { Oct. } \\
\text { Nov. } \\
\text { Dec. } \\
\text { Jan. } \\
\text { Feb. } \\
\text { Mar. } \\
\text { Apr. } \\
\text { May } \\
\text { June } \\
\text { July }\end{array}$ & $\begin{array}{ll}1, & 1957 \\
1 & \\
1 & \\
1 & \\
3 & \\
4 & \\
1 & \\
2 & \\
3, & 1958 \\
3 & \\
3 & \\
1 & \\
1 & \\
2 & \\
1 & \end{array}$ & $\begin{array}{c}38.54 \\
37.87 \\
42.82 \\
43.91 \\
43.09 \\
40.81 \\
39.52 \\
38.56 \\
37.76 \\
37.13 \\
36.64 \\
(j) \\
33.09 \\
36.66 \\
37.07\end{array}$ & $\begin{array}{l}\text { Aug. } \\
\text { Sept. } \\
\text { Oct. } \\
\text { Nov. } \\
\text { Dec. } \\
\text { Jan. } \\
\text { Feb. } \\
\text { Mar. } \\
\text { Apr. } \\
\text { May } \\
\text { June } \\
\text { July } \\
\text { Aug. } \\
\text { Sept. }\end{array}$ & \begin{tabular}{ll|}
1, & 1958 \\
2 & \\
3 & \\
3 & \\
1 & \\
2, & 1959 \\
2 & \\
2 & \\
1 & \\
1 & \\
4 & \\
1 & \\
1 & \\
4 & \\
1 &
\end{tabular} & $\begin{array}{l}38.98 \\
36.78 \\
34.95 \\
34.12 \\
34.30 \\
33.79 \\
33.35 \\
33.00 \\
34.32 \\
40.02 \\
40.69 \\
40.07 \\
42.81 \\
43.97 \\
43.00\end{array}$ & $\begin{array}{l}\text { Oct. } \\
\text { Nov. } \\
\text { Dec. } \\
\text { Jan. } \\
\text { Feb. } \\
\text { Mar. } \\
\text { Apr. } \\
\text { May } \\
\text { June } \\
\text { July } \\
\text { Aug. } \\
\text { Sept. } \\
\text { Oct. } \\
\text { Nov. } \\
\text { Dec. }\end{array}$ & $\begin{array}{l}5,1959 \\
3 \\
1 \\
5,1960 \\
1 \\
1 \\
1 \\
1 \\
1 \\
1 \\
1 \\
1 \\
5 \\
1 \\
1\end{array}$ & \begin{tabular}{|l|}
41.16 \\
40.23 \\
39.73 \\
39.21 \\
38.32 \\
38.05 \\
37.99 \\
44.20 \\
44.13 \\
48.34 \\
51.96 \\
46.92 \\
44.82 \\
43.99 \\
43.26
\end{tabular} \\
\hline
\end{tabular}

4N/7-31N5. Jacob Goehring. About $1.0 \mathrm{mi}$ northeast of Lodi. Drilled observation watertable well in alluvium, diam $4 \mathrm{in}$, depth $25 \mathrm{ft}$, cased to 25, perforated 15-25. Lsd $44.12 \mathrm{ft}$ above msl. MP top of casing, $2.90 \mathrm{ft}$ above lsd. Highest water level 1.73 below lsd, Apr. 30, 1943; lowest 14.63 below 1sd, Mar. 1, 1948. Records available: 1935-60.

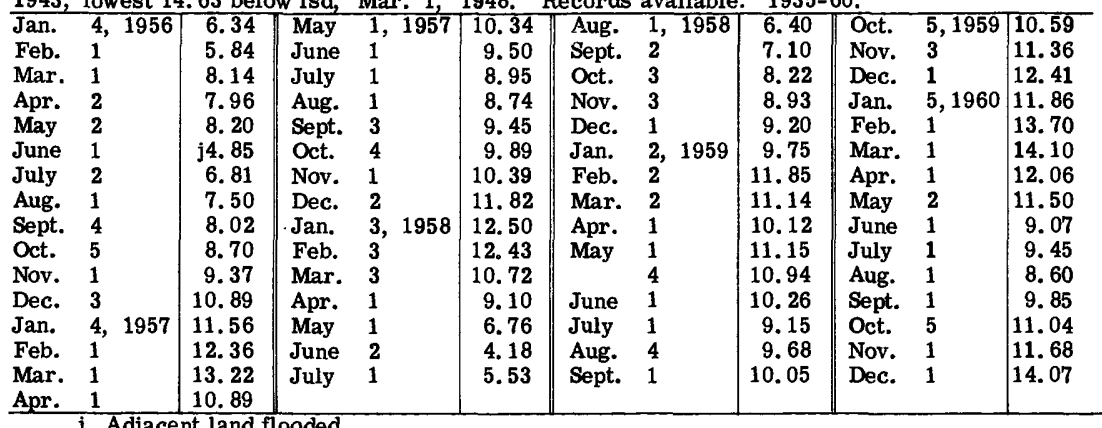

4N/7-34F11. John J. Schmiedt. About 4.2 mi east of Lodi. Drilled observation watertable well in alluvium, diam $4 \mathrm{in}$, depth $24 \mathrm{ft}$. Lsd $61.76 \mathrm{ft}$ above msl. MP top west side of casing, $1.35 \mathrm{ft}$ above lsd. Highest water level 6.09 below lsd, Jan. 6, 1956; lowest 18. 96 below lsd, May 2, 1960. Records available: 1952-60. 
4N/7-34F11--Continued.

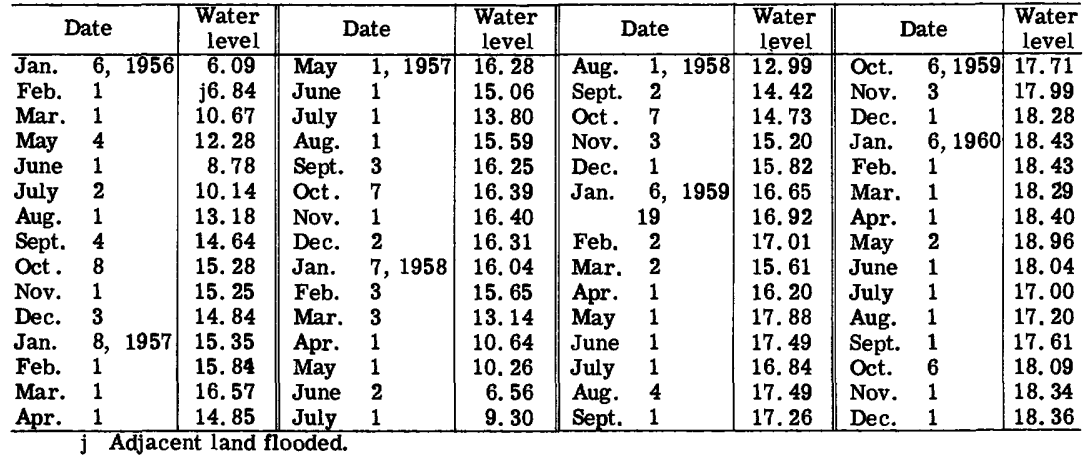

\section{Santa Barbara County}

\section{Carpinteria Basin}

4/25-19J5. Lyman \& Young. Drilled unused artesian well in alluvium, diam 8 in, depth $100 \mathrm{ft}$. Lsd about $55 \mathrm{ft}$ above $\mathrm{msl}$. MP top of casing, $0.18 \mathrm{ft}$ above $1 \mathrm{sd}$. Highest water level 39. 41 below lsd, Apr. 23, 1942; lowest 92. 95 below 1sd, Sept. 25, 1951. Records available: 1941-60.

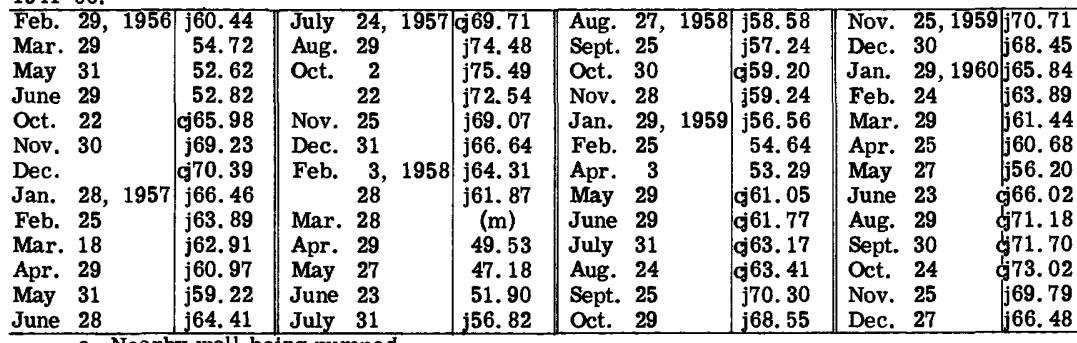

\section{c Nearby well being pumped.}

j Water level below sea level.

m Recharging.

4/25-26A1. Moses Mesa Associates Co. Drilled unused water-table well in Casitas Formation of Pleistocene age, diam $10 \mathrm{in}$, depth $480 \mathrm{ft}$, cased to 480 , perforated 228-380. Lsd about $420 \mathrm{ft}$ above msl (previously reported $412 \mathrm{ft}$ above msl). MP lower lip of $1 \frac{1}{4}$-in pipe, through concrete foundation, $1.00 \mathrm{ft}$ above $1 \mathrm{sd}$. Highest water level 230.09 below lsd, Feb. 8 , 1946; lowest 387.00 below lsd, Nov. 28, 1955. Records avallable: 1946-60.

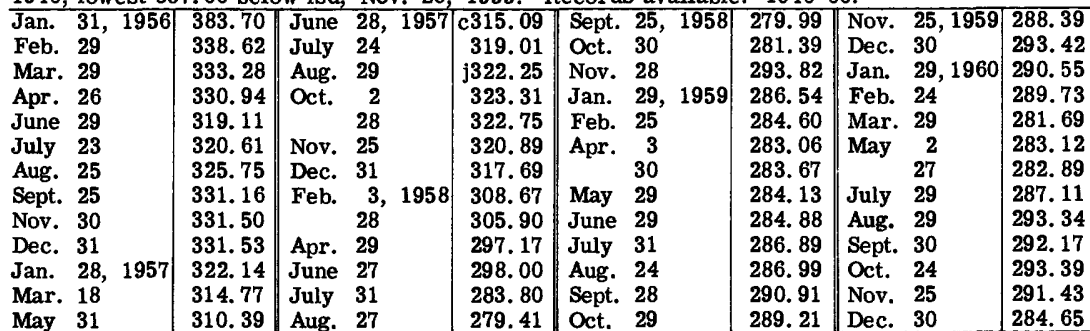

May 31

\section{c Nearby well being pumped.}

j Electric sounder. 
4/25-27Q2. A. F. Heimlich. Drilled unused artesian well in Casitas Formation of Pleistocene age, diam $10 \mathrm{in}$, depth $198 \mathrm{ft}$. Lsd about $127 \mathrm{ft}$ above msl. MP top east side of casing, $0.68 \mathrm{ft}$ above lsd. Highest water level 92.86 below lsd, Apr. 30, 1945; lowest 175.42 below lsd, Sept. 25, 1951. Records available: 1941-60.

\begin{tabular}{|c|c|c|c|c|c|c|c|}
\hline Date & $\begin{array}{l}\text { Water } \\
\text { level }\end{array}$ & Date & $\begin{array}{c}\text { Water } \\
\text { level }\end{array}$ & Date & $\begin{array}{l}\text { Water } \\
\text { level }\end{array}$ & Date & $\begin{array}{c}\text { Water } \\
\text { level }\end{array}$ \\
\hline an. 31,1956 & j141. 22 & Feb. 25,1957 & $\mathrm{j} 146.70$ & Apr. 29,1958 & j141. 77 & Dec. 30,1959 & $\overline{\mathrm{j} 152.36}$ \\
\hline Feb. 29 & $\mathrm{j} 141.76$ & Mar. 18 & j145. 58 & May 27 & j138. 26 & Jan. & \\
\hline Mar. 29 & j140. 80 & Apr. 29 & $\mathrm{j} 144.53$ & June 23 & 68 & Feb. 24 & j139. 63 \\
\hline Apr. 26 & $\mathrm{j} 140.17$ & May 31 & j143. 50 & July 31 & j136. 95 & Mar. 29 & $\mathbf{j} 137.68$ \\
\hline May 31 & $\mathrm{j} 138.40$ & July 24 & j1 145.46 & Aug. 27 & 50 & May & 23 \\
\hline June 29 & c่ 138.38 & Aug. 29 & cj147. 68 & Sept. 25 & j137. 53 & 27 & $\mathrm{j} 136.51$ \\
\hline July 23 & $\mathrm{j} 139.80$ & Oct. & git & Oct. 30 & j1: & June 23 & 95 \\
\hline Aug. 25 & j140.77 & 28 & j148. 81 & Nov. 28 & j141. 67 & Aug. 29 & 36.12 \\
\hline Sept. 25 & 01 & Nov. 25 & 83 & Feb. 25,1959 & 72 & Sept. 30 & 43 \\
\hline Det. 22 & 39 & Dec. 31 & .18 & Apr. 30 & j136. 42 & Oct. 24 & 8. 21 \\
\hline Tov. 30 & cj1 14 & 3,1958 & j1 & May & 92 & Nov. 25 & .28 \\
\hline ec. 31 & cj1 & 28 & .99 & Sept. 25 & cis6. 80 & Dec. 27 & j135. 32 \\
\hline an. 28,1957 & j1 147.16 & Mar. 28 & j143. 48 & & & & \\
\hline
\end{tabular}

c Nearby well being pumped.

j Water level below sea level.

4/25-29L1. A. P. Salzgeber. Drilled unused artesian well in alluvium, diam 2 in, depth $110 \mathrm{ft}$. Lsd about $18 \mathrm{ft}$ above msl. MP top of casing, $1.60 \mathrm{ft}$ above lsd. Highest water level 7.43 below 1sd, May 27, 1958; lowest 51.24 below lsd, Sept. 25, 1951. Records available: $1950-60$.

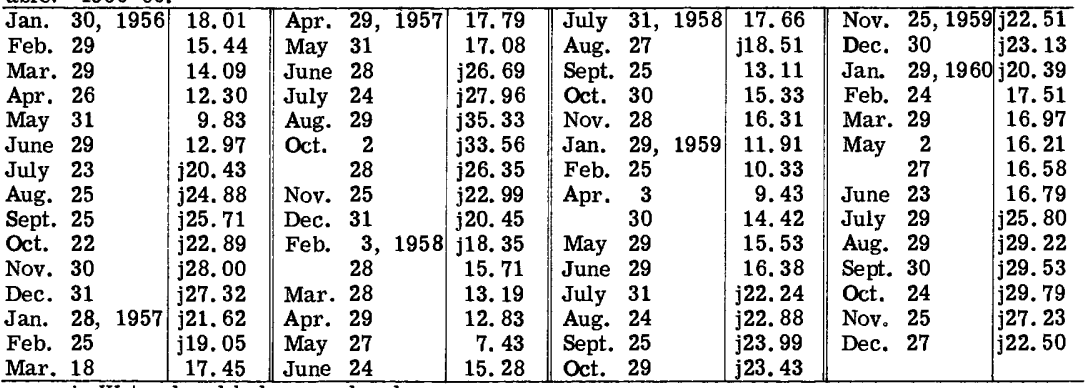

$\mathrm{j}$ Water level below sea level.

Goleta Basin

4/28-9E1. A. T. Spaulding. Fairview and Encina Rds. Drilled domestic artesian well in Santa Barbara Formation of early Pleistocene age, diam 12 in, depth $310 \mathrm{ft}$. Lsd $43.58 \mathrm{ft}$ above msl. MP top north side of casing, $0.20 \mathrm{ft}$ above lsd. Highest water level 27.64 below Isd, June 7, 1941; lowest 78.66 below lsd, Oct. 29, 1954. Records available: 1941, 1943-60.

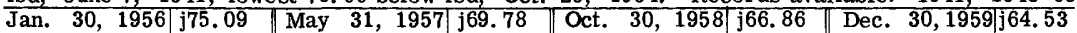

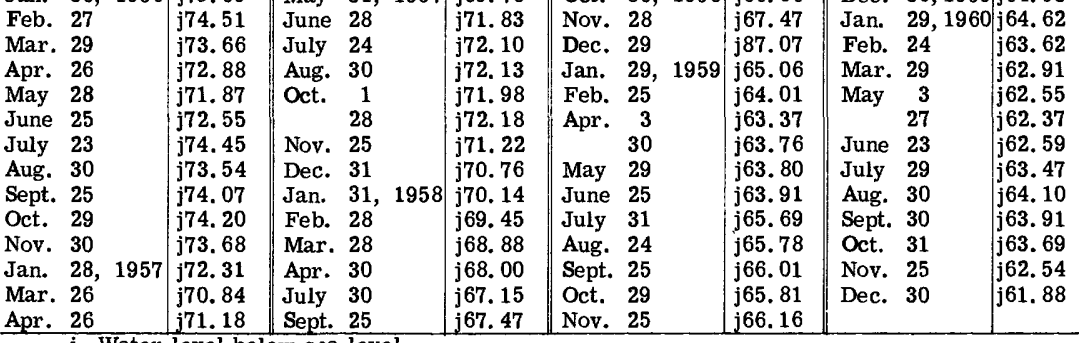

Water level below sea level.

4/28-10F1. J. S. Edwards. Patterson Ave. and Maria Ygnacio Creek. Drilled unused (previously reported domestic and irrigation) artesian well in Santa Barbara Formation of early Pleistocene age, diam 12 in, depth $459 \mathrm{ft}$, cased to 459, perforated 72-198, 312-459. Lsd $79.90 \mathrm{ft}$ above msl. MP top of hole in southeast side of pumpbase, $0.70 \mathrm{ft}$ above lsd. Highest water level 56. 44 below lsd, Apr. 28, 1943; lowest 103.27 below lsd, July 24, 1956. Records available: $1932-33,1937-38,1941-60$. 
4/28-10F1--Continued.

\begin{tabular}{|c|c|c|c|c|c|c|c|}
\hline Date & $\begin{array}{c}\text { Water } \\
\text { level }\end{array}$ & Date & $\begin{array}{c}\text { Water } \\
\text { level }\end{array}$ & Date & $\begin{array}{c}\text { Water } \\
\text { level }\end{array}$ & Date & $\begin{array}{c}\text { Water } \\
\text { level }\end{array}$ \\
\hline Jan. 30,1956 & $\mathrm{j} 80.34$ & May 31,1957 & 77.98 & Aug. 29,1958 & 73.05 & Dec. 30,1959 & $7 \overline{2.63}$ \\
\hline Feb. 27 & j79.95 & July 24 & 79.33 & Sept. 25 & 73.51 & Jan. 29,1960 & 72.96 \\
\hline Mar. 29 & 79.03 & Aug. 30 & 79.27 & Nov. 28 & 71.28 & Feb. 24 & 73.15 \\
\hline Apr. 26 & j87. 67 & Oct. & 79.03 & Dec. 29 & 70.38 & Mar. 29 & 72.88 \\
\hline May 28 & j89. 40 & 28 & 79.25 & Feb. 25, 1959 & 70.94 & May & 77.05 \\
\hline June 25 & j93, 18 & Nov. 25 & 78.45 & Apr. 3 & 69.76 & 27 & 76.87 \\
\hline July 24 & $\mathrm{j} 103.27$ & Dec. 31 & 78.05 & May 29 & 71.08 & June 23 & 77.90 \\
\hline Sept. 25 & $j 102.41$ & Jan. 31,1958 & 79.06 & June 25 & 71.10 & July 29 & 77.80 \\
\hline Nov. 30 & $j 102.97$ & Feb. 28 & 77.47 & July $\mathbf{3 1}$ & 71.38 & Aug. 30 & 74.33 \\
\hline Dec. 31 & j101. 81 & Mar. 28 & 77.81 & Aug. 24 & 71.68 & Sept. 30 & 74.31 \\
\hline Jan. 28, 1957 & j98. 07 & Apr. 30 & 76.15 & Sept. 25 & 72.13 & Oct. 31 & 74.44 \\
\hline Feb. 25 & j88. 80 & May 26 & 76.20 & Oct. 29 & 72.00 & Nov. 25 & 73.97 \\
\hline Mar. 25 & j85. 56 & June 24 & 76.31 & Nov. 25 & 71.91 & Dec. & 73.71 \\
\hline Apr. 26 & j85. 50 & July 30 & 72.55 & & & & \\
\hline
\end{tabular}

4/29-14A3. Ben Romer. Formerly Frank Baker. Glen Annie Rd. and Southern Pacific RR. Drilled domestic and irrigation water-table well in Santa Barbara Formation of early Pleistocene age, diam $12 \mathrm{in}$, depth $126 \mathrm{ft}$. Lsd about $51 \mathrm{ft}$ above msl. MP edge of hole in casing cover, $1.00 \mathrm{ft}$ above 1sd. Highest water level 51.02 below 1sd, Dec. 30, 1960; lowest 87. 46 below lsd, July 30, 1951. Records available: 1941-58, 1960. No measurement made in 1959. Jan. 30, 1956 j68.65

Feb. $27 \quad$ j68.45

Mar. 29

Apr. 26

May 28

June 25

July 24

Aug. 30

Oct. 29

Nov. 30

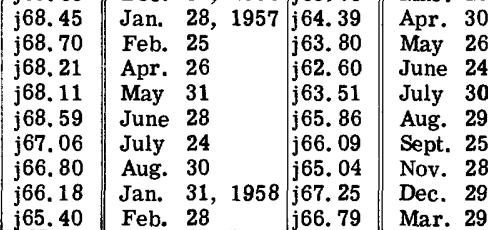

j60. 19

j60.20 June 23 j51.99

j60.42 July $29 \quad$ j52.00

j61.12 Aug. 30 j51. 74

j61.31 Sept. $30 \quad$ j51.39

j61.99 Oct. 31 j51.52

j56.99 Nov. 25 j52. 26

j56.41 Dec. $30 \quad 51.02$

$\mathrm{j}$ Water level below sea level.

\section{Upper Santa Ynez Valley}

6/30-9N1. San Lucas Ranch. Near Santa Ynez. Drilled stock water-table well in Paso Robles (?) Formation of Pliocene and Pleistocene (?) age, diam $8 \mathrm{in}$, depth $160 \mathrm{ft}$. Lsd $660.01 \mathrm{ft}$ above $\mathrm{msl}$ (previously reported about $653 \mathrm{ft}$ above msl). MP top north side of casing collar, $1.10 \mathrm{ft}$ above 1sd. Highest water level 24. 06 below lsd, July 27, 1960; lowest 41.54 below 1sd, Jan. 30, 1956. Records available: 1941-60.

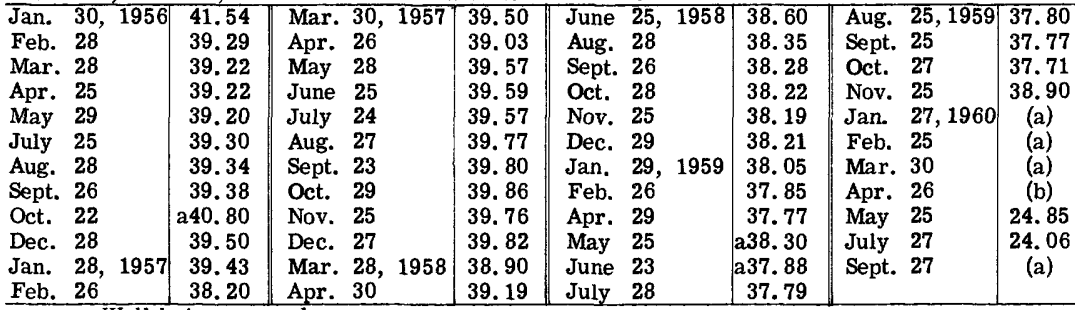

\section{a Well being pumped.}

b Well pumped recently.

6/30-29E1. Rancho Juan y Lolita. Near Santa Ynez. Drilled unused water-table well in alluvium, diam $10 \mathrm{in}$, depth $52 \mathrm{ft}$. Lsd $465.02 \mathrm{ft}$ above msl (previously reported about $461 \mathrm{ft}$ above msl). MP top of recorder shelf, $0.41 \mathrm{ft}$ above $1 \mathrm{sd}$. Highest water level 7.90 below $1 \mathrm{sd}$, Mar. 10, 1941; lowest 24.00 below 1sd, May 20-21, 1951. Records available: 1933-60.

Water level at noon, from recorder graph, 1956

\begin{tabular}{l|l|l|l|l|l|l|l|l|l|l|l|l}
\hline Day & Jan. & Feb. & Mar. & Apr. & May & June & July & Aug. & Sept. & Oct. & Nov. & Dec. \\
\hline 5 & 22.92 & 21.20 & 17.08 & 17.33 & $\mathbf{1 3 . 6 7}$ & $\mathbf{1 4 . 1 8}$ & 14.75 & 18.40 & 21.06 & $\mathbf{2 1 . 4 9}$ & $\mathbf{2 1 . 8 6}$ & $\ldots .3$ \\
10 & 22.95 & 19.80 & 16.94 & 17.42 & $\mathbf{1 3 . 8 4}$ & 13.55 & $\mathbf{1 5 . 0 5}$ & 18.98 & $\mathbf{2 1 . 1 7}$ & $\mathbf{2 1 . 5 6}$ & $\mathbf{2 1 . 9 5}$ & $\mathbf{2 2 . 3 0}$ \\
15 & 22.96 & 18.90 & 16.90 & 16.80 & 13.71 & 13.31 & 15.82 & 19.30 & $\mathbf{2 1 . 0 5}$ & $\mathbf{2 1 . 6 7}$ & $\mathbf{2 2 . 0 4}$ & $\mathbf{2 2 . 3 5}$ \\
20 & 22.92 & 18.16 & 16.98 & 15.74 & 13.87 & 13.35 & 16.46 & 19.78 & 21.20 & $\mathbf{2 1 . 7 5}$ & 22.10 & 22.40 \\
25 & 22.45 & 17.61 & 17.12 & 14.52 & 14.11 & 13.76 & 16.57 & 20.26 & 21.32 & 21.75 & $\mathbf{2 2 . 2}$ & $\mathbf{2 2 . 4 5}$ \\
Eom & 21.82 & 17.31 & 17.25 & 13.80 & 14.60 & 14.26 & 17.65 & 20.77 & $\mathbf{2 1 . 4 2}$ & $\mathbf{2 1 . 8 0}$ & $\mathbf{2 2 . 2 6}$ & $\mathbf{2 2 . 4 8}$ \\
\hline
\end{tabular}


6/30-29E1-- Continued.

1957

\begin{tabular}{|c|c|c|c|c|c|c|c|c|c|c|c|c|}
\hline Day & Jan. & Feb. & Mar. & Apr. & May & June & July & Aug. & Sept. & Oct. & Nov. & Dec. \\
\hline 5 & 22.51 & 22.62 & 22.43 & 22.07 & 21.85 & & 22.35 & 22.90 & 23.38 & 23.56 & 17.81 & 19.34 \\
\hline 10 & 22.56 & 22.59 & 22.40 & 22.07 & 21.80 & 21.80 & 22. 47 & 23.02 & 23.45 & 19.20 & & \\
\hline 15 & 22.56 & 22.55 & $\cdots$ & 22.06 & 21. 75 & 21.93 & 22.57 & 23.03 & 23.52 & 17.70 & 18.43 & 19.73 \\
\hline 20 & 22.63 & 22.56 & & 22.02 & 21.70 & 22.04 & 22.63 & 23.11 & 23.58 & 17. 17 & 18.77 & 19.61 \\
\hline 25 & 22.62 & .... & 22. 17 & 21.95 & 21.68 & 22.14 & 22.70 & 23.21 & 23. 67 & 17.13 & 18.93 & 18.80 \\
\hline Eom & 22.62 & & 22.09 & 21.93 & 21. 71 & 22.24 & 22. 82 & 23. 31 & 23.66 & 17.51 & 19.16 & 17.82 \\
\hline \multicolumn{13}{|c|}{1958} \\
\hline 5 & 17.47 & & 14.44 & 11.36 & 11.05 & 11.95 & 12.53 & 12.83 & 14.49 & 15.17 & 17.10 & \\
\hline 10 & 17.27 & 15.63 & 14.72 & $\cdots$ & 11.26 & 12.01 & 12.73 & 13.10 & $\cdots \cdots$ & 15.53 & 17. 14 & \\
\hline 15 & 17. 20 & & 14.94 & 10.31 & 11.48 & 12. 05 & 12.87 & 13.30 & & 16.13 & 17.02 & 15.31 \\
\hline 20 & & 15.20 & 14. 82 & 10.23 & 11.64 & 12.13 & 12.93 & 13.38 & & 16.56 & 16. 66 & $\cdots$ \\
\hline 25 & 17. 29 & 14.64 & 14.00 & 10.53 & 11.72 & 12. 21 & 13.00 & 13.29 & 14. 96 & 16. 79 & 16. 31 & $\ldots$ \\
\hline Eom & 17.02 & 14. 58 & 13.38 & 10.78 & 11.79 & & 12. 75 & 13.94 & 15.18 & 16.92 & 16.03 & \\
\hline \multicolumn{13}{|c|}{1959} \\
\hline 5 & & & 12.96 & 13.71 & 13,36 & 14.45 & 16.50 & 19.15 & 20.76 & 21.55 & & \\
\hline 10 & & & 12. 93 & 14.06 & 13.49 & 15.05 & 16.90 & 19.69 & 20.89 & • & $\cdots$ & $\cdots$ \\
\hline 15 & & 14.45 & 13.13 & 13.90 & 13. 80 & 15.47 & & 19.90 & 20.92 & $\ldots \ldots$ & . & \\
\hline 20 & & 14.01 & & 13.43 & 13. 88 & 15.97 & 17. 68 & 19.79 & 21.25 & & & 22.73 \\
\hline 25 & ..... & 13.57 & 13.44 & 13.24 & 13. 75 & 16. 37 & 18.05 & 20.08 & 21.40 & $\cdots \cdots$ & $\cdots$ & 22.79 \\
\hline Eon & & 13.25 & 13.48 & 13. & 13. 96 & 16.09 & 18.86 & 20.52 & 21. 51 & & & 22. 84 \\
\hline \multicolumn{13}{|c|}{1960} \\
\hline 5 & 22.90 & 22.92 & 17.75 & 14.11 & 14.08 & . & 16.22 & 18.68 & ..... & 121.31 & • & \\
\hline 10 & 22. & 22.86 & 15.91 & 13.94 & 13.85 & & 16.60 & 19.32 & $\cdots$ & $\cdots \cdots$ & $\cdots$ & $\cdots$ \\
\hline 15 & & 22.73 & 15. 32 & 13.70 & 13. 64 & 14. 66 & 17. 53 & 19.75 & $\cdots \cdots$ & $\cdots \cdots$ & $\cdots$ & \\
\hline 20 & 22. 95 & 21.71 & 14.81 & 13.51 & 13. 62 & 15.15 & 18.04 & 20.09 & .. & $\ldots \ldots$ & $\cdots$ & $\cdots$ \\
\hline 25 & 22.95 & 18.91 & 14.40 & 13. 67 & 13. 79 & 15.16 & 18. 39 & 20.41 & . & $\ldots$ & & .. \\
\hline Eom & 22.94 & 17.95 & 14. 12 & 13.91 & 13.78 & 15.65 & 17.93 & $\ldots$ & $\ldots$ & $\ldots \ldots$ & - & $\ldots$ \\
\hline
\end{tabular}

6/32-9A1. Owen E. Hollister. Near Buellton. Drilled domestic water-table well in alluvium, diam $8 \mathrm{in}$, depth $58 \mathrm{ft}$. Lsd $307.67 \mathrm{ft}$ above msl (previously reported $309.33 \mathrm{ft}$ above msl). MP bottom edge west side of pumpbase, $0.90 \mathrm{ft}$ above lsd. Highest water level 26. 20 below 1sd, Jan. 21, 1942; lowest 39. 55 below 1sd, July 10, 1956. Records available: 1943-58. Measurement discontinued.

\begin{tabular}{|c|c|c|c|c|c|c|c|}
\hline Date & $\begin{array}{c}\text { Water } \\
\text { level }\end{array}$ & Date & $\begin{array}{c}\text { Water } \\
\text { level }\end{array}$ & Date & $\begin{array}{c}\text { Water } \\
\text { level }\end{array}$ & Date & $\begin{array}{c}\text { Water } \\
\text { level }\end{array}$ \\
\hline Jan. 26, 1956 & 32.83 & Aug. 28, 1956 & 32.90 & Apr. 26, 1957 & 33.84 & Jan. 30,1958 & 33.67 \\
\hline Feb. 28 & $\begin{array}{l}32.50 \\
33\end{array}$ & Sept. 26 & b43. 90 & June 25 & $\begin{array}{l}34.20 \\
36.75\end{array}$ & June 25 & 33. 23 \\
\hline Apr. 25 & 34.60 & Nov. 27 & 33.80 & Aug. 27 & 37.23 & Sept. 26 & 35.58 \\
\hline July 10 & 39.55 & Dec. 19 & 33.54 & Sept. 4 & 37.20 & Oct. 28 & 34.53 \\
\hline Aug. & 35.63 & Feb. 26,1957 & 32.81 & Oct. 29 & 34.39 & & \\
\hline
\end{tabular}

b Well pumped recently.

6/33-9P1. Hollister Estate. Near Lompoc. Drilled unused water-table well in alluvium, diam $16 \mathrm{in}$, depth $83 \mathrm{ft}$. Lsd $203.03 \mathrm{ft}$ above msl (previously reported about $200 \mathrm{ft}$ above msl). MP top hole at center on south side of steel cover, at lsd. Highest water level 21.80 below lsd, Apr. 3, 1941; lowest 54.61 below Isd, Nov. 30, 1950. Records available: 1932-60.

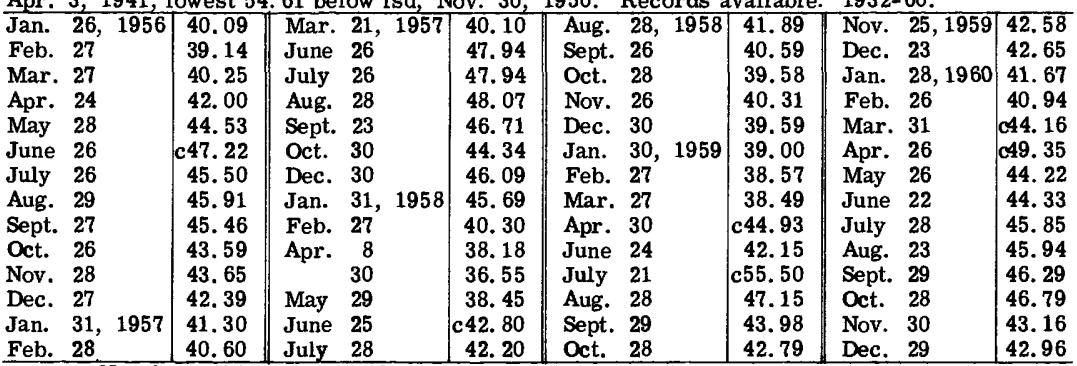

c Nearby well being pumped. 


\section{Lower Santa Ynez Valley}

7/31-36L2. D. B. Kilbourne. Near Ballard. Baseline and Grand (Refugio) Aves. Drilled domestic and irrigation water-table well in Paso Robles Formation, diam 12 in, depth $230 \mathrm{ft}$. Lsd $720.62 \mathrm{ft}$ above msl (previously reported about $715 \mathrm{ft}$ above msl). MP bottom of notch in northwest side of casing, $0.82 \mathrm{ft}$ above lsd. Highest water level 16.54 below lsd, Apr. 7, 1943; lowest 91.31 below lsd, Aug. 23, 1960 . Records available: 1942-60.

\begin{tabular}{|c|c|c|c|c|c|c|c|}
\hline Date & $\begin{array}{l}\text { Water } \\
\text { level }\end{array}$ & Date & $\begin{array}{l}\text { Water } \\
\text { level }\end{array}$ & Date & $\begin{array}{l}\text { Water } \\
\text { level }\end{array}$ & Date & \begin{tabular}{|l} 
Water \\
level
\end{tabular} \\
\hline Ian. 30,1956 & 54.02 & May 28,1957 & 64.42 & Aug. 28,1958 & 77.42 & Jan. 27,1960 & 72.36 \\
\hline Mar. 27 & 61.16 & June 26 & 73.66 & Sept. 26 & 79 & Feb. 25 & 69.93 \\
\hline Apr. 25 & 61.43 & July 24 & 75.35 & Oct. 28 & 76.83 & Mar. 30 & (a) \\
\hline May 29 & 63.38 & Aug. 27 & 80.85 & Nov. 25 & 73.18 & Apr. 26 & (a) \\
\hline June 25 & 61.86 & Sept. 23 & b76.93 & Dec. 29 & 74.70 & May 25 & 688.38 \\
\hline Aug. 28 & 66.60 & Oct. 29 & 74.38 & Jan. 29,1959 & 68.81 & June 21 & (a) \\
\hline Bept. 26 & 68.08 & Dec. 27 & 69.76 & Feb. 26 & 68.20 & July 26 & 90.03 \\
\hline Oct. 22 & 76.57 & Jan. 30,1958 & 67.67 & Apr. 29 & 72.07 & Aug. 23 & 91.31 \\
\hline Nov. 27 & 69.38 & Feb. 27 & 65.72 & May 25 & 75.37 & Sept. 27 & (a) \\
\hline Dec. 28 & 70.58 & Mar. 28 & 63.75 & June 23 & 77.22 & Oct. 28 & (a) \\
\hline Jan. 29, & 62.84 & Apr. 14 & 62.06 & July 28 & 78.07 & Nov. 30 & 81.65 \\
\hline $\begin{array}{l}\text { Mar. } 20 \\
\text { Apr. } 26\end{array}$ & $\begin{array}{l}60.05 \\
62.32\end{array}$ & $\begin{array}{ll}\text { May } & 28 \\
\text { July } & 28\end{array}$ & $\begin{array}{l}63.08 \\
71.50\end{array}$ & Nov. 25 & & Dec. 29 & (a) \\
\hline 40 & & & & & & & \\
\hline
\end{tabular}

a Well being pumped.

b Well pumped recently.

7/34-12E1. U.S. Geol. Survey. Union Oil Co., Purisima Lease. Near Lompoc. Drilled observation water-table well in Careaga Sand of Canfield (1939), diam 8 to 16 in, depth $385 \mathrm{ft}$, cased to 385, perforated 145-385 (previously reported 345-385). Lsd $385.83 \mathrm{ft}$ above msl. MP top of recorder shelf, $2.07 \mathrm{ft}$ above lsd. Highest water level 301.70 below $1 \mathrm{sd}$, June 25, 1949; lowest 306.54 below lsd, Aug. 9, 1960. Records available: 1949-60.

Highest water level for the day, from recorder graph, 1956

\begin{tabular}{|c|c|c|c|c|c|c|c|c|c|c|c|c|}
\hline Day & Jan. & Feb. & $\mathbf{M a}$ & Apr & May & June & July & Aug & Sept. & Oct. & Nov. & Dec. \\
\hline 5 & & 304.41 & 304.54 & 304.62 & & & $-\frac{1}{2}$ & 304.85 & 304.8 & 304.90 & 304.94 & 304.77 \\
\hline 10 & 304.57 & 304.65 & 304 & 304.50 & 304.70 & & $\cdots \cdot$ & 304.73 & ग & 8 & r & 04.95 \\
\hline 15 & 304.48 & 30 & & $\mid 304.77$ & 304.68 & & - & 304.81 & 79 & 87 & 304.90 & 304.77 \\
\hline 20 & 304.37 & 30 & & 304.69 & .65 & & 304.8 & 3 & & 93 & & 304.94 \\
\hline 25 & 304.42 & 55 & 3 & 304,60 & 304.52 & & 304. 67 & $3 c$ & & 90 & 304.95 & 305.10 \\
\hline Eom & 30 & & & 30 & 304.72 & & 304 & & & & 8 & 304.80 \\
\hline
\end{tabular}

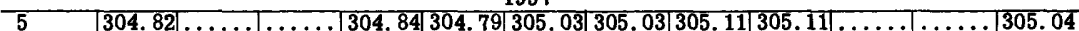

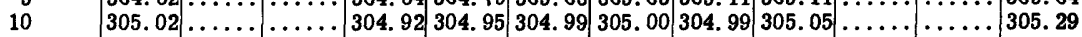

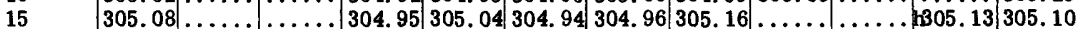

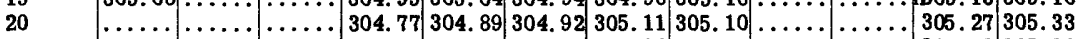

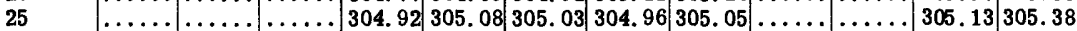

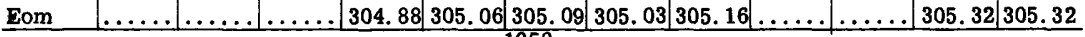

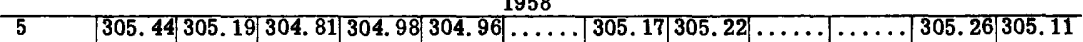

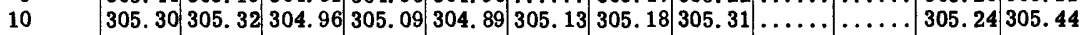

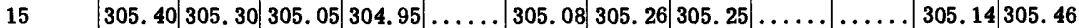

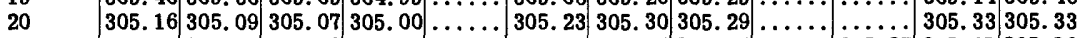

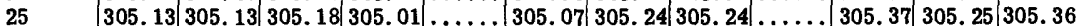

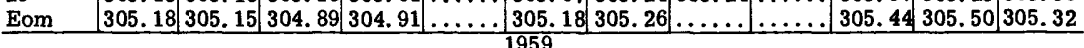

\begin{tabular}{l|l|l|l|l|l|l|l|l|l|l|l|l|}
\hline 5 & 304.84 & 305.18 & 305.27 & 305.21 & 305.46 & 305.34 & 305.47 & 305.33 & $\ldots \ldots$ & 305.73 & 305.78 & 305.79 \\
\hline
\end{tabular}

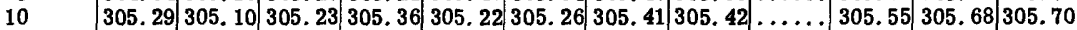

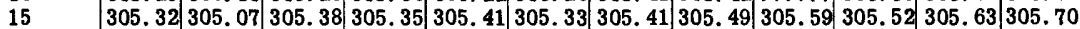

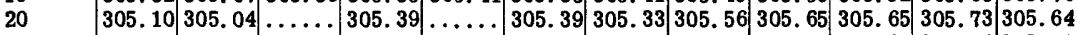
\begin{tabular}{l|l|l|l|l|l|l|l|l|l|l|l|l|l}
25 & 305.39 & 305.33 & 305.40 & 305.33 & $\ldots \ldots \ldots$ & 305.34 & 305.32 & 305.55 & 305.58 & 305.62 & 305.54 & 305.53
\end{tabular} \begin{tabular}{l|l|l|l|l|l|l|l|l|l|l|l|l|l|} 
Eom & 305.34 & 305.31 & 305.31 & 305.22 & 305.39 & 305.46 & 305.38 & $\ldots \ldots$ & 305.35 & 305.71 & 305.73 & 305.67 \\
\hline
\end{tabular}

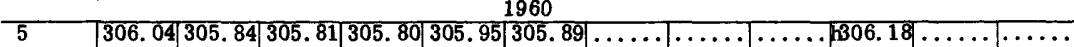

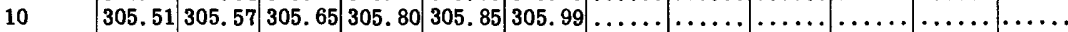

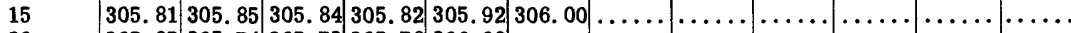

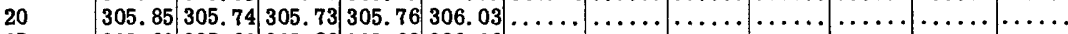
\begin{tabular}{l|l|l|l|l|l|l|l|l|l|l|l|l|}
25 & 305.69 & 305.60 & 305.80 & 305.80 & 306.06 & $\ldots \ldots$ & $\ldots \ldots$ & $\ldots \ldots \ldots$ & $\ldots \ldots$ & $\ldots \ldots \ldots$ & $\ldots \ldots \ldots$ & $\ldots \ldots \ldots$ \\
\hline
\end{tabular}

h Tape measurement.

7/34-28H2. T. M. Parks. Near Lompoc. Central Ave. and H St. Drilled unused artesian well in alluvium, diam 6 in, depth $78 \mathrm{ft}$. Lsd $89.55 \mathrm{ft}$ above msl. MP top of hole in board, $0.57 \mathrm{ft}$ above lsd. Highest water level 21. 74 below lsd, Mar. 10, 1943; lowest 43.14 below lsd, May 28, 1951. Records available: 1930-39, 1942-60. 
7/34-28H2-- Continued.

\begin{tabular}{|c|c|c|c|c|c|c|c|}
\hline Date & $\begin{array}{l}\text { Water } \\
\text { level }\end{array}$ & Date & $\begin{array}{l}\text { Water } \\
\text { level }\end{array}$ & Date & $\begin{array}{l}\text { Water } \\
\text { level }\end{array}$ & Date & $\begin{array}{l}\text { Water } \\
\text { level }\end{array}$ \\
\hline Jan. 26,1956 & 29.78 & Apr. 30,1957 & 36.15 & Sept. 29,1958 & c32. 23 & Nov. 25,1959 & 36. 86 \\
\hline Feb. 27 & 29.13 & May 29 & 33.34 & Oct. 28 & c31.31 & Dec. 22 & c35. 60 \\
\hline Mar. 27 & 32.43 & June 26 & 37.55 & Nov. 25 & c30. 75 & Jan. 27, 1960 & c34. 11 \\
\hline Apr. 24 & 34.88 & Aug. 28 & 37.33 & Dec. 30 & 31.31 & Feb. 25 & 33.79 \\
\hline May 28 & 33.73 & Sept. 27 & 38.11 & Jan. 29,1959 & 30.40 & Mar. 30 & c38.56 \\
\hline June 26 & c37.50 & Oct. 30 & 35.86 & Feb. 26 & 28.27 & Apr. 26 & 40.15 \\
\hline uly 26 & 35.07 & Nov. 26 & 34.72 & Mar. 26 & 34.77 & May 25 & 39.23 \\
\hline Aug. 29 & 32.20 & Dec. 30 & 33.87 & Apr. 29 & 34.27 & June 21 & 041.58 \\
\hline ept. 27 & 31.62 & Jan. 31,1958 & 33.25 & May 26 & $c 33.73$ & July 27 & 043.16 \\
\hline Oct. 26 & 31.37 & Feb. 28 & 31.68 & June 23 & c34.95 & Aug. 23 & c42. 31 \\
\hline Nov. 30 & 32.09 & Apr. 8 & 28. 27 & July 28 & $\mathrm{c} 35.54$ & Sept. 27 & 040.13 \\
\hline an. 31,1957 & 30.81 & 28 & 27.32 & Aug. 25 & c36.50 & Oct. 28 & 040.04 \\
\hline eb. 27 & 30.29 & June 24 & 29.06 & Sept. 25 & c36. 88 & Nov. 29 & 37.82 \\
\hline ar. 21 & 32.46 & Aug. 29 & 27.12 & Oct. 27 & c37.39 & Dec. 29 & 38.34 \\
\hline
\end{tabular}

c Nearby well being pumped.

7/34-34H1. Johns-Manville Corp. Lompoc. Pine Ave. and First St. Drilled unused (previously reported irrigation) water-table well in alluvium, diam $12 \mathrm{in,} \mathrm{depth} 160 \mathrm{ft}$, perforated 118-156. Lsd $112.10 \mathrm{ft}$ above msl. MP hole in floor of recorder shelter, $0.40 \mathrm{ft}$ above lsd. Highest water level 33.46 below Isd, May 8, 1941; lowest 56.71 below lsd, July 26, 1951. Records available: 1941-59. Recorder removed Mar. 14, 1959. Mar. 27, 1959, 40.08; Apr. 29, 44.27. Well destroyed; measurement discontinued.

Highest water level for the day, from recorder graph, 1956

\begin{tabular}{|c|c|c|c|c|c|c|c|c|c|c|c|c|}
\hline Day & Jan. & Feb. & Mar. & Apr. & May & June & July & Aug. & Sept. & Oct. & Nov. & Dec. \\
\hline 5 & 44.54 & 41.42 & 40.22 & 41.80 & 41.00 & 40.70 & 41.87 & 43.50 & 44.05 & 44.45 & 44.75 & 45.19 \\
\hline 10 & 43.88 & 41.16 & 40.37 & 41.62 & 40.73 & 41.20 & 42.14 & 43.36 & 44.10 & 44.50 & 44.95 & 45.27 \\
\hline 15 & 43.22 & 40.95 & 40.65 & 41.33 & 40.74 & 41.97 & 42.46 & 43.60 & 44. 18 & 44.55 & 45.03 & \\
\hline 20 & 42.79 & 40.72 & 41.10 & 41.17 & 40.58 & 42.23 & 42.85 & 43.98 & 44. 38 & 44.60 & 45.09 & 45.61 \\
\hline 25 & 42.28 & 40.51 & 41.10 & 41.31 & 40.68 & $\ldots \ldots$ & $\ldots \ldots$ & 43 & 44.59 & 44.75 & 45.09 & \\
\hline Eom & 41.84 & 40.34 & 41.67 & 40.96 & 40.45 & & 43.16 & 44.10 & 44 & 44.73 & 45.15 & 45.67 \\
\hline \multicolumn{13}{|c|}{1957} \\
\hline 5 & 45.50 & 45.04 & 43.35 & 42.74 & 44.44 & 45.78 & 47.15 & 48.90 & 49.98 & 50.17 & 50.64 & 50.22 \\
\hline 10 & 45.49 & 44.92 & 42.79 & 43.15 & 44.86 & 45.84 & 47.54 & 49.08 & 50.00 & 50.32 & 50.27 & .05 \\
\hline 15 & 45.50 & 44.74 & 42.32 & 43.65 & 44.86 & 45.90 & 47.76 & h52.03 & 50.02 & 50.08 & h50.17 & 49.93 \\
\hline 20 & 45.45 & 44.65 & 41.93 & 43.78 & 44.88 & 46.29 & 48.16 & 49.74 & 50.08 & 50.12 & 50.13 & 49.86 \\
\hline 25 & 45.42 & 44.30 & 41.84 & 43.89 & 45.17 & 46.48 & 48.42 & 49.91 & 50.05 & 50.28 & 50.45 & 49.77 \\
\hline Eom & 45.20 & 43.95 & 41.96 & 43.98 & 45.36 & 47.01 & 48.73 & 50.12 & 50.17 & 50.28 & 50.40 & 49.69 \\
\hline \multicolumn{13}{|c|}{1958} \\
\hline 5 & 49.68 & & 43.15 & & & 40.04 & 42.33 & & 42.44 & 42.55 & 42.96 & $\overline{43.43}$ \\
\hline 10 & 49. & 45.68 & & & 39.97 & 40.07 & . & 41.75 & 42.13 & 42.67 & 43.04 & 43.45 \\
\hline 15 & .. & 45.37 & . & $\cdots$ & 40.10 & 40.07 & & 42.02 & 42.10 & 42.75 & 43.02 & 43.49 \\
\hline 20 & $\ldots \ldots$ & 44.53 & .... & . & 40.19 & 40.27 & & 42.13 & 42.24 & 42.86 & 43.47 & 43.37 \\
\hline 25 & & 43.88 & $\cdots$ & $\ldots$ & 40.04 & 40.47 & 43.52 & 42.05 & $\cdots$ & 42.87 & 43.65 & 43.00 \\
\hline Eom & & & & & 40.22 & 40.63 & 41.30 & 42.16 & 42.29 & 42.86 & 43.38 & 42.65 \\
\hline \multicolumn{13}{|c|}{1959} \\
\hline 5 & 42.17 & 40.34 & 39.37 & $\cdots \cdots$ & $\cdots$ & $\cdot$ & $\cdots \cdots$ & ... & $\cdots \cdots$ & $\ldots \ldots$ & $\ldots \ldots$ & • \\
\hline 10 & 41.65 & 40.17 & 39.39 & 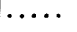 & . & $\ldots \ldots$ & $\ldots \ldots$ & $\cdots \cdots$ & $\cdots$ & $\cdots \cdots$ & $\ldots \ldots$ & $\cdots$ \\
\hline 15 & 41.32 & 39.96 & - & $\cdots$ & - & $\cdots \cdots$ & $\cdots$ & . & $\cdots$ & $\cdots \cdots$ & $\cdots$ & $\cdots$ \\
\hline 20 & 40.96 & 39.73 & & & & & & & & & & \\
\hline $\begin{array}{l}25 \\
\text { Eom }\end{array}$ & $\begin{array}{l}40.84 \\
40.53\end{array}$ & $\begin{array}{l}39.56 \\
39.46\end{array}$ & & $\begin{array}{l}\cdots \cdots \\
\ldots \ldots\end{array}$ & & $\cdots$ & $\cdots$ & ..... & $\cdots \cdots$ & * & & \\
\hline & \\
\hline
\end{tabular}

7/35-23J2. Union Sugar Co. Near Lompoc. Central and Artesia Aves. Drilled irrigation artesian well in alluvium, diam $12 \mathrm{in}$, depth $158 \mathrm{ft}$. Lsd $43.58 \mathrm{ft}$ above msl (previously reported $43.93 \mathrm{ft}$ above $\mathrm{msl}$ ). MP top of hole in north side of pumpbase, $1.00 \mathrm{ft}$ above $1 \mathrm{sd}$. Highest water level 11. 40 below 1sd, Apr. 8, 1958; lowest 31.11 below Isd, July 27, 1950 (previously reported 29.92 below 1sd, Aug. 26, 1948). Records available: 1947-60.

\begin{tabular}{|c|c|c|c|c|c|c|c|}
\hline Date & $\begin{array}{l}\text { Water } \\
\text { level }\end{array}$ & Date & $\begin{array}{l}\text { Water } \\
\text { level }\end{array}$ & Date & $\begin{array}{l}\text { Water } \\
\text { level }\end{array}$ & Dat & $\begin{array}{l}\text { Water } \\
\text { level }\end{array}$ \\
\hline Jan. 30,1956 & 13.24 & Jan. 21,1957 & 14.26 & Jan. 31,1958 & 16.90 & Sept. 29,1958 & $17 . \overline{72}$ \\
\hline eb. 27 & 13 & Mar. 21 & 16. & Feb. 28 & 13 & Oct. 29 & 99 \\
\hline ar. 27 & 16.36 & Apr. 30 & 23.55 & Apr. & 11.40 & Jan. 30,1959 & 14.22 \\
\hline aly 26 & 19.90 & May 29 & 19.13 & 28 & 18.20 & Feb. 27 & 13.17 \\
\hline ept. 27 & 17 & July 26 & b28. 43 & May 29 & 21.16 & June 24 & 69 \\
\hline ct. 26 & 18.28 & Sept. 27 & 18.62 & July 29 & 18.84 & Sept. 29 & 19.37 \\
\hline ov. 30 & 16.05 & Nov. 26 & 18.37 & Aug. 29 & 16.17 & Jan. 28,1960 & 16.27 \\
\hline
\end{tabular}


7/35-23J2--Continued.

\begin{tabular}{|c|c|c|c|c|c|c|c|}
\hline Date & $\begin{array}{l}\text { Water } \\
\text { level }\end{array}$ & Date & $\begin{array}{l}\text { Water } \\
\text { level }\end{array}$ & Date & $\begin{array}{l}\text { Water } \\
\text { level }\end{array}$ & Date & $\begin{array}{l}\text { Water } \\
\text { level }\end{array}$ \\
\hline $\begin{array}{l}\text { Feb. } 26,1960 \\
\text { Mar. 31 } \\
\text { Apr. }\end{array}$ & $\begin{array}{c}15.28 \\
\text { (a) }\end{array}$ & $\begin{array}{ll}\text { May } 27,1960 \\
\text { June } 23\end{array}$ & $\begin{array}{l}\text { (a) } \\
23.37\end{array}$ & $\begin{array}{l}\text { Aug. 25, } 1960 \\
\text { Sept. 29 } \\
\text { Oct. }\end{array}$ & $\begin{array}{c}\text { (a) } \\
\text { (a) }\end{array}$ & $\begin{array}{l}\text { Nov. } 29,1960 \\
\text { Dec. } 28\end{array}$ & $\begin{array}{l}18.47 \\
17.11\end{array}$ \\
\hline
\end{tabular}

a Well being pumped.

b Well pumped recently.

7/35-23J3. U. S. Geol. Survey, Union Sugar Co. property. Near Lompoc. Drilled observation water-table well in alluvium, diam 2 in, depth $32 \mathrm{ft}$, cased to 32 . Lsd $43.43 \mathrm{ft}$ above msl. MP top east side of casing, $1.00 \mathrm{ft}$ above $1 \mathrm{sd}$. Highest water l evel 9.16 below lsd, Apr. 28, 1958; lowest 26.56 below 1sd, Oct. 29, 1951. Records available: 1947-58. Well destroyed; measurement discontinued.

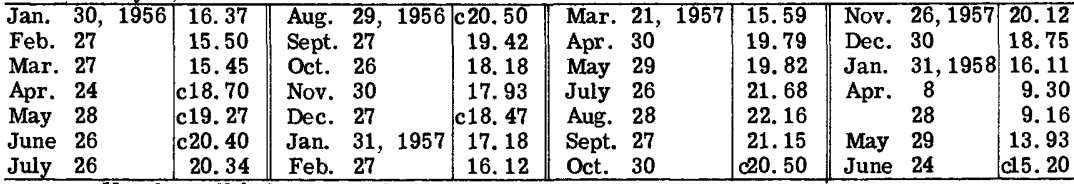

c Nearby well being pumped.

7/35-26J4. County of Santa Barbara, Artesia School District. Near Lompoc. Artesia and Central Aves. Drilled public-supply artesian well in alluvium, diam $8 \mathrm{in}$, depth $141 \mathrm{ft}$, perforated 132-140. Lsd $40.86 \mathrm{ft}$ above msl. MP top southwest side of 1 -in pipe, $1.50 \mathrm{ft}$ above 1sd. Highest water level 5.76 below lsd, Apr. 29, 1958; lowest 33.63 below 1sd, July 26, 1951. Records available: $1947-60$.

\begin{tabular}{ll|l||l|l||l|l||l|l|}
\hline Jan. 30,1956 & 8.28 & Apr. 30, 1957 & 17.91 & July 29, 1958 & 16.40 & Oct. 28, 1959c21.30
\end{tabular}

\begin{tabular}{|c|c|c|c|c|c|c|c|c|c|c|c|}
\hline & & & & & & & & & & & \\
\hline Feb. & 27 & 9.27 & May & 29 & 16. 30 & Aug. & 29 & 11.53 & Nov. & 25 & 15.67 \\
\hline Mar. & 27 & 24.75 & June & 26 & 22. 72 & Sept. & 29 & 13.46 & Dec. & 23 & c19. 85 \\
\hline Apr. & 24 & 23.25 & July & 26 & 19. 54 & Oct. & 29 & b16. 80 & Jan. & 28,1960 & 11.08 \\
\hline May & 28 & b14.58 & Aug. & 28 & 26.15 & Nov. & 26 & b11. 30 & Feb. & 26 & 10.18 \\
\hline June & 26 & 15.63 & Sept. & 26 & 14.55 & Dec. & 30 & 11. 22 & Mar. & 30 & 26.46 \\
\hline uly & 26 & 15.53 & Oct. & 30 & 13. 62 & Jan. & $30, \quad 1959$ & 13. 29 & Apr. & 29 & 18. 29 \\
\hline Aug. & 29 & 15.96 & Nov. & 26 & 18. 17 & Feb. & 28 & 7.33 & May & 27 & c21. 64 \\
\hline Sept. & 27 & 17.06 & Dec. & 27 & 10.37 & Mar. & 27 & c21. 27 & June & 23 & 21.84 \\
\hline Oct. & 27 & 10.84 & Jan. & 31,1958 & 16.08 & Apr. & 30 & 18.93 & July & 28 & 29.22 \\
\hline Nov. & 28 & 12.07 & Feb. & 28 & b8. 99 & May & 26 & b25. 14 & Aug. & 25 & 22.83 \\
\hline Dec. & 27 & 17. 60 & Mar. & 31 & 6.56 & June & 24 & 18. 78 & Sept. & 29 & 25.05 \\
\hline Jan. & 31,1957 & 9.13 & Apr. & 29 & 5. 76 & July & 31 & 26.43 & Oct. & 27 & b20. 10 \\
\hline Feb. & 27 & 7.98 & May & 29 & 18. 26 & Aug. & 28 & 22.09 & Nov. & 29 & b1 3.30 \\
\hline Mar. & 21 & 13.76 & June & 24 & 13. 26 & Sept. & 25 & b16. 67 & Dec. & 28 & 11.47 \\
\hline
\end{tabular}

b Well pumped recently.

c Nearby well being pumped.

San Antonio Valley

8/32-30K2. John Parma. Los Alamos. U. S. Highway 101 and Den St. Drilled unused artesian well in alluvium, diam $16 \mathrm{in}$, depth $100 \mathrm{ft}$. Lsd about $558 \mathrm{ft}$ above msl (previously reported $555 \mathrm{ft}$ above msl). MP bottom hole on north side of casing, $1.45 \mathrm{ft}$ above lsd. Highest water level 1.16 above lsd, Feb. 29, 1944; lowest 34.00 below lsd, Aug. 30, 1955. Records available: $1943-60$.

\begin{tabular}{l|r||l|r||l|r||l|r|}
\hline Jan. 26, 1956 & 10.11 & Apr. 23, 1957 & 16.96 & Aug. 26, 1958 & 11.44 & Nov. 24, 1959 & 10.67 \\
Feb. 29 & 8.62 & May 29 & 24.43 & Sept. 23 & 11.75 & Dec. 29 & 9.39 \\
Mar. 29 & 13.65 & June 26 & c27.71 & Oct. 29. & 11.25 & Feb. 2,1960 & 7.95 \\
Apr. 24 & 12.37 & Aug. 22 & 31.92 & Nov. 26 & 9.76 & 26 & 7.66 \\
May 31 & c9.25 & Sept. 27 & 24.52 & Dec. 31 & $\mathbf{8 . 4 8}$ & Mar. 28 & 7.16 \\
June 26 & 20.60 & Oct. 31 & 19.10 & Jan. 26, 1959 & 6.61 & Apr. 26 & 8.52 \\
July 25 & 28.78 & Nov. 26 & 17.17 & Feb. 26 & 5.74 & May 26 & 7.99 \\
Aug. 30 & 19.24 & Dec. 27 & 17.20 & Mar. 31 & 6.84 & June 21 & 9.39 \\
Sept. 25 & 22.37 & Jan. 30, 1958 & 14.55 & Apr. 28 & 6.42 & July 28 & 27.93 \\
Oct. 25 & 15.66 & Feb. 25 & 12.22 & May 27 & 7.55 & Aug. 31 & 30.34 \\
Nov. 29 & 13.68 & Mar. 25 & 10.38 & June 22 & 11.84 & Sept. 28 & 29.66 \\
Dec. 27 & 12.41 & Apr. 30 & 7.26 & July 27 & 15.38 & Oct. 26 & 11.53 \\
Jan. 31, 1957. & 11.08 & May 28 & c9.61 & Aug. 25 & $\mathbf{1 4 . 6 3}$ & Nov. 23 & 8.58 \\
Feb. 26 & 10.40 & June 26 & 12.94 & Sept. 30 & 11.00 & Dec. 29 & 11.30 \\
Mar. 20 & 9.92 & July 30 & 19.40 & Oct. 28 & 11.23 & & \\
\hline
\end{tabular}


8/34-23B1. Josephine Harris Estate. Near Los Alamos. Harris-Los Alamos Rd. and State Highway 1. Drilled unused artesian well in alluvium, diam $12 \mathrm{in}$, depth $150 \mathrm{ft}$. Lsd about $315 \mathrm{ft}$ above msl (previously reported about $310 \mathrm{ft}$ above msl). MP top east side of casing, $2.00 \mathrm{ft}$ above lsd. Highest water level 12.19 below 1sd, Feb. 29, 1944; lowest 20. 30 below 1sd, Mar. 27, 1953. Records available: 1943-60.

\begin{tabular}{|c|c|c|c|c|c|c|c|}
\hline Date & $\begin{array}{l}\text { Water } \\
\text { level }\end{array}$ & Date & $\begin{array}{l}\text { Water } \\
\text { level }\end{array}$ & Date & $\begin{array}{l}\text { Water } \\
\text { level }\end{array}$ & Date & $\begin{array}{l}\text { Water } \\
\text { level }\end{array}$ \\
\hline Jan. 26,1956 & $17 . \overline{30}$ & Apr. 23, 1957 & 20.10 & July 30,1958 & c20.00 & Oct. 28,1959 & c17.45 \\
\hline Feb. 29 & c16.95 & May 29 & c18. 96 & Aug. 26 & c16.68 & Nov. 24 & c17. 36 \\
\hline Mar. 29 & c20.65 & June 26 & c19. 65 & Sept. 23 & c16. 37 & Dec. 29 & 17. 04 \\
\hline pr. 24 & c20.77 & July 24 & c23. 07 & Oct. 29 & c16. 21 & 2,1960 & c16.76 \\
\hline May 31 & c17. 61 & Aug. 22 & c19. 85 & Nov. 26 & c16.14 & 26 & c16.56 \\
\hline June 26 & $\mathrm{c} 17.65$ & Sept. 27 & c19. 28 & Dec. 31 & 16.09 & Mar, 28 & 16.52 \\
\hline uly 25 & c21. 64 & Oct. 31 & c18. 85 & Jan. 26, 1959 & c16.05 & Apr. 26 & 16.56 \\
\hline Aug. 30 & c18.91 & Nov, 26 & c18. 74 & Feb. 26 & c15. 92 & May 26 & c17. 77 \\
\hline ept. 25 & c18.19 & Dec. 27 & c18. 52 & Mar. 31 & c19.47 & June 21 & c18.25 \\
\hline ct. 25 & c17.91 & Jan. 30,1958 & 18.40 & Apr. 28 & $\mathrm{c} 20.47$ & July 28 & 21.76 \\
\hline ov. 29 & c17. 81 & Feb. 25 & c17. 79 & May 27 & c17. 38 & Aug. 31 & 20.99 \\
\hline Dec. 27 & c17. 63 & Mar. 25 & 17.06 & June 22 & c17.06 & Sept. 28 & 19.99 \\
\hline an. 31,1957 & c17. 50 & Apr. 30 & c15. 66 & July 27 & c20 04 & Oct. 26 & c18.98 \\
\hline $\begin{array}{l}\text { Feb. } 28 \\
\text { Mar. } 20\end{array}$ & $\begin{array}{l}\text { c17. } 32 \\
\text { c17. } 31\end{array}$ & May 28 & c17. 76 & Aug. 24 & c17. 88 & Nov. 23 & c18.11 \\
\hline 1.200 & 017.31 & June 20 & & sept. 3 & ICI & Dec. & c17.42 \\
\hline
\end{tabular}

c Nearby well being pumped.

\section{Santa Maria Valley}

9/33-2A1. Santa Maria Realty Co. Garey. Wicks and Andrews Aves. Drilled irrigation water-table well in alluvium and Paso Robles Formation of Pliocene and Pleistocene (?) age, diam $12 \mathrm{in}$, depth $168 \mathrm{ft}$. Lsd $378.72 \mathrm{ft}$ above msl. MP north side of west edge of steel pumpbase support, $0.60 \mathrm{ft}$ above lsd. Highest water level 23.62 below lsd, June 4, 1941; lowest 96. 19 below lsd, June 21, 1960 . Records available: $1930-33,1936,1938-60$.

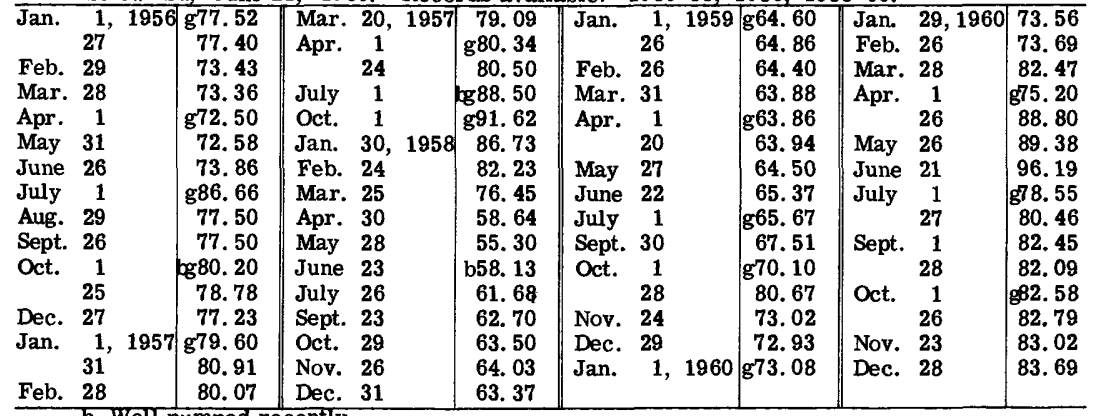

b well pumped recently.

g Measurement made by Santa Maria Valley Water Conservation District.

9/34-3N1. Formerly 9/32-3N1. City of Santa Maria. Near Orcutt. Drilled unused watertable well in Paso Robles Formation of Pliocene and Pleistocene (?) age, diam 16 in, depth $248 \mathrm{ft}$, perforated 163-190. Lsd about $258 \mathrm{ft}$ above msl. MP top of 2-in pipe, $0.90 \mathrm{ft}$ above $1 \mathrm{sd}$. Highest water level 142 below lsd, June 30, July 31, 1937, June 30, 1938; lowest 184. 99 below 1sd, Dec. 28, 1960. Records available: 1932-34, 1937-41, 1955-60.

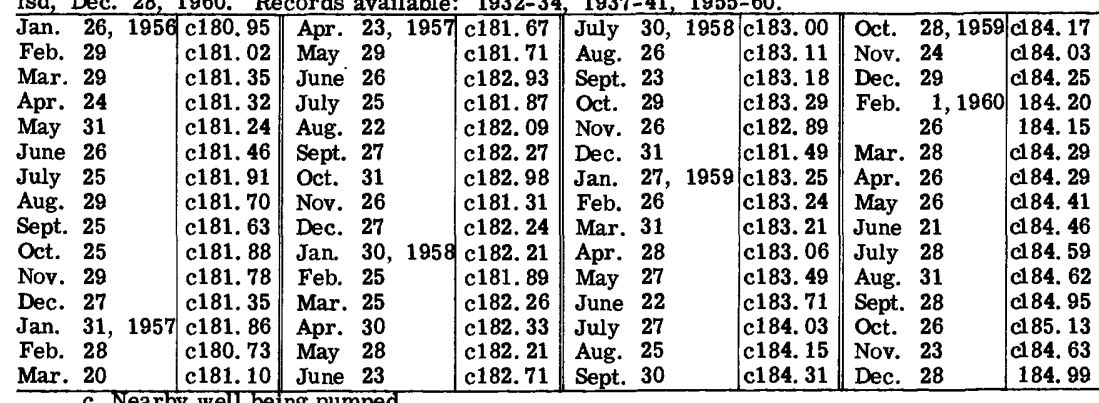


10/33-19B1. Owen T. Rice. Near Santa Maria. Battles and East Stowell Rds. Drilled irrigation water-table well in alluvium and Paso Robles Formation of Pliocene and Pleistocene (?) age, diam 16 in, depth $307 \mathrm{ft}$, perforated 92-97, 116-125, 190-215, 238-248. Lsd about $275 \mathrm{ft}$ above $\mathrm{msl}$. MP bottom west edge of pumpbase through hole in concrete foundation, $0.20 \mathrm{ft}$ above 1sd. Highest water level 73. 31 below 1sd, Sept. 2, 1943; lowest 168.40 below Isd, July 1, 1960. Records available: 1927, 1929-60.

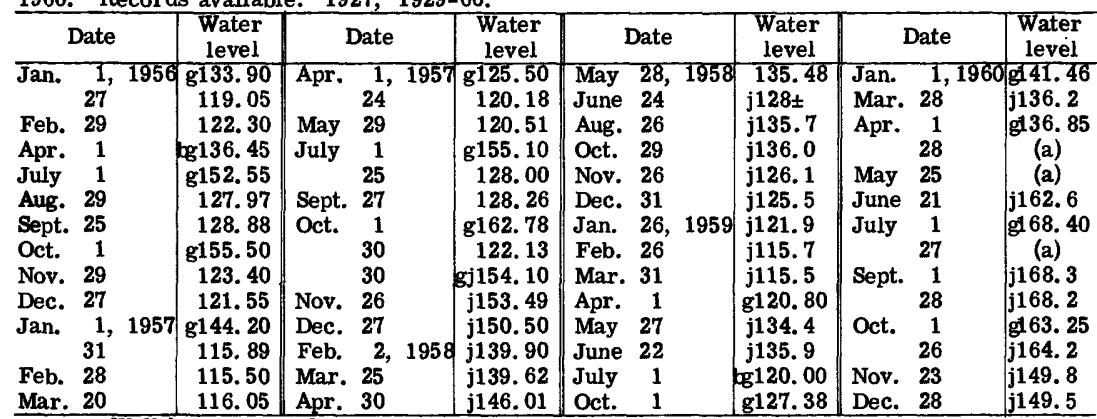

a Well being pumped.

b Well pumped recently.

g Measurement made by Santa Maria Valley Water Conservation District.

j Sounder measurement.

10/34-14E2. City of Santa Maria. Santa Maria Valley RR. and U. S. Highway 101. Drilled unused (previously reported public-supply) water-table well in Paso Robles Formation of Pliocene and Pleistocene (?) age, diam 16 in, depth $182 \mathrm{ft}$. Lsd about $225 \mathrm{ft}$ above msl. MP top of $1 \frac{1}{2}$-in pipe, $0.30 \mathrm{ft}$ below lsd. Highest water level 104.10 below Isd, Jan. 7, 1942; lowest 162.94 below lsd, Oct. 31, 1957. Records available: 1942, 1955-60.

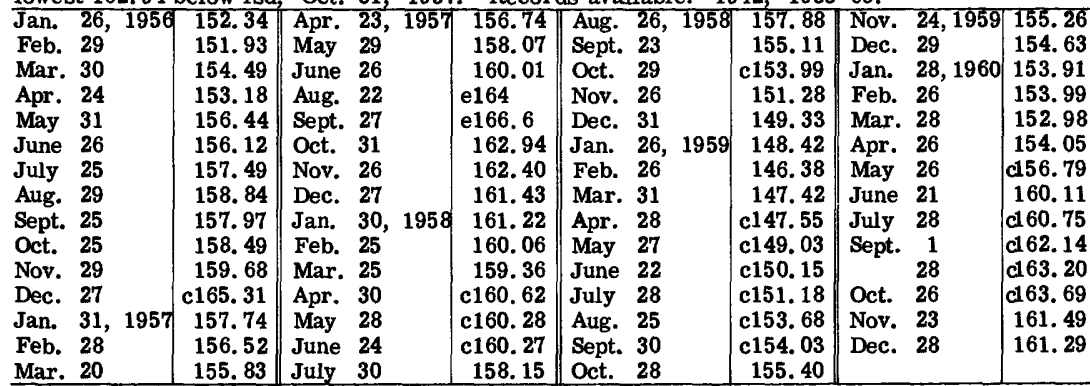

c Nearby well being pumped.

e Estimated.

10/35-7F1. M. J. Ellis. Near Guadalupe. Drilled domestic and irrigation artesian well in alluvium and Paso Robles Formation of Pliocene and Pleistocene (?) age, diam 12 in, depth $249 \mathrm{ft}$, perforated 140-145, 200-225. Lsd about $48 \mathrm{ft}$ above msl. MP top east side of pumpbase through hole, $1.80 \mathrm{ft}$ above lsd. Highest water level flowing, Dec. 30, 1943, Feb. 29, 1944; lowest 24.55 below 1sd, July 1, 1956. Records available: 1929-36, 1938-60.

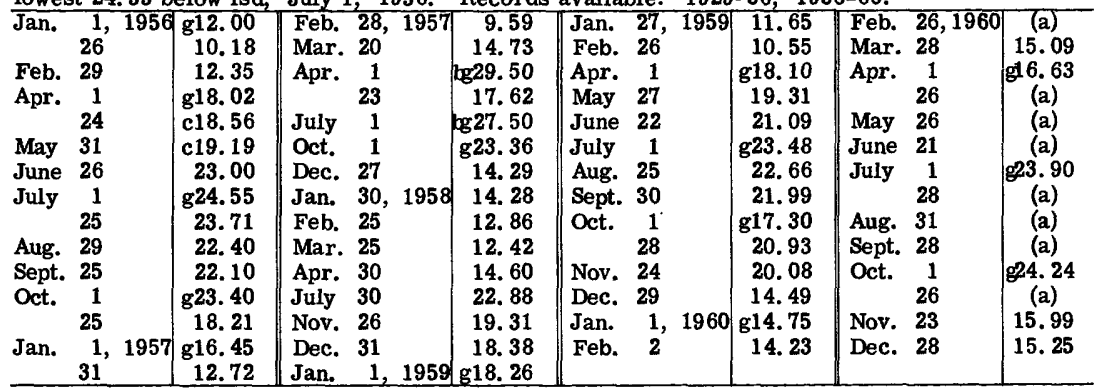

a Well being pumped.

b Well pumped recently.

c Nearby well being pumped.

g Measurement made by Santa Maria Valley Water Conservation District. 
Cuyama Valley

9/24-19Q1. Sam Knittle. Drilled unused water-table well in alluvium, diam 6 in, depth $90 \mathrm{ft}$. Lsd 2, 784. $19 \mathrm{ft}$ above msl. MP top of $1 \frac{1}{4}-i n$ pipe, $0.52 \mathrm{ft}$ above lsd. Highest water level 16.13 below lsd, May 30, 1944; lowest 83.58 below lsd, Jan. 29, 1958 . Records available: $1941-60$.

\begin{tabular}{|c|c|c|c|c|c|c|c|}
\hline Date & $\begin{array}{l}\text { Water } \\
\text { level }\end{array}$ & Date & $\begin{array}{c}\text { Water } \\
\text { level }\end{array}$ & Date & $\begin{array}{c}\text { Water } \\
\text { level }\end{array}$ & Date & $\begin{array}{l}\text { Water } \\
\text { level }\end{array}$ \\
\hline Jan. 27, 1956 & 76.48 & Apr. 24,1957 & 78.46 & July 29,1958 & 71.38 & Oct. 29,1959 & 71.94 \\
\hline Feb. 28 & 76.31 & May 28 & 78.67 & Aug. 27 & 71.18 & Nov. 23 & 67.70 \\
\hline Mar. 27 & 76.62 & June 25 & 80.02 & Sept. 22 & 70.91 & Dec. 30 & 67.32 \\
\hline Apr. 26 & 76.66 & July 24 & 79.44 & Oct. 27 & 70.46 & Jan. 27,1960 & 67.20 \\
\hline May 29 & 76.95 & Aug. 21 & 80.15 & Nov. 24 & 70.01 & Feb. 25 & 67.01 \\
\hline June 28 & 77.27 & Sept. 26 & 80.36 & Dec. 30 & 69.67 & Mar. 29 & 67.53 \\
\hline July 24 & 77.73 & Oct. 29 & 80.31 & Jan. 28,1959 & 70.97 & Apr. 27 & 67.77 \\
\hline Aug. 28 & 77.93 & Nov, 27 & 80.30 & Feb. 27 & 68.91 & May 24 & 67.77 \\
\hline Sept. 27 & 77.89 & Dec. 26 & 80.32 & Mar. 30 & 68.48 & June 22 & 67.93 \\
\hline Oct. 24 & 77.90 & Jan. 29,1958 & 83.58 & Apr. 24 & 68.04 & July 26 & 68.15 \\
\hline Nov. 27 & 77.95 & Feb. 24 & 80.30 & May 25 & 68.15 & Aug. 30 & 669.56 \\
\hline Dec. 28 & 77.83 & Mar. 24 & 79.65 & June 24 & c68.58 & Sept. 27 & 68.84 \\
\hline Jan. 30,1957 & 79.86 & Apr. 28 & 75.02 & July 29 & 67.99 & Oct. 25 & c71. 37 \\
\hline Feb. 26 & 78.53 & May 26 & 72.67 & Aug. 26 & 68.14 & Nov. 22 & 69.15 \\
\hline Mar. 21 & 77.97 & June 24 & 71.57 & Sept. 28 & 67.98 & Dec. 27 & 73.24 \\
\hline
\end{tabular}

10/25-30P1. Presseler and Kirschenmann. Two mi southeast of Cuyama. Drilled unused water-table well in alluvium and older continental deposits, diam 16 in, depth $400 \mathrm{ft}$. Lsd about 2, $340 \mathrm{ft}$ above msl. MP edge of steel plate, at lsd. Highest water level 109.55 below lsd, Jan. 30, 1957; lowest 141.91 below lsd, Sept. 27, 1960. Records available: 1955-60.

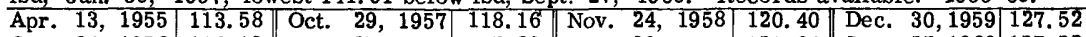
Oct. 24, 1956 116.13 Nov, 27 Nov. 27 Dec. 28 Jan. 30, 1957 Feb. 26 Mar. 21 Apr. 24 May 28 June 25 July 24 Aug. 21 Sept. 26

\begin{tabular}{|c|c|c|c|c|}
\hline 116. 13 & Nov. 2 & $\begin{array}{l}27 \\
26\end{array}$ & $\begin{array}{r}117.29 \\
\text { c116. } 00\end{array}$ & $\begin{array}{l}\text { Dec. } \\
\text { Jan. }\end{array}$ \\
\hline $\begin{array}{l}11.98 \\
111.16\end{array}$ & Jan 2 & $\begin{array}{ll}20 & 1958 \\
29 & 1958\end{array}$ & c116. 115.25 & \\
\hline 109.55 & Feb. 2 & 24 & 115.08 & Apr. \\
\hline 112.34 & Mar. 2 & 24 & 115.37 & May \\
\hline 113.97 & Apr. 2 & 28 & c117.01 & June \\
\hline 117.54 & May & 26 & c122. 34 & July \\
\hline 117.99 & July & 29 & c129.96 & Aug. \\
\hline 118.30 & Aug. 2 & 27 & 130.87 & Sept. \\
\hline 9.02 & Sept. 2 & 22 & 130.99 & Oct. \\
\hline 30.08 & Oct. & 27 & 122.49 & Nov. \\
\hline
\end{tabular}
\begin{tabular}{l|ll|l}
121.84 & Jan. 27,1960 & 127.22 \\
126.10 & Feb. 25 & c126.79
\end{tabular} 124. 48 Mar. $29 \quad 133.08$ 124.84 Apr. $27 \quad$ c130.86 138. 66 May $24 \quad$ c138. 61 133. 62 June $22 \quad 139.50$ 135. 25 Aug. $30 \quad 141.29$ 135. 49 Sept. $27 \quad 141.91$ 135.94 Oct. $25 \quad 136.75$ 128. 98 Nov. $22 \quad 136.43$ \begin{tabular}{l|l|l}
129.59 & Dec. 27 & 129.60
\end{tabular}

10/27-12R1. William Kirschenmann Estate. Drilled domestic and irrigation water-table well in alluvium, diam $12 \mathrm{in}$, depth $131 \mathrm{ft}$, cased to 131, perforated 53-128. Lsd about 2, $036 \mathrm{ft}$ above msl. MP inner edge of bottom hand hole east side of pumpbase, $0.90 \mathrm{ft}$ above lsd. Highest water level 38.58 below lsd, Apr. 28, 1942; lowest 72. 99 below 1sd, Aug. 26, 1959. Records available: 1941-60.

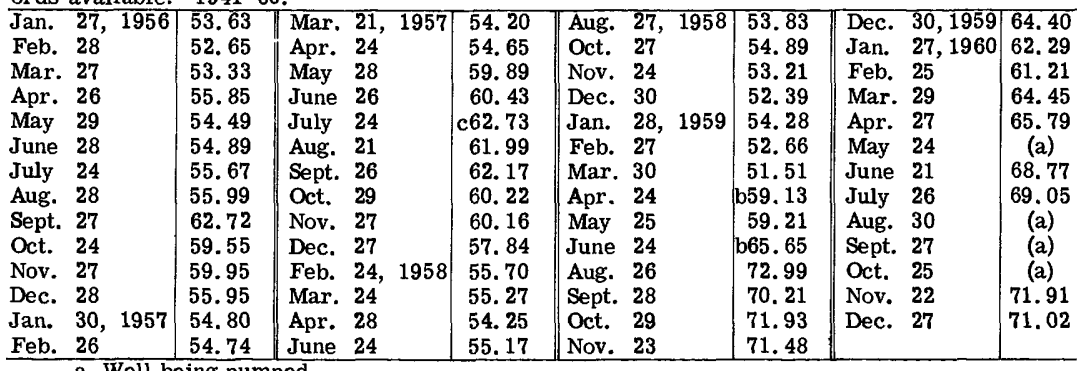




\section{Siskiyou County}

\section{Scott Valley}

44/9-28P1. F. R. Simpson. About $3 \mathrm{mi}$ northeast of Ft. Jones. Drilled unused watertable well in younger alluvium of Quaternary age, diam $8 \mathrm{in}$, depth $65 \mathrm{ft}$. Lsd 2,711 ft above msl. MP top of casing, $0.50 \mathrm{ft}$ above 1sd. Highest water level 3.8 below 1sd, Apr. 26, 1954; lowest 26.7 below lsd, Dec. 17, 1959, Jan. 28, 1960. Records available: 1953-54, 1958-60. No measurement made 1955-57.

\begin{tabular}{|c|c|c|c|c|c|c|c|}
\hline Date & $\begin{array}{l}\text { Water } \\
\text { level }\end{array}$ & Date & $\begin{array}{l}\text { Water } \\
\text { level }\end{array}$ & Date & $\begin{array}{c}\text { Water } \\
\text { level }\end{array}$ & Date & $\begin{array}{c}\text { Water } \\
\text { level }\end{array}$ \\
\hline Oct. 12,1953 & 17.8 & Apr. 23,1959 & 12.1 & Nov. 19,1959 & 25.8 & June 22,1960 & 8.5 \\
\hline Apr. 26, 1954 & 3.8 & May 20 & 5.3 & Dec. 17 & 26.7 & July 21 & 7.5 \\
\hline Oct. 16,1958 & 21.8 & June 24 & 8.6 & Jan. 28, 1960 & 26.7 & Aug. 24 & 15.5 \\
\hline Nov. 19 & 24.1 & July 22 & 9.7 & Feb. 18 & 17.8 & Sept. 21 & 15.4 \\
\hline Dec. 18 & 24.7 & Aug. 26 & 16.9 & Mar. 29 & 11.2 & Oct. 26 & 24.0 \\
\hline Jan. 21, 1959 & 21.9 & Sept. 23 & 18.7 & Apr. 27 & 9.5 & Nov. 16 & 25.8 \\
\hline $\begin{array}{l}\text { Feb. } 17 \\
\text { Mar. } 19\end{array}$ & $\begin{array}{r}15.7 \\
9.6\end{array}$ & Oct. 28 & 24.2 & May 25 & 8.1 & Dec. 29 & 21.9 \\
\hline
\end{tabular}

42/9-27N1. Joe Starr. About $1 \mathrm{mi}$ east of Etna. Dug unused water-table well in younger alluvium of Quaternary age, size $5 \times 5 \mathrm{ft}$, depth $19 \mathrm{ft}$. Lsd 2,930 ft above msl. MP top of board cover, $1.10 \mathrm{ft}$ above 1sd. Highest water level 1.3 below lsd, May 20, 1959, May 25, 1960 ; lowest 9.8 below 1sd, Sept. 23, 1959. Records available: 1953-54, 1958-60. No measurement made 1955-57.

\begin{tabular}{l|l||l|l||l|l||l|l}
\hline July 14, 1953 & 1.6 & Jan. 21, 1959 & 2.8 & Sept. 23, 1959 & 9.8 & May 25, 1960 & 1.3 \\
Aug. 3 & 2.2 & Feb. 17 & 2.8 & Oct. 28 & 7.7 & June 22 & 2.1 \\
Sept. 14 & 6.0 & Mar. 19 & $\mathbf{2 . 9}$ & Nov. 19 & 6.7 & July 21 & 4.8 \\
Oct. 5 & 6.7 & Apr. 23 & 2.3 & Dec. 17 & 6.3 & Aug. 24 & 7.7 \\
Apr. 30, 1954 & 3.0 & May 20 & 1.3 & Jan. 28, 1960 & 4.8 & Sept. 21 & 8.7 \\
Oct. 16, 1958 & 5.1 & June 24 & 2.4 & Feb. 18 & 2.1 & Oct. 26 & $\mathbf{8 . 6}$ \\
Nov. 17 & 5.3 & July 22 & 6.4 & Mar. 29 & 4.6 & Nov. 16 & 6.8 \\
Dec. 18 & 5.7 & Aug. 26 & 8.6 & Apr. 27 & 2.8 & Dec. 29 & 3.6 \\
\hline
\end{tabular}

\section{Shasta Valley}

45/6-19E1. George Weldon. $3 \mathrm{mi}$ east of Yreka. Drilled domestic water-table well in Chicot Formation of Late Cretaceous age, and perhaps into basement complex, diam 16 in, depth $425 \mathrm{ft}$. Lsd 2,538 ft above msl. MP top of casing, $1.00 \mathrm{ft}$ above lsd. Highest water level 17. 5 below 1sd, Apr. 28, 1960; lowest 22. 6 below 1sd, Oct. 5, 1953. Records available: 1953-54, 1958-60. No measurement made 1955-57.

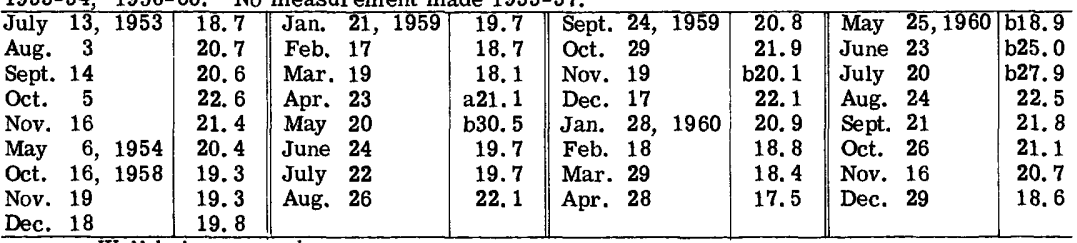

\section{a Well being pumped.}

\section{b Well pumped recently.}

44/5-34H1. Henry Silva, $1.75 \mathrm{mi}$ north of Big Springs. Drilled irrigation water-table well in Plutos Cave Basalt of Recent age, diam 16 in, depth $96 \mathrm{ft}$. Lsd 2,637 ft above msl. MP north side of pumpbase, at lsd. Highest water level 24.9 below lsd, July 22, Aug. 27, 1959 ; lowest 30.0 below 1sd, Nov. 19, 1959. Records available: 1953-54, 1958-60. No measurement made 1955-57.

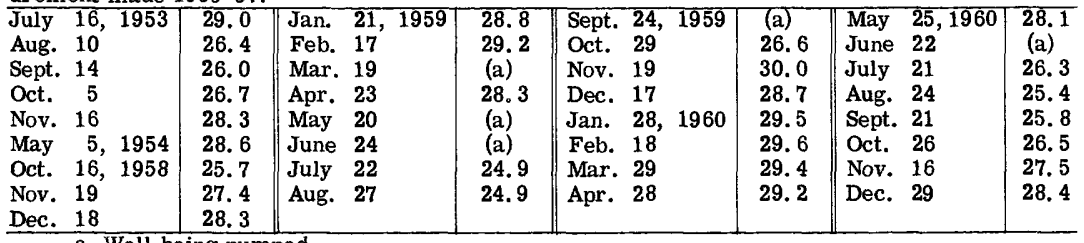

a Well being pumped. 
42/6-10J1. G. G. Maxwell. About $8 \mathrm{mi}$ northwest of Weed. Drilled domestic watertable well in pyroclastics, diam $6 \mathrm{in}$, depth $110 \mathrm{ft}$. Lsd 2, $835 \mathrm{ft}$ above msl. MP top of casing, $0.30 \mathrm{ft}$ above lsd. Highest water level 2.9 below lsd, May 3, 1954; lowest 15.9 below Isd, Sept. 21,1960 . Records available: $1953-54,1958-60$. No measurement made 1955-57.

\begin{tabular}{|c|c|c|c|c|c|c|c|}
\hline Date & $\begin{array}{l}\text { Water } \\
\text { level }\end{array}$ & Date & $\begin{array}{l}\text { Water } \\
\text { level }\end{array}$ & Date & $\begin{array}{l}\text { Water } \\
\text { level }\end{array}$ & Date & $\begin{array}{l}\text { Water } \\
\text { level }\end{array}$ \\
\hline July 16,1953 & 3.0 & Jan. 21,1959 & 8.9 & Sept. 24, 1959 & 14.4 & May 26,1960 & 4.3 \\
\hline Aug. 3 & 5.8 & Feb. 17 & 8.8 & Oct. 29 & 14.5 & June 22 & 6.9 \\
\hline Sept. 14 & 7.7 & Mar. 19 & 7.9 & Nov. 19 & 13.7 & July 21 & 11.8 \\
\hline Oct. 5 & 14.2 & Apr. 23 & b5. 6 & Dec. 17 & 11.5 & Aug. 24 & 15.1 \\
\hline & 9.3 & May 20 & 5.1 & Jan. 28, 1960 & 11.3 & Sept. 21 & 15.9 \\
\hline 3,1954 & 2.9 & June 24 & 9.6 & Feb. 18 & 10.3 & Oct. 26 & 15.3 \\
\hline Oct. 16,1958 & 11.2 & July 22 & 11.5 & Mar. 29 & 5.1 & Nov. 16 & 14.2 \\
\hline $\begin{array}{l}\text { Nov. } 19 \\
\text { Dec. } 18\end{array}$ & $\begin{array}{l}9.8 \\
9.8\end{array}$ & Aug. 27 & 14.5 & Apr. 28 & 5.3 & Dec. 29 & 8.7 \\
\hline
\end{tabular}

\section{Butte Valley}

46/1E-6N1. Leo Luzzi. About $4 \mathrm{mi}$ west-northwest of Macdoel. Drilled irrigation watertable well in lake deposits, diam $18 \mathrm{in}$, depth $200 \mathrm{ft}$. Lsd 4, 242.4 ft above msl. MP top east side of concrete base, $1.00 \mathrm{ft}$ above $1 \mathrm{sd}$. Highest water level 17.5 below 1sd, Mar. 19, 1959; lowest 29. 9 below lsd, Aug. 24, 1960. Records available: 1953-54, 1958-60. No measurement made 1955-57.

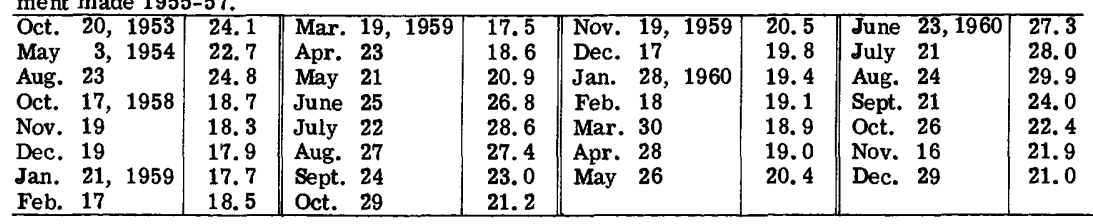

47/1W-14B1. U. S. Bureau of Reclamation. About 3. $4 \mathrm{mi}$ southwest of Dorris. Drilled observation water-table well in lake deposits, diam $2 \mathrm{in}$, depth $50 \mathrm{ft}$. Lsd 4, 233. $7 \mathrm{ft}$ above msl. MP top of casing, $0.50 \mathrm{ft}$ above lsd. Highest water level 11.4 below Isd, Jan. 21, 1959; lowest 18. 5 below lsd, Nov. 26, 1951. Records available: 1951-54, 1958-60. No measurement made 1955-57.

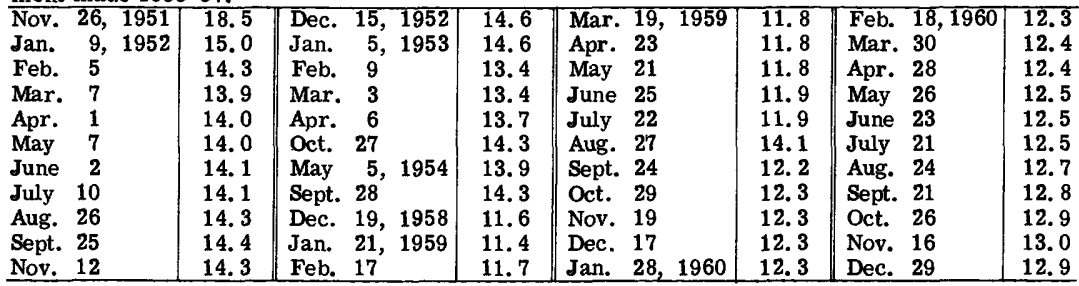

48/1W-26N1. Fairless. About $2 \mathrm{mi}$ west of Dorris. Drilled unused water-table well in lake deposits, diam $10 \mathrm{in}$, depth $375 \mathrm{ft}$, open bottom. Lsd 4,244.2 ft above msl. MP top of casing, at lsd. Highest water level 9.2 below lsd, May 5, 1954; lowest 20.8 below lsd, Oct. 26, 1960. Records available: $1953-54,1958-60$. No measurement made 1955-57.

\begin{tabular}{lr|r||l|l||l|l||l|l|l}
\hline Nov. 1,1953 & 13.7 & Apr. 23, 1959 & 15.6 & Nov. 19, 1959 & 18.5 & June 23, 1960 & 18.9 \\
May & 5,1954 & 9.2 & May 21 & 15.8 & Dec. 17 & 18.4 & July 21 & 19.8 \\
Nov. 20, 1958 & 15.4 & June 25 & 16.3 & Jan. 28, 1960 & 18.2 & Aug. 24 & 20.4 \\
Dec. 19 & & 15.8 & July 22 & 17.0 & Feb. 18 & 17.7 & Sept. 21 & 20.4 \\
Jan. 21, 1959 & $\mathbf{1 5 . 8}$ & Aug. 27 & 17.6 & Mar. 30 & 18.2 & Oct. 26 & 20.8 \\
Feb. 17 & 15.9 & Sept. 24 & 17.6 & Apr. 28 & 18.1 & Nov. 16 & 20.4 \\
Mar. 19 & 15.7 & Oct. 29 & 18.4 & May 26 & 18.1 & Dec. 29 & 20.6 \\
\hline
\end{tabular}

\section{Solano County}

Putah and Suisun-Fairfield Area

6/1W-11G1. Richfield Oil. About $8 \mathrm{mi}$ southwest of Dixon. Drilled domestic water-table well in alluvium of Recent age, diam 8 in, depth $93 \mathrm{ft}$. Lsd $87 \mathrm{ft}$ above msl. MP top of casing, $3.50 \mathrm{ft}$ below lsd. Highest water level 12.1 below lsd, May 6, 1938; lowest 38.9 below lsd, Oct. 6, 1960. Records available: 1931-42, 1949-51, 1958-60. Well destroyed; measurement discontinued. 
6/1W-11G1--Continued.

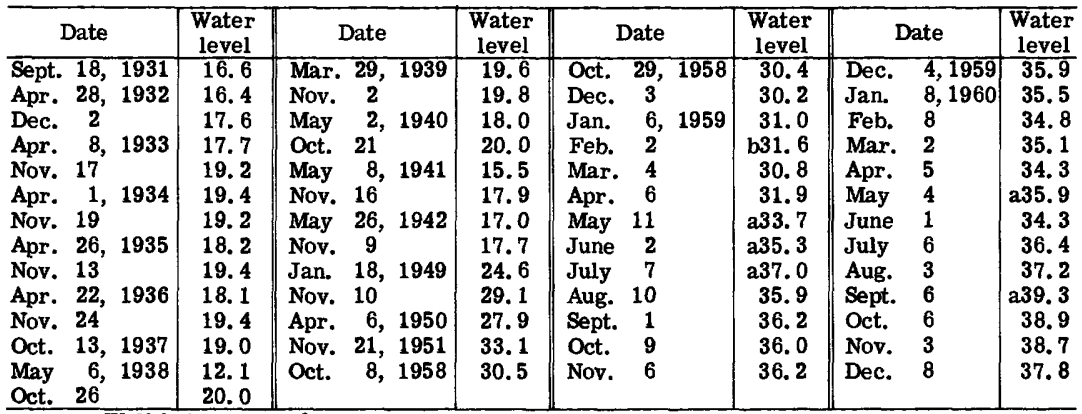

a Well being pumped.

b Well pumped recently.

\section{Sonoma County}

\section{Sonoma Valley}

5/5-29N1. Moll. About $3 \mathrm{mi}$ south of Sonoma. Drilled irrigation water-table well in younger and older alluvium, diam $10 \mathrm{in}$, depth $100 \mathrm{ft}$. Lsd $16 \mathrm{ft}$ above msl. MP top east side of casing, $0.10 \mathrm{ft}$ above 1sd. Highest water level 6.7 below lsd, Apr. 3, 1956; lowest 15.8 below lsd, Sept. 1, 1959. Records available: 1951-60.

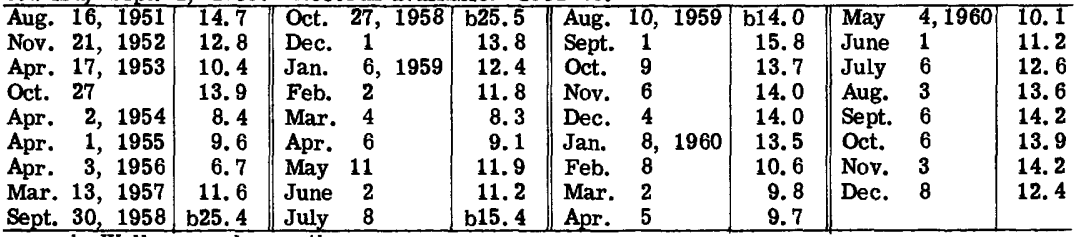

$\frac{\text { Sept. } 30,1958 \text { b25. } 4 \text { July }}{\text { b Well pumped recently. }}$

5/5-8Q1. O. H. Thomas. $0.5 \mathrm{mi}$ east of Sonoma. Drilled irrigation water-table well in younger and older alluvium, diam $8 \mathrm{in}$, depth $500 \mathrm{ft}$. Lsd $107 \mathrm{ft}$ above msl. MP top east side of casing, $0.50 \mathrm{ft}$ above Isd. Highest water level 0.5 above lsd, Apr. 4, 1950; lowest 30.8 below lsd, Dec. 1, 1958. Records available: 1950, 1952-54, 1956-60. No measurement made in 1951 or 1955 .

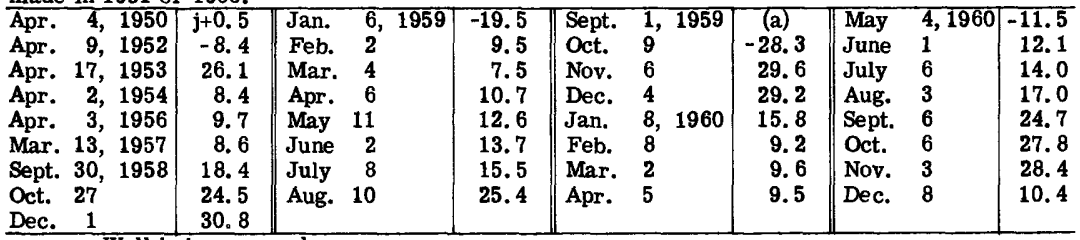

a Well being pumped.

j Flowing.

Santa Rosa-Petaluma Area

6/8-7P2. Joseph Kardohely. About $5 \mathrm{mi}$ southwest of Santa Rosa. Drilled domestic and irrigation water-table well in Merced Formation of Pliocene and Pleistocene (?) age, diam 8 in, depth $120 \mathrm{ft}$. Lsd $95 \mathrm{ft}$ above msl. MP top west side of casing, $1.30 \mathrm{ft}$ above $1 \mathrm{sd}$. Highest water level 10.6 below 1sd, Mar. 22, 1950, Apr. 4, 1952; lowest 33.5 below 1sd, Sept. 15, 1950. Records available: $1945,1949-60$.

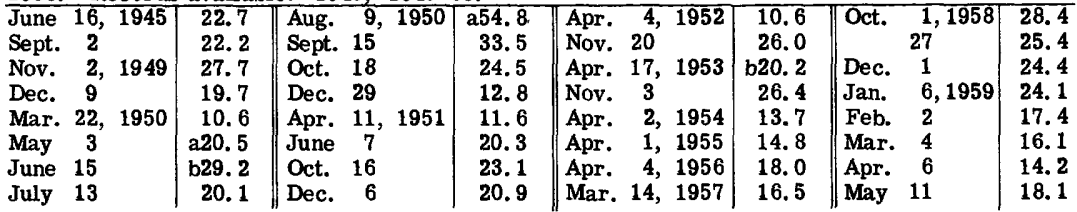


6/8-7P2--Continued.

\begin{tabular}{|c|c|c|c|c|c|c|c|}
\hline Date & $\begin{array}{l}\text { Water } \\
\text { level }\end{array}$ & Date & $\begin{array}{c}\text { Water } \\
\text { level }\end{array}$ & Date & $\begin{array}{c}\text { Water } \\
\text { level }\end{array}$ & Date & $\begin{array}{l}\text { Water } \\
\text { level }\end{array}$ \\
\hline June 3,1959 & 20.1 & 7,1959 & 27.6 & 6,1960 & 17.5 & Sept. 7,1960 & 26.3 \\
\hline July 8 & 32.6 & Dec. & 29.3 & May & 15.3 & Oct. 6 & 25.7 \\
\hline Aug. 11 & 27.3 & 9,1960 & 29.7 & June & b40. 6 & Nov. 3 & 27.3 \\
\hline Sept. 1 & 30.9 & Feb. & 19.1 & July & 25.0 & Dec. & 23.7 \\
\hline Oct. 10 & 31.6 & Mar. & 17.0 & Aug. & 26.4 & & \\
\hline
\end{tabular}

b Well pumped recently.

\section{Lower Russian River Area}

7/11-14E1. Morrill. About $5 \mathrm{mi}$ southwest of Gurneville. Drilled unused water-table well in younger alluvium, diam $10 \mathrm{in}$, depth $47 \mathrm{ft}$. Lsd $25 \mathrm{ft}$ above msl. MP top of casing, $4.00 \mathrm{ft}$ above 1sd. Highest water level 6.9 below lsd, Mar. 4, 1959; lowest 21.2 below lsd, Oct. 2, 1958. Records available: 1951, 1958-60.

\begin{tabular}{|c|c|c|c|c|c|c|c|c|c|c|}
\hline Sept. 1 & 12,1951 & 19.1 & Apr. 6,1959 & 17.6 & Nov & 7,1959 & 19.0 & July & 7,1960 & 19.1 \\
\hline Oct. & 2,1958 & 21. 2 & May 11 & 14.0 & Dec & & 15. 2 & Aug. & 3 & 19.4 \\
\hline & 28 & 18.2 & June & 19.3 & Jan. & 9,1960 & 15.9 & Sept. & 7 & 19.2 \\
\hline Dec. & 2 & 16. 5 & July & 19.3 & Mar & 2 & 17. 8 & Oct. & 6 & 19.2 \\
\hline Jan. & 6,1959 & 14.8 & Aug. 11 & 19.3 & Apr & 6 & 17. 2 & Nov. & 3 & 17.0 \\
\hline Feb. & 2 & 17.5 & Sept. 2 & 19.2 & May & 5 & 18.4 & Dec. & 8 & 17.5 \\
\hline Mar. & 4 & 6.9 & Oct. 10 & 19.0 & June & 2 & 18.9 & & & \\
\hline
\end{tabular}

Healdsburg Area

9/9-28N1. G. P. Brown. $1 \mathrm{mi}$ south of Healdsburg. Drilled stock water-table well in younger alluvium, diam $10 \mathrm{in}$, depth $53 \mathrm{ft}$. Lsd $90 \mathrm{ft}$ above msl. MP top of casing, $1.00 \mathrm{ft}$ above 1sd. Highest water level 7.6 below lsd, Feb. 9, 1960; lowest 21.8 below 1sd, Nov. 4, 1960. Records available: $1953-54,1958-60$. No measurement made 1955-57.

\begin{tabular}{|c|c|c|c|c|c|c|c|c|c|c|}
\hline Aug. & 1953 & 19.0 & Mar. 5, 1959 & 12.6 & Nov. & 7,1959 & 20.9 & June & 2,1960 & 14.2 \\
\hline May & 21,1954 & 12.9 & Apr. & 13.6 & Dec. & 5 & 21.5 & July & 7 & 16.8 \\
\hline June & 23 & a15. 4 & May 12 & 14.2 & Jan. & 9,1960 & 15.3 & Aug. & 4 & 18.2 \\
\hline Oct. & 1,1958 & a19. 5 & June & a16. 2 & Feb. & 9 & 7.6 & Sept. & 7 & 20.3 \\
\hline & 28 & 20.4 & July & 18.3 & Mar. & 3 & 13.3 & Oct. & 7 & 21.4 \\
\hline Dec. & 2 & 21.1 & Aug. 11 & 20.3 & Apr. & 6 & 13.2 & Nov. & 4 & 21.8 \\
\hline $\begin{array}{l}\text { Jan. } \\
\text { Feb. }\end{array}$ & $\begin{array}{ll}7, & 1959 \\
3 & \end{array}$ & $\begin{array}{l}12.4 \\
12.2\end{array}$ & $\begin{array}{l}\text { Sept. } 2 \\
\text { Oct. } 10\end{array}$ & $\begin{array}{r}\text { a22.1 } \\
20.5\end{array}$ & May & 5 & 13. 8 & Dec. & 9 & 13.1 \\
\hline
\end{tabular}

\section{Cloverdale Area}

11/10-19F2. State Division of Forestry. $1 \mathrm{mi}$ south of Cloverdale. Drilled unused artesian well in Franciscan Formation of Late Jurassic to Late Cretaceous age, and Knoxville Formation of Late Jurassic age, diam 8 in, depth $160 \mathrm{ft}$, cased to 135, perforated 116-135. Lsd $346 \mathrm{ft}$ above msl. MP top west side of casing, 1.50 ft above 1sd. Highest water level 3.0 below lsd, Apr. 1, 1954; lowest 14.1 below lsd, Nov. 7, Dec. 5, 1959. Records available: 1952-55, 1958-60. No measurement made 1956-57.

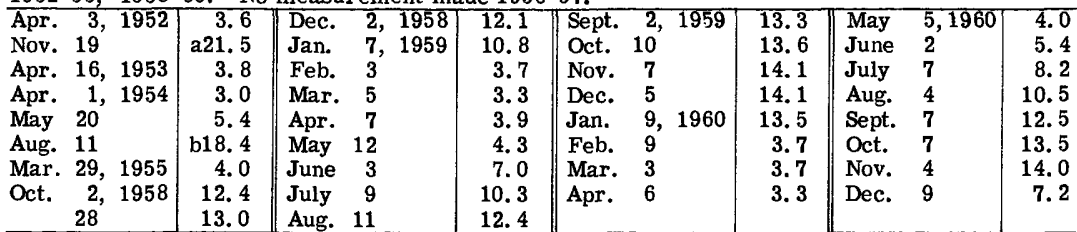

a Well being pumped.

b Well pumped recently.

\section{Yolo County}

Putah and Suisun-Fairfield Area

8/1E-15B1. Frank E. Russell. About $6 \mathrm{mi}$ west of Davis. Drilled stock water-table well in alluvium of Recent age, diam 10 in, depth $116 \mathrm{ft}$. Lsd $83.48 \mathrm{ft}$ above msl. MP top south side of casing, 2. $00 \mathrm{ft}$ above 1sd. Highest water level 13. 8 below 1sd, May 16, 1941; lowest 33. 1 below lsd, Nov. 3, Dec. 8, 1960. Records available: 1931-42, 1948-51, 1958-60. 
8/1E-15B1--Continued.

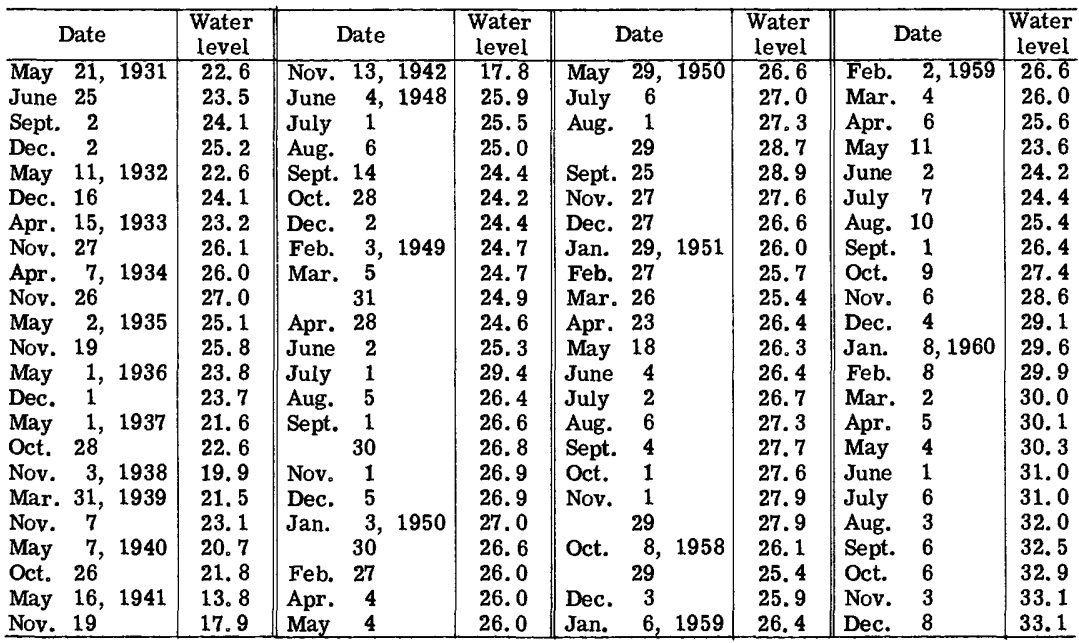

\section{References}

Evenson, R. E., 1958, Water levels in observation wells in Santa Barbara County, California, in 1957: U.S. Geol. Survey open-file rept., $46 \mathrm{p}$.

Merritt, P. M. , 1961, Water levels in observation wells in Santa Barbara County, California, in 1960: U. S. Geol. Survey open-file rept., 51 p., maps.

Miller, G. A., 1959, Water levels in observation wells in Santa Barbara County, California, in 1958: U.S. Geol. Survey open-file rept., 47 p., maps.

Muir, K. S. , and Merritt, P. M., 1957, Water levels in observation wells in Santa Barbara County, California, in 1956: U.S. Geol. Survey open-file rept., 68 p.

Muir, K. S., Merritt, P. M. , and Miller, G. A., 1960, Water levels in observation wells in Santa Barbara County, California, in 1959: U. S. Geol. Survey open-file rept., 46 p. , maps.

State of California, 1958, Water conditions in southern California during 1956-57: California Dept. of Water Resources Bull. No. 39-57, 3 vol. 1960, Water conditions in southern California during 1957-58: California Dept. of Water Resources Bull. No. 39-58, 3 vol.

1961, Water conditions in southern California during 1958-59: California Dept. of Water Resources Bull. No. 39-59, 3 vol. 1961, Water conditions in southern California during 1959-60: California Dept. of Water Resources Bull. No. 39-60, 3 vol.

1959, Ground-water conditions in central and northern California in 1957-58: California Dept. of Water Resources Bull. No. 77-58, $79 \mathrm{p}$.

1962, Ground-water conditions in central and northern California in 1958-59: California Dept. of Water Resources Bull. No. 77-59, 95 p. 
Systematic water-level measurements in Hawaii were begun in 1910 by the Public Works Department of the Territory of Hawaii. In 1917 the measurements were taken over by the Division of Hydrography of the Department of Public Lands, and since 1930 the observations have been made in a cooperative program carried on by the U.S. Geological Survey and the Division of Hydrography. Beginning in 1935 the records of measurements were published in the annual series of water-supply papers issued by the Geological Survey. Earlier records are published in bulletins issued by the Hawaii Division of Hydrography and prepared in cooperation with the Geological Survey. Figure 4 shows the location of observation wells included in this report.

\section{Well-Numbering System}

Beginning with number 1 at some point on each island, drilled wells of Hawaii are numbered consecutively as they occur in geographic sequence around the island. Single wells separated from others and pumped separately are numbered individually. Wells in a closely spaced group used to supply a central pumping plant are included under a single number with each well distinguished by a letter. In some areas certain numbers are left unassigned for the designation of new wells when they are drilled. Holes drilled especially for test or observation purposes are called test borings. Test borings on each island are numbered consecutively as they are drilled, beginning with " 1 " and are distinguished by a " $T$ " before each number. Shaft-type wells are numbered consecutively as they are dug and are distinguished by the word "shaft" before each number.

\section{Well Descriptions and Water-Level Measurements}

All ground-water levels in Hawaii are reported in feet above mean sea level. A landsurface datum is an arbitrary plane for each well, test hole, or shaft which approximates the ground surface and which may be above or below the point from which the water level is measured.

\section{Island of Hawaii}

Shaft 6. Laupahoehoe Sugar Co. Formerly Kaiwiki Sugar Co. Ookala. Lat $20^{\circ} 01^{\prime} 05^{\prime \prime}$, long $155^{\circ} 17^{\circ} 15^{\prime \prime}$. Dug domestic and irrigation basal water-table well in basalt of Hamakua Volcanic Series, Quaternary and late Tertiary age, size $6 \frac{1}{2} \times 6 \mathrm{ft}$, vertical depth of 30 -degree inclined shaft $300 \mathrm{ft}$; two infiltration tunnels, size $4 \times 6 \mathrm{ft}$, total length $650 \mathrm{ft}$. Lsd $300 \mathrm{ft}$ above msl. Highest water level 7.04 above msl, June 20, 1938; lowest 2.92 above msl, Apr. 27, 1946. Records available: 1937-60. Records furnished by Laupahoehoe Sugar Co.

Mean water level for the day, above msl, from recorder graph, 1956

\begin{tabular}{l|l|l|l|l|l|l|l|l|l|l|l|l|l}
\hline Day & Jan. & Feb. & Mar. & Apr. & May & June & July & Aug. & Sept. & Oct. & Nov. & Dec. \\
\hline $\mathbf{5}$ & $\mathbf{5 . 4}$ & $\mathbf{5 . 5}$ & $\mathbf{5 . 3}$ & $\mathbf{5 . 4}$ & $\mathbf{5 . 5}$ & $\mathbf{5 . 2}$ & $\mathbf{5 . 4}$ & $\mathbf{5 . 6}$ & $\mathbf{5 . 6}$ & $\mathbf{5 . 6}$ & $\mathbf{5 . 7}$ & $\mathbf{5 . 7}$ \\
10 & $\mathbf{5 . 3}$ & $\mathbf{5 . 2}$ & $\mathbf{5 . 3}$ & $\mathbf{5 . 4}$ & $\mathbf{5 . 4}$ & $\mathbf{5 . 3}$ & $\mathbf{5 . 4}$ & $\mathbf{5 . 5}$ & $\mathbf{5 . 5}$ & $\mathbf{5 . 6}$ & $\mathbf{5 . 6}$ & $\mathbf{5 . 7}$ \\
$\mathbf{1 5}$ & $\mathbf{5 . 6}$ & $\mathbf{5 . 4}$ & $\mathbf{5 . 1}$ & $\mathbf{5 . 5}$ & $\mathbf{5 . 4}$ & $\mathbf{5 . 4}$ & $\mathbf{5 . 6}$ & $\mathbf{5 . 3}$ & $\mathbf{5 . 5}$ & $\mathbf{5 . 6}$ & $\mathbf{5 . 6}$ & $\mathbf{5 . 7}$ \\
$\mathbf{2} 0$ & $\mathbf{5 . 2}$ & $\mathbf{5 . 2}$ & $\mathbf{5 . 1}$ & $\mathbf{5 . 4}$ & $\mathbf{5 . 5}$ & $\mathbf{5 . 2}$ & $\mathbf{5 . 4}$ & $\mathbf{5 . 4}$ & $\mathbf{5 . 4}$ & $\mathbf{5 . 7}$ & $\mathbf{5 . 6}$ & $\mathbf{5 . 7}$ \\
$\mathbf{2 5}$ & $\mathbf{5 . 3}$ & $\mathbf{5 . 5}$ & $\mathbf{5 . 4}$ & $\mathbf{5 . 5}$ & $\mathbf{5 . 3}$ & $\mathbf{5 . 4}$ & $\mathbf{5 . 5}$ & $\mathbf{5 . 5}$ & $\mathbf{5 . 5}$ & $\mathbf{5 . 6}$ & $\mathbf{5 . 7}$ & $\mathbf{5 . 8}$ \\
Eom & $\mathbf{5 . 3}$ & $\mathbf{5 . 2}$ & $\mathbf{5 . 5}$ & $\mathbf{5 . 5}$ & $\mathbf{5 . 3}$ & $\mathbf{5 . 5}$ & $\mathbf{5 . 5}$ & $\mathbf{5 . 5}$ & $\mathbf{5 . 6}$ & $\mathbf{5 . 7}$ & $\mathbf{5 . 8}$ & $\mathbf{5 . 8}$ \\
\hline $\mathbf{1 0}$ \\
\hline $\mathbf{5}$ & $5 . \mathbf{7}$ & $\mathbf{5 . 4}$ & $\mathbf{5 . 3}$ & $\mathbf{5 . 1}$ & $\mathbf{5 . 4}$ & $\mathbf{5 . 2}$ & $\mathbf{5 . 2}$ & $\mathbf{5 . 4}$ & $\mathbf{5 . 4}$ & $\mathbf{5 . 6}$ & $\mathbf{5 . 4}$ & 5.5 \\
10 & $\mathbf{5 . 6}$ & $\mathbf{5 . 5}$ & $\mathbf{5 . 4}$ & $\mathbf{5 . 3}$ & $\mathbf{5 . 2}$ & $\mathbf{5 . 3}$ & $\mathbf{5 . 2}$ & $\mathbf{5 . 4}$ & $\mathbf{5 . 4}$ & $\mathbf{5 . 4}$ & $\mathbf{5 . 6}$ & $\mathbf{5 . 4}$ \\
15 & $\mathbf{5 . 9}$ & $\mathbf{5 . 6}$ & $\mathbf{5 . 4}$ & $\mathbf{5 . 4}$ & $\mathbf{5 . 2}$ & $\mathbf{5 . 3}$ & $\mathbf{5 . 2}$ & $\mathbf{5 . 2}$ & $\mathbf{5 . 6}$ & $\mathbf{5 . 4}$ & $\mathbf{5 . 6}$ & $\mathbf{5 . 6}$ \\
$\mathbf{2 0}$ & $\mathbf{5 . 8}$ & $\mathbf{5 . 7}$ & $\mathbf{5 . 4}$ & $\mathbf{5 . 2}$ & $\mathbf{5 . 3}$ & $\mathbf{5 . 2}$ & $\mathbf{5 . 4}$ & $\mathbf{5 . 3}$ & $\mathbf{5 . 5}$ & $\mathbf{5 . 8}$ & $\mathbf{5 . 6}$ & $\mathbf{5 . 6}$ \\
$\mathbf{2 5}$ & $\mathbf{5 . 6}$ & $\mathbf{5 . 7}$ & $\mathbf{5 . 4}$ & $\mathbf{5 . 2}$ & $\mathbf{5 . 1}$ & $\mathbf{5 . 1}$ & $\mathbf{5 . 3}$ & $\mathbf{5 . 5}$ & $\mathbf{5 . 5}$ & $\mathbf{5 . 6}$ & $\mathbf{5 . 5}$ & $\mathbf{5 . 6}$ \\
Eom & $\mathbf{5 . 3}$ & $\mathbf{5 . 4}$ & $\mathbf{5 . 4}$ & $\mathbf{5 . 4}$ & $\mathbf{5 . 2}$ & $\mathbf{5 . 3}$ & $\mathbf{5 . 4}$ & $\mathbf{5 . 5}$ & $\mathbf{5 . 5}$ & $\mathbf{5 . 3}$ & $\mathbf{5 . 6}$ & $\mathbf{5 . 8}$ \\
\hline
\end{tabular}


Shaft 6--Continued.

\begin{tabular}{|c|c|c|c|c|c|c|c|c|c|c|c|c|}
\hline \multicolumn{13}{|c|}{1958} \\
\hline$\overline{\text { Day }}$ & Jan. & Feb. & Mar. & Apr. & May & June & July & Aug. & Sept. & Oct. & Nov. & Dec. \\
\hline 5 & 5.7 & 6.0 & 6.2 & 5.9 & 6.0 & 6.1 & 6.0 & 6.0 & 6.2 & 6.3 & 6.2 & 6.2 \\
\hline 10 & 5.7 & 5.9 & 6.2 & 5.9 & 6.0 & 6.1 & 5.9 & 6.1 & 6.1 & 6.1 & 6.2 & 6.3 \\
\hline 15 & 5.6 & 6. 0 & 6.1 & 5.7 & 6.0 & 6.0 & 5.9 & 6.1 & 6. 3 & 6.2 & 6.3 & 6.2 \\
\hline 20 & 5.7 & 6.0 & 6.0 & 5.9 & 6.0 & 5.9 & 6.1 & 6.1 & 6.2 & 6.3 & 6.2 & 6.3 \\
\hline 25 & 5.5 & 5.8 & 6.0 & 5.9 & 6.0 & 6.0 & 6.1 & 6.2 & 6.1 & 6.4 & 6.1 & 6.3 \\
\hline Eom & 5.6 & 6.1 & 5.9 & 6.0 & 6.0 & 5.9 & 6.0 & 6. 2 & 6.0 & 6. 2 & 6.3 & 6.5 \\
\hline \multicolumn{13}{|c|}{1959} \\
\hline 5 & 6.4 & 6.1 & 6.2 & 6.3 & 6.3 & 6.0 & 6.1 & 6.3 & 6.3 & 6.1 & 5.8 & 6.0 \\
\hline 10 & 6.5 & 6.1 & 6.1 & 6.1 & 6.2 & 6.0 & 6.0 & 6.3 & 6.3 & 6.1 & 6.1 & 6.0 \\
\hline 15 & 6.1 & 6. 3 & 6.3 & 6.1 & 6.1 & 6.0 & 6.1 & 6. 3 & 6.0 & 6.0 & 6.1 & 6.0 \\
\hline 20 & 6.1 & 5.9 & 5.9 & 6.3 & 6.2 & 6.1 & 6.2 & 6.2 & 6.2 & 5.9 & 5.9 & 5.9 \\
\hline 25 & e6. 1 & 6.2 & 6.2 & 6. 3 & 6.1 & 6.1 & 6.1 & 6.3 & 6.1 & 6.1 & 5.9 & 6. 1 \\
\hline Eom & 6.1 & 6.2 & 6.2 & 6.3 & 6.2 & 6.0 & 6.1 & 6. 3 & 6.1 & 6. 1 & 6.0 & 6.0 \\
\hline \multicolumn{13}{|c|}{1960} \\
\hline 5 & 5.9 & 5.6 & 5.9 & 5.8 & 5.7 & 6.3 & 6.2 & 5.9 & 6.3 & 6.2 & e6. 0 & 6.3 \\
\hline 10 & 5.8 & 5.5 & 5.6 & 6.0 & 6.0 & 6.0 & 6.1 & 5.9 & 6.3 & 6.1 & e6. 0 & 6.1 \\
\hline 15 & 5.7 & 5. 6 & 5.7 & 6.0 & 6.1 & 6.1 & 5.9 & 6.0 & 6.0 & 6.1 & 6.0 & 6. 1 \\
\hline 20 & 5. 6 & 5.7 & 5.8 & 5.7 & 6.1 & 6.3 & 6.1 & 6.0 & 6.0 & 6.0 & 6.2 & 6.2 \\
\hline 25 & 5.7 & 5. 6 & 5.8 & 5. 8 & 6.0 & 6. 2 & 6.2 & 6.1 & 6.3 & 6.1 & 6.2 & 6.1 \\
\hline Eom & 5.8 & 5. 7 & 5.8 & 5.9 & 6.1 & 6.0 & 6.2 & 6.1 & 6.1 & 6.0 & 6.2 & 6.1 \\
\hline
\end{tabular}

Shaft 7. Olaa Sugar Co. Olaa. Lat $19^{\circ} 37^{\prime} 50^{\prime \prime}$, long $155^{\circ} 02^{\prime} 00^{\prime \prime}$. Dug domestic and irrigation basal water-table well in basalt of Kahuku Volcanic Series, Pleistocene age, size $10 \times 10 \mathrm{ft}$, depth $203 \mathrm{ft}$; three infiltration tunnels, total length $48 \mathrm{ft}$. Lsd $220 \mathrm{ft}$ above msl. MP pump room floor, 22. $86 \mathrm{ft}$ above msl. Highest water level 25. 86 above msl, Mar. 6, 1939; lowest 12.53 above msl, Dec. 5, 1953. Records available: 1936-60. Records furnished by Olaa Sugar Co.

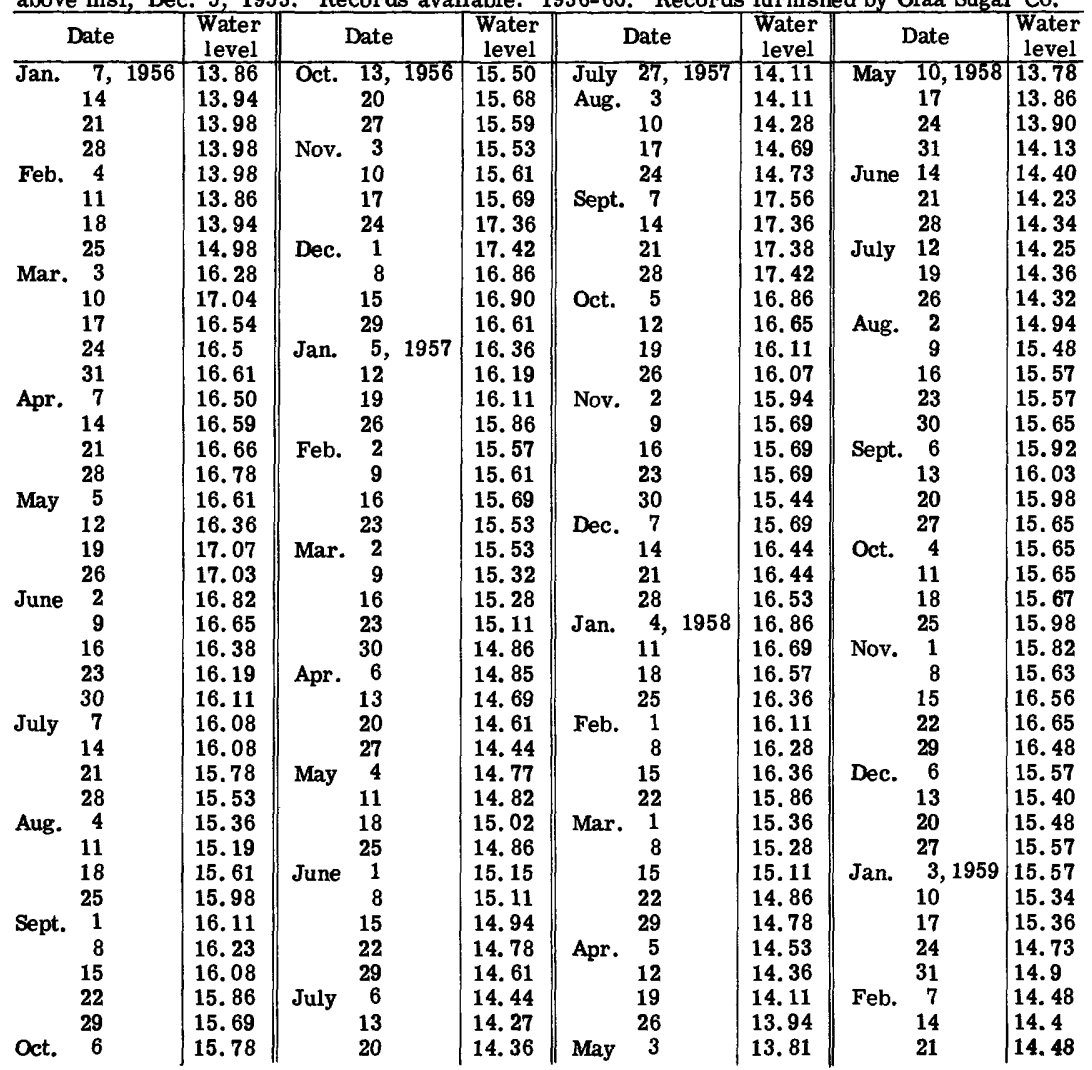




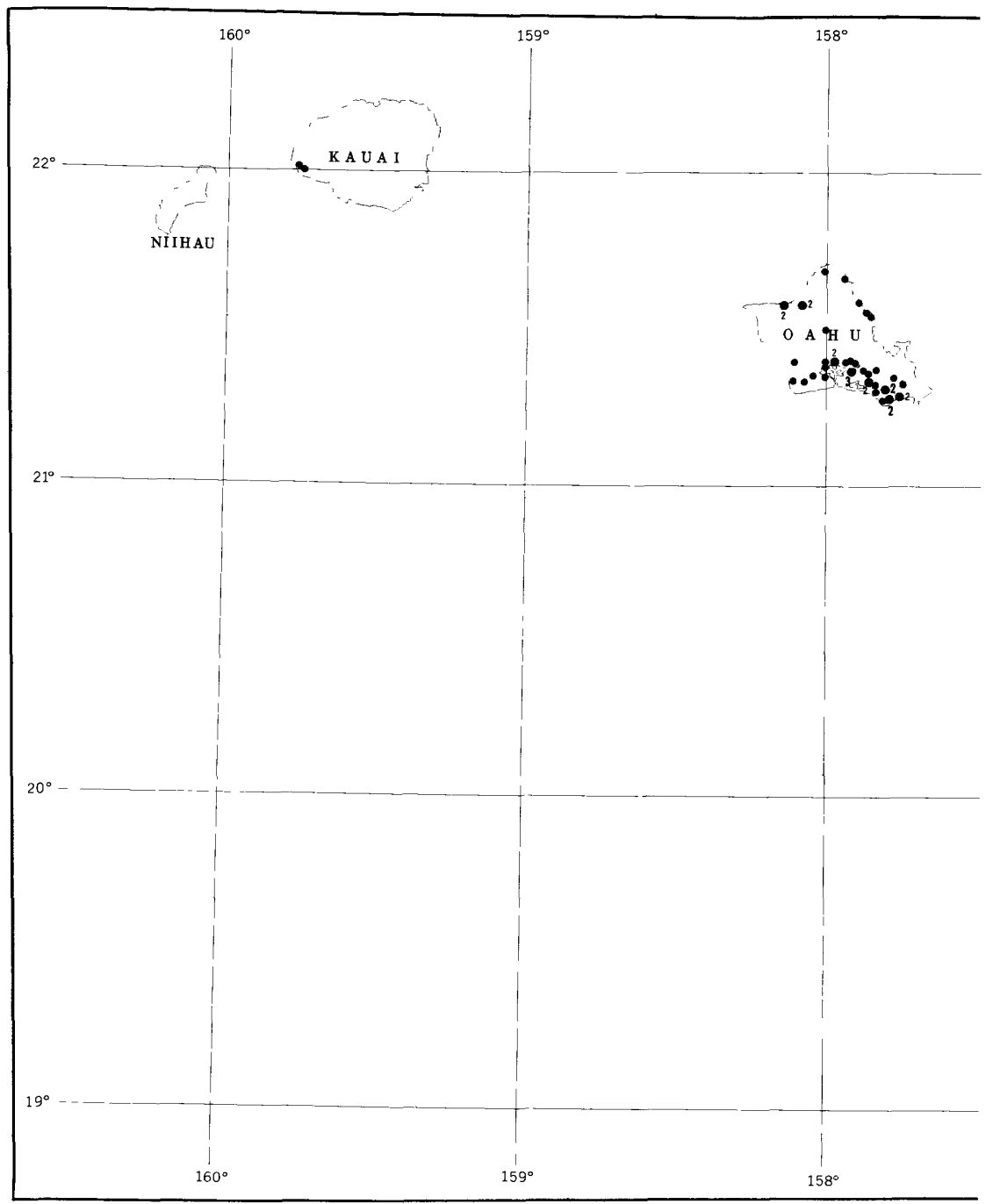




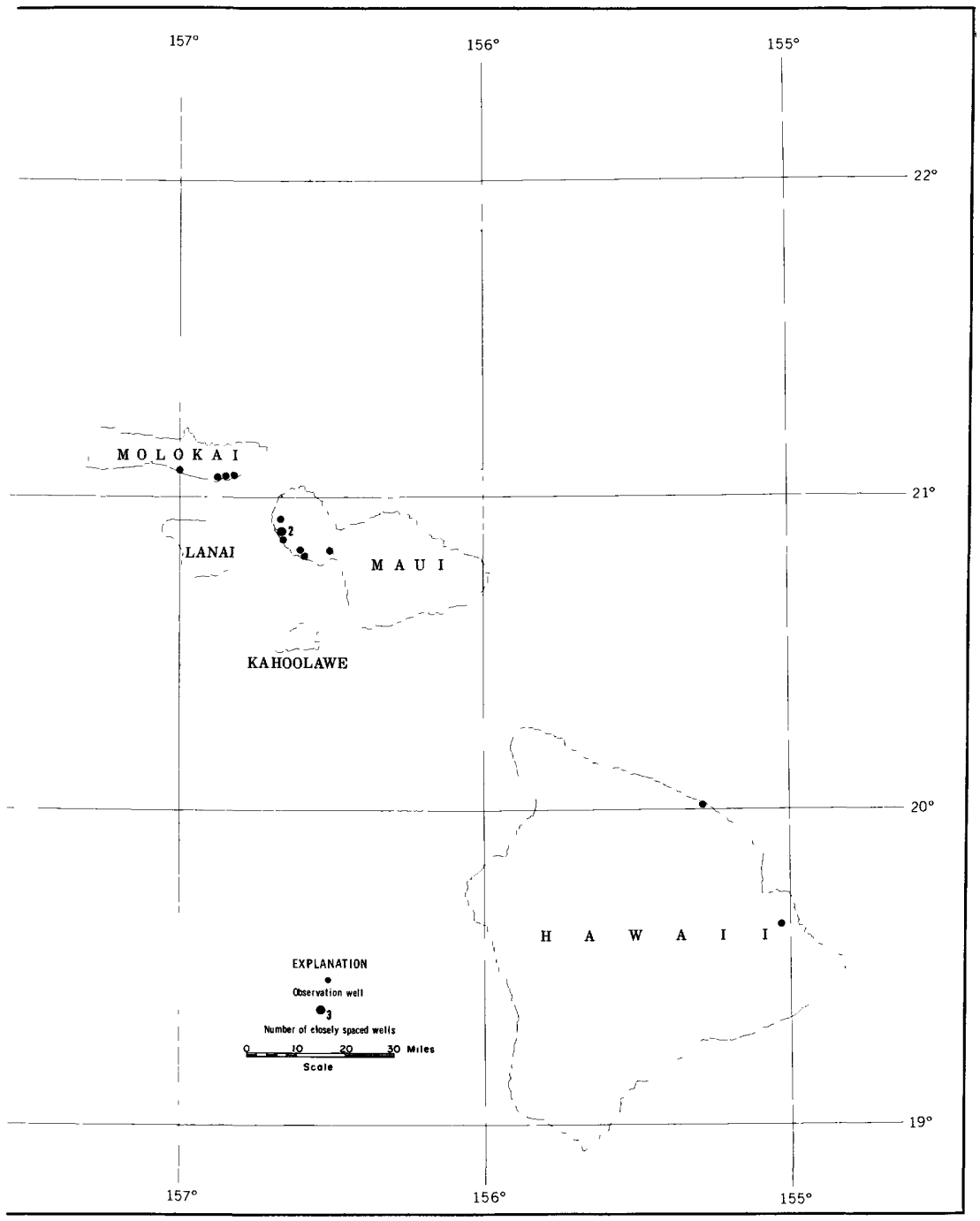

wells in Hawaii, 1956-60. 
Shaft 7--Continued.

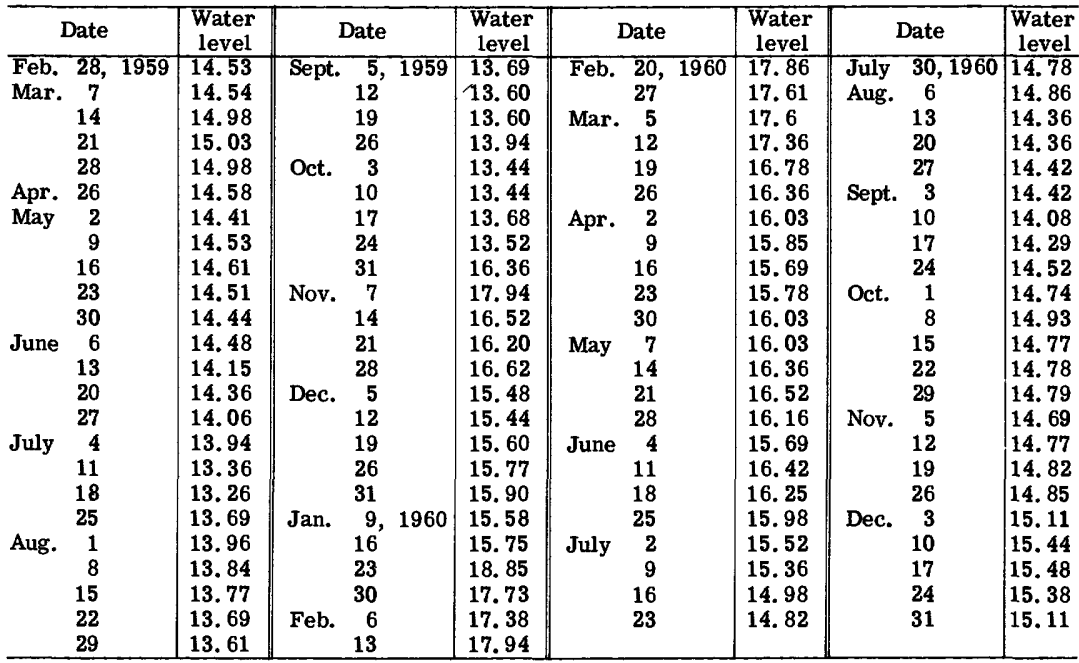

\section{Island of Kauai}

35. Kekaha Sugar Co. Near Kekaha. Lat $22^{\circ} 00^{\prime} 10^{\prime \prime}$, long $159^{\circ} 44^{\prime} 50^{\prime \prime}$. Drilled irrigation artesian basal-water well in basalt, diam $12 \mathrm{in}$, depth $245 \mathrm{ft}$, cased to 168 . Lsd 7. $82 \mathrm{ft}$ above msl. Highest water level 11.32 above msl, Dec. 20, 1937; lowest 7.63 above msl, Apr. 17, 1944. Records available: 1937-60. Records furnished by Kekaha Sugar Co.

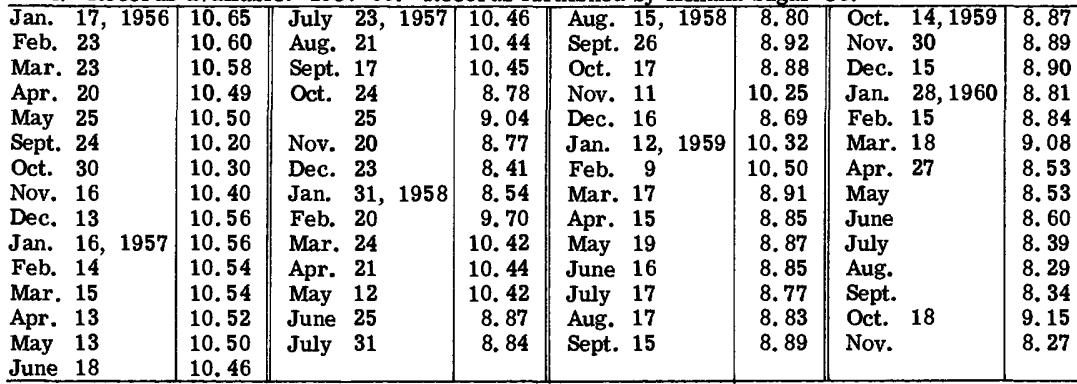

37. Kekaha Sugar Co. Near Kekaha. Lat $22^{\circ} 00^{\prime} 45^{\prime \prime}$, long $159^{\circ} 45^{\prime} 20^{\prime \prime}$. Drilled irrigation artesian basal-water well in basalt, diam $12 \mathrm{in}$, depth $262 \mathrm{ft}$, cased to 188 . Lsd 9.98 ft above msl. MP top of flange on 12-in T, $7.48 \mathrm{ft}$ above msl. Highest water level 11.08 above msl, Feb. 15, 1943; lowest 7.93 above msl, June 14, 1947. Records available: 1937-50, 1952-60. Records furnished by Kekaha Sugar Co.

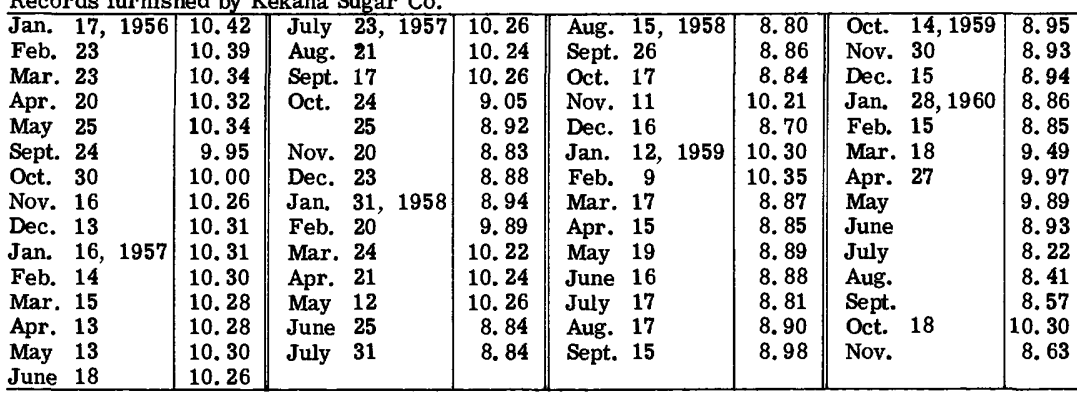




\section{Island of Maui}

T-110. Wailuku Sugar Co. Near Puu Hele. Lat $20^{\circ} 49^{\prime} 20^{\prime \prime}$, long $156^{\circ} 31^{\prime} 01^{\prime \prime}$. Drilled observation basal water-table well in basalt of Wailuku Volcanic Series, Pliocene (?) age, diam $\frac{3}{4}$ in, depth $325 \mathrm{ft}$, cased to 313 , perforated $309-313$. Lsd $312.67 \mathrm{ft}$ above msl. MP top of casing, $312.67 \mathrm{ft}$ above msl. Highest water level 8.9 above msl, Sept. 15, 1950; lowest 3.6 above msl, Oct. 19, 1955. Records available: 1939-57. Well abandoned; measurement discontinued. Records furnished by owner.

\begin{tabular}{c|c|c|c|c|c||c|c}
\hline \multicolumn{1}{c|}{ Date } & $\begin{array}{c}\text { Water } \\
\text { level }\end{array}$ & Date & $\begin{array}{c}\text { Water } \\
\text { level }\end{array}$ & \multicolumn{1}{|c|}{ Date } & $\begin{array}{c}\text { Water } \\
\text { level }\end{array}$ & Date & $\begin{array}{c}\text { Water } \\
\text { level }\end{array}$ \\
\hline Jan. 17, 1956 & 8.3 & July 19, 1956 & 5.9 & Nov. 15, 1956 & 5.8 & Mar. 19, 1957 & 4.6 \\
Apr. 17 & 5.8 & Aug. 20 & 5.4 & Dec. 14 & 6.8 & Apr. 16 & 5.9 \\
May 21 & 5.8 & Sept. 24 & 5.9 & Jan. 16, 1957 & 4.1 & May 20 & 3.9 \\
June 24 & 5.8 & Oct. 23 & 4.8 & Feb. 22 & 4.8 & July 17 & 4.6 \\
\hline
\end{tabular}

Shaft 3. Pioneer Mill Co., Ltd. Kaanapali. Lat $20^{\circ} 56^{\prime} 30^{\prime \prime}$, long $156^{\circ} 41^{\prime} 30^{\prime \prime}$. Dug irrigation basal water-table well in basalt of Wailuku Volcanic Series, Pliocene (?) age, depth $25 \mathrm{ft}$; two infiltration tunnels, total length of tunnels $1,561 \mathrm{ft}, 11$ supplemental drilled wells at bottom of shaft. Lsd $27 \mathrm{ft}$ above msl. Highest water level 2.98 above msl, Dec. 31, 1948; lowest 1.43 above msl, Dec. 31, 1949. Records available: 1937-58, 1960. No measurement made in 1959. Records furnished by Pioneer Mill Co., Ltd. Dec. 31, 1956, 1.9; Dec. 3, 1957, 2. 0; Dec. 3, 1.4 (well being pumped); Dec. 5, 1958, 1.5 (well being pumped); Dec. 31, 2.5; Dec. 30, 1960, 1.9 (well being pumped); Dec. 31, 2.1.

Shaft 5. Pioneer Mill Co., Ltd. Kahoma. Lat $20^{\circ} 53^{\prime} 50^{\prime \prime}$, long $156^{\circ} 40^{\prime} 00^{\prime \prime}$. Dug irrigation basal water-table well in basalt of Wailuku Volcanic Series, Pliocene (?) age, depth $323 \mathrm{ft}$; two infiltration tunnels, total length of tunnels $3,801 \mathrm{ft}$. Lsd $322 \mathrm{ft}$ above msl. Highest water level 3.78 above msl, Dec. 31, 1948; lowest 1.93 above msl, Dec. 31, 1945. Records available: 1937-58, 1960. No measurement made in 1959. Records furnished by Pioneer Mill Co., Ltd. Dec. 31, 1956, 2. 4; Dec. 1, 1957, 3.1; Dec. 1, 2. 0 (well being pumped); Dec. 29, 1958, 2.82 (well being pumped); Dec. 31, 3.20; Dec. 29, 1960, 1.8 (well being pumped); Dec. 31, 2. 2.

Shaft 7. Pioneer Mill Co., Ltd. Plantation mill in Lahaina. Lat $20^{\circ} 53^{\prime} 00^{\prime \prime}$, long $156^{\circ}$ $40^{\prime} 40^{\prime \prime}$. Dug irrigation basal water-table well in basalt of Wailuku Volcanic Series, Pliocene (?) age, depth $39 \mathrm{ft}$; one infiltration tunnel, length $768 \mathrm{ft}, 3$ supplemental drilled wells at bottom of shaft. Lsd $34 \mathrm{ft}$ above msl. Highest water level 3.63 above msl, Dec. 31, 1940; lowest 2.6 above msl, Dec. 31, 1953. Records available: 1937-58, 1960. No measurement made in 1959. Records furnished by Pioneer Mill Co., Ltd. Dec. 31, 1956, 3.0; Dec. 31, 1957, 3.1; Dec. 31, 0.5 (well being pumped); Dec. 31, 1958, 1.50 (well being pumped); Dec. 31, 3. 43; Dec. 23, 1960, 0.6 (well being pumped); Dec. $31,3.0$.

Shaft 9. Pioneer Mill Co., Ltd. Lahaina. Lat $20^{\circ} 52^{\prime} 25^{\prime \prime}$, long $156^{\circ} 40^{\prime} 15^{\prime \prime}$. Dug irrigation basal water-table well in basalt of Wailuku Volcanic Series, Pliocene (?) age, depth $31 \mathrm{ft}$; one infiltration tunnel, length $1,094 \mathrm{ft}, 10$ supplemental drilled wells at bottom of shaft. Lsd $30 \mathrm{ft}$ above msl. Highest water level 2. 83 above msl, Dec. 31, 1948; lowest 1.5 above msl, Dec. 31, 1953. Records available: 1937-58, 1960. No measurement made in 1959. Records furnished by Pioneer Mill Co., Ltd. Dec. 31, 1956, 1.7; Dec. 27, 1957, 2.1; Dec. 27, 1. 2 (well being pumped); Dec. 24, 1958, 1. 20 (well being pumped); Dec. 31, 2.52; Dec. 27, 1960, 0.8 (well being pumped); Dec. $31,1.8$.

Shaft 10. Pioneer Mill Co., Ltd. Olowalu. Lat $20^{\circ} 49^{\prime} 30^{\prime \prime}$, long $156^{\circ} 37^{\prime} 15^{\prime \prime}$. Dug irrigation basal water-table well in basalt of Wailuku Volcanic Series, Pliocene (?) age, vertical depth of 30-degree inclined shaft $300 \mathrm{ft}$; one infiltration tunnel, length $239 \mathrm{ft}$. Lsd $165 \mathrm{ft}$ above msl. Highest water level 4.3 above msl, Dec. 31, 1950; lowest 3.1 above msl, Dec. 31, 1953. Records available: 1937-58, 1960. No measurement made in 1959. Records furnished by Pioneer Mill Co., Ltd. Dec. 31, 1956, 3.2; Nov. 20, 1957, 3. 8; Nov. 20, 1.5 (well being pumped); Dec. 29, 1958, 3.14 (well being pumped); Dec. 31, 3. 80; Dec. 27, 1960, 2.5 (well being pumped); Dec. 31, 3.3.

Shaft 12. Pioneer Mill Co., Ltd. Ukumehame. Lat $20^{\circ} 48^{\prime} 45^{\prime \prime}$, long $156^{\circ} 35^{\prime} 50^{\prime \prime}$. Dug irrigation basal water-table well in basalt of Wailuku Volcanic Series, Pliocene (?) age, vertical depth of 30-degree inclined shaft $143 \mathrm{ft}$; one infiltration tunnel, length $428 \mathrm{ft}$. Lsd $79 \mathrm{ft}$ above msl. Highest water level 6.7 above msl, Dec. 31, 1950; lowest 4.27 above msl, Dec. 31, 1943. Records available: 1937-58, 1960. No measurement made in 1959. Records furnished by Pioneer Mill Co., Ltd. Dec. 31, 1956, 5.6; Nov. 20, 1957, 5.8; Nov. 20, 3.4 (well being pumped); Dec. 23, 1958, 4.98 (well being pumped); Dec. 31, 5.61; Dec. 12, 1960, 4.0 (well being pumped); Dec. $31,5.0$. 


\section{Island of Molokai}

Shaft 4. Molokai Ranch Co. Kawela Gulch. Lat $21^{\circ} 04^{\prime} 20^{\prime \prime}$, long $157^{\circ} 57^{\prime} 00^{\prime \prime}$. Dug publicsupply basal water-table well in basalt of East Molokai Volcanic Series, Pliocene (?) age, size $4 \times 4 \mathrm{ft}$, depth $38 \mathrm{ft}$, lined with concrete; two infiltration tunnels, total length $229 \mathrm{ft}$. Lsd $37.64 \mathrm{ft}$ above msl. MP top of steel I-beam under pump, $37.07 \mathrm{ft}$ above msl. Highest water level 2.57 above msl, Dec. 19, 1947; lowest 1.47 above msl, June 24, 1955 . Records available: 1947-60.

\begin{tabular}{|c|c|c|c|c|c|c|c|c|}
\hline \multicolumn{2}{|r|}{ Date } & $\begin{array}{c}\text { Water } \\
\text { level }\end{array}$ & Date & $\begin{array}{c}\text { Water } \\
\text { level }\end{array}$ & Date & $\begin{array}{c}\text { Water } \\
\text { level }\end{array}$ & Date & $\begin{array}{l}\text { Water } \\
\text { level }\end{array}$ \\
\hline Jan. & 3,1956 & 2.04 & Feb. 24,1956 & 2.00 & Jan. 20,1957 & 2.09 & Jan. 29,1959 & 2.14 \\
\hline & 13 & 1.56 & Mar. 6 & 1.68 & May 20 & 1. 80 & Mar. 25 & 2,12 \\
\hline & 14 & 2.00 & 7 & 1.83 & July & 1. 88 & July 30 & a2. 08 \\
\hline & 16 & 2. 00 & 15 & 1. 80 & Nov. & 1. 68 & Jan. 27,1960 & a1. 99 \\
\hline & 23 & 1.87 & May 21 & 1.85 & Feb. 24,1958 & 1.83 & May & 1.84 \\
\hline & 26 & 1.93 & July 22 & 1.75 & Apr. 28 & 1.72 & Aug. 16 & 1.74 \\
\hline & 31 & 2. 00 & Sept. 11 & 1. 87 & June 23 & 1.87 & Oct. & 1.99 \\
\hline Feb. & $\begin{array}{l}10 \\
16\end{array}$ & $\begin{array}{l}1.96 \\
1.70\end{array}$ & Nov. 21 & 2. 01 & Aug. 11 & 2. 20 & Nov. 30 & 2.14 \\
\hline
\end{tabular}

a Well being pumped.

Shaft 6. County of Maui. Ualapue. Lat $21^{\circ} 04^{\prime} 00^{\prime \prime}$, long $156^{\circ} 50^{\prime} 00^{\prime \prime}$. Dug public-supply basal water-table well in basalt of East Molokai Volcanic Series, Pliocene (?) age, size $4 \mathrm{x} 6 \mathrm{ft}$, depth $42 \mathrm{ft}$, lined with concrete; two infiltration tunnels, total length $214 \mathrm{ft}$. Lsd $43.71 \mathrm{ft}$ above msl. MP top of steel plate supporting gage, $44.42 \mathrm{ft}$ above msl. Highest water level 6.05 above msl, Jan. 19, 1950; lowest 4.57 above msl, June 21, 1955. Records available: 1938-60.

\begin{tabular}{|c|c|c|c|c|c|c|c|c|c|c|}
\hline Jan. & 31,1956 & 5.50 & Jan. 25,1957 & 5.33 & Apr. & 30,1958 & 4.84 & Mar. & 14,1960 & 5.17 \\
\hline Mar. & 7 & 5.29 & Mar. 23 & 5.17 & June & 23 & 4.99 & May & 2 & 4.95 \\
\hline May & 21 & 5.14 & May 20 & 5.05 & Aug. & 11 & 5.63 & & 6 & 4. 88 \\
\hline July & 22 & 5.16 & 29 & 4. 95 & Jan. & 29,1959 & 5.55 & & 9 & 4. 88 \\
\hline Aug. & 17 & 5.14 & July & 4. 87 & Mar. & 25 & 5.36 & & 13 & 4.88 \\
\hline Sept. & 11 & 5.20 & Sept. 10 & 5.14 & May & 27 & 5.29 & June & 3 & 4.81 \\
\hline Nov. & 21 & 5.29 & Nov. & 4. 87 & July & 30 & 5.70 & Aug. & 16 & 4. 68 \\
\hline ec. & 26 & 5.30 & 6,1958 & 5.08 & Jan. & 27,1960 & 5.14 & Oct. & 4 & 5.01 \\
\hline an. & 20,1957 & 5.25 & Feb. 24 & 4.92 & & & & & & \\
\hline
\end{tabular}

11. Molokai Ranch. Kaunakakai Gulch. Lat $21^{\circ} 06^{\prime}$, long $157^{\circ} 01^{\prime}$. Drilled unused basal water-table well in basalt of East Molokai Volcanic Series, Pliocene (?) age, diam 12 in, depth $59 \mathrm{ft}$, cased to 20 . Lsd $51 \mathrm{ft}$ above msl. MP top of surface casing, $51.95 \mathrm{ft}$ above msl. Highest water level 2.75 above msl, Nov. 21, 1956; lowest 1.87 above msl, Aug. 16, 1960. Records available: $1954-60$.

\begin{tabular}{l|r||l|l||ll|l||l|r|}
\hline Jan. 23, 1956 & 2.31 & Mar. 24, 1957 & 2.42 & May 1, 1958 & 2.05 & Dec. 3,1959 & 2.40 \\
Mar. 7 & 2.29 & May 14 & 2.26 & June 23 & 2.15 & Jan. 28, 1960 & 2.25 \\
May 11 & 2.23 & July 9 & 2.40 & Aug. 11 & 2.20 & Mar. 14 & 2.30 \\
July 11 & 2.20 & Aug. 20 & 2.40 & Jan. 26, 1959 & 2.50 & May 9 & 2.30 \\
& 22 & 2.20 & Sept. 13 & 2.45 & Mar. 23 & 2.35 & Aug. 16 & 1.87 \\
Sept. 21 & 2.35 & Nov. 5 & 2.05 & May 27 & 2.30 & Oct. 4 & 2.51 \\
Nov. 21 & 2.75 & Jan. 7,1958 & 2.05 & July 27 & 2.40 & Nov. 26 & 2.54 \\
Jan. 21, 1957 & 2.32 & Feb. 24 & 2.08 & Sept. 30 & 2.55 & & & \\
\hline
\end{tabular}

42. County of Maui. Kamalo. Lat $21^{\circ} 03^{\prime} 30^{\prime \prime}$, long $156^{\circ} 52^{\prime} 25^{\prime \prime}$. Dug unused (previously reported public-supply) basal water-table well in basalt of East Molokai Volcanic Series, Pliocene (?) age, size $4 \times 4 \mathrm{ft}$, depth $40 \mathrm{ft}$, lined with boulders. Lsd $43.23 \mathrm{ft}$ above msl. MP south edge of well cover opening, $42.67 \mathrm{ft}$ above msl. Highest water level 5. 40 above msl, Dec. 5, 1950; lowest 4.10 above msl, May 19, 1951. Records available: 1938-60.

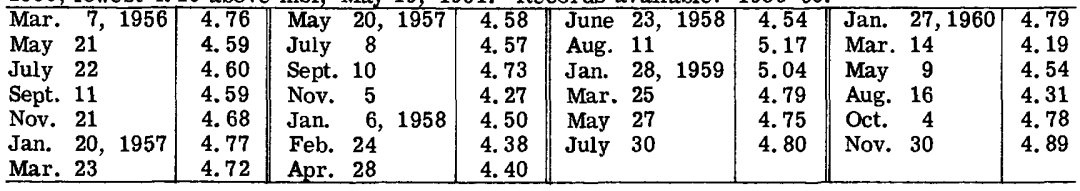

\section{Island of Oahu}

1A. B. P. Bishop Estate. Waialae Golf Links, Honolulu. Lat $21^{\circ} 16^{\prime} 45^{\prime \prime}$, long $157^{\circ} 46^{\prime} 45^{\prime \prime}$. Drilled unused artesian basal-water well in basalt of Koolau Volcanic Series, Pliocene (?) age, diam $4 \mathrm{in}$, depth $131 \mathrm{ft}$, cased to 100 . Lsd $18 \mathrm{ft}$ above msl. MP top of casing, 13. $84 \mathrm{ft}$ above msl. Highest water level 9.10 above msl, Feb. 4, 1940; lowest 7.55 above msl, June 14, 1946. Records available: 1933-44, 1947-60 (previously reported 1933-55). Records furnished by Honolulu Board of Water Supply. Jan. 5, 1959, 8.77; Jan. 10, 8.80; Jan. 15, 8.84; Jan. 20, 8.81; Aug. 26, 8.76; July 21, $1960,7.76$. 
1A--Continued.

Mean water level for the day, above msl, from recorder graph, 1956

\begin{tabular}{|c|c|c|c|c|c|c|c|c|c|c|c|c|}
\hline Day & Jan. & Feb. & Mar. & Apr. & May & June & July & Aug. & Sept. & Oet. & Nov. & Dec. \\
\hline 5 & 8.38 & 8.41 & 8.31 & 8.22 & 8.18 & 8.12 & 8.05 & 8.09 & 8.06 & 8.15 & 8.20 & 8.38 \\
\hline 10 & 8.36 & 8. 32 & 8.26 & 8.21 & 8. 17 & 8,15 & & 8.09 & ... & 8.15 & 8. 21 & $\ldots$ \\
\hline 15 & 8.41 & 8. 38 & 8. 22 & 8.21 & 8.21 & 8.12 & 8.06 & 8.03 & ... & 8. 18 & 8. 22 & 8. 37 \\
\hline 20 & 8. 39 & 8. 38 & 8. 18 & 8. 15 & 8.16 & 8.09 & 8.02 & 8.03 & $\ldots$ & 8.17 & 8.28 & 8. 36 \\
\hline 25 & 8.42 & 8. 38 & 8.25 & 8. 19 & 8.13 & $\cdots$ & & & & 8. 18 & 8.31 & 8. 34 \\
\hline Eom & 8.41 & 8. 37 & 8.25 & 8.20 & 8.14 & & 8.02 & 8.05 & 8.14 & 8.18 & 8.38 & 8.33 \\
\hline \multicolumn{13}{|c|}{1957} \\
\hline 5 & 8.32 & 8.57 & 8.46 & 8.24 & $8 . \overline{19}$ & 8.12 & 8.10 & 8.14 & 8.18 & 8.15 & 8.04 & 8.10 \\
\hline 10 & 8.28 & 8.53 & 8. 44 & 8. 23 & 8.12 & 8.11 & 8.10 & 8.18 & 8.16 & 8. 09 & 8.07 & 8. 08 \\
\hline 15 & 8.33 & 8. 54 & 8. 36 & 8.27 & 8.17 & 8. 10 & 8.11 & 8.15 & 8.17 & 8. 09 & 8.08 & 8. 10 \\
\hline 20 & 8.43 & 8.5 & 8.46 & 8. 20 & 8.17 & 8.09 & 8.13 & 8.14 & 8.17 & 8.11 & 8.10 & e8.11 \\
\hline 25 & 8.61 & 8. 51 & 8.31 & 8.20 & 8.12 & 8.07 & 8. 12 & 8.18 & 8.17 & 8. 08 & 8.12 & e8. 09 \\
\hline Eom & 8.59 & 8.50 & 8.30 & 8.22 & 8.13 & e8.08 & 8.15 & 8.18 & 8.16 & 8.03 & 8.11 & e8.07 \\
\hline \multicolumn{13}{|c|}{1958} \\
\hline 5 & 8.07 & 8.03 & 8.15 & 8.20 & 8.03 & 8.02 & 8.12 & & & .... & & e8.57 \\
\hline 10 & & 8.05 & 8.41 & 8. 15 & 8.08 & 8.04 & 8. 09 & $\cdots$ & $\cdots$ & $\cdots$ & . & 8. 70 \\
\hline 15 & 8.05 & 8.08 & 8.37 & 8.11 & 8.08 & 8. 04 & e8. 08 & $\cdots$ & & & • & 8.74 \\
\hline 20 & 8.06 & 8.13 & 8.31 & 8.13 & 8.07 & 8.02 & .... & - & e8. 48 & ... & $\ldots$ & 8.70 \\
\hline 25 & 8.00 & 8. 09 & 8.28 & 8.07 & 8.05 & 8.0 & .... & $\ldots$ & $\cdots$ & $\cdots$ & $\ldots$ & 8. 72 \\
\hline Eom & 7.99 & 8.14 & 8. 21 & 8. 06 & 8.05 & 8.07 & & & & & & e8. 77 \\
\hline
\end{tabular}

Estimated.

1B. B. P. Bishop Estate. Waialae Golf Links, Honolulu. Lat $21^{\circ} 16^{\prime} 45^{\prime \prime}$, long $157^{\circ} 46^{\prime} 50^{\prime \prime}$. Drilled domestic and irrigation artesian basal-water well in basalt of Koolau Volcanic Series, Pliocene (?) age, diam $8 \mathrm{in}$, depth $120 \mathrm{ft}$, cased to 43 . Lsd $18.22 \mathrm{ft}$ above msl. MP top of blind flange on well, $8.22 \mathrm{ft}$ above msl. Highest water level 8.94 above msl, Jan. 27, 1943; lowest 6.45 above msl, Oct. 20, 1933 . Records available: 1919, 1929-34, 1936-60.

\begin{tabular}{|c|c|c|c|c|c|c|c|}
\hline Date & $\begin{array}{l}\text { Water } \\
\text { level }\end{array}$ & Date & $\begin{array}{l}\text { Water } \\
\text { level }\end{array}$ & Date & $\begin{array}{c}\text { Water } \\
\text { level }\end{array}$ & Date & $\begin{array}{l}\text { Water } \\
\text { level }\end{array}$ \\
\hline Jan. 26,1956 & 8.41 & Apr. 24, 1957 & a8.06 & July 28,1958 & 8.17 & Oct. 26,1959 & 8.49 \\
\hline Feb. 28 & 8.33 & May 24 & a7. 87 & Aug. 28 & a8. 17 & Nov. 19 & 8.43 \\
\hline Mar. 28 & 8.23 & June 27 & 8. 07 & Sept. 26 & 8. 36 & Dec. 24 & 8.39 \\
\hline Apr. 25 & 8.18 & July 24 & 8.14 & Oct. 28 & a8. 41 & Jan. 26, 1960 & 8.24 \\
\hline May 24 & 8.10 & Aug. 28 & 8.28 & Nov. 26 & a8. 42 & Feb. 24 & 8.26 \\
\hline June 28 & 8.09 & Sept. 26 & 8.21 & Dec. 29 & 8.73 & Mar. 25 & 8.07 \\
\hline July 26 & 8. 02 & Oct. 24 & 8.05 & Jan. 26, 1959 & a8. 59 & Apr. 28 & 8.02 \\
\hline Aug. 29 & 8.01 & Nov. 26 & 8. 14 & Feb. 26 & 8. 73 & May 24 & 8.04 \\
\hline Sept. 27 & a8. 04 & Dec. 24 & 8.09 & Mar. 27 & 8.63 & June 24 & 7.94 \\
\hline Oct. 25 & 8.22 & Jan. 28, 1958 & 8. 02 & Apr. 23 & a8. 42 & July 21 & a7. 69 \\
\hline Nov. 21 & a8.26 & Feb. 25 & 8.06 & May 28 & 8.62 & Aug. 24 & 7. 87 \\
\hline Dec. 20 & 8.44 & Mar. 28 & 8.15 & June 22 & 8.70 & Sept. 21 & 7.85 \\
\hline Jan. 24, & 8.52 & Apr. 24 & 8.06 & July 24 & a8. 45 & Oct. 25 & a7. 81 \\
\hline Feb. 20 & 8.58 & May 28 & 7.97 & Aug. 27 & 8.77 & Dec. 29 & 8.04 \\
\hline Mar. 21 & 8.32 & June 25 & 8.06 & Sept. 23 & 8. 68 & & \\
\hline
\end{tabular}

a Well being pumped.

2. B. P. Bishop Estate. Kalei Rd., Honolulu. Lat $21^{\circ} 17^{\prime} 50^{\prime \prime}$, long $157^{\circ} 48^{\prime} 55^{\prime \prime}$. Drilled unused artesian basal-water well in basalt of Koolau Volcanic Series, Pliocene (?) age, diam $8 \mathrm{in}$, depth unknown, cased. Lsd $37 \mathrm{ft}$ above msl. MP top of casing, $37.38 \mathrm{ft}$ above msl.

Highest water level 31.55 above msl, Jan. 28, 1940; lowest 19.66 above msl, Sept. 14, 1944.

Records available: 1916, 1919, 1923, 1926, 1929-60. Records furnished by Honolulu Board of Water Supply.

Mean water level for the day, above msl, from recorder graph, 1956

\begin{tabular}{|c|c|c|c|c|c|c|c|c|c|c|c|c|}
\hline Day & Jan. & Feb. & Mar. & Apr. & May & June & July & Aug. & Sept. & Oct. & Nov. & Dec. \\
\hline 5 & & & & 29.06 & 28.49 & 28.14 & 27.43 & 26.37 & 25.64 & 25.45 & 26.57 & \\
\hline 10 & $\cdots$ & & & 28.89 & 28. 29 & 28.07 & 27. 24 & 26.06 & 25. 65 & 25.57 & 26.50 & 27.90 \\
\hline 15 & $\cdots$ & 29.79 & $\cdots$ & 28. 76 & 28. 30 & 28.11 & 27. 30 & 25.92 & 25.57 & 25.83 & 26.76 & 27.95 \\
\hline 20 & & $\cdots$ & $\cdots$ & 28. 87 & 28. 28 & 27.81 & 26.95 & 26.00 & 25.71 & 26.10 & 27.13 & 28. 05 \\
\hline 25 & 29.66 & & & 28. 96 & 28.06 & 27.81 & 26.75 & 25.85 & 25.57 & 26.31 & 27.37 & 28.18 \\
\hline Eom & & 29. 71 & 29.15 & 28.79 & 28.22 & 27.72 & 26.50 & 25.77 & 25.41 & 26.50 & 27.52 & 28.10 \\
\hline \multicolumn{13}{|c|}{1957} \\
\hline 5 & 27.90 & 28.90 & 28.88 & 27.75 & 28.43 & 27.45 & 26.90 & 26.38 & 26.58 & 26.42 & 26.01 & 26.41 \\
\hline 10 & 27.84 & 29.01 & 28. 67 & 27.91 & 28. 10 & 27.27 & 26.72 & 26.51 & 26.41 & 25.98 & 26.26 & 26.46 \\
\hline 15 & 28.0 & 29.08 & 28.53 & 28.03 & 28. 26 & 27.00 & 26.58 & 26.33 & 26. 51 & 25.96 & 26.30 & 26.48 \\
\hline 20 & 28. 28 & 29.11 & 28. 38 & 28. 17 & 28. 17 & 27.00 & 26.26 & 26.32 & 26. 54 & 26.05 & 26. 37 & 26.54 \\
\hline 25 & 28. 61 & 28. 99 & 28. 25 & 28.26 & 27.87 & 26.85 & 26.25 & 26.32 & 26. 55 & 25.97 & 26.39 & 26.54 \\
\hline Eom & 28.72 & 28. 95 & 28.12 & 28.37 & 27.67 & 26.88 & 26.35 & 26.54 & 26. 66 & 25.95 & 26.42 & 26.57 \\
\hline
\end{tabular}


2--Continued.

1958

\begin{tabular}{|c|c|c|c|c|c|c|c|c|c|c|c|c|}
\hline$\overline{\text { Day }}$ & Jan. & Feb. & Mar. & Apr. & $\overline{\text { May }}$ & June & July & Aug. & Sept. & Oct. & Nov. & Dec. \\
\hline 5 & 65 & 27 & e27 & 26.22 & 25.42 & 25.72 & e25. 30 & 25.34 & 25.51 & 25.48 & 26.46 & 05 \\
\hline 10 & 26. 75 & 27.12 & 27.62 & 26.02 & 25.30 & 25.76 & 25.30 & e25. 40 & 25.33 & 25.60 & 26. 54 & 27.08 \\
\hline 15 & 26.83 & 27.20 & 27.24 & 25.87 & 25.30 & 25.58 & 25.32 & 25.66 & 25.43 & 25.78 & 26. 58 & 27.10 \\
\hline 20 & 26.93 & 27.23 & 26.88 & 25.76 & e25. 20 & 25.37 & 25.41 & 25.41 & 25 & e26.00 & 26. 77 & .07 \\
\hline 25 & 26 & 27.20 & 26. & 25 & e25 & 25.29 & 25. 39 & 25.44 & 25.25 & 26 & e26.80 & 14 \\
\hline Eom & 27.01 & 27.30 & 26.36 & 25.52 & 25.59 & 25.31 & 25.20 & 25.64 & 25.27 & 26.36 & 26.90 & 27.8 \\
\hline
\end{tabular}

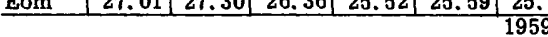

\begin{tabular}{|c|c|c|c|c|c|c|c|c|c|c|c|c|}
\hline & & & & & & & & & & & & \\
\hline 5 & 27.37 & $2 \overline{7} . \overline{7}$ & 27.76 & 27.44 & 26.70 & & & & & & & 24.80 \\
\hline 10 & 27.44 & 27.82 & 27.71 & 27.39 & 26.52 & . & .. & & $\ldots$ & . & .. & 25.04 \\
\hline 5 & 27.53 & 27.86 & 27.69 & 27.29 & 26.06 & $\cdots$ & ..... & . & & $\cdots$ & .. & 25 \\
\hline 0 & 27.61 & 27 & 27 & 27.20 & 25. & $\cdots$ & . & $\cdots$ & 24.43 & .... & $\ldots$ & 49 \\
\hline 25 & 27. & 27. & 27. & 26.94 & 26.10 & , . . & & $\ldots \ldots$ & $\cdots \cdots$ & - & 57 & 25.72 \\
\hline Eom & 27.73 & 27. & 27.35 & 26.90 & 26.16 & & & $\cdots$ & & ..... & 24. 83 & 25.49 \\
\hline
\end{tabular}

\begin{tabular}{l|l|l|l|l|l|l|l|l|l|l|l|l}
\hline $\mathbf{5}$ & 25.60 & 25.51 & 25.63 & 25.41 & 24.73 & 24.64 & 23.54 & 21.49 & 21.30 & 21.94 & 22.74 & 23.63 \\
10 & 25.69 & 25.39 & 25.84 & 25.48 & 24.65 & 24.15 & 23.23 & 21.30 & 21.52 & 21.94 & 22.91 & 23.82 \\
15 & 25.69 & 25.55 & 25.91 & 25.45 & 24.86 & 23.90 & 22.90 & 21.13 & 21.40 & 22.23 & 23.18 & 24.23 \\
20 & 25.58 & 25.60 & 25.80 & 25.45 & 24.88 & 23.77 & 22.60 & 20.91 & 21.46 & 22.12 & 23.19 & 24.46 \\
25 & 25.30 & 25.50 & 25.80 & 25.42 & 24.98 & 23.49 & 22.45 & 20.75 & 21.83 & 22.60 & 23.34 & 24.50 \\
Eom & 25.58 & 25.47 & 25.56 & 24.97 & 24.88 & 23.52 & 21.85 & 21.16 & 22.03 & 22.57 & 23.35 & 24.75 \\
\hline
\end{tabular}
e Estimated.

9. J. J. Gouveia. Kapahulu Ave, and Olu St., Honolulu. Lat $21^{\circ} 17^{\prime} 10^{\prime \prime}$, long $157^{\circ} 49^{\prime} 00^{\prime \prime}$. Drilled industrial artesian basal-water well in basalt of Koolau Volcanic Series, Pliocene (?) age, diam $6 \mathrm{in}$, depth $270 \mathrm{ft}$, cased to 256 . Lsd $16.08 \mathrm{ft}$ above msl. MP top of concrete pumpbase, $12.97 \mathrm{ft}$ above msl. Highest water level 30.92 above msl, Feb. 16, 1940; lowest 18.40 above msl, Aug. 17, 1926. Records available: 1921, 1923-60.

\begin{tabular}{|c|c|c|c|c|c|c|c|}
\hline Date & $\begin{array}{c}\text { Water } \\
\text { level }\end{array}$ & Date & $\begin{array}{c}\text { Water } \\
\text { level }\end{array}$ & Date & $\begin{array}{c}\text { Water } \\
\text { level }\end{array}$ & Date & $\begin{array}{l}\text { Water } \\
\text { level }\end{array}$ \\
\hline Jan. 25, 1956 & 29.57 & Feb. 20,1957 & 29.18 & Mar. 28, 1958 & 26.37 & Apr. 23, 1959 & 25.93 \\
\hline Feb. 28 & 29.67 & Mar. 21 & 28.37 & Apr. 24 & 25.63 & May 28 & 4.86 \\
\hline Mar. 28 & 29.12 & Apr. 24 & 28.12 & May 28 & 25.25 & June 22 & 24.13 \\
\hline Apr. 27 & 28.89 & May 24 & 27.97 & June 25 & 25.07 & July 30 & 22.58 \\
\hline May 24 & 28.05 & June 27 & 26.71 & July 28 & 25.27 & Aug. 27 & 23.40 \\
\hline June 28 & 27.67 & July 30 & 26.37 & Aug. 28 & 25.43 & Sept. 23 & 23.28 \\
\hline July 26 & 26.67 & Aug. 28 & 26. 60 & Sept. 26 & 25.01 & Oct. 26 & 23.46 \\
\hline Aug. 29 & 25.53 & Sept. 26 & 26.57 & Oct. 28 & 26.12 & Nov. 19 & 23.20 \\
\hline Sept. 27 & 25.32 & Oct. 24 & 25.97 & Nov. 26 & 26.73 & Dec. 24 & 21.78 \\
\hline Oct. 26 & 26.43 & Nov. 26 & 26. 27 & Dec. 29 & 27.13 & 4,1960 & 21.06 \\
\hline Nov. 21 & 27.18 & Dec. 24 & 26.27 & Jan. 26, 1959 & 27.53 & 24 & 21.26 \\
\hline Dec. 20 & 27.99 & Jan. 28, 1958 & 26.87 & Feb. 26 & 26.62 & Dec. 29 & 24.60 \\
\hline Jan. 24,1957 & 28.57 & Feb. 25 & 27.20 & Mar. 27 & 26.13 & & \\
\hline
\end{tabular}

36A. Honolulu Board of Water Supply. Wilder Ave. and Clement St., Honolulu. Lat $21^{\circ} 18^{\prime} 10^{\prime \prime}$, long $157^{\circ} 49^{\prime} 45^{\prime \prime}$. Drilled unused artesian basal-water well in basalt of Koolau Volcanic Series, Pliocene (?) age, diam 12 in, depth $395 \mathrm{ft}$. Lsd $43 \mathrm{ft}$ above msl. MP top of casing, $35.85 \mathrm{ft}$ above msl. Highest water level 33.35 above msl, Mar. 11, 1938; lowest 22.41 above msl, Oct. 13, 1945. Records available: 1924, 1929-32, 1934, 1938-58. Measurement suspended. Records furnished by Honolulu Board of Water Supply.

Mean water level for the day, above msl, from recorder graph, 1956

\begin{tabular}{|c|c|c|c|c|c|c|c|c|c|c|c|c|}
\hline$\overline{\text { Day }}$ & Jan. & Feb. & Mar. & Apr. & May & June & July & Aug. & Sept. & Oct. & Nov. & Dec. \\
\hline 5 & 30.71 & 31.38 & $\mathrm{e} 31.8$ & 31.30 & 30.55 & 30.40 & 29.39 & 28.86 & 28.70 & 28.66 & $29 . \overline{37}$ & 30.23 \\
\hline 10 & 30.84 & 31.42 & & 31.18 & 30.46 & 30.37 & 29.24 & 28.70 & 28. 69 & 28.82 & 29.40 & 30.36 \\
\hline 15 & 30 & 31.58 & 31.68 & 31.05 & 30. & 30. & 29.15 & 28. & 28.66 & 28.89 & 29.44 & 30.50 \\
\hline 20 & 31 & 31. & 31.52 & 30. & 30.39 & 30 & 29.10 & 28. & 28 & 28 & 34 & 30.62 \\
\hline 25 & 31.13 & 31.75 & 31.52 & 30.86 & 30.28 & 30.04 & 29.06 & 28. & 28 & 29.12 & 29.84 & - \\
\hline Eon & 31 & & & & 30. & 29.66 & 28. 87 & & & 29.27 & 30.14 & 30.72 \\
\hline \\
\hline 5 & 30.79 & 3 & 31.71 & 30.90 & 30.41 & 29.40 & 28.42 & & 27.42 & 14 & 26.91 & 28.12 \\
\hline 0 & 30.8 & 31 & 31.53 & 30.79 & 30.16 & 29.18 & 28. 20 & 27.42 & 27.37 & 27.05 & 27.10 & 28.44 \\
\hline 15 & & 31.80 & 31.35 & 30.71 & 30.11 & 29.00 & 28.15 & 27.32 & 27.30 & 26.97 & 27.11 & 28.76 \\
\hline 20 & 31.00 & 31.93 & 31.34 & 30.57 & 30.00 & 28.85 & 27.86 & 27.28 & 27.24 & 26.94 & 27.19 & 29.01 \\
\hline 25 & 31.23 & 31. & e31. 15 & 30.50 & 29.78 & 28.68 & 27.70 & 27.34 & 27.14 & 26.86 & 27.48 & 29.20 \\
\hline tor & 31.43 & 31.77 & 30.95 & 30.50 & 29. 63 & 28.52 & 27.58 & 27.30 & 27.19 & 26.87 & 27.92 & 29.23 \\
\hline
\end{tabular}


36A--Continued.

\begin{tabular}{|c|c|c|c|c|c|c|c|c|c|c|c|c|}
\hline \multicolumn{13}{|c|}{1958} \\
\hline Day & Jan. & Feb. & Mar. & Apr. & May & June & July & Aug. & Sept. & Oct. & Nov. & Dec. \\
\hline 5 & 29.23 & 28.89 & 29.08 & 29.54 & 29.09 & 28.50 & 27.72 & $\cdots \cdots$ & $\cdots \cdots$ & $\cdots \cdots$ & $\cdots$ & $\cdots \cdots$ \\
\hline 10 & 29.18 & 28. 85 & 29.39 & 29.19 & 28.88 & 28.22 & $\cdots$ & $\cdots \cdots$ & $\ldots . .$. & $\because$ & & \\
\hline 15 & e29. 10 & 28. 87 & 29.53 & 29.30 & 28.70 & 28.06 & $\ldots .$. & $\ldots$ & . & $\cdots$ & $\cdot$ & . \\
\hline 20 & $\cdots$ & 28.79 & 29. 71 & 29.24 & 28. 66 & 27.88 & .... & $\cdots \cdots$ & $\ldots \ldots$ & ..... & $\cdots$ & $\cdots$ \\
\hline 25 & 28.97 & 28. 81 & 29.59 & 29.13 & 28.56 & 27.99 & • & $\ldots$ & $\cdots$ & $\ldots \ldots$ & $\cdots \cdots$ & $\ldots$. \\
\hline Eom & 28.88 & 28.86 & 29.60 & 29.04 & 28.65 & 27.80 & $\ldots \ldots$ & $\ldots \ldots$ & $\ldots$ & $\ldots$ & $\ldots$ & ... \\
\hline
\end{tabular}

81. R. Sakimoto. Young and Victoria Sts., Honolulu. Lat $21^{\circ} 18^{\prime} 20^{\prime \prime}$, long $157^{\circ} 50^{\prime} 55^{\prime \prime}$.

Drilled domestic artesian basal-water well in basalt of Koolau Volcanic Series, Pliocene (?) age, diam $8 \mathrm{in}$, depth $505 \mathrm{ft}$, cased to 475 . Lsd $18.04 \mathrm{ft}$ above msl. MP top of vertical flange on main valve, $16.43 \mathrm{ft}$ above $\mathrm{msl}$. Highest water level 33.04 above msl, Feb. 28, 1938; lowest 21.99 above msl, Aug. 28, 1946. Records available: 1916, 1923-24, 1926, $1929-60$.

\begin{tabular}{|c|c|c|c|c|c|c|c|}
\hline Date & $\begin{array}{l}\text { Water } \\
\text { level }\end{array}$ & Date & $\begin{array}{c}\text { Water } \\
\text { level }\end{array}$ & Date & $\begin{array}{c}\text { Water } \\
\text { level }\end{array}$ & Date & $\begin{array}{c}\text { Water } \\
\text { level }\end{array}$ \\
\hline Jan. 25,1956 & 31.03 & Apr. 24,1957 & 30.08 & July 28,1958 & 27.03 & Oct. 26,1959 & 25.01 \\
\hline Feb. 28 & 31.53 & May 24 & 29.45 & Aug. 28 & 26.88 & Nov. 19 & 25.63 \\
\hline Mar. 28 & 31.28 & June 27 & 28.21 & Sept. 26 & 27.21 & Dec. 24 & 25.93 \\
\hline Apr. 27 & 30.53 & July 30 & 27.14 & Oct. 28 & 27.92 & Jan. 26,1960 & 26.08 \\
\hline May 24 & 29.86 & Aug. 28 & 27.06 & Nov. 26 & 28.58 & Feb. 24 & 26.03 \\
\hline June 28 & 29.33 & Sept. 26 & 26.83 & Dec. 29 & 29.03 & Mar. 25 & 25.69 \\
\hline July 26 & 28.63 & Oct. 24 & 26.53 & Jan. 26,1959 & 29.43 & Apr. 28 & .13 \\
\hline Aug. 29 & 28.24 & Nov. 26 & 27.23 & Feb. 26 & 29.83 & May 24 & 25.13 \\
\hline Sept. 27 & 28.18 & Dec. 24 & 28. 84 & Mar. 27 & 29.18 & June 24 & 4.53 \\
\hline Oct. 26 & 28.89 & Jan. 28,1958 & 28.43 & Apr. 23 & 28. 74 & July 21 & 24.03 \\
\hline Nov. 21 & 29.37 & Feb. 25 & 28. 63 & May 28 & 27.67 & Aug. 24 & 23.23 \\
\hline Dec. 20 & 30.13 & Mar. 28 & 29.41 & June 22 & 26.73 & Sept. 22 & 23.63 \\
\hline Jan. 24,1957 & 30.83 & Apr. 24 & 28.93 & July 24 & 25.68 & Oct. 25 & 24.31 \\
\hline Feb. 20 & 31.65 & May 28 & 28. 30 & Aug. 27 & 25.35 & Dec. & 24.85 \\
\hline Mar. 21 & 30.83 & June 25 & 27.63 & Sept. 23 & 25.33 & 29 & 26.25 \\
\hline
\end{tabular}

83. Honolulu Board of Water Supply. Formerly City and County of Honolulu. Beretania and Kapiolani Sts., Honolulu. Lat $21^{\circ} 18^{\prime} 20^{\prime \prime}$, long 157 $51^{\prime} 05^{\prime \prime}$. Drilled unused artesian basalwater well in basalt of Koolau Volcanic Series, Pliocene (?) age, diam 8 to 6 in, depth $509 \mathrm{ft}$, cased to 460 . Lsd $27 \mathrm{ft}$ above msl. MP top of 12-in standpipe, $32.60 \mathrm{ft}$ above msl. Highest water level 33. 29 above msl, Mar. 10, 1938; lowest 22. 07 above msl, Aug. 10, 1946. Records available: $1923,1925-48,1952-54,1957-60$. No measurement made in 1955 or 1956 . Records furnished by Honolulu Board of Water Supply.

Mean water level for the day, above msl, trom recorder graph, 1957

\begin{tabular}{|c|c|c|c|c|c|c|c|c|c|c|c|c|}
\hline$\overline{\text { Day }}$ & Jan. & Feb. & Mar. & Apr. & May & June & July & Aug. & Sept. & Oct. & Nov. & Dec. \\
\hline 5 & 30.69 & 31.47 & 31.60 & 30.80 & 30.30 & 29.30 & 28.30 & 27.50 & 27.30 & 27.00 & 26.84 & 28.13 \\
\hline 10 & 30.73 & 31.62 & 31.44 & 30.69 & 30.04 & 29.10 & 28.15 & 27.33 & 27.25 & 26.98 & 27.08 & 28.42 \\
\hline 15 & 30.81 & 31.70 & 31.25 & 30.63 & 30.00 & 28.90 & 28.09 & 27.20 & 27.25 & 26.91 & 27. 06 & 8. 74 \\
\hline 20 & 30.90 & 31.81 & 31. 25 & 30.44 & 29.90 & 28.77 & 27.75 & 27.17 & 27.16 & 26.89 & 15 & 95 \\
\hline 25 & 31.13 & 31.80 & 31.10 & 30.40 & 29. 70 & 28.62 & 27.58 & 27.30 & 27.02 & 26.81 & 27. 48 & 29.19 \\
\hline Eom & 31.36 & 31.65 & 30.86 & 30.36 & & 28.43 & 27 & 27.20 & 27.10 & 26.81 & 27.79 & 29.17 \\
\hline \multicolumn{13}{|c|}{1958} \\
\hline 10 & 29.19 & 28.84 & 29.06 & $\mathrm{e} 29.49$ & 28.94 & 28.54 & 27.72 & e27.00 & 27.28 & & & \\
\hline 10 & 29.13 & 28.82 & 29.35 & 29.29 & 28.80 & 28.31 & 27.74 & 27.26 & 27.30 & 27.48 & 45 & .86 \\
\hline 15 & 29.02 & 28.80 & 29.43 & e29. 22 & 28. 66 & 28.03 & 27.57 & 27.18 & e27. 30 & 27.57 & 58 & .04 \\
\hline 20 & 29.07 & 28.72 & 29.63 & 29.18 & e28. 60 & 27.89 & 27. 62 & e27. 10 & 27.51 & 27.85 & 76 & 29.11 \\
\hline 25 & 28. 92 & 28.72 & 29.50 & 29.09 & 28. 49 & 27.83 & 27 & 00 & 27.47 & 28.00 & 28. 76 & 29.14 \\
\hline Eom & 28. 83 & 28.77 & 29.60 & 29.05 & 28.59 & 27.83 & 27.07 & 20 & 27 & 28 & 28.80 & 29.27 \\
\hline \multicolumn{13}{|c|}{1959} \\
\hline 5 & $29.4 \overline{3}$ & 29.76 & 29.95 & 29.41 & 28.83 & 27.79 & 26.65 & 25.56 & 25.69 & 25.49 & 25.64 & .07 \\
\hline 10 & & e29 & 29.90 & 29.32 & 28.74 & 27.54 & 26.39 & 25.74 & 25.73 & 25.46 & 25. 87 & 11 \\
\hline 15 & 29.60 & 29.90 & 29.80 & 29.30 & 28.47 & 27.42 & 26.26 & 25.74 & 25.69 & 51 & 14 & .16 \\
\hline 20 & e29.60 & 29.90 & 29.66 & 29.20 & 28. 27 & 27.16 & 25.97 & 64 & 25.62 & 44 & .09 & .22 \\
\hline 25 & 29.60 & $\mathrm{e} 30.00$ & 29.42 & 29.07 & 28.10 & 26.92 & 25.65 & 25.70 & 25.49 & 47 & 26.17 & 26.34 \\
\hline Eom & 74 & 10 & e29. 30 & 28.94 & 27.96 & 26.77 & 59 & 25.76 & 25.52 & 25.56 & 26.18 & 26.28 \\
\hline \multicolumn{13}{|c|}{1960} \\
\hline 5 & 26.30 & 26.08 & 26.06 & 25.80 & 25.41 & 25.37 & 24.90 & 23.95 & 23.78 & 24 & .61 & $\overline{5} .07$ \\
\hline 10 & 26. 30 & 25.92 & 26.18 & 25.70 & 25.34 & 25.19 & 24.73 & 23. 80 & 23.82 & 16 & 68 & 25.20 \\
\hline 15 & 26.37 & 25 & 26. 18 & 25.74 & 25.40 & 25.12 & 24. 61 & 23.73 & 23.94 & 24.24 & 24.79 & 25.46 \\
\hline 20 & 44 & & 26.09 & 25.60 & 25.46 & 24.98 & 24.40 & 23. 64 & 23.98 & 24.28 & 24.86 & 25.58 \\
\hline 25 & & 25.95 & 25.97 & 25.69 & 25.48 & 24.84 & 24. 30 & 23.57 & 24.01 & 24.40 & 24.96 & 25 \\
\hline Eom & 26.29 & 91 & 25. 85 & 25.48 & 25.49 & 24.85 & 05 & 23.73 & 24.12 & 24.56 & 24.96 & 25.90 \\
\hline
\end{tabular}


119. Honolulu Gas Co. Honolulu Gas Works, Honolulu. Lat $21^{\circ} 19^{\prime} 05^{\prime \prime}$, long $157^{\circ} 52^{\prime} 25^{\prime \prime}$. Drilled industrial artesian basal-water well in basalt of Koolau Volcanic Series, Pliocene (?) age, diam $8 \mathrm{in}$, depth $682 \mathrm{ft}$, cased to 613 . Lsd $4.22 \mathrm{ft}$ above msl. MP top of concrete floor near pumps, $5.14 \mathrm{ft}$ above msl. Highest water level 32.55 above msl, Mar. 16, 1933; lowest 19.96 above msl, July 28, 1945. Records available: 1923-60.

\begin{tabular}{|c|c|c|c|c|c|c|c|}
\hline Date & $\begin{array}{l}\text { Water } \\
\text { level }\end{array}$ & Date & $\begin{array}{r}\text { Water } \\
\text { level }\end{array}$ & Date & $\begin{array}{r}\text { Water } \\
\text { level }\end{array}$ & Date & $\begin{array}{r}\text { Water } \\
\text { level }\end{array}$ \\
\hline Jan. 25,1956 & 27.34 & Apr. 24,1957 & 27.29 & July 28,1958 & 23.44 & Oct. $\quad 26,1959$ & 22.44 \\
\hline Feb. 28 & 28.74 & May 24 & 25.46 & Aug. 28 & 23. 69 & Nov. 19 & 22.74 \\
\hline Mar. 28 & 27.99 & June 27 & 24. 52 & Sept. 26 & 24.42 & Dec. 24 & 2.74 \\
\hline Apr. 27 & 26.94 & July 30 & 24.15 & Oct. 28 & 25.73 & Jan. 26, 1960 & 22.79 \\
\hline May 24 & 26.97 & Aug. 28 & 23.56 & Nov. 26 & 25.61 & Feb. 24 & 22.64 \\
\hline June 28 & 26.24 & Sept. 26 & 23.94 & Dec. 29 & 25.64 & Mar. 25 & 22.50 \\
\hline July 26 & 24.84 & Oct. 24 & 25.34 & Jan. 26,1959 & 26.44 & Apr. 28 & 22.24 \\
\hline Aug. 29 & 24.26 & Nov. 26 & 24.14 & Feb. 26 & 26.26 & May 24 & 22.14 \\
\hline Sept. 27 & 25.04 & Dec. 24 & 25.44 & Mar. 27 & 25. 86 & June 24 & 21.74 \\
\hline Oct. 26 & 25.88 & Jan. 28, 1958 & 25.04 & Apr. 23 & 24.44 & July 21 & 20.74 \\
\hline Nov. 21 & 26.14 & Feb. 25 & 25.44 & May 28 & 23.14 & Aug. 24 & 0.24 \\
\hline Dec. 20 & 27.34 & Mar. 28 & 25.02 & June 22 & 23.04 & Sept. 22 & 20.72 \\
\hline Jan. 24, & 27.04 & Apr. 24 & 26.07 & July 24 & 22. 20 & Oct. 25 & 21. 26 \\
\hline Feb. 20 & 28.16 & May 28 & 25.38 & Aug. 27 & 22.04 & Dec. & 22.56 \\
\hline Mar. 21 & 27.54 & June 25 & 24.24 & Sept. 23 & 21.99 & 29 & 23.06 \\
\hline
\end{tabular}

132. B. P. Bishop Estate. Old Kamehameha School, Honolulu. Lat $21^{\circ} 20^{\prime} 05^{\prime \prime}$, long $157^{\circ} 52^{\prime} 25^{\prime \prime}$. Drilled unused artesian basal-water well in basalt of Koolau Volcanic Series, Pliocene (?) age, diam 12 to $10 \mathrm{in}$, depth $346 \mathrm{ft}$, cased to 265 . Lsd $43 \mathrm{ft}$ above msl. MP top of casing, $37.31 \mathrm{ft}$ above msl. Highest water level 32.60 above msl (previously reported 32.70 above msl), Mar. 7, 1938; lowest 21.57 above msl, July 2, 1946. Records available: 1924, 1926, 1928-58. Measurement discontinued. Records furnished by Honolulu Board of Water Supply.

Mean water level for the day, above msl, from recorder graph, 1956

\begin{tabular}{|c|c|c|c|c|c|c|c|c|c|c|c|c|}
\hline Day & Jan. & Feb. & Mar. & Apr & May & June & July & Aug. & Sept. & Oct. & Nov. & Dec. \\
\hline 5 & 28.76 & 29.45 & 29.75 & 29.05 & 28.53 & 28.30 & 27.22 & 26.82 & 26.82 & 26.61 & 27.49 & 28.13 \\
\hline 10 & . & 29. 39 & 29.56 & $\ldots \ldots$ & 28. 36 & 28.29 & 27.10 & 26.60 & 26.78 & 26.81 & 27.53 & 28.45 \\
\hline 15 & & 29.55 & 29. 35 & & 28. 49 & 28.04 & 27.07 & 26.70 & 26. 67 & 26.95 & 27.48 & 28. 66 \\
\hline 20 & 28. 97 & 29.57 & 29.25 & 28.75 & 28. 47 & 27.90 & 26.88 & 26.85 & 26. 62 & 27.10 & 27.70 & 28.73 \\
\hline 25 & 29.20 & 29.58 & 29. 30 & 28.78 & 28.25 & 27.87 & 26.84 & 26.72 & 26. 60 & 27.27 & 27.98 & 28.90 \\
\hline Eom & 29.30 & 29.71 & 29.13 & 28.73 & 28.35 & 27.40 & 26. 79 & 26.65 & 26. 61 & 27.41 & 28.13 & 28. 89 \\
\hline \multicolumn{13}{|c|}{1957} \\
\hline 5 & 28.73 & 29.50 & 29.45 & 28.52 & $2 \overline{28.40}$ & 27.30 & 26.38 & 25.55 & 25.55 & 25.23 & 25.13 & 26.18 \\
\hline 10 & 28. 87 & 29.66 & 29.40 & 28.60 & 28.00 & 27.28 & 26.15 & 25.32 & 25. 51 & 25.10 & 25.37 & 26.46 \\
\hline 15 & 29.02 & 29.60 & 29.10 & 28.63 & 28. 07 & 26.90 & 26. 10 & 25.22 & 25.49 & 25.08 & 25.32 & 26.73 \\
\hline 20 & 29.04 & 29.69 & 29.23 & 28.42 & 28.04 & 26.72 & e25. 80 & 25.23 & 25. 30 & 25.21 & 25.44 & 26. 82 \\
\hline 25 & 29.17 & 29.69 & 29.03 & 28. 39 & 27.73 & 26.60 & 25.56 & 25.40 & 25.17 & 25.00 & 25.72 & 27.11 \\
\hline Eom & 29.30 & 29.48 & 28. 88 & & 27.58 & 26. 60 & 25.45 & 25.38 & 25.35 & 12 & 26.03 & 27.29 \\
\hline \multicolumn{13}{|c|}{1958} \\
\hline 5 & 7.32 & 26.71 & 27.11 & 27.66 & $\overline{27.39}$ & 26.72 & 25.64 & 24.97 & 25.39 & 25.81 & 26.66 & \\
\hline 10 & 27.06 & 26.79 & 27. 34 & 27.43 & 27.36 & 26.49 & 25.52 & 25.30 & 25.52 & 25.63 & 26.8 & \\
\hline 15 & 26. 95 & 26.81 & 27.37 & 27.57 & 27.35 & 26.36 & 25.46 & 25.10 & 25.64 & 25.82 & 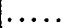 & \\
\hline 20 & 26.97 & 26.78 & 27.41 & 27.63 & 27. 27 & 26.01 & 25.57 & 25.09 & 25. 63 & 25.95 & & \\
\hline 25 & 26. 66 & 26.78 & 27.72 & 27.32 & 27.17 & 25.90 & 25.13 & 25. 27 & 25.70 & 26.1 & & \\
\hline Eom & 26. 65 & 26.87 & 27. 70 & 27.30 & 26.98 & 25.89 & 24. 89 & 25. 31 & 25.66 & 26.34 & . & $\cdots$ \\
\hline
\end{tabular}

153. Sam Damon Estate. Moanalua Gardens, Honolulu. Lat $21^{\circ} 21^{\prime} 05^{\prime \prime}$, long $157^{\circ} 53^{\prime} 40^{\prime \prime}$. Drilled domestic and irrigation artesian basal-water well in basalt of Koolau Volcanic Series, Pliocene (?) age, diam $10 \mathrm{in}$, depth $289 \mathrm{ft}$, cased to 79 . Lsd $20.38 \mathrm{ft}$ above msl. MP top of flange on 10-in T, 20.49 ft above msl. Highest water level 31.88 above msl, April 1917; lowest 19.39 above msl, Sept. 26, 1945. Records available: 1910-60.

\begin{tabular}{|c|c|c|c|c|c|c|c|}
\hline Date & $\begin{array}{l}\text { Water } \\
\text { level }\end{array}$ & Date & $\begin{array}{l}\text { Water } \\
\text { level }\end{array}$ & Date & $\begin{array}{l}\text { Water } \\
\text { level }\end{array}$ & Date & $\begin{array}{c}\text { Water } \\
\text { level }\end{array}$ \\
\hline Jan. 25,1956 & 25.93 & 24,1957 & 25.97 & Jan. 28,1958 & 23.77 & Jan. 26,1959 & 24.93 \\
\hline Feb. 28 & 26.43 & Feb. 19 & 26.59 & Feb. 25 & 24.20 & Feb. 26 & 25.08 \\
\hline Mar. 28 & 25.74 & Mar. 21 & 25.71 & Mar. 28 & 24.97 & Mar. 27 & 24.47 \\
\hline Apr. 24 & 25.60 & Apr. 24 & 25.25 & Apr. 24 & 25.09 & Apr. 23 & 24.18 \\
\hline May 23 & 25.23 & May 24 & 24.83 & May 28 & 25.11 & May 28 & 3.17 \\
\hline une 28 & 24.99 & June 25 & 24.09 & June 25 & 23.57 & June 22 & 84 \\
\hline uly 26 & 24.55 & July 30 & 23.24 & July 28 & 22.97 & July 24 & 21.97 \\
\hline Aug. 27 & 24. 33 & Aug. 28 & 23.05 & Aug. 27 & 23.38 & Aug. 27 & 22.21 \\
\hline Sept. 26 & 24.20 & Sept. 26 & 22.78 & Sept. 26 & 23.17 & Sept. 23 & 21.85 \\
\hline Det. 25 & 24. 61 & Oct. 24 & 22.49 & Oct. 28 & 23.74 & Oct. 26 & 21. 64 \\
\hline ov. 21 & 24.91 & Nov. 26 & 23.05 & Nov. 26 & 24.12 & Nov. 19 & 22.19 \\
\hline 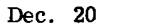 & 2 & 24 & 24.07 & 9 & 24.12 & D & 22 \\
\hline
\end{tabular}


153---Continued.

\begin{tabular}{|c|c|c|c|c|c|c|c|}
\hline Date & Water & Date & Water & Date & $\begin{array}{l}\text { Water } \\
\text { level }\end{array}$ & Date & $\begin{array}{l}\text { Water } \\
\text { level }\end{array}$ \\
\hline $\begin{array}{ll}\text { Jan. } & 26,1960 \\
\text { Feb. } & \mathbf{2 4} \\
\text { Mar. } & 25 \\
\text { Apr. } & 28 \\
\text { May } & 24 \\
\text { June } & 24 \\
\text { July } & 21\end{array}$ & $\begin{array}{l}22.17 \\
21.96 \\
21.93 \\
21.50 \\
21.67 \\
21.02 \\
20.89\end{array}$ & $\begin{array}{ll}\text { Aug. } & 16,1960 \\
23 & \\
\text { Sept. } 1 & \\
8 & \\
15 & \\
21 & \\
29 & \end{array}$ & $\begin{array}{l}20.39 \\
20.37 \\
20.59 \\
20.58 \\
20.61 \\
20.59 \\
20.69\end{array}$ & $\begin{array}{lrr}\text { Oct. } & 7,1960 \\
13 & \\
20 & \\
& 27 & \\
\text { Nov. } & 2 & \\
& 9 & \end{array}$ & $\begin{array}{l}20.71 \\
20.80 \\
20.88 \\
21.07 \\
21.05 \\
21.08\end{array}$ & $\begin{array}{ll}\text { Nov. } 18,1960 \\
29 \\
\text { Dec. } 7 \\
14 \\
22 \\
27\end{array}$ & $\begin{array}{l}21.20 \\
21.46 \\
21.61 \\
21.81 \\
21.94 \\
22.08\end{array}$ \\
\hline
\end{tabular}

187B. U. S. Navy. Aiea. Lat $21^{\circ} 22^{\prime} 40^{\prime \prime}$, long $157^{\circ} 56^{\prime} 05^{\prime \prime}$. Drilled industrial artesian basal-water well in basalt of Koolau Volcanic Series, Pliocene (?) age, diam $12 \mathrm{in}$, depth $173 \mathrm{ft}$, cased to 143. Lsd 9.93 ft above msl. MP top of flange on 12-in T, $8.76 \mathrm{ft}$ above msl. Highest water level 27. 10 above msl, Feb. 23, 1937; lowest 15.06 above msl, Aug. 19, 1945. Records available: $1923,1928-60$.

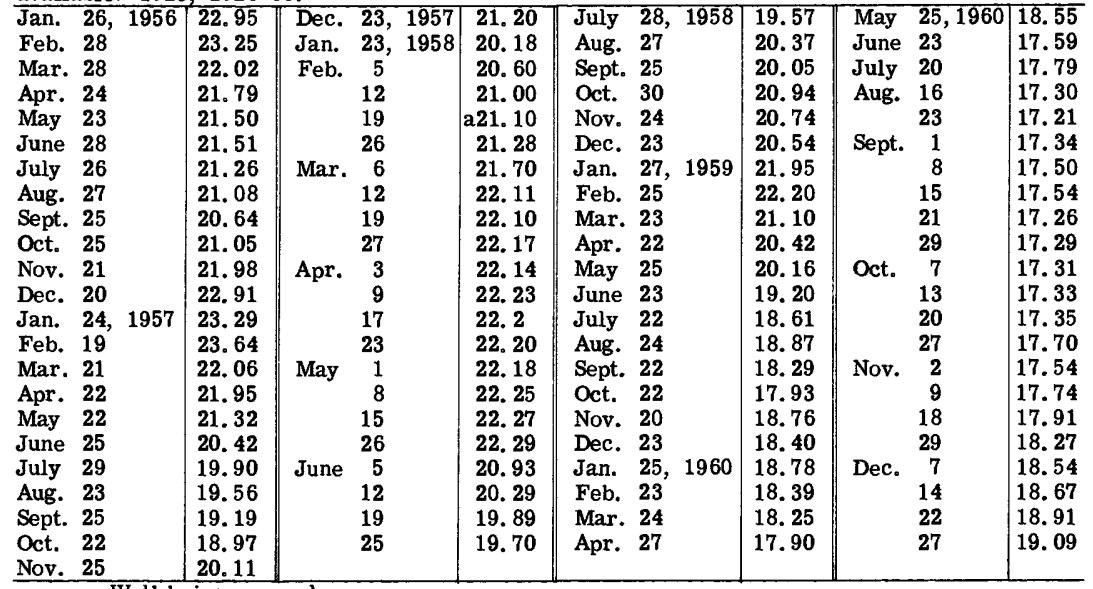

\section{a Well being pumped.}

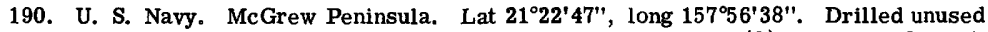
artesian basal-water we 11 in basalt of Koolau Volcanic Series, Pliocene (?) age, diam 6 in, depth $300 \mathrm{ft}$, cased to 200. Lsd 22.73 ft above msl. MP top of $\frac{1}{4}$-in nipple, $19.98 \mathrm{ft}$ above msl. Highest water level 25. 41 above msl, Feb. 23, 1937; lowest 15.38 above msl, Aug. 24, 1945. Records available: 1910, 1918-19, 1929-59. Measurement discontinued.

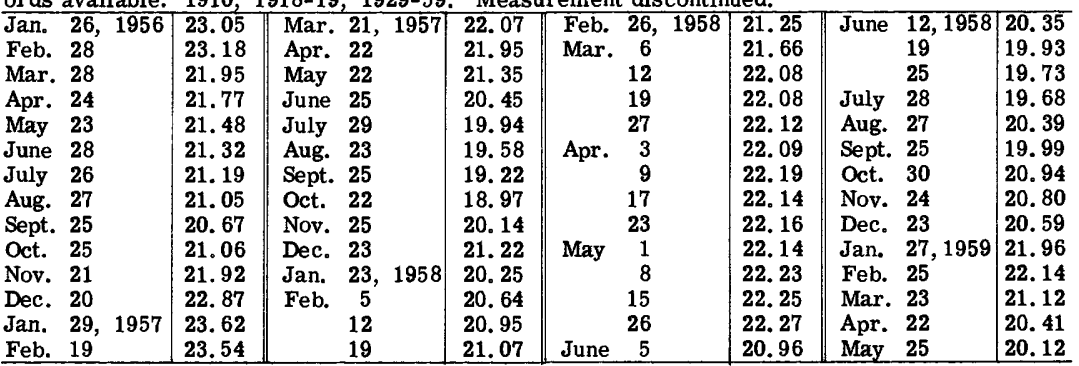

193. L. L. Mc Candless Estate. Waimalu Valley. Lat $21^{\circ} 23^{\prime} 37^{\prime \prime}$, long $157^{\circ} 56^{\prime} 52^{\prime \prime}$. Drilled domestic artesian basal-water well in basalt of Koolau Volcanic Series, Pliocene (?) age, diam $10 \mathrm{in}$, depth $363 \mathrm{ft}$, cased to 61 . Lsd $13.05 \mathrm{ft}$ above msl. MP top of cap on well, $22.93 \mathrm{ft}$ above msl. Highest water level 28.88 above msl, March 1916; lowest 14.65 above msl, Sept. 25, 1945. Records available: $1902,1910-59$. Well sealed; measurement discontinued. Replaced by T- 75 .

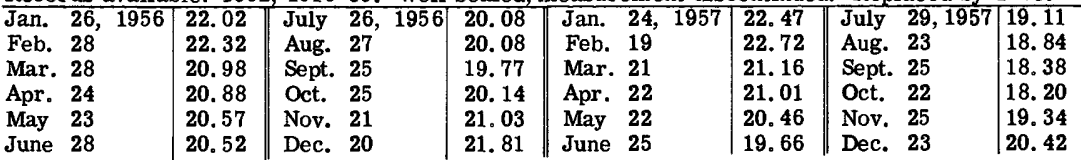


193--Continued.

\begin{tabular}{|c|c|c|c|c|c|c|c|}
\hline Date & $\begin{array}{l}\text { Water } \\
\text { level }\end{array}$ & Date & $\begin{array}{c}\text { Water } \\
\text { level }\end{array}$ & Date & $\begin{array}{c}\text { Water } \\
\text { level }\end{array}$ & Date & $\begin{array}{r}\text { Water } \\
\text { level }\end{array}$ \\
\hline $\begin{array}{ccc}\text { Jan. } & 23, & 1958 \\
\text { Feb. } & 5 & \\
12 & \\
19 & \end{array}$ & $\begin{array}{l}19.32 \\
19.86 \\
20.16 \\
20.31\end{array}$ & $\begin{array}{cc}\text { Feb. } & 26,1958 \\
\text { Mar. } 6 \\
12 \\
19\end{array}$ & $\begin{array}{l}20.43 \\
21.10 \\
21.30 \\
21.18\end{array}$ & $\begin{array}{ll}\text { Mar. } 25,1958 \\
& 27 \\
& \\
\text { Apr. } & 3 \\
& 9\end{array}$ & $\begin{array}{l}21.37 \\
21.37 \\
21.34 \\
21.40\end{array}$ & $\begin{array}{ll}\text { Apr. } & 17,1958 \\
& 23 \\
\text { May } & 1 \\
& 8\end{array}$ & $\begin{array}{l}21.38 \\
21.37 \\
21.39 \\
21.42\end{array}$ \\
\hline
\end{tabular}

Mean water level for the day, above msl, from recorder graph, 1958

\begin{tabular}{|c|c|c|c|c|c|c|c|c|c|c|c|c|}
\hline Day & Jan. & Feb. & Mar. & Apr. & May & June & July & Aug. & Sept. & Oct. & Nov. & Dec. \\
\hline 5 & $\cdots$ & $\cdots$ & & $\cdots$ & $\ldots$ & 20.22 & e19.1 & e19.2 & 19.40 & 19.25 & e20.2 & 20.32 \\
\hline 10 & $\ldots \ldots$ & $\ldots \ldots$ & & $\cdots$ & $\cdots$ & 19.76 & 18.75 & e19. 9 & 19. 33 & 19.15 & 20.19 & 20.00 \\
\hline 15 & & & & $\ldots \ldots$ & $\ldots \ldots$ & e19. 4 & 18. 69 & e19. 8 & 19. 29 & e19. 1 & 20.13 & e20. 2 \\
\hline 20 & $\cdots$ & & & $\ldots$ & 21.44 & 19.17 & 19.01 & e19. 6 & 19.39 & e19. 3 & 19.99 & e20. 1 \\
\hline 25 & & & & $\ldots$ & 21. 44 & 18.94 & 18.89 & 19.54 & 19.14 & e20. 0 & 20.12 & 20.39 \\
\hline Eom & ..... & $\ldots \ldots$ & $\ldots \ldots$ & ..... & 21.31 & e18.7 & 18.76 & 19.73 & 19.12 & e20.3 & 20.07 & 20.77 \\
\hline \multicolumn{13}{|c|}{1959} \\
\hline 5 & 20.92 & 20.71 & 20.64 & 20.36 & $1 \overline{9.42}$ & 19.13 & 18.47 & 17.84 & 17.89 & $\mathrm{e} 17.40$ & - & - \\
\hline 10 & 20.87 & 20.66 & 20.57 & 20.18 & 19.63 & 18.96 & 18. 18 & 18.77 & 17.86 & e17. 34 & $\cdots$ & $\cdots$ \\
\hline 15 & 20.75 & 21.22 & 20.88 & 19. 86 & 19. 32 & 18.78 & 18.05 & 18.56 & 17.78 & e17. 26 & $\ldots$ & - \\
\hline 20 & 21. 26 & 21.30 & 20. 46 & 19.83 & 19. 13 & 18.71 & 18.06 & 18.10 & 17. 71 & & & \\
\hline 25 & e21. 30 & 21.42 & 20.06 & 19.90 & 19.28 & 18. 33 & 17.96 & 17.96 & e17. 61 & .... & - & $\cdots$ \\
\hline Eom & 21. 17 & 21. 37 & 20.11 & 19,45 & 19.42 & 18.29 & 17.85 & 17.91 & e17. 54 & & & $\cdots$ \\
\hline
\end{tabular}

\begin{tabular}{|c|c|c|c|c|c|c|c|}
\hline Date & $\begin{array}{c}\text { Water } \\
\text { level }\end{array}$ & Date & $\begin{array}{c}\text { Water } \\
\text { level }\end{array}$ & Date & $\begin{array}{l}\text { Water } \\
\text { level }\end{array}$ & Date & $\begin{array}{c}\text { Water } \\
\text { level }\end{array}$ \\
\hline $\begin{array}{ll}\text { Jan. } & \mathbf{2 7}, 1959 \\
\text { Feb. } & \mathbf{2 5} \\
\text { Mar. } & \mathbf{2 3}\end{array}$ & $\begin{array}{l}21.23 \\
21.44 \\
20.37\end{array}$ & $\begin{array}{lll}\text { Apr. } & \text { 22, } & 1959 \\
\text { May } & \mathbf{2 5} \\
\text { June } & \mathbf{2 3}\end{array}$ & $\begin{array}{l}19.69 \\
19.30 \\
18.45\end{array}$ & $\begin{array}{lll}\text { July } & 22,1959 \\
\text { Aug. } & 24\end{array}$ & $\begin{array}{l}17.94 \\
18.06\end{array}$ & $\begin{array}{l}\text { Sept. } 22,1959 \\
\text { Oct. } 22\end{array}$ & $\begin{array}{l}17.62 \\
17.29\end{array}$ \\
\hline
\end{tabular}

201. U. S. Navy. Pearl City. Lat $21^{\circ} 23^{\prime} 35^{\prime \prime}$, long $157^{\circ} 58^{\prime} 20^{\prime \prime}$. Drilled irrigation artesian basal-water well in basalt of Koolau Volcanic Series, Pliocene (?) age, diam 12 in, depth $336 \mathrm{ft}$, cased to 58 . Lsd $9.17 \mathrm{ft}$ above msl. MP top of upper flange on valve, $13.17 \mathrm{ft}$ above msl. Highest water level 31.21 above msl, February 1916; lowest 14.18 above msl, Aug. 28, 1946. Records available: 1910-60.

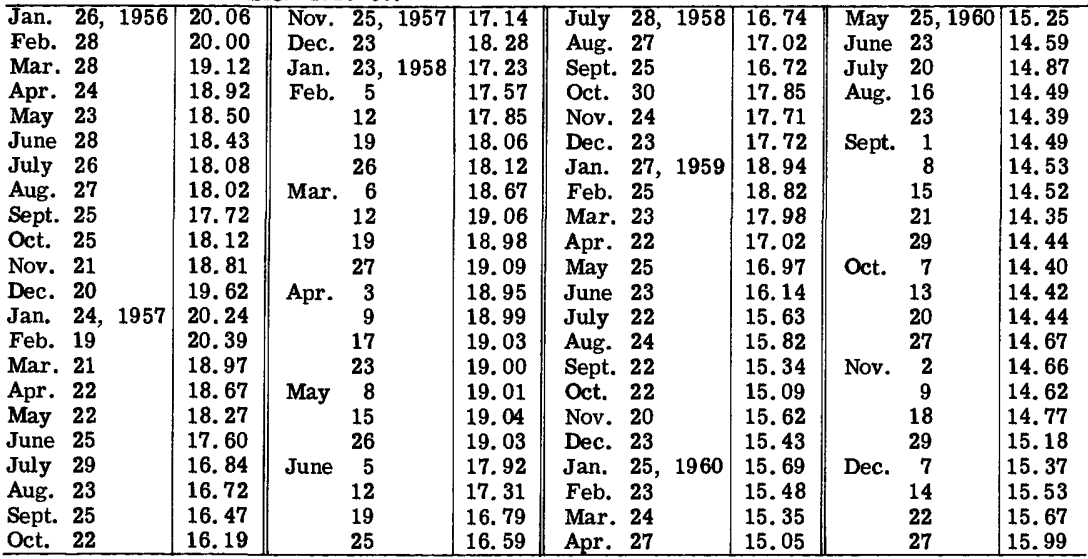

244. B. P. Bishop Estate. Waipahu. Lat $21^{\circ} 23^{\prime} 18^{\prime \prime}$, long $158^{\circ} 00^{\prime} 32^{\prime \prime}$. Drilled domestic artesian basal-water well in basalt of Koolau Volcanic Series, Pliocene (?) age, diam 12 in, depth $225 \mathrm{ft}$, cased to 58. Lsd $10.47 \mathrm{ft}$ above msl. MP top of plate on main valve, 12.47 ft above msl. Highest water level 30.02 above msl, February 1916; lowest 14.80 above msl, July 26, 1945. Records available: 1910-21, 1923-60.

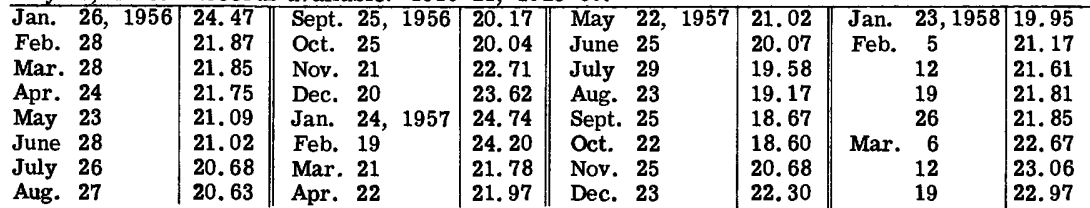


244--Continued.

\begin{tabular}{|c|c|c|c|c|c|c|c|}
\hline Date & $\begin{array}{c}\text { Water } \\
\text { level }\end{array}$ & Date & $\begin{array}{c}\text { Water } \\
\text { level }\end{array}$ & Date & $\begin{array}{c}\text { Water } \\
\text { level }\end{array}$ & Date & $\begin{array}{l}\text { Water } \\
\text { level }\end{array}$ \\
\hline Mar. 27, 1958 & 22.97 & Sept. 25, 1958 & 19.39 & Nov. 20,1959 & 17.95 & Sept. 21,1960 & 16.22 \\
\hline Apr. 3 & 22.87 & Oct. 30 & 21.27 & Dec. 23 & 17.75 & 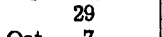 & 16. 27 \\
\hline 9 & 22.90 & Nov. 24 & 20.81 & Jan. 25, 1960 & 18.24 & Oct. & 16.22 \\
\hline 17 & 22.97 & Dec. 23 & 20.81 & Feb. 23 & 17.98 & 13 & 16. 27 \\
\hline 23 & 22.88 & Jan. 27,1959 & 23.05 & Mar. 24 & 17.82 & 20 & 16.28 \\
\hline May & 23.27 & Feb. 25 & 22.90 & Apr. 27 & 17. 25 & 27 & 16. 27 \\
\hline 8 & 23.01 & Mar. 23 & 21.13 & May 25 & 17.60 & Nov. & 16.60 \\
\hline 15 & 23.02 & Apr. 22 & 20.07 & June 23 & 16. 77 & 9 & 16.59 \\
\hline 26 & 23.00 & May 25 & 19.94 & July 20 & 17.08 & 18 & 16.74 \\
\hline June & 20.77 & June 23 & 18.65 & Aug. 16 & 16.39 & 29 & 17.50 \\
\hline 12 & 19.92 & July 22 & 17.91 & 23 & 16. 31 & Dec. & 17.82 \\
\hline 19 & 19.33 & Aug. 24 & 18. 22 & Sept. 1 & 16.09 & 14 & 17.98 \\
\hline 25 & 19.08 & Sept. 22 & 17. 58 & 8 & 16.52 & 22 & 18 \\
\hline $\begin{array}{ll}\text { July } & 28 \\
\text { Aug. } & 27\end{array}$ & $\begin{array}{l}18.92 \\
20.04\end{array}$ & Oct. 22 & 17.10 & 15 & 16.42 & 27 & 18.94 \\
\hline
\end{tabular}

266. Hawaii Meat Co. Honouliuli. Lat $21^{\circ} 21^{\prime} 55^{\prime \prime}$, long $158^{\circ} 01^{\prime} 52^{\prime \prime}$. Drilled irrigation artesian basal-water well in basalt of Koolau Volcanic Series, Pliocene (?) age, diam 10 in (previously reported $12 \mathrm{in}$ ), depth $355 \mathrm{ft}$, cased to 165 . Lsd $12.66 \mathrm{ft}$ above msl. MP top of blind flange on well, $15.16 \mathrm{ft}$ above msl. Highest water level 29.16 above msl, April 1918; lowest 12. 54 above $\mathrm{msl}$, Sept. 24, 1945 . Records available: 1910-60.

\begin{tabular}{|c|c|c|c|c|c|c|c|c|c|c|c|}
\hline Jan. & 26,1956 & 24.46 & Dec. 2 & 23,1957 & 21.50 & July & 28,1958 & 17.82 & May & 25,1960 & 15.98 \\
\hline Feb. & 28 & 22.60 & Jan. 2 & 23,1958 & 18.52 & Aug. & 27 & 18. 63 & June & & 14.96 \\
\hline Mar. & 28 & 20.69 & Feb. & 5 & 21.04 & Sept. & 25 & 18.14 & July & 20 & 15.51 \\
\hline Apr. & 24 & 20.43 & & 12 & 21.77 & Oct. & 30 & 21.33 & Aug. & 16 & 14.71 \\
\hline fay & 23 & 19.83 & & 19 & 22.09 & Nov. & 24 & 19.88 & & 23 & 4.58 \\
\hline June & 28 & 19.70 & & 26 & 22.17 & Dec. & 23 & 19.76 & Sept. & 1 & 14.59 \\
\hline July & 26 & a19.17 & Mar. & 6 & 22.99 & Jan. & 27,1959 & 22.07 & & 8 & 14.79 \\
\hline Aug. & 27 & 19.18 & & 12 & 23.44 & Feb. & 25 & 21.69 & & 15 & 4.58 \\
\hline Sept. & 25 & 18.66 & & 19 & 23.44 & Mar. & 23 & 20.20 & & 21 & 14.42 \\
\hline ct. & 25 & 19.50 & & 27 & 23.60 & Apr. & 22 & 18.74 & & 29 & 1. 46 \\
\hline Nov. & 21 & 22.54 & Apr. & 3 & 23. 24 & May & 25 & 18.95 & Oct. & 7 & 14.40 \\
\hline Dec. & 20 & 22.41 & & 9 & 23.29 & June & 23 & 17.29 & & 13 & .42 \\
\hline Jan. & 24,1957 & 25.13 & & 17 & 23.42 & July & 22 & 16.39 & & 20 & 14.43 \\
\hline Feb. & 19 & 23.38 & & 23 & 23.25 & Aug. & 24 & 16.75 & & 27 & 14.70 \\
\hline ar. & 21 & 20.56 & May & 1 & 23.36 & Sept. & 22 & 15.96 & Nov. & 2 & .91 \\
\hline Apr. & 22 & 20.86 & & 8 & 23.36 & Oct. & 22 & 15.47 & & 9 & .92 \\
\hline May & 22 & e19. 8 & & 15 & 23.36 & Nov. & 20 & 16.60 & & 18 & 15.07 \\
\hline June & 25 & e18.1 & & 26 & 23.32 & Dec. & 23 & 16.35 & & 29 & 16.04 \\
\hline July & 29 & e17. 6 & June & 5 & 19.44 & Jan. & 25,1960 & 17.12 & Dec. & 7 & 16.17 \\
\hline Aug. & 23 & e17. 0 & & 12 & 18.29 & Feb. & 23 & 16.54 & & 14 & 38 \\
\hline Sept. & 25 & e16.3 & & 19 & 18.02 & Mar. & 24 & 16.37 & & 22 & 16.73 \\
\hline Oct. & 22 & e16. 2 & & 25 & 17.76 & Apr. & 27 & 15.81 & & 27 & 17.83 \\
\hline ov. & 25 & $\mathrm{e} 20.2$ & & & & & & & & & \\
\hline
\end{tabular}

a Well being pumped.

e Estimated.

276. Ewa Plantation Co. Gilbert. Lat $21^{\circ} 20^{\prime} 16^{\prime \prime}$, long $158^{\circ} 06^{\prime} 35^{\prime \prime}$ (previously reported long $\left.158^{\circ} 16^{\prime} 35^{\prime \prime}\right)$. Drilled battery of four irrigation artesian basal-water wells in basalt of Waianae Volcanic Series, Pliocene (?) age, diam $12 \mathrm{in}$, average depth $160 \mathrm{ft}$. Lsd $40.58 \mathrm{ft}$ above msl. Highest water level 16. 7 above msl, February 1909; lowest 10.83 above msl, Aug. 27, 1960. Records available: 1905, 1908-60. Records furnished by Ewa Plantation Co.

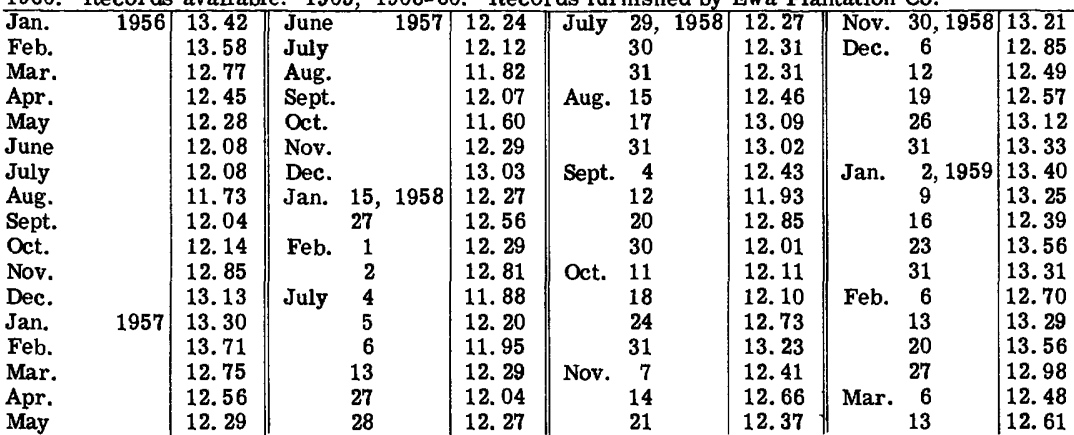


276--Continued.

\begin{tabular}{|c|c|c|c|c|c|c|c|}
\hline Date & $\begin{array}{c}\text { Water } \\
\text { level }\end{array}$ & Date & $\begin{array}{l}\text { Water } \\
\text { level }\end{array}$ & Date & $\begin{array}{l}\text { Water } \\
\text { level }\end{array}$ & Date & $\begin{array}{l}\text { Water } \\
\text { level }\end{array}$ \\
\hline Mar. 20, 1959 & 12.87 & June 6,1959 & 12.06 & Oct. 17,1959 & 11.73 & Mar. 27, 1960 & 12.23 \\
\hline 27 & 12.77 & 13 & 12.06 & Nov. 6 & 11. & Apr. 3 & 11.81 \\
\hline Apr. 3 & 12.73 & 20 & 12. 29 & 13 & 11. & May 30 & 12.12 \\
\hline 10 & 11.98 & 22 & 12.57 & 21 & 11. & June 27 & 11.89 \\
\hline 17 & 12.72 & July & 12.14 & 27 & 12. & July 31 & 11.32 \\
\hline 24 & 12.23 & 11 & 11. 71 & Dec. 5 & 11.79 & Aug. 27 & 10.83 \\
\hline May & 12.23 & 19 & 12.09 & 11 & 11 & 29 & 11.73 \\
\hline 9 & 12.50 & Aug. & 12.04 & 19 & 11 & Sept. 25 & 10.84 \\
\hline 16 & 12.38 & 7 & 12.56 & 25 & 12 & Oct. 31 & 11.79 \\
\hline 22 & 11.77 & 17 & 12.09 & Jan. 30,1960 & 11.73 & Nov. 30 & 11.29 \\
\hline 30 & 12.21 & Sept. 6 & 12.24 & Feb. 28 & 12.46 & Dec. 31 & 11.38 \\
\hline
\end{tabular}

286. Waialua Agricultural Co. Kawaihapai. Lat $21^{\circ} 34^{\prime} 46^{\prime \prime}$, long $158^{\circ} 10^{\prime} 49^{\prime \prime}$. Drilled unused artesian basal-water well in basalt of Waianae Volcanic Series, Pliocene (?) age, diam $1 \mathrm{in}$, depth $447 \mathrm{ft}$, cased to 447 , perforated $410-447$. Lsd $11.54 \mathrm{ft}$ above $\mathrm{msl}$. MP top of vertical flange on 1-in T, $14.17 \mathrm{ft}$ above msl. Highest water level 19. 23 above msl, Oct. 30, 1952; lowest 16.34 above msl, June 26, 1936. Records available: 1929-60.

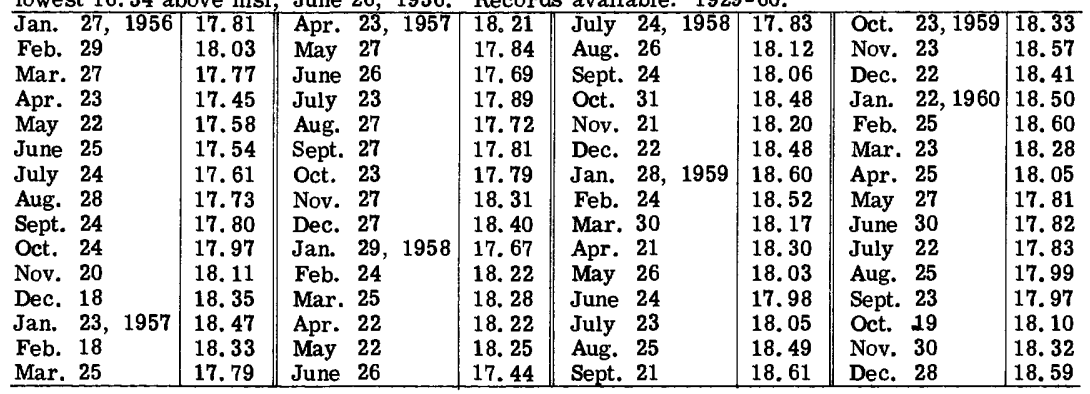

308. J. F. Mendonca. Mokuleia. Lat $21^{\circ} 34^{\prime} 35^{\prime \prime}$, long $158^{\circ} 09^{\prime} 11^{\prime \prime}$. Drilled irrigation artesian basal-water well in basalt of Waianae Volcanic Series, Pliocene (?) age, diam 10 to 8 in, depth $548 \mathrm{ft}$, cased to 440 . Lsd $8.46 \mathrm{ft}$ above msl. MP top of flange on well $307,7.51 \mathrm{ft}$ above msl. Highest water level 20.64 above msl, Oct. 26, 1939; lowest 16.81 above msl, July 25, 1927. Records available: $1924-60$.

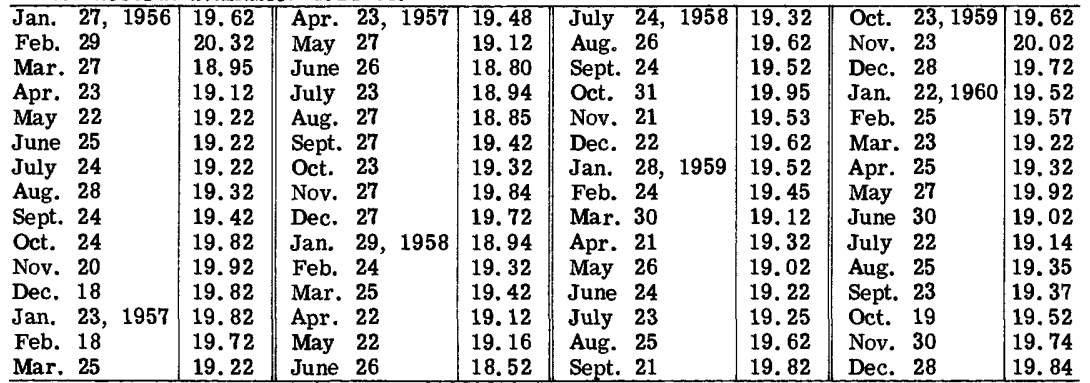

326. Waialua Agricultural Co. Waialua. Lat $21^{\circ} 34^{\prime} 56^{\prime \prime}$, long $158^{\circ} 06^{\prime} 52^{\prime \prime}$. Drilled irrigation artesian basal-water well in basalt of Koolau Volcanic Series, Pliocene (?) age, diam 8 in, depth $201 \mathrm{ft}$, cased to 114 . Lsd $6.19 \mathrm{ft}$ above msl. MP top of blind flange on well, $4.69 \mathrm{ft}$ above msl. Highest water level 13. 35 above msl, December 1914; lowest 9.19 above msl, Apr. 24, 1946. Records available: 1911-21, 1924-60.

\begin{tabular}{l|l||l|l||l|l||l|l|}
\hline Jan. 27, 1956 & 11.96 & Nov. 20, 1956 & 12.39 & Sept. 27, 1957 & 11.24 & July 24, 1958 & 11.69 \\
Feb. 29 & 12.03 & Dec. 18 & 12.56 & Oct. 23 & 11.13 & Aug. 26 & 12.07 \\
Mar. 27 & 11.64 & Jan. 23, 1957 & 12.69 & Nov. 27 & 11.95 & Sept. 24 & 11.78 \\
Apr. 23 & 11.59 & Feb. 18 & 12.59 & Dec. 27 & 11.89 & Oct. 31 & 12.22 \\
May 22 & $\mathbf{1 1 . 5 5}$ & Mar. 25 & 12.22 & Jan. 29, 1958 & 11.14 & Nov. 21 & 12.10 \\
June 25 & 11.82 & Apr. 23 & 11.84 & Feb. 24 & 11.92 & Dec. 22 & 12.24 \\
July 24 & 11.62 & May 27 & 11.48 & Mar. 25 & 12.04 & Jan. 28, 1959 & 12.19 \\
Aug. 28 & 11.82 & June 26 & 11.03 & Apr.22 & 11.76 & Feb. 24 & 12.14 \\
Sept. 24 & 11.94 & July 23 & 11.15 & May 22 & 11.79 & Mar. 30 & 11.69 \\
Oct. 24 & 12.02 & Aug. 27 & 11.37 & June 26 & 10.61 & Apr. 21 & 11.39
\end{tabular}


326--Continued.

\begin{tabular}{|c|c|c|c|c|c|c|c|}
\hline Date & $\begin{array}{r}\text { Water } \\
\text { level }\end{array}$ & Date & $\begin{array}{r}\text { Water } \\
\text { level }\end{array}$ & Date & $\begin{array}{l}\text { Water } \\
\text { level }\end{array}$ & Date & $\begin{array}{r}\text { Water } \\
\text { level }\end{array}$ \\
\hline May 26,1959 & 11.26 & Oct. 23,1959 & 10.87 & Mar. 23, 1960 & 11.28 & Aug. 25, 1960 & 10.22 \\
\hline June 24 & 10.89 & Nov. 23 & $\begin{array}{l}11.59 \\
11.57\end{array}$ & Apr. 25 & $\begin{array}{l}11.03 \\
10.92\end{array}$ & Sept. 23 & 10.47 \\
\hline Aug. 25 & 11.41 & Jan. 22, 1960 & 11.03 & June 30 & 10.46 & Nov. 30 & 11.30 \\
\hline Sept. 21 & 11.43 & Feb. 25 & 11.48 & July 22 & 10.26 & Dec. 28 & 11.59 \\
\hline
\end{tabular}

337. State of Hawaii. Waialee. Lat $21^{\circ} 41^{\prime} 30^{\prime \prime}$, long $158^{\circ} 01^{\prime} 25^{\prime \prime}$. Drilled unused artesian basal-water well in basalt of Koolau Volcanic Series, Pliocene (?) age, diam $8 \mathrm{in}$, depth $63 \mathrm{ft}$, cased to 36 . Lsd $21.45 \mathrm{ft}$ above msl. MP top of 4-in pipe, $21.89 \mathrm{ft}$ above msl. Highest water level 15.60 above msl, Nov. 14, 1932; lowest 11.70 above msl, May 27, 1947. Records available: $1929-60$.

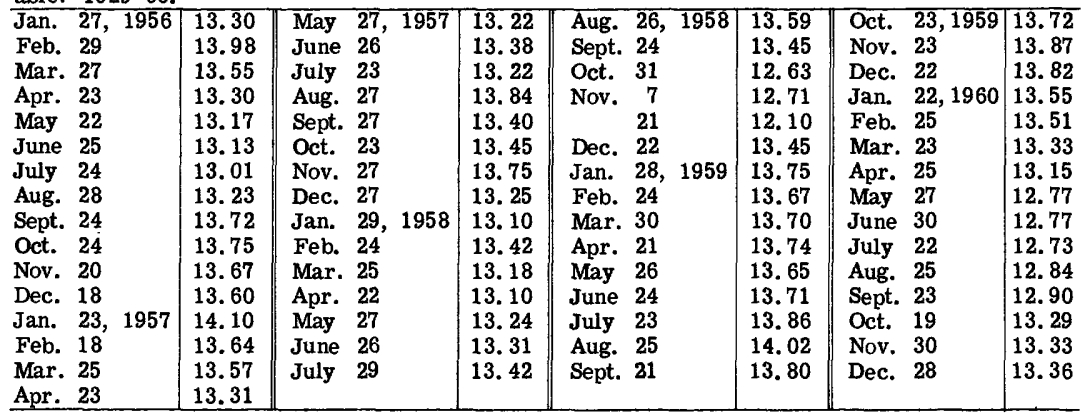

356. Kahuku Plantation Co. Kahuku. Lat $21^{\circ} 40^{\prime} 54^{\prime \prime}$, long $157^{\circ} 57^{\prime} 04^{\prime \prime}$. Drilled industrial artesian basal-water well in basalt of Koolau Volcanic Series, Pliocene (?) age, diam 12 in, depth $420 \mathrm{ft}$, cased to 156 . Lsd $8.83 \mathrm{ft}$ above $\mathrm{msl}$. MP top of vertical flange on valve of fire hydrant, $9.83 \mathrm{ft}$ above msl. Highest water level 17.12 above msl, January 1916; lowest 8.46 above msl, Aug. 25, 1960 . Records available: 1908, 1911-18, 1921, 1924-60.

\begin{tabular}{l|r||l|r||l|r|r||r|r|}
\hline Jan. 27, 1956 & 12.21 & Apr. 23, 1957 & 11.19 & July 24, 1958 & 9.29 & Oct. 23, 1959 & 8.71 \\
Feb. 29 & 12.48 & May 27 & 9.75 & Aug. 26 & 10.18 & Nov. 23 & 10.45 \\
Mar. 27 & 10.41 & June 26 & 8.89 & Sept. 24 & 9.34 & Dec. 22 & 10.27 \\
Apr. 23 & 11.36 & July 23 & 8.95 & Oct. 31 & 10.89 & Jan. 22, 1960 & 10.87 \\
May 22 & 9.86 & Aug. 27 & 8.77 & Nov. 21 & 10.57 & Feb. 25 & 11.05 \\
June 25 & 9.85 & Sept. 25 & 8.69 & Dec. 22 & 11.70 & Mar. 23 & 11.49 \\
July 24 & 9.57 & Oct. 23 & 9.63 & Jan. 28, 1959 & 12.04 & Apr. 25 & 10.01 \\
Aug. 28 & 9.58 & Nov. 27 & 11.39 & Feb. 24 & 11.37 & May 27 & 9.02 \\
Sept. 24 & 9.83 & Dec. 27 & 12.25 & Mar. 30 & 10.25 & June 30 & 8.92 \\
Oct. 24 & 9.35 & Jan. 29, 1958 & 11.20 & Apr. 21 & 9.63 & July 22 & 8.80 \\
Nov. 20 & 11.40 & Feb. 24 & 12.03 & May 26 & 9.23 & Aug. 25 & 8.46 \\
Dec. 18 & 12.27 & Mar. 25 & 12.28 & June 24 & 8.92 & Sept. 23 & 8.89 \\
Jan. 23, 1957 & 12.10 & Apr. 22 & 12.18 & July 23 & 9.51 & Oct. 19 & 8.74 \\
Feb. 18 & 11.69 & May 27 & 12.50 & Aug. 25 & 9.56 & Nov. 30 & 10.05 \\
Mar. 25 & 10.36 & June 26 & 9.29 & Sept. 21 & 9.26 & Dec. 28 & 10.63 \\
\hline
\end{tabular}

396. Kahuku Plantation Co. Hauula. Lat $21^{\circ} 36^{\prime} 22^{\prime \prime}$, long $157^{\circ} 54^{\prime} 36^{\prime \prime}$. Drilled domestic and irrigation artesian basal-water well in basalt of Koolau Volcanic Series, Pliocene (?) age, diam $8 \mathrm{in}$. Lsd $10.36 \mathrm{ft}$ above msl. MP top of blind flange on well, $15.52 \mathrm{ft}$ above msl. Highest water level 24.98 above msl, June 1918 ; lowest 16.68 above msl, Sept. 23, 1953 . Records available: $1911-19,1921,1924-60$.

\begin{tabular}{l|l|l|l||l|l||l|l}
\hline Jan. 27, 1956 & 19.78 & Apr. 23, 1957 & 19.78 & July 24, 1958 & 18.95 & Oct. 23, 1959 & 18.30 \\
Feb. 29 & 20.46 & May 27 & 19.19 & Aug. 26 & 19.04 & Nov. 23 & 18.95 \\
Mar. 27 & 20.20 & June 26 & 18.02 & Sept. 24 & 19.26 & Dec. 22 & 18.30 \\
Apr. 23 & 19.57 & July 23 & 18.21 & Oct. 31 & 19.48 & Jan. 22, 1960 & 18.55 \\
May 22 & 18.94 & Aug. 27 & 18.02 & Nov. 21 & 19.25 & Feb. 25 & 18.52 \\
June 25 & 19.34 & Sept. 27 & 17.88 & Dec. 22 & 19.66 & Mar. 23 & 19.56 \\
July 24 & 19.02 & Oct. 23 & 18.05 & Jan. 28, 1959 & 20.10 & Apr. 25 & 19.46 \\
Aug. 28 & 19.25 & Nov. 27 & 19.04 & Feb. 24 & 19.98 & May 27 & 19.12 \\
Sept. 24 & 18.77 & Dec. 27 & 20.04 & Mar. 30 & 19.59 & June 28 & 18.35 \\
Oct. 24 & 19.17 & Jan. 29, 1958 & 19.33 & Apr. 21 & 19.73 & July 22 & 18.60 \\
Nov. 20 & 19.11 & Feb. 24 & 19.47 & May 26 & 18.94 & Aug. 25 & 17.95 \\
Dec. 18 & 19.97 & Mar. 25 & 19.97 & June 24 & 18.67 & Sept. 23 & 18.08 \\
Jan. 23, 1957 & 19.80 & Apr. 22 & 19.84 & July 23 & 18.48 & Oct. 19 & 18.17 \\
Feb. 18 & 20.24 & May 27 & 20.01 & Aug. 25 & 18.49 & Nov. 29 & 18.56 \\
Mar. 25 & 19.18 & June 26 & 18.20 & Sept. 21 & 18.67 & Dec. 21 & 18.73 \\
\hline
\end{tabular}


405. M. E. Foster Estate. Kahana. Lat $21^{\circ} 33^{\prime} 27^{\prime \prime}$, long $157^{\circ} 52^{\prime} 44^{\prime \prime}$. Drilled domestic artesian basal-water well in basalt of Koolau Volcanic Series, Pliocene (?) age, diam 10 in, depth $441 \mathrm{ft}$, cased to 177 . Lsd $5.76 \mathrm{ft}$ above $\mathrm{msl}$. MP top of flange on well, $5.76 \mathrm{ft}$ above msl. Highest water level 21.07 above msl, July 25, 1938; lowest 14.80 above msl, Oct. 28, 1946. Records available: $1936-60$.

\begin{tabular}{l|c||c|c||c|c||c|c}
\hline Date & $\begin{array}{c}\text { Water } \\
\text { level }\end{array}$ & Date & $\begin{array}{c}\text { Water } \\
\text { level }\end{array}$ & Date & $\begin{array}{c}\text { Water } \\
\text { level }\end{array}$ & Date & $\begin{array}{c}\text { Water } \\
\text { level }\end{array}$ \\
\hline Jan. 27, 1956 & $\mathbf{1 8 . 0 6}$ & Apr. 23, 1957 & 19.22 & July 24, 1958 & 17.76 & Oct. 23, 1959 & 16.66 \\
Feb. 29 & 20.06 & May 27 & 18.76 & Aug. 26 & 18.08 & Nov. 23 & 16.56 \\
Mar. 27 & 18.75 & June 26 & 18.23 & Sept. 24 & 18.16 & Dec. 28 & 16.36 \\
Apr. 23 & 18.96 & July 23 & 18.18 & Oct. 31 & 18.09 & Jan. 22, 1960 & 16.46 \\
May 22 & 18.76 & Aug. 27 & 17.58 & Nov. 21 & 18.38 & Feb. 25 & 16.36 \\
June 25 & 18.36 & Sept. 27 & 17.66 & Dec. 22 & 18.21 & Mar. 23 & 17.06 \\
July 24 & 18.26 & Oct. 23 & 17.26 & Jan. 28, 1959 & 18.55 & Apr. 25 & 17.86 \\
Aug. 28 & 18.26 & Nov. 27 & 17.28 & Feb. 24 & 18.56 & May 27 & 18.06 \\
Sept. 24 & 18.06 & Dec. 27 & 18.38 & Mar. 30 & 18.30 & June 28 & 18.12 \\
Oct. 24 & 18.06 & Jan. 29, 1958 & 18.28 & App. 21 & 18.16 & July 22 & 17.58 \\
Nov. 20 & 18.06 & Feb. 24 & 18.06 & May 26 & 17.79 & Aug. 25 & 18.30 \\
Dec. 18 & 18.46 & Mar. 25 & 18.36 & June 24 & 17.76 & Sept. 23 & 17.36 \\
Jan. 23, 1957 & 18.56 & Apr. 22 & 18.36 & July 23 & 17.31 & Oct. 19 & 17.54 \\
Feb. 18 & 18.96 & May 27 & 18.48 & Aug. 25 & 17.26 & Nov. 29 & 17.49 \\
Mar. 25 & 19.16 & June 26 & 18.08 & Sept. 21 & 16.95 & Dec. 21 & 17.26 \\
\hline
\end{tabular}

406. Mrs. F. M. Swanzy. Kaaawa. Lat $21^{\circ} 32^{\prime} 41^{\prime \prime}$, long $157^{\circ} 51^{\prime} 00^{\prime \prime}$. Drilled irrigation artesian basal-water well in basalt of Koolau Volcanic Series, Pliocene (?) age, diam 9 in, depth 500 (?) ft. Lsd $10.27 \mathrm{ft}$ above msl. MP chiseled cross on top of concrete box, $12.67 \mathrm{ft}$ above msl. Highest water level 18. 37 above msl, July 25, 1938; lowest 12.35 above msl, Aug. 27, 1946. Records available: 1929-60.

\begin{tabular}{l|l||l|l||l|l|l||l|}
\hline Jan. 27, 1956 & 15.53 & Apr. 23, 1957 & 17.05 & July 24, 1958 & 15.81 & Oct. 23, 1959 14.72 \\
Feb. 29 & 15.94 & May 27 & 16.13 & Aug. 26 & 15.87 & Nov. 23 & 14.30 \\
Mar. 27 & 16.13 & June 26 & 16.54 & Sept. 24 & 15.81 & Dec. 22 & 14.34 \\
Apr. 23 & 16.36 & July 23 & 16.34 & Oct. 31 & 15.85 & Jan. 22, 1960 & 14.31 \\
May 22 & 16.43 & Aug. 27 & 16.03 & Nov. 21 & 15.92 & Feb. 25 & 14.18 \\
June 25 & 16.31 & Sept. 27 & 15.87 & Dec. 22 & 15.96 & Mar. 23 & 14.55 \\
July 24 & 16.21 & Oct. 23 & 15.55 & Jan. 28, 1959 & 16.07 & Apr. 25 & 14.98 \\
Aug. 28 & 15.85 & Nov. 27 & 15.50 & Feb. 24 & 16.12 & May 27 & 15.37 \\
Sept. 24 & 15.97 & Dec. 27 & 15.85 & Mar. 30 & 15.89 & June 28 & 15.45 \\
Oct. 24 & 15.96 & Jan. 29, 1958 & 15.87 & Apr. 21 & 15.84 & July 22 & 15.52 \\
Nov. 20 & 16.02 & Feb. 24 & 15.74 & May 26 & 15.72 & Aug. 25 & 15.45 \\
Dec. 18 & 17.17 & Mar. 25 & 16.21 & June 24 & 15.48 & Sept. 23 & 15.38 \\
Jan. 23, 1957 & 16.54 & Apr. 22 & 16.18 & July 23 & 15.23 & Oct. 19 & 15.38 \\
Feb. 18 & 16.76 & May 27 & 16.21 & Aug. 25 & 15.11 & Nov. 29 & 15.22 \\
Mar. 25 & 17.03 & June 26 & 15.94 & Sept. 21 & 14.99 & Dec. 21 & 15.11 \\
\hline
\end{tabular}

T-5. Honolulu Board of Water Supply. Formerly Honolulu Suburban Water System. Near Makaiwa Gulch, Lat $21^{\circ} 20^{\prime} 55^{\prime \prime}$, long $158^{\circ} 07^{\prime} 05^{\prime \prime}$. Drilled observation basal water-table well in basalt of Waianae Volcanic Series, Pliocene (?) age, diam 6 in, depth $100 \mathrm{ft}$, cased to 85 . Lsd $79.13 \mathrm{ft}$ above msl. MP top of casing, $80.20 \mathrm{ft}$ above msl. Highest water level 5.48 above msl, Mar. 27, 1951; lowest 2.53 above msl, June 27, 1939. Records available: 1939-60.

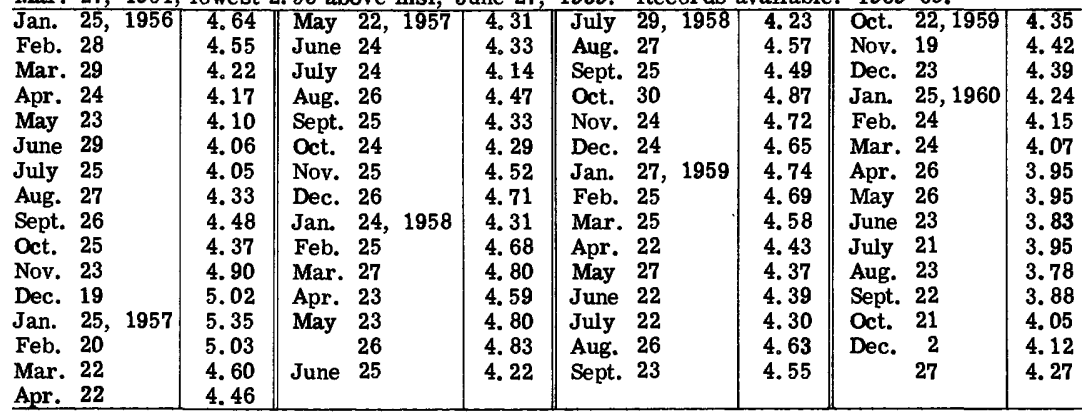

T-15. Honolulu Board of Water Supply. Formerly Honolulu Suburban Water System. Nanakuli Valley. Lat $21^{\circ} 23^{\prime} 50^{\prime \prime}$, long $158^{\circ} 07^{\prime} 20^{\prime \prime}$. Drilled observation basal water-table well in basalt of Waianae Volcanic Series, Pliocene (?) age, diam $\frac{3}{4}$ in, depth $489 \mathrm{ft}$, cased to 488 , perforated 468-488. Lsd 478. $64 \mathrm{ft}$ above msl. MP top of casing, 479.64 ft above msl. Highest water level 3.14 above msl, Feb. 25, 1943; lowest 1.57 above msl, Dec. 15, 1960. Records available: $1940-60$. 
T-15--Continued.

\begin{tabular}{l|c||c|c||c|c||c|c}
\hline \multicolumn{1}{c|}{ Date } & $\begin{array}{c}\text { Water } \\
\text { level }\end{array}$ & Date & $\begin{array}{c}\text { Water } \\
\text { level }\end{array}$ & Date & $\begin{array}{c}\text { Water } \\
\text { level }\end{array}$ & Date & $\begin{array}{c}\text { Water } \\
\text { level }\end{array}$ \\
\hline Mar. 29, 1956 & 2.63 & June 24, 1957 & 2.32 & Oct. 30, 1958 & 2.60 & Dec. 21, 1959 & 2.22 \\
June 29 & 2.18 & Sept. 25 & 2.16 & Jan. 29, 1959 & 3.06 & Apr. 26, 1960 & 1.98 \\
Sept. 26 & 2.11 & Dec. 27 & 2.22 & Apr. 28 & 2.64 & Sept. 7 & 1.79 \\
Dec. 19 & 2.10 & Apr. 21, 1958 & 2.73 & Aug. 26 & 2.12 & Dec. 15 & 1.57 \\
Mar. 22, 1957 & 2.74 & July 30 & 2.42 & & & & \\
\hline
\end{tabular}

T-20. U. S. Navy. Near Ewa. Lat $21^{\circ} 21^{\prime} 36^{\prime \prime}$, long 158 $03^{\prime} 45^{\prime \prime}$. Drilled observation artesian basal-water well in basalt of Koolau Volcanic Series, Pliocene (?) age, diam 6 in, depth $137 \mathrm{ft}$, cased to 9 . Lsd $139.50 \mathrm{ft}$ above msl. MP top of casing, $139.50 \mathrm{ft}$ above msl. Highest water level 19. 28 above msl, Jan. 28, 1943; lowest 15.57 above msl, Sept. 22, 1960 . Records available: $1942-60$.

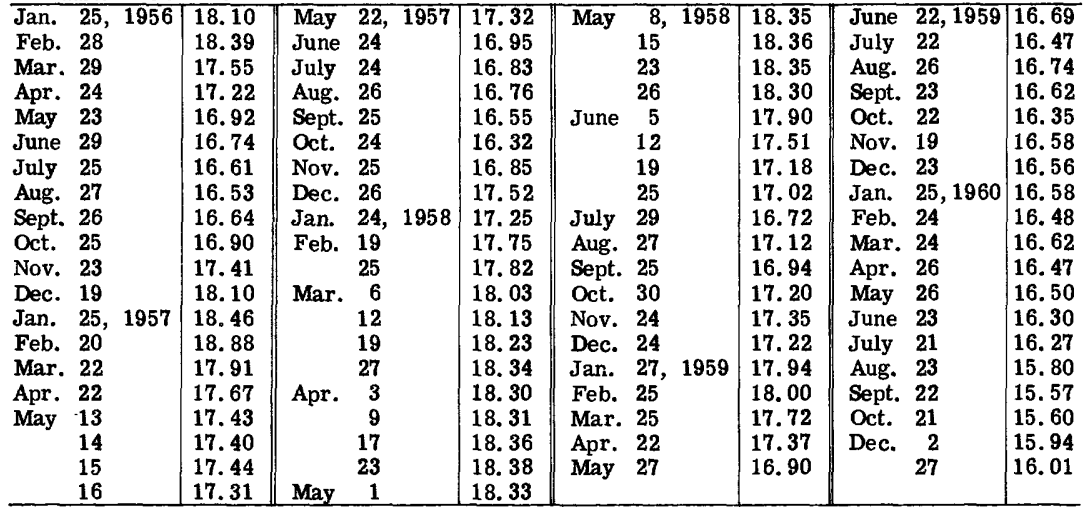

T-24. Honolulu Board of Water Supply. Manaiki Gulch. Lat $21^{\circ} 21^{\prime} 27^{\prime \prime}$, long $157^{\circ} 53^{\prime} 10^{\prime \prime}$. Drilled observation artesian basal-water well in basalt of Koolau Volcanic Series, Pliocene (?) age, diam $12 \mathrm{in}$, depth $115 \mathrm{ft}$, cased to 66 . Lsd $58.40 \mathrm{ft}$ above msl. MP top of casing, $61.05 \mathrm{ft}$ above msl. Highest water level 26.50 above msl, Feb. 11, 1957; lowest 20.32 above msl, Aug. 26, 1960. Records available: 1945-60. Records furnished by Honolulu Board of Water Supply.

Mean water level for the day, above msl, from recorder graph, 1956

\begin{tabular}{|c|c|c|c|c|c|c|c|c|c|c|c|c|}
\hline Day & Jan. & Feb. & Mar. & Apr. & May & June & July & Aug. & Sept. & Oct. & Nov. & Dec. \\
\hline 5 & 25.68 & 26.20 & 26.18 & 25.70 & 25.28 & 25.08 & 24.82 & 24.43 & 24.32 & 24.09 & 24.63 & 25.44 \\
\hline 10 & 73 & 26.13 & 6. 03 & 25.63 & 25. 23 & 25.10 & 24.72 & 24.23 & 24.24 & 24.14 & 24.54 & 25.49 \\
\hline 15 & 25.80 & 26. 26 & 25.86 & 25.52 & 25.22 & 25.08 & 24.63 & 24. 17 & 24.12 & 24. 24 & 24.58 & 25.64 \\
\hline 20 & 25.87 & 26.32 & 25.76 & 25.40 & 25.18 & 25.04 & 24.54 & 24. 25 & 24. 10 & 24. 39 & 24.75 & 25.72 \\
\hline 25 & 25.94 & 26.29 & 25.74 & 25.42 & 25.07 & 25.05 & & 24.13 & 24. 09 & 24.48 & 25.02 & 25.78 \\
\hline Eom & 26.08 & & 25.71 & 25.41 & 25.10 & 24.90 & 24.49 & & 24.10 & 24.55 & 25 & 25.69 \\
\hline \multicolumn{13}{|c|}{1957} \\
\hline 5 & e25.64 & 26.37 & 26.00 & $25 . \overline{27}$ & 25.12 & $24 . \overline{51}$ & 23.94 & 23.20 & 23.11 & 22.64 & 22.54 & 23.51 \\
\hline 10 & 25.45 & 26.47 & 25.86 & 25.20 & 24.94 & 24.42 & 23.84 & 23.16 & 23.00 & 22.54 & 22.62 & 23.66 \\
\hline 15 & e25. 57 & 26.45 & 25.68 & 25.25 & 24.91 & 24.26 & 23.75 & 23. 07 & 22.95 & 22.52 & 22.68 & 23.84 \\
\hline 20 & e25. 78 & 26.48 & 25.65 & 25.21 & 24. 90 & 24.18 & 23.62 & 23.02 & 22.87 & 22.52 & 22.71 & 23.98 \\
\hline 25 & 25.91 & 26. 36 & 25. 61 & 25.19 & 24.78 & 24.08 & 23.40 & 22.98 & 22.76 & 22.46 & 23.01 & 24.07 \\
\hline Eom & 26.19 & 26.16 & 25.42 & 25.20 & & 23.97 & 23. 25 & 23.01 & 22. 72 & 22.44 & 23.33 & 24. 28 \\
\hline \multicolumn{13}{|c|}{1958} \\
\hline 5 & 24.34 & 23.79 & 24.41 & 25.03 & 25.04 & 24.52 & 23.22 & 22.98 & 23.34 & 23.25 & 23.98 & 24.09 \\
\hline 10 & 24. 26 & 23.97 & 24.70 & 25.02 & 25.08 & 24.21 & 23.17 & 23.17 & 23.30 & 23.23 & 24.04 & 24.13 \\
\hline 15 & 24.19 & 24.10 & 24.87 & 25.03 & 25.18 & 23.98 & 23.10 & 23.28 & 23.23 & 23.27 & 24.00 & 24.16 \\
\hline 20 & 24.06 & 24.15 & 24. 91 & 25.10 & 25.15 & 23.62 & 23.12 & 23. 29 & 23. 15 & 23.37 & 24.09 & 23. 88 \\
\hline 25 & 23.78 & 24.17 & 24.94 & 25.03 & 25.06 & 23.44 & 23.01 & 23.34 & 23.12 & e23.40 & 24.09 & 23.77 \\
\hline Eom & 23.61 & & & & 24.88 & 23.29 & 22.90 & 23.29 & 23.17 & 23.82 & 24.17 & 24.07 \\
\hline \multicolumn{13}{|c|}{1959} \\
\hline 5 & 24.24 & 24.81 & 24.90 & $24 . \overline{44}$ & 23.76 & 23.11 & 22.55 & $\overline{21 . \overline{9}}$ & 22.00 & 21.71 & 21.99 & $\overline{22.08}$ \\
\hline 10 & 24. 24 & 24.79 & 24.81 & 24. 26 & 23.57 & 23.13 & 22.28 & 22. 28 & 22.10 & 21.53 & 22. 14 & 22.03 \\
\hline 15 & 24.32 & 24.84 & 24.73 & 24. 32 & 23.30 & 23.03 & 22.25 & 22.35 & 22.00 & 21.65 & 22. 18 & 22. 10 \\
\hline 20 & 24.65 & 24.93 & 24.55 & 24.25 & 23.25 & 22.80 & 22. 16 & 22.23 & 21.90 & 21.60 & 22. 10 & 22. 07 \\
\hline 25 & 24.84 & 25.06 & 24.48 & 24.05 & 23. 26 & 22.63 & 21.94 & 22.14 & 21.74 & 21.59 & 22.08 & 22.12 \\
\hline Eom & 24.83 & 25.04 & 24.43 & 23.95 & 23. 28 & 22.58 & 21.87 & 22.44 & 21.75 & 21.56 & 22. 24 & 22. 16 \\
\hline
\end{tabular}


T-24--Continued.

\begin{tabular}{l|l|l|l|l|l|l|l|l|l|l|l|l}
\hline Day & Jan. & Feb. & Mar. & Apr. & May & June & July & Aug. & Sept. & Oct. & Nov. & Dec. \\
\hline 5 & 22.22 & 21.94 & 21.93 & 21.69 & 21.3 & 21.4 & 21.15 & 20.57 & 20.43 & 20.66 & 20.94 & 21.48 \\
10 & 22.17 & 21.86 & 22.17 & 21.68 & 21.31 & 21.24 & 21.0 & 20.50 & 20.58 & 20.76 & 20.94 & 21.54 \\
15 & 22.08 & 21.88 & 22.21 & 21.58 & 21.48 & 21.14 & 21.07 & 20.43 & 20.53 & 20.76 & 21.13 & 21.72 \\
20 & 22.19 & 21.96 & 22.0 & 21.68 & 21.65 & 21.13 & 20.98 & 20.34 & 20.53 & 20.78 & 21.22 & 21.84 \\
25 & 22.18 & 21.92 & 21.90 & 21.64 & 21.69 & 20.97 & 20.87 & 20.34 & 20.5 & 20.93 & 21.30 & 21.96 \\
Eom & 22.10 & 21.91 & 21.76 & 21.38 & 21.65 & 21.01 & 20.67 & 20.41 & 20.61 & 20.97 & 21.35 & 22.05 \\
\hline
\end{tabular}

T-25. Honolulu Board of Water Supply. Waimalu Valley, near Pearl Harbor. Lat $21^{\circ}$ $23^{\prime} 35^{\prime \prime}$, long $157^{\circ} 56^{\prime} 48^{\prime \prime}$. Drilled observation artesian basal-water well in basalt of Koolau Volcanic Series, Pliocene (?) age, diam $12 \mathrm{in}$, depth $177 \mathrm{ft}$, cased to 42 . Lsd $24.40 \mathrm{ft}$ above msl. MP top of casing, 26.91 ft above msl. Highest water level 21.10 above msl, Mar. 23, 1955; lowest 16.10 above msl, Sept. 25, 1953. Records available: 1945-59. Measurement discontinued. Records furnished by Honolulu Board of Water Supply.

Mean water level for the day, above msl, from recorder graph, 1956

\begin{tabular}{|c|c|c|c|c|c|c|c|c|c|c|c|c|}
\hline Day & Jan. & Feb. & Mar. & Apr. & May & June & July & Aug & Sept. & Oct. & Nov. & Dec. \\
\hline 5 & 0.01 & 20.46 & 20.35 & 19.60 & 19.42 & 19.20 & 19.15 & 18.95 & 18.70 & 18.45 & 19.06 & 20.08 \\
\hline 10 & 19.82 & 20.55 & 20.05 & 19.57 & 19.20 & 19.45 & 19.00 & 18.87 & 18.67 & 18.48 & 18.85 & 20.21 \\
\hline 15 & 20.25 & 20.65 & 19.60 & 19.50 & 19.20 & 19.42 & 19.15 & 18.80 & 18. 60 & 18.95 & 18.70 & 20.25 \\
\hline 20 & 20.12 & 20.62 & 19.51 & 19.20 & 19.30 & 19.25 & 19.00 & 18.90 & 18. 52 & 19.05 & 19.46 & 20.20 \\
\hline 25 & 20 & 20.70 & 19.62 & 19.35 & 19.10 & 19.36 & 18.92 & 18.75 & 18.52 & 18.80 & 19.75 & 20.23 \\
\hline Eom & 20.42 & 20 & 19. & 19 & 19.06 & 19.30 & 18.87 & & 18.62 & 18.98 & 20.00 & 20.16 \\
\hline \multicolumn{13}{|c|}{1957} \\
\hline 5 & 19.90 & 20.97 & 19.93 & 19.35 & 19.45 & 18.60 & 18.35 & 18.00 & 17.80 & 17.35 & 17.35 & 18.60 \\
\hline 10 & 19.44 & 20.96 & 20.05 & 19.35 & 18.95 & 18.60 & 18.20 & 18.15 & 17.78 & 17.26 & 17.70 & 18.75 \\
\hline 15 & 19.78 & 20.82 & 19.55 & 19.56 & 19.05 & 18 & 18.20 & 17.85 & 17.72 & 17.22 & 17.55 & 18.90 \\
\hline 20 & 20.45 & 20.80 & 19.62 & 19.55 & 19.15 & 18. & 18.15 & 17.85 & 17.48 & 17.24 & 17.55 & 19.00 \\
\hline 25 & 20.10 & 20.40 & 19.65 & 19.45 & 18.95 & 18.35 & 18.04 & 18.02 & 17.42 & 17. 20 & 18.20 & 19.00 \\
\hline Eom & 20.92 & & 19.57 & & 18.70 & 18.32 & 18.02 & & 17.45 & 17.15 & 18.40 & 19.00 \\
\hline \multicolumn{13}{|c|}{1958} \\
\hline 5 & 19.10 & 18.58 & & 19.86 & 19.86 & 18.96 & 18.05 & 18 & 18.24 & 18.14 & 18.90 & 18.62 \\
\hline 10 & 18. 85 & 18.77 & 19.96 & 19.82 & 19.89 & 18.60 & 17.77 & 18.67 & 18. 21 & 18.09 & 18.88 & 18.68 \\
\hline 15 & 18.85 & 18.87 & 19.92 & 19.84 & 19.90 & 18.35 & 17.76 & 18.59 & 18. 26 & 18.09 & 18.85 & 18.95 \\
\hline 20 & 18 & & 89 & 19.88 & 19.88 & 18.12 & 18.08 & 44 & & 23 & 18. 75 & 18.83 \\
\hline 25 & 18.08 & 19.06 & 19. 89 & 19.85 & 19.86 & 17.96 & 17.96 & & 11 & 18.78 & 18. 77 & 18.92 \\
\hline Eom & 17.92 & 19.41 & 19.85 & 19.85 & 19.76 & 17.86 & 17.77 & 18 & 18.11 & 18.98 & 19.00 & 19.27 \\
\hline \multicolumn{13}{|c|}{$195 \overline{9}$} \\
\hline 5 & 19.50 & 19.25 & 19.25 & 19.17 & 18.30 & 18.05 & 17.52 & 17.04 & \multicolumn{2}{|c|}{$17.10 \mathrm{e} 17.0$} & & \\
\hline 10 & 19.42 & 19.24 & 19.23 & 18.83 & 18.47 & 17.95 & 17. 35 & 17.75 & 17.05 & & & \\
\hline 15 & 19.33 & 19.70 & 19.48 & 18. 70 & 18.15 & 17.87 & 17.18 & 17.55 & 17.00 & & & \\
\hline 20 & 19.73 & 19.70 & 19.00 & 18.77 & 18.10 & 17.70 & 17.18 & 17.20 & 16.90 & & & \\
\hline 25 & 19.77 & 19.90 & 18. 85 & 18.70 & 18. 25 & 17.40 & 17.13 & 17.12 & 16.80 & & $\ldots$ & \\
\hline Eom & 19.64 & 19.82 & 18.85 & 18.30 & 18.30 & 17.35 & 17.08 & 17.12 & 16.73 & & & \\
\hline
\end{tabular}

T-27. Honolulu Board of Water Supply. Pearl City. Lat $21^{\circ} 23^{\prime} 55^{\prime \prime}$, long $157^{\circ} 58^{\prime} 30^{\prime \prime}$. Drilled observation basal water-table well in basalt of Koolau Volcanic Series, Pliocene (?) age, diam $12 \mathrm{in}$, depth $71 \mathrm{ft}$, cased to 60 . Lsd $47 \mathrm{ft}$ above msl. MP top of casing, $48.10 \mathrm{ft}$ above msl. Highest water level 22.42 above msl, Mar. 29, 1955; lowest 15.22 above msl, Sept. 21, 1960. Records available: 1946-60. Records furnished by Honolulu Board of Water Supply.

Mean water level for the day, above msl, from recorder graph, 1956

\begin{tabular}{|c|c|c|c|c|c|c|c|c|c|c|c|c|}
\hline Day & Jan. & Feb. & Mar. & Apr. & May & June & July & Aug. & Sept. & Oct. & Nov. & Dec. \\
\hline 5 & 21.30 & 21.80 & 21.50 & 20.65 & 20.20 & 20.06 & 19.95 & 19.48 & 19.43 & 18.98 & 19.76 & 21.04 \\
\hline 10 & 24 & 21.70 & 21.10 & 20.60 & 20.06 & 0 & 19.72 & 19 & 19.40 & 19 & 19 & 18 \\
\hline 15 & 21.45 & 21.88 & 20.82 & 20.50 & 20.15 & 20 & 19.70 & 19 & 19. & 19 & 19. & 21.25 \\
\hline 20 & 21. 49 & 21.90 & 20.70 & 20.17 & 20.15 & 19.99 & 19.46 & 19.60 & 19.07 & 19.55 & 20.12 & 21.17 \\
\hline 25 & 21.56 & 21.73 & 20.75 & 20.35 & 19.87 & 20.08 & 19.50 & 19.37 & 19.10 & 19 & 20.56 & 21.17 \\
\hline Eom & & & 20.82 & 20.52 & 19. & 19.85 & & & 19 & & 20 & 21 \\
\hline \multicolumn{13}{|c|}{1957} \\
\hline 5 & 20.70 & 22.35 & 21.10 & 20.04 & $20 . \overline{23}$ & 19 & 18.90 & 18.32 & 18.12 & 17.47 & & .00 \\
\hline 10 & 20.34 & 22.35 & 21.05 & 20.00 & 19.72 & 19 & 18.65 & 18 & 17.96 & 17.41 & 17. & 9.20 \\
\hline 15 & 20.48 & 22.08 & 20.55 & 20.27 & 19.75 & 19 & 18. 60 & 18 & 17.95 & 17. 35 & 17. & 19.35 \\
\hline 20 & 20.75 & 21.95 & 20.60 & 20.37 & 19 & 18 & 18.46 & 12 & 17.72 & 17 & 17 & 19.50 \\
\hline 25 & & 21.70 & 20.60 & 20.16 & 19. & 18. & & 18 & 17.62 & 17. & & \\
\hline Eom & 22 & & 20.45 & 20.25 & & 18.82 & 18. & & & 17.35 & 18.65 & 19.60 \\
\hline
\end{tabular}


T-27--Continued.

1958

\begin{tabular}{|c|c|c|c|c|c|c|c|c|c|c|c|c|}
\hline Day & Jan. & Feb. & Mar. & Apr. & May & June & July & Aug. & Sept. & Oct. & Nov. & Dec. \\
\hline 5 & 19.60 & 18.93 & e19.83 & 20.41 & 20.40 & 19.35 & 18.00 & 17.92 & 18.32 & 18.22 & 19.20 & 18.83 \\
\hline 10 & 20 & 19.16 & 20.35 & 20.39 & 20. 42 & 18 & 17.74 & e18. 30 & 18. 27 & 18.02 & 19. 20 & 18.88 \\
\hline 15 & 5 & 19.33 & 20.41 & 20.39 & 20.45 & 13 & 17.69 & 18.59 & 18.36 & 18.09 & 18 & 19.17 \\
\hline 20 & 19.00 & 19.42 & 20.41 & 20.40 & 20.42 & 18.13 & 17.93 & 18.48 & 18.09 & 18.27 & 18.90 & 19.07 \\
\hline 25 & 18.43 & 19.44 & 20.44 & 20.37 & 20.40 & 17.93 & 17.72 & 18.48 & 18.06 & 18.68 & 19.02 & 19.15 \\
\hline Eom & 18.20 & 19. & 20.43 & 20.39 & 20.16 & 17. 83 & & 47 & 18 & 19.15 & 19.30 & \\
\hline \multicolumn{13}{|c|}{1959} \\
\hline 5 & 19.95 & 19.85 & 19.80 & 19.27 & 18.42 & 17.87 & 17.33 & 16.68 & e16.60 & 16.37 & 16.70 & 16.75 \\
\hline 10 & 19.82 & 19.67 & 19. 67 & 18.87 & 18. 57 & 17. 85 & 17.05 & 17.50 & e16.70 & 16.23 & 17.02 & 16.55 \\
\hline 15 & 19.87 & 20.12 & 19.72 & e18.70 & 18.20 & 17.80 & 16.95 & 17.38 & 16.65 & 16.17 & 17.07 & 16.62 \\
\hline 20 & e20.10 & 20.27 & 19. 22 & 18.92 & 18. 25 & 17.48 & 17.00 & 17.05 & 52 & 16. 20 & 16.73 & 16.78 \\
\hline 25 & 20.37 & 20.30 & 19.15 & 18.70 & 18.42 & e17. 20 & 16.76 & 16.88 & 16.38 & 16.17 & 16.72 & 16.75 \\
\hline Eom & 20.14 & 20.17 & e19.30 & 18. 37 & 18.28 & e17.00 & 16.68 & 16.87 & 16.33 & 16.18 & 17.13 & 16.45 \\
\hline \multicolumn{13}{|c|}{1960} \\
\hline 5 & 16.85 & 16.40 & 16.65 & 16.35 & 15.88 & 16.10 & 15.95 & 15.48 & 15.52 & 15.28 & 15.53 & 16.42 \\
\hline 10 & 16 & 16.38 & 16.98 & 16.45 & 15.82 & 15.75 & 15.95 & 15.45 & 15.42 & 15.32 & 15. 45 & 16.45 \\
\hline 15 & 16 & 16.48 & 17.00 & 16.38 & 16. 30 & 15.70 & 16.00 & 15.45 & 15.30 & 15. 32 & 15.72 & 16.47 \\
\hline 20 & 16 & 16.62 & 16.82 & 16.32 & 16.40 & 15.75 & 15.78 & 15.32 & 15.25 & 15.28 & 15.99 & 16.70 \\
\hline 25 & 16.88 & 16.48 & 16.45 & 16.30 & 16. 35 & 15.60 & 15.67 & 15.25 & 15.30 & 15.57 & 16. 25 & 16.75 \\
\hline Eom & 16.80 & 16.65 & 16.35 & 15.98 & 16. 20 & 15.58 & 15.80 & 15.30 & 15.28 & 15.66 & 16.14 & 16.86 \\
\hline
\end{tabular}

T-28. Honolulu Board of Water Supply. Halemano Gulch, near Waialua. Lat $21^{\circ} 34^{\prime} 40^{\prime \prime}$, long $158^{\circ} 06^{\prime} 07^{\prime \prime}$. Drilled observation basal water-table well in basalt of Koolau Volcanic Series, Pliocene (?) age, diam 12 in, depth $60 \mathrm{ft}$, cased to 39 . Lsd $35 \mathrm{ft}$ above msl. MP top of casing, $38.83 \mathrm{ft}$ above msl. Highest water level 12. 84 above msl, Jan. 24, 1957; lowest 9.53 above msl, June 12, 1947. Records available: 1947-60. Records furnished by Honolulu Board of Water Supply.

Mean water level for the day, above msl, from recorder graph, 1956

\begin{tabular}{|l|l|l|l|l|l|l|l|l|l|l|l|l}
\hline Day & Jan. & Feb. & Mar. & Apr. & May & June & July & Aug. & Sept. & Oct. & Nov. & Dec. \\
\hline 5 & 11.92 & 11.82 & 12.11 & 11.74 & 11.30 & 11.87 & 11.95 & 11.97 & 12.00 & 11.46 & 12.17 & 12.56 \\
10 & 11.81 & 11.79 & 11.99 & 11.65 & 11.25 & 12.06 & 11.63 & 12.05 & 12.00 & 11.63 & 11.95 & 12.67 \\
15 & 12.04 & 11.90 & 11.79 & 11.57 & 11.55 & 11.91 & 11.80 & 11.91 & 11.80 & 11.96 & 11.90 & 12.50 \\
20 & 11.92 & 11.83 & 11.75 & 11.26 & 11.80 & 11.89 & 11.62 & 12.10 & 11.77 & 12.20 & 12.30 & 12.53 \\
25 & 11.93 & 12.08 & 11.82 & 11.39 & 11.63 & 12.03 & 11.71 & 11.90 & 11.82 & 11.91 & 12.42 & 12.48
\end{tabular}

\begin{tabular}{l|l|l|l|l|l|l|l|l|l|l|l|l|} 
Eom & 11.86 & 12.12 & 11.91 & 11.55 & 11.75 & 11.93 & 11.73 & 11.77 & 11.90 & 11.98 & 12.55 & 12.44 \\
\hline
\end{tabular}

\begin{tabular}{l|l|l|l|l|l|l|l|l|l|l|l|l}
\hline 5 & 12.36 & 12.67 & $\ldots \ldots$ & $\ldots \ldots$ & $\ldots \ldots$ & 11.25 & 11.34 & 11.23 & 11.63 & 11.19 & 11.08 & 12.05 \\
\hline
\end{tabular}

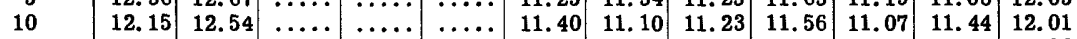

\begin{tabular}{ll|l|l|l|l|l|l|l|l|l|l|l}
15 & 12.33 & 12.60 & $\ldots \ldots$ & $\ldots \ldots$ & $\ldots \ldots$ & 11.40 & 11.10 & 11.23 & 11.56 & 11.07 & 11.44 & 12.01 \\
& 12.42 & 12.6 & $\ldots$ & $\ldots$. & $\ldots$. & 11.13 & 11.19 & 11.11 & $\ldots$. & 11.15 & 11.36 & 11.98
\end{tabular}

\begin{tabular}{ll|l|l|l|l|l|l|l|l|l|l|l|l}
20 & 12.42 & 12.73 & $\ldots$ & $\ldots \ldots$ & $\ldots \ldots$ & 11.19 & 11.10 & 11.16 & 11.37 & 11.43 & 11.34 & 11.91
\end{tabular}

\begin{tabular}{l|l|l|l|l|l|l|l|l|l|l|l|l|l|l}
25 & 12.83 & $\ldots \ldots$ & $\ldots \ldots$ & $\ldots \ldots$ & $\ldots \ldots$ & 11.32 & 11.18 & 11.04 & 11.42 & 11.33 & 11.10 & $\ldots \ldots$ & 11.89
\end{tabular}

\begin{tabular}{l|l|l|l|l|l|l|l|l|l|l|l|l|l|l} 
Eom & 12.71 & $\ldots \ldots$ & $\ldots \ldots$ & $\ldots \ldots$ & 11.19 & 11.35 & 11.12 & 11.63 & 11.55 & 10.93 & 11.99 & 11.94 \\
\hline
\end{tabular}

\begin{tabular}{l|l|l|l|l|l|l|l|l|l|l|l|l}
\hline 5 & 11.97 & 11.68 & 12.10 & 11.85 & 11.78 & e10.80 & $\ldots \ldots$ & $\ldots \ldots$ & $\ldots$ & 12.07 & 12.32 & 12.27 \\
\hline
\end{tabular}

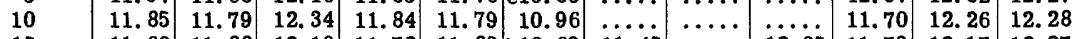
\begin{tabular}{l|l|l|l|l|l|l|l|l|l|l|l|l|l|l}
15 & 11.60 & 11.88 & 12.18 & 11.72 & 11.83 & 10.69 & 11.45 & $\ldots \ldots$ & 12.05 & 11.79 & 12.17 & 12.27
\end{tabular} \begin{tabular}{l|l|l|l|l|l|l|l|l|l|l|l|l}
20 & 11.60 & 11.94 & 12.07 & 11.80 & 11.85 & 10.55 & $\ldots \ldots$ & 12.04 & 12.02 & 12.02 & 12.14 & $\ldots \ldots$
\end{tabular} \begin{tabular}{llll|l|l|l|l|l|l|l|l|l}
25 & 11.32 & 11.92 & 12.00 & 11.77 & 11.84 & 10.58 & $\ldots \ldots$ & 12.16 & 11.81 & $\ldots \ldots$ & 12.18 & $\ldots \ldots$
\end{tabular} \begin{tabular}{l|l|l|l|l|l|l|l|l|l|l|l|l|l|} 
Eom & 11.18 & 11.98 & 11.91 & 11.75 & 11.31 & 10.77 & 11.66 & 12.23 & 11.89 & $\ldots \ldots$ & 12.30 & $\ldots \ldots$ \\
\hline
\end{tabular}

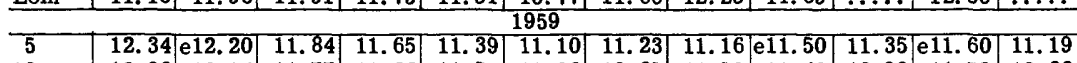
\begin{tabular}{l|l|l|l|l|l|l|l|l|l|l|l|l|l|l|}
\hline 10 & 12.38 & 12.14 & 11.77 & 11.66 & 11.54 & 11.08 & 10.85 & e11.50 & e11.40 & 10.99 & 11.72 & 10.88
\end{tabular} \begin{tabular}{l|l|l|l|l|l|l|l|l|l|l|l|l|l|}
15 & 12.37 & 12.23 & 11.88 & 11.72 & 11.11 & 11.30 & 10.93 & 11.79 & 11.27 & 10.95 & 11.81 & 11.20
\end{tabular} \begin{tabular}{l|l|l|l|l|l|l|l|l|l|l|l|l}
20 & 12.40 & 12.08 & 11.63 & 11.74 & 11.26 & 11.07 & 11.15 & e11.40 & 11.49 & 10.97 & 11.39 & 11.41
\end{tabular}

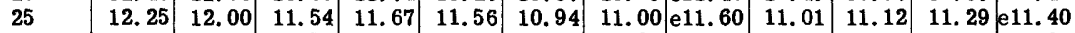
\begin{tabular}{l|l|l|l|l|l|l|l|l|l|l|l|l|l|} 
Eom & e12.20 & 11.94 & 11.57 & 11.23 & 11.55 & 11.06 & e10.90 & 11.54 & 11.08 & 11.07 & 11.41 & e 11.30 \\
\hline
\end{tabular} 1960

\begin{tabular}{l|r|r|r|r|r|r|r|r|r|r|r|r}
\hline 5 & e11.10 & 11.26 & 11.55 & 11.21 & 10.70 & $\ldots \ldots$ & 10.93 & 10.44 & 10.78 & 10.85 & 12.01 & 11.96 \\
10 & 11.02 & 10.92 & 11.52 & 11.20 & e10.80 & $\ldots \ldots$ & 10.86 & 10.46 & 10.38 & 11.00 & 11.82 & 11.97 \\
15 & 10.93 & 11.21 & 11.58 & 11.00 & e11.00 & $\ldots \ldots$ & 10.40 & 10.62 & 10.51 & 10.93 & 11.94 & 11.84 \\
20 & 11.02 & 11.39 & 11.33 & 11.10 & $\ldots \ldots$ & 10.82 & 10.39 & 10.33 & 10.62 & 11.12 & 11.98 & 11.90 \\
25 & 11.20 & 11.39 & 11.21 & 11.14 & $\ldots \ldots$ & 10.54 & 10.67 & 10.23 & 10.80 & 11.92 & 11.80 & 11.90 \\
Eom & 11.30 & 11.41 & 11.20 & 10.99 & $\ldots$. & 10.53 & 10.60 & 10.39 & 10.94 & 11.98 & 11.71 & 11.99 \\
\hline
\end{tabular}


T-41. Honolulu Board of Water Supply. Near Waipahu. Lat $21^{\circ} 22^{\prime} 45^{\prime \prime}$, long $158^{\circ} 01^{\prime} 50^{\prime \prime}$. Drilled observation artesian basal-water well in basalt of Koolau Volcanic Series, Pliocene (?) age, diam $12 \mathrm{in}$, depth $113 \mathrm{ft}$, cased to 92 . Lsd $84 \mathrm{ft}$ above msl. MP top of casing, 83.93 $\mathrm{ft}$ above msl. Highest water level 26.55 above msl, Feb. 2, 1957; lowest 14.78 above msl, Sept. 22, 1960. Records available: 1949-60. Records furnished by Honolulu Board of Water Supply.

\begin{tabular}{|c|c|c|c|c|c|c|c|c|c|c|c|c|}
\hline \multicolumn{13}{|c|}{ Mean water level for the day, above $\mathrm{msl}$, from recorder graph, 195} \\
\hline$\overline{\text { Day }}$ & Jan. & Feb. & Mar. & Apr. & May & June & July & Aug. & Sept. & Oct. & Nov. & Dec. \\
\hline $\mathbf{5}$ & 24.70 & 25.25 & 23.70 & 21.50 & 21.45 & 20.65 & 21.10 & 20.10 & 20.20 & 19.30 & 21.40 & 24.27 \\
\hline 10 & 24.44 & 24.95 & 23.25 & 21.65 & 20.62 & 21. 08 & 20.45 & 19.75 & 19.90 & 19.42 & 21.00 & 24.45 \\
\hline 15 & 24.82 & 25.41 & 22.20 & 21.85 & 20.90 & 20.60 & 20.55 & 19.85 & 19.65 & 20.70 & 19.85 & 24.52 \\
\hline 20 & 24.96 & 25.35 & 22. 10 & 20.90 & 21.10 & 20.55 & 19.85 & 20.25 & 19.30 & 21. 10 & 22.75 & 23.40 \\
\hline 25 & 24.83 & 24.40 & 22.85 & 21.10 & 20.50 & 20.85 & 19.93 & 19.85 & 19. 35 & 20.25 & 23.60 & 24.10 \\
\hline Eom & 25.21 & 23.30 & 23.00 & 21.70 & 20.40 & 20.70 & 19.85 & 19.70 & 19.65 & 20.50 & 23.91 & 23.96 \\
\hline \multicolumn{13}{|c|}{1957} \\
\hline 5 & 22.12 & 26.40 & $\overline{22.50}$ & 20.80 & 21.60 & 19.90 & 19.90 & 18.55 & 18.52 & 17.43 & 18.29 & 21.95 \\
\hline 10 & 21.72 & 26.36 & 23. 35 & 20.75 & 20.45 & 20.00 & 19. 10 & 18.55 & 18.16 & 17.30 & 19.40 & 22. 20 \\
\hline 15 & 22.05 & 24.25 & 21.60 & 21.40 & 20.40 & 19.75 & 19.00 & 18.42 & 18.12 & 17. 28 & 17. 80 & 22.50 \\
\hline 20 & 23. 20 & 23. 80 & 21.60 & 22.50 & 20.85 & 19.35 & 18.80 & 18. 30 & 17.74 & 17.97 & 17.90 & 22.60 \\
\hline 25 & 25.85 & 23.85 & 22. 25 & 20.90 & 20.75 & 19.25 & 18.60 & 18. 35 & 17.62 & 17. 71 & 20.65 & 22.50 \\
\hline Eom & 26,50 & 23.55 & 22.50 & 21.00 & 19.95 & 19.45 & 18.55 & 18.24 & 17.63 & 17. 76 & 21.10 & 21.80 \\
\hline \multicolumn{13}{|c|}{1958} \\
\hline 5 & 22.30 & 21.38 & 22.76 & 23.75 & 23.78 & 20.06 & 18.82 & 18,45 & 19.30 & 19.85 & 21.83 & 19.73 \\
\hline 10 & 20.45 & 21.96 & 23. 78 & 23.77 & 23. 79 & 19.29 & 18.12 & e19.10 & 19.24 & 18.50 & 20.90 & 00 \\
\hline 5 & 20.17 & 22.38 & 23. 89 & 23.74 & 23. 74 & 18.84 & 18.26 & 20.00 & e18.90 & 18.60 & 20.40 & 19.80 \\
\hline 20 & 20.40 & 22. 43 & 23. 88 & 23.74 & 23. 74 & 18. 49 & 18.62 & 19.42 & 19.35 & 19.30 & 19.63 & 20.60 \\
\hline 25 & 19.25 & 22.46 & 23. 88 & 23.72 & 23.75 & 18.26 & 18.18 & 19.57 & e19.80 & 20.85 & 20.05 & 21.00 \\
\hline Eom & 18.42 & 22.59 & 23.81 & & 22.00 & 18.14 & 18.20 & e19 & 30 & 21.83 & 21.50 & 21.77 \\
\hline \multicolumn{13}{|c|}{1959} \\
\hline 5 & 22.32 & 21.30 & 21.05 & 21.05 & 19.23 & 18.35 & 18.20 & 16.83 & 16.85 & 16.40 & 17.45 & 17.90 \\
\hline 10 & 22.00 & 21.52 & 20.90 & 19.40 & 20.30 & 18.40 & 17.30 & 19.75 & 17.05 & 16. 28 & 18. 87 & 16.95 \\
\hline 15 & 22.55 & 23.05 & e21.00 & 19.60 & 18.78 & 18.65 & 17.13 & 18.28 & 16.75 & e16.00 & 18.95 & 17.10 \\
\hline 20 & e23. 60 & 23. 30 & 19.87 & 20.20 & 18.90 & 18.07 & 17.18 & 17.23 & 16.75 & e15.90 & 17. 25 & 18.15 \\
\hline 25 & 23.85 & 22. 40 & 19.97 & 19.95 & 19. 70 & 17.55 & 16.85 & 17.13 & 16. 33 & 16.45 & 17.45 & 17.85 \\
\hline Eom & 22.62 & 22. 40 & 20.25 & 18. & 19.82 & 17.60 & 16.70 & 17.12 & 28 & 16.42 & 18.60 & 17.20 \\
\hline \multicolumn{13}{|c|}{1960} \\
\hline 5 & 17.65 & 16.60 & 17.82 & 16.98 & 15.92 & 16.60 & 16.42 & 15.40 & 16.10 & 14.88 & 16.00 & $\overline{16.86}$ \\
\hline 10 & 18. 35 & 16.45 & 18.40 & 17.68 & 16.08 & 15.75 & 16.25 & 15.30 & 15.22 & 15.00 & 15.28 & 17.42 \\
\hline 15 & 17.05 & 17. 35 & 18. 35 & 17.55 & 17.70 & 15.78 & 17.82 & 15. 30 & 14.92 & 15.00 & 15. 77 & 16. 72 \\
\hline 20 & 17. 35 & 17.70 & 18.40 & 16.75 & 16. 75 & e15. 50 & 15.92 & 15.10 & 14.88 & 14.85 & 16.85 & \\
\hline 25 & 17.75 & 16. 88 & 16.85 & 16.77 & 16.48 & 15.68 & 15. 48 & 14.95 & 15.08 & 15.18 & 17. 75 & \\
\hline Eom & 18.30 & 17.80 & 16.87 & 16.35 & 16.42 & 15.90 & 16. 35 & 15.00 & 14.88 & 15.88 & 16.38 & e17. 60 \\
\hline
\end{tabular}

T-44. Honolulu Board of Water Supply. 16th Ave. and Claudine St., Honolulu. Lat $21^{\circ} 17^{\prime} 17^{\prime \prime}$, long $15^{\circ} 7^{\circ} 7^{\prime} 39^{\prime \prime}$. Drilled observation basal water-table well in basalt of Koolau Volcanic Series, Pliocene (?) age, diam $1 \mathrm{in}$, depth $171 \mathrm{ft}$, cased to 168, perforated 158, 163, 167. Lsd $152.04 \mathrm{ft}$ above msl. MP top of casing, $151.90 \mathrm{ft}$ above msl. Highest water level 11.07 above msl, Aug. 12, 1937; lowest 8.12 above msl, July 8, 1947. Records available: 1936-60.




T-44--Continued.

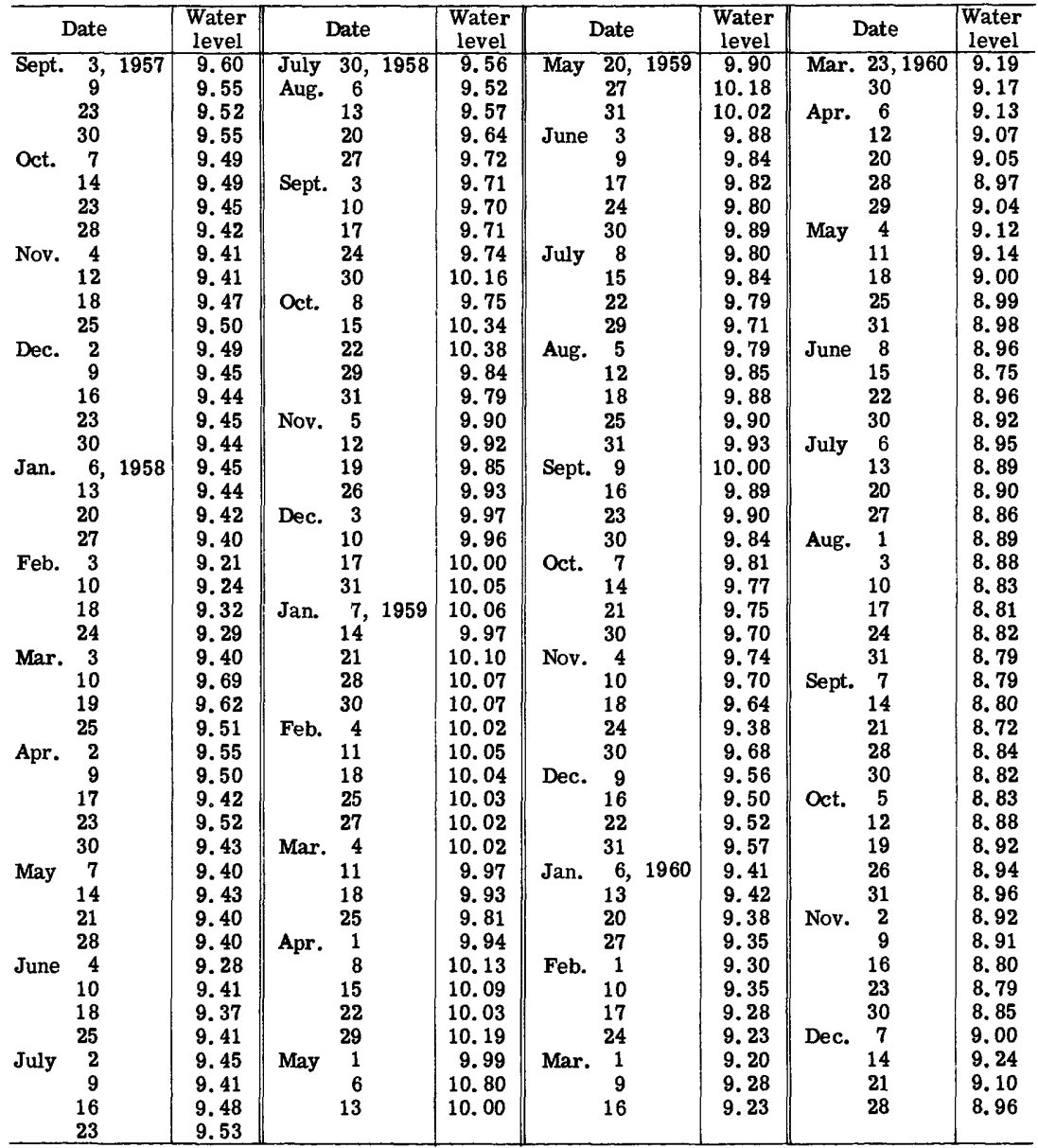

T-45. Honolulu Board of Water Supply. Near North Halawa Valley. Lat $21^{\circ} 22^{\prime} 34^{\prime \prime}$, long $157^{\circ} 55^{\prime} 26^{\prime \prime}$. Drilled observation basal water-table well in basalt of Koolau Volcanic Series, Pliocene (?) age, diam $12 \mathrm{in}$, depth $85 \mathrm{ft}$, cased to 59 . Lsd $57 \mathrm{ft}$ above $\mathrm{msl}$. MP top of casing, $58.06 \mathrm{ft}$ above msl. Highest water level 24.08 above msl, Feb. 12, 1957; lowest 17. 27 above msl, Aug. 27, 1960. Records available: 1954-60.

Mean water level for the day, above msl, from recorder graph, 1956

\begin{tabular}{l|l|l|l|l|l|l|l|l|l|l|l|l}
\hline Day & Jan. & Feb. & Mar. & Apr. & May & June & July & Aug. & Sept. & Oct. & Nov. & Dec. \\
\hline 5 & 23.11 & 23.52 & 23.40 & 22.70 & 22.34 & 21.95 & 21.82 & 21.62 & 21.20 & 20.92 & 21.68 & 22.91 \\
10 & 22.96 & 23.57 & 23.02 & 22.62 & 22.02 & 22.25 & 21.60 & 21.40 & 21.20 & 20.93 & 21.56 & 23.02 \\
15 & 23.17 & 23.62 & 22.64 & 22.50 & 21.95 & 22.08 & 21.78 & 21.34 & 21.02 & 21.40 & 21.26 & 23.13 \\
20 & 23.18 & 23.70 & 22.58 & 22.08 & 22.21 & 21.98 & 21.56 & 21.30 & $\ldots .$. & 21.58 & 22.06 & 23.14 \\
25 & 23.22 & 23.62 & 22.80 & 22.18 & 21.93 & 22.10 & 21.56 & 21.29 & $\ldots .$. & 21.38 & 22.48 & 23.15 \\
Eom & 23.42 & 23.54 & 22.86 & 22.40 & 21.92 & 21.96 & 21.52 & 21.06 & 21.21 & 21.55 & 22.73 & 23.05 \\
\hline \multicolumn{10}{|c|}{19.9} \\
\hline 5 & 22.70 & 23.98 & 22.90 & 22.04 & 22.22 & 21.24 & 20.84 & 20.38 & 20.00 & 19.50 & 19.45 & 20.94 \\
10 & 22.21 & 24.06 & 23.04 & 22.05 & 21.70 & 21.23 & 20.58 & 20.48 & 19.94 & 19.47 & 19.88 & 21.12 \\
15 & 22.54 & 23.96 & 22.42 & 22.40 & 21.64 & 21.10 & 20.50 & 20.05 & 19.92 & 19.50 & 19.54 & 21.24 \\
20 & 22.77 & 23.90 & 22.50 & 22.44 & 21.92 & 20.93 & 20.40 & 20.10 & 19.69 & 19.48 & 19.56 & 21.36 \\
25 & 23.66 & 23.50 & 22.50 & 22.18 & 21.72 & 20.78 & 20.30 & 20.20 & 19.59 & 19.34 & 20.38 & 21.46 \\
Eom & 23.90 & 23.06 & 22.50 & 22.22 & 21.34 & 20.74 & 20.26 & 20.20 & 19.60 & 19.30 & 20.70 & 21.50 \\
\hline
\end{tabular}


T-45-- Continued.

1958

\begin{tabular}{|c|c|c|c|c|c|c|c|c|c|c|c|c|}
\hline Day & Jan. & Feb. & Mar. & Apr. & May & June & July & Aug. & Sept. & Oct. & Nov. & Dec. \\
\hline 5 & 21.56 & 21.10 & e21. 84 & 22.44 & 22.42 & & e19.94 & 20.33 & 20.36 & 20.27 & $\mathrm{e} 21.00$ & 20.88 \\
\hline 10 & 21.18 & 21.13 & 22.36 & 22.46 & 22.52 & 20.80 & 19.90 & 20.64 & 20.34 & 20.19 & 21. 22 & 20.99 \\
\hline 15 & 21.12 & 21.26 & 22. 41 & 22. 49 & 22.53 & 20.58 & 19.84 & 20.76 & 20.46 & 20.14 & 21. 11 & 21.32 \\
\hline 20 & 20.80 & 21.36 & 22. 40 & 22.49 & 22.51 & 20.27 & 20.24 & 20.69 & 20.26 & 20.41 & 20.98 & 21.10 \\
\hline 25 & 20.40 & 21.44 & 22. 39 & 22.45 & 22.48 & 20.08 & 20.09 & 20.70 & 20. 27 & 20.71 & 21.05 & 21.00 \\
\hline Eom & 20.13 & 21.55 & 22. 37 & 22.42 & 22. 36 & 20.10 & 19 & 20 & 20.25 & 21.12 & 21. 31 & 21.50 \\
\hline \multicolumn{13}{|c|}{1959} \\
\hline 5 & 21.76 & 21.77 & 21.72 & 21.51 & 20.50 & 20.16 & 19.58 & 18.80 & 18.84 & 18.51 & 19.11 & 19.05 \\
\hline 10 & 21.74 & 21.74 & 21.66 & 21.18 & 20.72 & 20.05 & 19.23 & 19.66 & 18.92 & 18.49 & e19.10 & 18.77 \\
\hline 15 & 21.52 & 22.16 & 21.96 & 21.08 & 20.09 & 19.84 & 19.11 & 19.42 & 18. 86 & 18. 39 & 18.96 & 18.83 \\
\hline 20 & 22.04 & 22.23 & 21.41 & 21.18 & 20.02 & 19.68 & 19. 12 & 19.10 & 18. 59 & 18. 42 & 18. 99 & 19.16 \\
\hline 25 & 22. 28 & 22. 39 & 21.19 & 20.96 & 20.41 & 19.39 & 19.00 & 19.02 & 18. 61 & 18.43 & 18.75 & 19.02 \\
\hline Eom & 22.10 & 22.33 & 21. 23 & 20.58 & 20.51 & 19.35 & 18.81 & 19.04 & 18. 47 & 18.42 & 19.10 & 18.90 \\
\hline \multicolumn{13}{|c|}{1960} \\
\hline 5 & 18.93 & 18.52 & 18.86 & 18.44 & 18.11 & 18.44 & 18.25 & 17.55 & 17.46 & 17.59 & 17.80 & .64 \\
\hline 10 & 19. 24 & 18.60 & 19.11 & 18.66 & 18.07 & 17.97 & 18. 29 & 17.60 & 17.63 & 17.60 & 17. 84 & 18.80 \\
\hline 15 & 18.83 & 18.67 & 18.99 & 18.60 & 18.73 & 17.92 & 18.14 & 17.49 & 17.58 & 17.59 & 18.05 & 18.89 \\
\hline 20 & 19.04 & 18.97 & 19.11 & 18.40 & 18.82 & 17.94 & 18.04 & 17.36 & 17.47 & 17.56 & 18.32 & 19.10 \\
\hline 25 & 19.14 & 18.69 & 18.58 & 18.46 & 18.77 & 18.03 & 17.92 & 17. 42 & 17.46 & 17.86 & 18.47 & 19.08 \\
\hline Eom & 19.12 & 18.83 & 18.44 & 18.30 & 18. 63 & 17.94 & 17.74 & 17.42 & 17.45 & 17.84 & 18.51 & 19.2 \\
\hline
\end{tabular}

T-48A. Honolulu Board of Water Supply. Formerly Honolulu Suburban Water System. At head of Maunawili Valley. Lat $21^{\circ} 20^{\prime} 42^{\prime \prime}$, long $157^{\circ} 47^{\prime} 14^{\prime \prime}$. Drilled observation high-level watertable well in basalt of Koolau Volcanic Series, Pliocene (?) age, diam $\frac{3}{4}$ in, depth 1, $230 \mathrm{ft}$, cased to 1,120 . Lsd $1,272 \mathrm{ft}$ above msl. MP top of $\frac{3}{4}$-in nipple on top of $\frac{3}{4}$-in pipe, $1,273.56 \mathrm{ft}$ above msl. Highest water level 685.3 above msl, June 26, 1954; lowest 612.91 above msl, Apr. 7, 1960. Records available: 1953-56, 1960. No measurement made in 1957-59. Apr. 26, 1956, 624. 0; Aug. 23, 623.1; Apr. 7, 1960, 612.91; Aug. 3, 617. 56.

T-49. Honolulu Board of Water Supply. Formerly Honolulu Suburban Water System. At head of Maunawili Valley. Lat $21^{\circ} 20^{\prime} 48^{\prime \prime}$, long $157^{\circ} 46^{\prime} 57^{\prime \prime}$. Drilled observation high-level watertable well in basalt of Koolau Volcanic Series, Pliocene (?) age, diam 1 in, depth $805 \mathrm{ft}$, cased to 805, perforated 784-805. Lsd $777 \mathrm{ft}$ above msl. MP top of 1-in pipe, $776.84 \mathrm{ft}$ above msl. Highest water level 635.9 above msl, Mar. 6, 1954; lowest 625.17 above msl, Dec. 23, 1960. Records available: 1953-56, 1960. No measurement made in 1957-59. Apr. 26, 1956, 630. 3; Aug. 23, 628. 6; Mar. 29, 1960, 627.0; Apr. 7, 626.9; Apr. 29, 626.97; Aug. 3, 626.54; Dec. 23, 625.17 .

T-52. Honolulu Board of Water Supply. Waimalu Valley, near Pearl Harbor. Lat $21^{\circ}$ $24^{\prime} 16^{\prime \prime}$, long $157^{\circ} 55^{\prime} 42^{\prime \prime}$. Drilled observation basal water-table well in basalt of Koolau Volcanic Series, Pliocene (?) age, diam $16 \mathrm{in}$, depth $321 \mathrm{ft}$, cased to 180, perforated 170-180. Lsd $168 \mathrm{ft}$ above $\mathrm{msl}$. MP top of casing, $169.13 \mathrm{ft}$ above msl. Highest water level 24.64 above msl, Feb. 12, 1957; lowest 17.55 above msl, Sept. 23, 1960. Records available: 1956-60. Recording gage installed August 1956 by Honolulu Board of Water Supply.

Mean water level for the day, above msl, from recorder graph, 1956

\begin{tabular}{|c|c|c|c|c|c|c|c|c|c|c|c|c|}
\hline Day & Jan. & Feb. & Mar. & Apr. & May & June & July & Aug. & Sept. & Oct. & Nov. & Dec. \\
\hline 5 & & & & & & & & & 22.03 & 21.58 & 22. 21 & 23.35 \\
\hline 10 & & & & . & & • & & & 21.96 & 21.61 & 22. 01 & 23. 41 \\
\hline 15 & & & & & & & & & 21. 81 & 21.85 & $\ldots$ & 23.55 \\
\hline 20 & & & & & & $\cdots$ & & & 21.76 & 22.04 & 22. 37 & 23.58 \\
\hline 25 & & & & & & & & 21.98 & 21.70 & 22.02 & 22.83 & 23.60 \\
\hline Eom & $\ldots$ & & $\ldots$ & & & & & 21.92 & 21.74 & 22.14 & 23.08 & 23. 47 \\
\hline \multicolumn{13}{|c|}{1957} \\
\hline 5 & 23.25 & 24.48 & 23.70 & 22.63 & $22 . \overline{71}$ & 21.88 & 21.24 & 20.76 & 20.50 & 19.86 & 19.70 & 20.99 \\
\hline 10 & 22.89 & 24.61 & 23.58 & 22.59 & 22.32 & 21.81 & 21.10 & 20.80 & 20.41 & 19.77 & 19.86 & 21. 21 \\
\hline 15 & 23. 01 & 24.54 & 23. 18 & 22. 68 & 22. 28 & 21.61 & 20.98 & 20.58 & 20.24 & 19.72 & 19. 81 & 21.33 \\
\hline 20 & 23. 12 & 24.51 & 23. 19 & 22.82 & 22.38 & 21.44 & 20.89 & 20.55 & 20.07 & 19.65 & 19.85 & 21.48 \\
\hline 25 & 23.85 & 24.20 & 23.17 & 22.73 & 22. 17 & 21.34 & 20.82 & 20.56 & 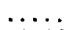 & 19. 60 & 20.37 & 21.58 \\
\hline Eom & 24.32 & 23.90 & 22.72 & 22. 80 & 21. 98 & 21.20 & 20.75 & 20.51 & 19.99 & 19.58 & 20.75 & 21.65 \\
\hline \multicolumn{13}{|c|}{1958} \\
\hline 5 & 21.74 & 20.92 & 21.71 & 22.65 & 22.72 & 21.98 & $20 . \overline{30}$ & 20.41 & 20.74 & 20.60 & 21.51 & 21.42 \\
\hline 10 & 21.51 & 21.14 & 22. 35 & 22. 66 & 22.75 & 21.45 & 20.27 & 20.68 & 20.74 & 20.60 & 21.61 & 21. 46 \\
\hline 15 & 21. 35 & 21.36 & 22. 50 & 22. 67 & 22.78 & 21.09 & 20.18 & 20.95 & 20.79 & 20.65 & 21.55 & 21.71 \\
\hline 20 & 21.18 & 21.46 & 22.56 & 22.70 & 22.79 & 20.75 & 20.34 & 20.92 & 20. 62 & 20.78 & 21.55 & 21.60 \\
\hline 25 & 20.82 & 21.55 & 22.59 & 22.68 & 22.76 & 20.52 & 20.23 & 20.86 & 20.67 & 21.02 & 21. 61 & 21.51 \\
\hline Eom & 20. 44 & 21.62 & 22.63 & 22.65 & 22. 70 & 20.39 & e20.10 & 20.81 & 20.68 & 21. 39 & 21.64 & 22.06 \\
\hline
\end{tabular}


T-52--Continued.

\begin{tabular}{|c|c|c|c|c|c|c|c|c|c|c|c|c|}
\hline \\
\hline$\overline{\text { Day }}$ & Jan. & Feb. & Mar. & Apr. & May & June & July & Aug. & Sept. & Oet. & Nov. & Dec. \\
\hline 5 & 22.28 & 22.33 & $22 . \overline{36}$ & 21.80 & e21.00 & 20.52 & 19.76 & 19.14 & 19.11 & 18.75 & 19.00 & 19.12 \\
\hline 10 & 22. 26 & 22.24 & 22.24 & 21.57 & 21.14 & 20.44 & 19.57 & e19.68 & 19.19 & 18.66 & 19.14 & 18.98 \\
\hline 15 & 22. 29 & 22.44 & 22.28 & 21.49 & e20.90 & 20.32 & 19.47 & 19.68 & 19.12 & 18.60 & 19.15 & 19.01 \\
\hline 20 & 22.56 & 22. 59 & 21.92 & 21.59 & e20.70 & 20.03 & 19.39 & 19.45 & 18.96 & 18.57 & 19.16 & 19.09 \\
\hline 25 & 22.63 & 22.76 & 21.74 & 21.38 & 20.78 & 19.83 & 19.24 & 19.33 & 18.85 & 18.54 & 19.09 & 19.03 \\
\hline Eom & 22. 49 & 22. 69 & 21.73 & 21. & 20.72 & 19.72 & 19.15 & 19.26 & 18.78 & 18.53 & 19.30 & 19.05 \\
\hline \multicolumn{13}{|c|}{1960} \\
\hline 5 & 19.12 & 18.77 & 18.84 & 18.60 & 18.24 & 18.37 & 18.18 & 17.81 & 17.67 & 17.64 & 17.82 & 18.30 \\
\hline 10 & 19.14 & 18.74 & 19.05 & 18.56 & 18.16 & 18.17 & 18. 23 & 17.80 & 17. 70 & 17.63 & 17.79 & 18.44 \\
\hline 15 & 18. 92 & 18.73 & 19.15 & 18.60 & 18.46 & 18.09 & 18.18 & 17.74 & 17.72 & 17.62 & 18.01 & 18.57 \\
\hline 20 & 19.08 & 18.94 & 19.04 & 18. & 18. 74 & 18.06 & 18.15 & 17.69 & 17.62 & 17.62 & . & 18.72 \\
\hline 25 & 19.15 & 18.69 & 18.78 & 18. & 18.74 & 17.97 & 18.01 & 17.59 & 17.57 & 17.80 & ... & 18.91 \\
\hline Eom & 19.08 & 18.76 & 18. 61 & 18 & 18.62 & 17.92 & 17.95 & 17.63 & 17.58 & 17.88 & 18.15 & 19.09 \\
\hline
\end{tabular}

T-69. Honolulu Board of Water Supply. Kapalama Elementary School. Lat $21^{\circ} 23^{\prime} 35^{\prime \prime}$, long $157^{\circ} 56^{\prime} 55^{\prime \prime}$. Drilled observation artesian basal-water well in basalt of Koolau Volcanic Series, Pliocene (?) age, diam 8 in, depth $283 \mathrm{ft}$, cased to 233 . Lsd $81 \mathrm{ft}$ above msl. MP top of casing, $81.82 \mathrm{ft}$ above msl. Highest water level 24.56 above msl, Dec. 27, 1959; lowest 21. 85 above msl, Aug. 26, 1960. Records available: 1959-60. Recording gage installed Nov. 20, 1959. Records furnished by Honolulu Board of Water Supply.

Mean water level for the day, above msl, from recorder graph, 1959

\begin{tabular}{|c|c|c|c|c|c|c|c|c|c|c|c|c|}
\hline$\overline{\text { Day }}$ & Jan. & $\overline{\text { Feb. }}$ & Mar. & Apr. & May & June & July & Aug. & Sept. & Oct. & Nov. & Dec. \\
\hline 5 & $\cdots$ & . & & & $\cdots$ & & & & & & & 24.35 \\
\hline 10 & $\ldots$ & ..... & $\ldots$ & $\ldots$ & $\ldots$ & ..... & $\cdots \cdots$ & ..... & ..... & $\ldots \ldots$ & $\ldots$. & 28 \\
\hline 15 & $\ldots \ldots$ & $\ldots \ldots$ & $\ldots \ldots$ & $\ldots$ & $\ldots \ldots$ & ..... & $\ldots \ldots$ & $\cdots$ & $\ldots \ldots$ & $\ldots$ & $\cdots$ & 4.42 \\
\hline 20 & & & & & & & & & & & & 24.45 \\
\hline 25 & $\ldots \ldots$ & $\ldots \ldots$ & $\ldots \ldots$ & $\ldots \ldots$ & $\ldots \ldots$ & ..... & . & $\ldots$ & $\ldots \ldots$ & . & 24. 30 & 24.54 \\
\hline Eom & $\ldots \ldots$ & $\ldots \ldots$ & $\ldots \ldots$ & & 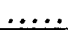 & & & $\ldots \ldots$ & $\ldots \ldots$ & $\ldots$ & 24.48 & 42 \\
\hline \multicolumn{13}{|c|}{1960} \\
\hline 5 & 24.48 & 24.11 & 24.28 & 23.95 & 23.53 & $2 \overline{3} .50$ & 23.09 & 22.10 & 22.21 & 22.54 & 23.01 & 23.63 \\
\hline 10 & 24. 53 & 24. 11 & 24.28 & 24.00 & 23. 50 & 23.26 & 22.80 & 22. 00 & 22.22 & 22. 69 & 23. 19 & 23.64 \\
\hline 15 & 24. 35 & 24.19 & 24.34 & 23.85 & 23. 74 & 23.16 & 22. 70 & 22. 06 & 22.30 & 22. 69 & 23. 10 & 23.70 \\
\hline 20 & 24. 39 & 24.15 & 24.31 & 23.93 & 23.62 & 23.10 & 22.58 & 21.87 & 22. 40 & 22.74 & 23.36 & 23.90 \\
\hline 25 & 24.40 & 24.17 & 24.03 & 23.92 & 23.68 & 23.00 & 22. 47 & 21.90 & 22.40 & 22.90 & 23. 42 & 23.88 \\
\hline Eom & 24.36 & 24.24 & 23.90 & 23.71 & 23.70 & 22.98 & 22. 28 & 21.99 & 22. 47 & 23.03 & 23.42 & 24.25 \\
\hline
\end{tabular}

T-75. Honolulu Board of Water Supply. Waimalu, Lat $21^{\circ} 20^{\prime} 15^{\prime \prime}$, long $157^{\circ} 52^{\prime} 15^{\prime \prime}$. Drilled observation artesian basal-water well in basalt of Koolau Volcanic Series, Pliocene (?) age, diam 6 in, depth $250 \mathrm{ft}$, cased to 75 . Lsd $26 \mathrm{ft}$ above msl. MP top of casing, $27.35 \mathrm{ft}$ above msl. Highest water level 18.19 above msl, Dec. 26, 1959; lowest 16. 20 above msl, Aug. 22, 1960. Records available: 1959-60. Recording gage installed Oct. 14, 1959. Records furnished by Honolulu Board of Water Supply.

Mean water level for the day, above msl, from recorder graph, 1959

\begin{tabular}{|c|c|c|c|c|c|c|c|c|c|c|c|c|}
\hline Day & Jan. & Feb. & Mar. & Apr. & May & June & July & Aug. & Sept. & Oct. & Nov. & Dec. \\
\hline 5 & - & & & & & & & & & & 17.89 & 17.86 \\
\hline 10 & & & & $\ldots \ldots$ & & & & & & & 17.93 & 17.59 \\
\hline 15 & $\ldots \ldots$ & & & $\cdots \cdots$ & - & $\cdots$ & . & • & . & 17.24 & 17. 91 & 17. 62 \\
\hline 20 & & & & $\ldots \ldots$ & & & & & & 17.20 & 17.83 & 17.90 \\
\hline 25 & & & & & & & & & & 17. 24 & e17. 60 & 18.05 \\
\hline Eom & & & & & & & & & & 17. 27 & e 17.70 & 17.78 \\
\hline \multicolumn{13}{|c|}{1960} \\
\hline 5 & 17.75 & 17.50 & 17.75 & 17.21 & 16.82 & 17.17 & 16.94 & 16.46 & 16.48 & 16.43 & $\overline{16.64}$ & 17.54 \\
\hline 10 & 17.99 & 17.40 & 17.79 & 17.40 & 16.80 & 16.83 & 17.12 & 16. 49 & 16.52 & 16.43 & 16.58 & 17.56 \\
\hline 15 & 17.67 & 17.45 & 17.78 & 17. 47 & 17. 57 & 16.77 & 16.92 & 16.37 & 16. 45 & 16.43 & 16.85 & 17.81 \\
\hline 20 & 17.75 & 17.83 & 17. 75 & 17. 18 & 17. 77 & 16.77 & 16.77 & 16. 25 & 16.32 & 16.43 & 17,05 & 17. 91 \\
\hline 25 & 17.86 & 17.42 & 17. 37 & 17.18 & 17.75 & 16.75 & 16.69 & 16. 22 & 16. 36 & 16.64 & 17.35 & 18.06 \\
\hline Eom & 17.92 & 17.55 & 17.20 & 17.01 & 17.34 & 16.62 & 16.66 & 16.35 & 16.36 & 16.71 & 17.14 & 18.20 \\
\hline
\end{tabular}

Shaft 4. U. S. Army. Near Wahiawa. Lat $21^{\circ} 29^{\prime} 30^{\prime \prime}$, long $158^{\circ} 01^{\prime} 45^{\prime \prime}$. Dug domestic high-level water-table well in basalt of Koolau Volcanic Series, Pliocene (?) age, size $8 \times 8 \mathrm{ft}$, vertical depth of 30 -degree inclined shaft $563 \mathrm{ft}$, lined with concrete in upper part, pump chamber at bottom of shaft. Lsd $850 \mathrm{ft}$ above msl. Highest water level 284.13 above msl, Sept. 4, 1937; lowest 273.17 above msl, Mar. 11, 1946. Records available: 1936-60. 
Shaft 4-- Continued.

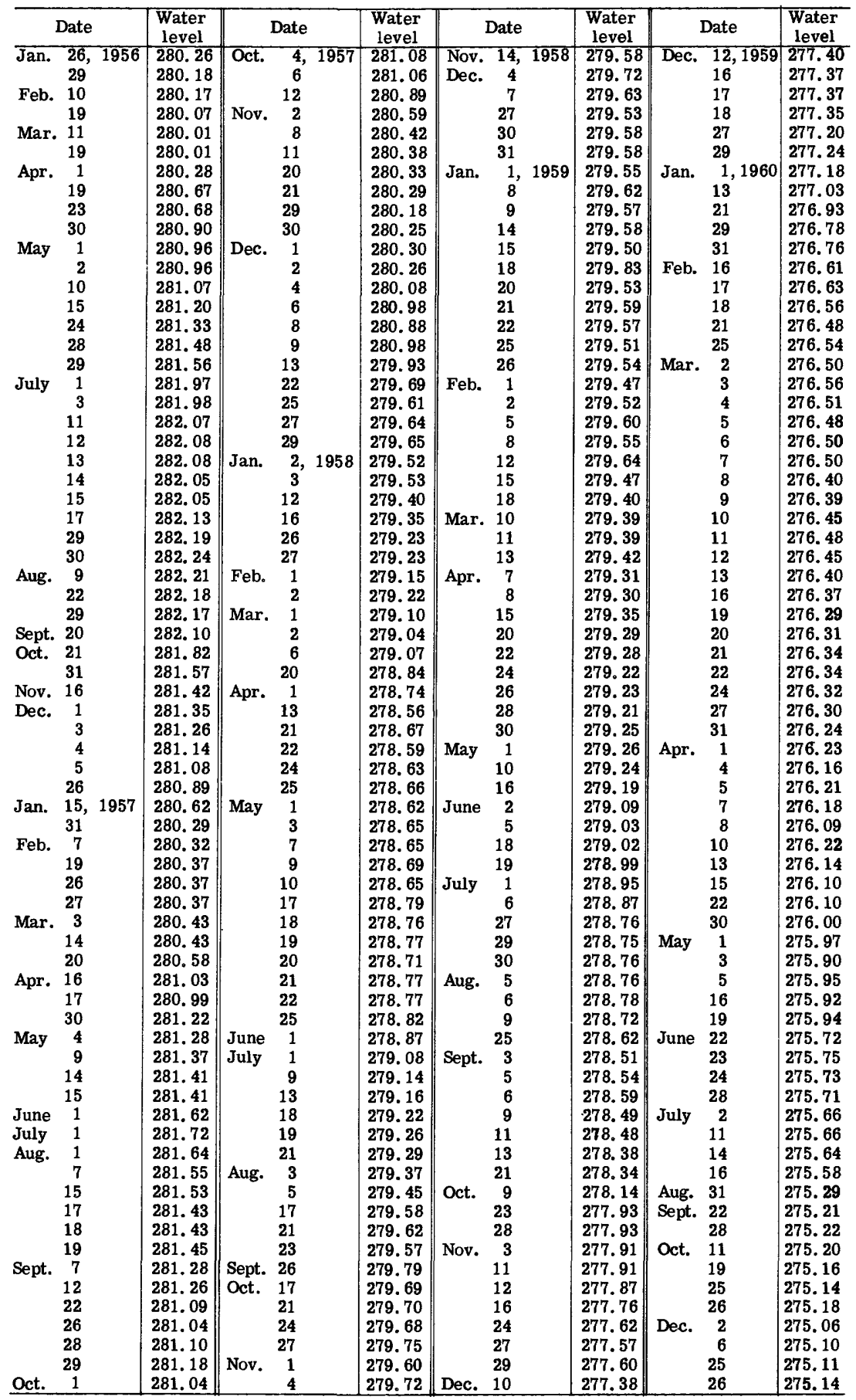




\section{References}

Board of Water Supply, City and County of Honolulu, Hawaii, Biennial reports, 1925-26, et seq. Macdonald, G. A., Davis, D. A., and Cox, D. C., 1960, Geology and ground-water resources of the island of Kauai, Hawaii: Hawaii Div. Hydrography Bull. 13, 212 p.

Stearns, H. T., 1938, Records of the drilled wells of Oahu, Hawaii: Hawaii Div. Hydrography Bull. 4, 213 p.

1940, Supplement to the geology and ground-water resources of the island of Oahu, Hawaii: Hawaii Div. Hydrography Bull. 5, 164 p.

1946, Geology and ground-water resources of the island of Hawaii: Hawaii Div. Hydrography Bull. 9, $363 \mathrm{p}$.

1947, Geology and ground-water resources of the island of Molokai, Hawaii: Hawaii Div. Hydrography Bull. 11, $113 \mathrm{p}$.

Stearns, H. T., and Macdonald, G. A., 1942, Geology and ground-water resources of the island of Maui, Hawaii: Hawaii Div. Hydrography Bull. 7, $344 \mathrm{p}$.

Stearns, H. T., and Vaksvik, K. N., 1935, Geology and ground-water resources of the island of Oahu, Hawaii: Hawaii Div. Hydrography Bull. 1, 479 p. 
By R. A. Lyman, Jr.

The observation-well program in Nevada was continued during the period 1956 through 1960 in cooperation with the Department of Conservation and Natural Resources, State of Nevada. Figure 5 shows the location of observation wells included in this report.

\section{Well-Numbering System}

The number assigned to a well is both an identification and a location number. It is based on the Mount Diablo base line and meridian network of surveys established by the General Land Office (now known as the U. S. Bureau of Land Management). A typical well number is composed of three segments. The first segment indicates the township north of the Mount Diablo base line; if the township is south of the base line, the number is preceded by the letter " $\mathrm{S}$." The second segment, separated from the first by a slant, indicates the range east of the Mount Diablo meridian. The third segment, separated from the second by a hyphen, indicates the section and the location of the well within the section. Lowercase letters--a, b, c, and d--assigned in a counterclockwise direction, designate the northeast, northwest, southwest, and southeast quarter sections, quarter-quarter sections, and quarter-quarter-quarter sections (160-acre, 40-acre, and 10-acre tracts). If two or more wells are in the subdivision, consecutive numbers are assigned in the order in which the well data are recorded. Thus, well number S10/60-4dab1 designates the first well recorded in the $\mathrm{NW} \frac{1}{4} \mathrm{NE} \frac{1}{4} \mathrm{SE} \frac{1}{4}$ sec. 4, T. $10 \mathrm{~S}$, , R. $60 \mathrm{E}$.

\section{Well Descriptions and Water-Level Measurements}

Water levels are reported in feet below land-surface datum unless otherwise indicated. When some measurements in a table are above and others below the plane of reference, a plus $(+)$ or minus (-) sign is placed immediately before the first entry in each column. Measurements between plus signs are above the plane of reference, and those between minus signs are below.

\section{Churchill County}

\section{Dixie Valley}

21/34-27dc1. Gregory Homestead. Drilled unused well, diam $11 \mathrm{in}$, depth $114 \mathrm{ft}$, cased. MP top of casing, $0.50 \mathrm{ft}$ above lsd. Highest water level 28.04 below lsd, June 7, 1956; lowest 32. 62 below lsd, Aug. 21, 1951. Records available: 1951, 1955-60.

\begin{tabular}{|c|c|c|c|c|c|c|c|}
\hline Date & $\begin{array}{l}\text { Water } \\
\text { level }\end{array}$ & Date & $\begin{array}{l}\text { Water } \\
\text { level }\end{array}$ & Date & $\begin{array}{l}\text { Water } \\
\text { level }\end{array}$ & Date & $\begin{array}{l}\text { Water } \\
\text { level }\end{array}$ \\
\hline Aug. 21, 1951 & 32.62 & Mar. 7,1957 & 28.06 & 6,1958 & 28.31 & Mar. 18,1959 & 28.46 \\
\hline 3,1955 & 28.08 & May 15 & 28.09 & July 16 & 28.32 & May 11 & 28.30 \\
\hline 19 & 28.66 & July 12 & 28.20 & Sept. 25 & 28.54 & Aug. 4 & 28.50 \\
\hline June 7,1956 & 28.04 & Sept. 4 & 28.29 & Nov. 24 & 28.48 & June 22, 1960 & 29.03 \\
\hline $\begin{array}{l}\text { Nov. } 26 \\
\text { Jan. } 16,1957\end{array}$ & $\begin{array}{l}28.09 \\
28.15\end{array}$ & $\begin{array}{l}\text { Nov. } 19 \\
\text { Jan. } \quad 6,1958\end{array}$ & $\begin{array}{l}28.31 \\
28.34\end{array}$ & Jan. 14,1959 & 28.52 & Sept. 26 & \\
\hline
\end{tabular}

21/35-19ac2. Howard Turley. Drilled irrigation well, diam $14 \mathrm{in,} \mathrm{depth} 173 \mathrm{ft}$, cased to 155. MP top of casing, at lsd. Highest water level flowing, June 22, Sept. 26, 1960; lowest 4. 39 below lsd, Oct. 28, 1955. Records available: 1951, 1955-60.

\begin{tabular}{|c|c|c|c|c|c|c|c|c|c|c|}
\hline May & 195 & & & & & & 19.1957 & $\overline{90}$ & Mar. 18,1959 & 1.60 \\
\hline $\begin{array}{l}\text { liay } \\
\text { Jan. }\end{array}$ & $\begin{array}{r}1,190 \\
26,195\end{array}$ & 3.36 & June 2 & 6,1900 & 2.99 & Jan. & 6,1958 & 2. 37 & May 11 & $\begin{array}{r}1.00 \\
.34\end{array}$ \\
\hline Iar. 1 & $\begin{array}{l}20,180 \\
18\end{array}$ & $\begin{array}{l}1.00 \\
1.70\end{array}$ & Jan. 1 & 16,1957 & 3.12 & May & 6 & 1.42 & Aug. 4 & .46 \\
\hline pr. 1 & $\begin{array}{l}10 \\
18\end{array}$ & 3.08 & Mar. & 7 & 2. 64 & July & 16 & 2.38 & Dec. 16 & .07 \\
\hline ane 1 & 13 & 3.12 & May 1 & 15 & 2. 86 & Sept. & 25 & 3.26 & June 22,1960 & (j) \\
\hline ct. 2 & 28 & 4. 39 & July 1 & 12 & 3.02 & Nov. & 24 & 2.83 & Sept. 26 & (j) \\
\hline an. 1 & 19 , & 3.25 & Sept. & 4 & 3.44 & Jan. & 14,1959 & 2.39 & & \\
\hline
\end{tabular}

Jan. $19,1956,3$ 
21/35-31d1. C. B. Stark. Drilled stock well, diam 8 in, depth $50 \mathrm{ft}$, cased. MP top of casing collar, $4.00 \mathrm{ft}$ above lsd. Highest water level 31.72 below lsd, Sept. 26, 1960; lowest 34.92 below lsd, Dec. 11, 1951. Records available: 1951, 1955-60.

\begin{tabular}{|c|c|c|c|c|c|c|c|}
\hline Date & Water & Date & $\begin{array}{l}\text { Water } \\
\text { level }\end{array}$ & Date & $\begin{array}{r}\text { Water } \\
\text { level }\end{array}$ & Date & $\begin{array}{r}\text { Water } \\
\text { level }\end{array}$ \\
\hline Dec. 11,1951 & 34.92 & June 7,1956 & 34.03 & Nov. 19,1957 & 33.74 & Jan. 14,1959 & 33.62 \\
\hline Jan. 19,1955 & 34.44 & Nov. 26 & 33.92 & 6,1958 & 33.74 & May 11 & 33.54 \\
\hline Mar. 18 & 34.37 & Jan. 16,1957 & 33.94 & May & 33.67 & Aug. & 33.71 \\
\hline Apr. 13 & 34.35 & May 15 & 33.84 & July 16 & 33.66 & June 22,1960 & 33.59 \\
\hline July 19 & 34.39 & July 12 & 33.78 & Sept. 25 & 33.65 & Sept. 26 & 31.72 \\
\hline Oct. 28 & 34.27 & Sept. 4 & 33.75 & Nov. 24 & 33.60 & & \\
\hline
\end{tabular}

\section{Fairview Valley}

16/33-2dc1. C. B. Stark. Drilled stock well, diam 8 in, depth $441 \mathrm{ft}$, cased. Lsd about 4, $160 \mathrm{ft}$ above msl. MP top of casing, $0.50 \mathrm{ft}$ above lsd. Highest water level 216. 68 below lsd, Jan. 13, 1955; lowest 221. 65 below 1sd, June 22, 1960. Records available: 1950, 1955-60.

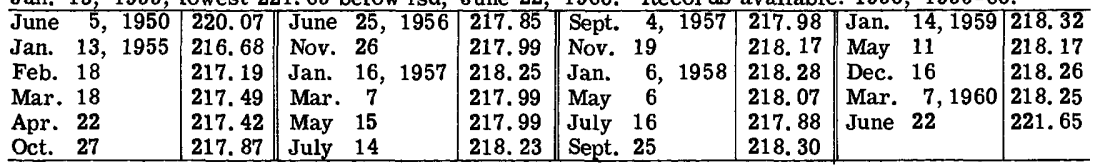

17/35-32dc1. U. S. Government. Drilled unused well, diam 6 in, depth $99 \mathrm{ft}$, cased. Lsd about 4, $470 \mathrm{ft}$ above msl. MP top of casing, $0.40 \mathrm{ft}$ above lsd. Highest water level 45.75 below Isd, Oct. 27, 1955; lowest 60.38 below lsd, Jan. 26, 1955. Records available: 1946-47, $1951,1955-60$.

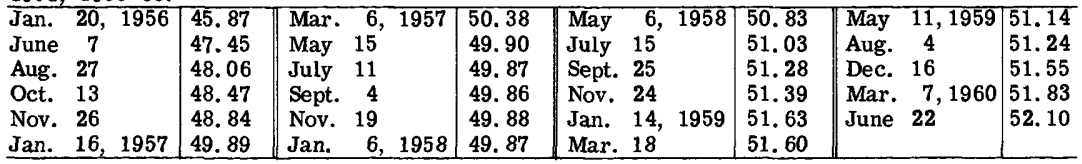

\section{Clark County}

\section{Indian Spring Valley}

S16/56-9bc2. Tim Harnedy. Drilled domestic and irrigation well in alluvium of Quaternary age, diam $8 \mathrm{in}$, depth $582 \mathrm{ft}$, cased. MP top south side of casing, $0.20 \mathrm{ft}$ above Isd. Highest water level 2.20 below Isd, Feb. 18, 1959; lowest 6.70 below lsd, Aug. 5, 1949. Records available: 1946-51, 1955-60. Feb. 10, 1956, 3.34; Feb. 7, 1957, 3.37; Sept. 1, 5. 39; Feb. 12, 1958, 3.15; Feb. 18, 1959, 2. 20; Feb. 14, 1960, 3.60.

\section{Las Vegas Valley}

S17/59-20bcl. Formerly S17/59-16bc1. U. S. Bureau of Land Management. Drilled stock well in alluvium of Quaternary age, diam 6 in, depth $300 \mathrm{ft}$, cased. MP top of casing, $0.30 \mathrm{ft}$ above lsd. Highest water level 26. 44 below lsd, June 20, 1958; lowest 31.01 below lsd, Sept. 12, 1944. Records available: 1944-60.

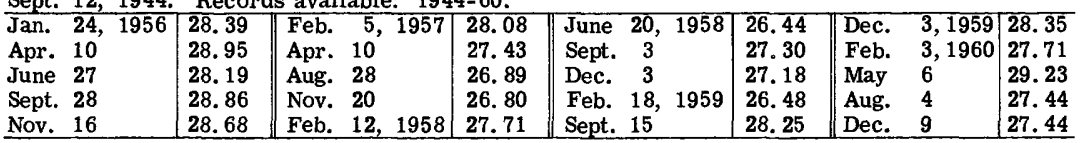

S19/60-9bcc1. P. J. Goumond (State Engineer No. 427). Drilled unused artesian well, diam $10 \mathrm{in}$, depth $830 \mathrm{ft}$, cased to 140 . MP top of casing, $0.50 \mathrm{ft}$ above lsd. Highest water level 43. 65 below lsd, June 3, 1944; lowest 99.78 below Isd, Aug. 18, 1959. Records available: $1944-60$.

Water level at noon, from recorder graph, 1956

\begin{tabular}{|c|c|c|c|c|c|c|c|c|c|c|c|c|}
\hline$\overline{\text { Day }}$ & Jan. & Feb. & $\operatorname{Mar}$ & $\overline{\mathrm{Apr}}$ & May & June & July & $\mathrm{Au}$ & Sept. & Oct. & Nov. & Dec. \\
\hline 5 & 86.06 & 86.59 & 87.59 & 89.24 & & 90.12 & 91.65 & 93.48 & 94.38 & 95.22 & 92.98 & 91.76 \\
\hline 10 & 87.08 & 89.21 & 87.13 & $\ldots$ & 89.84 & 91.71 & 92. 30 & 93.71 & 94.57 & 95.35 & ... & 92.24 \\
\hline 15 & 88.66 & 89.57 & 87.30 & 89.86 & 88.26 & 91.99 & 93.24 & 93.78 & 94.20 & 94.29 & 92.69 & 92.10 \\
\hline 20 & 87.30 & 87. & 88.60 & 90.61 & 89.74 & 92 & 91.99 & 95 & 94.78 & 45 & 91.80 & .74 \\
\hline 25 & 86 & 87. & 87.67 & 9 & & 3 & 70 & & 95 & 93 & 92 & 9 \\
\hline Eom & 87.39 & 87. & 88 & 90.16 & 89.27 & 92.73 & 92.17 & 22 & 95.15 & 94.17 & 93.01 & 91.69 \\
\hline
\end{tabular}




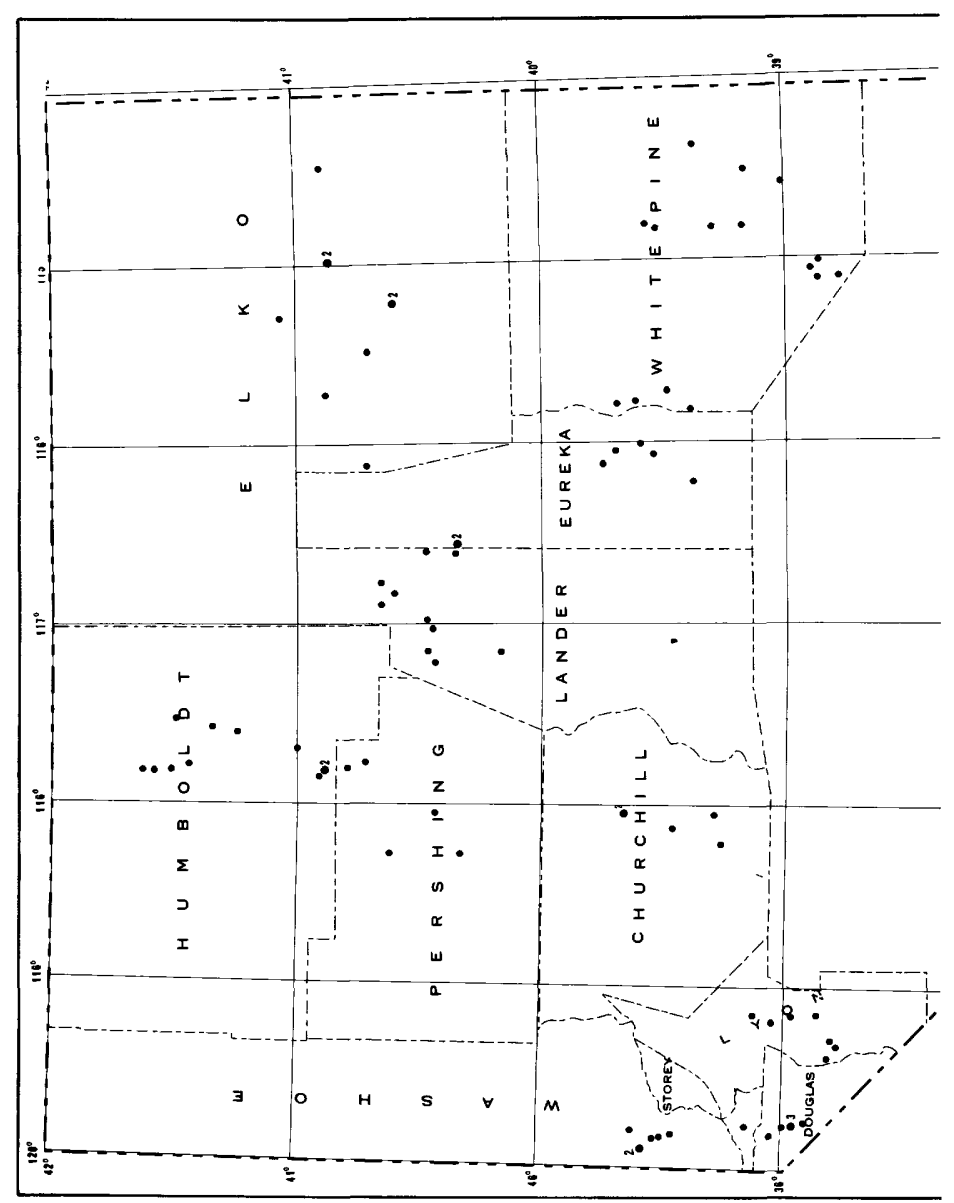




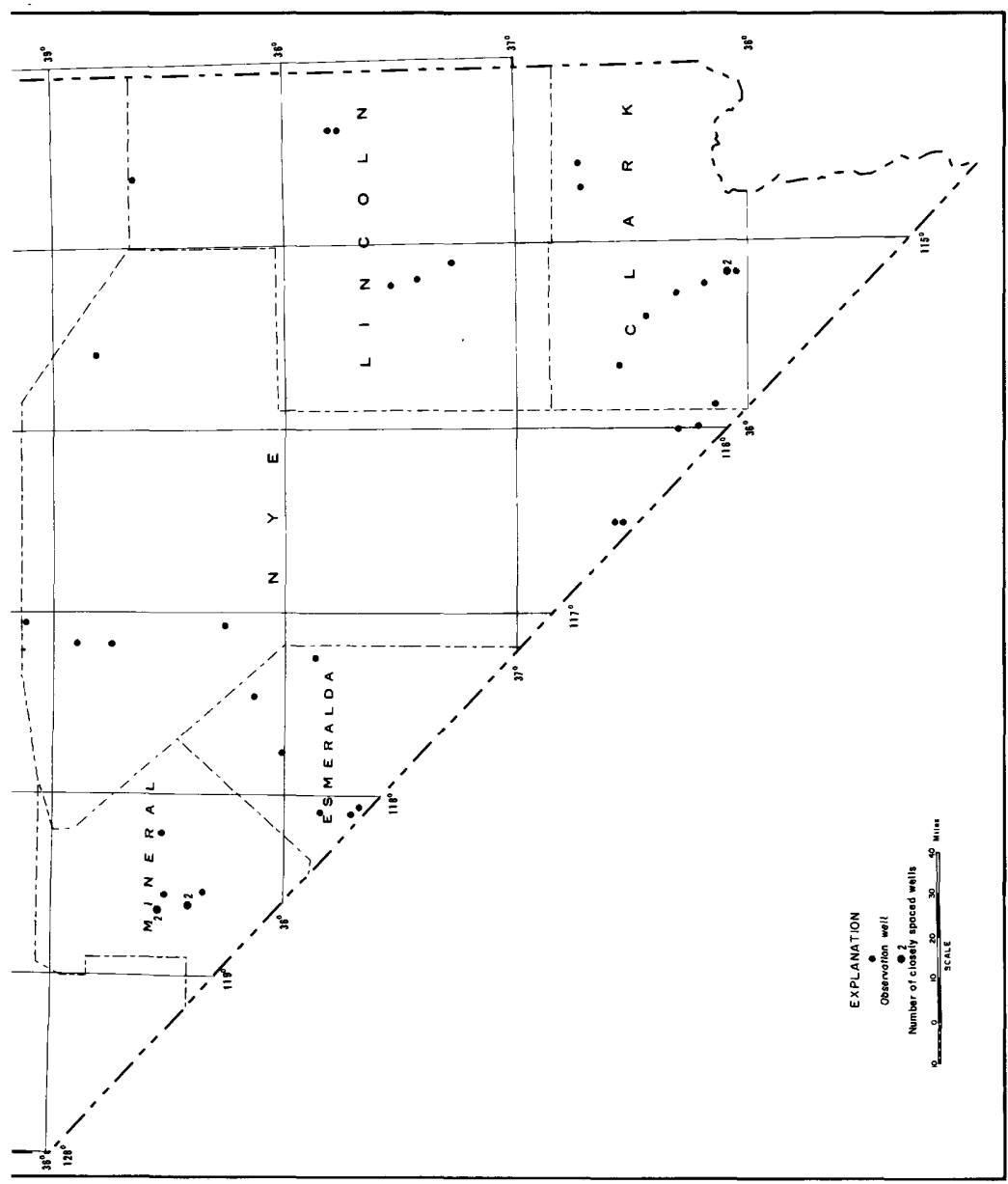

宔 
S19/60-9bcc1--Continued.

1957

\begin{tabular}{l|l|l|l|l|l|l|l|l|l|l|l|l}
\hline Day & Jan. & Feb. & Mar. & Apr. & May & June & July & Aug. & Sept. & Oct. & Nov. & Dec. \\
\hline 5 & 01. & 89.5 & 91 &
\end{tabular}

\begin{tabular}{l|l|l|l|l|l|l|l|l|l|l|l|l|l|l|}
10 & $\ldots \ldots$ & 89.29 & 91.16 & 93.24 & $\ldots$. & 96.86 & 97.50 & 97.30 & 98.40 & 95.42 & 93.92 & 92.30
\end{tabular}

\begin{tabular}{l|l|l|l|l|l|l|l|l|l|l|l|l|l|l|l|l|l|l|l|}
15 & 91.02 & 91.18 & 91.33 & 94.18 & 96.08 & 96.95 & 96.66 & 97.29 & 98.55 & 95.88 & 93.60 & 92.09
\end{tabular}

\begin{tabular}{l|l|l|l|l|l|l|l|l|l|l|l|l|}
20 & 91.33 & 91.54 & 90.95 & 94.27 & 95.11 & 97.00 & 97.76 & 97.24 & 98.74 & 95.72 & 93.35 & 91.99
\end{tabular}

\begin{tabular}{l|l|l|l|l|l|l|l|l|l|l|l|l|l|l|l|l|}
25 & 90.24 & 89.86 & 91.02 & 94.38 & 96.21 & 97.30 & 97.86 & 97.07 & 97.97 & 95.43 & 92.99 & 91.89 \\
\hline
\end{tabular}

\begin{tabular}{l|l|l|l|l|l|l|l|l|l|l|l|l|l|} 
Eom & 89.85 & 90.54 & 90.61 & 95.25 & 96.44 & 96.77 & 97.64 & 97.48 & 98.23 & 95.27 & 92.77 & 91.99 \\
\hline
\end{tabular}

\begin{tabular}{l|l|l|l|l|l|l|l|l|l|l|l|l|}
\hline 5 & 91.75 & 91.69 & 92.34 & 91.14 & 93.16 & 95.32 & $\ldots$ & 95.95 & 97.92 & 95.60 & 94.49 & 93.26 \\
\hline
\end{tabular}

\begin{tabular}{l|l|l|l|l|l|l|l|l|l|l|l|l|l|}
10 & 91.42 & 91.30 & 92.77 & 90.89 & 93.78 & 94.55 & 96.88 & 97.22 & 97.07 & 95.91 & 95.61 & 93.34
\end{tabular}

\begin{tabular}{l|l|l|l|l|l|l|l|l|l|l|l|l|l|l|l|}
15 & 91.30 & 91.10 & 91.22 & 90.86 & 93.70 & 95.75 & 96.17 & 96.58 & 97.24 & 94.84 & 94.46 & 93.77
\end{tabular}

\begin{tabular}{l|l|l|l|l|l|l|l|l|l|l|l|l|l|l|}
20 & 91.17 & 91.74 & 91.06 & 93.17 & 94.14 & 95.92 & 96.10 & 97.52 & 97.59 & 95.75 & 94.20 & 92.96
\end{tabular}

\begin{tabular}{l|l|l|l|l|l|l|l|l|l|l|l|l|l|l|}
25 & 91.13 & 92.02 & 92.33 & 92.71 & 93.86 & 95.82 & 96.23 & 97.63 & 96.88 & 96.01 & 93.89 & 92.73 \\
\hline
\end{tabular}

\begin{tabular}{l|l|l|l|l|l|l|l|l|l|l|l|l|l} 
Eom & 92.15 & 91.27 & 91.29 & 92.88 & 95.13 & 96.59 & 95.97 & 97.83 & 95.62 & 95.19 & 94.42 & 92.56 \\
\hline
\end{tabular} 1959

\begin{tabular}{l|l|l|l|l|l|l|l|l|l|l|l|l}
\hline 5 & 92.50 & 93.03 & $\ldots \ldots$ & 93.66 & 95.84 & $\ldots \ldots$ & $\ldots \ldots$ & 98.44 & 97.88 & 97.07 & 94.51 & 93.80 \\
10 & 92.37 & 92.08 & 93.43 & $\ldots$. & 95.39 & 96.80 & 97.42 & $\ldots \ldots$ & 98.57 & 95.87 & 93.97 & 93.02 \\
15 & 92.11 & 92.31 & 93.51 & $\ldots \ldots$ & $\ldots \ldots$ & 96.36 & 97.97 & 99.37 & 97.23 & 97.10 & 93.76 & 93.29 \\
20 & 92.38 & 91.40 & $\ldots \ldots$ & $\ldots \ldots$ & $\ldots \ldots$ & 97.29 & $\ldots \ldots$ & 98.93 & 97.03 & 97.15 & 93.85 & $\ldots .$. \\
25 & 92.34 & 91.40 & 93.61 & 93.86 & 96.90 & 96.22 & 97.61 & 97.98 & 97.95 & 95.84 & 93.23 & 92.07 \\
Eom & 91.56 & 92.23 & 93.99 & 94.63 & 96.60 & 97.71 & 99.03 & 96.68 & 96.88 & 95.45 & 94.79 & 92.06 \\
\hline \multicolumn{10}{|c|}{1960} \\
\hline 5 & 91.98 & 90.96 & $\ldots$ & 92.71 & 94.53 & 94.98 & 96.35 & 96.43 & 95.80 & 97.37 & 96.10 & 93.50 \\
10 & 91.66 & 91.18 & 90.92 & 93.04 & 94.66 & 94.90 & 96.14 & 96.15 & 96.10 & 96.15 & 94.46 & 93.04 \\
20 & 91.40 & 90.69 & 92.29 & $\ldots \ldots$ & 94.60 & 96.65 & 97.76 & 95.91 & 96.05 & 96.26 & 95.27 & 92.83 \\
25 & 91.89 & 90.56 & 92.71 & 94.41 & $\ldots \ldots$ & 95.21 & 96.18 & 96.01 & 95.91 & 95.58 & 95.32 & 92.72 \\
Eom & 91.06 & $\ldots \ldots$ & 93.03 & 93.58 & $\ldots \ldots$ & 95.96 & 96.39 & 95.80 & 96.20 & 95.94 & 93.70 & 92.75 \\
\hline
\end{tabular}

S20/60-25adb1. Jones (State Engineer No. 602). Drilled irrigation and domestic artesian well, diam 10 to $8 \mathrm{in}$, depth $438 \mathrm{ft}$, cased, 8-in 0-318, 6-in 318-430. MP top of casing, $1.00 \mathrm{ft}$ above 1sd. Highest water level 25.00 below lsd, July 7, 1948 (previously reported 30.81 below Isd, July 31, 1948); lowest 75.42 below lsd, June 26, 1959. Records available: 1948-59. Measurement discontinued.

Water level at noon, from recorder graph, 1956

\begin{tabular}{|c|c|c|c|c|c|c|c|c|c|c|c|c|}
\hline Day & Jan. & Feb. & Mar. & Apr. & May & June & July & Aug. & Sept. & Oct. & Nov. & Dec. \\
\hline 5 & 50.43 & 50.02 & 51.51 & 54.10 & 55.79 & 59.59 & 61.30 & 61.09 & 63.58 & 60.57 & & 57.34 \\
\hline 10 & 50.64 & 50.46 & 50.98 & 54.46 & 55.92 & 59.86 & 61.57 & 62.36 & 62.60 & 60.90 & 59.05 & 56.96 \\
\hline 15 & 50.26 & 50.70 & 52.46 & 53.88 & 57.06 & 60.66 & 62.11 & 62.70 & 63.02 & 60.40 & 38.78 & 56.90 \\
\hline 20 & 50. 26 & & 52.56 & 54.51 & 57.63 & 60.61 & 62.54 & 61.98 & 62.95 & 61.00 & 58.84 & .86 \\
\hline 25 & 50.58 & 50.40 & 52.82 & 55.26 & 58.91 & 60.88 & 60.64 & 62.60 & 61.90 & 59.03 & 58.24 & 56.50 \\
\hline Eom & 49.99 & 51.14 & 53.42 & 55.94 & 58.69 & 61.42 & 60.09 & 63.99 & 61.52 & 60.20 & 58.07 & 56.60 \\
\hline \multicolumn{13}{|c|}{1957} \\
\hline 5 & 56.60 & 55.98 & 57.32 & 60.04 & 60.86 & 64.07 & & 67.90 & 66.44 & 66.52 & 62.16 & 60.57 \\
\hline 10 & 56. 38 & 55.92 & 56.96 & 60.08 & 61.20 & 64.57 & 67.18 & 67.69 & 66.89 & 66.23 & 61.87 & 61.36 \\
\hline 15 & 56.31 & 56.50 & 57.42 & 60.74 & 60.62 & 65.56 & 66.95 & 68.03 & 66.24 & 64.92 & 61.58 & 60.35 \\
\hline 20 & 55.45 & 56.97 & & 59.79 & 61. 32 & 65.54 & 67.03 & 66.27 & 66.16 & 63.80 & 61.54 & 60.30 \\
\hline 25 & 55.86 & 56.46 & & 58.94 & 61.10 & 66.13 & 67.88 & 64.72 & 66.69 & 63.90 & 62.01 & 60.31 \\
\hline Eom & 55.78 & 56.82 & 58.92 & 60.31 & 63.28 & 66.55 & 66.04 & 66.15 & & 62.70 & 61.70 & 60.35 \\
\hline \multicolumn{13}{|c|}{1958} \\
\hline 5 & 60.20 & 59.56 & 61.16 & 60.40 & 65.20 & 29.13 & 71.41 & $\overline{72.75}$ & 73.44 & 71.30 & 69.82 & 66.26 \\
\hline 10 & 59.92 & 59.58 & 60.54 & 60.97 & 66.06 & 69.72 & 71.98 & 72.47 & 71.92 & 71.96 & 58.77 & 66.47 \\
\hline 15 & 60.14 & 60.32 & 59.72 & 62.20 & 65.20 & 70.03 & 72.61 & 70.38 & 71.67 & 72.28 & 67.27 & 66.13 \\
\hline 20 & 60.10 & 60.04 & 60.80 & 63.10 & 66.97 & 70.09 & 72. 41 & 72.24 & 72. 42 & 71.71 & 66.99 & 65.90 \\
\hline 25 & 59.78 & 59.84 & 61.44 & 63.69 & 68.19 & 70.84 & 72.46 & 73.00 & 70.00 & 69.85 & 67.12 & 65.52 \\
\hline Eom & 59.72 & 60.43 & 61.04 & 64.17 & 68.73 & 70.90 & 71.54 & 72.86 & 71.74 & 69.76 & & 65.44 \\
\hline \multicolumn{13}{|c|}{1959} \\
\hline 5 & 65.25 & $\overline{65 . \overline{3} 8}$ & & & 69.30 & 72.78 & & & & & & \\
\hline 10 & 64.84 & 64.46 & $\cdots$ & & 68.82 & 73.79 & & $\ldots \ldots$ & . & & & \\
\hline 15 & 64.85 & & & & 70.38 & 74.28 & & . & & & & \\
\hline 20 & 64.76 & 63.89 & & & 70.39 & 74.54 & & & & & & \\
\hline 25 & 64.72 & 64.59 & 66.78 & 68.50 & 70.60 & 75.04 & $\cdots$ & $\ldots \ldots$ & $\ldots \ldots$ & $\ldots \ldots$ & $\ldots$ & .. \\
\hline Eom & & 64.32 & 66.45 & 69.38 & 72.16 & 74.96 & & & & & & \\
\hline
\end{tabular}

S21/61-4aad1. Opaco Lumber Co. (State Engineer No. 386). Drilled unused artesian well, diam $10 \mathrm{in}$, depth $793 \mathrm{ft}$, cased to 770 , perforated 338-438, 642-770. Lsd 2, 038.76 ft above msl. MP top of 8-in plug, 3.00 ft above 1sd. Highest water level 46.7 above 1sd, Feb. 8, 1950 (previously reported 46.5 above lsd, Feb. 21, 1952); lowest 5.98 below Isd, Aug. 3, 1960. Records available: $1944-60$. 
S21/61-4aad1--Continued.

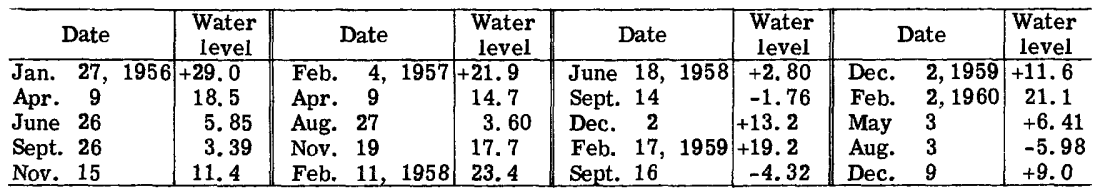

S21/61-22ccc1. A. P. Baker (State Engineer No. 117). Drilled unused artesian well, diam 6 in, depth $500 \mathrm{ft}$, cased. Lsd 2, 070. $8 \mathrm{ft}$ above msl. MP $1 / 8$-in plug in casing, $1.00 \mathrm{ft}$ above lsd. Highest water level 35.65 above lsd, Dec. 20, 1942, Jan. 24, 1943; lowest 23.45 below Isd, Aug. 3, 1960. Records available: 1940-60.

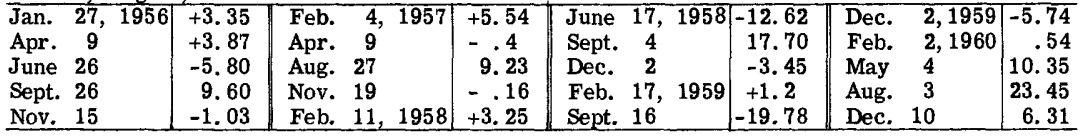

S22/61-4bcc1. Fitzpatrick (State Engineer No. 41). Drilled unused well, diam 8 in, depth $355 \mathrm{ft}$, cased. Lsd 2, 224. $91 \mathrm{ft}$ above msl. MP top of casing, $3.80 \mathrm{ft}$ above $1 \mathrm{sd}$. Highest water level 74.4 below lsd, Jan. 25, 1939; lowest 101.97 below lsd, Sept. 1, 1960 . Records available: $1938-60$.

\begin{tabular}{|c|c|c|c|c|c|c|c|c|c|c|c|c|}
\hline & & & & & & & & & & & & \\
\hline bay & Jan. & Feb. & Mar. & Apr. & May & June & July & Aug. & Sept. & Oct. & Nov. & Dec. \\
\hline 5 & 88.93 & 88.63 & 89.44 & 90.05 & 91.03 & 92.32 & 93.52 & 93.10 & & 93.43 & & 91.09 \\
\hline 10 & 89.00 & 89.03 & 89.61 & 90.43 & 91.12 & 92.24 & 93.50 & & 94.54 & 93.73 & 92.13 & $\ldots .$. \\
\hline 15 & 88.96 & 89.03 & 89.96 & 90.42 & 91.22 & 92.23 & 93.61 & 93.44 & & & 91.88 & 0.84 \\
\hline 20 & 88.72 & 89.31 & & 90.71 & & 92. 61 & 93.68 & 93.88 & 94.56 & 93.11 & 91.37 & 90.88 \\
\hline 25 & 88.81 & 89.49 & 89.70 & 90.83 & 91.80 & 92.88 & 92.91 & $\ldots$ & 94.02 & 92.58 & 91.14 & 90.81 \\
\hline Eom & 88.73 & 89.44 & 89.76 & 91.03 & 92. 38 & 92.92 & 92.63 & & 94.24 & 92.20 & 91.11 & 90.68 \\
\hline & & & & & & 1957 & & & & & & \\
\hline 5 & 1.02 & 50 & 91.69 & 2.88 & & 94.71 & $\overline{95.54}$ & 96.09 & $\overline{95.59}$ & $\longdiv { 5 . 7 1 }$ & $\overline{93.19}$ & .93 \\
\hline 10 & .06 & 90.55 & 91.49 & 93. 01 & 93.49 & 95.10 & 95.66 & 96.38 & 95.87 & 95.33 & 93.15 & 3.17 \\
\hline 15 & 90.83 & 90.99 & 92.37 & 93.17 & 93.63 & 11 & 95.89 & 96.46 & 95.45 & 95.06 & 92.93 & 93.59 \\
\hline 20 & 90.65 & 91.39 & 92.15 & 92.76 & 93.38 & 95.81 & 96.17 & 96.39 & 95.89 & 94.65 & 92.84 & 93.55 \\
\hline 25 & 90.48 & 91.50 & 91.81 & 92.48 & 93.73 & 95.83 & 96.32 & 94.87 & 95.93 & 94.15 & 92.82 & 93.44 \\
\hline Eom & 90.40 & 91.47 & & 92.87 & 94.52 & 95.86 & 95.61 & 95.09 & 96.02 & 93.58 & 93.37 & 93.19 \\
\hline & & & & & & 195 & & & & & & \\
\hline 5 & 93.16 & 92.23 & 92.63 & 93.48 & 94.95 & 96.12 & 97.55 & 97.64 & 97.82 & 97.68 & 96.09 & 95.20 \\
\hline 10 & 93.09 & 92.11 & 92.48 & 93.54 & 95.15 & 96.38 & 97.89 & 97.44 & 97.65 & 97.84 & 96.30 & 95.10 \\
\hline 15 & 92. 79 & 92.11 & 92.49 & 93.37 & 94.61 & 97.12 & 97.63 & 97.67 & 97.84 & 97.28 & & 4.78 \\
\hline 20 & 92.38 & 92.12 & 92.82 & 94.21 & 95 & 97.39 & 97.81 & 98.12 & 97.70 & 97.20 & & 94.60 \\
\hline 25 & 92. 35 & 92.11 & 93.29 & 94.39 & 96.18 & 97.88 & 97.68 & 98.04 & 97.47 & 97.13 & & 94.43 \\
\hline Eon & 92.39 & 92.45 & 93.90 & & & 97.58 & & 97.65 & 97.42 & 96.34 & 95.33 & 94.39 \\
\hline & & & & & & 1959 & & & & & & \\
\hline 5 & 94.29 & & & 35 & 96.62 & 97.57 & 98.60 & & 99.42 & & 97.35 & 96.63 \\
\hline 0 & 24 & 82 & $\ddot{94} \ddot{99}$ & 95,28 & 96.89 & 98.17 & 98.50 & 98.82 & 99.38 & 98.25 & 96.99 & 96.53 \\
\hline 15 & .61 & 94.72 & 95.34 & & 97.08 & 98.41 & 98.53 & 98.94 & 98.99 & 98.17 & 97.03 & 96.20 \\
\hline 20 & 94.65 & 94.71 & & & 97.15 & 98.54 & 98.57 & 98.92 & 98.77 & 97.86 & 97.08 & 96.25 \\
\hline 25 & 69 & 94.67 & 95.52 & 95.98 & 97.33 & 98.45 & 98.61 & 99.00 & 98.76 & 97.99 & 97.08 & 95.91 \\
\hline Eom & 9 & 8 & 95.54 & 96.12 & & 98.66 & 98.88 & 99.10 & 98.54 & 97.84 & 96.83 & 95.88 \\
\hline & & & & & & & & & & & & \\
\hline 5 & 96.06 & 95.96 & 96.40 & 98.19 & 98.30 & 99.61 & 100.86 & 101.40 & 00.91 & 100.67 & 99.35 & 98.42 \\
\hline 10 & 95.92 & 95.69 & 96.98 & 98.31 & 98.70 & 99 & 100.71 & 101.20 & 101.12 & 100.40 & 98.86 & 98.20 \\
\hline 15 & 95.91 & 95.84 & 96.62 & 98.09 & 98.91 & 99.72 & 81 & 100.93 & 101.26 & 99.74 & 98.66 & 98.23 \\
\hline 20 & 95.94 & 95. & 97.47 & 98.33 & & & 100 & 100.68 & 28 & 99.66 & 98.33 & 98.41 \\
\hline 25 & 95.72 & 95.69 & 98.02 & 98.33 & 98,96 & 99.92 & 100.60 & 100.91 & 100.98 & 99.52 & 98.47 & 98.42 \\
\hline Eom & 9 & 96.05 & 98.32 & 20 & 99.08 & 100 & 14. & 40 & 100.82 & 99.64 & 98.45 & 98.25 \\
\hline
\end{tabular}

\section{Muddy River Valley}

S14/65-23ac2. V. V. Perkins. Drilled unused well, diam 6 in, depth $50 \mathrm{ft}$, cased. MP top of casing, $1.90 \mathrm{ft}$ above lsd. Highest water level 0.01 below lsd, Mar. 23, 1956; lowest 7. 27 below Isd, Sept. 23, 1948. Records available: 1948-53, 1955-59. No measurement made in 1960. Mar. 23, 1956, 0.01; Oct. 22, 1957, 1.90; Sept. 5, 1958, 4. 36; Sept. 18, 1959, 4. 82.

S14/66-35dd1. Jay Robb. Drilled irrigation well, diam 16 in, depth $118 \mathrm{ft}$, cased 0-98, perforated 65-88. MP bottom of slot in casing, $0.80 \mathrm{ft}$ above lsd. Highest water level 11.16 below lsd, Dec. 18, 1951; lowest 38.75 below lsd, Aug. 2, 1960. Records available: 1948, 1950$54,1957-60$. No measurement made 1955-56. 
S14/66-35dd1--Continued.

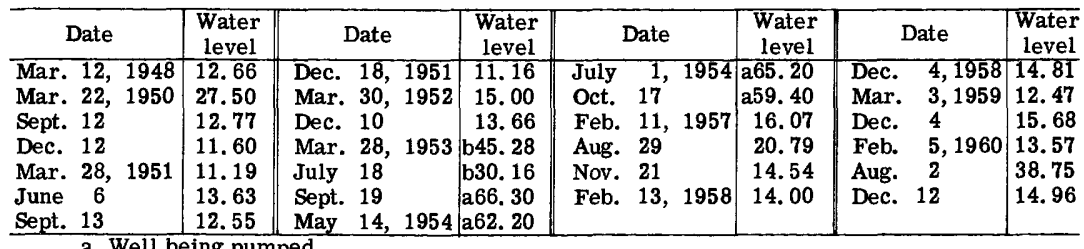

a Well being pumped.

b Well pumped recently.

\section{Pahrump Valley}

S21/54-10aac1. Bowman (State Engineer No. 22). Drilled unused well, diam 14 in, depth $800 \mathrm{ft}$, cased to 472 , perforated $100-450$. Lsd 2,885. $68 \mathrm{ft}$ above msl. MP top of casing, $0.30 \mathrm{ft}$ above lsd. Highest water level 25.99 below lsd, Jan. 5, 1945; lowest 52.53 below lsd, Aus. 4, 1958. Records available: 1944-58. Measurement discontinued.

Water level at noon, from recorder graph, 1956

\begin{tabular}{|c|c|c|c|c|c|c|c|c|c|c|c|c|}
\hline Day & Jan. & Feb. & Mar. & Apr. & May & June & July & Aug. & Sept. & Oct. & Nov. & Dec. \\
\hline 5 & & & 41.57 & 42.10 & 42.44 & $\cdots$ & $\Rightarrow$ & 43.54 & 43.98 & $\cdots \cdots$ & $\cdots \cdots$ & .13 \\
\hline 10 & & & 41.70 & & 42.27 & & & - & $\cdots$ & & & $\cdots$ \\
\hline 15 & & 41.11 & & & & & & & 43.89 & •• & . & $\ldots$ \\
\hline 20 & & 41.48 & 40.99 & 42. 47 & & & & & 45.01 & & & 54 \\
\hline 25 & & & 40.98 & 42.41 & 42.84 & & & . & & $\ldots$ & & 83 \\
\hline Eon & & & & 42.48 & & & & & & & & \\
\hline \multicolumn{13}{|c|}{1957} \\
\hline 5 & 0.82 & 1.00 & & & 3.96 & 43.92 & 4.50 & 4.93 & $75 . \overline{27}$ & 5.31 & 13.64 & 42.03 \\
\hline 10 & 40.22 & .89 & & & 44.10 & 25 & & .96 & 45.30 & 45.21 & 8 & 41. 85 \\
\hline 15 & & & & 44.14 & 44.08 & 44.16 & & 45.10 & 45. 26 & 45.06 & 42.85 & 41.79 \\
\hline 20 & 1. 44 & & 43.78 & 44.03 & 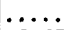 & 26 & & & & 44.63 & 42 & 42.18 \\
\hline 25 & 41.61 & 42.36 & 43.99 & 44.26 & 43.65 & 44.42 & & 45 & 45 & 45.06 & 42.32 & 41.77 \\
\hline on & 41.35 & 42.54 & 43.95 & 44. 56 & 44.24 & 44.57 & 44.67 & 45.31 & & 44.52 & 42 & 41.63 \\
\hline \multicolumn{13}{|c|}{1958} \\
\hline 5 & 41.56 & .66 & 90 & $47 . \overline{28}$ & $45 . \overline{38}$ & 47.92 & .43 & 52.50 & 51.58 & 49.50 & 46.43 & 45.59 \\
\hline 10 & 42 & 41. & 43. & 47.81 & 45.86 & & 28 & & & & & \\
\hline 15 & 42.23 & 41.32 & 43. 94 & & & 47 & 50.52 & & & & & 46 \\
\hline 20 & 42.04 & 41.09 & 45.42 & 47.12 & 45.24 & & & & 51.81 & & 45.59 & 46. 64 \\
\hline 25 & 41.74 & 40.74 & & 46.81 & 45.79 & & & 51.56 & 49.61 & 47.10 & 45.29 & 48.40 \\
\hline Eom & 41.81 & 41.24 & 47.61 & 46.04 & 47.88 & 49.90 & 52.12 & 52.12 & & 48.22 & 45.24 & $\cdots$ \\
\hline
\end{tabular}

\section{Douglas County}

\section{Carson Valley}

12/20-17ba1. John Helwinkel, Jr. Drilled irrigation water-table well in alluvium of Quaternary age, diam $18 \mathrm{in}$, depth $365 \mathrm{ft}$, cased. Lsd about 4, $775 \mathrm{ft}$ above msl. MP slot in pumpbase, $0.10 \mathrm{ft}$ above lsd. Highest water level 8.82 below lsd, July 9, 1949; lowest 20.19 below lsd, Mar. 22, 1960. Records available: 1948-56, 1959-60. No measurement made 1957-58. Apr. 6, 1956, 18. 60; Mar. 31, 1959, 18. 47; Oct. 5, 17.59; Mar. 22, 1960, 20. 19.

13/20-8ca1. C. W. Godecke. Drilled stock and irrigation well, diam 18 to 12 in, depth $300 \mathrm{ft}$, cased, 18-in 0-60, 12-in 60-300. MP slot in pumpbase, $0.80 \mathrm{ft}$ above 1sd. Highest water level flowing, Mar. 30, 1950, Sept. 18, Dec. 23, 1952, Apr. 3, 1953; lowest 6.36 below 1sd, Sept. 27, 1954. Records available: 1942, 1948-52, 1954-60.

\begin{tabular}{l|r||c|c||c|c|c|c}
\multicolumn{1}{c|}{ Date } & $\begin{array}{r}\text { Water } \\
\text { level }\end{array}$ & Date & $\begin{array}{r}\text { Water } \\
\text { level }\end{array}$ & Date & $\begin{array}{r}\text { Water } \\
\text { level }\end{array}$ & Date & $\begin{array}{r}\text { Water } \\
\text { level }\end{array}$ \\
\hline Apr. 6,1956 & $\mathbf{0 . 9 0}$ & Oct. 2, 1957 & $\mathbf{3 . 5 0}$ & Sept. 23, 1958 & $\mathbf{0 . 8 8}$ & Oct. 5, 1959 & $\mathbf{2 . 8 0}$ \\
Oct. 2 & .50 & Mar. 28, 1958 & 1.54 & Mar. 16, 1959 & .50 & Mar. 22, 1960 & 1.84 \\
Mar. 19, 1957 & .32 & & & & & &
\end{tabular}

13/20-29aab1. H. F. Dangberg Co. Drilled irrigation artesian well, diam 12 in, reported depth $320 \mathrm{ft}$, reported plugged at $125 \mathrm{ft}$. MP top of discharge pipe, $4.85 \mathrm{ft}$ above lsd. Highest water level 0.10 above 1sd, May 11, 1948; lowest 4.04 below Isd, Aug. 15, 1950. Records available: $1948-60$.

\begin{tabular}{|l|l||l|l|l|l|l||l|l}
\hline Apr. 6, 1956 & 1.98 & Oct. 2, 1957 & 1.57 & Mar. 16, 1959 & 2.13 & Mar. 22, 1960 & 2.14 \\
Oct. 2 & 1.44 & Mar. 28, 1958 & 1.98 & Oct. 5 & 2.38 & Aug. 26 & 2.35 \\
Mar. 19, 1957 & 1.83 & Sept. 23 & 1.41 & & & & \\
\hline
\end{tabular}


13/20-31bdl. H. Dangberg. Drilled irrigation water-table well in alluvium of Quaternary age, diam 16 in, depth $413 \mathrm{ft}$, cased to 400 , perforated 60-400. MP end of 6-in discharge pipe, $7.00 \mathrm{ft}$ above lsd. Highest water level 2.12 below lsd, Dec. 19, 1950, Sept. 18, 1952; lowest 7.52 below lsd, Aug. 15, 1950. Records available: 1950-60.

\begin{tabular}{c|c||c|c||c|c||c|c}
\hline \multicolumn{1}{c|}{ Date } & $\begin{array}{c}\text { Water } \\
\text { level }\end{array}$ & Date & $\begin{array}{c}\text { Water } \\
\text { level }\end{array}$ & Date & $\begin{array}{c}\text { Water } \\
\text { level }\end{array}$ & \multicolumn{1}{|c|}{ Date } & $\begin{array}{c}\text { Water } \\
\text { level }\end{array}$ \\
\hline Apr. 6, 1956 & $\mathbf{3 . 1 7}$ & Oct. 2, 1957 & $\mathbf{3 . 9 6}$ & Mar. 16, 1959 & 2.26 & Mar. 22, 1960 & 3.40 \\
Oct. 2 & 2.83 & Mar. 28, 1958 & 2.97 & Oct. 5 & $\mathbf{3 . 8 4}$ & Aug. 26 & $\mathbf{5 . 5 0}$ \\
Mar. 19, 1957 & $\mathbf{2 . 9 8}$ & Sept. 23 & 6.70 & & & \\
\hline
\end{tabular}

13/20-32dc1. Mack Land \& Cattle Co. Drilled irrigation water-table well in alluvium of Quaternary age, diam $18 \mathrm{in}$, reported depth $420 \mathrm{ft}$. Lsd about $4,733 \mathrm{ft}$ above msl. MP top of casing, $0.40 \mathrm{ft}$ above 1sd. Highest water level 7.83 below 1sd, May 24, 1950; lowest 14.17 below 1sd, Aug. 26, 1960. Records available: 1948-60.

\begin{tabular}{|c|c|c|c|c|c|c|c|}
\hline $\begin{array}{lrr}\text { Apr. } & 6, & 1956 \\
\text { Oct. } & 2 & \\
\text { Mar. } & 19, & 1957\end{array}$ & $\begin{array}{r}10.28 \\
8.65 \\
9.92\end{array}$ & $\begin{array}{l}\text { Oct. 2, 1957 } \\
\text { Mar. 28, 1958 } \\
\text { Sept. 23 }\end{array}$ & $\begin{array}{r}10.57 \\
10.06 \\
8.21\end{array}$ & $\begin{array}{l}\text { Mar. } \\
\text { Oct. } 5\end{array}$ & \begin{tabular}{|l|}
10.30 \\
10.64
\end{tabular} & $\begin{array}{l}\text { Mar. 22,1960 } \\
\text { Aug. } 26\end{array}$ & $\begin{array}{l}10.76 \\
14.17\end{array}$ \\
\hline
\end{tabular}

14/19-25ba1. Carson Indian Agency. Drilled irrigation water-table well in alluvium of Quaternary age, diam $12 \mathrm{in}$, depth $239 \mathrm{ft}$, cased. MP top of casing, $0.50 \mathrm{ft}$ above lsd. Highest water level 10. 49 below 1sd, May 10, 1946 (previously reported 10.82 below lsd, Apr. 2, 1951); lowest 20.09 below 1sd, Aug. 3, 1948. Records available: 1946, 1948-60. Apr. 6, 1956, 14. 23; Mar. 19, 1957, 11.19; Mar. 26, 1958, 14. 34; Mar. 16, 1959, 12. 58; Oct. 5, 20.52 (well pumped recently); Mar. 22, 1960, 20.90 (well pumped recently).

\section{Elko County}

\section{Clover Valley}

35/62-27b1. U. S. Geol. Survey. Drilled test and observation well, diam 6 in, depth $286 \mathrm{ft}$, cased 0-197. MP top of cap on casing, $0.45 \mathrm{ft}$ above lsd. Highest water level 6.70 below lsd (previously reported 6.65 below 1sd), Mar. 27, 1951; lowest 11. 07 below 1sd, May 3, 1955. Records available: 1949-60.

\begin{tabular}{|c|c|c|c|c|c|c|c|c|}
\hline $\begin{array}{lrl}\text { Jan. } & 31, & 1956 \\
\text { Feb. } & 25 \\
\text { Apr. } & 1 \\
\text { June } & 15\end{array}$ & $\begin{array}{l}9.52 \\
9.86 \\
9.67 \\
7.73\end{array}$ & $\begin{array}{l}\text { Aug. } \\
\text { Oct. } \\
\text { Jan. }\end{array}$ & $\begin{array}{rr}21, & 1956 \\
29 & \\
31 & \\
3, & 1957\end{array}$ & $\begin{array}{l}8.71 \\
8.78 \\
9.38 \\
9.39\end{array}$ & $\begin{array}{ll}\text { Mar. } & \text { 2, } 1957 \\
& 29 \\
& \\
\text { June } & 29 \\
\text { Sept. } 10 & \end{array}$ & $\begin{array}{l}9.37 \\
9.33 \\
6.99 \\
8.21\end{array}$ & $\begin{array}{lr}\text { Dec. } & 27,1957 \\
\text { Sept. } & 4,1958 \\
\text { Sept. } & 13,1959 \\
\text { Aug. } 14,1960\end{array}$ & $\begin{array}{l}8.72 \\
8.07 \\
9.85 \\
9.86 \\
\end{array}$ \\
\hline
\end{tabular}

35/62-27b2. U. S. Geol. Survey. Drilled observation water-table well, diam 1 in, depth $15 \mathrm{ft}$, cased. MP top of casing, $0.47 \mathrm{ft}$ above lsd. Highest water level 6.53 below lsd, June 9 , 1949 (previously reported 7.10 below lsd, Sept. 29, 1952); lowest 10.91 below isd, May 3, 1955. Records available: $1949-60$.

\begin{tabular}{|c|c|c|c|c|c|c|c|c|}
\hline $\begin{array}{lcc}\text { Jan. } & 31, & 1956 \\
\text { Feb. } & \mathbf{2 5} \\
\text { Apr. } & 1 \\
\text { June } & 15\end{array}$ & $\begin{array}{l}8.87 \\
9.44 \\
9.45 \\
8.98\end{array}$ & $\begin{array}{l}\text { Aug. } \\
\text { Oct. } \\
\text { Jan. }\end{array}$ & \begin{tabular}{rr|}
21, & 1956 \\
29 & \\
31 & \\
3, & 1957
\end{tabular} & $\begin{array}{l}9.22 \\
9.29 \\
9.45 \\
9.31\end{array}$ & $\begin{array}{ll}\text { Mar. } & 2,1957 \\
& 29 \\
\text { June } & 29 \\
\text { Sept. } 10 & \end{array}$ & $\begin{array}{l}9.19 \\
9.15 \\
7.89 \\
9.01\end{array}$ & $\begin{array}{lr}\text { Dec. } & 27,1957 \\
\text { Sept. } & 4,1958 \\
\text { Sept. } & 13,1959 \\
\text { Aug. } & 14,1960\end{array}$ & $\begin{array}{l}8.75 \\
8.36 \\
9.27 \\
9.73 \\
\end{array}$ \\
\hline
\end{tabular}

Goshute-Antelope Valley

34/67-6a2. Western Pacific RR. Co. Shafter. Drilled industrial water-table well, diam 16 in, reported depth $250 \mathrm{ft}$, cased. MP top of curb, at lsd (since Mar. 27, 1956). Highest water level 26. 85 below 1sd, Mar. 27, 1951; lowest 30.51 below 1sd, Mar. 28, 1949. Records available: 1948-60. Mar. 27, 1956, 27. 29; Aug. 29, 28. 22; Sept. 11, 1957, 28. 75; Sept. 5, 1958, 29.02; Sept. 12, 1959, 29.18; Aug. 14, 1960, 29.17.

\section{Humboldt River Valley}

[See also Humboldt, Lander, and Pershing Counties]

33/52-27d1. Carlin Town Government. Drilled unus ed water-table well, diam 20 in, depth $500 \mathrm{ft}$, cased to 125 . MP top of casing, $0.75 \mathrm{ft}$ above 1sd. Highest water level 2.77 below 1sd, Feb. 20, 1951; lowest 8.88 below 1sd, Jan. 20, 1955. Records available: 1938-60.

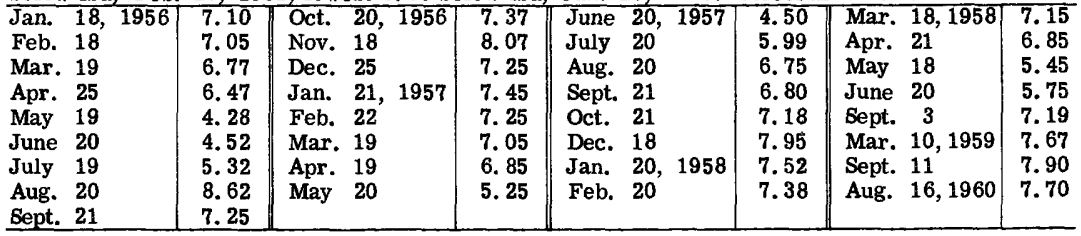


35/56-30c1. Fernald. Dug unused water-table well, depth $20 \mathrm{ft}$. MP roofing tack at top of 4 - $\times 6$-in wooden pipe clamp, $0.90 \mathrm{ft}$ above lsd. Highest water level 5. 20 below 1sd, May 28, 1950; lowest 18. 5 below 1sd, Sept. 28, 1956. Records available: 1938-60.

\begin{tabular}{|c|c|c|c|c|c|c|c|}
\hline Date & $\begin{array}{r}\text { Water } \\
\text { level }\end{array}$ & Date & $\begin{array}{l}\text { Water } \\
\text { level }\end{array}$ & Date & $\begin{array}{c}\text { Water } \\
\text { level }\end{array}$ & Date & $\begin{array}{l}\text { Water } \\
\text { level }\end{array}$ \\
\hline Mar. 30,1956 & 13.0 & Dec. 28,1956 & 16.0 & Sept. 28, 1957 & 15.4 & Apr. 28, 1958 & 13.4 \\
\hline Apr. 30 & 15.0 & Feb. 28,1957 & 13.4 & Oct. 28 & 18.3 & May 28 & 8.5 \\
\hline May 30 & 10.8 & Mar. 28 & 13.9 & Nov. 28 & 16.1 & June 28 & 9.1 \\
\hline June 30 & 10.7 & Apr. 28 & 12.7 & Dec. 28 & 14.8 & Sept. 5 & 15.35 \\
\hline July 30 & 11.7 & May 29 & 8.3 & Jan. 28,1958 & 7.9 & Mar. 10,1959 & 14.06 \\
\hline Aug. 28 & 15.2 & June 28 & 10.8 & Feb. 28 & 13.6 & Sept. 12 & 16.40 \\
\hline Sept. 28 & 18.5 & July 28 & 11.1 & Mar. 28 & 13.5 & Aug. 16,1960 & 6.37 \\
\hline Oct. 28 & 18.0 & Aug. 28 & 15.0 & & & & \\
\hline
\end{tabular}

37/59-26a1. Deeth. Dug domestic water-table well, diam $4 \mathrm{ft}$, depth $14 \mathrm{ft}$, cased. MP top of elbow welded on well cover, $1.70 \mathrm{ft}$ above Isd (since Mar. 10, 1959). Highest water level 2.9 below Isd, May 28, 1952; lowest 10.8 below 1sd, Mar. 30, Sept. 28, 1955. Records available: $1938-60$.

\begin{tabular}{l|r||ll||l||l||l||l|l|}
\hline Jan. 28, 1956 & 10.2 & Oct. 28, 1956 & 9.5 & July 28, 1957 & 6.1 & Mar. 28, 1958 & 7.6 \\
Feb. 29 & 8.5 & Nov. 28 & 9.9 & Aug. 28 & 8.8 & Apr. 28 & 6.1 \\
Mar. 30 & $\mathbf{5 . 7}$ & Dec. 28 & 10.2 & Sept. 28 & 9.0 & May 28 & $\mathbf{5 . 3}$ \\
Apr. 30 & $\mathbf{5 . 1}$ & Jan. 28, 1957 & 10.3 & Oct. 28 & 9.5 & June 28 & 6.8 \\
May 30 & 4.3 & Feb. 28 & 9.7 & Nov. 28 & 9.65 & Sept. 5 & 7.84 \\
June 30 & 4.0 & Mar. 28 & 8.3 & Dec. 28 & 9.8 & Mar. 10, 1959 & $\mathbf{8 . 6 4}$ \\
July 30 & $\mathbf{8 . 2}$ & Apr. 28 & 7.8 & Jan. 28, 1958 & 10.3 & Sept. 12 & 8.32 \\
Aug. 28 & $\mathbf{8 . 8}$ & May 29 & 5.1 & Feb. 26 & 7.6 & Aug. 15, 1960 & 7.57 \\
Sept. 28 & 9.5 & June 28 & 4.9 & & & & \\
\hline
\end{tabular}

\section{Lamoille Valley}

33/58-19ad1. H. Conrad. Known as Lamoille Church. Dug domestic water-table well, diam $4 \mathrm{ft}$, depth $16 \mathrm{ft}$, cased. MP top of wood curb, at lsd. Highest water level 0.6 below lsd, July 1, 1936; lowest 15.1 below 1sd, Dec. 15, 1940. Records available: 1934-60.

\begin{tabular}{l|r||l|l||l|l||r|r|r}
\hline Jan. 28, 1956 & 11.4 & Oct. 28, 1956 & 10.5 & July 28, 1957 & 11.1 & Mar. 28, 1958 & 10.1 \\
Feb. 29 & 10.6 & Nov. 28 & 11.8 & Aug. 28 & 7.1 & Apr. 28 & 11.3 \\
Mar. 30 & 10.0 & Dec. 28 & 12.3 & Sept. 28 & 7.3 & May 28 & 1.8 \\
Apr. 30 & 11.1 & Jan. 28, 1957 & 8.0 & Oct. 28 & 10.1 & June 28 & 1.0 \\
May 30 & 3.3 & Feb. 28 & 10.8 & Nov. 28 & 11.5 & Sept. 6 & 6.73 \\
June 30 & 3.4 & Mar. 28 & 11.0 & Dec. 28 & 11.7 & Mar. 11, 1959 & 12.85 \\
July 30 & 2.4 & Apr. 28 & 11.3 & Jan. 28, 1958 & 12.3 & Sept. 12 & 10.37 \\
Aug. 28 & 3.8 & May 29 & 3.4 & Feb. 26 & 10.3 & Aug. 16, 1960 & 6.47 \\
Sept. 28 & 8.9 & June 28 & 1.3 & & & & & \\
\hline
\end{tabular}

\section{Ruby Valley}

32/60-29c1. U.S. Geol. Survey. Drilled test and observation well, diam 6 in, depth $202 \mathrm{ft}$, cased to 137. MP top of cap on casing, $0.70 \mathrm{ft}$ above isd. Highest water level 1.38 below $1 \mathrm{sd}$, Mar. 28, 1951; lowest 5.21 below lsd, Sept. 20, 1955 (previously reported 4.81 below lsd, Sept. 15, 1954). Records available: 1949-60. Mar. 27, 1956, 2.73; Aug. 28, 3.92; Sept. 10, 1957, 4. 23; Sept. 4, 1958, 3.79; Sept. 13, 1959, 4.81; Aug. 15, 1960, 4.84.

$32 / 60-29 c 2$. U.S. Geol. Survey. Driven observation water-table well, diam $1 \frac{1}{2}$ in, depth $15 \mathrm{ft}$, cased to 15. MP top of casing, 2. $05 \mathrm{ft}$ above lsd. Highest water level 3.65 below $1 \mathrm{sd}$, Mar. 28, 1951; lowest 7. 35 below lsd, Sept. 20, 1955. Records available: 1949-60. Aug. 28, 1956, 6. 44; Sept. 10, 1957, 6.86; Sept. 4, 1958, 6. 47; Sept. 13, 1959, 7. 07; Aug. 15, 1960, 7.10.

\section{Esmeralda County}

\section{Alkali Flat}

2/38-20dc1. Goldfield and Tonopah RR. Dug unused well, size $4 \times 5 \mathrm{ft}$, depth $114 \mathrm{ft}$, cribbed with wood. MP top of wood cribbing, at Isd. Highest water level 102.34 below Isd, Dec. 1, 1960; lowest 103.04 below Isd, Feb. 1, 1960. Records available: 1957-60. Oct. 23, 1957, 102. 76; Sept. 2, 1958, 102.69; Sept. 14, 1959, 102.50; Feb. 1, 1960, 103.04; Dec. 1, 102. 34 .

\section{Alkali Springs Valley}

S1/42-3. Owner unknown. Drilled unused well, diam 6 in, depth unknown, cased. MP top of casing, $0.50 \mathrm{ft}$ above lsd. Highest water level 196.69 below lsd, Feb. 1, 1960; lowest 197. 08 below lsd, Oct. 23, 1957. Records available: 1957-60. Oct. 23, 1957, 197. 08; Feb. 15, 1958, 196.90; Sept. 2, 196.90; Sept. 14, 1959, 196.81; Feb. 1, 1960, 196.69; Dec. 1, 196. 81. 


\section{Fish Lake Valley}

S1/35-28a1. Rex B. Clark. Drilled stock water-table well, diam 16 in, depth $624 \mathrm{ft}$, cased to 600, perforated 150-600. MP top of hole in plug in casing, $0.50 \mathrm{ft}$ above lsd. Highest water level 25. 45 below lsd, Jan. 21, 1948; lowest 32.51 below 1sd, Mar. 27, 1952 (previously reported 30.89 below 1sd, Sept. 30, 1955). Records available: 1945-56, 1958-59. No measurement made in 1957 or 1960 . Mar. 20, 1956, 31. 20; Aug. 31, 30.83; Oct. 15, 1958, 29.94; Oct. $18,1959,30.78$.

S3/35-4d3. Sigurd Folwick. Drilled irrigation water-table well, diam 14 in, reported depth $132 \mathrm{ft}$, perforated 70-132. MP top of casing, $0.50 \mathrm{ft}$ above lsd. Highest water level 44.95 below lsd, Mar. 22, 1950; lowest 48.33 below Isd, Oct. 18, 1959. Records available: 1950-57, 1959. No measurement made in 1958 or 1960. Mar. 20, 1956, 47. 89; Aug. 31, 48. 27; Oct. 22, 1957, 48. 17; Oct. 18, 1959, 48.33.

S3/35-14c4. U. S. Bureau of Land Management. Drilled unused water-table well, diam $12 \mathrm{in,} \mathrm{depth} \mathrm{unknown,} \mathrm{cased.} \mathrm{MP} \mathrm{top} \mathrm{of} \mathrm{casing,} 0.40 \mathrm{ft}$ above lsd. Highest water level 38.6 below lsd, Jan. 21, 1948; lowest 43. 24 below lsd, Oct. 18, 1959. Records available: 1945, 194759. No measurement made in 1960. Mar. 20, 1956, 42.07; Aug. 31, 42.38; Oct. 22, 1957, 42. 66; Oct. 15, 1958, 41.87; Oct. 18, 1959, 43. 24.

\section{Tonopah and Vicinity}

3/40-2c1. Millers Mill. Dug unused water-table well, size $8 \times 5 \mathrm{ft}$, depth $61 \mathrm{ft}$, cribbed with wood. MP arrow carved on southwest corner of cribbing, at 1sd. Highest water level 39.10 below 1sd, Nov. 10, 1948 (previously reported 39. 11 below lsd, Sept. 8, 1952); lowest 39. 92 below 1sd, Dec. 1, 1960 . Records available: 1948-55, 1957-60. No measurement made in 1956. Oct. 23, 1957, 39.56; Sept. 2, 1958, 39. 43; Sept. 14, 1959, 39. 38; Dec. 1, 1960, 39. 92.

\section{Eureka County}

\section{Antelope Valley}

18/51-34d1. Bartholemae Corp. Drilled stock water-table well, diam 6 in, depth $134 \mathrm{ft}$, cased. MP top of casing, $0.60 \mathrm{ft}$ above lsd. Highest water level 93.96 below isd, Oct. 1, 1952; lowest 94.23 below 1sd, Dec. 17, 1959. Records available: 1949-53, 1955, 1959 . No measurement made in 1956-58 or 1960 . Dec. 17, 1959, 94.23.

\section{Crescent Valley}

29/48-34c1. Dan Filippini. Drilled stock water-table well, diam 6 in, depth unknown, cased. Lsd 4,731. $3 \mathrm{ft}$ above msl. MP lower edge of pumpbase, $0.40 \mathrm{ft}$ above lsd. Highest water level 4.17 below 1sd, Mar. 12, 1957; lowest 13.52 below 1sd, Mar. 27, 1956. Records available: 1948-52, 1954-58. Measurement discontinued. Mar. 27, 1956, 13.52; Aug. 25, 6.93; Mar. 12, 1957, 4.17; Sept. 9, 7.81; Mar. 17, 1958, 6.56.

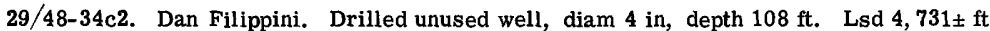
above msl. MP top of casing, at lsd. Highest water level 2.68 below 1sd, Mar. 10, 1959; lowest 6.13 below lsd, Sept. 11, 1959. Records available: 1958-60. Replaces well 29/48-34c1. Sept. 3, 1958, 5.46; Mar. 10, 1959, 2.68; Sept. 11, 6.13; Aug. 16, 1960, 5. 07.

\section{Diamond Valley}

20/53-15b1. U. S. Bureau of Land Management. Dug stock water-table well, diam $4 \mathrm{ft}$, reported depth $99 \mathrm{ft}$, cribbed with concrete. MP inside edge of concrete curb marked with arrow, 2. $90 \mathrm{ft}$ above 1sd. Highest water level 71.75 below lsd, Apr. 30, 1948; lowest 76. 49 below 1sd, Mar. 24, 1949. Records available: 1947-59. No measurement made in 1960.

\begin{tabular}{l|c||c|c||c|c||c|c}
\multicolumn{1}{c|}{ Date } & $\begin{array}{c}\text { Water } \\
\text { level }\end{array}$ & Date & $\begin{array}{c}\text { Water } \\
\text { level }\end{array}$ & Date & $\begin{array}{c}\text { Water } \\
\text { level }\end{array}$ & Date & $\begin{array}{c}\text { Water } \\
\text { level }\end{array}$ \\
\hline Jan. 20, 1956 & 74.51 & Jan. 15, 1957 & 74.84 & Sept. 6, 1957 & 75.12 & Nov. 23, 1958 & 75.76 \\
Mar. 26 & 74.60 & Mar. 6 & 75.13 & Jan. 7,1958 & 75.14 & Jan. 15, 1959 & 75.95 \\
May 10 & 74.84 & May 2 & 74.96 & May 7 & 75.27 & May 12 & 76.22 \\
June 20 & 74.65 & July 11 & 75.03 & July 15 & 75.43 & Dec. 18 & 75.21 \\
Aug. 7 & $\mathbf{7 4 . 6 4}$ & & & & & & \\
\hline
\end{tabular}

20/53-31d1. A. C. Florio. Drilled stock well, diam 6 in, depth unknown, cased. MP top of casing, 4.00 ft above lsd. Highest water level 155.46 below 1sd, Sept. 6, 1957; lowest 165.90 below lsd, Sept. 13, 1949. Records available: 1947-60. 
20/53-31d1--Continued.

\begin{tabular}{|c|c|c|c|c|c|c|c|}
\hline Date & $\begin{array}{c}\text { Water } \\
\text { level }\end{array}$ & Date & $\begin{array}{c}\text { Water } \\
\text { level }\end{array}$ & Date & $\begin{array}{c}\text { Water } \\
\text { level }\end{array}$ & Date & $\begin{array}{l}\text { Water } \\
\text { level }\end{array}$ \\
\hline Jan. 20,1956 & 157.43 & Nov. 19,1956 & 156.59 & Sept. & 155.46 & Jan. 15,1959 & 157.03 \\
\hline Mar. 26 & 157.39 & Jan. 15, 1957 & 156.92 & 7,1958 & 155.89 & May 12 & 157.42 \\
\hline May 10 & 157.59 & Mar. & 156.42 & May & 155.77 & Dec. 12 & 163.07 \\
\hline June 20 & 157.28 & May & 156.22 & July 15 & 156.06 & Mar. & 155.74 \\
\hline Aug. 7 & 157.70 & July 11 & 155.99 & Nov. 23 & 156.72 & Sept. 27 & 158.45 \\
\hline
\end{tabular}

21/53-5c1. A. C. Florio. Drilled stock water-table well, diam $4 \mathrm{ft}$, depth $42 \mathrm{ft}$, cased. MP top of casing, 4. $00 \mathrm{ft}$ above 1sd. Highest water level 27.61 below 1sd, Dec. 18, 1959; lowest 29.00 below 1sd, Aug. 28, 1956. Records available: 1947-56, 1959. No measurement made 1957-58 or 1960. Mar. 26, 1956, 28.93; Aug. 28, 29.00; Dec. 18, 1959, 27.61.

22/54-27 ca1. Formerly 22/54-27a1. Robert Stucki. Drilled domestic and irrigation well, diam 12 in, depth $94 \mathrm{ft}$, cased to 93 , perforated 46-93. Lsd 5, $858 \mathrm{ft}$ above msl. MP top of casing, $0.80 \mathrm{ft}$ above 1sd. Highest water level 5.49 below lsd, Aug. 11, 1949; lowest 13.60 below lsd, Dec. 18, 1959. Records available: $1949-55,1957$, 1959. No measurement made in 1956, 1958 or 1960 . Sept. $6,1957,12.44$; Dec. $18,1959,13.60$.

\section{Humboldt County}

\section{Grass Valley}

\section{[See also Pershing County]}

35/37-28b1. U. S. Bureau of Land Management. Button Sage well. Drilled unused watertable well, diam 12 in, depth $73 \mathrm{ft}$, perforated 0-73. Lsd 4,300 ft above msl. MP top of casing, $1.40 \mathrm{ft}$ above lsd. Highest water level 33.30 below lsd, Sept. 11, 1952; lowest 39. 69 below 1sd, Aug. 29, 1960. Records available: 1946-60.

\begin{tabular}{|l|l||l|l||l||l|l|l|l|}
\hline Mar. 21, 1956 & 38.23 & Mar. 20, 1958 & 37.59 & Mar. 9, 1959 & 37.58 & Mar. 29, 1960 38.76 \\
Sept. 27 & 38.60 & Sept. 9 & 37.55 & Sept. 17 & 39.45 & Aug. 29 & 39.69 \\
Sept. 8, 1957 & 37.99 & & & & & & \\
\hline
\end{tabular}

35/37-34a2. Owner unknown. Drilled unused water-table well, diam $10 \mathrm{in}$, depth $83 \mathrm{ft}$, cased. Lsd 4,301.5 ft above msl. MP top of casing, $1.00 \mathrm{ft}$ above lsd. Highest water level 17. 68 below lsd, May 16, 1946; lowest 24.94 below lsd, Aug. 29, 1960. Records available: 1946-60.

\begin{tabular}{l|l||l|l||l|l|l|l|l}
\hline Mar. 21, 1956 & 22.05 & Mar. 20, 1958 & 21.34 & Mar. 9, 1959 & 21.33 & Mar. 29, 1960 & 22.93 \\
Sept. 27 & 22.96 & Sept. 9 & 20.50 & Sept. 14 & 24. 28 & Sept. 29 & 24.94 \\
Sept. 14, 1957 & 21.14 & & & & & & & \\
\hline
\end{tabular}

Humboldt River Valley

[See also Elko, Lander, and Pershing Counties]

35/37-8d2. D. H. McNinch. Drilled unused water-table well, diam $16 \mathrm{in}$, depth $77 \mathrm{ft}$, cased. Lsd 4,301 ft above msl. MP top of casing, $1.20 \mathrm{ft}$ above lsd. Highest water level 50.74 below lsd, Mar. 17, 1953; lowest 59.55 below 1sd, Sept. 2, 1960. Records available: 1947-60.

\begin{tabular}{l|l||l|l||l|l||l|l|l|}
\hline Mar. 24, 1956 & 57.20 & Mar. 15, 1957 & 57.36 & Sept. 9, 1958 & 57.68 & Mar. 29, 1960 58.44 \\
Aug. 30 & $\mathbf{5 7 . 5 9}$ & Sept. 14 & $\mathbf{5 7 . 9 8}$ & Mar. 12, 1959 & $\mathbf{5 7 . 7 8}$ & Sept. 2 & $\mathbf{5 9 . 5 5}$ \\
Sept. 25 & $\mathbf{5 8 . 1 6}$ & Mar. 20, 1958 & $\mathbf{5 6 . 6 9}$ & Sept. 17 & $\mathbf{5 9 . 1 6}$ & & & \\
\hline
\end{tabular}

36/38-16c1. George Hay Co. Drilled irrigation water-table well, diam 12 in, depth $55 \mathrm{ft}$, cased. Lsd 4, 291.6 ft above msl. MP top of $\frac{1}{2}$-in hole in pumpbase, $0.30 \mathrm{ft}$ above lsd. Highest water level 15. 08 below lsd, May 14, 1951; lowest 21.53 below 1sd, Oct. 29, 1951. Records available: 1947-59. No measurement made in 1960. Mar. 21, 1956, 17. 83; Sept. 25, 19.19; Mar. 15, 1957, 18. 03; Sept. 14, 18. 77; Mar. 20, 1958, 17. 35; Mar. 9, 1959, 19. 09.

\section{Paradise Valley}

39/39-16d1. Dwight C. Vedder. Drilled stock water-table well, diam 12 in, depth $46 \mathrm{ft}$, cased. Lsd 4, 331. $7 \mathrm{ft}$ above msl. MP bottom edge of pumpbase, $0.30 \mathrm{ft}$ above lsd. Highest water level 3.82 below lsd, May 28, 1951; lowest 10.69 below lsd, Sept. 14, 1948. Records available: $1947-60$.

\begin{tabular}{|c|c|c|c|c|c|c|c|}
\hline $\begin{array}{l}\text { Mar. 22, } 1956 \\
\text { Sept. 26 } \\
\text { Mar. 14, } 1957\end{array}$ & $\begin{array}{l}8.93 \\
9.09 \\
8.10\end{array}$ & $\begin{array}{lrr}\text { Sept. } & 13, & 1957 \\
\text { Sept. } & 8, & 1958\end{array}$ & $\begin{array}{l}8.28 \\
8.24\end{array}$ & $\begin{array}{l}\text { Mar. 2, } 1959 \\
\text { Sept. 27 }\end{array}$ & $\begin{array}{l}6.33 \\
8.86\end{array}$ & $\begin{array}{l}\text { Mar. } 16,1960 \\
\text { Aug. } 30\end{array}$ & $\begin{array}{l}7.71 \\
8.68\end{array}$ \\
\hline
\end{tabular}


40/39-10d1. Owner unknown. Drilled unused water-table well, diam $12 \mathrm{in}$, depth $55 \mathrm{ft}$, cased with stovepipe. Lsd 4, $422 \mathrm{ft}$ above msl. MP top of casing, $0.20 \mathrm{ft}$ above $1 \mathrm{sd}$. Highest water level 37.82 below 1sd, Mar. 19, 1958; lowest dry, Sept. 8, 1958. Records available: 1945-58. Measurement discontinued. Mar. 22, 1956, 44.43; Sept. 26, 43. 22; Mar. 14, 1957, 43. 03; Mar. 19, 1958, 37. 82; Sept. 8, dry.

42/39-25c1. U. S. Bureau of Land Management. Dug unused water-table well, diam $6 \mathrm{ft}$, depth $18 \mathrm{ft}$, cased with iron. Lsd 4,523 ft above msl. MP top of concrete floor, 5. $20 \mathrm{ft}$ below 1sd. Highest water level 1.80 below lsd, July 8, 1958; lowest 10.95 below 1sd, Jan. 14, 1956. Records available: $1945-60$.

\begin{tabular}{|c|c|c|c|c|c|c|c|}
\hline Date & $\begin{array}{l}\text { Water } \\
\text { level }\end{array}$ & Date & $\begin{array}{c}\text { Water } \\
\text { level }\end{array}$ & Date & $\begin{array}{c}\text { Water } \\
\text { level }\end{array}$ & Date & $\begin{array}{l}\text { Water } \\
\text { level }\end{array}$ \\
\hline Jan. 14,1956 & 10.95 & July 9,1957 & 4.13 & Sept. 12, 1958 & 7.97 & Nov. 11,1959 & 10.05 \\
\hline Feb. 3 & 8.50 & Aug. 12 & 6.58 & Oct. 17 & 8. 48 & Dec. 10 & 10.09 \\
\hline Mar. 8 & 8.01 & Sept. 27 & 8. 17 & Nov. 13 & 8.57 & Jan. 20,1960 & 10.16 \\
\hline Apr. 6 & 7.41 & Oct. 19 & 8. 38 & Dec. 11 & 8. 63 & Feb. 18 & 9.76 \\
\hline 24 & 6.10 & Nov. 21 & 8. 19 & Jan. 9,1959 & 8. 61 & Mar. 14 & 9.21 \\
\hline May 24 & 4.06 & Dec. 12 & 8. 18 & Feb. 18 & 8. 39 & 16 & 9.08 \\
\hline June 14 & 2.93 & Jan. 23,1958 & 8.07 & Mar. 11 & 8.19 & 30 & 8.77 \\
\hline July 19 & 5.85 & Feb. 20 & 6.49 & 12 & 8.26 & Apr. & 8.82 \\
\hline Aug. & 7.11 & Mar. 19 & 5.25 & Apr. 15 & 8. 10 & 27 & 8.80 \\
\hline Sept. 26 & 8.65 & 29 & 5.12 & May 14 & 8.29 & May 26 & 6.80 \\
\hline 28 & 8.70 & Apr. 23 & 4. 31 & June & 8. 46 & Aug. 12 & 9.46 \\
\hline Nov. 1 & 8.93 & May & 4. 48 & July & 8.48 & 30 & 9.80 \\
\hline Dec. & 8.94 & 17 & 2. 08 & Aug. 11 & 9.25 & Sept. 15 & 10.04 \\
\hline Jan. 16, 1957 & 8.70 & June 10 & 2. 86 & Sept. 15 & 9.99 & Oct. 22 & 10.30 \\
\hline Feb. 20 & 8. 41 & July 8 & 1.80 & & 10.00 & Nov. 15 & 10.27 \\
\hline Mar. 14 & 8.48 & Aug. 29 & 2. 90 & Oct. 15 & 9.97 & Dec. 12 & 10.22 \\
\hline Apr. 11 & 6.74 & Sept. 8 & 7.96 & & & & \\
\hline
\end{tabular}

Quinn River Valley

42/37-3b1. George Reed. Drilled irrigation water-table well, diam $12 \mathrm{in}$, depth $160 \mathrm{ft}$, cased to 160. MP top of casing, $1.20 \mathrm{ft}$ above 1sd. Highest water level 16.55 below 1sd, Jan. 20, 1950; lowest 41.45 below 1sd, Sept. 1, 1960. Records available: 1949-60.

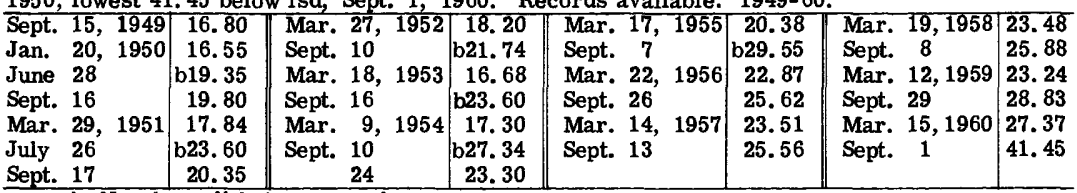

b Nearby well being pumped.

42/37-33b2. Hassenyager. Drilled irrigation water-table well, diam $18 \mathrm{in}$, depth $95 \mathrm{ft}$, cased to 68. MP top of slot in east side of casing, 2. $00 \mathrm{ft}$ above lsd. Highest water level 35.42 below lsd, Sept. 16, 1953; lowest 45.56 below lsd, Sept. 1, 1960. Records available: 1948-60. \begin{tabular}{l|l||l||l||l|l|l|}
\hline Mar. 22, 1956 & 40.78 & Sept. 13, 1957 & 39.85 & Mar. 12, 1959 & 36.23 & Mar. 15, 1960 39.57
\end{tabular}

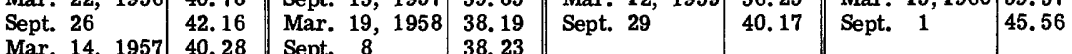

43/37-4c2. Owner unknown. Drilled unused water-table well, diam 6 in, depth $42 \mathrm{ft}$, cased. Lsd 4, $230 \mathrm{ft}$ above msl. MP top of casing, $0.40 \mathrm{ft}$ above lsd. Highest water level 30.41 below 1sd, Sept. 10, 1952; lowest 35. 76 below lsd, Mar. 14, 1957. Records availa ble: 1947-59. No measurement made in 1960. Mar. 22, 1956, 35. 22; Sept. 26, 34.98; Mar. 14, 1957, 35. 76; Sept. 13, 34. 92; Mar. 19, 1958, 34. 62; Sept. 8, 34.75; Mar. 12, 1959, 35. 42.

43/37-28a1. Elmo Bowly. Dug and drilled irrigation water-table well, size $5 \times 6 \mathrm{ft}$, depth $57 \mathrm{ft}$, wood cribbed to 12, 12-in diam 12-57. Lsd 4, $234 \mathrm{ft}$ above msl. MP top of well curb, $1.00 \mathrm{ft}$ above 1sd. Highest water level 8.13 below lsd, Nov. 5, 1947; lowest 21.10 below $1 \mathrm{sd}$, Sept. 13, 1957. Records available: 1946-59. Measurement discontinued. Mar. 22, 1956, 14.69; Sept. 13, 1957, 21.10; Mar. 19, 1958, 16.80; Mar. 12, 1959, 16.90.

\section{Lander County}

\section{Crescent Valley}

29/48-29c2. Beowawe Farms. Drilled irrigation well, diam $14 \mathrm{in}$, depth $300 \mathrm{ft}$, cased to 300. MP top of casing, $0.60 \mathrm{ft}$ above lsd. Highest water level 63.56 below lsd, Mar. 17, 1958; lowest 64.45 below lsd, Sept. 3, 1958. Records available: 1958-60. Mar. 17, 1958, 63. 56; Sept. 3, 64. 45; Mar. 10, 1959, 63. 67; Sept. 11, 63.77; Aug. 16, 1960, 63.84. 
30/48-33c1. H. J. Buchaneau. Drilled irrigation well, diam $14 \mathrm{in}$, reported depth $300 \mathrm{ft}$, cased to 300 . Lsd 4, 804. $36 \mathrm{ft}$ above msl. MP top of air line slot, $0.50 \mathrm{ft}$ above lsd (since Mar. 17, 1958). Highest water level 58.40 below lsd, Mar. 6, 1953; lowest 70.78 below lsd, Sept. 11, 1959. Records available: 1953, 1958-59. No measurement made 1954-57, 1960. Mar. 6, 1953, 58.40; Oct. 21, 68. 84; Mar. 17, 1958, 69. 48; Sept. 28, 70. 44; Mar. 10, 1959, 69. 54; Sept. 11, 70.78.

\section{Humboldt River Valley}

[See also Elko, Humboldt, and Pershing Counties]

32/45-11d1. U.S. Geol. Survey. Drilled test and observation well, diam 6 in, depth $197 \mathrm{ft}$, cased to 171. MP top of casing, $2.00 \mathrm{ft}$ above lsd. Highest water level 4.08 below 1sd, July 10, 1952; lowest 10.58 below 1sd, Sept. 21, 1955. Records available: 1949-60.

\begin{tabular}{|c|c|c|c|c|c|c|c|}
\hline Date & $\begin{array}{l}\text { Water } \\
\text { leve] }\end{array}$ & Date & $\begin{array}{l}\text { Water } \\
\text { level }\end{array}$ & Date & $\begin{array}{l}\text { Water } \\
\text { level }\end{array}$ & Date & $\begin{array}{l}\text { Water } \\
\text { level }\end{array}$ \\
\hline $\begin{array}{lll}\text { Aug. } 29, & 1956 \\
\text { Mar. } & 12,1957 \\
\text { Sept. } 12 & \end{array}$ & $\begin{array}{l}8.00 \\
7.17 \\
7.77\end{array}$ & $\begin{array}{l}\text { Mar. } \\
\text { Sept. } 2\end{array}$ & $\begin{array}{l}7.60 \\
8.91\end{array}$ & $\begin{array}{l}\text { Mar. 11, } 1959 \\
\text { Sept. 11 }\end{array}$ & $\begin{array}{r}8.77 \\
10.27\end{array}$ & $\begin{array}{l}\text { Mar. } 14,1960 \\
\text { Aug. } 17\end{array}$ & $\begin{array}{r}9.22 \\
10.30\end{array}$ \\
\hline
\end{tabular}

32/46-11d1. U. S. Bureau of Reclamation. Dug stock water-table well, size $4 \times 5 \mathrm{ft}$, depth $13 \mathrm{ft}$, cribbed with wood. Lsd 4,543 ft above msl. MP top of 8- x 14-in timber pump support, $0.80 \mathrm{ft}$ above 1sd. Highest water level 3.77 below lsd, Apr. 11, 1946; lowest 9.77 below lsd, Sept. 6, 1955. Records available: 1945-59. No measurement made in 1960. Mar. 28, 1956, 6.06; Aug. 29, 8.97; Mar. 12, 1957, 6.52; Sept. 11, 8.49; Mar. 18, 1958, 6. 64; Sept. 2, 8. 30; Mar. 11, 1959, 7.18.

32/46-31 bb1. Humboldt Petroleum Co. Drilled oil test well, diam 6 in, reported depth $126 \mathrm{ft}$, cased. Lsd 4,529 ft above msl. MP top of casing, $0.90 \mathrm{ft}$ above 1sd. Highest water level 10.75 below Isd, Apr. 3, 1952; lowest 12.58 below 1sd, Sept. 21, 1955 (previously reported 12.19 below lsd, Sept. 19, 1949). Records available: 1947-60.

\begin{tabular}{l|l||l|l||l|l||l|l|}
\hline Mar. 28, 1956 & $\begin{array}{l}\text { 11.53 } \\
\text { Aug. 29 }\end{array}$ & $\begin{array}{l}\text { Sept. 11, 1957 } \\
\text { 12.04 }\end{array}$ & $\begin{array}{l}\text { Mar. 17, 1958 } \\
\text { Mar. 11.71 }\end{array}$ & $\begin{array}{l}\text { Mar. 11, 1959 } \\
\text { Sept. 11 }\end{array}$ & $\begin{array}{l}11.61 \\
12.46\end{array}$ & $\begin{array}{l}\text { Mar. 15, 1960 } \\
\text { Aug. 11. 17 }\end{array}$ & 12.08 \\
\hline
\end{tabular}

\section{Reese River Valley}

27/43-33cd1. Owner unknown. Watts. Drilled unused well, diam $6 \mathrm{in}$, depth $114 \mathrm{ft}$, cased. Lsd 4,810 ft above msl. MP top of 2-in plank cover, $0.40 \mathrm{ft}$ above 1sd. Highest water level 12.36 below lsd, Apr. 22, 1948; lowest 15.12 below 1sd, Sept. 3, 1958. Records available: 1947-58. No measurement made 1959-60. Mar. 27, 1956, 13. 75; Sept. 12, 1957, 15.06; Sept. 3, 1958, 15.12.

30/42-24cc1. U. S. Bureau of Land Management. Drilled stock water-table well, diam 6 in, depth $54 \mathrm{ft}$, cased. Lsd 4,634 $\mathrm{ft}$ above msl. MP $\frac{1}{2}$-in hole in pumpbase, $0.10 \mathrm{ft}$ above 1sd. Highest water level 10.30 below lsd, Mar. 16, 1949; lowest 13.25 below lsd, Sept. 21, 1955. Records available: $1947-60$.

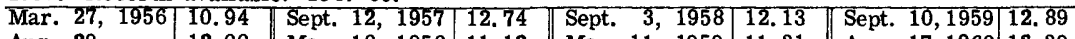

\begin{tabular}{l|l|l|l|l|l|l|l|l|l|l|l|} 
Aug. 29 & 12.00 & Mar. 18, 1958 & 11.12 & Mar. 11, 1959 & 11.31 & Aug. 17, 1960 & 12.39 \\
\hline
\end{tabular}

30/43-9aa1. Copper Canyon Mining Co. Drilled unused well, diam $12 \mathrm{in,} \mathrm{depth} 201 \mathrm{ft}$, cased to 192 . Lsd 4,767 ft above msl. MP top of casing, $0.30 \mathrm{ft}$ above lsd. Highest water level 134.56 below lsd, May 22, 1947; lowest 138.27 below 1sd, Mar. 16, 1951. Records available: $1947-60$.

\begin{tabular}{l||l||l||ll||l|l|l|}
\hline Mar. 28, 1956 & 136.15 & Sept. 12, 1957 & 135.57 & Sept. 3, 1958 & 135.63 & Sept. 10, 1959 & 135.90
\end{tabular}

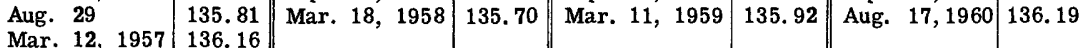

Mar. 12, $1957 \mid 136.16$

30/44-22cb1. Owner unknown. Dillon. Drilled unused water-table well, diam 6 in, depth $80 \mathrm{ft}$. Lsd 4, $676 \mathrm{ft}$ above msl. MP top of casing, $0.90 \mathrm{ft}$ above $1 \mathrm{sd}$ (since Sept. 3, 1958). Highest water level 26.64 below lsd, Nov. 8, 1947; lowest 30.65 below 1sd, Aug. 17, 1960. Records available: $1947-60$.

\begin{tabular}{l|l||l|l||l|l||l|l|l|}
\hline Mar. 27, 1956 & 28.95 & Mar. 12, 1957 & 28.76 & Mar. 18, 1958 & 29.13 & Mar. 11, 1959 29.48 \\
Aug. 29 & 28.73 & Sept. 12 & 28.91 & Sept. 3 & 30.33 & Aug. 17, 1960 & 30.65 \\
\hline
\end{tabular}

30/45-18aa1. U. S. Bureau of Land Management. Dug stock water-table well, size $4 \mathrm{x}$ $4 \mathrm{ft}$, reported depth $60 \mathrm{ft}$, cribbed with wood. Lsd 4,635 ft above msl. MP top of plank platform, at lsd. Highest water level 23.69 below lsd, Jan. 8, 1948; lowest 32.18 below lsd, Aug. 17, 1960. Records available: $1947-60$.

\begin{tabular}{l|l||l||l|l||l|l|l|}
\hline Mar. 27, 1956 & 29.03 & Sept. 12, 1957 & 28.77 & Sept. 3, 1958 & 29.68 & Sept. 10, 1959 31.01
\end{tabular} \begin{tabular}{|l|l|l||l|l||l|l|l|} 
Aug. 29 & 27. & Mar. 18, 1958 & 28. 80 & Mar. 11, 1959 & 29.71 & Aug. 17, 1960 & 32.18 \\
\hline
\end{tabular}

Mar. 12, $1957 \quad 27.64$ 


\title{
Lincoln County
}

\author{
Lake Valley
}

\section{[See also White Pine County]}

9/65-1b1. Fred Twisselman. Drilled irrigation well, diam $12 \mathrm{in}$, depth $165 \mathrm{ft}$, cased. MP top of casing, at Isd. Highest water level 23.64 below 1sd, Apr. 16, 1947; lowest 41.99 below 1sd, Oct. 25, 1957. Records available: 1947-55, 1957-60. No measurement made in 1956. Oct. 25, 1957, 41.99; Sept. 5, 1958, 36.00; Sept. 19, 1959, 38. 36; Dec. 7, 1960, 38. 04.

\section{Meadow Valley}

S1/68-33b1. Lafe Matthews Estate. Drilled irrigation well in alluvium of Quaternary age, diam 10 in, reported depth $120 \mathrm{ft}$, cased to 80, perforated 60-80. Lsd 4, 784. $7 \mathrm{ft}$ above msl. MP top south side of casing, at lsd. Highest water level 30.32 below 1sd, Apr. 25, 1946; lowest 40.50 below isd, Sept. 19, 1959. Records available: 1946-55, 1957-60. No measurement made in 1956. Oct. 23, 1957, 36.83; Sept. 7, 1958, 38.03; Sept. 19, 1959, 40.50; Dec. 7, 1960, 37. 87 .

S2/68-8b5. U. S. Geol. Survey. Drilled test and observation well in alluvium of Quaternary age, diam $8 \mathrm{in}$, depth $110 \mathrm{ft}$, cased to 110 . MP top of recorder platform, $1.20 \mathrm{ft}$ above Isd. Highest water level 10.72 below 1sd, Mar. 20, 1950; lowest 17. 48 below 1sd, Sept. 19, 1959. Records available: 1949-59. No measurement made in 1960. Mar. 16, 1956, 11. 71; Apr. 8, 12. 85; Oct. 23, 1957, 16. 83; Sept. 7, 1958, 15. 37; Sept. 19, 1959, 17. 48.

\section{Pahranagat Valley}

S4/60-34a2. W. U. Schofield, Jr. Drilled unused well, diam $10 \mathrm{in}$, reported depth $96 \mathrm{ft}$, cased to 96, perforated 60-96. MP top of casing, $1.30 \mathrm{ft}$ above $1 \mathrm{sd}$. Highest water level 58.12 below 1sd, Aug. 8, 1946; lowest 64.31 below 1sd, Feb. 19, 1948. Records available: 1946, 1948-60. Mar. 21, 1956, 60.48; Aug. 30, 61.29; Oct. 23, 1957, 62. 20; Sept. 6, 1958, 63. 66; Sept. 18, 1959, 63.78; Dec. 7, 1960, 64. 02.

S6/61-18d2. Gardner Chism. Drilled unused well, diam 6 in, depth $41 \mathrm{ft}$, cased. MP top of flange on casing, 1.70 ft above 1sd. Highest water level 5.35 below lsd (previously reported 5.55 below $1 \mathrm{sd}$ ), Mar. 14, 1951; lowest 9.66 below isd, Sept. 8, 1954 . Records available: 1946-60. Mar. 21, 1956, 7.56; Aug. 30, 8. 19; Oct. 22, 1957, 7. 86; Sept. 6, 1958, 7. 45; Sept. 18, 1959, 8.09; Dec. 7, 1960, 8.06.

S8/61-2c1. J. H. Hail. Drilled irrigation well, diam $10 \mathrm{in}$, depth $92 \mathrm{ft}$, sand-filled to 30 . MP top edge of 1-in hole in pumpbase, $0.80 \mathrm{ft}$ above 1sd. Highest water level 19.37 below lsd, Mar. 21, 1950; lowest 26.98 below lsd, Mar. 24, 1955. Records available: 1946-60. Mar. 21, 1956, 25. 22; Aug. 30, 24. 72; Oct. 23, 1957, 23. 30; Sept. 6, 1958, 22.73; Sept. 18, 1959, 23.87; Dec. 17, 1960, 22.97.

\section{Lyon County}

\section{Mason Valley}

12/25-35dc1. John C. Baker. Drilled irrigation well, diam 16 in, reported depth $253 \mathrm{ft}$, cased to 242. MP top of slot in casing, $1.50 \mathrm{ft}$ above isd. Highest water level 3.39 below $1 \mathrm{sd}$, Aug. 18, 1958; lowest 10.19 below Isd, Mar. 22, 1960 . Records available: 1953, 1957-60. No measurement made 1954-56.

\begin{tabular}{|c|c|c|c|c|c|c|c|}
\hline Date & $\begin{array}{l}\text { Water } \\
\text { level }\end{array}$ & Date & $\begin{array}{l}\text { Water } \\
\text { level }\end{array}$ & Date & $\begin{array}{l}\text { Water } \\
\text { level }\end{array}$ & Date & $\begin{array}{l}\text { Water } \\
\text { level }\end{array}$ \\
\hline ept. & $\begin{array}{l}4.32 \\
8.69\end{array}$ & Oct. & 3.43 & Aug. 18, 1958 & 3.39 & Sept. & 7.11 \\
\hline
\end{tabular}

13/25-14bc1. Yerington High School. Drilled irrigation well, diam 8 in, depth unknown. cased. MP top of casing, $1.20 \mathrm{ft}$ below $1 \mathrm{sd}$. Highest water level 3.35 below 1sd, Apr. 22, 1957; lowest 5.72 below 1sd, Oct. 4, 1960. Records available: 1957-60. Apr, 22, 1957, 3.35; Oct. 1, 3. 43; Mar. 25, 1958, 3. 38; Mar. 24, 1959, 3.63; Sept. 21, 4. 36; Oct. 4, 1960, 5. 72.

14/25-28d1. School District. Drilled unused well, diam 6 in, depth $38 \mathrm{ft}$, cased. MP top of casing, $0.70 \mathrm{ft}$ above 1sd. Highest water level 1.75 below lsd, July 13, 1953; lowest 6.73 below lsd, Oct. 4, 1960. Records available: 1947-60.

\begin{tabular}{l|c||l|l||l|l||l|l}
\hline Apr. 3, 1956 & 5.41 \\
Mar. 21, 1957 & 5.28 \\
Oct. 1 & Mar. 25, 1958 & Aug. 18 & 3.63 & Mar. 24, 1959 & 5.50 & Mar. 22, 1960 & 6.04 \\
\hline
\end{tabular}


15/25-26c1. Mason Valley Ranch. Drilled unused well, diam 8 in, depth $49 \mathrm{ft}$, cased. MP top of casing, 2. $60 \mathrm{ft}$ above lsd. Highest water level 3.45 below lsd, Mar. 23, 1959; lowest 7.41 below lsd, Oct. 4, 1960. Records available: 1945, 1947-55, 1957-60. No measurement made in 1956.

\begin{tabular}{l|c||c|c||c|c||c|c}
\hline \multicolumn{1}{c|}{ Date } & $\begin{array}{c}\text { Water } \\
\text { level }\end{array}$ & Date & $\begin{array}{c}\text { Water } \\
\text { level }\end{array}$ & Date & $\begin{array}{c}\text { Water } \\
\text { level }\end{array}$ & Date & $\begin{array}{c}\text { Water } \\
\text { level }\end{array}$ \\
\hline Mar. 21, 1957 & 4.07 & Mar. 24, 1958 & $\begin{array}{c}4.38 \\
\text { Oct. 1 }\end{array}$ & $\mathbf{5 . 3 8}$ & Aug. 18 & Mar. 23, 1959 & 3.45 \\
Sept. 21 & Mar. 22, 1960 & 5.05 \\
\hline
\end{tabular}

\section{Smith Valley}

11/23-3dc1. R. B. Day. Drilled irrigation well, diam $12 \mathrm{in}$, depth $242 \mathrm{ft}$, cased to 164 , perforated 0-164. Lsd 4, $830 \mathrm{ft}$ above msl. MP top of casing, $1.20 \mathrm{ft}$ above lsd. Highest water level 45. 21 below lsd, June 17, 1957; lowest 53.11 below Isd, June 9, 1960. Records available: 1948-57, 1959-60. No measurement made in 1958. Sept. 29, 1956, 50. 75; Dec. 20, 48. 48; June 17, 1957, 45. 21; Sept. 25, 47. 60; Dec. 10, 48. 27; Mar. 19, 1959, 50. 89; June 9, 1960, 53.11.

11/24-22dc1. Fred Fulstone. Dug unused water-table well, size $18 \times 30$ in, reported depth $130 \mathrm{ft}$, cribbed with concrete. Lsd 4,888.46 ft above msl. MP top of hole in floor of recorder platform, 1.40 ft above lsd. Highest water level 53.46 below lsd, Feb. 3, 1953; lowest 67. 24 below lsd, Dec. 20, 1960. Records available: 1948-60.

Water level at noon, from recorder graph, 1956

\begin{tabular}{|c|c|c|c|c|c|c|c|c|c|c|c|c|}
\hline Day & Jan. & Feb. & Mar. & Apr. & May & June & July & Aug. & Sept. & Oct. & Nov. & Dec. \\
\hline 5 & 60.44 & 60.58 & 60.77 & 61.00 & 57.85 & & & 58.04 & & 57.85 & 57.35 & \begin{tabular}{|l}
55.55 \\
\end{tabular} \\
\hline 10 & $\ldots$ & 60.62 & 60.80 & 61.00 & 57.57 & 56.78 & & 58.16 & 57.66 & 57.60 & 57.18 & 55.81 \\
\hline 15 & 60.45 & 60.64 & & 60.94 & 57.08 & 56.88 & $\cdots$ & 58.28 & 57.81 & 57.35 & 56.86 & 56.01 \\
\hline 20 & 60.48 & 60.68 & 60.88 & 60.74 & 3.88 & 56.28 & ..... & 42 & 57.93 & 57.32 & 56.52 & 56.15 \\
\hline 25 & 60.51 & 60.71 & 60.91 & $\ldots \ldots$ & .58 & 55.50 & & 56 & 58.01 & 57.33 & 10 & 53 \\
\hline Eom & 60.55 & 60.73 & 60.96 & $\ldots$ & 56.64 & & .. & & 57.99 & 46 & 78 & 63 \\
\hline \multicolumn{13}{|c|}{1957} \\
\hline 5 & 56.83 & 57.59 & 57.73 & 58.39 & $5 \overline{8.84}$ & 58.88 & 58.58 & 58.99 & 59.39 & 59.69 & 9.12 & 59.14 \\
\hline 10 & & 57.77 & 57.81 & 58.52 & 58.91 & 58.90 & 58.61 & 59.08 & 59.43 & 59.62 & 59.11 & $\ldots \ldots$ \\
\hline 15 & & 57.70 & 57.93 & 58.60 & 01 & .95 & 58.64 & 59.15 & 59.48 & $\ldots \ldots$ & 59.10 & ?... \\
\hline 20 & & 57.58 & 58.02 & 58.66 & 07 & 02 & & 22 & 59.55 & $\ldots \ldots$ & 59. 22 & $\ldots$ \\
\hline 25 & 57.44 & 57.60 & 58.20 & 58.73 & 59.14 & 58.82 & 58.80 & 59.26 & 59.56 & … & 59.21 & 57.14 \\
\hline om & 57.51 & 57.54 & 58.32 & 58.75 & 97 & 58.62 & 58.92 & $\ldots$ & 59.63 & 59.08 & 59.31 & … \\
\hline \\
\hline 5 & & 89 & & 57.54 & 57.71 & 56.07 & 53.77 & 55.49 & 6.65 & $\cdot$ & & - . \\
\hline 10 & & 57.00 & $\cdots$ & 57.75 & 56.89 & 56.13 & 53. 64 & $\ldots \ldots$ & 6.73 & & 57.49 & ... \\
\hline 5 & & 57.13 &. & 57.88 & 56.78 & $\ldots \ldots$ & 54.02 & $\cdots$ & 6.93 & $\ldots \ldots$ & $\cdots \cdots$ & ..... \\
\hline 0 & & 57.29 & $\cdots$ & 58.01 & 56.58 & $\cdots \cdots$ & 54. 49 & $\ldots \ldots$ & 57.05 & $\cdots \cdots$ & $\cdots$ & 58.12 \\
\hline 25 & 56.61 & -. & . & 58.12 & 5 & & 54.86 & $\cdots$ & $\cdots \cdots$ & $\cdot$ & - & 58.22 \\
\hline Eom & 56.78 & & & 58.05 & & 53.58 & 55.23 & & & & & 58.31 \\
\hline \multicolumn{13}{|c|}{1959} \\
\hline 5 & 58.30 & 58.88 & 59.28 & 59.74 & & & & 60.79 & 61.24 & 61.64 & 62.04 & 62.27 \\
\hline 10 & $5 \varepsilon$ & 58.94 & 59 & 59.80 & & & & 60.86 & 61.33 & 61.71 & 62.08 & 62.32 \\
\hline 5 & 58.59 & 58.98 & & & & $\ldots$ & & 60.93 & 61.39 & 61.77 & 62.12 & 62.35 \\
\hline 20 & 58.66 & 59.11 & 59.52 & . & & .. & 60.57 & 61.03 & 61.46 & 61.83 & 62.17 & 62.37 \\
\hline 25 & 58.74 & 59.20 & 59.61 & $\ldots$ & . & $\ldots$ & 60.63 & 61.08 & 61.53 & & 62.23 & 62.47 \\
\hline Eom & 83 & 59.23 & 59.72 & & & & 60.72 & 61.18 & 61.57 & 62.00 & 62.27 & 62.49 \\
\hline \multicolumn{13}{|c|}{1960} \\
\hline 5 & & 62.77 & 62.90 & 63.27 & 63.62 & & 64 & 65.05 & & 6.30 & ... & 67.10 \\
\hline 10 & 62. 54 & .79 & 63.03 & ..... & 63.67 & 64.16 & 64.59 & ... & ..... & 66.37 & $\ldots$. & 67.16 \\
\hline 15 & 62. 64 & 62.82 & 63.11 & & 63.70 & 64.28 & 64.69 & $\cdots \cdots$ & . & 66.48 & & 67.20 \\
\hline 20 & 62.66 & 62.89 & 63.13 & & 63 & 34 & & $\cdots \cdots$ & & 66.54 & 66.91 & .24 \\
\hline-1 & 62. 71 & 62.86 & 63.16 & 63.48 & $\cdots$ & 64.40 & 64.87 & & $\cdots$ & 66.61 & 66.95 & - \\
\hline Gom & 62.70 & 62.89 & 63.25 & 63.55 & 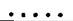 & 64.45 & 64.97 & & $\ldots \ldots$ & 66.71 & 67.03 & \\
\hline
\end{tabular}

11/24-32dc1. A. Nuti. Drilled irrigation well, diam $16 \mathrm{in}$, reported depth $390 \mathrm{ft}$, cased. Lsd 4, $865 \mathrm{ft}$ above msl. MP bottom of torch-cut slot, $0.20 \mathrm{ft}$ above lsd (since Sept. 21, 1959). Highest water level 23.62 below lsd, Mar. 3, 1948; lowest 60.56 below lsd, Oct. 4, 1960. Records available: $1948-60$.

\begin{tabular}{|c|c|c|c|c|c|c|c|}
\hline Date & $\begin{array}{c}\text { Water } \\
\text { level }\end{array}$ & Date & $\begin{array}{c}\text { Water } \\
\text { level }\end{array}$ & Date & $\begin{array}{l}\text { Water } \\
\text { level }\end{array}$ & Date & $\begin{array}{c}\text { Water } \\
\text { level }\end{array}$ \\
\hline 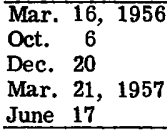 & $\begin{array}{l}32.85 \\
33.54 \\
31.93 \\
32.12 \\
37.76 \\
\end{array}$ & $\begin{array}{ll}\text { Sept. } & 25,1957 \\
\text { Dec. } 10 & \\
\text { Mar. } & \mathbf{3 1}, 1958 \\
\text { June } & 25 \\
\text { Sept. } 22 & \\
\end{array}$ & $\begin{array}{l}39.46 \\
32.55 \\
32.98 \\
36.00 \\
36.92 \\
\end{array}$ & $\begin{array}{lrr}\text { Dec. } & 16, & 1958 \\
\text { Mar. } & 19, & 1959 \\
\text { June } & 10 & \\
\text { Sept. } & \mathbf{2 1} & \\
\text { Jan. } & 6, & 1960 \\
\end{array}$ & $\begin{array}{r}32.40 \\
32.28 \\
\text { c62. } 59 \\
46.70 \\
36.58 \\
\end{array}$ & $\begin{array}{lr}\text { Mar. } & 22,1960 \\
\text { June } & 9 \\
\text { Aug. } & 15 \\
\text { Oct. } & 4\end{array}$ & $\begin{array}{r}36.15 \\
\$ 32.76 \\
66.40 \\
60.56\end{array}$ \\
\hline
\end{tabular}

c Nearby well being pumped. 


\section{Mineral County}

\section{Lake Valley}

8/30-3da1. U. S. Naval Ammunition Depot. Drilled domestic and industrial well, diam $18 \mathrm{in}$, depth $852 \mathrm{ft}$, cased. Lsd 4,125 $\mathrm{ft}$ above msl. MP top of casing, $1.00 \mathrm{ft}$ above lsd. Highest water level 47.20 below lsd, Dec. 9, 1954; lowest 49.48 below lsd, Nov. 22, 1960. Records available: $1954,1957-60$. No measurement made 1955-56. Oct. 21, 1957, 48. 19; Sept. 25, 1958, 48.57; Sept. 20, 1959, 49.00; Nov. 22, 1960, 49.48.

8/30-18aad1. U. S. Naval Ammunition Depot. Drilled irrigation well, diam 18 in, depth $345 \mathrm{ft}$, cased. MP top of measuring pipe, $1.00 \mathrm{ft}$ above lsd. Highest water level 95.1 below lsd, Nov. 21, 1952; lowest 100.83 below lsd, Nov. 22, 1960. Records available: 1952, 1957-60. No measurement made 1953-56. Nov. 21, 1952, 95.1; Oct. 21, 1957, 99.13; Sept. 25, 1958, 99.38; Oct. 20, 1959, 100.16; Nov. 22, 1960, 100.83.

8/30-21ddb1. U. S. Naval Ammunition Depot. Drilled domestic and irrigation well, diam $18 \mathrm{in}$, depth $394 \mathrm{ft}$, cased. MP top of casing cover, $1.20 \mathrm{ft}$ above $1 \mathrm{sd}$. Highest water level 199. 9 below 1sd, Nov. 21, 1952; lowest 209. 18 below 1sd, Nov. 22, 1960. Records available: 1952, 1957-60. No measurement made 1953-56. Nov. 21, 1952, 199.9; Oct. 21, 1957, 204.83; Sept. 25, 1958, 207. 19 (well being pumped); Oct. 20, 1959, 205.6; Nov. 22, 1960, 209. 18.

8/30-26dda1. U. S. Naval Ammunition Depot. Drilled industrial well, diam 18 in, depth $423 \mathrm{ft}$, cased. MP top of measuring pipe, $1.00 \mathrm{ft}$ above lsd. Highest water level 246.5 below 1sd, Nov. 21, 1952; lowest 250. 86 below 1sd, Nov. 22, 1960. Records available: 1952, 1957-60. No measurement made 1953-56. Nov. 21, 1952, 246.5; Oct. 21, 1957, 249.13; Sept. 25, 1958, 249.96; Oct. 20, 1959, 250.22; Nov. 22, 1960, 250. 86.

8/31-32bab1. U. S. Naval Ammunition Depot. Drilled industrial well, diam 18 in, depth $452 \mathrm{ft}$, cased. Lsd 4,374 ft above msl. MP top of measuring pipe, $0.20 \mathrm{ft}$ above lsd. Highest water level 242.6 below lsd, Nov. 21, 1952; lowest 244.68 below lsd, Nov. 22, 1960. Records available: 1952, 1957-58, 1960. No measurement made 1953-56 or 1959. Nov. 21, 1952, 242. 6; Oct. 21, 1957, 243. 52; Sept. 25, 1958, 244.27; Nov. 22, 1960, 244.68.

\section{Soda Spring Valley}

8/34-28c1. Basic Magnesium Plant. Drilled unused well, diam $8 \mathrm{in}$, depth unknown, cased. MP lower edge of hole in east side of casing, $0.30 \mathrm{ft}$ above lsd. Highest water level 136. 77 below 1sd, Feb. 1, 1960; lowest 137. 72 below 1sd, Mar. 20, 1950. Records available: 1949-60. Mar. 20, 1956, 137. 02; Aug. 31, 137.03; Oct. 23, 1957, 137. 02; Feb. 15, 1958, 136. 87; Sept. 2, 137.14; Sept. 14, 1959, 136.95; Feb. 1, 1960, 136. 77.

\section{Whiskey Flat}

6/31-33b2. W. F. Merchant. Drilled unused well, diam 8 in, depth $69 \mathrm{ft}$, cased. MP top of casing, at lsd. Highest water level 42. 23 below 1sd, Sept. 19, 1950; lowest 44.97 below lsd, Sept. 25, 1958. Records available: 1948-51, 1957-58. No measurement made 1952-56 or 1959-60. May 11, 1948, 42.30; Nov. 28, 1949, 42.26; Jan. 19, 1950, 42. 28; Sept. 19, 42. 23; Mar. 12, 1951, 42. 37; Oct. 21, 1957, 44. 72; Sept. 25, 1958, 44. 97.

\section{Nye County}

\section{Amargosa Desert}

S16/49-18dc1. U. S. Bureau of Land Management. Formerly U. S. Government. Drilled observation well, diam $12 \mathrm{in}$, depth $348 \mathrm{ft}$, cased. Lsd 2,375.1 ft above msl. MP top of casing collar, at lsd. Highest water level 103.10 below 1sd, Feb. 12, 1955, May 24, 1956; lowest 104.73 below Isd, Sept. 15, 1959. Records available: 1953, 1955-59. No measurement made in 1960. May 24, 1956, 103.10; Dec. 6, 103.60; Aug. 28, 1957, 103.25; Feb. 15, 1958, 103. 37; Sept. 3, 103.74; Sept. 15, 1959, 104.73.

S16/49-31ba1. Gordon Bettles. Drilled unused well, diam 16 in, depth $162 \mathrm{ft}$, cased. Lsd 2,326, $33 \mathrm{ft}$ above msl. MP top of casing, at lsd. Highest water level 66. 10 below 1sd, May 7, 1952; lowest 67.10 below lsd, Sept. 15, 1959. Records available: 1952-59. No measurement made in 1960. Apr. 21, 1956, 66.40; Aug. 9, 66.44; Dec. 6, 66.35; Aug. 28, 1957, 66. 42; Sept. 3, 1958, 66. 46; Sept. 15, 1959, 67. 10 .

\section{Big Smoky Valley}

10/43-5aa1. C. J. Howard. Drilled unused well, diam $14 \mathrm{in}$, depth $70 \mathrm{ft}$, cased. MP top of casing, $0.80 \mathrm{ft}$ above lsd. Highest water level 1.02 below lsd, Dec. 1, 1960; lowest 9.48 below lsd, Sept. 3, 1957. Records available: 1957-60. 
10/43-5aa1--Continued.

\begin{tabular}{|c|c|c|c|c|c|c|c|}
\hline Date & $\begin{array}{l}\text { Water } \\
\text { level }\end{array}$ & Date & $\begin{array}{c}\text { Water } \\
\text { level }\end{array}$ & Date & $\begin{array}{c}\text { Water } \\
\text { level }\end{array}$ & Date & $\begin{array}{c}\text { Water } \\
\text { level }\end{array}$ \\
\hline $\begin{array}{lll}\text { May } & 2, & 1957 \\
\text { Sept. } & 3 & \\
\end{array}$ & $\begin{array}{l}9.24 \\
9.48\end{array}$ & $\begin{array}{l}\text { May } 7,1958 \\
\text { Nov. } 23\end{array}$ & $\begin{array}{l}8.09 \\
8.22\end{array}$ & $\begin{array}{l}\text { May } 12,1959 \\
\text { Dec. } 17\end{array}$ & $\begin{array}{l}8.27 \\
6.62 \\
\end{array}$ & $\begin{array}{ll}\text { Mar. } & 3,1960 \\
\text { Dec. } & 1\end{array}$ & $\begin{array}{l}6.94 \\
1.02 \\
\end{array}$ \\
\hline
\end{tabular}

12/43-9ca1. Carl Haas. Drilled unused well, diam $12 \mathrm{in}$, depth $190 \mathrm{ft}$, cased. MP top of casing, $2.50 \mathrm{ft}$ above 1sd. Highest water level 22. 72 below lsd, Sept. 3, 1957; lowest 35.87 below 1sd, Mar. 7, 1960. Records available: 1957-60.

\begin{tabular}{l|l|l|l|l||ll||l|l|l|}
\hline May 2, & 1957 & 31.62 & May & 7, 1958 & 32.19 & May 12, 1959 & 33.41 & Mar. 7, 1960 35.87
\end{tabular}

\begin{tabular}{l|l|l|l|l|l|l|l|l|} 
Sept. 3 & 22.72 & Nov. 23 & 27.08 & Dec. 17 & 34.21 & Dec. 1 & 35.62 \\
\hline
\end{tabular}

15/44-20ba1. A. B. Robertson. Drilled unused well, diam 6 in, depth $57 \mathrm{ft}$, cased. MP top of casing, $0.60 \mathrm{ft}$ above 1sd. Highest water level 24.92 below 1sd, Sept. 3, 1957; lowest 39.60 below 1sd, Mar. 7, 1960. Records available: 1957-60.

\begin{tabular}{|l|l||l|l|l|l|l||ll|l}
\hline May 2, 1957 & 33.75 & May & 7, & 1958 & 32.58 & May 12, 1959 & 34.14 & Mar. 7,1960 & 39.60 \\
Sept. 3 & 24.92 & Nov. 23 & 31.33 & Dec. 17 & 37.93 & Dec. 1 & 38.61 \\
\hline
\end{tabular}

\section{Pahrump Valley}

[See also Clark County]

S19/53-22acd1. Stavers (State Engineer No. 31). Drilled domestic and irrigation well, diam $16 \mathrm{in}$, reported depth $540 \mathrm{ft}$, cased to 280 , perforated 112-124, cemented at 280. MP top of pumpbase, at lsd. Highest water level 41.27 below 1sd, Apr. 11, 1947; lowest 78.5 below 1sd, August 1959. Records available: 1947-60.

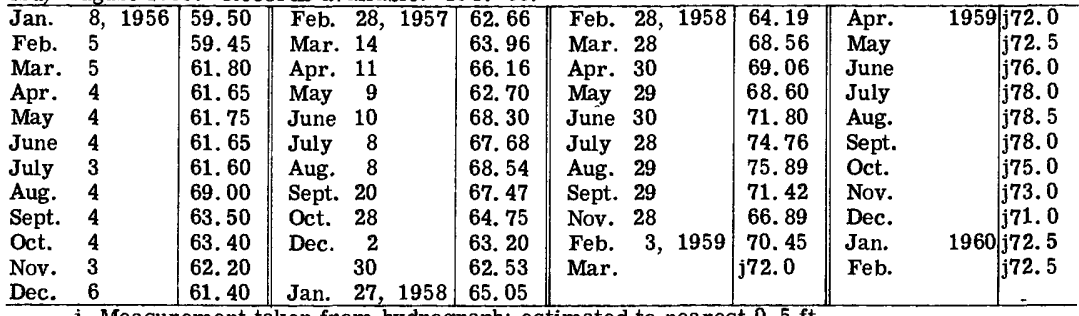

j Measurement taken from hydrograph; estimated to nearest $0.5 \mathrm{ft}$.

S20/53-24caa1. Ray Thomas (State Engineer No. 40). Drilled unused artesian well, diam $10 \mathrm{in}$, depth $570 \mathrm{ft}$, cased. MP top of casing, at lsd. Highest water level 25.10 below lsd, Mar. 17, 1945; lowest 59. 24 below 1sd, July 24, 1958. Records available: 1945, 1948-59. Measurement discontinued. Recorder removed Apr. 27, 1958.

Water level at noon, from recorder graph, 1956

\begin{tabular}{|c|c|c|c|c|c|c|c|c|c|c|c|c|}
\hline Day & Jan. & Feb. & Mar. & Apr. & May & June & July & Aug. & Sept. & Oct. & Nov. & Dec. \\
\hline 5 & 40.18 & $\mathbf{3 9} .80$ & & 42.70 & 42.93 & 41.89 & 44.37 & 45.86 & 44.82 & 42.84 & & 41.33 \\
\hline 10 & 40.14 & 39.85 & & $\ldots \ldots$ & 42. 73 & 42. 07 & 44.54 & 45.93 & 44. 21 & $\ldots \ldots$ & & 41.29 \\
\hline 15 & 40.04 & 39.78 & & & & 42.73 & 44. 71 & 47.17 & 44.02 & & & 41.28 \\
\hline 20 & 40.04 & 39.82 & & 42.99 & 41.82 & 43.10 & 44.52 & 46.24 & 43.70 & 42.29 & 41.67 & 41.25 \\
\hline 25 & $\ldots \ldots$ & $\ldots$ & 42.67 & 43.12 & 42.10 & 43.94 & 45.02 & 46.43 & 43.54 & $\ldots \ldots$ & 41.55 & 41.16 \\
\hline Eom & 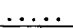 & $\ldots$ & 43. 20 & 42. 80 & 41.92 & 44. 37 & 45.56 & 46.63 & 43.15 & $\ldots$ & 41.40 & 41.05 \\
\hline \multicolumn{13}{|c|}{1957} \\
\hline 5 & 41.03 & 44.87 & 46.23 & 43.30 & & 42.63 & 48.42 & 50.63 & 50.35 & 49.49 & 44.35 & $\cdots$ \\
\hline 10 & 41.01 & 45.25 & 44. 22 & & & 43.39 & 48.72 & 50.53 & 51.77 & 47.63 & 44.07 & \\
\hline 15 & 41.10 & 45.53 & 45.82 & $\ldots$ & 44.14 & 45.61 & 49.60 & .. & & & 43. 79 & * \\
\hline 20 & 41.15 & 45.77 & 44.13 & $\ldots$. & 43. 31 & 46.61 & 49.50 & $\ldots \ldots$ & 49.68 & 46.42 & 43. 61 & \\
\hline 25 & 43.57 & 45.83 & 43.59 & $\ldots \ldots$ & 43.04 & 49.03 & 47.08 & 51.74 & 49.87 & 45.28 & 43.51 & ... \\
\hline Eom & 44.52 & 45.93 & 43.13 & & 42. 75 & 48.90 & 50.72 & 51.16 & 49.62 & 44.61 & 43.41 & 42.62 \\
\hline \multicolumn{13}{|c|}{$195 \overline{8}$} \\
\hline 5 & 42.50 & $42.2 \overline{2}$ & 42.43 & 51.11 & & $\cdots$ & $\cdots$ & ... & & $\cdots$ & $\cdots$ & • \\
\hline 10 & 42.46 & 42.09 & 43.99 & 52.34 & . & $\ldots \ldots$ & $\ldots \ldots$ & $\cdots$ & .... & $\cdots$ & $\cdots$ & $\cdots$ \\
\hline 15 & 42.36 & 42.23 & 44.46 & 52.92 & .. & $\ldots \ldots$ & $\cdots \cdots$ & $\ldots \ldots$ & $\ldots \ldots$ & $\cdots \cdots$ & & . \\
\hline 20 & 42.28 & 42.43 & 47.09 & 53.27 & & $\ldots$ & $\cdots$ & $\because$ & $\cdots$ & & & \\
\hline 25 & 42. 21 & 42. 21 & 49.07 & $\cdots$ & & $\ldots \ldots$ & $\cdots$ & . & & & & \\
\hline Eom & 42.22 & 42.14 & 51.01 & & & & & & & & & \\
\hline
\end{tabular}

\begin{tabular}{|c|c|c|c|c|c|c|c|}
\hline Date & $\begin{array}{c}\text { Water } \\
\text { level }\end{array}$ & Date & $\begin{array}{c}\text { Water } \\
\text { level }\end{array}$ & Date & $\begin{array}{l}\text { Water } \\
\text { level }\end{array}$ & Date & $\begin{array}{l}\text { Water } \\
\text { level }\end{array}$ \\
\hline $\begin{array}{l}\text { May } 23,1958 \\
\text { June } 23\end{array}$ & $\begin{array}{l}50.38 \\
55.27 \\
\end{array}$ & $\begin{array}{l}\text { July } 24,1958 \\
\text { Aug. } 22\end{array}$ & $\begin{array}{l}59.24 \\
58.98\end{array}$ & $\begin{array}{l}\text { Sept. } 22,1958 \\
\text { Oct. } 23\end{array}$ & $\begin{array}{l}51.54 \\
48.83 \\
\end{array}$ & $\begin{array}{ll}\text { Nov. } & 24,1958 \\
\text { Feb. } & 19,1959 \\
\end{array}$ & $\begin{array}{l}48.79 \\
47.36 \\
\end{array}$ \\
\hline
\end{tabular}




\section{Railroad Valley}

11/57-16a1. U. S. Bureau of Land Management. Drilled stock well, diam 6 in, depth $354 \mathrm{ft}$, cased. MP top of casing, $0.50 \mathrm{ft}$ above Isd. Highest water level 172. 32 below $1 \mathrm{sd}$, Oct. 25, 1957; lowest 177.61 below lsd, Sept. 16, 1949. Records available: 1948-57. No measurement made 1958-60.

\begin{tabular}{l|c||c|c||c|c||c|c}
\multicolumn{1}{c|}{ Date } & $\begin{array}{c}\text { Water } \\
\text { level }\end{array}$ & Date & $\begin{array}{c}\text { Water } \\
\text { level }\end{array}$ & Date & $\begin{array}{c}\text { Water } \\
\text { level }\end{array}$ & Date & $\begin{array}{c}\text { Water } \\
\text { level }\end{array}$ \\
\hline Feb. 13, 1948 & 175.2 & Sept. 15, 1950 & 174.03 & Sept. 9, 1952 & 173.89 & Sept. 13, 1955 & 173.30 \\
Apr. 25 & 174.94 & Mar. 13, 1951 & 174.62 & Sept. 18, 1953 & 174.51 & Aug. 30, 1956 & 172.93 \\
Sept. 16, 1949 & 177.61 & Sept. 11 & 174.04 & Sept. 10, 1954 & 173.79 & Oct. 25, 1957 & 172.32 \\
Mar. 27, 1950 & 174.40 & Mar. 26, 1952 & 174.32 & & & & \\
\hline
\end{tabular}

\section{Ralston Valley}

5/44-32bb1. Owner unknown. Dug unused water-table well, diam unknown, depth $18 \mathrm{ft}$, cribbed with wood. MP top south side of cribbing, at lsd. Highest water level 11.85 below Isd, Mar. 23, 1956; lowest 12.85 below lsd, Sept. 11, 1951. Records available: 1948-60. Mar. 23, 1956, 11. 85; Aug. 30, 12.54; Oct. 22, 1957, 12.47; Sept. 2, 1958, 12.78; Sept. 14, 1959, 12. 65 ; Dec. 1, 1960, 12.50.

\section{Ormsby County}

\section{Eagle Valley}

15/20-17c1. State Children's Home. Drilled irrigation well, diam 18 to 12 to $10 \mathrm{in,} \mathrm{depth}$ $595 \mathrm{ft}$, cased to 595 . MP concrete floor of pumphouse, $0.60 \mathrm{ft}$ above 1 sd. Highest water level 1.84 below 1sd, Mar. 13, 1952; lowest 17.90 below 1sd, Aug. 26, 1960 . Records available: 1946, 1948-56, 1959-60. No measurement made 1957-58. Mar. 29, 1956, 2. 65; Mar. 7, 1959, 4. 14; Mar. 22, 1960, 7.02; Aug. 26, 17.90.

\section{Pershing County}

\section{Buena Vista Valley}

30/35-27b1. Neill Talcott. Drilled observation and stock well, diam 8 in, reported depth $100 \mathrm{ft}$, cased to 100 , perforated 25-100. MP top of casing, $0.60 \mathrm{ft}$ above Isd. Highest water level 21.91 below 1sd, Jan. 7, 1948; lowest 29. 28 below 1sd, Sept. 27, 1959. Records available: 1948-50, 1952-59. No measurement made in 1960.

\begin{tabular}{l|l||l|l||l|l||l|l|}
\hline Mar. 24, 1956 & 29.22 & Mar. 16, 1957 & 27.39 & Mar. 21, 1958 & 27.95 & Mar. 13, 1959 & 27.81 \\
Sept. 25 & 27.00 & Sept. 15 & 26.24 & Sept. 10 & 25.68 & Sept. 27 & 29.28 \\
\hline
\end{tabular}

\section{Grass Valley}

\section{[See also Humboldt County]}

33/37-24a1. Lloyd Sweeney. Dug and drilled unused well, size $6 \times 8 \mathrm{ft}$ to $11 \mathrm{ft}, 10 \mathrm{in}$ to $63 \mathrm{ft}$. Lsd about 4, $400 \mathrm{ft}$ above msl. MP top of concrete curb, $0.50 \mathrm{ft}$ above 1sd. Highest water level 1.80 below lsd, Apr. 24, 1946; lowest 14.74 below lsd, Sept. 22, 1955. Records available: $1945-60$.

\begin{tabular}{|c|c|c|c|c|c|c|c|}
\hline $\begin{array}{l}\text { Mar. 21, } 1956 \\
\text { Sept. 27 } \\
\text { Mar. 13, } 1957\end{array}$ & $\begin{array}{l}12.78 \\
11.30 \\
12.49\end{array}$ & $\begin{array}{lll}\text { Sept. } & 14, & 1957 \\
\text { Mar. } 20,1958 \\
\text { Sept. } 9\end{array}$ & $\begin{array}{r}10.51 \\
11.44 \\
9.16\end{array}$ & $\begin{array}{l}\text { Mar. 9, } 1959 \\
\text { Sept. } 14\end{array}$ & $\begin{array}{l}10.95 \\
12.66\end{array}$ & $\begin{array}{l}\text { Mar. } 30,1960 \\
\text { Aug. } 29\end{array}$ & $\begin{array}{l}12.72 \\
13.83\end{array}$ \\
\hline
\end{tabular}

34/37-22a1. J. Ballard. Drilled unused well, diam 6 in, depth $50 \mathrm{ft}$, cased. Lsd $4,329 \mathrm{ft}$ above msl. MP top of casing, at Isd. Highest water level 9.31 below Isd, Mar. 21, 1956; lowest 12.97 below 1sd, Sept. 20, 1951. Records available: 1946-60.

\begin{tabular}{l|r||l|l|l|l|l|l}
\hline Mar. 21, 1956 & 9.31 & Sept. 14, 1957 & 11.54 & Mar. 9, 1959 & 10.71 & Mar. 29, 1960 & 11.20 \\
Sept. 27 & 12.15 & Mar. 20, 1958 & 11.02 & Sept. 14 & 12.46 & Aug. 29 & 12.58 \\
Mar. 13, 1957 & 11.05 & Sept. 9 & 11.62 & & & & \\
\hline
\end{tabular}

Humboldt River Valley

[See also Elko, Humboldt, and Lander Counties]

29/33-33c1. Southern Pacific RR. Co. Drilled industrial and municipal well, diam 12 in, reported depth $432 \mathrm{ft}$, cased. Lsd 4, $264 \mathrm{ft}$ above msl. MP bottom of hole in pump shell, $1.00 \mathrm{ft}$ above 1sd. Highest water level 65.16 below Isd, May 19, 1947; lowest 79.15 below 1sd, Sept. 27, 1959. Records available: 1945-54, 1956-59. No measurement made in 1960. Mar. 29, 1956, 72.75; Sept. 25, 74.52; Mar. 16, 1957, 76.73; Mar. 21, 1958, 77.87; Mar. 13, 1959, 77. 41; Sept. 27, 79.15. 
32/33-28d1. Cliff and Cecil Campbell. Humboldt. Drilled irrigation well, diam 14 in, reported depth $288 \mathrm{ft}$, cased 0-236. MP end of discharge pipe, $8.10 \mathrm{ft}$ above lsd. Highest water level 34. 37 below lsd, Apr. 26, 1950; lowest 39.42 below 1sd, Aug. 27, 1960. Records available: $1950-54,1956-60$.

\begin{tabular}{l|c||c|c||c|c||c|c}
\multicolumn{1}{c|}{ Date } & $\begin{array}{c}\text { Water } \\
\text { level }\end{array}$ & Date & $\begin{array}{c}\text { Water } \\
\text { level }\end{array}$ & Date & $\begin{array}{c}\text { Water } \\
\text { level }\end{array}$ & Date & $\begin{array}{c}\text { Water } \\
\text { level }\end{array}$ \\
\hline Mar. 21, 1956 & 36.81 & Sept. 8, 1957 & 37.86 & Sept. 2, 1958 & 38.10 & Sept. 17, 1959 38.60 \\
Sept. 27 & 37.34 & Mar. 16, 1958 & $\mathbf{3 7 . 5 8}$ & Mar. 8, 1959 & $\mathbf{3 7 . 4 8}$ & Aug. 27, 1960 & 39.42 \\
Mar. 11, 1957. & $\mathbf{3 7 . 3 2}$ & & & & & & \\
\hline
\end{tabular}

\section{Washoe County}

\section{Truckee Meadows}

18/19-12db2. Paul Christman. Drilled unused artesian well in alluvium of Quaternary age, diam $14 \mathrm{in}$, depth $376 \mathrm{ft}$, cased to 299. MP top of casing, $1.00 \mathrm{ft}$ above lsd. Highest water level 16.17 below 1sd, Aug. 31, 1956; lowest 48.17 below 1sd, Apr. 23, 1952. Records available: $1950-60$.

\begin{tabular}{|c|c|c|c|c|c|c|c|c|c|c|c|c|}
\hline$\overline{\text { May }}$ & 16 , & 1950 & 41.91 & June 2 & $26, \overline{1953}$ & 44.66 & Jan. & 30,1956 & 17.98 & Aug. & 22,1958 & 17.29 \\
\hline June & 13 & & 39.14 & July 2 & 24 & 44.17 & Feb. & 28 & 18. 33 & Sept. & 23 & 16.62 \\
\hline aly & 17 & & 38.04 & Aug. 3 & 31 & 42.34 & Apr. & 4 & 19.05 & Oct. & 28 & 16.30 \\
\hline dug. & 14 & & 37.93 & Sept. 2 & 24 & 41.91 & & 30 & 19.29 & Nov. & 22 & 17.05 \\
\hline Sept. & 18 & & 36.30 & Oct. 2 & 26 & 41.88 & May & 31 & 18.12 & Dec. & 29 & 18.78 \\
\hline Det. & 16 & & 35.50 & Nov. & 25 & 42.73 & June & 28 & 17.40 & Jan. & 27,1959 & 18.94 \\
\hline lov. & 13 & & 36.29 & Dec. & 30 & 45.09 & July & 30 & 16.59 & Feb. & 25 & 19.72 \\
\hline ec. & 18 & & 38.38 & Jan. 2 & 29,1954 & 46.39 & Aug. & 31 & 16.17 & Mar. & 25 & 20.48 \\
\hline an. & 2 & 1951 & 38.64 & Feb. 2 & 26 & 46.93 & Nov. & 30 & 17.44 & Apr. & 29 & 20.16 \\
\hline lar. & 27 & & 41.98 & Mar. 2 & 24 & 47.64 & Dec. & 26 & 18. 32 & June & 1 & 19.03 \\
\hline pr. & 24 & & 44.46 & Apr. 2 & 29 & 47. 89 & Jan. & 28,1957 & 19.08 & & 29 & 18.55 \\
\hline lay & 28 & & 45.19 & May 2 & 28 & 47. 01 & Feb. & 27 & 19.66 & July & 30 & 18.04 \\
\hline uly & 24 & & 43.22 & June 3 & 30 & 46.12 & Mar. & 26 & 20.24 & Aug. & 26 & 17.50 \\
\hline ug. & 27 & & 41.74 & July & 26 & 44.87 & Apr. & 30 & 20.14 & Oct. & 5 & 6.88 \\
\hline pt. & 25 & & 40.87 & Aug. & 27 & 43.62 & May & 28 & 18.73 & & 29 & .87 \\
\hline in. & 28, & 1952 & 44. & Sept. 2 & 27 & 42.55 & June & 28 & 17.89 & Nov. & 30 & 18.06 \\
\hline eb. & 27 & & 46.15 & Nov. & 1 & 42.25 & July & 30 & 17.25 & Dec. & 29 & 19.23 \\
\hline lar. & 21 & & 47.38 & Dec. 2 & 29 & 45.10 & Aug. & 30 & 16.91 & Jan. & 26,1960 & 20.06 \\
\hline pr. & 23 & & 48.17 & Jan. 2 & 27,1955 & 46. 52 & Sept. & 30 & 16.37 & Feb. & 23 & 20.38 \\
\hline ine & 24 & & 45.83 & Mar. 2 & 22 & 47.46 & Oct. & 28 & 16.67 & Mar. & 25 & 21.24 \\
\hline ly & 21 & & 44.28 & Apr. 2 & 29 & 47.92 & Dec. & 2 & 17.98 & Apr. & 27 & 20.93 \\
\hline Aug. & 22 & & 44,07 & June & 1 & 46.98 & & 23 & 18.44 & May & 25 & 19.75 \\
\hline pt. & 19 & & 42.90 & & 27 & 45.49 & Jan. & 29,1958 & 19.49 & June & 23 & 18.47 \\
\hline ct. & 22 & & 39.66 & July & 28 & 44.58 & Mar. & 4 & 19.94 & July & 22 & 18.14 \\
\hline ov. & 25 & & 40.48 & Sept. & 1 & 27.74 & Apr. & 2 & 20.22 & Sept. & 30 & 17.04 \\
\hline ec. & 18 & & 41.10 & & 30 & 21.51 & & 30 & 20.41 & Oct. & 26 & 17.06 \\
\hline eb. & 25 , & 1953 & 44.31 & Nov. & 1 & 18.90 & May & 28 & 19.58 & Nov. & 29 & 18.36 \\
\hline $\mathbf{r}$ & 28 & & 46.05 & Dec. & 1 & 18.87 & July & 29 & 17.88 & Dec. & 27 & 19.12 \\
\hline & & & & & & & & & & & & \\
\hline
\end{tabular}

18/20-7dcb1. Mrs. Martin Estate. Sierra Manor subdivision. Drilled unused artesian well in alluvium of Quaternary age, diam $12 \mathrm{in}$, reported depth $203 \mathrm{ft}$, cased. Lsd 4,568 ft above msl. MP top of steel plate casing cover, $1.00 \mathrm{ft}$ above lsd. Highest water level 7. 32 below 1sd, Oct. 28, 1957; lowest 15.88 below 1sd, Apr. 18, 1950. Records available: 1949-60.

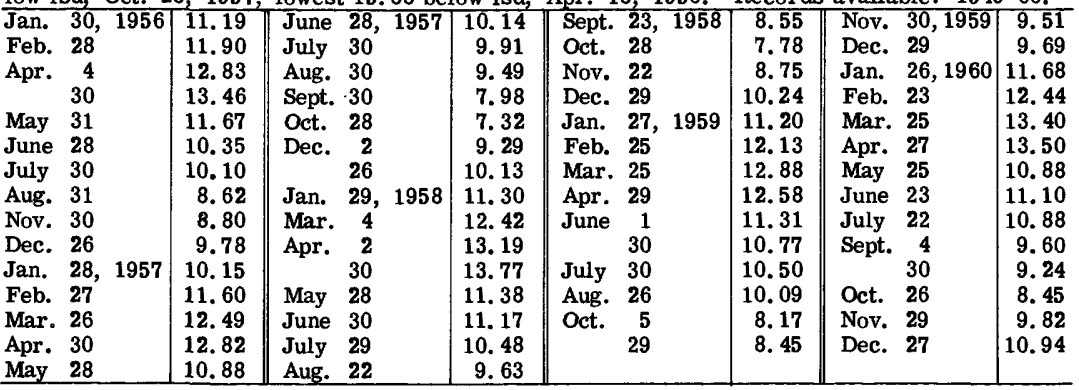

19/19-11b1. Reno High School. Fifth and West Sts. Drilled unused water-table well, diam $4 \mathrm{in}$, depth $49 \mathrm{ft}$, cased. MP top of iron curb, $0.30 \mathrm{ft}$ above lsd. Highest water level 28. 48 below lsd, Aug. 3, 1949; lowest 33.10 below 1sd, Feb. 28, 1950. Records available: 1949-60. Measurement discontinued. 
19/19-11b1--Continued.

\begin{tabular}{|c|c|c|c|c|c|c|c|}
\hline Date & $\begin{array}{c}\text { Water } \\
\text { level }\end{array}$ & Date & $\begin{array}{l}\text { Water } \\
\text { level }\end{array}$ & Date & $\begin{array}{l}\text { Water } \\
\text { level }\end{array}$ & Date & $\begin{array}{l}\text { Water } \\
\text { level }\end{array}$ \\
\hline Jan. 30,1956 & 30.34 & Apr. 30,1957 & 32.85 & May 28,1958 & 30.60 & June 30,1959 & 30.83 \\
\hline Feb. 28 & 30.99 & May 28 & 31. 78 & June 30 & 29.68 & July 3 & 29.19 \\
\hline Apr. $\quad 2$ & 31. 89 & June 28 & 30.28 & July 29 & 29.31 & Aug. 26 & 30.26 \\
\hline 30 & 32.29 & July 30 & 29.37 & Aug. 22 & 29.61 & Oct. & 5 \\
\hline May 31 & 30.89 & Aug. 30 & 29.43 & Sept. 23 & 29. & 28 & 9 \\
\hline June 28 & 29.58 & Sept. 30 & 29.69 & Oct. 28 & 30.53 & Nov. 30 & 31.47 \\
\hline July 30 & 29.47 & Oct. 28 & 30.39 & Nov. 22 & 31.46 & Dec. 29 & 32.37 \\
\hline Aug. 31 & 29.92 & Nov. 30 & 31.54 & Dec. 29 & 32.50 & Jan. 26, 1960 & 32.77 \\
\hline Nov. 30 & 31.68 & Dec. 26 & 32.37 & Jan. 27, 1959 & 32.77 & Feb. 23 & 32.76 \\
\hline Dec. 26 & 32.32 & Jan. 29,1958 & 32.78 & Feb. 25 & 32.88 & Mar. 25 & 32.76 \\
\hline Jan. 28, & 32.73 & Mar. 4 & 32. 82 & Mar. 25 & 32. 87 & Apr. 27 & 32.77 \\
\hline $\begin{array}{l}\text { Feb. } 27 \\
\text { Mar. } 26\end{array}$ & $\begin{array}{l}32.80 \\
32.83\end{array}$ & Apr. & 32.79 & Apr. 29 & 32.94 & May 25 & 31.82 \\
\hline
\end{tabular}

19/19-24cc1. J. B. Linabary. Drilled unused well, diam 6 in, depth $89 \mathrm{ft}$, cased to 89 .

MP top of casing, 2. $50 \mathrm{ft}$ below lsd. Highest water level 27. 45 below lsd, Aug. 27, 1959; lowest 30.80 below lsd, Mar. 29, 1960. Records available: 1956-60.

\begin{tabular}{|c|c|c|c|c|c|c|c|c|c|}
\hline $\begin{array}{lr}\text { ec. } & 14,1956 \\
\text { eb. } & 13,1957 \\
\text { pr. } & 15 \\
\text { une } & 12 \\
\text { ug. } & 9\end{array}$ & $\begin{array}{l}29 . \\
29 . \\
30 . \\
29 . \\
28 .\end{array}$ & $\begin{array}{ll}\text { Oct. } 1 \\
\text { Dec. } 2 \\
\text { Feb. } \\
\text { Apr. } 1 \\
\text { June } 1\end{array}$ & $\begin{array}{cc}15, & 1957 \\
20 & \\
6, & 1958 \\
15 & \\
10 & \end{array}$ & \begin{tabular}{|l|}
28.40 \\
29.69 \\
30.64 \\
30.63 \\
29.35
\end{tabular} & $\begin{array}{l}\text { Aug. } \\
\text { Nov. } \\
\text { Dec. } \\
\text { Apr. }\end{array}$ & $\begin{array}{ll}15, & 1958 \\
28 & \\
29 & \\
15, & 1959\end{array}$ & & .4 & \begin{tabular}{l|l|l} 
June 30,1959 & 2 \\
Aug. 27 & 2 \\
Mar. 29,1960 & 3 \\
Aug. 25 & 2
\end{tabular} \\
\hline
\end{tabular}

19/20-30bc2. Edward Peckham. Drilled unused well, diam $12 \mathrm{in}$, depth $120 \mathrm{ft}$, cased to 120. MP top of casing, $0.80 \mathrm{ft}$ above lsd. Highest water level 23.42 below lsd, Sept. 28, 1948; lowest 28. 60 below lsd, Apr. 14, 1959. Records available: 1948, 1956-60.

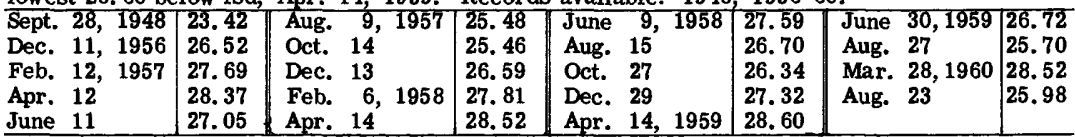

19/20-33bd1. Howard Hamilton. Drilled domestic well, diam 6 in, depth $70 \mathrm{ft}$, cased. MP top of casing, $0.40 \mathrm{ft}$ above lsd. Highest water level 8. 89 below lsd, Feb. 6, 1958; lowest 10. 42 below 1sd, June 30, 1959. Records available: 1957-60.

\begin{tabular}{|l|l||l|l||l|l|l|l|l|}
\hline Apr. 25, 1957 & 9.63 & Feb. 6, 1958 & 8.89 & Oct. 27, 1958 & 9.51 & June 30, 1959 & 10.42 \\
June 11 & 9.77 & Apr. 14 & 9.08 & Dec. 29 & 9.76 & Aug. 27 & 9.92 \\
Oct. 14 & 9.33 & June 9 & 9.53 & Apr. 14, 1959 & 9.79 & Mar. 28, 1960 & 9.75 \\
Dec. 13 & 9.36 & Aug. 14 & 9.57 & & & & & \\
\hline
\end{tabular}

\section{White Pine County}

\section{Newark Valley}

[See also Lincoln County]

18/55-31c1. Formerly 18/55-31d1. Owner unknown. Dug stock water-table well, diam $36 \mathrm{in}$, depth $43 \mathrm{ft}$, cased with concrete. MP top of concrete curb, $1.00 \mathrm{ft}$ above lsd. Highest water level 33.19 below lsd, Sept. 15, 1954, Sept. 15, 1955; lowest 34. 65 below lsd, Dec. 21, 1946. Records available: 1946-59. No measurement made in 1960. Mar. 26, 1956, 33. 23; Aug. 28, 33. 27; Sept. 5, 1957, 33.50; Sept. 4, 1958, 33.59; Dec. 18, 1959, 33. 64.

19/56-30d2. Don Eldridge. Dug stock water-table well, diam $42 \mathrm{in}$, depth $37 \mathrm{ft}$, cribbed with concrete. Lsd 5,900 ft above msl. MP top of concrete curb, $1.50 \mathrm{ft}$ above $1 \mathrm{sd}$. Highest water level 31. 63 below 1sd, Oct. 1, 1952; lowest 35. 31 below 1sd, Dec. 21, 1959. Records available: 1948-60. Mar. 26, 1956, 31.87; Sept. 5, 1957, 31.91; Sept. 4, 1958, 32.32; Dec. 21, 1959, 35. 31; Aug. 24, 1960, 32.67.

20/55-10d1. U. S. Bureau of Land Management. Dug stock water-table well, diam 36 in, depth $22 \mathrm{ft}$, cased with concrete. Lsd 5, $871 \mathrm{ft}$ above msl. MP top of plank well cover, $1.00 \mathrm{ft}$ above lsd. Highest water level 8.08 below lsd, Mar. 24, 1949; lowest 9.48 below 1sd, Dec. 21, 1959. Records available: $1948-59$. No measurement made in 1960. Mar. 26, 1956, 9.16;

Aug. 28, 9. 40; Sept. 5, 1957, 9. 34; Sept. 4, 1958, 9. 26; Dec. 21, 1959, 9. 48.

21/55-10c1. Formerly 21/55-9b1. R. W. Hooper. Dug domestic water-table well, size $5 \times 5 \mathrm{ft}$, depth $34 \mathrm{ft}$, cribbed with wood. Lsd 5,950 ft above msl. MP top of wood cribbing, $3.50 \mathrm{ft}$ above 1sd. Highest water level 10.67 below 1sd, Mar. 26, 1956; lowest 19.26 below lsd, Sept. 5, 1957. Records available: 1948-59. No measurement made in 1960. Mar, 26, 1956, 10. 67; Aug. 28, 18. 89; Sept. 5, 1957, 19. 26; Sept. 4, 1958, 19. 08; Dec. 21, 1959, 18.88. 


\section{Spring Valley}

13/67-8d1. A. Schaurman. Dug stock water-table well, diam 36 in, reported depth $45 \mathrm{ft}$, cased. MP top of concrete curb, $2.50 \mathrm{ft}$ above lsd. Highest water level 11.60 below $1 \mathrm{sd}$, Mar. 4, 1953 (previously reported 11.70 below Isd, Mar. 29, 1949); lowest 15.11 below lsd, Sept. 10, 1952. Records available: 1947-57, 1959-60. No measurement made in 1958. Aug. 29, 1956, 14. 68; Sept. 5, 1957, 13.84; Dec. 19, 1959, 13.37; Sept. 27, 1960, 14. 30.

15/66-13d1. J. P. Johanson. Drilled domestic well, diam 6 in, depth $82 \mathrm{ft}$, cased. MP lowest point of break on east side of pumpbase, $1.00 \mathrm{ft}$ above $1 \mathrm{sd}$ (since Dec. 19, 1959). Highest water level 13.68 below 1sd, Sept. 10, 1952; lowest 23. 29 below lsd, Sept. 27, 1960. Records available: $1947,1949-57,1959-60$. No measurement made in 1958. Mar. 22, 1956, 17. 75; Aug. 29, 20.58; Sept. 5, 1957, 19.34; Dec. 19, 1959, 22. 21; Sept. 27, 1960, 23. 29.

17/68-6d1. U. S. Bureau of Land Management. Dug stock water-table well, diam $4 \mathrm{ft}$, depth $28 \mathrm{ft}$, cribbed with concrete. MP top of concrete curb, $2.50 \mathrm{ft}$ above lsd. Highest water level 21. 69 below 1sd, Mar. 18, 1950; lowest 26. 42 below 1sd, Sept. 27, 1960. Records available: $1948-55,1957,1959-60$. No measurement made in 1956 or 1958 . Sept. 15, 1957, 25. 29; Dec. 18, 1959, 25.41; Sept. 27, 1960, 26.42.

\section{Steptoe Valley}

15/64-7a1. Lloyd Sorenson. Drilled irrigation well, diam $16 \mathrm{in}$, reported depth $200 \mathrm{ft}$, cased. MP top of casing, $1.00 \mathrm{ft}$ above lsd. Highest water level 32.70 below lsd, Mar. 4, 1953; lowest 39. 06 below lsd, Mar. 14, 1951. Records available: 1948-57, 1959. No measurement made in 1958 and 1960. Aug. 29, 1956, 34.18; Sept. 4, 1957, 33. 82; Dec. 19, 1959, 37. 42.

16/63-1b1. Owner unknown. Drilled unused well, diam 6 in, depth unknown, cased. MP top of cap on casing, $0.80 \mathrm{ft}$ above lsd. Highest water level 59.10 below lsd, Mar. 10, 1954; lowest 71. 77 below lsd, Sept. 27, 1960 . Records available: 1949-57, 1959-60. No measurement made in 1958. Mar. 26, 1956, 61.06; Aug. 28, 63.08; Sept. 4, 1957, 66. 32; Dec. 19, 1959, 71. 07; Sept. 27, 1960, 71.77.

19/63-12a1. U.S. Geol. Survey. Drilled test well in sand and gravel, diam 12 to 8 in, depth $915 \mathrm{ft}$, cased, 12-in 0-181, 8-in 0-540. MP top of concrete curb, $1.60 \mathrm{ft}$ above lsd. Highest water level 9.6 below lsd, 1918 (previously reported 13.81 below lsd, Mar. 4, 1953); lowest 21. 20 below lsd, July 5, 1949 . Records available: 1918, 1949-57, 1959. No measurement made in 1958 and 1960. Mar. 26, 1956, 14. 57; Aug. 28, 14. 75; Sept. 4, 1957, 15. 00; Dec. 19, 1959, 15. 20.

20/64-32c2. U.S. Geol. Survey. Drilled test well, diam $10 \mathrm{in}$, depth $110 \mathrm{ft}$, cased. MP top of casing, $1.00 \mathrm{ft}$ above lsd. Highest water level 13. 45 below 1sd, Mar. 9, 1955; lowest 14. 72 below 1sd, Sept. 4, 1957. Records available: 1918, 1949-57, 1959. No measurement made in 1958 or 1960 . Sept. 26, 1956, 13.53; Sept. 4, 1957, 14.72; Dec. 19, 1959, 13.99.

\section{White River Valley}

[See also Nye County]

11/61-35a1. Public domain. Drilled stock well, diam 6 in, depth unknown, cased. MP top of casing, $0.40 \mathrm{ft}$ above Isd (since Aug. 30, 1956). Highest water level 10.49 below lsd, Sept. 18, 1953; lowest 13.08 below lsd, Sept. 11, 1951. Records available: 1947-57, 1959. No measurement made in 1958 and 1960. Aug. 30, 1956, 12.76; Sept. 4, 1957, 12. 74; Dec. 20, 1959, 12.66.

12/61-34a1. U. S. Bureau of Land Management. Drilled stock well, diam 7 in, depth $72 \mathrm{ft}$, cased. MP top of casing collar, $1.50 \mathrm{ft}$ above $1 \mathrm{sd}$. Highest water level 57.65 below lsd, Dec. 15, 1949; lowest 62. 43 below 1sd, Dec. 20, 1959. Records available: 1947-57, 1959-60. No measurement made in 1958. Aug. 30, 1956, 60.42; Sept. 4, 1957, 60.60; Dec. 20, 1959, 62. 43; Sept. 27, 1960, 61.95.

12/62-18d1. U. S. Geol. Survey. Drilled test and observation well, diam 6 in, depth $108 \mathrm{ft}$, cased to 105 . MP top of casing collar, $0.50 \mathrm{ft}$ above $1 \mathrm{sd}$. Highest water level 43.56 below lsd, Sept. 9, 1952; lowest 50.73 below lsd, Dec. 18, 1947. Records available: 1947-53, 1955-57, 1959. No measurement made in 1958 and 1960. Aug. 30, 1956, 47.84; Sept. 4, 1957, 48. 11; Dec. 20, 1959, 47.41.

12/62-33a5. Wayne Gardner. Lund. Dug domestic water-table well, size $4 \mathrm{x} 4 \mathrm{ft}$, depth $31 \mathrm{ft}$, cased with concrete. Lsd 5,578.45 ft above msl. MP top edge of south side of iron manhole, $0.50 \mathrm{ft}$ above 1sd. Highest water level 17.56 below 1sd, Dec. 20, 1959; lowest 25.44 below lsd, Mar. 15, 1950. Records available: 1947-57, 1959-60. No measurement made in 1958. Mar. 22, 1956, 22. 20; Aug. 30, 24.97; Sept. 4, 1957, 23.13; Dec. 20, 1959, 17. 56; Sept. 27, $1960,23.31$. 


\section{References}

Eakin, T. E., 1960, Ground-water appraisal of Newark Valley, White Pine County, Nevada: Nevada Dept. Conserv. Nat. Resources, Reconn. Ser. Rept. 1.

Eakin, T. E., and others, 1951, Contributions to the hydrology of eastern Nevada: Nevada State Engineer, Water Resources Bull. 12.

Eakin, T. E., and Robinson, T. W., 1950, Ground-water conditions in Whiskey Flat, Mineral County, Nevada: U.S. Geol. Survey open-file rept.

Loeltz, O. J., and Eakin, T. E., 1953, Geology and water resources of Smith Valley, Lyon and Douglas Counties, Nevada: U.S. Geol. Survey Water-Supply Paper 1228. [1954].

Loeltz, O. J., Phoenix, D. A., and Robinson, T. W., 1949, Ground water in Paradise Valley, Humboldt County, Nevada: Nevada State Engineer, Water Resources Bull. 10.

Loeltz, O. J., and Phoenix, D. A., 1955, Geology and ground-water resources of Buena Vista Valley, Pershing County, Nevada: Nevada State Engineer, Water Resources Bull. 13.

Maxey, G. B., and Eakin, T. E., 1949, Ground water in White River Valley, White Pine, Nye, and Lincoln Counties, Nevada: Nevada State Engineer, Water Resources Bull. 8.

Maxey, G. B., and Jameson, C. H, , 1946, Well data in Las Vegas and Indian Spring Valleys, Nevada: Nevada State Engineer, Water Resources Bull. 4. 1948, Geology and water resources of Las Vegas, Pahrump, and Indian Spring Valleys, Clark and Nye Counties, Nevada: Nevada State Engineer, Water Resources Bull. 5.

Phoenix, D. A. , 1948, Geology and ground water in the Meadow Valley Wash drainage area, Nevada, above the vicinity of Caliente: Nevada State Engineer, Water Resources Bull. 7.

Robinson, T. W., and others, 1947, Water levels and artesian pressures in wells in Las Vegas Valley and in other valleys in Nevada, 1913-45: Nevada State Engineer, Water Resources Bull. 3.

Robinson, T. W., and Fredericks, J. C., 1946, Ground water in Lovelock Valley, Nevada: Nevada State Engineer, Water Resources Bull. 2.

Visher, F. N., 1957, Geology and ground-water resources of Quinn River Valley, Humboldt County, Nevada: Nevada State Engineer, Water Resources Bull. 14.

Zones, C. P., 1959, Ground-water potentialities in Crescent Valley, Eureka and Lander Counties, Nevada: U.S. Geol. Survey open-file rept., 92 p. 
The observation-well program, which has been in progress in parts of New Mexico since 1925, was continued during 1956 through 1960 in cooperation with the State Engineer of New Mexico. However, the total observation-well program is much more extensive than the wells covered in this report. Other data collected are published annually in the Technical Report series of the State Engineer. Measurements of water levels in observation wells in the Virden Valley in Hidalgo County were made by the Arizona office of the U.S. Geological Survey. Figure 6 shows the location of observation wells included in this report.

\section{We1l-Numbering System}

The system of numbering wells in New Mexico, except the thermal wells in the Truth or Consequences area in Sierra County, is based on the common subdivision of public lands into sections. The well number is based on the New Mexico principal meridian and base line except in the Sangre de Cristo Grant in Taos County, where it is based on a system of sectionized land used in Colorado and extended into that part of the land grant in New Mexico.

The well number, in addition to designating the well, locates its position to the nearest 10 acre tract in the land net. The number is divided by periods into four segments. The first segment denotes the township north or south of the New Mexico base line; the second, the range east or west of the New Mexico principal meridian; the third, the section; and the fourth, the particular tract in which the well is situated. In a county where wells are situated both north and south of the base line, such as Roosevelt County, an $\mathrm{N}$ is added to the first segment of the well number if the well is north of the base line. Similarly, in a county where wells are both east and west of the meridian, an $\mathrm{E}$ is added to the second segment of the number if the well is east of the meridian. In counties entirely within one quadrant of the principal meridian and base line, the direction north or south of the base line or east or west of the meridian is not given.

The fourth segment of the number, which consists of three digits, denotes the particular 10 -acre tract, in which the well is situated. For this purpose, the section is divided into four quarters, numbered $1,2,3$, and 4 , in the normal reading order, for the northwest, northeast, southwest, and southeast quarters. The first digit of the fourth segment gives the quarter section. Similarly, the quarter section is divided into four 40 -acre tracts numbered in the same manner, and the second digit denotes the 40-acre tract. Finally, the 40-acre tract is divided into four 10 -acre tracts, and the third digit denotes the 10-acre tract. Thus, well 12.36. 24.342 in Lea County is located in the $\mathrm{NE}_{\frac{1}{4}} \mathrm{SE} \frac{1}{4} \mathrm{SW} \frac{1}{4} \mathrm{sec} .24, \mathrm{~T} .12 \mathrm{~S}$, , R. $36 \mathrm{E}$. If a well cannot be located within a 10-acre tract, a zero is used as the third digit, and if it cannot be located within a 40acre tract, zeros are used for both the second and third digits. If the well cannot be located more closely than the section, the fourth segment of the well number is omitted. When it becomes possible to locate more accurately a well in whose number zeros have been used, the proper digit or digits are substituted for the zeros. In Water-Supply Paper 911 and earlier reports, the digits corresponding to unknown 10-acre and 40-acre tracts were simply omitted; but this practice caused some confusion in cataloging the wells. In Water-Supply Paper 941 and subsequent reports, wells whose numbers end in one or two zeros correspond to wells whose numbers in earlier reports are the same except for the omission of such zeros. Letters a, b, c, etc., are added to the last segment to designate the second, third, fourth, and succeeding wells in the same 10-acre tract.

\section{We1l Descriptions and Water-Level Measurements}

All measurements of water level in this report are given in feet above or below landsurface datum, which approximates the land surface at the well. 


\section{Bernalillo County}

\section{Albuquerque Area}

9. 2E. 34. 322. Denison Farm. Drilled irrigation water-table well in Santa Fe Group of middle (?) Miocene to Pleistocene (?) age, diam 12 in, depth unknown. MP top of $\frac{3}{2}$ in hole in north side of pumpbase, $1.60 \mathrm{ft}$ above lsd. Highest water level 12.47 below lsd, July 8, 1957; lowest 15.08 below lsd, Jan. 24, 1957. Records available: 1956-60.

\begin{tabular}{|c|c|c|c|c|c|c|c|}
\hline Date & $\begin{array}{c}\text { Water } \\
\text { level }\end{array}$ & Date & $\begin{array}{c}\text { Water } \\
\text { level }\end{array}$ & Date & $\begin{array}{r}\text { Water } \\
\text { level }\end{array}$ & Date & $\begin{array}{l}\text { Water } \\
\text { level }\end{array}$ \\
\hline July 11,1956 & 14.45 & Jan. 29,1958 & 14.46 & Feb. 25, 1959 & 13.90 & Feb. 2,1960 & 13.77 \\
\hline Oct. 9 & 14.82 & Mar. 11 & 14.49 & Mar. 18 & 13.82 & Mar. 3 & 13.89 \\
\hline Nov. 13 & 14.88 & Apr. 21 & 14.15 & Apr. 15 & 13.14 & Apr. & 13.68 \\
\hline Dec. 11 & 14.98 & May 11 & 13.96 & May & 14.97 & May & 13.46 \\
\hline Jan. 24, 1957 & 15.08 & June 10 & 13.64 & June & 12.90 & June & 13.05 \\
\hline Mar. 19 & 14.96 & July 10 & 13.40 & July & 13.03 & July 12 & 12.91 \\
\hline May 7 & 14.59 & Aug. 11 & 13.59 & Aug. 18 & 13.32 & Aug. $\quad 2$ & 13.18 \\
\hline June & 14.48 & Sept. 5 & 13.34 & Sept. 10 & 13.63 & Sept. & 13.61 \\
\hline July & 12.47 & Oct. 23 & 13.49 & Oct. & 13.67 & Oct. 19 & 13.44 \\
\hline Aug. & 13.66 & Nov. 20 & 13.52 & Nov. & 13.44 & Nov. 10 & 13.44 \\
\hline Sept. 19 & 14.03 & Dec. 12 & 13.61 & Dec. 1 & 13.57 & Dec. 13 & 13.54 \\
\hline Oct. 29 & 13.79 & Jan. 23,1959 & 13.81 & 5,1960 & 13.68 & & \\
\hline
\end{tabular}

10. 2E.12. 223. City of Albuquerque. Drilled observation water-table well in alluvium and Santa Fe Group of middle (?) Miocene to Pleistocene (?) age, diam 6 in. MP top north side of casing, 6. $00 \mathrm{ft}$ above 1sd. Highest water level 12.10 below 1sd, Apr. 16, 1953; lowest 28.28 below 1sd, Dec. 12, 1960. Records available: 1953, 1957-60. No measurement made 1954-56.

\begin{tabular}{|c|c|c|c|c|c|c|c|c|c|c|c|c|}
\hline or. & 16 , & $\overline{53}$ & 2.10 & Apr. & 22,1958 & 20 & Mar. & 18,1959 & 21.55 & Feb. & 2,1 & 17.30 \\
\hline an. & 31 , & 1957 & 15.54 & May & 11 & 23.48 & Apr. & 15 & 25.27 & Mar. & 3 & 17.88 \\
\hline Aar. & 21 & & 15.92 & June & 10 & 26.10 & May & 6 & 22.68 & Apr. & 5 & 24.05 \\
\hline lay & 9 & & 17.40 & July & 10 & 27.97 & June & 3 & 24.89 & May & 3 & 25.50 \\
\hline ine & 7 & & 26.43 & Aug. & 11 & 25.87 & July & 7 & 26. & July & 6 & 26. 61 \\
\hline uly & 8 & & 25.52 & Sept. & 5 & 25.99 & Aug. & 18 & 25.82 & & 12 & 27.18 \\
\hline Aug. & 1 & & 20.50 & Oct. & 23 & 16.53 & Sept. & 10 & 24.65 & Sept. & 7 & 28 \\
\hline ept. & 19 & & & Nov. & 20 & 15. & Oct. & 7 & 23. & Oct. 1 & 19 & 2 \\
\hline t. & 30 & & 19.37 & Dec. & 12 & 17. & Nov. & 9 & 24.72 & Nov. & 9 & 23 \\
\hline n & 30 , & 1958 & 14.43 & Jan. & 23,1959 & 21.02 & Dec. & 1 & 18.84 & Dec. 1 & 12 & 28.28 \\
\hline ar. & 11 & & 14.22 & Feb. & 25 & 19.33 & Jan. & 5,1960 & 25.03 & & & \\
\hline
\end{tabular}

10. 2E. 24. 413. City of Albuquerque. Drilled observation water-table well in alluvium and Santa Fe Group of middle (?) Miocene to Pleistocene (?) age, diam 6 in. MP top east side of casing, $5.50 \mathrm{ft}$ above lsd. Highest water level 20.69 below 1sd, Nov. 13, 1956; lowest 22. 09 below 1sd, Nov. 10, Dec. 13, 1960. Records available: 1956-60.

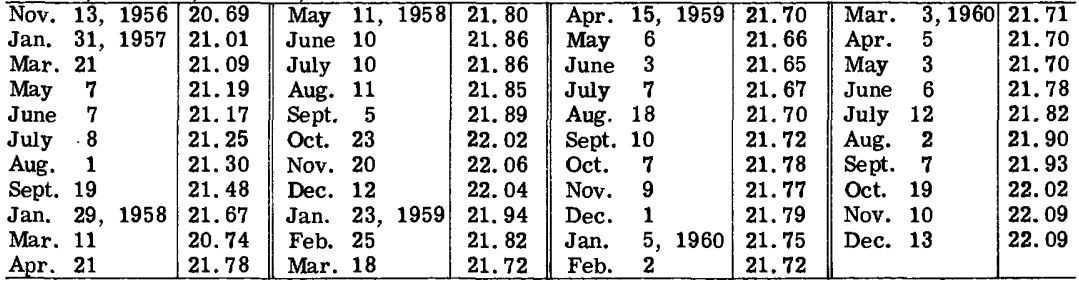

10. 4E. 3. 242. Mr. Gutierrez. Drilled unused water-table well in Santa Fe Group of middle (?) Miocene to Pleistocene (?) age, diam 6 in, depth $320 \mathrm{ft}$. MP top north side of steel pipe clamp, $1.50 \mathrm{ft}$ above 1sd. Highest water level 252. 37 below 1sd, Dec. 18, 1956; lowest 257.01 below lsd, Dec. 12, 1960. Records available: 1956-60.

\begin{tabular}{|c|c|c|c|c|c|c|c|c|c|c|c|}
\hline in. & 26,1956 & 255.35 & Apr. 2 & 22,1958 & 253.90 & Apr. 1 & 15,1959 & 255.07 & Mar. & 3,1 & 256.16 \\
\hline ec. 1 & 18 & 252. 37 & May 1 & 12 & 19 & May & 6 & 255.20 & Apr. & 5 & 256 \\
\hline an. & 25,1957 & 252.55 & June 1 & 12 & 25 & July & 7 & 255.48 & May & 3 & 25 \\
\hline ar. 2 & 22 & 252 & July 1 & 11 & & Aug. 1 & 19 & 25 & June & 6 & \\
\hline ay 1 & 14 & 252.85 & Aug. 1 & 12 & & Sept. 1 & 10 & 255 & July 1 & 12 & 25 \\
\hline & 6 & & Sept. & 8 & & Oct. & 7 & & Aug. & 2 & 25 \\
\hline ly & 9 & 0 & Nov. 2 & 21 & & Nov. & 9 & 2 & Sept. & 6 & 255.85 \\
\hline ig. & 2 & 3 & Dec. 1 & 12 & 25 & Dec. & 1 & 25 & Oct. 1 & 18 & m257.12 \\
\hline pt. 2 & 20 & 2 & Jan. & 26 & $\ln 256$ & Jan. & 6,1960 & 255.94 & Nov. 1 & 10 & 256.7 \\
\hline 3 & 31 & 253.37 & Feb. 2 & 25 & 254.98 & Feb. & 2 & 255.74 & Dec. 1 & 12 & 257.01 \\
\hline 2 & 28, & 253.85 & Mar. 1 & 18 & & & & & & & \\
\hline
\end{tabular}

m Measurement uncertain. 


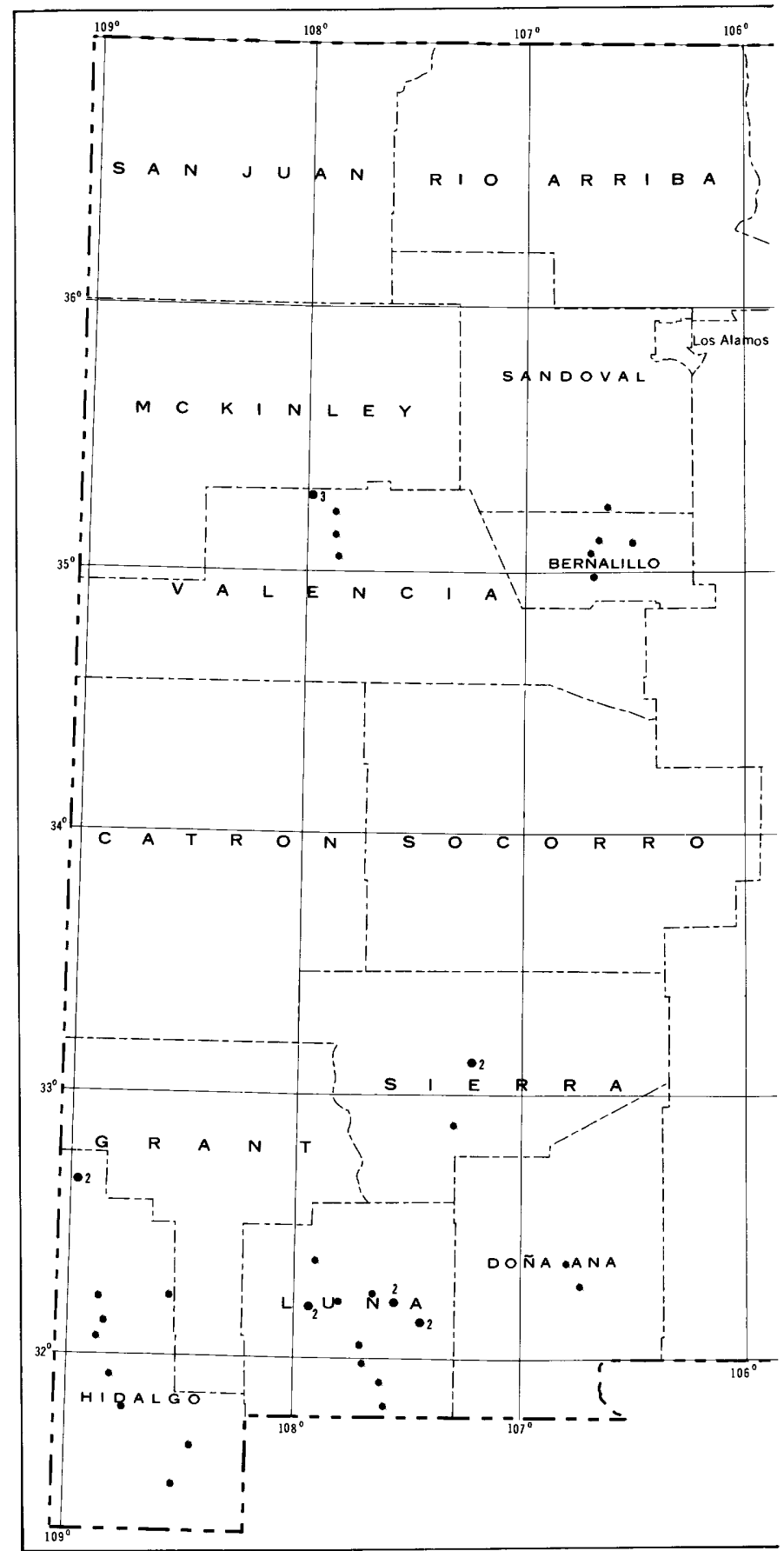

Figure 6. --Location of observation 


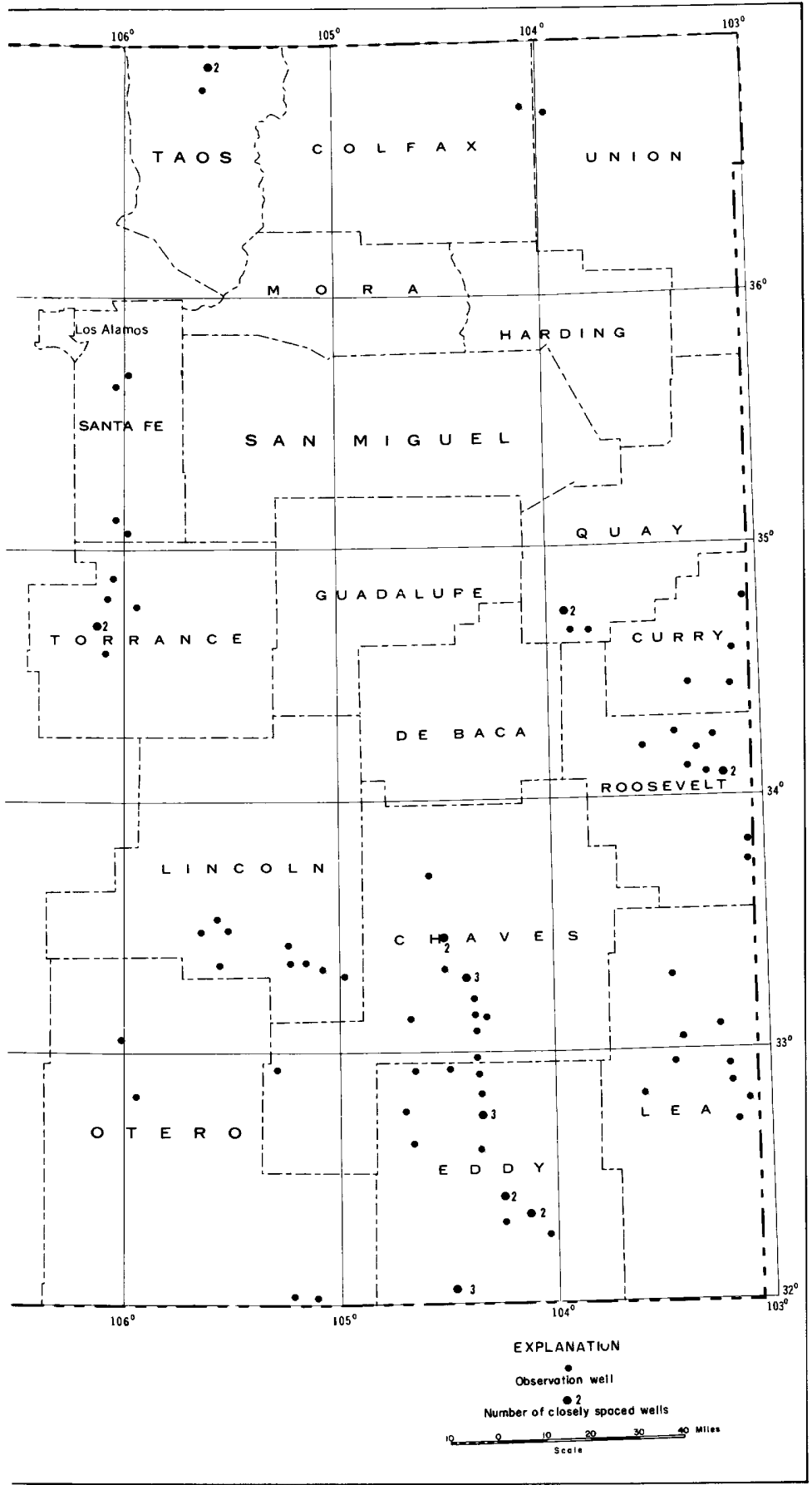

wells in New Mexico, 1956-60. 


\section{Chaves County}

\section{Roswell Basin}

7. 23. 23. 242. Jess Corn. Drilled irrigation artesian well in San Andres Limestone of Permian age, diam 14 in, depth $426 \mathrm{ft}$. Lsd 3,814 ft above msl. MP lower outer edge of mouth of discharge pipe, 3.71 ft above lsd. Highest water level 239.83 below 1sd, May 26, 1951; lowest 265.64 below Isd, Mar. 16, 1960. Records available: 1951-60.

\begin{tabular}{|c|c|c|c|c|c|c|c|}
\hline Date & $\begin{array}{l}\text { Water } \\
\text { level }\end{array}$ & Date & $\begin{array}{l}\text { Water } \\
\text { level }\end{array}$ & Date & $\begin{array}{l}\text { Water } \\
\text { level }\end{array}$ & Date & $\begin{array}{c}\text { Water } \\
\text { level }\end{array}$ \\
\hline Jan. 10,1956 & 250.17 & Mar. 7, 1957 & 257.85 & May 13,1958 & 262.24 & May 18,1959 & 262.59 \\
\hline Mar. 5 & 249.28 & May 2 & 255.71 & July 16 & (a) & July 13 & 264. 39 \\
\hline May & 250.61 & July 19 & (a) & Aug. 25 & (a) & Sept. 15 & (a) \\
\hline July 13 & (a) & Sept. 16 & (a) & Sept. 19 & 260.59 & Nov. 18 & (b) \\
\hline Sept. 13 & (a) & Nov. 19 & 263.94 & Nov. 14 & 261.93 & 4,1960 & 262.89 \\
\hline $\begin{array}{l}\text { Nov. } 2 \\
\text { Jan. } 23,1957\end{array}$ & $\begin{array}{c}\text { (a) } \\
254.49\end{array}$ & $\begin{array}{l}\text { Jan. 7, } 1958 \\
\text { Mar. 11 }\end{array}$ & $\begin{array}{l}259.27 \\
260.19\end{array}$ & $\begin{array}{l}\text { Jan. 5, } 1959 \\
\text { Mar. 16 }\end{array}$ & $\begin{array}{l}257.67 \\
257.59\end{array}$ & Mar. 16 & 265.64 \\
\hline
\end{tabular}

10. 24. 9. 333. Berrendo. Drilled observation artesian well in San Andres Limestone of Permian age, diam 10 in, depth $258 \mathrm{ft}$, cased $0-258$, depth to artesian aquifers 170,241 . Lsd $3,586.16 \mathrm{ft}$ above msl. MP upper surface of recorder-shelter floor, $0.83 \mathrm{ft}$ above Isd. Highest water level 11. 29 below 1sd, Dec. 19-20, 1926; lowest 53.48 below 1sd, Sept. 7, 1960. Records available: 1926-60. For additional measurements, see Reeder and others (1960b) (1960c).

Highest water level for the day, from recorder graph, 1956

\begin{tabular}{|c|c|c|c|c|c|c|c|c|c|c|c|c|}
\hline Day & an. & Feb. & Mar. & Apr. & May & June & July & Aug. & Sept. & Oct. & Nov. & Dec. \\
\hline 5 & 30.36 & 30.07 & 29.84 & 35.28 & 35.54 & 37.14 & 40.12 & 42.28 & 44.85 & 41.31 & 38.47 & 36.80 \\
\hline 10 & & 29.89 & 30.24 & 35.62 & 35.65 & 38.52 & 42.44 & 43.38 & 44.29 & 40.90 & & 46 \\
\hline 15 & & 60 & 31. & & 35. & & & & 43. 38 & 40. 37 & & .18 \\
\hline 20 & 32 & 29.55 & & & 35.82 & 38.83 & 43.52 & 43.28 & 42. 88 & 39.67 & 37.82 & 35.93 \\
\hline 25 & & 29.72 & 33.56 & 35.52 & 36.14 & 38.84 & 42.02 & 42.75 & 42.50 & 39.35 & 37.38 & 35.72 \\
\hline Eom & & 29.75 & 35.04 & 35.41 & 36. & 39.69 & & & 42.18 & 38.87 & 37.08 & 35.43 \\
\hline \multicolumn{13}{|c|}{1957} \\
\hline 5 & 35.49 & 35.17 & 35.33 & 40.11 & 39.62 & 40.92 & 46.27 & 45.36 & 46.03 & 44.34 & 40.10 & 7.81 \\
\hline 10 & & 35.28 & 36.02 & 41.15 & 39.65 & 41.56 & 46.05 & 47.36 & 47.01 & 43.52 & 39.67 & 37.67 \\
\hline 15 & 38 & 35.45 & 36.44 & 40.33 & 39.34 & 42.04 & 45.95 & 46.72 & 46. 36 & 42.65 & 39.24 & -37.47 \\
\hline 20 & & 35.22 & 36. 85 & 41.64 & 40.00 & 42. & 45.32 & 45.79 & 46.20 & 41.88 & 38.88 & 37.24 \\
\hline 25 & 5 & 35.12 & 37.30 & 40.48 & 40.53 & 43. & 47.09 & 45.89 & 45.18 & 41.25 & 38.52 & 37.03 \\
\hline Eon & & 35 & 40.63 & 41 & 42. 48 & 43.83 & 45.67 & 45.87 & 44.40 & 40.56 & 38.20 & 36.95 \\
\hline \multicolumn{13}{|c|}{1958} \\
\hline 5 & 36.62 & 35.42 & 5.22 & 38.23 & $3 \overline{9} .30$ & 41.52 & 5.15 & 46.48 & 46.93 & 42.60 & 39 & 37.62 \\
\hline 0 & 20 & & 35.18 & 38.80 & 39.20 & 42 & 46.23 & 47. & 47.25 & 41.99 & 39 & 37.45 \\
\hline 15 & 36.03 & 35 & 35.39 & 38.40 & 38.74 & 43. & 46.95 & 48.18 & 45.88 & 41. 35 & 38 & 37.28 \\
\hline 20 & 76 & 35.48 & 36.32 & 38.30 & 38.41 & 44.45 & 46.17 & 49.80 & 45.62 & 40.83 & 38.43 & 37.21 \\
\hline 25 & & 35.22 & 36.95 & 38.55 & 39.18 & 43. 68 & 47.15 & 48. & 40 & 40.38 & 38.14 & 37.02 \\
\hline Eom & & 35.32 & & & 40.27 & 43.10 & 46. 76 & & & 79 & 84 & 36.75 \\
\hline \multicolumn{13}{|c|}{$1 \overline{959}$} \\
\hline 5 & 36.59 & 36.87 & 38.67 & 42.62 & 43.29 & 45.92 & 45.80 & 47.82 & 47.59 & 45.87 & 43.55 & 41.96 \\
\hline 10 & 7 & 12 & 03 & 42.60 & 41.99 & 44. & 46. 89 & 48 & 35 & 45.28 & 02 & 41.65 \\
\hline 15 & & 37.17 & 39 & 42.13 & 41. 88 & 44. & 45.39 & 49 & 50 & 44.85 & 42.70 & 41.40 \\
\hline 20 & & 37.52 & 40 & 42.02 & 41.45 & 44. & 44. & & 49 & 44.46 & 42.47 & 41.03 \\
\hline 25 & & 37 & 41 & 42.26 & 41.91 & 45.42 & 44. & & 48 & 44.19 & 42 & 40.62 \\
\hline om & & 38.43 & 41.98 & 43.45 & 43.64 & 45.61 & 46.50 & 46.48 & & 43.88 & 42.03 & 40.30 \\
\hline \multicolumn{13}{|c|}{1960} \\
\hline 5 & 15 & 38.65 & 38.70 & 47.29 & 45.93 & 47.33 & 49.23 & 50.00 & 50.62 & 47.63 & 44.17 & 42.13 \\
\hline 10 & & 38.47 & 39.24 & 46.63 & 46.13 & 46.13 & 45.33 & 50.49 & 50.70 & 47.07 & 43.67 & 41.74 \\
\hline 15 & & 38.50 & 41.07 & 46.28 & 46.17 & 45.17 & 44.45 & 50. & 51 & 46 & .. & 41.47 \\
\hline 20 & 41 & 38.40 & 41.46 & 46.37 & 46.81 & 44.90 & 44. 07 & 50.68 & & 45.73 & & 41.20 \\
\hline 25 & & 38.41 & 43.19 & 46.01 & 48.43 & 47.45 & 44.23 & 50.23 & 50.00 & 45.07 & 42. 67 & 40.87 \\
\hline & & 38.51 & 44.97 & 46.88 & 48.93 & 49.18 & 46.86 & 49.33 & 48.11 & 44.47 & 42.52 & 40.55 \\
\hline
\end{tabular}

11. 24. 28.113. S. W. Skinner. Drilled domestic water-table well in valley fill, diam 6 in, depth $143 \mathrm{ft}$, cased $0-133$. MP lower inside edge of slot in casing, $0.55 \mathrm{ft}$ above isd. Highest water level 50.78 below Isd, Nov. 14, 1941; lowest 107.00 below Isd, Sept. 15, 1959 . Records available: $1938-60$.

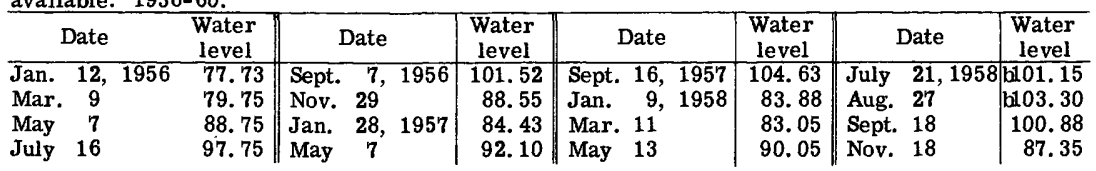


11. 24. 28.113-- Continued.

\begin{tabular}{|c|c|c|c|c|c|c|c|}
\hline Date & Water & Date & Water & Date & Water & Date & Water \\
\hline $\begin{array}{l}\text { Jan. } 27,1959 \\
\text { Mar. } 16 \\
\text { May } 18\end{array}$ & $\begin{array}{l}84.37 \\
91.10 \\
92.69\end{array}$ & $\begin{array}{ll}\text { July } & 15,1959 \\
\text { Sept. } & 15 \\
\text { Nov. } & 19\end{array}$ & $\begin{array}{r}100.25 \\
107.00 \\
93.08\end{array}$ & $\begin{array}{l}\text { Jan. } 8,1960 \\
\text { Mar. } 21 \\
\text { May } 17\end{array}$ & $\begin{array}{r}88.35 \\
93.78 \\
101.10\end{array}$ & \begin{tabular}{ll|} 
July & 19,1960 \\
Sept. & 29 \\
Nov. & 16
\end{tabular} & $\begin{array}{r}97.10 \\
\text { b1 } 05.00 \\
\text { b92. } 82\end{array}$ \\
\hline
\end{tabular}

11. 25. 29.444. G. C. Wheeler. Drilled unused water-table well in valley fill, diam 6 in, depth $30 \mathrm{ft}$, cased to 30 . MP top of casing, $0.92 \mathrm{ft}$ above lsd. Highest water level 3.14 below lsd, Mar. 15, 1942; lowest 15.49 below Isd, Nov. 3, 1948. Records available: 1937-60.

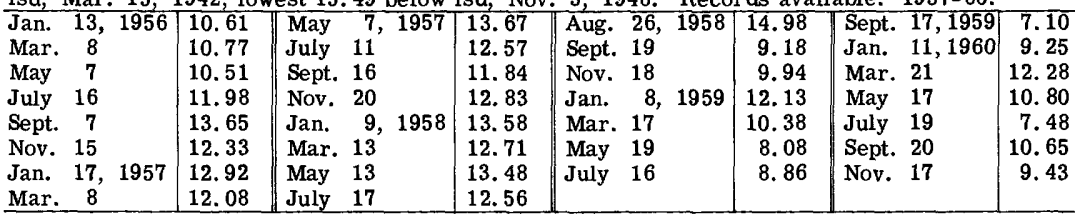

12. 25. 9. 422. Cumberland townsite. Drilled unused water-table well in valley fill, diam $10 \mathrm{in}$, reported depth $90 \mathrm{ft}$. MP top of $\frac{3}{4}$-in pipe collar, $0.62 \mathrm{ft}$ above lsd. Highest water level 38.64 below lsd, Oct. 16, 1941; lowest 73.96 below lsd, Sept. 20, 1960. Records available: 1937-60.

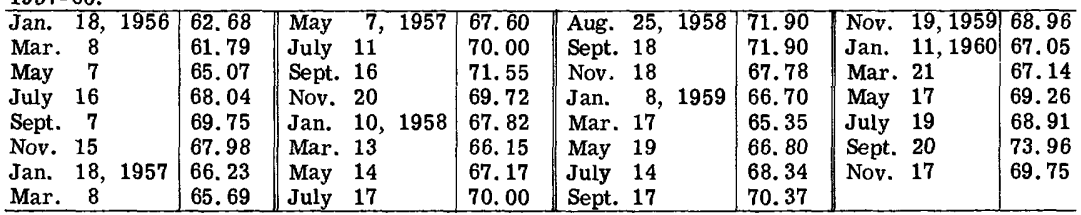

12. 25. 23.113. Formerly 12. 25. 23.110. Orchard Park. Drilled unused artesian well in San Andres Limestone of Permian age, diam 8 in, depth $810 \mathrm{ft}$, depth to artesian aquifers 600790, cased 0-645. Lsd $3,546.19 \mathrm{ft}$ above msl. MP top of casing, $0.40 \mathrm{ft}$ above lsd. Highest water level 1.74 below lsd, Jan. 15, 1942; lowest 134.79 below lsd, Aug. 19, 1959. Records available: 1925-60. For additional measurements, see Reeder and others (1960b and c).

Highest water level for the day, from recorder graph, 1956

\begin{tabular}{|c|c|c|c|c|c|c|c|c|c|c|c|c|}
\hline$\overline{\text { Day }}$ & Jan. & Feb. & Mar & Apr & May & June & July & $\mathrm{Aug}$ & Sept. & Oct. & Nov. & Dec. \\
\hline 5 & 23.35 & 18.35 & 31.84 & 93.53 & 79.68 & 99.64 & 112.00 & 109.70 & 113.95 & 77.30 & e47.53 & 33.60 \\
\hline 10 & 22,31 & 16.34 & 44.43 & 90.40 & 78.49 & 93.65 & 105.82 & 115.22 & 107.73 & 68.75 & 44.60 & 31.68 \\
\hline 15 & 20.27 & 17.84 & 63.08 & 79.68 & 70.40 & 96.79 & 103.14 & 118.68 & 114.78 & e59. 85 & 39.60 & 32.69 \\
\hline 20 & 21.40 & 23.35 & 72.30 & 77.74 & 65.30 & 95.29 & 107.61 & 107.60 & 108.53 & e57.73 & 37.33 & 31.72 \\
\hline 25 & 24.39 & 26.98 & e86.20 & 77.06 & 71.68 & 91.57 & 108.11 & 105.68 & 101.15 & 52.82 & 34.73 & 28.74 \\
\hline Eom & 31.02 & 26.07 & 96.04 & 71.37 & 87.37 & 104.58 & 11 & 11 & 84.22 & 48.10 & 35.81 & 27 \\
\hline & & & & & & 1957 & & & & & & \\
\hline 5 & 31.95 & 34.50 & 53.16 & 92.38 & 64.70 & & 115.33 & & 118.57 & 80.95 & & 2.93 \\
\hline 10 & 31.01 & 38.67 & 57.10 & 89.16 & e68.02 & & 119.65 & 126.50 & 118.53 & 65.58 & & 22.27 \\
\hline 15 & 31.55 & 45.72 & 80.71 & 74.03 & 61.29 & 99.70 & 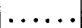 & 126.04 & & & & 20.90 \\
\hline 20 & 31.17 & 44.17 & 75.09 & 77.94 & & & 120.70 & 121.57 & 88.03 & 42.75 & & 20.55 \\
\hline 25 & 34.63 & 41.99 & 80.71 & 76.98 & e94. 40 & 99.33 & 102.77 & .40 & 95.86 & 38.98 & 24.40 & 20.52 \\
\hline Eom & 34.85 & 45.79 & 93.58 & 70.58 & 100.43 & 105.63 & 109.27 & 115 & 86.60 & 34.35 & 23.61 & 22.26 \\
\hline & & & & & & 1958 & & & & & & \\
\hline 5 & 19.67 & 18.71 & 31.87 & & 60.00 & 96.27 & $10 \mathrm{~s}$ & 40 & 84 & 39.37 & 25.40 & 22.13 \\
\hline 0 & 18.43 & 18.91 & 27.63 & $\ldots$ & 70.43 & 93.38 & 10 & 12 & 21.45 & 35.29 & 24.67 & 24.35 \\
\hline 5 & 16.75 & 26.17 & 33.11 & 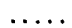 & 62.08 & 103.15 & 113.99 & 124.41 & & 31.68 & 23.22 & 22.63 \\
\hline 20 & 15.90 & 31.57 & 44.07 & & 60.37 & 108.08 & 12 & 129.44 & 62.60 & 30.19 & 23.01 & 24.28 \\
\hline 25 & 16.62 & 23.85 & 65.00 & 72.62 & 82.94 & 10 & & & 59.77 & 28.65 & 22.53 & 21.67 \\
\hline om & 16.43 & 29. & 75 & 64.32 & 92 & 109 & 123.15 & 105.65 & 44.90 & 26.22 & 21.37 & 21.59 \\
\hline & & & & & & 195 & & & & & & \\
\hline 5 & 20.42 & 33.52 & & & 93.86 & 92.63 & $\mathrm{el09.98}$ & $1 \overline{26.55}$ & 123.52 & 76.88 & $\overline{42.56}$ & 38.02 \\
\hline 10 & 21.54 & 34.52 & 58.63 & & 56.05 & & 112.93 & 125.22 & & 61.07 & e 39.90 & 39.42 \\
\hline 15 & 22.17 & 36.98 & & & 51.96 & & & 130.15 & 126.96 & 59.77 & 38.72 & 37.94 \\
\hline 20 & 22.82 & & & 74.20 & 50.22 & $\ldots$. & & 131. 46 & 116.98 & 54.77 & 37.18 & 34.87 \\
\hline 25 & 27.52 & e 41.95 & e99. 65 & 92.82 & 56.13 & 112.35 & 107.87 & 119.69 & 114.67 & 54.38 & 35.72 & 33.05 \\
\hline om & 34.25 & 45.78 & 105.32 & 99.88 & 77.38 & 113.17 & 113.84 & 117.25 & 103.32 & 48.49 & 35.67 & 35.38 \\
\hline
\end{tabular}


12. 25. 23.113--Continued.

1960

\begin{tabular}{|c|c|c|c|c|c|c|c|c|c|c|c|c|}
\hline Day & Jan & Feb & Mar & Ap & May & Jun & July & Aug & Sept. & Oct. & Nov & Dec. \\
\hline 5 & 30.33 & 23.47 & 39.89 & 102.20 & 79.60 & 87.88 & 99.70 & 116.80 & 118.68 & 82.58 & 35.32 & \\
\hline 10 & 30.90 & 23.73 & 70 & 106.51 & 74.04 & 66.64 & 53.98 & 121.21 & 121.32 & 72.90 & 34.01 & \\
\hline 15 & 26.98 & 25.42 & 67.60 & 106.77 & 77.80 & 48.82 & 59.25 & 117.57 & 118.15 & 62.64 & 31.32 & \\
\hline 20 & 24.05 & 32.20 & 77.10 & 96.35 & 92.05 & 50.18 & 54.78 & 119.60 & 107.30 & 46. 62 & 31.88 & 24.56 \\
\hline 25 & 22.98 & 30.34 & 101.54 & 86.20 & 110.56 & 77.18 & 67.55 & 115.98 & 99.05 & 40.40 & 31.34 & 23.27 \\
\hline Eom & 22. 37 & 31. & 77 & 92.49 & 98.12 & 99.90 & 103.71 & 11 & 98. 39 & 35.93 & 31.06 & 22. 37 \\
\hline
\end{tabular}

13. 26. 7. 333. Howard Amason. Drilled unused water-table well in valley fill, diam 6 in, depth $118 \mathrm{ft}$, cased 0-118. MP top of casing, $0.60 \mathrm{ft}$ above isd. Highest water level 4.45 below lsd, Oct. 1, 1941; lowest 30. 23 below Isd, Sept. 18, 19, 1958. Records available: 1941-60. For additional measurements, see Reeder and others (1960b and c).

\begin{tabular}{|c|c|c|c|c|c|c|c|}
\hline Date & $\begin{array}{l}\text { Water } \\
\text { level }\end{array}$ & Date & $\begin{array}{l}\text { Water } \\
\text { level }\end{array}$ & Date & $\begin{array}{l}\text { Water } \\
\text { level }\end{array}$ & Date & $\begin{array}{l}\text { Water } \\
\text { level }\end{array}$ \\
\hline Jan. 17,1956 & 23.49 & May 7,1957 & 26.57 & Sept. 18, 1958 & $\mathrm{k} 30.23$ & Sept. 17,1959 & 24.78 \\
\hline Mar. 8 & 24.90 & July 12 & 28.85 & 23 & 26.65 & Nov. 19 & 25.02 \\
\hline May 18 & 23.72 & Sept. 16 & 27. 90 & Nov. 19 & 25.83 & Jan. 13,1960 & 25.65 \\
\hline July 16 & 25.86 & Nov. 21 & 27.88 & Jan. 12,1959 & 24.96 & May 17 & 26.42 \\
\hline Sept. & 26.59 & Jan. 14,1958 & 28.45 & Mar. 18 & 24.85 & July 20 & 23.82 \\
\hline Nov. 15 & 26.06 & Mar. 13 & 28.43 & May 14 & 24.92 & Sept. 20 & 24.70 \\
\hline Jan. 15,1957 & 26.35 & May 14 & 27.14 & July 15 & 25.26 & Nov. 17 & 24.00 \\
\hline Mar. 8 & 26.55 & July 17 & 27.34 & & & & \\
\hline
\end{tabular}

14. 23. 8. 144. M. D. Kincaid. Drilled stock water-table well in San Andres Limestone of Permian age, diam $8 \mathrm{in}$, depth $460 \mathrm{ft}$, not cased. Lsd 3, $845 \mathrm{ft}$ above msl. MP top of casing, $1.00 \mathrm{ft}$ above 1sd. Highest water level 257.55 below 1sd, Feb. 9, 1943; lowest 299.07 below 1sd, Sept. 22, 1960. Records available: 1940-60. In intake area of artesian aquifer.

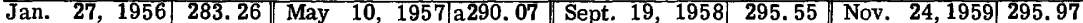
\begin{tabular}{l|l|ll|l|l|l|l|l|l|l|} 
Mar. 6 & 282. & July 22 & 292.22 & Nov. 25 & 292. 20 & Jan. 21, 1960 294.50
\end{tabular}

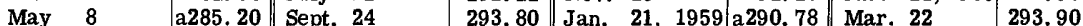
\begin{tabular}{l|l||l|l|l||l|l|l|l|} 
July 20 & 287.94 & Nov. 27 & a293.30 & Mar. 24 & a291.30 & May 25 & 296.73
\end{tabular} \begin{tabular}{l|l|l|l|l|l|l|l|l|l|} 
Sept. 10 & a289.53 & Jan. 21, 1958 290.25 & May 25 & a293.30 & July 21 & 297.04
\end{tabular} \begin{tabular}{l|l|l|l|l||l||l|l|} 
Nov. 21 & 289.41 & Mar. 25 & 289.58 & July 23 & 295.22 & Sept. 22 & 299.07
\end{tabular}

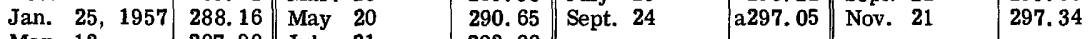
\begin{tabular}{l|l|l|l} 
Mar. 13 & 287. 98 & Jul \\
\hline a Well being pumped.
\end{tabular}

14. 26. 7. 433. Henry Johnson. Drilled unused water-table well in valley fill, diam 10 in, depth $126 \mathrm{ft}$. Lsd 3, 476.12 ft above $\mathrm{msl}$. MP top of casing, $0.40 \mathrm{ft}$ above lsd. Highest water level 88.15 below 1sd, Jan. 21, 1953; lowest 117.40 below lsd, Mar. 14, 1958 . Records available: 1953-58. Measurement discontinued. Jan. 25, 1956, 108. 95 (nearby well being pumped); Mar. 7, 104. 58 (nearby well being pumped); May 10, 112.51 (nearby well being pumped); Nov. 18, 116.17; Jan. 14, 1957, 109. 80; Mar. 8, 110.20; Mar. 14, 1958, 117. 40 (measurement uncertain).

14. 26. 12. 433b. Mr. Commins. Drilled irrigation water-table well in valley fill, diam 13 in, depth $125 \mathrm{ft}$, cased 0-125, perforated 50-115. MP top of casing, at 1sd. Highest water level 12. 50 below lsd, Jan. 22, 1942; lowest 20.10 below Isd, Sept. 10, 1951. Records available: $1940-60$.

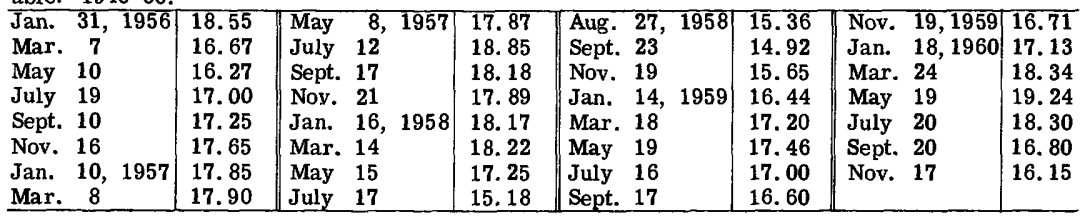

14. 26.30.111. Norris " $A$ " State Engineer and Pecos Valley Artesian Conservancy District. Drilled observation water-table well in sands and gravels in the Orchard Park Terrace of the Pecos Valley fill, diam 9 in, depth $210 \mathrm{ft}$, cased to 190, perforated 95-190. MP top of casing, $0.75 \mathrm{ft}$ above lsd. Highest water level 81.36 below lsd, July 15, 1953; lowest 101.73 below lsd, Nov. 17, 1960. Records available: 1953-60. For additional measurements, see Reeder and others (1960b and c).

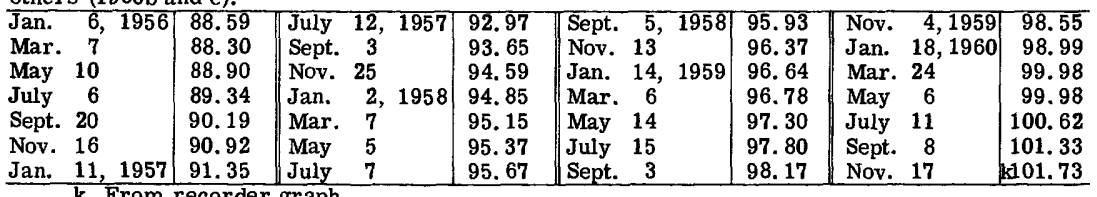

k From recorder graph. 
15.25. 35.111. M. M. Spence. Drilled domestic water-table well in valley fill, diam 6 in, depth $130 \mathrm{ft}$, cased to 130 . MP top of casing, $0.30 \mathrm{ft}$ above 1sd. Highest water level 12.48 below 1sd, Oct. 15, 1941; lowest 35.78 below 1sd, Sept. 16, 1953. Records available: 1938-60.

\begin{tabular}{|c|c|c|c|c|c|c|c|}
\hline Date & $\begin{array}{c}\text { Water } \\
\text { level }\end{array}$ & Date & $\begin{array}{l}\text { Water } \\
\text { level }\end{array}$ & Date & $\begin{array}{r}\text { Water } \\
\text { level }\end{array}$ & Date & $\begin{array}{l}\text { Water } \\
\text { level }\end{array}$ \\
\hline $\begin{array}{lrr}\text { Jan. } & 30, & 1956 \\
\text { Mar. } & 7 & \\
\text { May } & 10 & \\
\text { July } & 19 & \\
\text { Sept. } & 10 & \\
\text { Nov. } & 16 & \\
\text { Jan. } & 9, & 1957 \\
\text { Mar. } & 12 & \\
\end{array}$ & $\begin{array}{l}26.70 \\
26.89 \\
26.50 \\
18.34 \\
23.29 \\
21.98 \\
21.08 \\
20.20\end{array}$ & \begin{tabular}{lll} 
May & \multicolumn{1}{c}{$\mathbf{1 9 5 7}$} \\
July & $\mathbf{1 2}$ & \\
Sept. & $\mathbf{1 7}$ & \\
Nov. & $\mathbf{2 5}$ & \\
Jan. & 17, & 1958 \\
Mar. & 14 & \\
May & 15 & \\
July & 18 & \\
\end{tabular} & $\begin{array}{r}21.78 \\
23.43 \\
25.55 \\
\text { b26. } 42 \\
25.98 \\
25.67 \\
23.82 \\
25.35\end{array}$ & $\begin{array}{lll}\text { Aug. } & 27, & 1958 \\
\text { Sept. } 23 & \\
\text { Nov. } & 19 \\
\text { Jan. } & 19,1959 \\
\text { Mar. } & 18 & \\
\text { May } & 20 \\
\text { July } & 21 \\
\text { Sept. } & 17 & \end{array}$ & $\begin{array}{l}24.15 \\
26.06 \\
26.12 \\
27.83 \\
25.17 \\
24.50 \\
25.75 \\
26.35\end{array}$ & $\begin{array}{ll}\text { Nov. } & 20,1959 \\
\text { Jan. } & 19,1960 \\
\text { Mar. } & 24 \\
\text { May } & 19 \\
\text { July } & 20 \\
\text { Sept. } & 21 \\
\text { Nov. } & 17\end{array}$ & $\begin{array}{l}27.20 \\
27.75 \\
27.98 \\
27.50 \\
27.80 \\
25.84 \\
25.60\end{array}$ \\
\hline
\end{tabular}

b Well pumped recently.

16. 16. 3. 312. Charles Cleve. Two mi east of Elk Post Office on south side of State Highway 83. Drilled domestic water-table well in alluvium, diam $6 \mathrm{in}$, depth $85 \mathrm{ft}$, cased to 85 . MP top of casing, $1.00 \mathrm{ft}$ above 1sd. Highest water level 44.25 below isd, Nov. 2, 1954; lowest 49.98 below 1sd, Mar. 13, 1957. Records available: 1954-60.

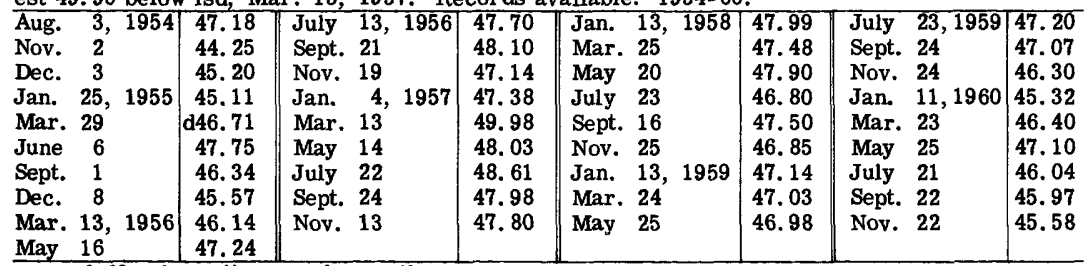

d Nearby well pumped recently.

\section{Colfax County}

\section{Capulin Basin}

29. 27. 16. 222. John King. $200 \mathrm{ft}$ north of U. S. Highway 64-87. Drilled unused watertable well in alluvium, diam 8 in, depth $120 \mathrm{ft}$. Lsd $6,821.5 \mathrm{ft}$ above msl. MP top of casing, $1.50 \mathrm{ft}$ above 1sd. Highest water level 4.65 below 1sd, Feb. 3, Aug. 24, 1960; lowest 6.24 below 1sd, Aug. 13, 1957. Records available: 1957-60.

\begin{tabular}{l|l||l|l||l|l|l||l|l}
\hline Feb. 18, 1957 & 5.69 & Feb. 19, 1958 & 5.70 & Feb. 12, 1959 & 5.05 & Feb. 3, 1960 & 4.65 \\
Aug. 13 & 6.24 & Aug. 14 & 5.38 & Aug. 20 & 5.64 & Aug. 24 & 4.65 \\
\hline
\end{tabular}

\section{Curry County}

\section{Clovis Area}

3. 34. 23. 433. Monte Matlock. Formerly Archie Baker. Drilled unused water-table well in Ogallala Formation of Pliocene age, diam 16 in, reported depth $418 \mathrm{ft}$, cased to 418 , perforated 365-418. MP top southwest side of casing, $0.90 \mathrm{ft}$ above 1sd. Highest water level 340.62 below lsd, Mar. 16, 1957; lowest 341.18 below lsd, Sept. 13, 1960. Records available: 1954-60.

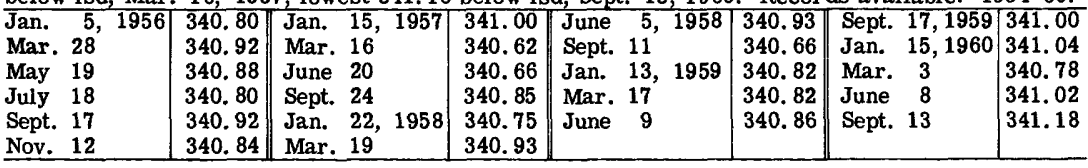

3.36. 27. 413. C. H. Moore-Oliver Co. Drilled irrigation water-table well in Ogallala Formation of Pliocene age, diam $16 \mathrm{in,} \mathrm{depth} 416 \mathrm{ft}$. MP top of casing, $1.41 \mathrm{ft}$ above $1 \mathrm{sd}$. Highest water level 286. 05 below lsd, Jan. 24, 1955; lowest 318. 33 below 1sd, Sept. 13, 1960. Records available: $1954-60$.

\begin{tabular}{|c|c|c|c|c|c|c|c|}
\hline $\begin{array}{lll}\text { Sept. } 23,1954 \\
\text { Jan. } 24,1955 \\
\text { Mar. } 23 \\
\text { May } 26 \\
\text { Juiy } & 25 \\
\text { Sept. } 16 \\
\text { Nov. } 17\end{array}$ & $\begin{array}{c}286.15 \\
286.05 \\
\text { (a) } \\
\text { (a) } \\
289.65 \\
\text { (a) } \\
299.57\end{array}$ & $\begin{array}{lll}\text { Jan. } & 12,1956 \\
\text { Mar. } & 27 \\
\text { May } & 18 \\
\text { July } & 19 \\
\text { Sept. } & 17 \\
\text { Nov. } & 12\end{array}$ & $\begin{array}{c}294.42 \\
\text { (a) } \\
\text { (a) } \\
296.72 \\
\text { (a) } \\
295.81\end{array}$ & $\begin{array}{l}\text { Jan. } 15,1957 \\
\text { Mar. } 15 \\
\text { June } 19 \\
\text { Sept. } 23 \\
\text { Jan. } 16,1958 \\
\text { Mar. 20 }\end{array}$ & $\begin{array}{l}298.64 \\
294.82 \\
298.05 \\
303.95 \\
298.05 \\
299.04\end{array}$ & $\begin{array}{l}\text { June } 5,1958 \\
\text { Sept. } 11 \\
\text { Mar. } 17,1959 \\
\text { Mar. } 3,1960 \\
\text { June } 9 \\
\text { Sept. } 13\end{array}$ & $\begin{array}{l}304.11 \\
303.46 \\
302.94 \\
303.52 \\
309.55 \\
318.33\end{array}$ \\
\hline
\end{tabular}


4.36. 10.111. O. E. Patterson (previously reported Pattison). Drilled irrigation watertable well in Ogallala Formation of Pliocene age, diam $18 \mathrm{in}$, reported depth $411 \mathrm{ft}$, cased to 411, perforated 286-411. MP top of 1-in hole in pumpbase, $2.20 \mathrm{ft}$ above lsd (since Jan. 13, 1956). Highest water level 328. 37 below lsd, July 26, 1954; lowest 345.82 below Isd, Nov. 13, 1956. Records available: $1954-60$.

\begin{tabular}{|c|c|c|c|c|c|c|c|}
\hline Date & $\begin{array}{c}\text { Water } \\
\text { level }\end{array}$ & Date & $\begin{array}{c}\text { Water } \\
\text { level }\end{array}$ & Date & $\begin{array}{c}\text { Water } \\
\text { level }\end{array}$ & Date & $\begin{array}{c}\text { Water } \\
\text { level }\end{array}$ \\
\hline Jan. 13, 1956 & 331.96 & Nov. 13,1956 & 345.82 & Jan. 23,1958 & 337.74 & Sept. 17,1959 & 337.60 \\
\hline Mar. 27 & 332.88 & Jan. 12,1957 & 336.73 & Mar. 20 & b339. 25 & Jan. 16,1960 & 339.22 \\
\hline May 18 & (a) & Mar. 16 & (a) & June & c340.90 & Mar. & 339.18 \\
\hline July 19 & (a) & June 19 & 337.61 & Jan. 15,1959 & 338.47 & June & 343.40 \\
\hline Sept. 17 & (a) & Sept. 23 & 339.33 & June & 339.03 & Sept. 13 & 341.37 \\
\hline
\end{tabular}

a Well being pumped.

b Well pumped recently.

c Nearby well being pumped.

7.37.32.131. R. M. Potts. Drilled irrigation water-table well in Ogallala Formation of Pliocene age. MP top of concrete pumpbase, $1.50 \mathrm{ft}$ above lsd. Highest water level 224.54 below 1sd, Jan. 27, 1955; lowest 235.47 below lsd, Mar. 28, 1956. Records available: 1954-60. \begin{tabular}{l||l|l|l|l||l|l|l|l|}
\hline Jan. 13, 1956 228.74 & Nov. 13, 1956 & 227.30 & Jan. 23, 1958 & 227. 19 & Oct. 22, 1959 229.80
\end{tabular} \begin{tabular}{l|l|ll|l|l|l|l|l|} 
Mar. 28 & 235.47 & Jan. 12, 1957 & 227.52 & Mar. 20 & 226.34 & Mar. 3, 1960m227.56
\end{tabular} \begin{tabular}{l|l|l|l|l|l|l|l|l|l|l|l|} 
May 18 & a251.80 & Mar. 15 & 225.87 & June 5 & 228.54 & June 8 & 232.83
\end{tabular}

\begin{tabular}{l|c||c||l|l|l|l|l|l|} 
July 19 & (a) & June 19 & (a) & Sept. 11 & 231.80 & Sept. 13 & 232.24 \\
\hline
\end{tabular}

\begin{tabular}{l|l|l|l} 
Sept. 17 & 231.50 & Sept. 23 \\
\hline
\end{tabular}

a Well being pumped.

m Measurement uncertain.

\section{Dona Ana County}

Rincon and Mesilla Valleys

22.1E. 26. 411. H. Worthheim Estate. Drilled irrigation water-table well in valley fill, diam $18 \mathrm{in.} \mathrm{MP} \mathrm{top} \mathrm{east} \mathrm{side} \mathrm{of} \mathrm{casing,} 1.50 \mathrm{ft}$ above lsd. Highest water level 14.09 below Isd, July 20, 1960; lowest 25.57 below lsd, Apr. 25, 1957. Records available: 1957-60. \begin{tabular}{|l|l|l|l|l|l|l|l|l|l|l|}
\hline Apr. 25, 1957 & 25.57 & Jan. & 8,1958 & 22.58 & June & 12,1959 & 18.26 & Jan. 8, 1960 & 17.69 \\
\hline
\end{tabular} \begin{tabular}{l|l|l|l|l|l|l|l|l|l|l|l|} 
July 24 & 25.48 & July 22 & 20.39 & July 28 & 17.92 & July 20 & 14.09 \\
\hline
\end{tabular}

23. 2E. 31.213. New Mexico State University. Drilled irrigation water-table well in valley fill, diam 14 in, reported depth $70 \mathrm{ft}$. MP top of $5 / 8$-in hole in basal flange of pump, $1.08 \mathrm{ft}$ above Isd. Highest water level 14.13 below Isd, Feb. 10, 1948; lowest 29.12 below Isd, Jan. 7, 1958. Records available: $1948,1957-60$.

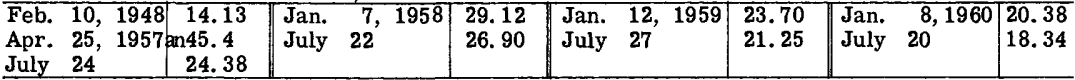

July 24

a Well being pumped.

n Measurement made with electric tape.

\section{Eddy County}

\section{Roswell Basin}

16. 23. 15.323. D. W. Runyan. Drilled stock water-table well in San Andres Limestone of Permian age, diam $10 \mathrm{in}$, depth $1,485 \mathrm{ft}$. MP top of casing, $0.70 \mathrm{ft}$ above $1 \mathrm{sd}$. Highest water level 211. 87 below Isd, Mar. 25, 1945; lowest 252.50 below Isd, July 26, 1960. Records available: $1940-60$. In intake area of artesian aquifer.

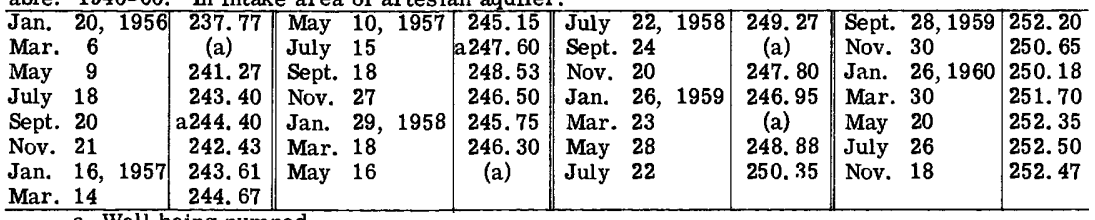

a Well being pumped.

16. 25.6. 313. Frank Childress. Drilled unused water-table well in valley fill, diam 20 in, depth $39 \mathrm{ft}$, cased to 39 . MP top of 20 -in wood cribbing, $0.40 \mathrm{ft}$ above lsd. Highest water level 27. 06 below lsd, Apr. 23, 1942; lowest 30.62 below Isd (previously reported 30.59 below lsd), Aug. $9-10,1954$. Records available: 1937-60. For additional measurements, see Reeder and others $(1960 \mathrm{~b}$ and $\mathrm{c})$. 
16. 25. 6. 313--Continued.

\begin{tabular}{|c|c|c|c|c|c|c|c|}
\hline Date & $\begin{array}{c}\text { Water } \\
\text { level }\end{array}$ & Date & $\begin{array}{c}\text { Water } \\
\text { level }\end{array}$ & Date & $\begin{array}{r}\text { Water } \\
\text { level }\end{array}$ & Date & $\begin{array}{c}\text { Water } \\
\text { level }\end{array}$ \\
\hline Jan. 24, 1956 & 28.64 & May 8, 1957 & 27.30 & Aug. 25, 1958 & 28.13 & Nov. 20,1959 & 27.44 \\
\hline May 9 & $\begin{array}{l}28.10 \\
28.49\end{array}$ & $\begin{array}{ll}\text { July } & 16 \\
\text { Sept. } & 17\end{array}$ & $\begin{array}{l}28.63 \\
28.63\end{array}$ & $\begin{array}{l}\text { sept. } 23 \\
\text { Nov. } 28\end{array}$ & $\begin{array}{l}28.00 \\
27.72\end{array}$ & Mar. 24 & $\begin{array}{l}27.47 \\
27.47\end{array}$ \\
\hline July & 28.95 & Nov. 26 & 28.10 & Jan. 20,1959 & 27.15 & May 19 & 27.50 \\
\hline Sept. 17 & 29.12 & Jan. 22,1958 & 27.84 & Mar. 19 & 27.25 & July 27 & 27.75 \\
\hline Nov. 16 & 28.93 & Mar. 19 & 27.97 & May 20 & 27.18 & Sept. 21 & 27.90 \\
\hline 9,1957 & 28.56 & May 15 & 28.00 & July 15 & 27.38 & Nov. 17 & 27.80 \\
\hline Mar. 12 & 28.56 & July & 27.96 & Sept. & 27.50 & & \\
\hline
\end{tabular}

16. 26. 19.211. H. V. Parker. Drilled irrigation water-table well in valley fill, diam

$12 \mathrm{in}$, depth $107 \mathrm{ft}$, cased to 107. MP top of casing, at 1sd. Highest water level 9. 34 below lsd, Jan. 15, 1942; lowest 90.00 below 1sd, Sept. 21, 1960. Records available: 1938-60.

\begin{tabular}{|c|c|c|c|c|c|c|c|c|c|c|c|}
\hline an. & 13,195 & 39.92 & Sept. & 7 , & 77.87 & July & 16,1957 & c94.84 & Mar. & 19,1 & 74.60 \\
\hline an. & $11, \quad 1952$ & 33.07 & Nov. & 8 & 62.55 & Sept. & 18 & c96.15 & May & 20 & 63 \\
\hline an. & 13,1953 & c75. 96 & Jan. & $30, \quad 1956$ & 50. & Nov. & 26 & 70. & July & 21 & \\
\hline an. & 1954 & 54.47 & Mar. & 7 & 52.00 & Jan. & 23,1958 & 58.28 & Sept. & 22 & \\
\hline Aay & 19 & c86. 42 & May & 9 & c79. 62 & Mar. & 17 & c68. 85 & Nov. & 20 & \\
\hline July & 15 & $\mathrm{~cm} 74$. & July 1 & 19 & c & May & 15 & 66 & Jan. & 21,1 & \\
\hline Jant & 15 & 85 & Sept. 1 & 17 & & July & 18 & (c & Mar. & 24 & \\
\hline Nov. & 16 & 66 & Nov. 1 & 16 & 68. 68 & Aug. & 25 & 79.42 & May & 19 & \\
\hline an. & 20,1955 & 58.51 & Jan. & 8, & c77. 44 & Sept. & 25 & 71. & July & 27 & 082.77 \\
\hline May & 11 & 74.20 & Mar. 1 & 12 & 65.81 & Nov. & 28 & 53.62 & Sept. & 21 & 00 \\
\hline uly & 7 & c90.91 & May & 8 & 78.00 & Jan. & $20, \quad 1959$ & 45.70 & Nov. & 17 & 23 \\
\hline
\end{tabular}

c Nearby well being pumped.

$m$ Measurement uncertain.

17. 26. 16. 333. Artesia Cemetery. Drilled unused (previously reported municipal) watertable well in valley fill, diam $6 \mathrm{in}$, depth $83 \mathrm{ft}$, cased to 83 . MP top of casing, $0.25 \mathrm{ft}$ above 1 sd. Highest water level 6.14 below lsd, Jan. 13, 1942; lowest dry at 83 below lsd, Sept. 22, 1959. Records available: $1937-60$.

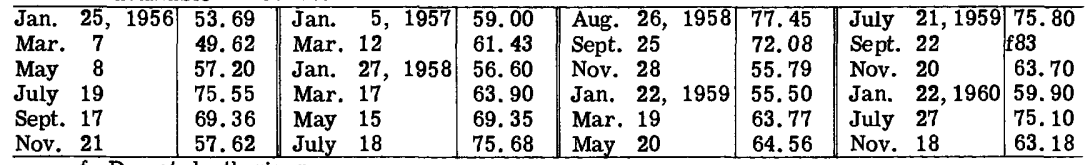

f Dry at depth given.

18. 23. 5. 333. Joe Clements. Drilled stock water-table well in San Andres Limestone of Permian age, diam 6 in, depth $500 \mathrm{ft}$, surface casing. MP top of casing, $0.40 \mathrm{ft}$ above lsd. Highest water level 385. 50 below lsd, July 21, 1945; lowest 449.04 below 1sd, Sept. 29, 1960. Records available: $1945-60$. In intake area of artesian aquifer.

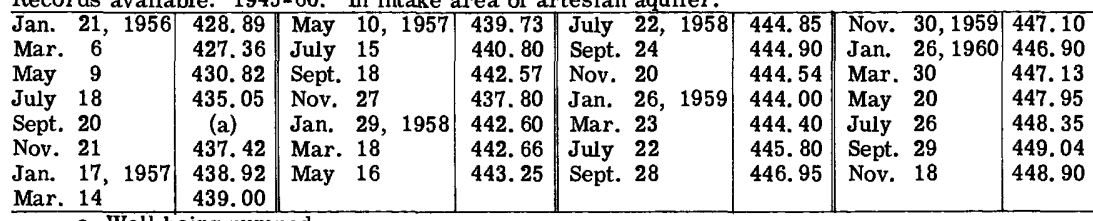

a Well being pumped.

18. 26.5. 333. Artesia. Drilled unused artesian well in San Andres Limestone of Permian age, diam 8 in, depth $1,056 \mathrm{ft}$, depth to artesian aquifers $750,820,905$, cased to 721 . Lsd $3,394.50 \mathrm{ft}$ above msl. MP top of casing, $1.94 \mathrm{ft}$ above $1 \mathrm{sd}$. Highest water level $8.30 \mathrm{below}$ Isd, Jan. 12, 1942; lowest 157. 80 below lsd, Aug. 28, 1957. Records available: 1931-60. For additional measurements, see Reeder and others (1960 b and $\mathrm{c}$ ).

Highest water level for the day, from recorder graph, 1956

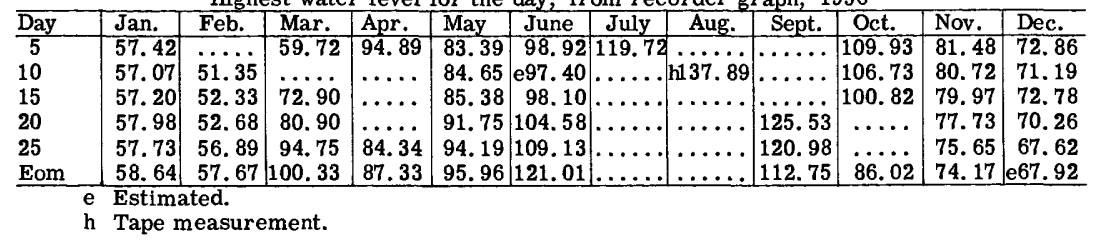


18. 26. 5. 333--Continued.

1957

\begin{tabular}{|c|c|c|c|c|c|c|c|c|c|c|c|c|}
\hline Day & Jan. & Feb. & Mar. & Apr. & May & June & July & Aug. & Sept. & Oct. & Nov. & Dec. \\
\hline 5 & 68.30 & $\overline{71.84}$ & 81.56 & 110.27 & 91.35 & 106.68 & & 146.88 & 154.56 & 129.63 & 90.10 & $7 \overline{76} .44$ \\
\hline 10 & 71.07 & 70.72 & 87 & 105.47 & 90.37 & 10 & 143. & , & 154.75 & 123.49 & 87.34 & 74.93 \\
\hline 15 & 71.32 & 73.97 & 89.00 & 98.07 & 92.47 & 115 & 144.82 & 1 & 15 & 115.00 & 84.41 & 72.70 \\
\hline 20 & 70.68 & 72.43 & 91.47 & 100.12 & 97.77 & 1 & 147.28 & 151.79 & 150.72 & 105.21 & 82.73 & 71.75 \\
\hline & 72. & 74.53 & 94.77 & 98.15 & 109.47 & & 145.88 & . & 141.38 & e98.66 & 79.55 & 70.89 \\
\hline Eom & 72.83 & 77.89 & 106. 67 & 94.66 & 109.93 & 137.40 & 14 & 155.10 & 136.32 & 93.44 & 78.06 & 71.60 \\
\hline
\end{tabular}

\begin{tabular}{l|l|l|l|l|l|l|l|l|l|l|l|l|}
\hline 5 & 68.84 & 66.40 & 67.22 & $\ldots \ldots$ & 84.88 & $\ldots \ldots$ & $\ldots \ldots$ & 139.89 & 126.48 & 88.31 & 73.65 & 65.38
\end{tabular}

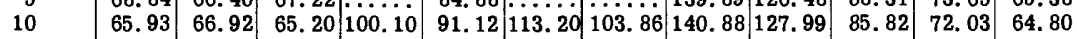
\begin{tabular}{l|l|l|l|l|l|l|l|l|l|l|l|l|l|l|l|}
15 & 66.36 & 67.50 & 69.08 & 87.77 & 95.38 & 122.96 & 119.82 & 148.37 & 115.95 & 83.05 & 70.18 & 64.43
\end{tabular} \begin{tabular}{l|l|l|l|l|l|l|l|l|l|l|l|l|l|l|l|}
20 & 65.39 & 69.19 & 81.04 & 80.40 & 98.47 & 129.81 & 133.54 & 148.15 & 110.40 & 80.47 & 69.46 & 65.73
\end{tabular} \begin{tabular}{l|l|l|l|l|l|l|l|l|l|l|l|l|l|l|}
25 & 65.96 & 65.02 & $\ldots$. & 84.73 & $\ldots \ldots$ & 113.80 & 142.23 & 126.22 & 107.87 & 79.17 & 67.83 & 64.67
\end{tabular} \begin{tabular}{l|c|c|c|c|c|c|c|c|c|c|c|c|c|} 
Eom & 65.82 & 66.17 & 100.17 & 84.75 & $\ldots \ldots$ & 106.47 & 142.48 & 122.73 & 94.32 & 75.57 & 66.40 & 63.91 \\
\hline
\end{tabular} \begin{tabular}{l|l|l|l|l|l|l|l|l|l|l|l|l|}
\hline 5 & 62.67 & 63.53 & $\ldots$. & 112.95 & 98.61 & 94.84 & 129.68 & 144.35 & 151.62 & 119.60 & 97.00 & 85.68 \\
\hline
\end{tabular} \begin{tabular}{l|l|l|l|l|l|l|l|l|l|l|l|l|l|l|l|}
10 & 62.57 & 64.45 & 83.07 & 106.86 & 90.08 & 98.09 & $\ldots$ & 148.05 & 151.03 & 113.35 & 92.18 & 84.03
\end{tabular} \begin{tabular}{l|l|l|l|l|l|l|l|l|l|l|l|l|l|l|}
15 & 63.32 & 66.79 & 92.52 & 99.76 & 87.15 & 104.42 & 123.07 & 148.95 & 148.70 & 106.66 & 89.53 & 81.25
\end{tabular} \begin{tabular}{l|l|l|l|l|l|l|l|l|l|l|l|l|l|l|l|l|l|l|l|}
20 & 62.66 & 68.68 & 103.30 & 94.45 & 82.09 & 110.72 & 117.15 & $\ldots \ldots$ & 145.74 & 103.31 & 88.22 & 81.03
\end{tabular} \begin{tabular}{l|l|l|l|l|l|l|l|l|l|l|l|l|l|l}
25 & 63.23 & 67.69 & 111.20 & 99.47 & 86.67 & 115.27 & 129.39 & 146.58 & $\ldots \ldots$ & 100.73 & 86.72 & 79.23 \\
\hline
\end{tabular} \begin{tabular}{l|l|l|l|l|l|l|l|l|l|l|l|l|l|} 
Eom & 66.62 & 73.45 & 114.46 & 101.53 & 94.34 & 124.72 & $\mathrm{e} 39.98$ & 143.77 & 127.65 & 98.60 & 85.18 & 78.73 \\
\hline
\end{tabular} 1960

\begin{tabular}{l|l|l|l|l|l|l|l|l|l|l|l|l|}
\hline 5 & 80.08 & 76.17 & 75.28 & 123.52 & 103.45 & $\ldots \ldots$ & 136.83 & 148.93 & 144.53 & 117.81 & 91.45 & 82.10 \\
\hline
\end{tabular}
\begin{tabular}{r|r|r|r|r|r|r|r|r|r|r|r|r|}
\hline 5 & 80.08 & 76.17 & 75.28 & 123.52 & 103.45 & $\ldots$ & 136.83 & 148.93 & 144.53 & 117.81 & 91.45 & 82.10 \\
10 & 79.02 & 74.84 & 81.20 & 120.90 & 102.52 & 119.09 & 110.92 & 151.28 & 144.47 & 112.23 & 88.72 & 79.14 \\
\hline
\end{tabular}

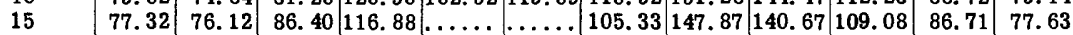
\begin{tabular}{l|l|l|l|l|l|l|l|l|l|l|l|l|l|l|l|l|l}
20 & 77.16 & 76.53 & 97.15 & 114.64 & $\ldots \ldots$ & $\ldots \ldots$ & 101.21 & 140.40 & 138.38 & 101.80 & 85.38 & 76.43
\end{tabular}

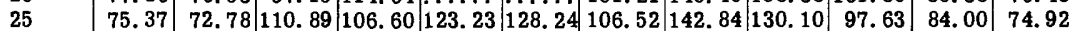
\begin{tabular}{l|l|l|l|l|l|l|l|l|l|l|l|l|l|l|} 
Eom & 75.42 & 73.40 & 121.04 & 105.93 & 127.66 & 140.47 & 136.12 & 143.25 & 124.40 & 93.73 & 83.02 & 73.30 \\
\hline
\end{tabular} e Estimated.

h Tape measurement.

18. 26. 7. 234a. C. H. Hutsonpiller. Drilled unused water-table well in valley fill, diam $8 \mathrm{in}$, depth $159 \mathrm{ft}$, cased to 159 . MP top of casing, $0.80 \mathrm{ft}$ above lsd. Highest water level 43.50 below Isd, Feb. 9, 1943; lowest 85.00 below 1sd, Mar. 29, 1960. Records available: 1937-60. For additional measurements, see Reeder and others (1960b) (1960c).

\begin{tabular}{|c|c|c|c|c|c|c|c|}
\hline Date & $\begin{array}{c}\text { Water } \\
\text { level }\end{array}$ & Date & $\begin{array}{c}\text { Water } \\
\text { level }\end{array}$ & Date & $\begin{array}{c}\text { Water } \\
\text { level }\end{array}$ & Date & $\begin{array}{c}\text { Water } \\
\text { level }\end{array}$ \\
\hline 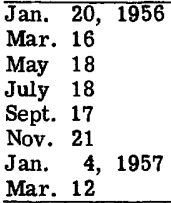 & $\begin{array}{l}77.88 \\
77.70 \\
78.82 \\
79.55 \\
80.20 \\
80.60 \\
80.69 \\
81.05\end{array}$ & $\begin{array}{lll}\text { May } & 8, & 1957 \\
\text { July } & 15 & \\
\text { Sept. } & 18 & \\
\text { Nov. } & 26 & \\
\text { Jan. } & 28, & 1958 \\
\text { Mar. } & 17 & \\
\text { May } & 15 & \\
\text { July } & 18 & \end{array}$ & $\begin{array}{l}81.40 \\
81.80 \\
82.17 \\
82.45 \\
82.67 \\
82.74 \\
82.92 \\
82.68\end{array}$ & $\begin{array}{lll}\text { Sept. } & 25, & 1958 \\
\text { Nov. } & 28 & \\
\text { Jan. } & 23, & 1959 \\
\text { Mar. } & 19 & \\
\text { May } & 20 & \\
\text { July } & 15 & \\
\text { Sept. } & 28 & \end{array}$ & $\begin{array}{l}83.25 \\
83.39 \\
83.33 \\
83.48 \\
83.83 \\
84.09 \\
84.52\end{array}$ & $\begin{array}{ll}\text { Nov. } & 20,1959 \\
\text { Jan. } & 25,1960 \\
\text { Mar. } & 29 \\
\text { May } & 20 \\
\text { July } & 27 \\
\text { Sept. } & 29 \\
\text { Nov. } & 18\end{array}$ & $\begin{array}{l}84.68 \\
84.85 \\
85.00 \\
84.18 \\
83.86 \\
83.57 \\
83.70\end{array}$ \\
\hline
\end{tabular}

18. 26. 28.121a. Town of Dayton. Drilled observation water-table well in valley fill, diam $8 \mathrm{in}$, depth $250 \mathrm{ft}$, cased to 182 , casing slotted 92-182. MP top of casing, $0.06 \mathrm{ft}$ above lsd. Highest water level 59. 79 below 1sd, Feb. 5, 1952; lowest 96. 98 below 1sd, Dec. 30-31, 1960. Records available: 1951-60. For additional measurements, see Reeder and others (1960b and c).

Highest water level for the day, from recorder graph, 1956

\begin{tabular}{|c|c|c|c|c|c|c|c|c|c|c|c|c|}
\hline Day & Jan. & Feb. & Mar & $\mathrm{Apr}$ & May & June & July & Aug & Sept. & Oct. & Nov. & Dec. \\
\hline 5 & 81.03 & 80.73 & 80.47 & 80.50 & 81.01 & $81 . \overline{36}$ & 81.73 & 82.29 & 82.97 & 83.57 & 84.14 & 84.52 \\
\hline 10 & 80.96 & 80.67 & 80.44 & 80.57 & 81.07 & 81.42 & 81.78 & 82.39 & 83. 07 & 83. 67 & 84.20 & 84.53 \\
\hline 15 & 80.93 & 80.56 & 80.45 & 80.67 & 81.17 & 81.45 & 81.87 & 82.50 & 83.18 & 83.77 & 84. 31 & 84.60 \\
\hline 20 & ..... & 80.52 & 80.39 & 80.79 & 81.22 & 81.50 & 81.97 & 82.61 & 83.26 & 83. 85 & 84.35 & 84 \\
\hline 25 & & 80.45 & 80.40 & 80.87 & 81.28 & 81.57 & 82.04 & & 83.39 & 83.96 & 84. 39 & 84.64 \\
\hline Eom & 80.76 & 80 & 80. & 80. & 81.34 & 81.63 & 82.18 & 82.86 & 83.48 & 84.04 & 84.47 & 84.64 \\
\hline \multicolumn{13}{|c|}{1957} \\
\hline 5 & 84.63 & $84 . \overline{64}$ & 84.61 & 85.00 & 85.40 & 85.84 & 86.27 & 86.82 & 87.36 & 87.90 & 88.41 & 88.75 \\
\hline 10 & 84.65 & 84.63 & 84 & 85 & 85.46 & 85 & 34 & 86.89 & 87.46 & 88.01 & 88.50 & 88.79 \\
\hline 15 & 84.67 & 84.57 & 84.77 & 85.08 & 85.51 & 85.98 & 86.42 & 86.99 & 87.56 & 88.07 & 88.52 & 88. 87 \\
\hline 20 & & 84.60 & 84.81 & 85.18 & 85.57 & 86.03 & 86.53 & 87.07 & 87.62 & 88.17 & 88.62 & 88.90 \\
\hline 25 & 84.66 & 84.59 & 84. 87 & 85.25 & & 86.11 & 86.61 & 87.15 & 87.71 & 88.23 & & $\cdots$ \\
\hline Eom & & & 84.92 & 85.32 & 75 & 86.20 & 86.72 & 87.28 & 87.81 & 88.32 & 88.79 & \\
\hline
\end{tabular}


18. 26. 28.121a---Continued.

1958

\begin{tabular}{|c|c|c|c|c|c|c|c|c|c|c|c|c|}
\hline Day & Jan. & Feb. & Mar. & Apr. & May & June & July & Aug. & Sept. & Oct. & Nov. & Dec. \\
\hline 5 & 10 & 12 & 89.19 & 89.25 & 89.51 & 89.75 & & 90.36 & 90.75 & 91.11 & 91.43 & 91. \\
\hline 10 & 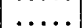 & 89.14 & 89.18 & 89.30 & 89.56 & 89.79 & 90.10 & 90.42 & 90.82 & 91.21 & 91.49 & 91 \\
\hline 15 & 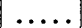 & 89.16 & 89.16 & 89.32 & 89.60 & 89.84 & 90.13 & 90.49 & 90.88 & 91.25 & 50 & 91 \\
\hline 20 & e89.08 & 89 & 89 & 89.37 & 89.64 & 89. & 90.19 & 5 & 94 & 28 & & . \\
\hline 25 & 89.10 & 89.12 & 89.18 & 89.41 & 89.64 & 89.91 & & & 91.01 & 91.37 & 91 & 91 \\
\hline Eom & 89.11 & 89 & 89.20 & 89.43 & 89.6 & & 90.32 & 68 & 91.03 & 91.41 & 91.64 & 91.7 \\
\hline
\end{tabular}

\begin{tabular}{l|l|l|l|l|l|l|l|l|l|l|l|l}
\hline 5 & 91.73 & 91.69 & 91.70 & $\ldots$ & 91.62 & 91.66 & 91.94 & 92.19 & 92.53 & 92.85 & 93.23 & 93.60 \\
\hline
\end{tabular}
\begin{tabular}{l|l|l|l|l|l|l|l|l|l|l|l|l}
5 & 91.71 & 91.71 & 91.73 & $\ldots \ldots$ & 91.60 & h91.72 & 91.96 & 92.25 & 92.59 & 92.90 & 93.31 & 93.65
\end{tabular}

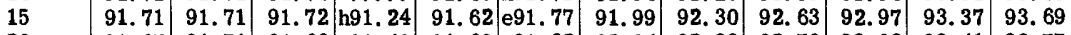
\begin{tabular}{l|l|l|l|l|l|l|l|l|l|l|l|l|l}
20 & 91.67 & 91.71 & 91.68 & 91.49 & 91.63 & $\mathrm{e} 91.82$ & 92.04 & 92.33 & 92.70 & 93.03 & 93.41 & e93. 77
\end{tabular} \begin{tabular}{l|l|l|l|l|l|l|l|l|l|l|l|l|l|}
25 & 91.71 & 91.69 & 91.72 & 91.53 & 91.65 & 91.84 & 92.09 & 92.39 & 92.76 & 93.09 & 93.48 & 93.79
\end{tabular}

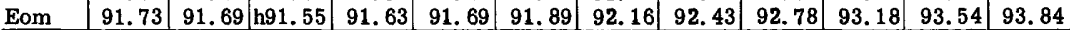

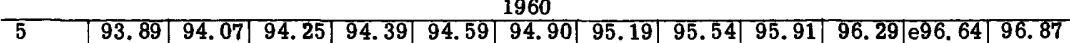
\begin{tabular}{l|r|r|r|r|r|r|r|r|r|r|r|r|}
\hline 10 & 93.93 & 94.11 & 94.27 & 94.43 & 94.65 & 94.97 & 95.26 & 95.59 & 95.95 & e96.34 & e96. 69 & 96.89 \\
15 & 93.95 & 94.15 & 94.28 & 94.46 & 94.69 & 95.01 & 95.31 & 95.65 & 96.01 & e96.41 & e96. 74 & 96.91 \\
20 & 93.99 & 94.17 & 94.32 & 94.50 & 94.74 & 95.06 & 95.35 & 95.71 & 96.08 & e96.47 & e96. 80 & 96.94 \\
25 & 94.03 & 94.21 & 94.34 & 94.51 & 94.79 & 95.11 & 95.39 & 95.77 & 96.15 & e96.53 & 96.83 & 96.97 \\
Eom & 94.05 & 94.23 & 94.37 & 94.56 & 94.85 & 95.15 & 95.49 & 95.85 & 96.22 & e96.59 & 96.85 & 96.98 \\
\hline
\end{tabular} e Estimated.

h Tape measurement.

19. 23. 27.111. C. R. Coffin. Drilled stock water-table well in San Andres Limestone of Permian age, diam 7 in (previously reported 6 in), depth $416 \mathrm{ft}$, surface casing. Lsd 3,940 ft above msl. MP top of casing, $0.40 \mathrm{ft}$ above lsd. Highest water level 368.75 below 1sd, Oct. 19, 1943; lowest 391.07 below Isd, May 10, 1957. Records available: 1940-60. In intake area of artesian aquifer.

\begin{tabular}{|c|c|c|c|c|c|c|c|}
\hline Date & $\begin{array}{l}\text { Water } \\
\text { level }\end{array}$ & Date & $\begin{array}{l}\text { Water } \\
\text { level }\end{array}$ & Date & $\begin{array}{l}\text { Water } \\
\text { level }\end{array}$ & Date & $\begin{array}{c}\text { Water } \\
\text { level }\end{array}$ \\
\hline $\begin{array}{lll}\text { Mar. } & 6,1956 \\
\text { July } & 18 & \\
\text { Sept. } 20 & \\
\text { Jan. } 17, & 1957 \\
\text { Mar. } 14 & \\
\text { May } 10 & \end{array}$ & $\begin{array}{r}367.27 \\
386.17 \\
381.83 \\
384.88 \\
389.77 \\
391.07\end{array}$ & $\begin{array}{lll}\text { July } & 15,1957 \\
\text { Sept. } & 18 \\
\text { Nov. } & 27 \\
\text { Jan. } & 29,1958 \\
\text { May } & 16 \\
\text { July } & 22\end{array}$ & $\begin{array}{r}387.93 \\
\text { a393. } \\
385.09 \\
386.60 \\
\mathrm{a} 391.40 \\
387.60\end{array}$ & $\begin{array}{l}\text { Sept. } 24,1958 \\
\text { Jan. 26, } 1959 \\
\text { Mar. 23 } \\
\text { May 28 } \\
\text { July } 22 \\
\text { Sept. 28 }\end{array}$ & $\begin{array}{r}384.65 \\
382.56 \\
a 384.30 \\
\mathrm{a} 388.20 \\
384.85 \\
384.80\end{array}$ & $\begin{array}{ll}\text { Nov. } & 30,1959 \\
\text { Jan. } & 26,1960 \\
\text { Mar. } & 30 \\
\text { May } & 20 \\
\text { July } & 26\end{array}$ & $\begin{array}{l}385.30 \\
385.15 \\
387.50 \\
386.60 \\
387.00\end{array}$ \\
\hline
\end{tabular}

\section{a Well being pumped.}

m Measurement uncertain.

19. 26. 27. 233. E. C. McGonagill. Drilled domestic water-table well in valley fill, diam $8 \mathrm{in}$, depth $127 \mathrm{ft}$, cased to 127 . MP top of casing, $1.58 \mathrm{ft}$ above lsd. Highest water level 37.63 below 1sd, May 11, 1942; lowest 84.02 below 1sd, Sept. 28, 1959. Records avallable: 1937-39, 1941-60.

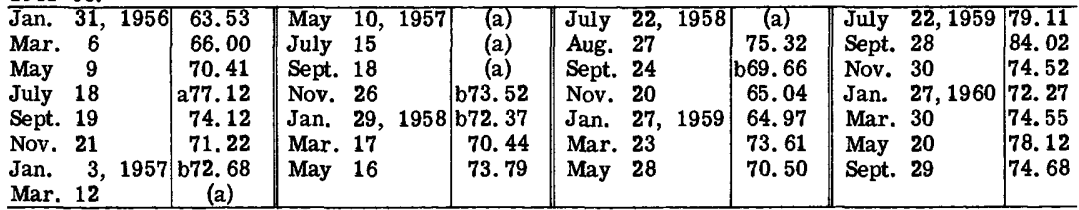

a Well being pumped.

b Well pumped recently.

\section{Carlsbad Area}

21. 27. 32.112. L. E. Loman. Drilled domestic and irrigation artesian well in Artesia Group of Permian age, diam 6 in, reported depth $305 \mathrm{ft}$. Lsd $3,112 \mathrm{ft}$ above $\mathrm{msl}$. MP top of casing, $0.40 \mathrm{ft}$ above 1sd. Highest water level 4.64 below Isd, Jan. 17, 1950; lowest 14. 24 below lsd, Nov. 22, 1960. Records available: 1947-60.

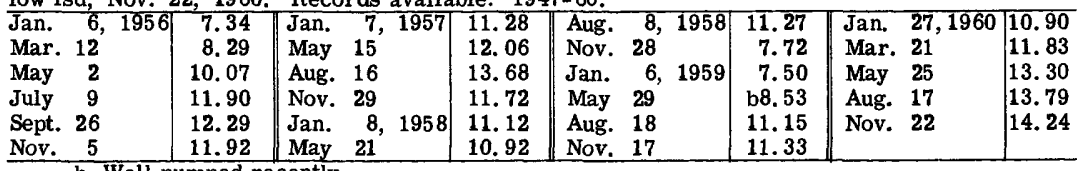


21. 27. 32.112a. S. Tracy. Drilled irrigation water-table well in alluvium, diam 15 in, reported depth $105 \mathrm{ft}$. Lsd $3,112 \mathrm{ft}$ above msl. MP top of casing, $1.00 \mathrm{ft}$ above lsd. Highest water level 9.13 below lsd, Nov. 22, 1960; lowest 16. 26 below lsd, Aug. 16, 1957. Records available: $1950-60$.

\begin{tabular}{|c|c|c|c|c|c|c|c|}
\hline Date & $\begin{array}{l}\text { Water } \\
\text { level }\end{array}$ & Date & $\begin{array}{l}\text { Water } \\
\text { level }\end{array}$ & Date & $\begin{array}{l}\text { Water } \\
\text { level }\end{array}$ & Date & $\begin{array}{c}\text { Water } \\
\text { level }\end{array}$ \\
\hline $6, \overline{1956}$ & 14.26 & 5,1957 & 14.89 & 8,1958 & 13.05 & Jan. $\quad 27,1960$ & 14.26 \\
\hline Mar. 12 & 14.17 & May 21 & 14.93 & Nov. 28 & 13.04 & Mar. 21 & 14.47 \\
\hline May & 13.90 & Aug. 16 & 16. 26 & 6,1959 & 13.40 & May 25 & 14.10 \\
\hline July & 14.12 & Nov. 29 & 14.40 & May 29 & 12.84 & Aug. 17 & 10.83 \\
\hline Sept. 26 & 14.22 & 8,1958 & 14.58 & Aug. 18 & 12.57 & Nov. 22 & 9.13 \\
\hline Nov. 5 & 14.60 & May 21 & 13.64 & Nov. 17 & 13.99 & & \\
\hline
\end{tabular}

22. 26. 36.111a. Carlsbad Airfield 2. Drilled unused water-table well in alluvium, diam $12 \mathrm{in}$, depth $260 \mathrm{ft}$. MP top of recorder platform, 2. $70 \mathrm{ft}$ above lsd. Highest water level 131.50 below 1sd, Oct. 14, 1942; lowest 205.67 below 1sd, Aug. 20, 1954 (previously reported 204.66 below lsd, Aug. 21, 1954). Records available: 1942-60. For additional measurements, see Reeder and others $(1960 \mathrm{~b}$ and $\mathrm{c})$.

Highest water level for the day, from recorder graph, 1956

\begin{tabular}{l|l|l|l|l|l|l|l|l|l|l|l|l}
\hline Day & Jan. & Feb. & Mar. & Apr. & May & June & July & Aug. & Sept. & Oct. & Nov. & Dec. \\
\hline 5 & 173.53 & 172.20 & 171.57 & 175.13 & 181.67 & 183.04 & 186.46 & 191.58 & 193.50 & 189.51 & 184.63 & 179.73 \\
10 & 173.45 & 171.76 & 171.44 & 176.80 & 182.01 & 182.70 & 187.38 & 192.25 & 193.28 & 188.75 & 183.53 & 178.67 \\
15 & 172.75 & 171.04 & 171.50 & 178.45 & 182.40 & 182.48 & 188.45 & 193.03 & 193.25 & 188.47 & $\ldots \ldots$ & 178.27 \\
20 & 172.46 & 171.14 & 171.79 & 179.98 & 182.65 & 183.40 & 189.75 & 193.54 & 192.48 & 187.66 & $\ldots \ldots$ & 177.92 \\
25 & 172.22 & 171.55 & 172.50 & 181.00 & 183.25 & 184.12 & 190.15 & 194.08 & 191.54 & 186.71 & 181.55 & 177.52 \\
Eom & 172.05 & 171.76 & 173.93 & 181.43 & 183.33 & 185.45 & 191.37 & 193.68 & 190.27 & 185.15 & 180.62 & 176.57 \\
\hline
\end{tabular}
\begin{tabular}{l|l|l|l|l|l|l|l|l|l|} 
Eom & 172.05 & 171.76 & 173.93 & 181.43 & 183.33 & 18 \\
\hline
\end{tabular}

\begin{tabular}{|c|c|c|}
\hline & & \\
\hline 5 & \begin{tabular}{|l|l|l|l|}
175.90 & 176.71 & 176.13 & 180.30 \\
\end{tabular} & \begin{tabular}{|l|l|l|l|l|l|}
190.77 & 195.95 & 194.68 & 195.57 & 189.57 & 183.24 \\
\end{tabular} \\
\hline 10 & \begin{tabular}{|l|l|l|l|l|}
175.58 & 176.63 & $\ldots \ldots$ & 181.59 & 186
\end{tabular} & 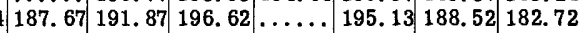 \\
\hline 15 & \begin{tabular}{|l|l|l|l|l|}
175.63 & 176.67 & 177.07 & 183.17 & 186.33 \\
\end{tabular} & 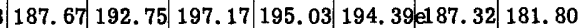 \\
\hline 20 & \begin{tabular}{|l|l|l|l|l}
175.67 & 176.67 & 177.77 & 184.60 & 186.76
\end{tabular} & 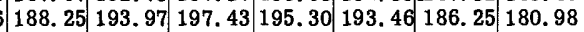 \\
\hline 25 & & \begin{tabular}{|l|l|l|l|l|l|l|l}
188.99 & 194.56 & 195.65 & 195.55 & 192.28 & 185.28 & 180.38 \\
\end{tabular} \\
\hline om & & \begin{tabular}{l|l|l}
.479 .61 & 179.61 \\
\end{tabular} \\
\hline & & 1958 \\
\hline 5 & \begin{tabular}{|l|l|}
9.02 & 175.46
\end{tabular} & \begin{tabular}{ll|l|l|l|}
4.65 & 189.72 & 180.95 & 175.36
\end{tabular} \\
\hline 10 & 3.78 & 58180.13 hi 74.60 169.61 \\
\hline 15 & 17 & 97179.23 \\
\hline 20 & 177.03 & \begin{tabular}{l|l|l|l|l}
22 & 178.15 & 172.87 & 168.39
\end{tabular} \\
\hline 25 & .46 & 86.47177 .43 \\
\hline on & .00 & \begin{tabular}{l|l|l|l|l|}
0 & 182.07 & 176.27 \\
\end{tabular} \\
\hline & & 1959 \\
\hline $\mathbf{5}$ & 8 & 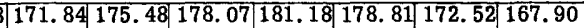 \\
\hline 10 & 301 & .431 \\
\hline 15 & 17 & $90176.52170 .51 \quad 167.08$ \\
\hline 20 & 172 & \begin{tabular}{l|l|l|l|l}
31.37 & 175.33 & 169.74 & 166.75
\end{tabular} \\
\hline 25 & $3 \mid 16$ & \begin{tabular}{l|l|l|l|l}
.09 & 180.67 & 174.26 & 168.73 & 165.84
\end{tabular} \\
\hline on & 67.72173 .8 & 87179.80173 .30 . \\
\hline & & 190 \\
\hline 5 & 164.70 & \begin{tabular}{l|l|l}
76.93 & $169.55 h 162.84$
\end{tabular} \\
\hline 10 & 16 & 178.111 \\
\hline 15 & \begin{tabular}{l|l|l}
11 & 172.66 & 176.01
\end{tabular} & 177.6317 \\
\hline 20 & \begin{tabular}{l|l|l}
58 & 175.13 & 176.79
\end{tabular} & \begin{tabular}{l|l|l}
.67 & 160.84
\end{tabular} \\
\hline 25 & 70175.43177 & \begin{tabular}{|l|l|l|l|l|l|l|l|l|}
177.62 & 174.69 & 179.31 & 178.27 & $\mathrm{e} 171.93$ & 164.58 & 160.04 \\
\end{tabular} \\
\hline & & \\
\hline
\end{tabular}

h Tape measurement.

22. 27. 22. 421. Enea Grandi. Drilled irrigation water-table well in alluvium, diam 16 in, reported depth $150 \mathrm{ft}$. Lsd 3,100 ft above msl. MP top of casing, $1.20 \mathrm{ft}$ above lsd (since Mar. 12, 1956). Highest water level 21.43 below lsd, Sept. 15, 1950; lowest 64.60 below lsd, Aug. 31, 1954. Records available: 1947-60.

\begin{tabular}{|c|c|c|c|c|c|c|c|}
\hline Date & $\begin{array}{c}\text { Water } \\
\text { level }\end{array}$ & Date & $\begin{array}{c}\text { Water } \\
\text { level }\end{array}$ & Date & $\begin{array}{c}\text { Water } \\
\text { level }\end{array}$ & Date & $\begin{array}{l}\text { Water } \\
\text { level }\end{array}$ \\
\hline 7,1956 & 37. & 5,1957 & 40.02 & 8,1958 & 41.41 & Nov. 17,1959 & 33 \\
\hline 12 & 38.26 & May 15 & 49.40 & Nov, 28 & 37.56 & Jan. & 35. \\
\hline Iay & 38.30 & Aug. & 14 & 8,1959 & 38.00 & Mar. 21 & 37.72 \\
\hline uly & 39.82 & Nov. 29 & 47 & May 29 & 35.06 & May 25 & 2 \\
\hline Sept. 26 & 41.40 & Jan. $\quad 8,1958$ & 45 & Aug. 18 & (a) & Aug. 17 & 78 \\
\hline Nov. & 39.47 & May 21 & 44.20 & 28 & 36.79 & Nov. 22 & 30.82 \\
\hline
\end{tabular}

a Well being pumped. 
23. 27. 2. 122. Jim Derrick. Drilled irrigation water-table well in alluvium, diam 18 in, depth $186 \mathrm{ft}$. MP top of air-line flange support, $2.10 \mathrm{ft}$ above isd. Highest water level 26. 40 below 1sd, Oct. 25, 1946; lowest 68.10 below 1sd, July 7, 1954. Records available: 1946-60.

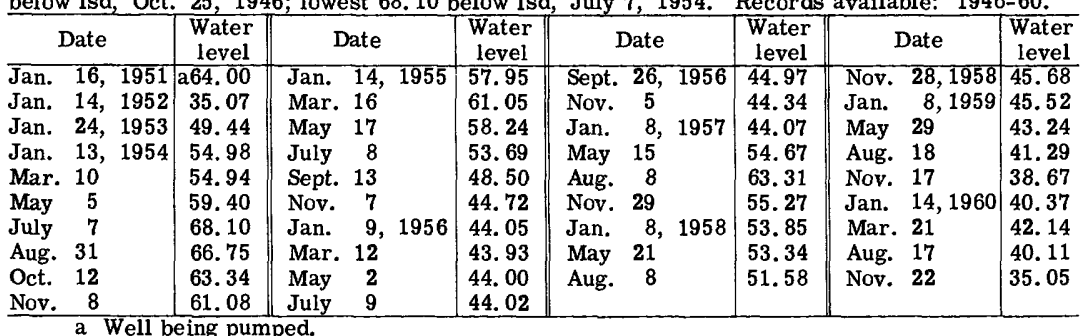

$$
\text { a Well being pumped. }
$$

23. 28. 23.133. A. R. Donaldson. Drilled irrigation water-table well in alluvium, diam $16 \mathrm{in}$, depth $148 \mathrm{ft}$. Lsd 3, $020 \mathrm{ft}$ above msl. MP bottom edge of north $\frac{1}{2}$-in hole in west side of pumpbase, $0.80 \mathrm{ft}$ above lsd. Highest water level 38.25 below lsd, Sept. 14, 1950; lowest 70.59 below Isd, Aug. 31, 1954. Records available: 1947-60.

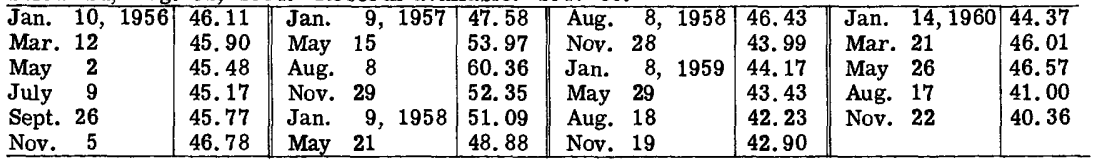

25. 24. 26.121. State Department of Game and Fish. Dug unused artesian well in alluvial conglomerate, diam 24 in, depth $15 \mathrm{ft}$. Lsd 3,636.39 ft above msl. MP top of casing, $3.00 \mathrm{ft}$ above lsd. Highest water level 0.76 above lsd, Feb. 19, 1953; lowest 3.62 below 1sd, Sept. 11, 1960. Records available: 1953-60. For additional measurements, see Reeder and others $(1960 \mathrm{~b}$ and $\mathrm{c})$.

Highest water level for the day, from recorder graph, 1956

\begin{tabular}{|c|c|c|c|c|c|c|c|c|c|c|c|c|}
\hline Day & Jan. & Feb. & Mar. & Apr. & May & June & July & Aug. & Sept. & Oct. & Nov. & Dec. \\
\hline 5 & +0.44 & +0.47 & +0.45 & & -0.44 & -0.05 & -1.85 & $\ldots$ & $\ldots$ & $\cdots$ & $\cdots$ & $\cdots$ \\
\hline 10 & .40 & .47 & +.45 & -0.35 & .18 & .01 & 1. 49 & $\ldots$ & $\ldots$ & $\ldots$ & $\ldots$ & $\ldots$ \\
\hline 15 & .50 & .48 & $\ldots$ & .74 & 1.08 & .93 & 1.71 & $\ldots$ & $\ldots$ & $\ldots$ & $\ldots$ & $\ldots$ \\
\hline 20 & .48 & .48 & $\ldots$ & .67 & 1. 19 & .96 & 1.75 & ... & $\ldots$ & $\cdots$ & $\ldots$ & $\ldots$ \\
\hline 25 & .42 & .46 & ... & .63 & .68 & 1. 51 & 1.84 & $\ldots$ & $\ldots$ & $\ldots$ & $\cdots$ & $\cdots$ \\
\hline Eom & .46 & .49 & ... & 1.05 & .22 & 1.33 & 1.29 & 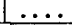 & $\ldots$ & $\ldots$ & .. & $\cdots$ \\
\hline \multicolumn{13}{|c|}{1957} \\
\hline 5 & $\bullet$ & $\cdots$ & $\cdots$ & $\cdots$ & $\cdots$ & -1.24 & -2.50 & $T-2.18$ & -2.25 & -1.82 & $\cdots$ & -0.18 \\
\hline 10 & $\ldots$ & $\cdots$ & $\cdots$ & $\ldots$ & $\ldots$ & e1.97 & 2. 73 & 2. 52 & 1.65 & 1.76 & $\ldots$ & .25 \\
\hline 15 & $\cdots$ & $\cdots$ & $\cdots$ & $\cdots$ & $\ldots$ & 2. 25 & 2. 26 & 2. 36 & 1. 81 & 1. 30 & $\ldots$ & .13 \\
\hline 20 & $\cdots$ & $\cdots$ & $\ldots$ & $\ldots$ & $\ldots$ & 1.96 & 2.03 & 1.70 & 2. 28 & .94 & $\ldots$. & . 26 \\
\hline 25 & & $\because$ & & $\ldots$ & -2.72 & 2.01 & 1.84 & 1.66 & 1.82 & $\cdots$ & & . 22 \\
\hline Eom & & & & & 1.26 & 1.81 & 2. 23 & 2.02 & 2.32 & & -0.27 & e. 17 \\
\hline \multicolumn{13}{|c|}{1958} \\
\hline$\overline{5}$ & -0.22 & & -0.26 & -1.35 & $\ldots$ & $T-2.40$ & $\cdots$ & -1.28 & $\ldots$ & .. & $\cdots$ & $\cdots$ \\
\hline 10 & .17 & -0.08 & .26 & 1. 56 & $\cdot$ & $\ldots$ & $\ldots$ & $\ldots$ & $\cdots$ & $\cdots$ & $\ldots$ & $\cdots$ \\
\hline 15 & .19 & ... & .29 & 1. 47 & 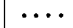 & & $\cdots$ & -. & $\cdots$ & $\cdots$ & $\cdots$ & $\cdots$ \\
\hline 20 & $\ldots$ & $\ldots$ & .28 & $\ldots$ & .. & h2. 47 & $\ldots$ & $\ldots$ & & $\cdots$ & $\ldots$ & $\ldots$ \\
\hline 25 & .28 & .12 & 1.37 & $\ldots$ & -1.70 & 2. 17 & -1.65 & $\ldots$ & $h-1.32$ & $\ldots$ & $\ldots$ & $\ldots$ \\
\hline Eom & .39 & .19 & h1.22 & $\ldots$ & 1.94 & 1.90 & 1.94 & $\cdots$ & $\cdots$ & $\ldots$ & $\cdots$ & $\ldots$ \\
\hline \multicolumn{13}{|c|}{1959} \\
\hline 5 & & $\cdots$ & $\cdots$ & ... & ... & $\ldots$ & . & -1.81 & -2.65 & -1.74 & -0.46 & -0.04 \\
\hline 10 & $\cdots$ & $\ldots$ & $\cdots$ & $\cdots$ & ... & ... & $\cdots$ & 2. 41 & & 1.14 & .36 & .08 \\
\hline 15 & & $\ldots$ & ... & ... & $\cdot$ & ... & $\cdots$ & $\cdots$ & 2. 43 & .86 & .32 & .00 \\
\hline 20 & et 0.08 & $\ldots$ & $\ldots$ & $\ldots$ & 0 & $\ldots$ & $\cdots$ & $\cdots$ & 2.44 & .78 & .31 & .03 \\
\hline 25 & $\ldots$ & $\cdots$ & ... & $\cdots$ & -1.21 & ... & & & 3.09 & .73 & .20 & -.01 \\
\hline Eom & & & & & & & -1.80 & 2. 41 & 2. 37 & .62 & .14 & +.04 \\
\hline \multicolumn{13}{|c|}{1960} \\
\hline 5 & -0.06 & 0.00 & -0.21 & -1.88 & -2.74 & -2.74 & -2.43 & -2.36 & -2.95 & $\mid-1.71$ & -0.70 & -0.46 \\
\hline 10 & +.04 & -.04 & .14 & 2. 45 & 2. 59 & 2.58 & 1.81 & 2. 94 & 3.53 & 1. 46 & .58 & .42 \\
\hline 15 & .03 & .03 & .09 & 2. 54 & 1.83 & 1.70 & 1.75 & 2. 91 & 2. 50 & 1. 27 & .59 & .39 \\
\hline 20 & .03 & .13 & .20 & 2. 54 & 1. 80 & 1. 28 & 2. 23 & 3.24 & $\cdots$ & 1. 07 & .58 & .34 \\
\hline 25 & .04 & .04 & 1.13 & 2. 36 & 2. 17 & 1.69 & 2.26 & 3.16 & e2. 76 & .95 & .49 & .30 \\
\hline Eom & .04 & .24 & 1.16 & 3.23 & 2. 10 & 2. 75 & 1.38 & 3.11 & 2. 71 & .85 & .49 & .26 \\
\hline
\end{tabular}

h Tape measurement. 
25. 24. 27. 421. Brumble and Caffie. Formerly McClure and Hellyer. Drilled irrigation well in alluvium, diam $16 \mathrm{in}$, depth $101 \mathrm{ft}$. Lsd 3,701 ft above msl. MP northwest corner of pumpbase, $1.00 \mathrm{ft}$ above lsd (since Sept. 25, 1958). Highest water level 55.68 below lsd, Apr. 4, 1952; lowest 59.97 below lsd, July 23, 1959. Records available: 1952-60.

\begin{tabular}{l|c||c|c||c|c||c|c}
\multicolumn{1}{c|}{ Date } & $\begin{array}{c}\text { Water } \\
\text { level }\end{array}$ & Date & $\begin{array}{c}\text { Water } \\
\text { level }\end{array}$ & Date & $\begin{array}{c}\text { Water } \\
\text { level }\end{array}$ & \multicolumn{1}{|c|}{ Date } & $\begin{array}{c}\text { Water } \\
\text { level }\end{array}$ \\
\hline Jan. 12, 1956 & $\mathbf{5 6 . 9 0}$ & Nov. 6, 1956 & $\mathbf{5 7 . 5 0}$ & Nov. 26, 1957 & $\mathbf{5 7 . 9 6}$ & July 23, 1959 59.97 \\
Mar. 13 & $\mathbf{5 6 . 2 3}$ & Jan. 11, 1957 & $\mathbf{5 7 . 4 7}$ & Jan. 27, 1958 & $\mathbf{5 7 . 8 0}$ & Nov. 23 & $\mathbf{5 7 . 5 9}$ \\
May 2 & $\mathbf{5 8 . 6 3}$ & Mar. 22 & $\mathbf{5 9 . 2 0}$ & Sept. 25 & $\mathbf{5 9 . 2 4}$ & Jan. 18, 1960 & $\mathbf{5 7 . 4 3}$ \\
July 10 & (a) & July 19 & (a) & Nov. 28 & $\mathbf{5 7 . 3 3}$ & Mar. 18 & $\mathbf{5 7 . 7 3}$ \\
Sept. 27 & $\mathbf{5 8 . 4 8}$ & Sept. 26 & (a) & Jan. 22, 1959 & $\mathbf{5 7 . 2 1}$ & Nov. 15 & $\mathbf{5 8 . 1 8}$ \\
\hline
\end{tabular}

a Well being pumped.

26. 24.9. 441. John Mayes, Drilled irrigation well in alluvium, diam $12 \mathrm{in,} \mathrm{depth} 100 \mathrm{ft}$. Lsd 3, 749. $4 \mathrm{ft}$ above msl. MP top of air-line flange support, $1.40 \mathrm{ft}$ above lsd. Highest water level 42. 29 below lsd, Nov. 8, 1955; lowest 50.20 below lsd, July 19, 1957. Records available: 1952-60.

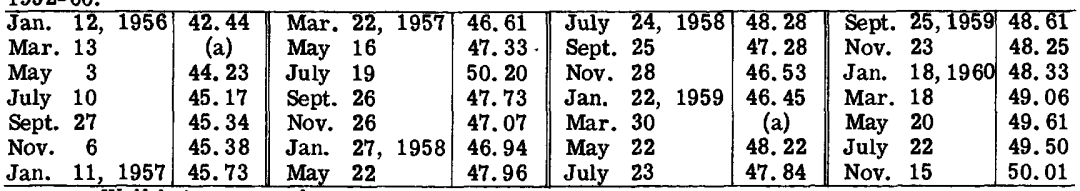

a Well being pumped.

\section{Hidalgo County}

Virden Valley

19.20.18.120. Floyd Johns. Drilled domestic water-table well, diam $8 \mathrm{in}$, depth $60 \mathrm{ft}$. Lsd 3, 803. $47 \mathrm{ft}$ above msl. MP top of casing, $0.60 \mathrm{ft}$ above lsd. Highest water level $20.05 \mathrm{be}-$ low lsd, Feb. 1, 1945; lowest 55. 00 below lsd, July 23, 1947. Records available: 1939-60. Measurements made by Arizona District Ground Water Office.

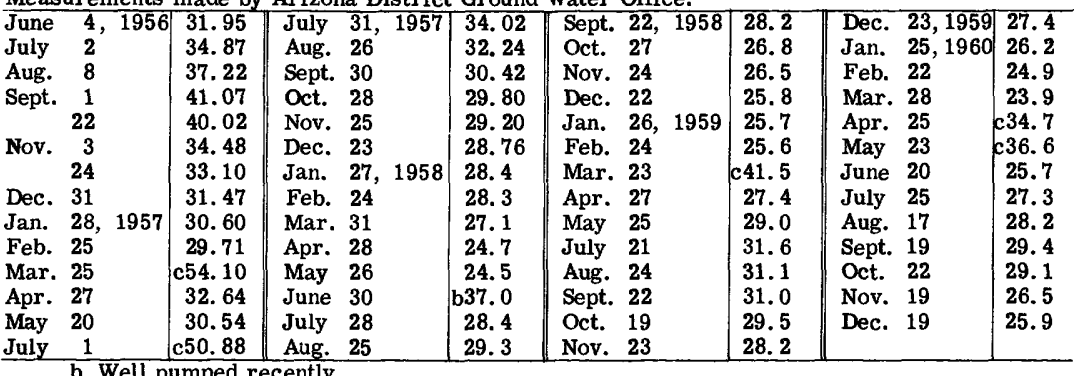

c Nearby well being pumped.

19.21. 2. 330a. W. O. Clouse. Formerly Byron Echols. Drilled irrigation water-table well, diam $20 \mathrm{in}$, depth $80 \mathrm{ft}$. Lsd about 3, $755 \mathrm{ft}$ above msl. MP hole in pumpbase, 3.00 ft above lsd. Highest water level 12.9 below lsd, Apr. 28, 1958; lowest 24. 25 below lsd, Nov. 3, 1956. Records available: 1948-60. Measurements made by Arizona District Ground Water Office.

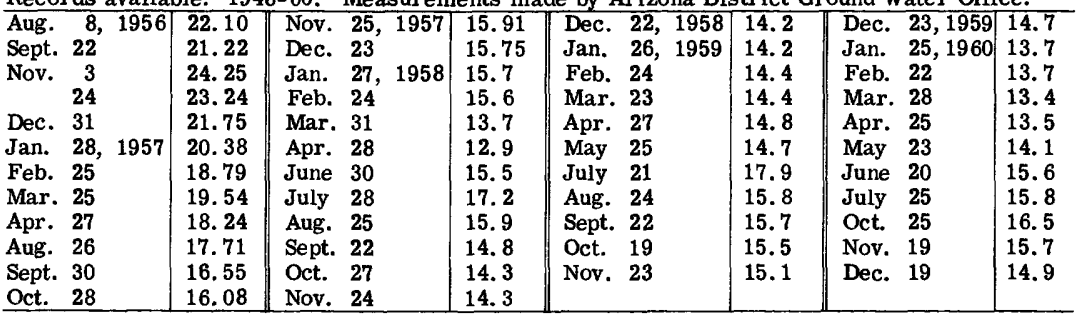




\section{Animas Valley}

24.17.11.233. W. V. Mitchell. Drilled irrigation water-table well in valley fill, diam $12 \mathrm{in}$, depth $250 \mathrm{ft}$. MP top of concrete base and steel casing, $1.00 \mathrm{ft}$ above $1 \mathrm{sd}$. Highest water level 77. 85 below lsd, Nov. 10, 1960; lowest 80.60 below lsd, May 14, 1956. Records available: $1955-60$.

\begin{tabular}{|c|c|c|c|c|c|c|c|}
\hline Date & $\begin{array}{l}\text { Water } \\
\text { level }\end{array}$ & Date & $\begin{array}{c}\text { Water } \\
\text { level }\end{array}$ & Date & $\begin{array}{c}\text { Water } \\
\text { level }\end{array}$ & Date & $\begin{array}{c}\text { Water } \\
\text { level }\end{array}$ \\
\hline $\begin{array}{ll}\text { Nov. } & 10,1955 \\
\text { Jan. } & 16,1956 \\
\text { Mar. } & 12 \\
\text { May } & 14 \\
\text { July } & 15 \\
\text { Sept. } & 13\end{array}$ & $\begin{array}{l}78.27 \\
78.00 \\
77.93 \\
80.60 \\
79.84 \\
79.27\end{array}$ & $\begin{array}{lrr}\text { Nov. } & 13, & 1956 \\
\text { Jan. } & 9, & 1957 \\
\text { Apr. } & 6 & \\
\text { Nov. } & 14 & \\
\text { Jan. } & 11, & 1958 \\
\text { Apr. } & 18 & \end{array}$ & $\begin{array}{l}78.37 \\
78.19 \\
78.64 \\
78.37 \\
78.20 \\
77.98\end{array}$ & \begin{tabular}{lcc|} 
July & 31, & 1958 \\
Nov. & 14 & \\
Jan. & 7,1959 \\
Apr. & 15 & \\
Aug. & 3 &
\end{tabular} & $\begin{array}{l}78.20 \\
78.14 \\
78.20 \\
78.25 \\
78.24\end{array}$ & $\begin{array}{lr}\text { Nov. } & 2,1959 \\
\text { Jan. } & 25,1960 \\
\text { Apr. } & 5 \\
\text { Aug. } & 1 \\
\text { Nov. } & 10\end{array}$ & $\begin{array}{l}78.31 \\
78.29 \\
77.96 \\
78.30 \\
77.85\end{array}$ \\
\hline
\end{tabular}

24. 20. 22. 222. W. W. Roark. Drilled unused water-table well in bolson deposits, diam $6 \mathrm{in}$, depth $28 \mathrm{ft}$. MP top edge of collar on casing, $0.95 \mathrm{ft}$ above $1 \mathrm{sd}$. Highest water level 17. 35 below lsd, May 21, 1948; lowest 24.85 below Isd, Nov. 9, 1960. Records available: 1948-60.

Mar. 14

July 16

Sept. 15

Nov. 13

\begin{tabular}{|r||rr|}
\hline 21.50 & Apr. 6 \\
21.60 & Aug. 6 \\
21.91 & Nov. 14 \\
22.20 & Jan. 8,1958 \\
22.32 & Apr. 17 & \\
\hline
\end{tabular}

\begin{tabular}{|l||rr|} 
22. 38 & Nov. 13 & \\
22. 80 & Jan. 9,1959 \\
22.95 & Apr. 16 \\
22.92 & Aug. 4 & \\
22.97 & & \\
\hline
\end{tabular}

23.03

23. 10

23. 30

Jan. 7, 1960

24. 11

Apr. 5

23. 88 Aug.

24. 15

25. 20. 24.313. Rudiger \& Jundt. Drilled irrigation water-table well in bolson deposits, diam $16 \mathrm{in}$, depth $97 \mathrm{ft}$. Lsd 4,221. $43 \mathrm{ft}$ above msl. MP top of casing, $0.43 \mathrm{ft}$ above lsd. Highest water level 42.43 below lsd, Apr. 1, 1948; lowest 82.04 below 1sd, Nov. 9, 1960. Records available: $1948-60$.

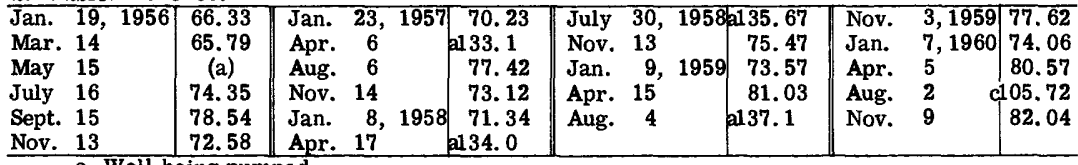

\section{a Well being pumped.}

c Nearby well being pumped.

25. 20.35.434. W. Veck. Drilled irrigation water-table well in bolson deposits, diam $16 \mathrm{in}$, depth $120 \mathrm{ft}$, cased to 108, perforated 60-108. Lsd 4, 245. $96 \mathrm{ft}$ above msl. MP top of casing, $0.50 \mathrm{ft}$ below 1sd. Highest water level 50.27 below 1sd, Apr. 2, 1948; lowest 88.07 below lsd, Apr. 6, 1957. Records available: 1948-60.

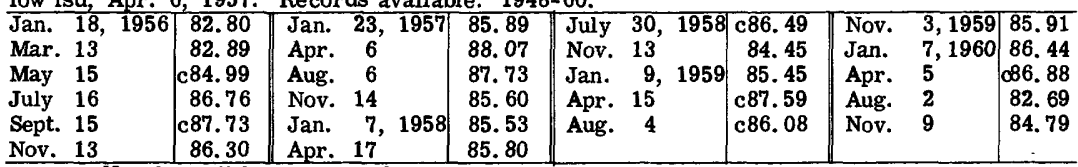

Nov. 13

c Nearby well being pumped.

27. 19. 20.343. Felix Gauthier. Drilled unused water-table well in bolson deposits, diam 16 in, depth $250 \mathrm{ft}$, cased to 250 , perforated $112-218$. MP top edge of $1 \frac{1}{4}$-in pipe in concrete pumpbase, $1.25 \mathrm{ft}$ above isd (since July 16, 1956). Highest water level 131.90 below lsd, July 29, 1949; lowest 153.58 below lsd, Nov. 10, 1960. Records available: 1949-60.

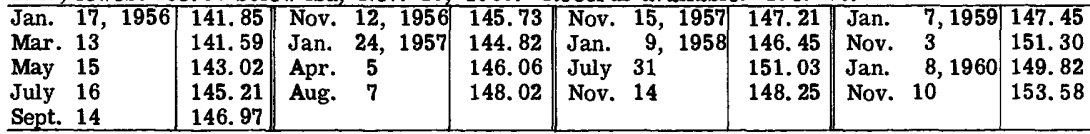

29. 19. 3. 100. T. B. Strickland. Dug stock water-table well in bolson deposits, diam 36 in, depth $30 \mathrm{ft}$, cribbed with wood. MP top of concrete well curb, $0.60 \mathrm{ft}$ above $1 \mathrm{sd}$. Highest water level 22. 40 below 1sd, Jan. 17, 1956; lowest 27.99 below 1sd, May 26, 1954. Records available: 1949-60.

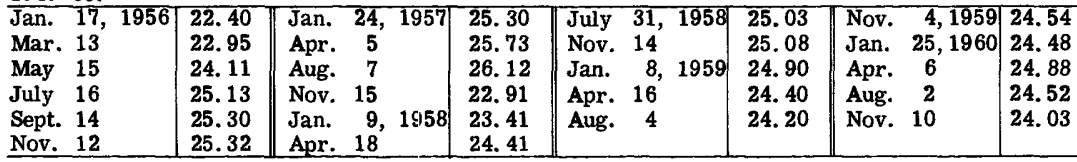


Playas Valley

30.16. 29. 422. H. Groessel. Formerly Myers Bros. Drilled irrigation water-table well in bolson deposits, diam 16 in, depth $160 \mathrm{ft}$. Lsd 4,347.5 ft above msl. MP top of 2-in pipe with threaded plug in plate over well, $0.71 \mathrm{ft}$ above $1 \mathrm{sd}$. Highest water level 43.85 below lsd, Feb. 4, 1949; lowest 61.17 below 1sd, Nov. 23, 1953. Records available: 1948-60.

\begin{tabular}{|c|c|c|c|c|c|c|c|}
\hline Date & Water & Date & Water & Date & Water & Date & $\begin{array}{l}\text { Water } \\
\text { level }\end{array}$ \\
\hline $\begin{array}{l}\text { Jan. } 16,1956 \\
\text { Mar. } 12\end{array}$ & $\begin{array}{l}57.04 \\
56.14\end{array}$ & $\begin{array}{ll}\text { Jan. } 22,1957 \\
\text { Apr. } 10\end{array}$ & $\begin{array}{l}58.65 \\
57.26\end{array}$ & $\begin{array}{l}\text { Aug. } 5,1958 \\
\text { Nov, } 15\end{array}$ & $\begin{array}{l}56.49 \\
56.27\end{array}$ & $\begin{array}{lr}\text { Nov. } & 4,1959 \\
\text { Jan } & 22,1960\end{array}$ & 54.68 \\
\hline May 12 & 50.14 & $\begin{array}{l}\text { Apr. } 10 \\
\text { Aug. } 14\end{array}$ & $\begin{array}{l}57.60 \\
58.67\end{array}$ & $\begin{array}{l}\text { Nov. 13 } \\
\text { Jan. } 13,1959\end{array}$ & 55.57 & Apr. 12 & 52.90 \\
\hline July 13 & 58.22 & Nov. 18 & 59.30 & Apr. 17 & 54.58 & Aug. & c55. 26 \\
\hline Sept. 13 & 59.46 & Jan. 10,1958 & 58.19 & Aug. & 55.19 & Nov. 16 & 53.07 \\
\hline Nov. 14 & 59.72 & Apr. 19 & 56.38 & & & & \\
\hline
\end{tabular}

32. 17. 23. 434. Mr. Timberlake. Drilled irrigation water-table well in bolson deposits, diam $12 \mathrm{in,} \mathrm{depth} 162 \mathrm{ft}$. Lsd 4,507.0 ft above msl. MP top of casing, at isd (since Jan. 16, 1956). Highest water level 96.09 below lsd, Mar. 23, 1949; lowest 100.19 below lsd, Aug. 3, 1960. Records available: $1948-53,1956-60$

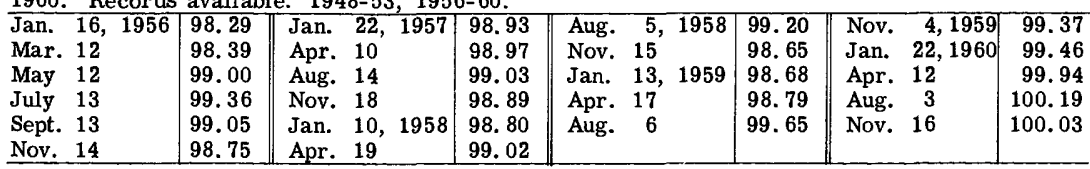

\section{Lea County}

\section{Tatum-Lovington- Hobbs Area}

12.34.11.413. A. D. Jones Estate. Drilled unused water-table well in Ogallala Formation of Pliocene age, diam $15 \mathrm{in}$, depth $87 \mathrm{ft}$, cased. MP top of concrete pumpbase, $0.80 \mathrm{ft}$ above 1sd. Highest water level 29.57 below 1sd, May 24, 1949; lowest 32.13 below 1sd, Sept. 27, 1955. Records available: $1949-60$.

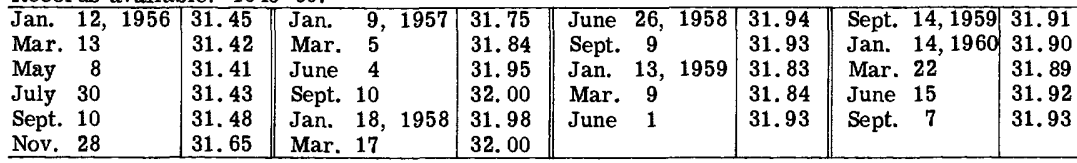

14.35.33.433. W. A. Anderson. Drilled unused water-table well in Ogallala Formation of Pliocene age, diam 6 in, depth $62 \mathrm{ft}$, not cased. Lsd 4,013.6 ft above msl. MP top of concrete collar on well, $1.00 \mathrm{ft}$ above lsd. Highest water level 39.65 below isd, May 21, July 25, 1951, Jan. 9, May 24, 1952; lowest 44.42 below 1sd, Nov, 27, 1956. Records available: 1929-60.

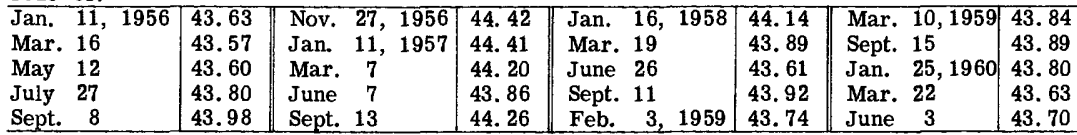

14. 36. 13. 211. Mrs. Mattie Field. Drilled unused water-table well in Ogallala Formation of Pliocene age, diam 12 in, depth $87 \mathrm{ft}$. Lsd 3,904.59 ft above msl. MP notch in north side of concrete well curb, at lsd. Highest water level 35.74 below lsd, Jan. 30, May 24, 1946; lowest 51. 66 below lsd, Sept. 8, 1960. Records available: 1929-60.

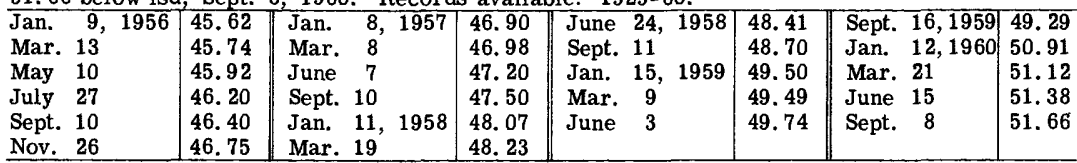

16. 36. 5 Lot 2. Miss Ethel Yadon. Drilled unused water-table well in Ogallala Formation of Pliocene age, diam $16 \mathrm{in}$, depth $97 \mathrm{ft}$. MP top of hole in recorder shelter shelf, $2.95 \mathrm{ft}$ above 1sd. Highest water level 53. 83 below lsd, Mar. 14, 1957; lowest 56.04 below lsd, Dec. 15, 1960. Records available: 1956-60. For additional measurements, see Reeder and others (1960b and c). Recording gage installed Nov. 29, 1956. 
16. 36. 5 Lot 2--Continued.

Highest water level for the day, from recorder graph, 1956

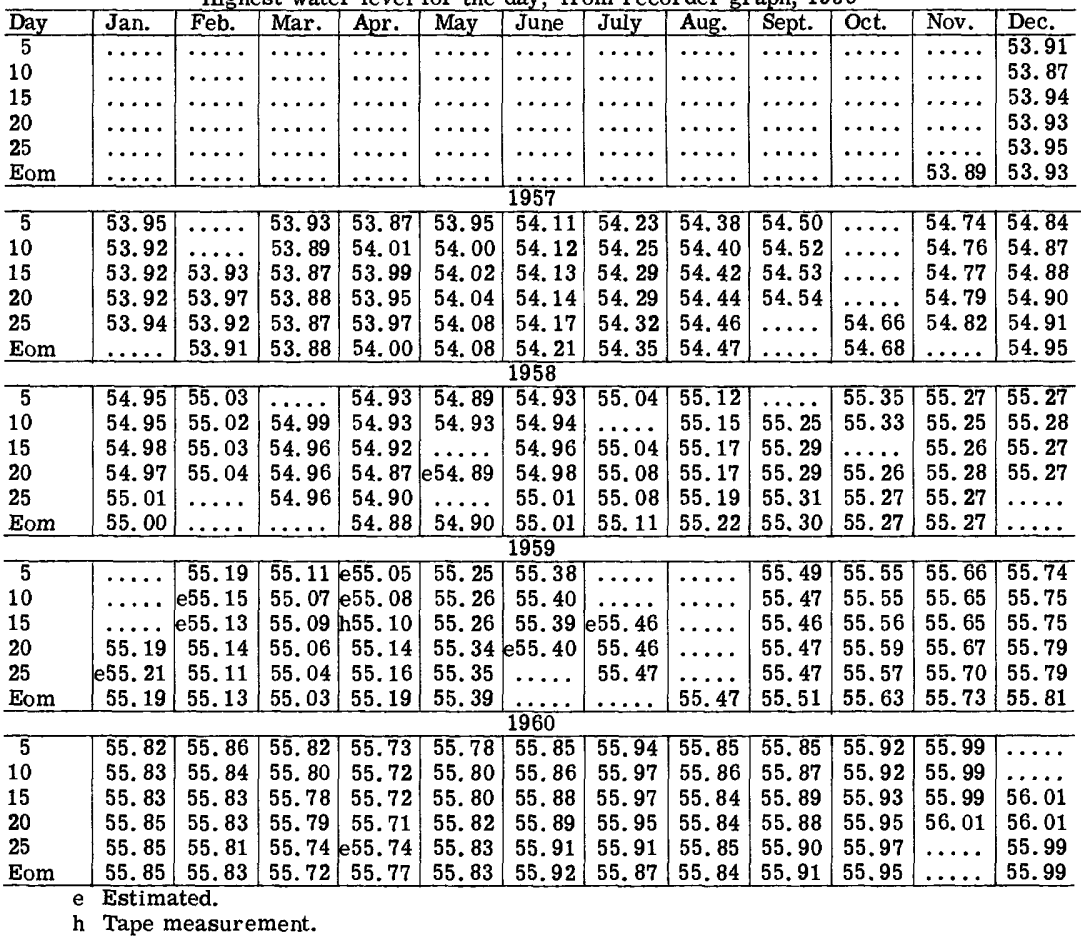

16. 37. 11.111. A. J. Birkshire. Drilled irrigation water-table well in Ogallala Formation of Pliocene age, diam 16 in, reported depth $118 \mathrm{ft}$. MP top of 1-in hole in southwest side of pump, $1.34 \mathrm{ft}$ above 1sd. Highest water level 31.93 below 1sd, Jan. 23, 1949; lowest 73.88 below 1sd, Sept. 2, 1960. Records available: 1949-60.

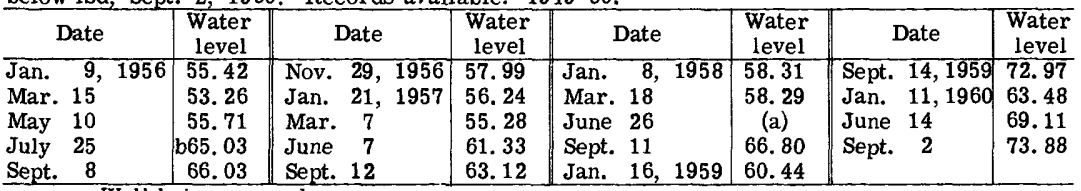

a Well being pumped.

b Well pumped recently.

17. 33. 13.341. Potash Co. of America. Drilled observation water-table well in Ogallala Formation of Pliocene age, diam 6 in, depth $252 \mathrm{ft}$, cased to 252 . Lsd 4,123.5 ft above msl. MP top of casing, $1.10 \mathrm{ft}$ above lsd. Highest water level 146.00 below Isd, Jan. 21, 1953; lowest 156. 90 below 1sd, Nov. 22, 1960. Records available: 1952-60. For additional measurements, see Reeder and others (1960b and $\mathrm{c})$.

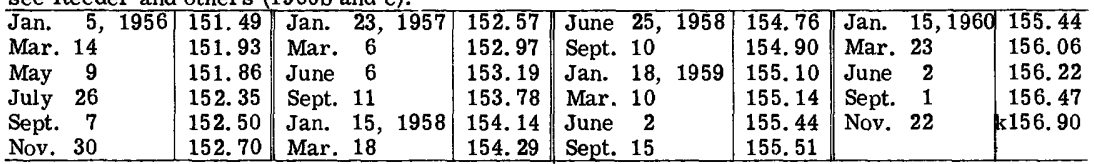

Nov. $30 \quad 152.70$ Mar. 18

17. 38. 7.111a. L. R. Sebings. Formerly Jim Cunningham. Drilled irrigation watertable well in Ogallala Formation of Pliocene age, diam 16 in, reported depth $125 \mathrm{ft}$ (previously reported $135 \mathrm{ft}$ ). MP top of concrete pumpbase, $0.95 \mathrm{ft}$ above lsd. Highest water level 35.59 below Isd, Mar. 21, 1952; lowest 53. 47 below Isd, Sept. 2, 1960. Records available: 1951-60. 
17. 38. 7. 111a--Continued.

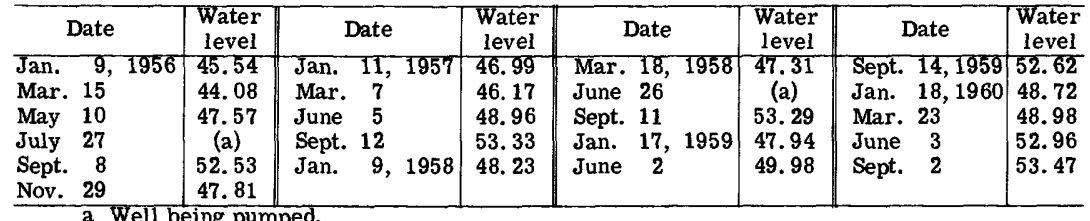

17. 38. 34.113. W. E. Busby. Drilled irrigation water-table well in Ogallala Formation of Pliocene age, diam 12 in, depth $125 \mathrm{ft}$ (previously reported $120 \mathrm{ft}$ ). MP top of $\frac{1}{2}$-in hole in pumpbase, $0.54 \mathrm{ft}$ above lsd. Highest water level 24.78 below 1sd, Jan. 15, 1944; lowest 32.90 below lsd, June 3, 1960. Records available: 1943-60.

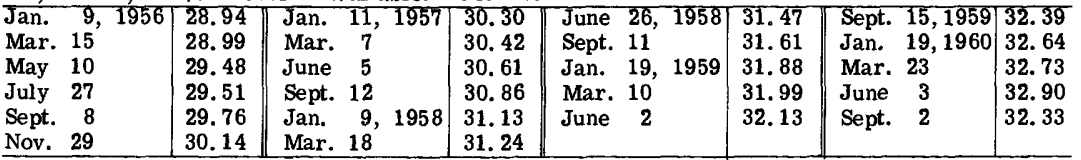

18.38. 30.223. J. T. Montgomery, Jr. Formerly Mrs. Sadie Davis. Drilled unused water-table well in Ogallala Formation of Pliocene age, diam 6 in, depth $50 \mathrm{ft}$. MP top of casing, $0.30 \mathrm{ft}$ above 1sd. Highest water level 23.01 below lsd, Nov. 17, 1947; lowest 30.64 below Isd, June 3, 1960. Records available: 1931-60.

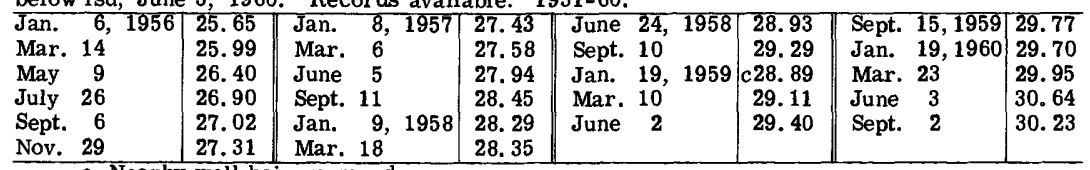

c Nearby well being pumped.

\section{$\underline{\text { Lincoln County }}$}

\section{Hondo Valley}

9.13.25.113. M. W. Coll. $0.4 \mathrm{mi}$ southwest of intersection of Magado Creek and State Highway 48. Drilled irrigation and domestic water-table well in alluvium, diam 8 in, depth $90 \mathrm{ft}$. Lsd about 6,750 ft above msl. MP top of casing, at lsd. Highest water level 18. 04 below lsd, Nov. 26, 1958; lowest 35.72 below lsd, Sept. 25, 1956. Records available: 1955-60.

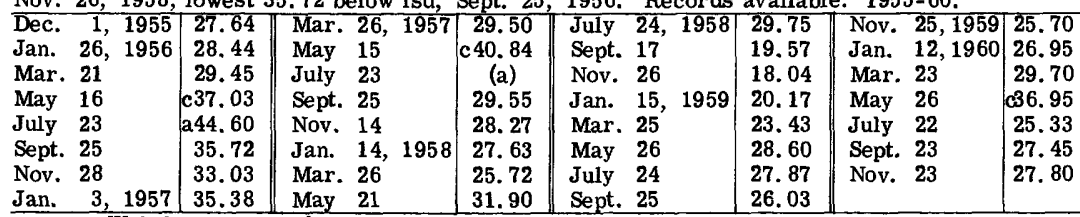
Jan. 3, 1957 35.38 May 21

a Well being pumped.

c Nearby well being pumped.

9.14.10.132. Village of Capitan. East end of village on south side of U. S. Highway 380 in wooden shed. Drilled public-supply water-table well in Mancos Shale of Upper Cretaceous age, diam $8 \mathrm{in}$, depth $1,261 \mathrm{ft}$. Lsd about 6,340 ft above $\mathrm{msl}$. MP top of breather hole on west side of pumpbase, $1.00 \mathrm{ft}$ above 1sd. Highest water level 45.69 below 1sd, Jan. 12, 1960; lowest 69.77 below lsd, Nov. 28, 1956. Records available: 1955-60.

\begin{tabular}{|c|c|c|c|c|c|c|c|c|c|c|}
\hline une & & & & & & & & & & \\
\hline & 15,1955 & 62.81 & Mar. 26, 1957 & (a) & May 2 & 21,1958 & 51.47 & July & 24,1959 & 46.71 \\
\hline Mar. & 26, & 59.36 & May 15 & (a) & July ? & 24 & 49.84 & Sept. & 25 & 46.04 \\
\hline May & 20 & 69. & $\begin{array}{l}\text { July } 23 \\
\text { Sent } 25\end{array}$ & 68.85 & Sept. & 17 & $\begin{array}{l}48.89 \\
47.90\end{array}$ & Jan. & 12 , & 45.69 \\
\hline uly & 23 & 65.33 & Nov 14 & (a) & Jan. 1 & 15 & 47 & May & 26 & 45 \\
\hline ept. & 25 & I & Jan. & 56,69 & Mar. & 25 & 47.13 & July & 22 & 45,95 \\
\hline Tov. & 28 & 69. & Mar. 26 & 53.30 & May 2 & 26 & 47.10 & Nov. & 23 & 47.10 \\
\hline & 2,1 & 64. & & & & & & & & \\
\hline
\end{tabular}

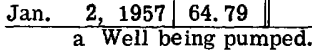


9. 14. 25. 324. Fort Stanton. On north bank of Rio Bonito, at west end of Medical Center's buildings. Drilled unused water-table well in alluvium, diam 8 in, depth $45 \mathrm{ft}$. Lsd about $6,210 \mathrm{ft}$ above msl. MP top of casing, 2, $00 \mathrm{ft}$ above lsd. Highest water level 12.30 below lsd, May 21, 1958; lowest 23. 20 below Isd, Nov. 14, 1957. Records available: 1955-60.

\begin{tabular}{|c|c|c|c|c|c|c|c|}
\hline Date & $\begin{array}{l}\text { Water } \\
\text { level }\end{array}$ & Date & $\begin{array}{r}\text { Water } \\
\text { level }\end{array}$ & Date & $\begin{array}{c}\text { Water } \\
\text { level }\end{array}$ & Date & $\begin{array}{l}\text { Water } \\
\text { level }\end{array}$ \\
\hline Apr. 28, 1955 & 21.95 & Jan. 2,1957 & 21.30 & Mar. 26, 1958 & 12.62 & May 26,1959 & 18.75 \\
\hline Jan. 27, & & Mar. 26 & 21.53 & May 21 & & July 24 & \\
\hline Mar. 20 & 20 & May 15 & 21.68 & July & & Sept. 25 & \\
\hline May 16 & & July 23 & & Sept. 17 & & Nov. 2 & 85 \\
\hline July & 20.41 & Sept. 25 & 20 & Nov. 26 & 18 & Jan. 12, & 20.98 \\
\hline Sept & 2 & Nov. 14 & 23.20 & Jan. 15,1959 & 16.42 & Mar. 23 & 20.95 \\
\hline Nov. 28 & 21.20 & Jan. 14,1958 & 17.37 & Mar. 25 & 18.00 & May 26 & 14.78 \\
\hline
\end{tabular}

10.17.16.433. Wilbur McKnight. $5.4 \mathrm{mi}$ north of U. S. Highway 70 at sheep corral in Chaves Canyon. Drilled stock water-table well in Glorieta Sandstone of Permian age, diam 8 in, depth $140 \mathrm{ft}$. Lsd about 5, $540 \mathrm{ft}$ above msl. MP top of casing, $1.50 \mathrm{ft}$ above $1 \mathrm{sd}$. Highest water level 121. 57 below Isd, Jan. 26, 1956; lowest 124. 05 below 1sd, May 26, 1960. Records available: $1955-60$.

\begin{tabular}{l|l||l|l|l|l|l|l|l|}
\hline May 23, 1955 & $\mathbf{1 2 2 . 0 9}$ & Jan. 2, 1957 & $\mathbf{1 2 2 . 1 8}$ & Mar. 26, 1958 & 122.84 & July 24, 1959 & 123.73 \\
Jan. 26, 1956 & 121.57 & Mar. 26 & 122.48 & May 21 & & 123.05 & Jan. 12, 1960 & 123.65 \\
Mar. 21 & 121.70 & May 15 & 122.47 & July 24 & 123.15 & Mar. 23 & 123.55 \\
May 15 & 121.99 & Sept. 25 & 122.02 & Jan. 14, 1959 & 123.43 & May 26 & 124.05 \\
Sept. 24 & 122.01 & Nov. 14 & 122.39 & Mar. 25 & 123.40 & Sept. 23 & a127. 25 \\
Nov. 28 & 122.20 & Jan. 15, 1958 & $\mathbf{1 2 2 . 8 9}$ & May 26 & 123.60 & Nov. 23 & 123.86 \\
\hline
\end{tabular}

a Well being pumped.

11.14.15. 431. E. H. Fuchs, $0.1 \mathrm{mi}$ west of Valley View Motel. Drilled unused watertable well in alluvium, diam $8 \mathrm{in}$, depth $90 \mathrm{ft}$. Lsd 6, $240 \mathrm{ft}$ above msl. MP top of east edge of casing, $1.00 \mathrm{ft}$ above lsd. Highest water level 57.16 below lsd, Mar. 26, 1958; lowest 61.60 below Isd, May 20, 1958. Records available: 1955-60.

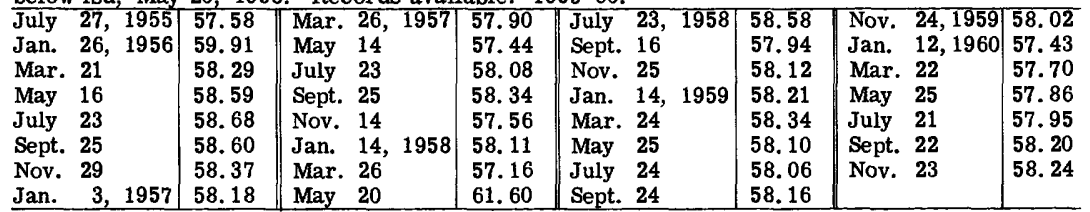

11.17.10.211. H. M. Dow. 0.1 mi southwest of U. S. Highway 70. Drilled unused watertable well in alluvium, diam $7 \mathrm{in}$, depth $260 \mathrm{ft}$. Lsd 5,190 ft above msl. MP top of 5-in casing, at lsd. Highest water level 28. 35 below lsd, July 22, 1960; lowest 35.08 below Isd, Jan. 15, 1958. Records available: $1955-60$.

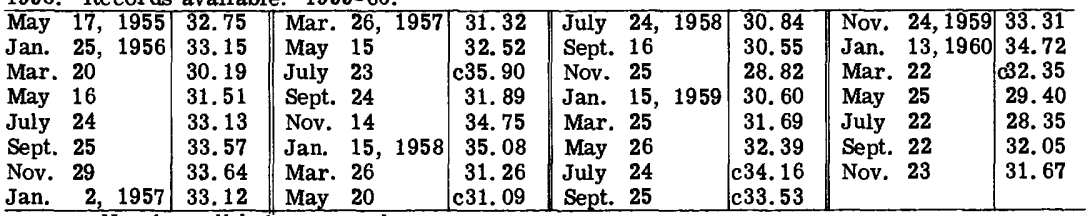

c Nearby well being pumped.

11.18.16.444. B. G. Robinson. 0.4 mi south of Picacho bridge on east side of Casey Canyon rd. Drilled domestic and stock water-table well in Yeso Formation of Lower Permian age, diam $12 \mathrm{in}$, depth $125 \mathrm{ft}$. Lsd $5,010 \mathrm{ft}$ above msl. MP top of casing, $0.50 \mathrm{ft}$ above isd. Highest water level 54.14 below lsd, Oct. 26, 1955; lowest 60.18 below lsd, Jan. 15, 1959. Records available: $1955-60$.

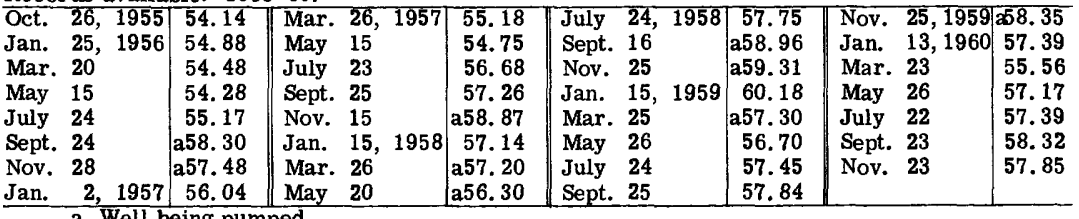
a Well being pumped.

11. 19. 20.344. Geo. Clements. $150 \mathrm{ft}$ north of house on north side of U. S. Highway 70, $1.5 \mathrm{mi}$ west of Riverside. Drilled domestic and stock water-table well in Yeso Formation of Lower Permian age, diam $5 \mathrm{in}$, depth $160 \mathrm{ft}$. Lsd 4, $830 \mathrm{ft}$ above msl. MP top of casing, $1.00 \mathrm{ft}$ above lsd. Highest water level 130.82 below lsd, Aug. 23, 1955; lowest 136. 85 below lsd, July 24, 1959. Records available: 1955-60. 
11. 19. 20. 344--Continued.

\begin{tabular}{|c|c|c|c|c|c|c|c|}
\hline Date & $\begin{array}{l}\text { Water } \\
\text { level }\end{array}$ & Date & $\begin{array}{l}\text { Water } \\
\text { level }\end{array}$ & Date & $\begin{array}{c}\text { Water } \\
\text { level }\end{array}$ & Da & $\begin{array}{c}\text { Water } \\
\text { level }\end{array}$ \\
\hline Aug. 23, 1955 & 130.82 & Jan. 2,1957 & 136.38 & May 21,1958 & 134.10 & Nov. 25,1959 & $\longdiv { 1 3 4 . 3 2 }$ \\
\hline Jan. $\quad 5,1956$ & 132. 25 & Mar. 26 & 134.87 & July 24 & 135.65 & Jan. 13,1960 & 134. 42 \\
\hline 25 & .77 & May 15 & 13 & Sept. 16 & 135 & Mar. 23 & 64 \\
\hline Mar. 20 & 132.62 & July 23 & 136.06 & Nov. 26 & 136.55 & May 26 & 134.44 \\
\hline May 15 & 133.61 & Sept. 25 & 135.42 & Jan. 15,1959 & 136 & July 22 & 70 \\
\hline July 24 & 134. 22 & Nov. 15 & 135 & May 26 & 136.54 & Sept. 23 & 15 \\
\hline Sept. 24 & 135.06 & Jan. 15,1958 & 135.28 & July 24 & 136.85 & Nov. 23 & 134.15 \\
\hline Nov. 28 & 135.75 & Mar. 26 & 136.70 & Sept. 25 & 136.22 & & \\
\hline
\end{tabular}

11. 20.31.123. J. P. White. $0.3 \mathrm{mi}$ west of Border Hills crossing at Rio Hondo. Drilled stock water-table well in Yeso Formation of Lower Permian age, diam 6 in, depth $260 \mathrm{ft}$. MP top of casing, 2. $50 \mathrm{ft}$ above Isd. Highest water level 136. 89 below Isd, Oct. 12, 1955; lowest 146. 97 below Isd, Nov. 23, 1960 . Records available: 1955-60.

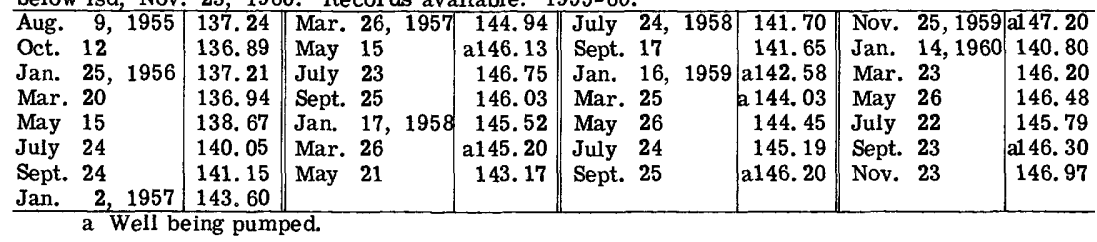

\section{Luna County}

Mimbres Valley

22. 10.18.121. State of New Mexico. Drilled unused water-table well in valley fill, diam $30 \mathrm{in}$, depth $223 \mathrm{ft}$. MP top of concrete well curb, at lsd. Highest water level 68.00 below lsd, Sept. 30, 1929; lowest 81.94 below Isd, Nov. 11, 1960. Records available: 1928-60.

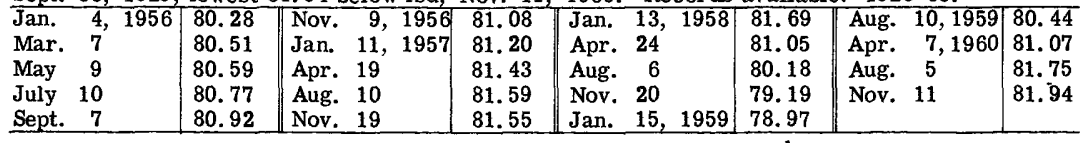

24. 7. 4. 424. G. D. Hatfield. Drilled stock and domestic water-table well in valley fill, diam $10 \mathrm{in}$, depth $107 \mathrm{ft}$. MP top of casing, $1.00 \mathrm{ft}$ above Isd. Highest water level 64.58 below 1sd, Apr. 16, 1929; lowest 98.69 below 1sd, Sept. 6, 1952. Records available: 1928-60.

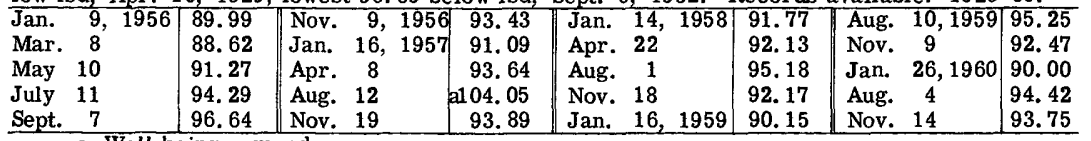

a Well being pumped.

24. 7.14.221. J. H. Winslow. Drilled unused water-table well in valley fill, diam 28 in, depth $118 \mathrm{ft}$. MP top inside edge of tile casing, $1.93 \mathrm{ft}$ above Isd. Highest water level 71.15 below Isd, Apr. 19-20, 1939; lowest 91.64 below Isd, Aug. 4, 1958. Records available: 1939-60.

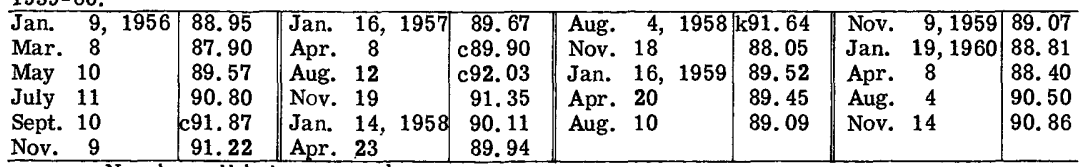

c Nearby well being pumped.

k From recorder graph.

24. 8. 4.111. Foy Riley. Drilled unused water-table well in valley fill, diam 24 in, reported depth $100 \mathrm{ft}$. MP top of 2- x 4-in holding hasp, $2.78 \mathrm{ft}$ above 1sd. Highest water level 35.06 below Isd, May 6, 1941; lowest 60.94 below Isd, Oct. 6, 1960. Records available: 1941-60. For additional measurements, see Reeder and others (1960b and c). 
24. 8. 4.111--Continued.

Highest water level for the day, from recorder graph, 1956

\begin{tabular}{|c|c|c|c|c|c|c|c|c|c|c|c|c|}
\hline$\overline{\text { Day }}$ & Jan. & Feb. & Mar & Apr & May & June & July & Aug. & Sept. & Oct. & Nov. & Dec. \\
\hline 5 & e54. 77 & 54.39 & 54.21 & 54.59 & 55.06 & 55.67 & 56.25 & 56.70 & 57.04 & 57.17 & 57.06 & 56.72 \\
\hline 10 & 54.69 & 54.35 & 54.34 & 54.74 & 55.09 & 55.70 & 56.34 & 56.79 & 57.08 & 57.16 & 57.05 & 56.66 \\
\hline 15 & 54.62 & 54.29 & 54.42 & 54.78 & 55.18 & 55.83 & 56.46 & 56.87 & 57.08 & 57.18 & 56.98 & 56.62 \\
\hline 20 & 54.56 & 54.27 & 54.32 & 54.83 & 55.31 & 55.96 & 56. 57 & 56.93 & 57.15 & 57.17 & 56.91 & 56.56 \\
\hline 25 & 52 & 54.20 & 54.35 & 54.86 & 55,43 & 56.05 & 56. 64 & 56.96 & 57.19 & 57.13 & 56. 86 & 56.54 \\
\hline Eom & & .17 & 54.50 & 54.95 & 55.61 & 56.11 & 56.65 & 57.01 & 57.19 & 57.07 & 56.79 & 56.43 \\
\hline \multicolumn{13}{|c|}{1957} \\
\hline 5 & 56.42 & 5.03 & 55.93 & 56.26 & 56.60 & 57.18 & 57.75 & 01 & 58.18 & 58.35 & 58.11 & $\overline{57.74}$ \\
\hline 10 & 56.36 & 56.01 & 55.93 & 56.36 & 56.69 & 57.24 & 57.85 & 58.00 & 58. 25 & 58.33 & 58.06 & 57.70 \\
\hline 15 & 56.27 & 55.97 & 55.96 & 56.46 & 56.87 & 57.33 & 57.94 & 58.04 & 58.25 & 58.30 & e57.99 & 57.66 \\
\hline 20 & 56.21 & 55.96 & 55.98 & 56.52 & 56.88 & 57.41 & 57.97 & 58.07 & 58.27 & 58.26 & 57.92 & 57.60 \\
\hline 25 & 56.16 & 55.93 & 56.09 & 56.54 & 57.00 & 57.59 & 58.04 & 58.09 & 58.33 & 58.22 & 57.86 & 57.53 \\
\hline Eom & 56.08 & 92 & 56.16 & 56.57 & 57.13 & 57.67 & 58.04 & 58.15 & 58.34 & 58.14 & 57.82 & 57.46 \\
\hline \multicolumn{13}{|c|}{1958} \\
\hline 5 & 57.42 & 57.21 & 56.92 & 56.92 & 57.43 & & & 82 & 59.14 & 58.88 & 58.39 & 57.94 \\
\hline 10 & 57.36 & 57.15 & 56.87 & 56.97 & 57.59 & & & 82 & 59.14 & 58.80 & 58.32 & 57.87 \\
\hline 15 & 57.31 & 57.10 & 56.80 & 57.08 & 67 & & & 58.95 & 59.15 & 58.71 & 58.24 & 57.80 \\
\hline 20 & 57.25 & 57.09 & 56.75 & 57.10 & & & & 01 & 59.09 & 58.63 & 58.19 & 7.72 \\
\hline 25 & 57.20 & 57.01 & 56.71 & 19 & & & & 01 & 59.03 & 58.56 & 58.10 & 57.64 \\
\hline Eom & 57.25 & 56.99 & 56.77 & 57.20 & & & & 05 & 58. 97 & 58.48 & 58.04 & 57.56 \\
\hline \multicolumn{13}{|c|}{1959} \\
\hline 5 & 57.49 & 57.23 & 56.99 & 57.37 & 57.68 & 58.25 & 58.80 & 59.26 & & & $\mathrm{e} 59.32$ & 58.80 \\
\hline 10 & 49 & 17 & 96 & 57.48 & 57.84 & 58.47 & 58. 91 & 30 & & & .25 & 69 \\
\hline 5 & 57.67 & 11 & 16 & 57.55 & & 58.64 & 58.95 & 40 & & & 18 & 65 \\
\hline 20 & 57.62 & 57.14 & 57.11 & 57.62 & & 58.70 & 59.01 & 59.42 & & & e59.08 & 58.57 \\
\hline 25 & 57.44 & 56.99 & 57.14 & 57.64 & e58.00 & 58.68 & 59.16 & & ..... & $\ldots \ldots$ & 58.97 & 58.49 \\
\hline Eon & & & & 62 & 58.13 & 58.72 & 59.24 & & & & 58.89 & 58.47 \\
\hline \multicolumn{13}{|c|}{1960} \\
\hline 5 & 58.43 & 57.98 & 57.86 & 58.64 & 59.07 & 59.68 & 59.96 & 60.14 & 60.66 & 60.92 & 60.77 & 60.47 \\
\hline 10 & & & 57.97 & 58.71 & 59. 13 & 59.74 & 59.99 & 60.23 & 60.81 & 60.91 & 60.72 & 60.37 \\
\hline 15 & & 57.95 & 57.88 & 58.80 & 59. 26 & 59.73 & 60.02 & 60.33 & 60.81 & 60.85 & 60.64 & 60.32 \\
\hline 20 & 58.18 & 57.76 & 58.04 & 58.91 & 59.40 & 59.79 & $\cdots$ & 60.40 & 60.90 & 60.80 & 60.54 & 60.25 \\
\hline 25 & 58.08 & 57.67 & 58.09 & 58.97 & 59.49 & 59.85 & & 60.43 & 60.85 & 60.75 & 60.50 & 60.18 \\
\hline Eom & 58.01 & 57.72 & 58.37 & 59.04 & 59.62 & 59.89 & & 60.49 & 60.88 & 60.72 & 60.52 & 60.11 \\
\hline
\end{tabular}

24. 10.12. 431. Steve Hrna. Dug and drilled unused water-table well in valley fill, diam 36 to $12 \mathrm{in}$, reported depth $132 \mathrm{ft}$. MP top of recorder shelter shelf, $1.36 \mathrm{ft}$ above lsd. Highest water level 77. 61 below 1sd, May 6-13, 1940; lowest 111.33 below 1sd, Sept. 1, 1960. Records available: 1939-60. For additional measurements, see Reeder and others (1960b and c).

Highest water level for the day, from recorder graph, 1956

\begin{tabular}{|c|c|c|c|c|c|c|c|c|c|c|c|c|}
\hline Day & $\operatorname{Tan}$ & Feb. & Mar & Āpr. & May & June & July & Aug. & Sept. & Oct. & Nov. & Dec. \\
\hline 5 & 99.83 & 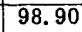 & 100.89 & 104.27 & 104.39 & 106.17 & 108.01 & 109.07 & 109.00 & 106.77 & 103.0 & 101.53 \\
\hline 10 & 99.72 & 98.83 & 101.75 & 104.22 & 105.04 & 107.23 & 108.44 & 109.07 & 108.56 & 106.15 & 102.70 & 101.25 \\
\hline 15 & 99.43 & 99.11 & 103.66 & 103.55 & 105.32 & 107.13 & 108.75 & 109.46 & 108.77 & 105.53 & 102.71 & 101.16 \\
\hline 20 & 48 & 99.53 & 11 & 103.97 & 105.60 & 107.46 & 108.78 & 109.53 & 108.07 & 104.70 & 102.2 & 101.1 \\
\hline 25 & 99.25 & 99.84 & 104 & 103.94 & 105.44 & 107.99 & 109.09 & 109.32 & 10 & 103.9 & 101. & 101.28 \\
\hline Eom & 99 & 100. & 77 & 10 & 02 & 107.82 & 1 & & & 10 & 10 & 69 \\
\hline \multicolumn{13}{|c|}{1957} \\
\hline 5 & 00.72 & 99.96 & 103.04 & 10 & 104.48 & 107.12 & & 30 & 10 & & & 6 \\
\hline 10 & 100.39 & 100. & & & 47 & 107.83 & 109.34 & 108.17 & & 80 & 102.69 & 101 \\
\hline 15 & 100.07 & 100.59 & 47 & 105.26 & 106.46 & 107.67 & 108.47 & 109.36 & $\mid 107.58$ & 104.87 & & 101.46 \\
\hline 20 & 99.97 & 101.03 & 50 & 15 & 106.87 & 107.98 & 109.39 & 108.75 & $\mid 107.99$ & 104.23 & 102.24 & 101.27 \\
\hline 25 & & 101.59 & 39 & 88 & 106.76 & 108.11 & 109.60 & 108.36 & 62 & 103.77 & 102.18 & 101.11 \\
\hline Eon & & & & & & 8.49 & & & & 103.33 & 102.0 & 01.33 \\
\hline \multicolumn{13}{|c|}{1958} \\
\hline 5 & & 11 & & & & 10 & & & $1 \times$ & & 2. & . \\
\hline 10 & 1 & 100.76 & 10 & 81 & 22 & 44 & 10 & 108.81 & 19 & 10 & 7 & .76 \\
\hline 15 & 10 & 101.30 & 40 & 104.96 & 104.67 & 106.94 & 108.91 & 109.26 & .90 & 103.71 & 102.44 & 101.62 \\
\hline 20 & & & & 103.59 & 32 & 107.21 & 108.46 & 40 & .08 & 103.36 & 102.49 & 101.46 \\
\hline 25 & & & & 103.82 & 15 & & 109.00 & 108.69 & .41 & 103.45 & 102.12 & 101.28 \\
\hline Eom & & & & & & 100 & & 10860 & 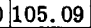 & $10 \%$ & 102. & 101.1 \\
\hline
\end{tabular}


24. 10.12.431---Continued.

1959

\begin{tabular}{c|l|l|l|l|l|l|l|l|l|l|l|l|}
\hline Day & Jan. & Feb. & Mar. & Apr. & May & June & July & Aug. & Sept. & Oct. & Nov. & Dec. \\
\hline 5 & 100.98 & 100.84 & 104.61 & 106.08 & 105.93 & 108.13 & 109.11 & 110.43 & 108.09 & 107.53 & 104.49 & 103.26 \\
10 & 100.94 & 101.38 & 105.09 & 106.30 & 106.54 & 108.76 & 109.74 & 109.70 & 108.85 & $\ldots \ldots$ & 104.32 & 103.14 \\
15 & 100.89 & 101.91 & 106.18 & 105.87 & 106.87 & 109.10 & 109.94 & 108.61 & 108.97 & $\ldots \ldots$. & 104.37 & 102.92 \\
20 & 100.61 & 102.53 & 106.52 & 105.35 & 107.46 & 109.20 & 109.91 & 108.13 & 108.22 & $\ldots \ldots .$. & 103.87 & 102.67 \\
25 & 100.78 & 103.66 & 106.51 & 105.31 & 107.87 & 109.46 & 110.02 & 107.58 & 108.00 & 105.55 & 103.66 & 102.47 \\
Eom & 100.87 & 104.17 & 106.57 & 105.77 & 107.69 & 109.55 & 110.38 & 107.03 & 108.03 & $\ldots \ldots$ & 103.60 & 102.55 \\
\hline
\end{tabular}

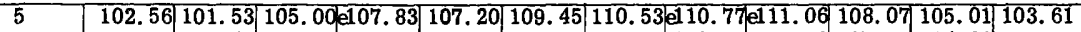

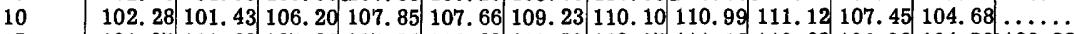

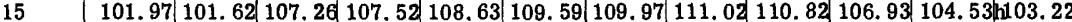

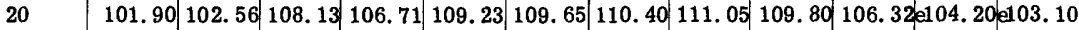

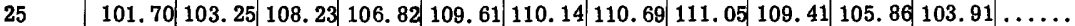

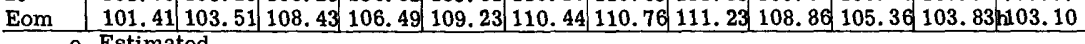

e Estimated.

h Tape measurement.

24. 11.14.122. Charles Waldrop. Drilled irrigation water-table well in valley fill, diam $12 \mathrm{in}$, reported depth $210 \mathrm{ft}$, cased to 198 . Lsd 4, $405 \mathrm{ft}$ above $\mathrm{msl}$. MP top of 1-in hole in pumpbase, $0.80 \mathrm{ft}$ above lsd. Highest water level 107.66 below 1sd, Jan. 23, 1952; lowest 190.38 below 1sd, May 11, 1956. Records available: 1951-60.

\begin{tabular}{|c|c|c|c|c|c|c|c|}
\hline Date & $\begin{array}{l}\text { Water } \\
\text { level }\end{array}$ & Date & $\begin{array}{l}\text { Water } \\
\text { level }\end{array}$ & Date & $\begin{array}{c}\text { Water } \\
\text { level }\end{array}$ & Date & $\begin{array}{l}\text { Water } \\
\text { level }\end{array}$ \\
\hline in. 5,1956 & 131.59 & Jan. 15,1957 & 135.50 & Apr. 22, 1958 & 2201.10 & Aug. 11,1959 & 155.71 \\
\hline ar. & 13 & Apr. 9 & a203.90 & Aug. & 37 & Nov. & bils \\
\hline lay 11 & 38 & Aug. 12 & 14 & Nov. 17 & 14 & Jan. 12,1960 & 0146. \\
\hline 12 & a183. 90 & Nov. 23 & b158.87 & Jan. 20, 1959 & 14 & Apr. & 209.20 \\
\hline eept. 11 & $\begin{array}{c}\text { (a) } \\
138.85\end{array}$ & Jan. 17,1958 & 139.45 & Apr. 21 & 156.11 & Nov. 14 & 157.09 \\
\hline
\end{tabular}

a Well being pumped.

b Well pumped recently.

24. 11. 24. 311. Madrid Bros. Drilled irrigation water-table well in valley fill, diam 16 in, depth $400 \mathrm{ft}$ (previously reported $200 \mathrm{ft}$ ), perforated 100-198. Lsd 4, $200 \mathrm{ft}$ above msl. MP top of casing, $0.83 \mathrm{ft}$ above lsd. Highest water level 87.71 below Isd, Jan. 23, 1952; lowest 114. 89 below 1sd, Apr. 21, 1959. Records available: 1951-59. Measurement discontinued.

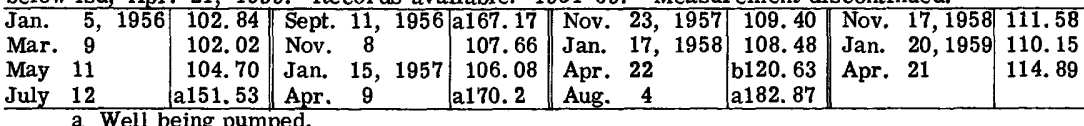

a Well being pumped.

b Well pumped recently.

25.6. 2. 111. C. W. Johnson, Jr. Drilled irrigation artesian well in valley fill, diam 16 in, depth $235 \mathrm{ft}$, perforated 180-235, gravel packed. MP top of casing, $1.30 \mathrm{ft}$ above lsd. Highest water level 0.45 below 1sd, Mar. 14, 1953; lowest 36.86 below 1sd, Nov. 12, 1953. Records available: 1952-60.

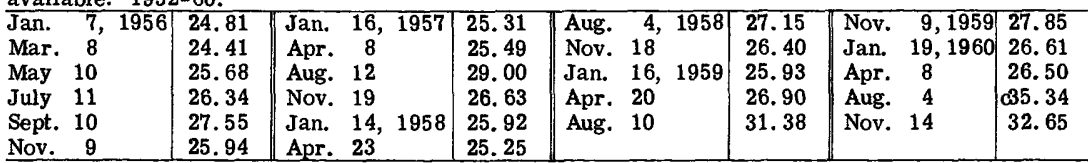

c Nearby well being pumped.

25.6. 7. 211. H. C. Telles. Drilled irrigation water-table well in valley fill, diam 16 in, depth $230 \mathrm{ft}$. MP top of casing, $0.50 \mathrm{ft}$ above $1 \mathrm{sd}$. Highest water level 65.34 below $1 \mathrm{sd}, \mathrm{Mar}, 14$, 1953; lowest 97.85 below 1sd, May 20, 1954. Records available: 1953-60.

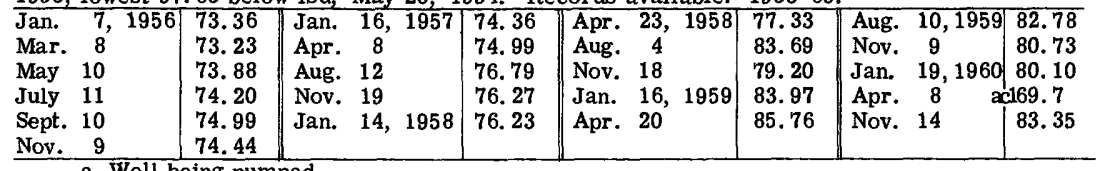

a Well being pumped.

c Nearby well being pumped.

26.9.11. 211. State of New Mexico. Dug and drilled unused water-table well in valley fill, diam $12 \mathrm{in,} \mathrm{depth} 80 \mathrm{ft}$. MP top of concrete well curb, at south side of well, at 1sd. Highest water level 36. 92 below 1sd, Apr. 15, 1939; lowest 57.40 below lsd, Nov. 15, 1960. Records available: 1939-60. 
26.9.11. 211--Continued.

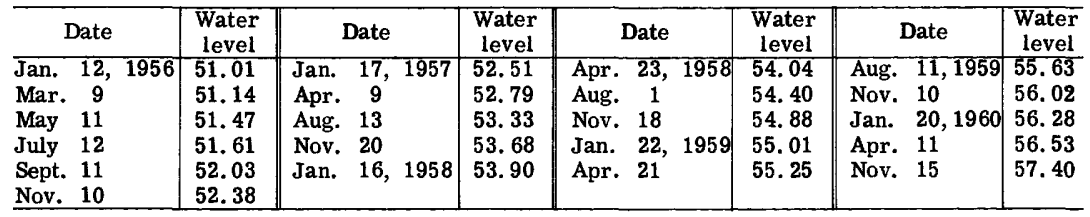

27. 8. 35. 122. Mrs. M. M. Gibson. Drilled irrigation water-table well in valley fill, diam 12 to 8 in, depth $550 \mathrm{ft}$, cased to 550, perforated 155-550. MP top of casing, $1.40 \mathrm{ft}$ above 1sd. Highest water level 20.84 below isd, Mar. 16, 1953; lowest 54.80 below lsd, Aug. 11, 1959. Records available: $1952-60$.

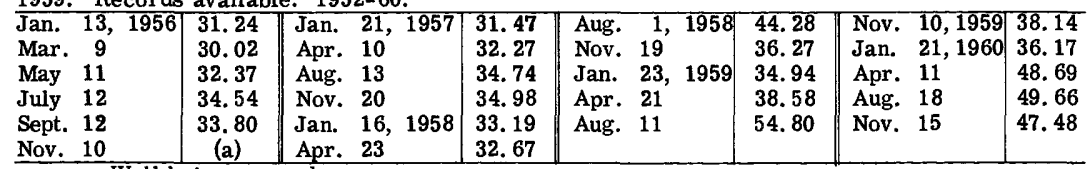

a Well being pumped.

27. 9.1.431. W. A. Prater. Drilled irrigation well in valley fill, diam $16 \mathrm{in}$, depth $62 \mathrm{ft}$. MP top edge of rectangular hole in pumpbase, $0.65 \mathrm{ft}$ above isd. Highest water level 30.61 below Isd, Jan. 19, 1954; lowest 41.68 below lsd, Sept. 14, 1954. Records available: 1954-60.

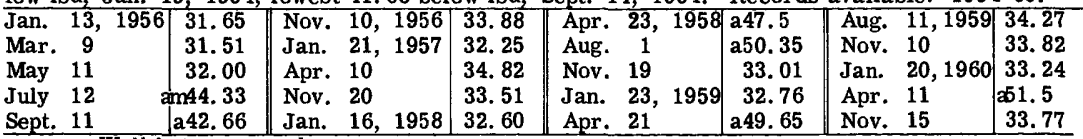

a Well being pumped.

m Measurement uncertain.

28. 8. 36.111. M. R. Hemley. Drilled irrigation artesian well in valley fill, diam 16 in, depth $270 \mathrm{ft}$, cased to 250 , gravel packed, casing perforated. Lsd 4, $020 \mathrm{ft}$ above msl. MP top of casing at high point on northwest side of well, $0.80 \mathrm{ft}$ above Isd (since Nov. 20, 1957). Highest water level 22. 61 below 1sd, Jan. 26, 1953; lowest 55.06 below 1sd, Aug. 8, 1960. Records available: $1952-60$

\begin{tabular}{|c|c|c|c|c|c|c|c|}
\hline $\begin{array}{lrr}\text { Jan. } & 14, & 1956 \\
\text { Mar. } & 9 & \\
\text { May } & 11 & \\
\text { Sept. } & 12 & \\
\text { Nov. } & 10 & \\
& & \end{array}$ & $\begin{array}{l}33.15 \\
31.73 \\
39.90 \\
43.87 \\
38.74 \\
34.80\end{array}$ & $\begin{array}{lll}\text { Apr. } & 10,1957 \\
\text { Aug. } & 13 \\
\text { Nov. } 20 \\
\text { Jan. } 18,1958 \\
\text { Apr. } 23\end{array}$ & $\begin{array}{l}38.10 \\
49.08 \\
38.44 \\
36.65 \\
39.00\end{array}$ & $\begin{array}{lrr}\text { Aug. } & 5, & 1958 \\
\text { Nov. } & 19 & \\
\text { Jan. } & 24, & 1959 \\
\text { Apr. } & 22 & \\
\text { Aug. } & 12 & \end{array}$ & $\begin{array}{l}47.36 \\
38.03 \\
36.32 \\
40.23 \\
47.00\end{array}$ & $\begin{array}{lr}\text { Nov. } & 10,1959 \\
\text { Jan. } & 21,1960 \\
\text { Apr. } & 11 \\
\text { Aug. } & 8 \\
\text { Nov. } & 15\end{array}$ & $\begin{array}{l}40.15 \\
37.75 \\
50.08 \\
55.06 \\
47.52\end{array}$ \\
\hline
\end{tabular}

\section{Otero County}

Tularosa-Alamogordo Area

14. 10. 31.144. Luther Watson. Drilled irrigation water-table well in bolson deposits, diam $17 \mathrm{in}$, depth $230 \mathrm{ft}$, cased, 16 to $14 \mathrm{in}, 0-130$. MP top edge of 1 -in hole in pumpbase, $0.70 \mathrm{ft}$ above isd. Highest water level 73.75 below isd, Apr, 8, 1952; lowest 108. 26 below Isd, Apr. 16, 1954. Records available: 1952-60.

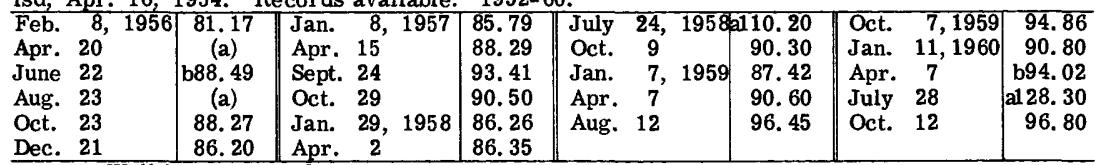

a Well being pumped.

b Well pumped recently.

17. 9. 24. 343. U. S. Air Force. About $0.75 \mathrm{mi}$ west of Holloman Air Force Base pumping station. Drilled public-supply water-table well in bolson deposits, diam 10 in, depth $236 \mathrm{ft}$, cased to 236. MP top of $1 \frac{1}{2}$-in pipe with screw plug at south side of concrete base, $2.10 \mathrm{ft}$ above Isd (since Aug. 12, 1959). Highest water level 61. 42 below 1sd, Apr. 7, 1960; lowest 78.72 below lsd, Oct. 23, 1956. Records available: 1955-60. 
17. 9. 24. 343--Continued.

\begin{tabular}{|c|c|c|c|c|c|c|c|}
\hline Date & $\begin{array}{c}\text { Water } \\
\text { level }\end{array}$ & Date & $\begin{array}{l}\text { Water } \\
\text { level }\end{array}$ & Date & $\begin{array}{c}\text { Water } \\
\text { level }\end{array}$ & Date & $\begin{array}{c}\text { Water } \\
\text { level }\end{array}$ \\
\hline $\begin{array}{lcc}\text { Apr. } & 29, & 1955 \\
\text { June } & 15 & \\
\text { Aug. } & 25 & \\
\text { Oct. } & 14 & \\
\text { Dec. } & 12 & \\
\text { Jan. } & 16, & 1956 \\
\text { Feb. } & 9 & \end{array}$ & $\begin{array}{c}62.46 \\
63.63 \\
c 70.56 \\
\text { (a) } \\
\text { (a) } \\
65.58 \\
63.42\end{array}$ & $\begin{array}{lll}\text { Apr. } & 20,1956 \\
\text { June } & \mathbf{2 2} & \\
\text { Aug. } & 23 & \\
\text { Oct. } & 23 & \\
\text { Jan. } & 16, & 1957 \\
\text { Sept. } & 24 & \\
\text { Oct. } & 29 & \end{array}$ & $\begin{array}{c}\text { (a) } \\
\text { b84. } 75 \\
75.88 \\
78.72 \\
73.91 \\
68.37 \\
\text { (a) }\end{array}$ & $\begin{array}{lrr}\text { Jan. } & 28, & 1958 \\
\text { Apr. } & 2 & \\
\text { July } & 24 & \\
\text { Oct. } & 9 & \\
\text { Jan. } & 7, & 1959 \\
\text { Apr. } & 7 & \end{array}$ & $\begin{array}{r}66.06 \\
66.32 \\
b 81.55 \\
64.27 \\
63.20 \\
62.55\end{array}$ & $\begin{array}{lr}\text { Aug. } & 12,1959 \\
\text { Oct. } & 7 \\
\text { Jan. } & 11,1960 \\
\text { Apr. } & 7 \\
\text { July } & 28 \\
\text { Oct. } & 12\end{array}$ & $\begin{array}{l}62.58 \\
62.45 \\
61.92 \\
61.42 \\
65.66 \\
63.95\end{array}$ \\
\hline
\end{tabular}

a Well being pumped

b Well pumped recently.

c Nearby well being pumped.

26. 17. 21.333. Bryce Dugger. Drilled irrigation artesian well in Bone Spring Limestone of Permian age (Leonard Series), diam $18 \mathrm{in}$, depth 1,100 ft. MP top of casing, $1.00 \mathrm{ft}$ above 1sd. Highest water level 182.52 below lsd, Feb. 14, 1956; lowest 200.76 below 1sd, Aug. 19, 1960. Records available: 1956-60.

\begin{tabular}{|l|l||l|l|l|ll||l|l|l|}
\hline Feb. 14, 1956 & 182.52 & Jan. 15, 1957 & 184.47 & Aug. 14, 1958 & 196. 21 & Jan. 25, 1960 & 189.18
\end{tabular} \begin{tabular}{l|l|l|l|l|l|l|l|l|l} 
Apr. 11 & 187.28 & Aug. 6 & 195.46 & Jan. 22, 1959 & 187.83 & Aug. 19 & 200.76 \\
Sept. 12 & 192.74 & Jan. 29, 1958 & 186.50 & Aug. 13 & 198.36 & & & \\
\hline
\end{tabular}

26.18. 28.113. Frank Gentry. Drilled irrigation artesian well in Bone Spring Limestone of Permian age (Leonard Series), diam $18 \mathrm{in}$, depth $394 \mathrm{ft}$. MP top of casing, 2.00 ft above Isd. Highest water level 31.50 below Isd, Feb. 13, 1956; lowest 42.12 below 1sd, Sept. 12, 1956. Records available: 1956-60.

\begin{tabular}{l|l||l|l|l||l|l|l|l|l|}
\hline Feb. 13, 1956 & 31.50 & Jan. 15,1957 & 33.33 & Jan. 22, 1959 & 37.00 & Jan. 25, 1960 & 38.23
\end{tabular} Apr. 12 Sept. 12 37. 60 Aug. 6 42.12 (a) Aug. 13 (a) Aug. 19

a Well being pumped.

\section{Quay County}

House Area

5.29.17.133. W. W. Kuykendall. Drilled unused water-table well in Ogallala Formation of Pliocene age, diam 12 in, depth $57 \mathrm{ft}$, cased 35-57. Lsd 4,748 ft above msl. MP top edge of U.S. Geol. Survey marker in west edge of east 6- x 8-in crosstie over well, at lsd. Highest water level 29.82 below 1sd, Jan. 21, 1942; lowest 48.24 below lsd, Oct. 30-31, 1954. Records available: 1941-60. For additional measurements, see Reeder and others (1960b and c).

Highest water level for the day, from recorder graph, 1956

\begin{tabular}{|c|c|c|c|c|c|c|c|c|c|c|c|c|}
\hline Day & Jan. & Feb. & Mar. & Apr. & May & June & July & Aug. & Sept. & Oct. & Nov. & Dec. \\
\hline 5 & 47.72 & 47.38 & 47.14 & 46.83 & 46.59 & 46.46 & 46.46 & 46.49 & 46.65 & 46.83 & 47.21 & 47.39 \\
\hline 10 & 47.59 & 47.34 & 47.12 & 46.81 & 46.56 & 46.47 & 46.45 & 46.50 & 46.67 & 46.87 & 47.29 & 47.38 \\
\hline 15 & 47.55 & 47.30 & 47.06 & 46.76 & 46.52 & 46.46 & 46.45 & 46.51 & 46.69 & 46.91 & 47.33 & 47. 38 \\
\hline 20 & 47.51 & 47. 27 & 46.95 & 46.73 & 46.50 & 46.46 & 46.44 & 46.54 & 46.71 & 46.97 & 47.36 & 47.36 \\
\hline 25 & 47.47 & 47.21 & 46.92 & 46.68 & 46.49 & 46.46 & 46.45 & 46.56 & 46.73 & 47.04 & 47.39 & 47.35 \\
\hline Eom & 47.42 & 47.18 & 46. 86 & 46.61 & 46.48 & 46.46 & 46.46 & 46.63 & 46.78 & 47.13 & 47.39 & 47.31 \\
\hline \multicolumn{13}{|c|}{1957} \\
\hline 5 & 47.27 & 47.12 & 46.90 & 46.69 & 46.55 & 46.79 & 46.95 & 47.33 & 47.83 & 48.04 & 48.23 & 48.05 \\
\hline 10 & 47.26 & 47.10 & 46.84 & 46.66 & 46.54 & 46.81 & 46. 99 & 47.40 & 47.89 & 48.08 & 48 & 48.02 \\
\hline 15 & 47.24 & 47.07 & 46.78 & 46.63 & 46.55 & 46.83 & 47.04 & 47.47 & 47.94 & 48.14 & $\cdots \cdots$ & 47.97 \\
\hline 20 & 47.20 & 47.04 & 46.75 & 46.60 & 46.59 & 46.86 & 47.09 & 47.57 & 47.98 & 48.21 & 48. 15 & 47.94 \\
\hline 25 & 47.17 & 47.00 & 46.74 & 46.59 & 46.65 & 46.88 & 47.16 & $\ldots \ldots$ & 48.01 & 48.22 & 48.12 & 47.91 \\
\hline Eom & 47.14 & 46 & 46.70 & 46.57 & 46.73 & 46.90 & 47.25 & 47.74 & 48.03 & 48.23 & 48.06 & 47.87 \\
\hline \multicolumn{13}{|c|}{1958} \\
\hline 5 & & 47.53 & & 47.08 & 46.90 & 47.08 & 47.36 & 47.50 & 47.53 & & 47.32 & 47.14 \\
\hline 10 & 47.69 & 47.50 & 47 & 47.04 & 46.89 & 47.13 & 47.39 & 47.48 & 47.49 & 47.41 & 47.29 & 47.09 \\
\hline 15 & 47.66 & 47.46 & 47.19 & 47.02 & 46.89 & 47.18 & & & 47.43 & 47.40 & 47.25 & 47.07 \\
\hline 20 & 47.62 & 47.43 & 47.16 & 46.98 & 46.94 & 47.22 & 47.48 & 47.49 & 47. 39 & & 47.22 & 47.03 \\
\hline 25 & 47.60 & 47.32 & 47.15 & 46.97 & 47.00 & 47.25 & 47.49 & 47.53 & 47.38 & 47.39 & 47.21 & 47.01 \\
\hline Eom & 47. & 47. & 47. & 46 & 47 & 47.31 & 50 & 47.55 & 47.39 & 47.37 & 18 & $\mathrm{e} 46.96$ \\
\hline \multicolumn{13}{|c|}{1959} \\
\hline 5 & 46.92 & 46.74 & 46.94 & 47.35 & 47.40 & 47.71 & 47.78 & 47.79 & 47.92 & 47.90 & 47.93 & $\mathrm{e} 47.93$ \\
\hline 10 & 46.87 & 46.73 & 47.06 & 47.37 & 47.45 & 47.74 & 47.80 & 47.83 & 47.91 & 47.92 & 47.95 & 47.90 \\
\hline 15 & 46. 83 & 46.73 & 47.14 & 47.37 & 47.48 & 47.75 & 47.85 & 47.87 & 47.88 & 47.94 & 47.97 & 47.88 \\
\hline 20 & 46.79 & 46.75 & 47.18 & 47.39 & 47.55 & 47.78 & 47.82 & 47.89 & 47. 85 & 47.91 & 47.97 & 47.84 \\
\hline 25 & 46. 74 & 46.81 & 47.24 & 47.36 & 47.60 & 47.78 & 47.80 & 47.93 & 47.83 & 47.88 & 47.95 & 47.80 \\
\hline Eom & 46.74 & 46. 86 & 47.31 & 47.36 & 47.67 & 47.82 & 47.78 & 47.92 & 47.86 & 47.89 & 47.95 & 47.76 \\
\hline
\end{tabular}


1960

\begin{tabular}{l|l|l|l|l|l|l|l|l|l|l|l|l}
\hline Day & Jan. & Feb. & Mar. & Apr. & May & June & July & Aug. & Sept. & Oct. & Nov. & Dec. \\
\hline 5 & 47.72 & 47.48 & 47.27 & 47.06 & 46.88 & 47.25 & 47.66 & 47.34 & 46.87 & 46.89 & 46.80 & 46.47 \\
10 & 47.68 & 47.43 & 47.22 & 47.03 & 46.90 & 47.35 & 47.74 & 47.28 & 46.84 & 46.89 & 46.73 & 46.43 \\
15 & 47.65 & 47.40 & 47.20 & 46.98 & 46.95 & 47.42 & 47.71 & 47.23 & 46.84 & 46.85 & 46.72 & 46.38 \\
20 & 47.61 & 47.36 & 47.18 & 46.95 & 47.02 & 47.51 & 47.58 & 47.09 & 46.89 & 46.87 & 46.62 & 46.34 \\
25 & 47.56 & 47.32 & 47.14 & 46.93 & 47.07 & 47.57 & 47.50 & 46.97 & 46.93 & 46.85 & 46.58 & 46.28 \\
Eom & e47.51 & 47.30 & 47.09 & 46.91 & 47.14 & 47.63 & 47.41 & 46.94 & 46.92 & 46.82 & 46.52 & 46.21 \\
\hline
\end{tabular}
e Estimated.

5.30.18.331. W. C. and H. J. Lee. Formerly W. M. Lee. Drilled irrigation watertable well in Ogallala Formation of Pliocene age, diam $16 \mathrm{in}$, depth $75 \mathrm{ft}$, cased to 60 . MP top edge of 2-in hole in drum over well, $0.50 \mathrm{ft}$ above lsd. Highest water level 34.76 below isd, Mar. 28, 1946; lowest 50.91 below Isd, Sept. 18, 1956. Records available: 1944-60.

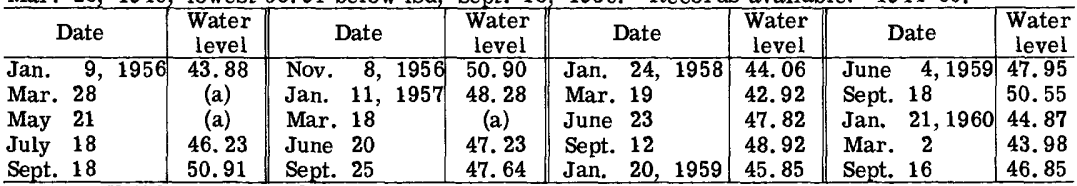

a Well being pumped.

6. 28. 24. 233. G. B. Irwin. Formerly Byers Irwin. Drilled irrigation water-table well in Ogallala Formation of Pliocene age, diam 16 in, reported depth $131 \mathrm{ft}$, cased 79-131. MP top of 2-in opening in concrete base, $1.21 \mathrm{ft}$ above lsd. Highest water level 77.97 below lsd, Mar. 27, 1944; lowest 95.49 below lsd, Sept. 15, 1955. Records available: 1944-60.

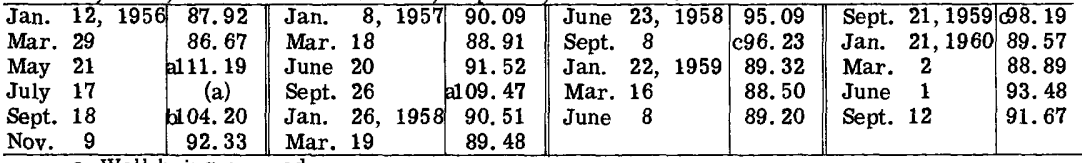

a Well being pumped.

b Well pumped recently.

c Nearby well being pumped.

6. 29.30.412. Grady and Irene Terrill. Formerly R. W. Dean. Drilled unused (previously reported irrigation) water-table well in Ogallala Formation of Pliocene age, diam 18 in, depth $122 \mathrm{ft}$, cased to 122 . MP top of casing at high point on southeast side of well, $0.78 \mathrm{ft}$ above lsd. Highest water level 73.63 below Isd, Apr. 1, 1947; lowest 86. 65 below Isd, Jan. 21, 1960. Records available: $1946-60$.

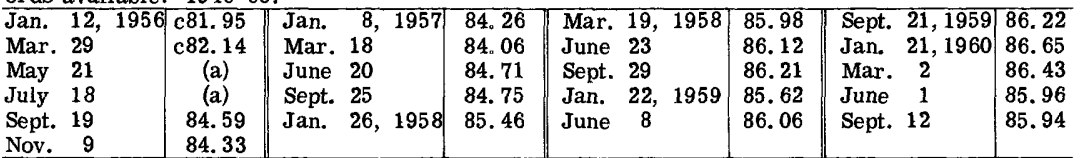

a Well being pumped.

c Nearby well being pumped.

\section{Roosevelt County}

Portales Valley

1N. 33. 36. 400c. Woodburn Bros. Drilled observation water-table well in valley fill, diam $8 \mathrm{in}$, depth $43 \mathrm{ft}$, cased to 40 , perforated 8-40. MP top of casing, 2. $86 \mathrm{ft}$ above lsd. Highest water level 7.88 below lsd, Apr. 9, 1953; lowest 16.12 below lsd, July 5-6, 1960 . Records available: $1953-60$. For additional measurements, see Reeder and others (1960b and c).

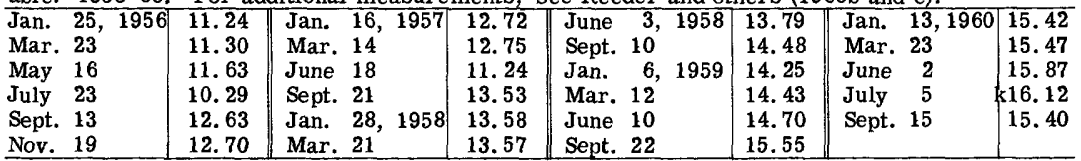

k From recorder graph. 
1.32.14. 431. Robert Morrison. Drilled unused water-table well in valley fill, diam 12 in, depth $104 \mathrm{ft}$, cased to 85. MP at lower edge of board cover over well, $1.14 \mathrm{ft}$ below lsd. Highest water level 43.55 below lsd, Apr. 6, 1945; lowest 66.80 below Isd, June 3, 1960. Records available: $1944-60$.

\begin{tabular}{|c|c|c|c|c|c|c|c|}
\hline Date & $\begin{array}{l}\text { Water } \\
\text { level }\end{array}$ & Date & $\begin{array}{l}\text { Water } \\
\text { level }\end{array}$ & Date & $\begin{array}{l}\text { Water } \\
\text { level }\end{array}$ & Date & $\begin{array}{c}\text { Water } \\
\text { level }\end{array}$ \\
\hline $\begin{array}{ll}\text { Jan. } & 26,1956 \\
\text { Mar. } & 23 \\
\text { May } & 16 \\
\text { July } & 23 \\
\text { Sept. } & 13 \\
\text { Nov. } & 19\end{array}$ & $\begin{array}{r}c 59.18 \\
56.96 \\
c 61.77 \\
60.72 \\
c 65.24 \\
60.28\end{array}$ & $\begin{array}{ll}\text { Jan. } & 18,1957 \\
\text { Mar. } & 14 \\
\text { June } & 17 \\
\text { Sept. } & 20 \\
\text { Jan. } & 27,1958\end{array}$ & \begin{tabular}{|r|}
59.69 \\
$c 61.60$ \\
$c 62.03$ \\
64.37 \\
60.62
\end{tabular} & $\begin{array}{lrl}\text { Mar. } & 21, & 1958 \\
\text { June } & 3 & \\
\text { Sept. } & 10 & \\
\text { Jan. } & 6, & 1959 \\
\text { Mar. } & 12 & \end{array}$ & $\begin{array}{l}60.04 \\
59.62 \\
64.59 \\
60.81 \\
60.27\end{array}$ & $\begin{array}{lr}\text { Sept. } & 21,1959 \\
\text { Jan. } & 7,1960 \\
\text { Mar. } & 23 \\
\text { June } & 3 \\
\text { Sept. } & 14\end{array}$ & $\begin{array}{l}65.13 \\
62.13 \\
61.42 \\
66.80 \\
65.95\end{array}$ \\
\hline
\end{tabular}

c Nearby well being pumped.

1.34.25.211. J. B. H. Young. Drilled unused water-table well in valley fill, diam 12 in, reported depth $101 \mathrm{ft}$. MP top of concrete base, at lsd. Highest water level 31.98 below lsd, May 16, 1933; lowest 70.76 below 1sd, Sept. 20, 1960. Records available: 1931-60. For additional measurements, see Reeder and others (1960b and c).

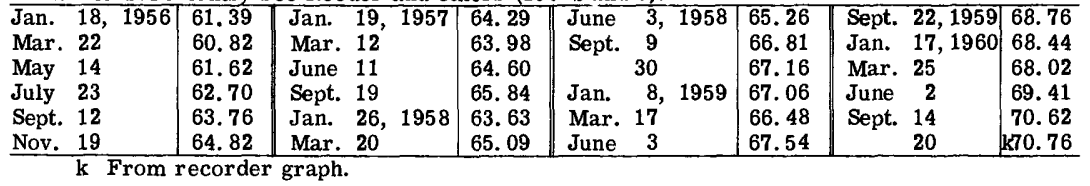

1.35.11.241. A. Hobbs Estate. Drilled unused water-table well in valley fill, diam 6 in, depth $51 \mathrm{ft}$. MP top of casing, 3.25 ft above lsd. Highest water level 13.98 below lsd, Mar. 27, 1943; lowest 20.09 below 1sd, Sept. 22, 1940. Records available: $1940-60$.

\begin{tabular}{ll|l|l|l|l|l|l|l|l|l}
\hline Jan. 18, 1956 & 17.88 & Jan. 19, 1957 & 18.17 & June 4, 1958 & 18.73 & Sept. 22, 1959 & 19.19 \\
Mar. 24 & 17.93 & Mar. 14 & 18.24 & Sept. 9 & & 19.01 & Jan. 14, 1960 & 19.06 \\
May 15 & 18.00 & June 18 & 18.33 & Jan. & $\mathbf{7}, 1959$ & 18.89 & Mar. 24 & 19.08 \\
July 20 & 18.14 & Sept. 21 & 18.65 & Mar. 12 & 18.95 & June 2 & 19.32 \\
Sept. 14 & 18.21 & Jan. 25, 1958 & 18.55 & June 10 & 19.10 & Sept. 13 & 19.44 \\
Nov. 14 & $\mathbf{1 8 . 1 7}$ & Mar. 21 & 18.59 & & & & & \\
\hline
\end{tabular}

2. 34. 2. 233. Louisa Trout. Drilled unused water-table well in valley fill, diam $12 \dot{\mathrm{in}}$, depth $89 \mathrm{ft}$. MP top of casing, at lsd. Highest water level 32.71 below lsd, Mar. 6-15, 1942; lowest 81.80 below lsd, Oct. 5, 1960. Records available: 1931-60. For additional measurements, see Reeder and others $(1960 \mathrm{~b}$ and $\mathrm{c})$.

\begin{tabular}{|c|c|c|c|c|c|c|c|c|c|c|c|c|}
\hline \\
\hline Day & Jan. & Feb. & Mar. & Apr. & May & June & July & Aug. & Sept. & Oct. & Nov. & Dec. \\
\hline 5 & 67.42 & 67.20 & 66.80 & & & & 70.00 & 70.85 & 71.56 & 71.08 & 70.89 & 70.56 \\
\hline 10 & 67.40 & 67.09 & 66.85 & 1. & & 69.15 & 70.22 & 71.02 & 71.42 & 71.27 & 70.88 & 70.53 \\
\hline 15 & 67.37 & 67.02 & 66.80 & & 68.35 & 69.43 & 70.37 & 71.14 & 71.58 & 71.25 & 70.80 & 70.98 \\
\hline 20 & 67.26 & 66.98 & 67.10 & e67.50 & 68.62 & 69.40 & 70.92 & 71.15 & 71.33 & 71.13 & 70.78 & 70.78 \\
\hline 25 & 67.18 & 66.88 & 67.00 & ... & $\ldots$ & 69.40 & 70.43 & 71.39 & 71.20 & 71.10 & 70.69 & 70.66 \\
\hline Eom & 67.30 & 66.88 & & & & 69.85 & 70.69 & 71.55 & 71.18 & 70.97 & 70.60 & 70.58 \\
\hline \multicolumn{13}{|c|}{1957} \\
\hline 5 & 70.55 & 70.52 & 70.55 & 70.26 & 70.83 & 71.07 & 71.80 & 72.73 & & 73.46 & $\overline{72.59}$ & 72.33 \\
\hline 10 & 70.49 & 70.70 & 70.39 & 70.22 & 71.27 & 70.98 & 71.74 & 72.90 & & 73.20 & 72.53 & 72. 32 \\
\hline 15 & 70.65 & 70.58 & 70.39 & 70.35 & 71.08 & 71.15 & 72.05 & 73.05 & & 73.08 & 72.46 & 72.18 \\
\hline 20 & 70.72 & 70.50 & 70.38 & 70.78 & 71.09 & 71.45 & 72.30 & 73.12 & e73. 25 & 72.92 & 72.48 & 72.10 \\
\hline 25 & 70.78 & 70.45 & 70.35 & 70.60 & 71. 27 & 71.75 & 72.43 & 73.28 & 73.50 & 72.85 & 72.42 & 72.14 \\
\hline Eom & 70.58 & 70.43 & 70.30 & 70.60 & 71.18 & 71. 89 & 72.59 & & 73. 30 & 72.68 & 72.38 & 72.44 \\
\hline \multicolumn{13}{|c|}{1958} \\
\hline 5 & 72.51 & 71.90 & 71.67 & 71.61 & 72.30 & 73.11 & 72.64 & 73.84 & 74.37 & 73.68 & $\overline{73.21}$ & 72.96 \\
\hline 10 & 72.35 & 71.86 & 71.62 & 71.58 & 72. 72 & 73.33 & 72. 64 & 73.97 & 74. 24 & 73.59 & 73.22 & 72.90 \\
\hline 15 & 72.19 & 71.81 & 71.57 & 71.51 & 72.57 & 73.22 & 73.13 & & 74.09 & 73.53 & 73. 17 & 72.89 \\
\hline 20 & 72.10 & 71.79 & 71.55 & 71.61 & 72.40 & 73.04 & 73.04 & 74.31 & 74.00 & 73.45 & 73.13 & 72.87 \\
\hline 25 & 72.00 & 71.69 & 71.51 & 71.63 & 72. 33 & 72.92 & 73.48 & 74.15 & 73.91 & 73.42 & 73.11 & 72.80 \\
\hline Eom & 71.98 & 71.70 & 71.51 & 72.08 & 73.04 & 72.74 & 73.59 & 74.20 & 73.82 & 73.28 & 73.04 & 72.79 \\
\hline \multicolumn{13}{|c|}{1959} \\
\hline 5 & 72.72 & 72.50 & & 72.70 & 74.13 & 74.14 & & & 75.53 & 75.60 & $\overline{75.32}$ & \\
\hline 10 & 72.72 & 72.48 & & 73.18 & 73.85 & 74.08 & & & 75.50 & 75.48 & 75.14 & \\
\hline 15 & 72. 67 & 72.39 & 72. 78 & 73.18 & 73.69 & 74.39 & & $\ldots$ & 76.06 & 75.34 & 75.07 & \\
\hline 20 & 72.59 & 72.39 & 72.79 & 73.40 & 73. 75 & e75.00 & h74.30 & & 76.02 & 75.31 & 75.00 & e74.58 \\
\hline 25 & 72.54 & & 72.82 & 73.70 & 73. 64 & $\ldots \ldots$ & 74.28 & 75.88 & 76.04 & 75.64 & e74. 98 & $\cdots$ \\
\hline Eom & 72.54 & & 73.17 & 73.78 & 74.08 & $\ldots$ & 74.90 & 75.59 & 75.30 & 75.48 & $\ldots$ & $\ldots$ \\
\hline
\end{tabular}




\section{Sandoval County}

\section{Albuquerque Area}

12. 3E. 22. 232. Mrs. Engle. Drilled unused water-table well in Santa Fe Group of middle (?) Miocene to Pleistocene (?) age, diam $14 \mathrm{in}$, depth $84 \mathrm{ft}$. MP top north side of casing, $3.50 \mathrm{ft}$ above 1sd. Highest water level 34.05 below 1sd, Sept. 19, Oct. 30, 1957; lowest 35.58 below Isd, Jan. 25, 1957. Records available: 1956-60.

\begin{tabular}{|c|c|c|c|c|c|c|c|}
\hline Date & $\begin{array}{l}\text { Water } \\
\text { level }\end{array}$ & Date & $\begin{array}{l}\text { Water } \\
\text { level }\end{array}$ & Date & $\begin{array}{r}\text { Water } \\
\text { level }\end{array}$ & Date & $\begin{array}{c}\text { Water } \\
\text { level }\end{array}$ \\
\hline Oct. 16,1956 & 34.12 & Apr. 21, 1958 & 35.14 & Mar. 19,1959 & 35.12 & Feb. $\quad 3,1960$ & 35.02 \\
\hline Nov. 20 & 34.15 & May 12 & 35.22 & Apr. 16 & 35.24 & Mar. 4 & 35.22 \\
\hline Jan. 25,1957 & 35.58 & June 13 & 35.02 & May & 8 & Apr. & 35 \\
\hline May & 34.80 & July 11 & 34.70 & June & 5 & May & 12 \\
\hline June & 34.65 & Aug. 12 & 34.30 & July & 34.60 & June & 34.81 \\
\hline July & 34.40 & Sept. 8 & 34.22 & Aug. 19 & 34.56 & July 13 & 34.30 \\
\hline Aug. & 34.19 & Oct. 24 & 34.30 & Sept. 11 & 34.71 & Aug. 3 & 34.30 \\
\hline Sept. 19 & 34.05 & Nov. 21 & 34.30 & Oct. 8 & 31 & Sept. 6 & 11 \\
\hline Oct. 30 & 34.05 & Dec. 15 & 34.45 & Nov, 10 & 34.48 & Oct. 19 & 34.24 \\
\hline Jan. 28, 1958 & 34.67 & Jan, 26, 1959 & 34.72 & Dec. & 34.59 & Nov. 9 & 34.28 \\
\hline Mar. 12 & 34.99 & Feb. 26 & 34.97 & 6,1960 & 34.87 & Dec. 12 & 34.54 \\
\hline
\end{tabular}

\section{Santa Fe County}

\section{Estancia Valley}

10. 8.13.133. W. R. Irby. Drilled irrigation water-table well in valley fill, reported depth $518 \mathrm{ft}$. MP lower inside edge of hole in south side of casing, $0.45 \mathrm{ft}$ above isd. Highest water level 86.75 below lsd, Feb. 22, 1950; lowest 106. 85 below lsd, Sept. 27, 1960. Records available: $1950-60$.

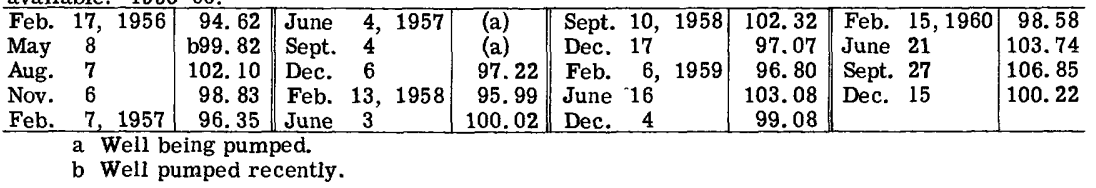

10.9.29. 130. Glen Terry. Drilled irrigation water-table well in Glorieta Sandstone of Permian age, diam $14 \mathrm{in}$, reported depth $200 \mathrm{ft}$, cased to 140 . MP top edge of 3-in pipe on north side of pump, $1.30 \mathrm{ft}$ above lsd. Highest water level 55.13 below lsd, Feb. 18, 1949; lowest 74.51 below Isd, Sept. 27, 1960. Records available: 1949-60.

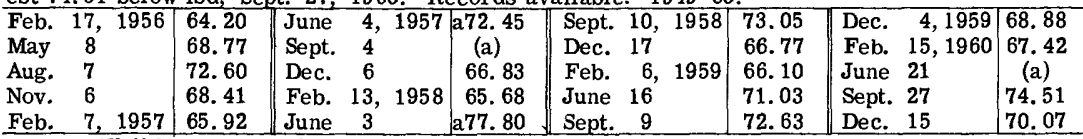

Feb. $7,1957 \quad 65.92 \quad$ June

a Well being pumped.

\section{Santa Fe Area}

16. 8.12.131. Golf Course. Drilled unused well in Ancha (?) Formation of upper Pliocene or Pleistocene age and Tesuque (?) Formation of middle (?) Miocene to lower Pliocene age, diam $5 \mathrm{in}$, depth $400 \mathrm{ft}$. MP top of 3/8-in hole in cover plate, $0.20 \mathrm{ft}$ above isd. Highest water level 259. 39 below lsd, Oct. 12, 1960; lowest 271.97 below 1sd, Jan. 9, 1959. Records available: $1951,1953-60$.

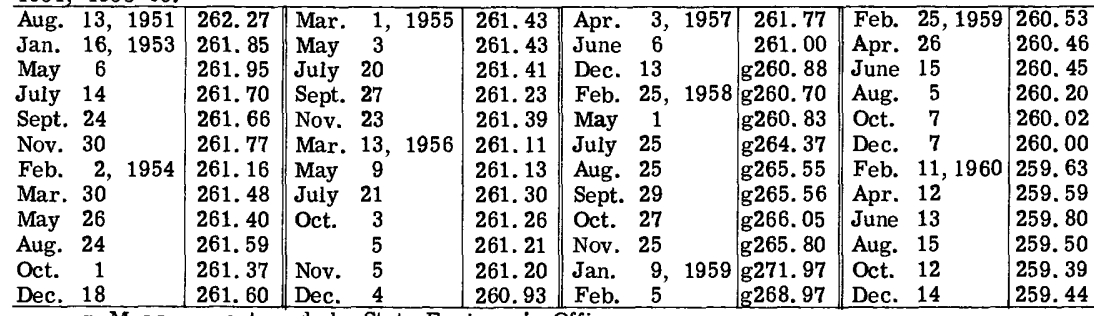

$\mathrm{g}$ Measurement made by State Engineer's Office. 
17. 9. 23. 334. J. Hanson \& Sons. Drilled unused well in Tesuque (?) Formation of middle (?) Miocene to lower Pliocene age, diam $7 \mathrm{in}$, depth $200 \mathrm{ft}$. MP top of recorder shelf, $3.84 \mathrm{ft}$ above lsd. Highest water level 140.84 below Isd, June 15, 1959; lowest 156.17 below Isd,

Mar. 15, 1960. Records available: 1959-60.

\begin{tabular}{|c|c|c|c|c|c|c|c|}
\hline Date & $\begin{array}{l}\text { Water } \\
\text { level }\end{array}$ & Date & $\begin{array}{l}\text { Water } \\
\text { level }\end{array}$ & Date & $\begin{array}{c}\text { Water } \\
\text { level }\end{array}$ & Date & $\begin{array}{l}\text { Water } \\
\text { level }\end{array}$ \\
\hline $\begin{array}{l}\text { Mar. } 4,1959 \\
\text { Apr. } 26 \\
\text { June } 15 \\
\text { Aug. } 16\end{array}$ & $\begin{array}{l}142.39 \\
141.79 \\
140.84 \\
142.87\end{array}$ & $\begin{array}{l}\text { Oct. } \quad 8,1959 \\
\text { Dec. } 7 \\
\text { Feb. } 11,1960\end{array}$ & $\begin{array}{l}145.89 \\
150.40 \\
155.64\end{array}$ & $\begin{array}{l}\text { Mar. 15, } 1960 \\
\text { Apr. } 12 \\
\text { June } 13\end{array}$ & $\begin{array}{r}\mathrm{k} 156.17 \\
154.69 \\
153.54\end{array}$ & $\begin{array}{ll}\text { Aug. } & 15,1960 \\
\text { Oct. } & 12 \\
\text { Dec. } & 14\end{array}$ & $\begin{array}{l}151.89 \\
150.41 \\
148.96\end{array}$ \\
\hline
\end{tabular}

\section{Sierra County}

\section{Hot Springs Area}

6. Harry Dakos. Lot 4 , block 8 in Truth or Consequences. Drilled unused artesian well in Magdalena Group of Pennsylvanian and lowermost Permian age, diam 7 in, depth $105 \mathrm{ft}$. Lsd 4, 243. $75 \mathrm{ft}$ above msl. MP top of extended casing, 3. $72 \mathrm{ft}$ above $1 \mathrm{sd}$. Highest water level 1.66 above Isd, Dec. 24, 1941, June 27, 1958; lowest 1. 00 below 1sd, Nov. 29, 1956. Records available: 1940-60. For additional measurements, see Reeder and others (1960b and c).

Highest water level for the day, from recorder graph, 1956

\begin{tabular}{|c|c|c|c|c|c|c|c|c|c|c|c|c|}
\hline Day & Jan. & Feb. & Mar. & Apr. & May & June & July & Aug. & Sept. & Oct. & Nov. & Dec. \\
\hline 5 & -0.18 & -0.15 & +0.04 & +0.43 & +0.13 & 0.00 & -0.03 & -0.15 & -0.02 & -0.57 & -0.70 & -0.70 \\
\hline 10 & .19 & .13 & -.15 & .38 & +.03 & +.11 & +.09 & .05 & .10 & .62 & .70 & .69 \\
\hline 15 & .15 & .12 & .00 & .31 & -.12 & .09 & +.09 & .01 & .30 & .62 & .74 & .71 \\
\hline 20 & .17 & .17 & .19 & .29 & .12 & .15 & .00 & .10 & .41 & .68 & .72 & .67 \\
\hline 25 & .19 & .14 & -.38 & .29 & .19 & .08 & .00 & .17 & .49 & .69 & .69 & .67 \\
\hline Eom & .17 & $e-.15$ & $\begin{array}{r}+.48 \\
\end{array}$ & .29 & -.22 & +.01 & 0.07 & .13 & .53 & .69 & .71 & .66 \\
\hline \multicolumn{13}{|c|}{1957} \\
\hline 5 & -0.66 & -0.68 & -0.59 & -0.19 & -0.36 & -0.54 & +0.02 & +0.28 & +0.30 & +0.39 & +0.69 & +0.80 \\
\hline 10 & .71 & .67 & .58 & .13 & .38 & -.16 & .13 & . 28 & .29 & .40 & .72 & .81 \\
\hline 15 & .67 & .64 & .60 & .09 & .42 & .00 & .20 & .37 & .58 & .47 & .79 & .81 \\
\hline 20 & .65 & .63 & .52 & .14 & .45 & +.10 & .20 & .30 & .55 & .54 & .78 & .80 \\
\hline 25 & .69 & .60 & .31 & .25 & .52 & +.02 & .30 & .27 & .42 & .56 & .79 & 81 \\
\hline Eom & .68 & .59 & .17 & .24 & .54 & -.04 & .16 & $\ldots$ & .38 & .64 & .79 & .82 \\
\hline \multicolumn{13}{|c|}{1958} \\
\hline 5 & +0.89 & +1.25 & +1.30 & +1.47 & +1.57 & +1.61 & +1.59 & +1.50 & & +1.02 & +1.05 & +1.11 \\
\hline 10 & .83 & 1.27 & . & 1.52 & 1.58 & 1.63 & 1.56 & 1.52 & +1.17 & 1.00 & 1.07 & 1.10 \\
\hline 15 & 1.07 & 1.27 & & 1.55 & 1.57 & 1.61 & 1.57 & 1.51 & 1.11 & 1.01 & 1.11 & 1.10 \\
\hline 20 & 1.17 & 1.28 & 1.46 & 1.60 & 1.55 & 1.60 & 1.52 & 1. 35 & 1.03 & 1.02 & 1.04 & 1.11 \\
\hline 25 & 1. 24 & 1. 29 & 1.46 & 1.55 & 1.54 & 1. 61 & & 1. 30 & 1.00 & 1.00 & 1.11 & 1.18 \\
\hline Eom & 1.17 & 1.29 & 1. 47 & 1.55 & 1.56 & 1.61 & 1.49 & 1.25 & .99 & 1.00 & 1.11 & 1.10 \\
\hline \multicolumn{13}{|c|}{1959} \\
\hline 5 & +1.09 & +1.15 & $1+1.13$ & +1.10 & +0.86 & +0.71 & +0.80 & +0.81 & +0.58 & +0.57 & +0.26 & +0.18 \\
\hline 10 & 1.06 & 1.13 & 1.15 & 1.04 & .87 & .71 & .81 & .81 & .55 & .55 & .28 & .20 \\
\hline 15 & 1.07 & 1.15 & 1.11 & 1.01 & 80 & .72 & .82 & .74 & .56 & 47 & .27 & 21 \\
\hline 20 & 1.15 & 1.12 & 1.09 & .98 & .75 & .71 & .82 & .69 & .57 & .43 & .23 & .17 \\
\hline 25 & 1.14 & 1.13 & 1.14 & .94 & .71 & .72 & .87 & .65 & .56 & .41 & .24 & .23 \\
\hline Eom & 1.16 & 1.10 & 1.16 & .95 & .66 & .72 & .81 & .57 & .60 & .31 & .21 & .21 \\
\hline \multicolumn{13}{|c|}{1960} \\
\hline 5 & +0.23 & +0.45 & +0.58 & +0.77 & +0.82 & +0.71 & +0.70 & +0.50 & +0.51 & -0.08 & -0.15 & -0.07 \\
\hline 10 & . 35 & .49 & .66 & .82 & .77 & .73 & .76 & .42 & .31 & .14 & .14 & .04 \\
\hline 15 & .40 & .46 & .70 & .82 & .78 & .69 & .67 & . 41 & .30 & .13 & .11 & .01 \\
\hline 20 & .40 & e+. 50 & .71 & .81 & .75 & .66 & .63 & .40 & .13 & .11 & .12 & -.03 \\
\hline 25 & .46 & .50 & .73 & .81 & .74 & .64 & .62 & . 48 & +.02 & .12 & .10 & +.03 \\
\hline Eom & .50 & .51 & .80 & .79 & .71 & .65 & .53 & .51 & -.03 & .11 & .10 & .00 \\
\hline
\end{tabular}

6a. Harry Dakos. Lot 4 , block 8 in Truth or Consequences. Dug unused water-table well in alluvium, diam $24 \mathrm{in}$, depth $6 \mathrm{ft}$. Lsd 4, 240.71 ft above msl. MP base of recorder, $3.97 \mathrm{ft}$ above isd. Highest water level 1.37 above isd, July $5,8,1960$; lowest 2.75 below Isd, Oct. 27, 31, Nov. 3, 1956. Records available: 1941-60. For additional measurements, see Reeder and others (1960b and $\mathrm{c})$.

Highest water level for the day, from recorder graph, 1956

\begin{tabular}{l|r|r|r|r|r|r|r|r|r|r|r|r}
\hline Day & Jan. & \multicolumn{1}{|c|}{ Feb. } & \multicolumn{1}{|c}{ Mar. } & \multicolumn{1}{|c}{ Apr. } & \multicolumn{1}{|c}{ May } & June & July & Aug. & Sept. & Oct. & Nov. & Dec. \\
\hline 5 & -2.29 & -2.30 & -2.13 & -1.69 & -1.90 & -2.24 & -1.84 & -0.92 & -2.18 & -2.60 & -2.74 & -2.69 \\
10 & 2.30 & .70 & 2.18 & 1.71 & 1.99 & 2.10 & 1.97 & 1.61 & 2.15 & 2.65 & 2.72 & 2.69 \\
15 & 2.29 & 1.35 & 2.18 & 1.75 & 2.10 & 2.03 & 2.02 & 1.85 & 2.25 & 2.70 & 2.71 & 2.68 \\
20 & 2.29 & 1.75 & 2.05 & 1.78 & 2.18 & 2.00 & 2.02 & 1.98 & 2.38 & 2.73 & 2.71 & 2.68 \\
25 & 2.30 & 1.95 & 1.86 & 1.81 & 2.23 & 2.00 & 2.05 & 2.12 & 2.49 & 2.74 & 2.71 & 2.67 \\
Eom & 2.30 & 2.05 & 1.72 & 1.83 & 2.29 & 1.56 & 1.00 & 2.20 & 2.55 & 2.75 & 2.71 & 2.66 \\
\hline
\end{tabular}


6a-- Continued.

\begin{tabular}{|c|c|c|c|c|c|c|c|c|c|c|c|c|}
\hline \multicolumn{13}{|c|}{1957} \\
\hline Day & Jan. & Feb. & Mar. & Apr. & $\overline{\text { May }}$ & June & July & Aug. & Sept. & Oct. & Nov. & Dec. \\
\hline 5 & -2.63 & -2.60 & -2.54 & -1.96 & -1.38 & -2.53 & -1.99 & -1.21 & -0.85 & -1.79 & -1.76 & -1.70 \\
\hline 10 & 2. 61 & 2. 59 & 2.53 & 2. 05 & 1.36 & 2. 34 & 1.94 & 1.13 & 1.32 & 1.82 & 1.74 & 1.70 \\
\hline 15 & 2. 60 & 2. 58 & 2.53 & 2. 08 & 1.84 & 2. 12 & .60 & 1. 44 & 1.50 & 1.84 & 1.76 & 1. 66 \\
\hline 20 & 2. 62 & 2.56 & .07 & 2.10 & 2.15 & 1.99 & -1.27 & 1.52 & 1.52 & 1.83 & 1.74 & \\
\hline 25 & 2. 62 & 2.56 & 1.36 & 2. 18 & 2. 34 & 1.96 & +1.20 & -1.60 & 1.60 & 1.80 & 1.74 & 1.61 \\
\hline Eom & 2. 61 & 2.54 & 1.78 & .40 & 2.45 & 1.98 & -.66 & +1.22 & 1.72 & 1.79 & 1.71 & 1.59 \\
\hline \multicolumn{13}{|c|}{$19 \overline{9} 8$} \\
\hline 5 & -1.35 & -1.20 & +0.06 & -0.89 & -0.96 & -0.57 & -0.93 & -0.78 & & -0.75 & -0.97 & -1.41 \\
\hline 10 & 1.42 & 1.17 & - & .91 & .98 & .79 & .98 & +.28 & -1.23 & 1.09 & 1.17 & 1. 41 \\
\hline 15 & 1.44 & 1.15 & & .92 & 1.00 & .91 & .99 & -.43 & +.99 & 1.25 & 1.30 & 1.39 \\
\hline 20 & 1.37 & 1.12 & -.52 & .62 & 1.00 & .95 & 1.00 & .79 & -.56 & 1.35 & 1.39 & 1.39 \\
\hline 25 & 1.29 & 1.09 & .71 & .78 & 1.01 & .96 & 1.01 & 1.00 & .99 & 1.41 & 1.41 & 1.40 \\
\hline Eom & 1.25 & -1.08 & .84 & .92 & 24 & 83 & .40 & 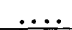 & .17 & .49 & 1.41 & ... \\
\hline \multicolumn{13}{|c|}{1959} \\
\hline 5 & $\mathrm{e}-1.42$ & -1.35 & -1.33 & -1.33 & -1.48 & -1.69 & -1.64 & -1.53 & -1.53 & -1.84 & -2.05 & -2.17 \\
\hline 10 & 1. 43 & 1.34 & 1.33 & 1.35 & 1.51 & 1.68 & 1.59 & 1.53 & 1.66 & 1.83 & 2.10 & 2. 17 \\
\hline 15 & 1.42 & 1.33 & 1.33 & 1. 39 & 1.54 & 1.68 & 1.57 & 1.32 & 1.75 & 1.88 & 2.11 & 2.15 \\
\hline 20 & 1.42 & 1. 32 & 1.34 & 1. 41 & 1.59 & 1.67 & 1.55 & 1.43 & 1.79 & 1.94 & 2.14 & 2.13 \\
\hline 25 & 1.40 & 1.33 & 1.34 & 1.43 & 1.64 & 1.66 & 1.53 & 1.08 & 1.81 & 1.97 & 2.14 & 2.13 \\
\hline Eom & 1.37 & 1.33 & 1.33 & 1. 44 & 1.68 & 1.68 & 1.51 & 1.37 & 1.83 & 2. 02 & 2.16 & 2.13 \\
\hline \multicolumn{13}{|c|}{1960} \\
\hline 5 & -2.12 & -1.79 & -1.69 & -1.45 & -1.43 & -1.54 & +1.37 & -1.26 & -1.67 & $\ldots$ & & 0.00 \\
\hline 10 & 2. 04 & 1.76 & 1.60 & 1.43 & 1.45 & +1.07 & +1.30 & 1.45 & … & $\cdots$ & -1.86 & +1.30 \\
\hline 15 & 1.96 & 1.76 & 1.55 & 1.42 & 1.45 & -.08 & .... & 1.59 & ..... & .. & 2.01 & 1. 27 \\
\hline 20 & 1.91 & 1.76 & 1.53 & e1. 42 & 1.48 & .59 & $\cdots$ & 1.68 & $\ldots$ & .. & 2. 14 & +.20 \\
\hline 25 & 1.86 & 1.75 & 1.48 & 1.43 & 1.51 & 1.03 & $\cdots$ & 1.74 & & & 2.21 & -.43 \\
\hline Eom & 1.81 & 1.74 & 1.46 & 1.43 & 1.52 & -1.28 & & 1.65 & & & -2.25 & 1.04 \\
\hline
\end{tabular}

\section{Rincon Valley}

16.5.25.211. U. S. Government. Drilled unused water-table well in valley fill, diam $10 \mathrm{in}$, depth $32 \mathrm{ft}$. MP top of casing, $3.00 \mathrm{ft}$ above $1 \mathrm{sd}$. Highest water level 11.30 below lsd, Apr. 17, 1947; lowest 27.78 below Isd, Jan. 6, 1958. Records available: 1946-48, 1957-60.

\begin{tabular}{|c|c|c|c|c|c|c|c|}
\hline Date & $\begin{array}{l}\text { Water } \\
\text { leveI }\end{array}$ & Date & $\begin{array}{l}\text { Water } \\
\text { level }\end{array}$ & Date & $\begin{array}{c}\text { Water } \\
\text { Ievel }\end{array}$ & Date & $\begin{array}{c}\text { Water } \\
\text { level }\end{array}$ \\
\hline $\begin{array}{lll}\text { Apr. } & 22, & 1957 \\
\text { July } & 26 \\
\end{array}$ & \begin{tabular}{|l|}
27.16 \\
26.62 \\
\end{tabular} & \begin{tabular}{|l} 
Jan. \\
July 21 \\
\end{tabular} & $\begin{array}{l}27.78 \\
26.42 \\
\end{array}$ & $\begin{array}{lrl}\text { Jan. } & 9, & 1959 \\
\text { July } & 27\end{array}$ & $\begin{array}{l}24.27 \\
19.11 \\
\end{array}$ & $\begin{array}{ll}\text { Jan. } & 4,1960 \\
\text { July } 20\end{array}$ & $\begin{array}{l}21.36 \\
21.75 \\
\end{array}$ \\
\hline
\end{tabular}

\section{Taos County}

\section{Sunshine Valley}

1S. 73. 19. 422. Spring Bros. Drilled unused water-table well in Santa Fe Group of middle (?) Miocene to Pleistocene (?) age, diam 16 in, depth $446 \mathrm{ft}$, cased to 446 . MP top of casing, $1.18 \mathrm{ft}$ above Isd. Highest water level 193.95 below Isd, June 5, 1957; lowest 207. 47 below lsd, Aug. 13, 1958. Records available: $1955-60$.

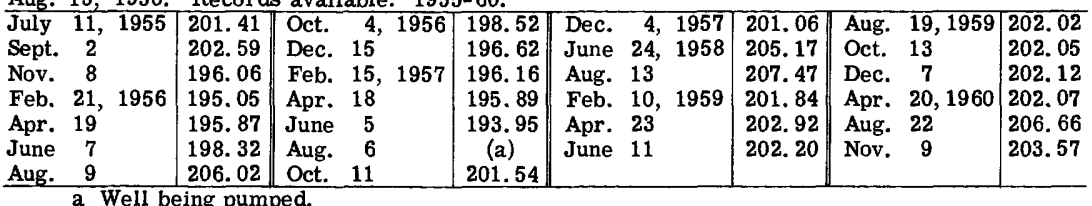

1S. 74. 26. 442. Top $O^{\prime}$ The World Farms, Inc. Drilled test hole water-table well in Santa Fe Group of middle (?) Miocene to Pleistocene (?) age, diam 2 in, depth $259 \mathrm{ft}$, cased to 259. Lsd 7, 587. $6 \mathrm{ft}$ above msl. MP top of casing, $1.20 \mathrm{ft}$ above Isd. Highest water level 228.80 below Isd, May 6, 1958; lowest 236.41 below Isd, Nov. 9, 1960. Records available: 1955-60. \begin{tabular}{l|l||l|l||ll||l|l|l|}
\hline July 10,1955 & 232.9 & Dec. 14, 1956 & 233.54 & May & 6,1958 & 228. 80 & Aug. 19, 1959 235. 44
\end{tabular} \begin{tabular}{l|l|lll|l|ll||l|l|l|l|} 
Aug. 22 & 233.05 & Feb. 15, 1957 & 233.80 & June 24 & 234. 65 & Oct. 13 & 235. 63 \\
\hline
\end{tabular} Nov. 8233.08 Apr. 18 233.75 Aug. 12 235.00

\begin{tabular}{l|l|l|l|l||l||l|l|l|l|} 
Feb. 21, 1956 & 232.78 & June & 5 & 233.93 & Oct. 14 & 235.30 & Feb. 2, 1960 & 235.47
\end{tabular}

\begin{tabular}{l|ll|l|l|l|l|} 
Apr. 19 & 232.65 & Aug. 6 & 234.10 & Dec. 4
\end{tabular}

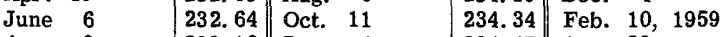

\begin{tabular}{ll|l|l|l|l|l|} 
Aug. 8 & 233.18 & Dec. 4 & 234.47 & Apr. 23 \\
\hline
\end{tabular}

\begin{tabular}{l|l|lll|l|l} 
Oct. 3 & 233.60 & Feb. 18,1958 & 234.58 & June 11 \\
\hline
\end{tabular} 235. 02 Apr. 20 \begin{tabular}{l|l|l}
235.25 & Aug. 22 &
\end{tabular} 235. 33 Nov. 9 236. 41 
30.12. 23.122. U. S. Army Corps of Engineers, Chifle damsite. Drilled test hole watertable well in Santa Fe Group of middle (?) Miocene to Pleistocene (?) age, diam 2 in, depth $266 \mathrm{ft}$, cased to 266. Lsd 7,511.7 ft above msl. MP top of inner casing, $1.70 \mathrm{ft}$ above lsd. Highest water level 167.12 below lsd, July 27, 1955; lowest 178.70 below 1sd, Nov. 9, 1960 . Records available: $1955-60$.

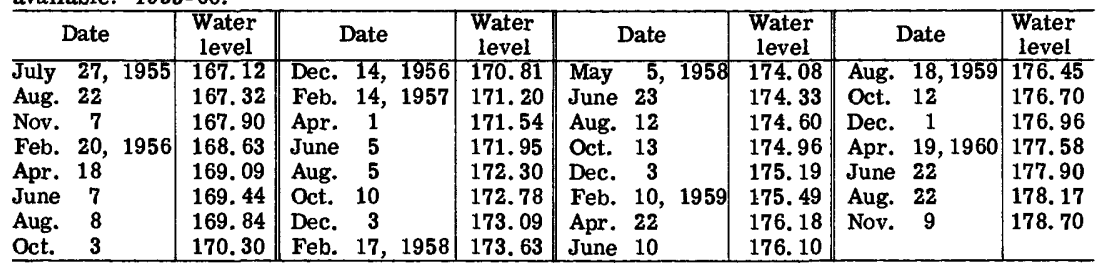

\section{Torrance County}

\section{Estancia Valley}

4. 8. 11. 433. F. D. Breedlove. Formerly R. B. Slease. Drilled unused water-table well in valley fill, diam $16 \mathrm{in}$, reported depth $180 \mathrm{ft}$, cased to 160 . MP top of casing at high point on northwest side of well, $0.70 \mathrm{ft}$ above 1sd. Highest water level 82.93 below 1sd, May 2, 1951; lowest 86.49 below 1sd, Sept. 26, 1960. Records available: 1950-60.

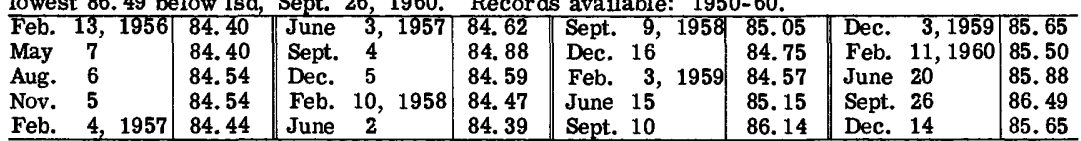

5. 8. 8. 424. A. T. Austin. Drilled irrigation water-table well in valley fill, diam $20 \mathrm{in}$, reported depth $204 \mathrm{ft}$, cased to 98 . MP top of casing, $0.80 \mathrm{ft}$ above lsd. Highest water level 62. 03 below 1sd, Mar. 23, 1948; lowest 89.95 below 1sd, Sept. 26, 1960. Records available: 1948-60.

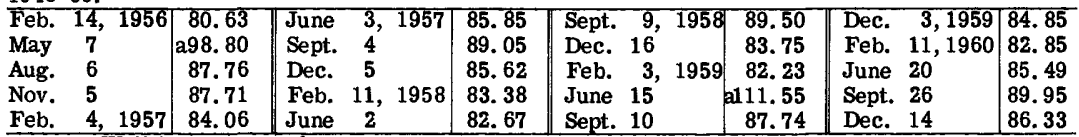

\begin{tabular}{l} 
Feb. 4,1957 \\
\hline a Well being pumped.
\end{tabular}

6. 8. 32. 212. Revis Strong. Formerly O. R. Ethridge. Drilled irrigation water-table well in valley fill, diam $18 \mathrm{in}$, reported depth $209 \mathrm{ft}$, cased to 84 . MP top of $1 \frac{1}{2}$-in hole in pumpbase, $0.04 \mathrm{ft}$ above lsd. Highest water level 23.22 below Isd, Feb. 18, 1947; lowest 42.24 below lsd, Sept. 26, 1960. Records available: 1947-60.

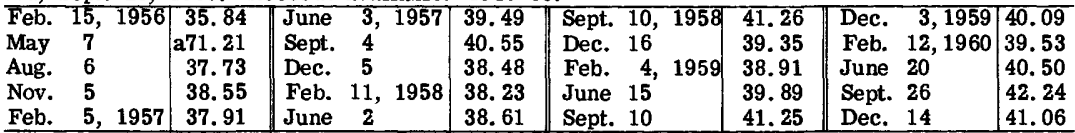

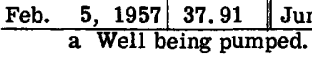

6.9.11.211. R. O. Brown. Formerly H. E. Means. Drilled irrigation water-table well in valley fill, diam $18 \mathrm{in}$, reported depth $148 \mathrm{ft}$, cased to 140 . MP top of casing, $0.75 \mathrm{ft}$ above 1sd. Highest water level 5.07 below isd, May 4, 1949; lowest 13.55 below 1sd, May 7, 1956. Records available: $1949-60$.

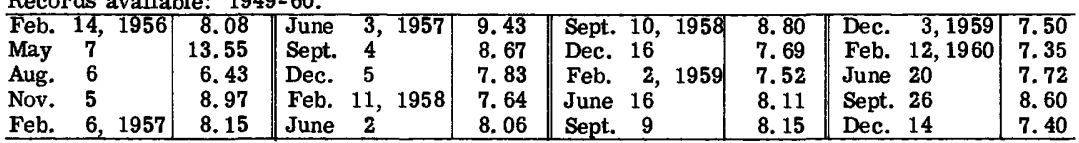

7. 8. 27.221. F. C. Pace. Drilled unused water-table well in valley flll, diam 6 in, depth $61 \mathrm{ft}$, cased to 15 . Lsd 6,185 ft above msl. MP top of recorder shelter shelf, $3.70 \mathrm{ft}$ above lsd. Highest water level 19.06 below 1sd, May 7-10, 1947; lowest 43. 42 below lsd, Oct. 11-13, 1960. Records available: 1941-60. For additional measurements, see Reeder and others $(1960 \mathrm{~b}$ and $\mathrm{c})$. 
7. 8. 27. 221--Continued.

Highest water level for the day, from recorder graph, 1956

\begin{tabular}{|c|c|c|c|c|c|c|c|c|c|c|c|c|}
\hline Day & Jan. & Feb. & Mar. & Apr. & May & June & July & Aug. & Sept. & Oct. & Nov. & Dec. \\
\hline 5 & 32.63 & 31.93 & 31.48 & 31.90 & 33.81 & 36.38 & & 39.61 & & & 39.91 & 38.04 \\
\hline 10 & 32.58 & 31.82 & 31.43 & 32.43 & 34.34 & 36.73 & & 39.87 & & 40.60 & 39.49 & 37.78 \\
\hline 15 & 32. 42 & 31.81 & 31.44 & 32.79 & 34.93 & ..... & $\ldots \ldots$ & 40.12 & .... & 40.45 & 39.18 & 37.64 \\
\hline 20 & 32. 30 & 31.80 & 31.30 & 33.11 & 35.37 & $\ldots \ldots$ & . & 40.34 & $\ldots \ldots$ & 40.29 & 38.89 & 37.43 \\
\hline 25 & 32.21 & 31.67 & 31.23 & 33.24 & 35.70 & .... & $\ldots$ & 40.54 & ..... & 15 & 38.60 & 37.36 \\
\hline Eom & 32.03 & 31.58 & 31.41 & 33.50 & 36.04 & & 39.32 & & & 40.00 & 38.31 & 37.08 \\
\hline
\end{tabular}

\begin{tabular}{l|l|l|l|l|l|l|l|l|l|l|l|l}
\hline 5 & 36.95 & 36.22 & $\mathbf{3 5 . 5 1}$ & $\mathbf{3 4 . 9 7}$ & 35.04 & $\mathbf{3 7 . 0 9}$ & 37.94 & 39.71 & 39.29 & $\mathbf{3 8 . 9 1}$ & 37.49 & 36.43 \\
10 & $\mathbf{3 6 . 8 3}$ & 36.10 & $\mathbf{3 5 . 3 9}$ & $\mathbf{3 4 . 8 3}$ & $\mathbf{3 5 . 4 6}$ & $\mathbf{3 7 . 4 4}$ & $\mathbf{3 8 . 1 6}$ & $\mathbf{3 9 . 6 9}$ & 39.07 & $\mathbf{3 8 . 6 7}$ & 37.33 & $\mathbf{3 6 . 3 9}$ \\
15 & $\mathbf{3 6 . 6 4}$ & $\mathbf{3 5 . 9 7}$ & 35.35 & $\mathbf{3 4 . 7 8}$ & $\mathbf{3 5 . 8 9}$ & $\mathbf{3 7 . 7 8}$ & $\mathbf{3 8 . 5 3}$ & $\mathbf{3 9 . 5 9}$ & $\mathbf{3 9 . 2 7}$ & $\mathbf{3 8 . 4 1}$ & $\mathbf{3 7 . 0 9}$ & $\mathbf{3 6 . 2 5}$ \\
$\mathbf{2 0}$ & $\mathbf{3 6 . 4 6}$ & $\mathbf{3 5 . 8 4}$ & $\mathbf{3 5 . 1 9}$ & $\mathbf{3 4 . 8 3}$ & $\mathbf{3 6 . 2 7}$ & $\mathbf{3 8 . 0 1}$ & $\mathbf{3 8 . 9 8}$ & $\mathbf{3 9 . 5 9}$ & $\mathbf{3 9 . 4 2}$ & $\mathbf{3 8 . 1 4}$ & $\mathbf{3 6 . 9 9}$ & $\mathbf{3 6 . 1 4}$ \\
$\mathbf{2 5}$ & $\mathbf{3 6 . 3 8}$ & $\mathbf{3 5 . 7 0}$ & $\mathbf{3 5 . 1 5}$ & $\mathbf{3 4 . 9 2}$ & $\mathbf{3 6 . 7 0}$ & $\mathbf{3 7 . 9 5}$ & $\mathbf{3 9 . 2 1}$ & $\mathbf{3 9 . 5 5}$ & 39.41 & $\mathbf{3 8 . 0 0}$ & $\mathbf{3 6 . 7 9}$ & 36.04 \\
Eom & $\mathbf{3 6 . 2 5}$ & $\mathbf{3 5 . 6 5}$ & $\mathbf{3 5 . 0 2}$ & $\mathbf{3 4 . 7 9}$ & $\mathbf{3 6 . 9 6}$ & $\mathbf{3 8 . 0 4}$ & $\mathbf{3 9 . 4 2}$ & $\mathbf{3 9 . 4 5}$ & $\mathbf{3 9 . 1 9}$ & $\mathbf{3 7 . 6 7}$ & $\mathbf{3 6 . 6 7}$ & $\mathbf{3 5 . 9 4}$ \\
\hline
\end{tabular}

\begin{tabular}{l|l|l|l|l|l|l|l|l|l|l|l|l}
\hline 5 & 35.83 & $\mathbf{3 5 . 2 3}$ & $\mathbf{3 4 . 8 7}$ & $\mathbf{3 4 . 5 3}$ & $\mathbf{3 4 . 2 5}$ & $\mathbf{3 5 . 3 3}$ & $\mathbf{3 7 . 8 9}$ & $\mathbf{4 0 . 3 6}$ & $\mathbf{4 1 . 6 5}$ & 39.91 & $\ldots \ldots$ & $\ldots \ldots$ \\
10 & $\mathbf{3 5 . 6 2}$ & $\mathbf{3 5 . 1 7}$ & $\mathbf{3 4 . 8 7}$ & $\mathbf{3 4 . 4 9}$ & $\mathbf{3 4 . 2 0}$ & $\mathbf{3 5 . 9 4}$ & $\mathbf{3 8 . 1 9}$ & $\mathbf{4 0 . 7 1}$ & $\mathbf{4 1 . 7 3}$ & $\mathbf{3 9 . 6 3}$ & $\ldots \ldots$ & $\ldots \ldots$ \\
15 & $\mathbf{3 5 . 6 4}$ & $\mathbf{3 5 . 1 9}$ & $\mathbf{3 4 . 7 5}$ & $\mathbf{3 4 . 4 4}$ & $\mathbf{3 4 . 1 3}$ & $\mathbf{3 6 . 5 7}$ & $\mathbf{3 8 . 6 0}$ & $\mathbf{4 1 . 0 2}$ & $\mathbf{4 1 . 4 3}$ & $\mathbf{3 9 . 3 6}$ & $\ldots \ldots$ & $\ldots \ldots$ \\
20 & $\mathbf{3 5 . 4 7}$ & $\mathbf{3 5 . 0 7}$ & $\mathbf{3 4 . 6 9}$ & $\mathbf{3 4 . 3 4}$ & $\mathbf{3 4 . 0 8}$ & $\mathbf{3 7 . 0 8}$ & $\mathbf{3 9 . 0 0}$ & $\mathbf{4 1 . 3 2}$ & $\mathbf{4 0 . 9 9}$ & $\mathbf{3 9 . 0 9}$ & $\ldots \ldots$ & $\mathbf{3 7 . 1 9}$ \\
$\mathbf{2 5}$ & 35.41 & $\mathbf{3 4 . 9 9}$ & $\mathbf{3 4 . 6 1}$ & $\mathbf{3 4 . 2 9}$ & $\mathbf{3 4 . 1 9}$ & $\mathbf{3 7 . 3 2}$ & $\mathbf{3 9 . 4 7}$ & $\mathbf{4 1 . 4 0}$ & $\mathbf{4 0 . 5 9}$ & $\mathbf{3 8 . 9 3}$ & $\ldots \ldots$ & $\mathbf{3 7 . 0 3}$ \\
Eom & $\mathbf{3 5 . 4 0}$ & $\mathbf{3 4 . 9 7}$ & $\mathbf{3 4 . 5 2}$ & $\mathbf{3 4 . 2 1}$ & $\mathbf{3 4 . 6 7}$ & $\mathbf{3 7 . 6 2}$ & $\mathbf{3 9 . 9 4}$ & $\mathbf{4 1 . 3 5}$ & $\mathbf{4 0 . 2 7}$ & $\ldots \ldots$ & $\ldots \ldots$ & $\mathbf{3 7 . 0 0}$ \\
\hline
\end{tabular}
\begin{tabular}{l|l|l|l|l|l|l|l|} 
Eom & 35.40 & $\mathbf{3 4 . 9 7}$ & $\mathbf{3 4 . 5 2}$ & $\mathbf{3 4 . 2 1}$ & $\mathbf{3 4 . 6 7}$ & $\mathbf{3 7 . 6}$ \\
\hline
\end{tabular}

\begin{tabular}{|c|c|c|c|c|c|c|c|c|c|c|c|c|}
\hline & & & & & \\
\hline 5 & & & & & & & 37.97 & 40.51 & & 41.07 & 39.51 & 38.49 \\
\hline 10 & & & & & & & 38.29 & $\cdots$ & h 41.12 & 40.75 & 39.27 & 38.32 \\
\hline 15 & .. & & & ..... & & h37. 23 & 38. 61 & $\ldots$ & 41.29 & 40.43 & 39.09 & 38. 21 \\
\hline 20 & ..... & . & .... & $\ldots$ & & 37.80 & 39.02 & $\cdots$ & 41.52 & 40.14 & ... & 38.12 \\
\hline 25 & $\cdots$ & $\cdots$ & $\cdots \cdots$ & $\cdots$ & - & 37.93 & 39.51 & $\cdots \cdots$ & 41.66 & 39.89 & $\cdots$ & 38.02 \\
\hline Eom & & & & & & 37.93 & 40.16 & & 41.36 & 39.69 & & 37.75 \\
\hline \\
\hline 5 & 37.75 & 37.29 & $\overline{36.85}$ & 36.52 & 37.45 & 40.45 & 40.82 & 40.91 & 42.52 & 43.31 & 41.92 & 40.57 \\
\hline 10 & 37.70 & 37.18 & 36.74 & 36.41 & 37.98 & 40.81 & 41.16 & 41.33 & 42.70 & 43.39 & 41.61 & 40.42 \\
\hline 15 & 37.57 & 37.12 & 36.65 & 36.36 & 38.35 & 40.65 & 41.01 & 41.61 & ..... & 43.35 & 41.39 & 40.25 \\
\hline 20 & 37.57 & 36.89 & 36.67 & 36.44 & 38.87 & 40.39 & 40.73 & 41.87 & & 43.02 & 41.19 & 40.09 \\
\hline 25 & 37.41 & 37.06 & 36.60 & 36.55 & 39.35 & 40.10 & 40.44 & 42.11 & $\ldots \ldots$ & 42.65 & 40.97 & 39.89 \\
\hline Eom & 37.30 & 36.92 & 36.47 & 37.08 & 39.96 & 40.16 & 40.49 & 42.33 & 43. 18 & 42.23 & 40.78 & 39.71 \\
\hline
\end{tabular}

8. 8. 35. 322. A. C. Hibner. Drilled irrigation water-table well in valley fill (?), diam $16 \mathrm{in}$, reported depth $228 \mathrm{ft}$, cased to 110 . Lsd $6,240 \mathrm{ft}$ above msl. MP top of casing, $0.75 \mathrm{ft}$ above Isd. Highest water level 50.12 below lsd, May 28, 1947; lowest 85.35 below lsd, Sept. 26, 1960. Records available: $1947-60$.

\begin{tabular}{|c|c|c|c|c|c|c|c|}
\hline Date & $\begin{array}{l}\text { Water } \\
\text { level }\end{array}$ & Date & $\begin{array}{c}\text { Water } \\
\text { level }\end{array}$ & Date & $\begin{array}{l}\text { Water } \\
\text { level }\end{array}$ & Date & $\begin{array}{l}\text { Water } \\
\text { level }\end{array}$ \\
\hline $\begin{array}{lcc}\text { Feb. } & 17, & 1956 \\
\text { May } & 8 & \\
\text { Aug. } & 7 \\
\text { Nov. } & 6 & \\
\text { Feb. } & 6, & 1957\end{array}$ & $\begin{array}{l}77.22 \\
79.28 \\
81.80 \\
81.50 \\
79.60\end{array}$ & $\begin{array}{lrl}\text { June } & 4, & 1957 \\
\text { Sept. } & 9 & \\
\text { Dec. } & 6 & \\
\text { Feb. } & 12, & 1958 \\
\text { June } & 3 & \end{array}$ & $\begin{array}{l}81.23 \\
82.27 \\
80.48 \\
79.51 \\
80.30\end{array}$ & $\begin{array}{lrl}\text { Sept. } & 10,1958 \\
\text { Dec. } & 17 & \\
\text { Feb. } & 5,1959 \\
\text { June } & 16 & \\
\text { Sept. } & 9 & \end{array}$ & $\begin{array}{l}83.85 \\
81.49 \\
80.64 \\
82.10 \\
83.48\end{array}$ & $\begin{array}{lr}\text { Dec. } & 4,1959 \\
\text { Feb. } & 15,1960 \\
\text { June } & 21 \\
\text { Sept. } & 26 \\
\text { Dec. } & 15\end{array}$ & $\begin{array}{l}82.00 \\
80.78 \\
81.99 \\
85.35 \\
81.60\end{array}$ \\
\hline
\end{tabular}

\section{Union County}

Capulin Basin

29. 28.18.341. City of Raton. $300 \mathrm{ft}$ north of U. S. Highway 67-87 at Capulin. Drilled irrigation water-table well in cinders, diam 16 in, depth $78 \mathrm{ft}$, cased to 78 . Lsd 6, 821. $2 \mathrm{ft}$ above msl. MP edge of 2-in hole in south side of casing, $1.20 \mathrm{ft}$ above lsd. Highest water level 30.80 below 1sd, Feb. 3, 1960; lowest 32.30 below lsd, Aug. 13, 1957. Records available: $1951,1957-60$.

\begin{tabular}{l|l||l|l||l|l||l|l|}
\hline July 11, 1951 & 30.94 & Feb. 19, 1958 & 32.04 & Feb. 12, 1959 & 31.71 & Feb. 3, 1960 30.80
\end{tabular}

\begin{tabular}{l|l|l|l|l|l|l|l|} 
Feb. 18, 1957 & 32.13 & Aug. 14 & 31.87 & Aug. 20 & 32.00 & Aug. 24 & 32.03 \\
\hline Aug. 13 & 32.30 & & & & &
\end{tabular}




\section{Valencia County}

\section{Grants-Bluewater Area}

10.10.26.331. Monico Mirabal. Drilled irrigation water-table well in Glorieta Sandstone of Permian age, diam $16 \mathrm{in}$, depth $216 \mathrm{ft}$, cased to 216 . Lsd 6, $455 \mathrm{ft}$ above msl. MP top of $\frac{1}{2}$-in hole in pumpbase, $1.00 \mathrm{ft}$ above lsd. Highest water level 22.18 below lsd, Feb. 21, 1952; lowest 28. 45 below lsd, Nov. 15, 1960. Records available: 1952-60.

\begin{tabular}{|c|c|c|c|c|c|c|c|}
\hline Date & $\begin{array}{l}\text { Water } \\
\text { level }\end{array}$ & Date & $\begin{array}{c}\text { Water } \\
\text { level }\end{array}$ & Date & $\begin{array}{c}\text { Water } \\
\text { level }\end{array}$ & Date & $\begin{array}{c}\text { Water } \\
\text { level }\end{array}$ \\
\hline Jan. 21,1956 & 24.89 & Dec. 11,1956 & 26.44 & Feb. $\quad 5,1958$ & 25.80 & Nov. $\quad 4,1959$ & 28.35 \\
\hline Apr. 17 & a47.79 & Feb. 12,1957 & 25.90 & Aug. 19 & 27.43 & Feb. $\quad 8,1960$ & 27.40 \\
\hline June 5 & 247.00 & May & 27.18 & Nov. 19 & 27.38 & May 11 & 249.52 \\
\hline Aug. & 27.28 & Aug. & 27.17 & Feb. 17,1959 & 26.71 & Aug. 26 & 249.59 \\
\hline Oct. & a44.59 & Nov. 13 & 26.08 & May & 27.85 & Nov. 15 & 28.45 \\
\hline
\end{tabular}

a Well being pumped.

11. 10. 27. 242. City of Grants. Formerly Ice Plant. Drilled industrial water-table well in San Andres Limestone of Permian age, diam 16 to $12 \mathrm{in}$, depth $158 \mathrm{ft}$, perforated 50-158. Lsd 6, $480 \mathrm{ft}$ above msl. MP top of 1 -in hole in pumpbase, $1.35 \mathrm{ft}$ above lsd. Highest water level 19.86 below lsd, Feb. 20, 1953; lowest 36.30 below 1sd, Aug. 26, 1960. Records available: $1953-60$.

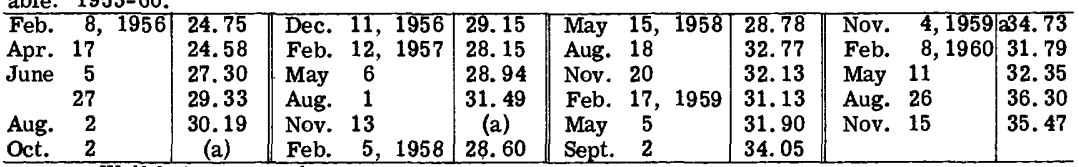

a Well being pumped.

12. 10. 29. 434. A. R. Card. Drilled unused artesian well in San Andres Limestone of Permian age (previously reported San Andres Formation), diam $18 \mathrm{in,} \mathrm{reported} \mathrm{depth} 205 \mathrm{ft}$, cased 0-150, perforated 93-130. MP lower edge of hole in north side of casing, 2. $20 \mathrm{ft}$ above lsd (since Aug. 1, 1956). Highest water level 65. 46 below lsd, Oct. 14, 1944; lowest 100.74 below lsd, Nov. 3, 1959. Records available: $1944,1946-60$.

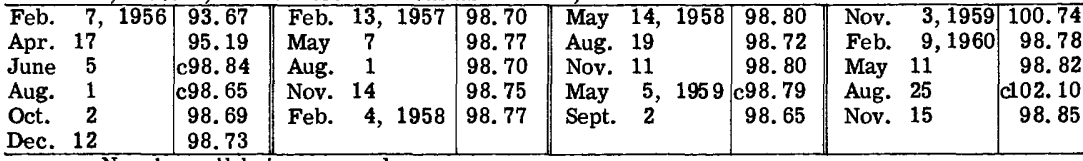

c Nearby well being pumped.

12.11.9. 221. Formerly 12.11.9.222. J. Church Co. Drilled unused water-table well in San Andres Limestone of Permian age (previously reported San Andres Formation), diam 18 in, depth $500 \mathrm{ft}$, cased to 500 . MP top of casing at low point, $2.22 \mathrm{ft}$ above lsd. Highest water level 115. 70 below lsd, Feb. 27, 1946; lowest 193.51 below lsd, June 30, 1957. Records available: 1946-60. For additional measurements, see Reeder and others (1960b and c).

Highest water level for the day, from recorder graph, 1956

\begin{tabular}{|c|c|c|c|c|c|c|c|c|c|c|c|c|}
\hline Day & Jan. & Feb. & Mar. & Apr. & May & June & July & & Sent & Oct. & Nov. & Dec. \\
\hline 5 & & & & & 171.66 & 177.90 & 183.93 & 56 & 00 & 186.18 & 181.00 & 176.9 \\
\hline 10 & & & & & 174.48 & 179.84 & & & & 185.56 & 180.15 & 176.66 \\
\hline 15 & & & $\ldots$ & & 174.32 & 180.02 & & & & 184.15 & .43 & 176.27 \\
\hline 20 & & & & 166.82 & 178.95 & 180.43 & 185.62 & & & 184.00 & 178.78 & 175.85 \\
\hline 25 & & & & 166.95 & 177.47 & 181.87 & 187.22 & & & 05 & 17 & 5.66 \\
\hline Eom & & & & 168.97 & 44 & 67 & 72 & & 186.68 & 68 & 1 & 01 \\
\hline & & & & & & & & & & & & \\
\hline 5 & 174.73 & 172.71 & 170.98 & 171.80 & & & 192.39 & 93 & 6.23 & & 43 & 3.44 \\
\hline 10 & 174.42 & 34 & 69 & 37 & & 47 & 192.52 & 51 & 94 & 180 & & 21 \\
\hline 15 & 173.92 & 98 & 170.52 & 44 & 18 & 18 & 190.76 & 35 & 186.07 & 179.23 & .29 & 83 \\
\hline 20 & 173.50 & 1.68 & 170.24 & 8.20 & 187.64 & .30 & 190.18 & 186.81 & 184.43 & 178.37 & 174.86 & 172.30 \\
\hline 25 & 173.23 & .42 & 170.17 & & 187.75 & 191.60 & 188.28 & 16 & .22 & .80 & .44 & .03 \\
\hline Eom & 172.97 & & & & 186.64 & 192.81 & 186.40 & & 11040 & 176.99 & & 171.49 \\
\hline & & & & & & & & & & & & \\
\hline 5 & 1 & 168.74 & 67.68 & 165.87 & & 33 & & & & & & 41 \\
\hline 10 & 170.44 & 168.59 & 167.49 & 165.81 & & 162.59 & 165.12 & 16 & 167.82 & 167.83 & & .09 \\
\hline 15 & 170.15 & 168.48 & 167.28 & 165.20 & 163.48 & 163.82 & 165.21 & 165.48 & 166.38 & 168.82 & & 163.88 \\
\hline 20 & 169.64 & 168.28 & 167.06 & & 163.42 & 164.12 & 165.41 & 165.79 & 167.67 & 167.68 & 164.39 & \\
\hline 25 & $\ldots$ & 167.91 & 166.74 & .. & 162.84 & 164.51 & 165.68 & 166.24 & 168.16 & 166.99 & 164.76 & \\
\hline om & & 167 & & & & & & & & & 164.83 & \\
\hline
\end{tabular}


12. 11.9. 221--Continued.

\begin{tabular}{|c|c|c|c|c|c|c|c|c|c|c|c|c|}
\hline & & & & & & 1959 & & & & & & \\
\hline$\overline{\text { Day }}$ & Jan. & Feb. & $\mathrm{Mar}$ & Apr & May & June & July & Aug & Sept. & Oct. & Nov. & Dec. \\
\hline 5 & & 161.29 & 158.93 & 162.91 & 166.51 & 170.21 & 169.45 & $\begin{array}{lll}5 & 167.67\end{array}$ & 167.27 & 167.93 & e167.90 & 165.94 \\
\hline 10 & .. & el 61.17 & 158.08 & 163.92 & 166.21 & 170,41 & 17 & 25 & 167.49 & 167.51 & 167.32 & 165.5 \\
\hline 15 & .. & $\cdots$ & 158. 11 & 164.25 & 167.04 & 169.07 & 717 & 3167.94 & 168.03 & 167.20 & 166.88 & 165.37 \\
\hline 20 & 161.68 & 8159.62 & 160.37 & 165.50 & 167.95 & 169.33 & 169.8 & 168.38 & 168.49 & 167.20 & 166.61 & 165.29 \\
\hline 25 & 161.7 & 6159.46 & 161.04 & 165.17 & 167.46 & 169.40 & 16 & 5 & 16 & 167.41 & 16 & 165.00 \\
\hline Eon & 161. & 41 & 161 & 1 & 16 & & & & 33 & 168.88 & 8 166.19 & 164.4 \\
\hline
\end{tabular}

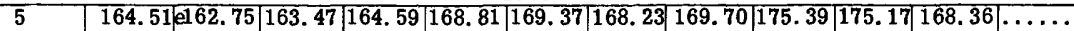
\begin{tabular}{l|l|l|l|l|l|l|l|l|l|l|l|l|l|l|l|l|l}
10 & 164.30 & 163.03 & 163.66 & 164.96 & 169.31 & 170.02 & 167.30 & 169.89 & 174.48 & 173.11 & 167.63 & $\ldots \ldots$
\end{tabular}

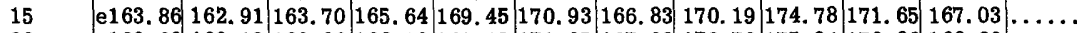

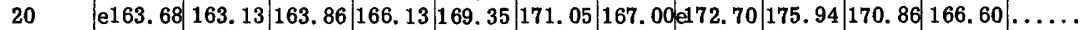

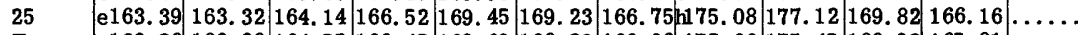

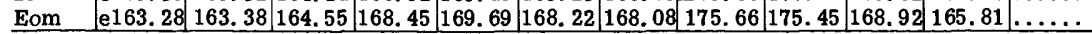

e Estimated.

h Tape measurement.

12. 11. 9. 424. George Rowley. Drilled unused artesian well in San Andres Limestone of Permian age and Yeso Formation of lower Permian age, diam 16 in, reported depth $505 \mathrm{ft}$. Lsd 6,642 ft above msl. MP top of casing, $3.05 \mathrm{ft}$ above lsd. Highest water level 93.75 below lsd, May 10, 1946; lowest 139. 05 below lsd, Aug. 1, 1957. Records available: 1946-60.

\begin{tabular}{|c|c|c|c|c|c|c|c|}
\hline Date & $\begin{array}{c}\text { Water } \\
\text { level }\end{array}$ & Date & $\begin{array}{l}\text { Water } \\
\text { level }\end{array}$ & Date & $\begin{array}{c}\text { Water } \\
\text { level }\end{array}$ & Date & $\begin{array}{c}\text { Water } \\
\text { level }\end{array}$ \\
\hline $\begin{array}{lrl}\text { Feb. } & 6,1956 \\
\text { Apr. } & 16 & \\
\text { June } & 4 \\
\text { Aug. } & 1 \\
\text { Oct. } & 1 \\
\text { Dec. } & 11 & \end{array}$ & $\begin{array}{l}129.40 \\
129.41 \\
131.88 \\
134.80 \\
136.45 \\
135.08\end{array}$ & $\begin{array}{lrl}\text { Feb. } & 12, & 1957 \\
\text { May } & 6 \\
\text { Aug. } & 1 \\
\text { Nov. } & 14 \\
\text { Feb. } & 4,1958 \\
\text { May } & 14 & \end{array}$ & $\begin{array}{l}134.50 \\
136.25 \\
139.05 \\
135.58 \\
133.92 \\
130.91\end{array}$ & $\begin{array}{lcc}\text { Aug. } & 18, & 1958 \\
\text { Nov. } & 11 & \\
\text { Feb. } & 16, & 1959 \\
\text { May } & 4 & \\
\text { Sept. } & 2 & \end{array}$ & $\begin{array}{l}129.69 \\
129.23 \\
127.57 \\
128.13 \\
127.65\end{array}$ & $\begin{array}{lr}\text { Nov. } & 3,1959 \\
\text { Feb. } & 8,1960 \\
\text { May } & 10 \\
\text { Aug. } & 25 \\
\text { Nov. } & 14\end{array}$ & $\begin{array}{l}129.23 \\
128.17 \\
127.99 \\
129.77 \\
131.93\end{array}$ \\
\hline
\end{tabular}

12. 11. 14. 213. Duane (previously reported Dyan) Berryhill. Drilled unused water-table well in alluvium of Quaternary age, diam $4 \mathrm{in}$, depth $130 \mathrm{ft}$, surface casing to 5 . Lsd 6,605. $4 \mathrm{ft}$ above msl. MP top of 4-in downspout, 2.95 ft above lsd (since Aug. 1, 1956). Highest water level 87.85 below lsd, May 4, 1959; lowest 101. 39 below lsd, June 10, 1954. Records available: 1949-60.

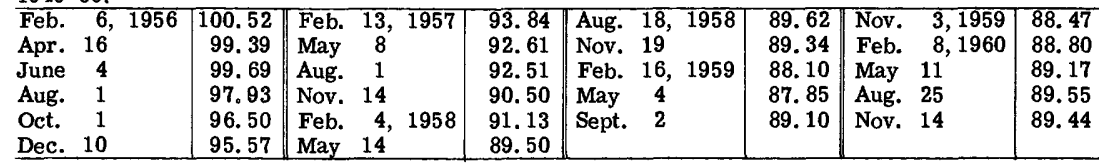

\section{References}

Bean, R. T., 1949, Geology of the Roswell artesian basin, New Mexico, and its relation to the Hondo Reservoir: New Mexico State Engineer Tech. Rept. 9, p. 1-31, 3 pls. , 2 figs. [1957].

Bjorklund, L. J., 1957, Reconnaissance of ground-water conditions in the Crow Flats area, Otero County, New Mexico: New Mexico State Engineer Tech. Rept. 8, 26 p., 1 pl., 4 figs. , 3 tables.

Bjorklund, L. J., and Motts, W. S., 1959, Geology and water resources of the Carlsbad area, Eddy County, New Mexico: New Mexico State Engineer open-file rept., 322 p. , 14 pls., 54 figs., 17 tables.

Conover, C. S., and Akin, P. D., 1942a, Progress report on the ground-water supply of Mimbres Valley, New Mexico, 1938-1941: New Mexico State Engineer 14th and 15th Bienn. Repts, , p. 235-282 [1953].

$1942 \mathrm{~b}$, Progress report on the ground-water supply of northern Lea County, New Mexico: New Mexico State Engineer 14th and 15th Bienn. Repts., p. 283-310 [1953]. 1942c, Progress report on the ground-water supply of Portales Valley, New Mexico, 1938-1941: New Mexico State Engineer 14th and 15th Bienn. Repts., p. 311-346 [1953]. 1943a, Progress report on the ground-water supply of the Mimbres Valley, New Mexico: U.S. Geol. Survey open-file rept., 46 p., 12 figs. $1943 b$, Progress report on the ground-water supply of northern Lea County, New Mexico: U.S. Geol. Survey open-file rept., 22 p. , 3 figs.

Conover, C. S., Reeder, H. O., and Willett, J. R., 1954, Summary of changes in water levels in observation wells, 1942-46, New Mexico: U.S. Geol. Survey open-file rept., 61 p.

Cooper, J. B., 1960, Ground water in the Causey-Lingo area, Roosevelt County, New Mexico: New Mexico State Engineer Tech. Rept. 14, 51 p., 2 pls., 13 figs., 4 tables. 
Doty, G. C., 1960, Reconnaissance of ground water in Playas Valley, Hidalgo County, New Mexico: New Mexico State Engineer Tech. Rept. 15, 40 p., 1 pl., 9 figs. , 7 tables.

Fiedler, A. G., 1926, Report on investigations of the Roswell artesian basin, Chaves and Eddy Counties, New Mexico: New Mexico State Engineer 7th Bienn. Rept., p. 21-60. 1928, Report on a reconnaissance of the ground-water area of the Mimbres Valley, Luna County, New Mexico: New Mexico State Engineer 8th Bienn. Rept., p. 159-171.

Fiedler, A. G. , and Nye, S. S., 1928, Ground-water investigations of the Roswell artesian basin, New Mexico: New Mexico State Engineer 8th Bienn. Rept., p. 81-107. 1932, Progress report of the Roswell artesian basin investigation: New Mexico State Engineer 10th Bienn. Rept., p. 162-182.

Galloway, Sherman E., 1956, Geology and ground-water resources of the Portales Valley area, Roosevelt and Curry Counties, New Mexico: New Mexico Univ., M. S. thesis, 127 p., app. A-D.

Gordon, E. D., 1960, Geology and ground-water resour ces of the Grants-Bluewater area, Valencia County, New Mexico, with sections on aquifer characteristics by H. O. Reeder and chemical quality of the ground water by J. L. Kunkler: New Mexico State Engineer openfile rept., 224 p., 17 figs., 12 tables.

Hale, W. E., 1945, Ground-water conditions in the vicinity of Carlsbad, New Mexico: U.S. Geol. Survey open-file rept., $77 \mathrm{p}$.

1955, Ground-water conditions in the vicinity of Rattlesnake Springs, Eddy County, New Mexico: New Mexico State Engineer Tech. Rept. 3, 54 p., 1 pl., 9 figs.

Hale, W. E. , and Theis, C. V., 1942, Memorandum on ground-water conditions in the vicinity of the city airport southwest of Carlsbad, New Mexico: New Mexico State Engineer 14th and 15th Bienn. Repts., p. 219-234. [1953]

Hendrickson, G. E., and Jones, R. S., 1952, Geology and ground-water resources of Eddy County, New Mexico: New Mexico Inst. Mineralogy and Technology State Bur. Mines and Mineral Resources Ground-Water Rept. 3, 169 p.

Herrick, E. H., 1951, Memorandum on the possibilities of irrigation in the Capulin area, Colfax and Union Counties, New Mexico: New Mexico State Engineer open-file rept. , 9 p. , 1 fig., 2 tables.

Howard, J. W. , Jr., 1954, Reconnaissance of ground-water conditions in Curry County, New Mexico: New Mexico State Engineer Tech. Rept. 1, 35 p., 1 fig., 3 tables [1.955].

Minton, E. G., Jr., 1941, Analysis of relationship between artesian pressure head and precipitation and consumptive use, Roswell artesian basin, New Mexico: New Mexico State Engineer 14th and 15th Bienn. Repts., p. 167-179 [1953].

1942, Report of artesian well supervisor, Roswell artesian basin, New Mexico: New Mexico State Engineer 14th and 15th Bienn. Repts., p. 367-379 [1953].

Morgan, A. M. , 1938, Geology and shallow-water resources of the Roswell artesian basin, New Mexico: New Mexico State Engineer 12th and 13th Bienn. Repts., p. 155-249.

Murray, C. R., 1959, Ground-water conditions in the nonthermal artesian-water basin south of Hot Springs, Sierra County, New Mexico: New Mexico State Engineer Tech. Rept. 10, 33 p. , 1 pl., 32 figs., 9 tables.

Nye, S. S., 1930, Shallow ground-water supplies in northern Lea County, New Mexico: New Mexico State Engineer 9th Bienn. Rept., p. 363-387.

1932, Progress report on the ground-water supply of northern Lea County, New Mexico: New Mexico State Engineer 10th Bienn. Rept., p. 229-251.

Reeder, H. O., 1957, Ground water in Animas Valley, Hidalgo County, New Mexico: New Mexico State Engineer Tech. Rept. 11, 101 p. , 4 pls., 39 figs., 6 tables [1958].

Reeder, H. O., and others, 1959, Annual water-level measurements in observation wells, 19511955, and atlas of maps showing changes in water levels for various periods from beginning of record through 1954, New Mexico: New Mexico State Engineer Tech. Rept. 13, 339 p., 161 figs., 26 tables.

1960a, Changes in water levels in 1955 and annual water-level measurements in January and February 1956 in observation wells in New Mexico: New Mexico State Engineer Tech. Rept. 16, 145 p., 31 figs., 16 tables.

1960b, Ground-water levels in New Mexico, 1956: New Mexico State Engineer Tech. Rept. 19, 251 p. , 19 figs., 52 tables.

1960c, Ground-water levels in New Mexico, 1957: New Mexico State Engineer open-file rept., 229 p., 19 figs. , 51 tables.

Smith, R. E., 1957, Geology and ground-water resources of Torrance County, New Mexico: New Mexico State Bur. of Mines and Mineral Resources, Ground-Water Rept. 5, 186 p., 5 pls., 7 figs., 16 tables.

Spiegel, Zane, and Baldwin, Brewster, 1958, Geology and water resources of the Santa Fe area, New Mexico, with contributions by F. E. Kottlowski and E. L. Barrows, and a section on geophysics by H. A. Winkler: New Mexico State Engineer open-file rept., 403 p., 13 pls., 50 figs., 23 tables.

Theis, C. V., 1932, Report on the ground water in Curry and Roosevelt Counties, New Mexico: New Mexico State Engineer 10th Bienn. Rept., p. 98-160. 1934a, Progress report on the ground-water supply of the Portales Valley, New Mexico: New Mexico State Engineer 11th Bienn. Rept., p. 87-108. $1934 \mathrm{~b}$, Progress report on the ground-water supply of Lea County, New Mexico: New Mexico State Engineer 11th Bienn. Rept., p. 127-153. 
Theis, C. V., 1938a, Progress report on the ground-water supply of the Portales Valley, New Mexico: New Mexico State Engineer 12th and 13th Bienn. Repts., p. 101-118. 1938b, Progress report on the ground-water supply of Lea County, New Mexico: New Mexico State Engineer 12th and 13th Bienn. Repts., p. 121-133. 1938c, Progress report on the ground-water supply of the Mimbres Valley, New Mexico: New Mexico State Engineer 12th and 13th Bienn. Repts., p. 135-153.

1938d, Origin of water in Major Johnson Springs, near Carlsbad, New Mexico: New Mexico State Engineer 12th and 13th Bienn. Repts., p. 251-252.

Theis, C. V., Taylor, C. G., Jr., and Murray, C. R., 1942, Thermal waters of the Hot Springs artesian basin, Sierra County, New Mexico: New Mexico State Engineer 14th and 15th Bienn. Repts., 1938-1942, p. 419-492 [1953].

White, W. N., 1930, Preliminary report on the ground-water supply of Mimbres Valley, New Mexico: New Mexico State Engineer 9th Bienn. Rept., p. 131-152. 1932, Progress report on the ground-water supply of the Mimbres Valley, New Mexico: New Mexico State Engineer 10th Bienn. Rept., p. 183-227.

1934, Progress report on the ground-water supply of the Mimbres Valley, New Mexico: New Mexico State Engineer 11th Bienn. Rept., p. 109-125.

Winograd, I. J., 1959, Ground-water conditions and geology of Sunshine Valley and western Taos County, New Mexico: New Mexico State Engineer Tech. Rept. 12, 70 p., 3 pls., 11 figs., 8 tables. 